\title{
DIGITALCOMMONS
}

\section{Vol. 6, No. 1 (Full Issue)}

JMASM Editors

Follow this and additional works at: http:// digitalcommons.wayne.edu/jmasm

\section{Recommended Citation}

Editors, JMASM (2007) "Vol. 6, No. 1 (Full Issue)," Journal of Modern Applied Statistical Methods: Vol. 6 : Iss. 1 , Article 36. DOI: $10.22237 /$ jmasm/1177994100

Available at: http://digitalcommons.wayne.edu/jmasm/vol6/iss1/36 


\title{
Journal Of Modern Applied Statistical Methods
}

\author{
Shlomo S. Sawilowsky
}

Editor

College of Education

Wayne State University

Harvey Keselman

Associate Editor

Department of Psychology

University of Manitoba

Bruno D. Zumbo

Associate Editor

Measurement, Evaluation, \& Research Methodology

University of British Columbia

Vance W. Berger

Assistant Editor

Biometry Research Group

National Cancer Institute

John L. Cuzzocrea

Assistant Editor

Educational Research

University of Akron

Todd C. Headrick Assistant Editor

Educational Psychology and Special Education

Southern Illinois University-Carbondale

Alan Klockars

Assistant Editor

Educational Psychology

University of Washington 


\section{Editorial Board}

Subhash Chandra Bagui

Department of Mathematics \& Statistics University of West Florida

J. Jackson Barnette

School of Public Health

University of Alabama at Birmingham

Vincent A. R. Camara

Department of Mathematics

University of South Florida

Ling Chen

Department of Statistics

Florida International University

Christopher W. Chiu

Test Development \& Psychometric Rsch

Law School Admission Council, PA

Jai Won Choi

National Center for Health Statistics

Hyattsville, MD

Rahul Dhanda

Forest Pharmaceuticals

New York, NY

John N. Dyer

Dept. of Information System \& Logistics Georgia Southern University

Matthew E. Elam

Dept. of Industrial Engineering

University of Alabama

Mohammed A. El-Saidi

Accounting, Finance, Economics \&

Statistics, Ferris State University

Felix Famoye

Department of Mathematics

Central Michigan University

Barbara Foster

Academic Computing Services, UT

Southwestern Medical Center, Dallas

Shiva Gautam

Department of Preventive Medicine

Vanderbilt University

Dominique Haughton

Mathematical Sciences Department

Bentley College

Scott L. Hershberger

Department of Psychology

California State University, Long Beach

Joseph Hilbe

Departments of Statistics/ Sociology

Arizona State University
Sin-Ho Jung

Dept. of Biostatistics \& Bioinformatics

Duke University

Jong-Min Kim

Statistics, Division of Science \& Math

University of Minnesota

Harry Khamis

Statistical Consulting Center

Wright State University

Kallappa M. Koti

Food and Drug Administration

Rockville, MD

Tomasz J. Kozubowski

Department of Mathematics

University of Nevada

Kwan R. Lee

GlaxoSmithKline Pharmaceuticals

Collegeville, PA

Hee-Jeong Lim

Dept. of Math \& Computer Science

Northern Kentucky University

Balgobin Nandram

Department of Mathematical Sciences

Worcester Polytechnic Institute

J. Sunil Rao

Dept. of Epidemiology \& Biostatistics

Case Western Reserve University

Karan P. Singh

University of North Texas Health

Science Center, Fort Worth

Jianguo (Tony) Sun

Department of Statistics

University of Missouri, Columbia

Joshua M. Tebbs

Department of Statistics

Kansas State University

Dimitrios D. Thomakos

Department of Economics

Florida International University

Justin Tobias

Department of Economics

University of California-Irvine

Dawn M. VanLeeuwen

Agricultural \& Extension Education

New Mexico State University

David Walker

Educational Tech, Rsrch, \& Assessment

Northern Illinois University
J. J. Wang

Dept. of Advanced Educational Studies

California State University, Bakersfield

Dongfeng $\mathrm{Wu}$

Dept. of Mathematics \& Statistics

Mississippi State University

Chengjie Xiong

Division of Biostatistics

Washington University in St. Louis

Andrei Yakovlev

Biostatistics and Computational Biology

University of Rochester

Heping Zhang

Dept. of Epidemiology \& Public Health

Yale University

\section{INTERNATIONAL}

Mohammed Ageel

Dept. of Mathematics, \& Graduate School King Khalid University, Saudi Arabia

Mohammad Fraiwan Al-Saleh

Department of Statistics

Yarmouk University, Irbid-Jordan

Keumhee Chough (K.C.) Carriere

Mathematical \& Statistical Sciences

University of Alberta, Canada

Michael B. C. Khoo

Mathematical Sciences

Universiti Sains, Malaysia

Debasis Kundu

Department of Mathematics

Indian Institute of Technology, India

Christos Koukouvinos

Department of Mathematics

National Technical University, Greece

Lisa M. Lix

Dept. of Community Health Sciences

University of Manitoba, Canada

Takis Papaioannou

Statistics and Insurance Science

University of Piraeus, Greece

Nasrollah Saebi

Computing, Information Systems \& Math

Kingston University, UK

Keming $\mathrm{Yu}$

Department of Statistics

University of Plymouth, UK 


\section{Journal Of Modern Applied Statistical Methods}

Invited Articles

$\begin{array}{lll}2-7 & \begin{array}{l}\text { Meng-Jia Wu, } \\ \text { Betsy Jane Becker, } \\ \text { Yael Netz }\end{array} & \begin{array}{l}\text { Effects of Physical Activity on Psychological Change } \\ \text { in Advanced Age: A Multivariate Meta-Analysis }\end{array} \\ 8-20 & \text { Thomas R. Knapp } & \text { Bimodality Revisited } \\ 21-29 & \begin{array}{l}\text { Bruno D. Zumbo, } \\ \text { Anne Gadermann, } \\ \text { Cornelia Zeisser }\end{array} & \text { Ordinal Versions of Coefficients Alpha and Theta for } \\ & \end{array}$

$30-35 \quad$ Rand R. Wilcox On Flexible Tests of Independence and Homoscedasticity

Regular Articles

$36-52$

Sean W. Mulvenon,

M. Austin Betz,

Application of a New Procedure for Power Analysis

Kening Wang,

and Comparison of the Adjusted Univariate and

Bruno Zumbo

Multivariate Tests in Repeated Measures Designs

$53-65$

Stephanie Wehry, James Algina

Analyses of Unbalanced Groups-Versus-Individual Research Designs Using Three Alternative Approximate Degrees of Freedom Tests: Test Development and Type I Error Rates

$66-80$

Miguel A. Padilla, James Algina

Type I Error Rates of the Kenward-Roger Adjusted Degree of Freedom F test for a Split Plot Design with Missing Values

81 - 90 Gibbs Y. Kanyongo Reliability and Statistical Power: How Measurement G. P. Brooks, Fallibility Affects Power and Required Sample Sizes Lydia K.-Blankson, for Several Parametric and Nonparametric Statistics Gulsah Gocmen

91 - 106 Gary E. Meek, Comparison of the t vs. Wilcoxon Signed-Rank Test Ceyhun Ozgur, for Likert Scale Data and Small Samples Kenneth Dunning

$107-116 \quad$ Bruno Lecoutre Another Look at Confidence Intervals for the Noncentral t Distribution

117 - 132 Robert A. Cribbie, Tests for Treatment Group Equality When Data are Rand R. Wilcox, Nonnormal and Heteroscedastic Carmen Bewell, H. J. Keselman 


$\begin{array}{lll}133-140 & \begin{array}{l}\text { Jamie A. Gruman, } \\ \text { Robert A. Cribbie, } \\ \text { Chantal A.-Cribbie }\end{array} & \text { The Effects of Heteroscedasticity on Tests of Equivaler } \\ 141-152 & \text { Vincent Camara } & \begin{array}{l}\text { Approximate Bayesian Confidence Intervals for the } \\ \text { Mean of an Exponential Distribution }\end{array} \\ 153-161 & \text { James F. Reed III } & \text { Better Binomial Confidence Intervals } \\ 162-172 & \text { David A. Walker } & \begin{array}{l}\text { A Comparison Of Eight Shrinkage Formulas Under } \\ \text { Extreme Conditions }\end{array} \\ 173-186 & \begin{array}{l}\text { Carl Lee, } \\ \text { Felix Famoye, } \\ \text { Olugbenga Olumolade }\end{array} & \begin{array}{l}\text { Applications to Censored Data } \\ \text { Beta-Weibull distribution: Some Properties and }\end{array}\end{array}$

$187-211 \quad$ Lingji Kong, On the Properties of Beta-Gamma Distribution

Carl Lee,

J. H. Sepanski

$212-218$ M. Shakil,

B. M. Golam Kibria

$219-227 \quad$ Stan Lipovetsky Optimal Lp-Metric for Minimizing Powered Deviations in Regression

228 - 238 Ani Shabri, LQ Moments For Statistical Analysis Of Extreme Events Abdul Aziz Jemain

239-247 Chin-Shang Li, A Spline-Based Lack-Of-Fit Test for Independent Wanzhu Tu Variable Effect in Poisson Regression

248 - 257 Adriana Peréz Using the Fractional Imputation Methodology to Evaluate Variance Due to Hot Deck Imputation in Survey Data

258 - 264 Kosei Fukuda Practical Unit-Root Analysis Using Information Criteria: Simulation Evidence

265 - 278 Senay Yolacan, A Fano-Huffman Based Statistical Coding Method Aladdin Shamilov

279-290 Mahesh Menon, A Comparison of One-High-Threshold and Two-HighTodd S. Woodward Threshold Multinomial Models of Source Monitoring 


\section{1 - $303 \quad$ Ilker Ercan, Examining Cronbach Alpha, Theta, Omega \\ Berna Yazici, Reliability Coefficients According to the Sample Size Deniz Sigirli, Bulent Ediz, Ismet Kan}

304 - 319 Ann A. O’Connell, Modeling Longitudinal Ordinal Response Variables Heather L. Doucette for Educational Data

320 - 323 Ajit Mukherjee, Risk for Developing Cardiac Problems from Type2 Ajit Mathur, Diabetes via Density Estimation

Rakesh Mittal,

$324-330$
K. Rajendran,
Multinomial Logistic Regression Model for the Inferential T. Ramamurthy. Dipika Sur
Risk Age Groups for Infection Caused by Vibrio Cholerae in Kolkata, India

Early Scholars
$331-335$ Tian Tian, Rand R. Wilcox Designs

\begin{abstract}
A Comparison of Two Rank Tests for Repeated Measures
\end{abstract}
Algorithms and Code

$\begin{aligned} & 336-340 \text { Paul Nakonezny, } \\ & \text { Robert D. Shull }\end{aligned}$

$341-349$

\author{
Yanyan Sheng, \\ Todd C. Headrick
}

JMASM26: Hettmansperger and McKean Linear Model Aligned Rank Test for the Single Covariate and One-Way ANCOVA Case (SAS)

JMASM27: An Algorithm for Implementing Gibbs

Sampling for 2PNO IRT Models (Fortran)

Translations, Ephemerals, \& Biographies

350 - 354 Shlomo Sawilowsky Mathematics in Volume I of Scritpa Universitatis

JMASM is an independent print and electronic journal (http://tbf.coe.wayne.edu/jmasm), publishing (1) new statistical tests or procedures, or the comparison of existing statistical tests or procedures, using computer-intensive Monte Carlo, bootstrap, jackknife, or resampling methods, (2) the study of nonparametric, robust, permutation, exact, and approximate randomization methods, and (3) applications of computer programming, preferably in Fortran (all other programming environments are welcome), related to statistical algorithms, pseudorandom number generators, simulation techniques, and self-contained executable code to carry out new or interesting statistical methods.

Editorial Assistant: John Cuzzocrea

Production Staff: Christina Gase

Internet Sponsor: Paula C. Wood, Dean, College of Education, Wayne State University

Cushing-Malloy, Inc.

Internet: www.cushing-malloy.com
(888) 295-7244 toll-free (Phone)

(734) 663-5731 (Fax)
Sales \& Information:

skehoe@cushing-malloy.com 


\section{Invited Articles \\ Effects of Physical Activity on Psychological Change in Advanced Age: A Multivariate Meta-Analysis}

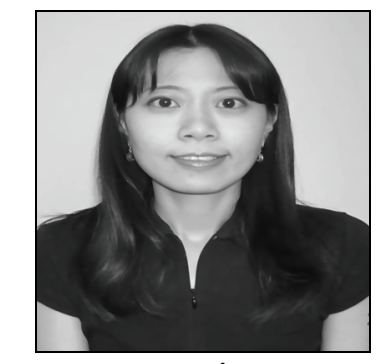

Meng-Jia $\mathrm{Wu}$

Loyola University Chicago

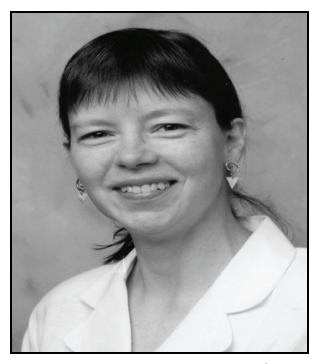

Betsy Jane Becker Florida State University

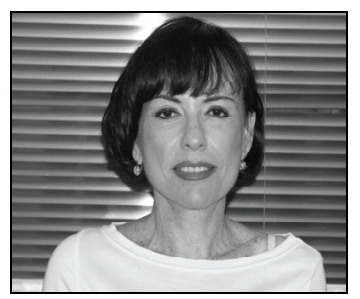

Yael Netz

Wingate Institute, Israel

An example of multivariate meta-analysis is demonstrated by synthesizing the treatment effects of exercise of 15 groups on six mood state changes in elders measured by the Profile of Mood States (POMS) scale. Two different methods were used to analyze this multivariate dataset. The SAS codes for two set of the analyses were provided. Results showed that exercise has a modest and positive impact on elders mood change.

Key words: multivariate meta-analysis, mood state, POMS, Psychological change, exercise

\section{Introduction}

In this article, an application of meta-analysis to multivariate data is demonstrated. The eight primary studies included in the current metaanalysis are a subset of studies from a larger meta-analysis of the impact of exercise on psychological change in the elderly. The full list of studies on this topic, the search process, and

Meng-Jia Wu is Assistant Professor of Research Methodology in the School of Education. Her current interests focus on connecting missing data techniques to meta-analyze regression studies. Email her at mwu2@luc.edu. Betsy Jane Becker is Professor of Measurement and Statistics in the College of Education. Her research interests are in meta-analysis. Email her at bjbecker@coe.fsu.edu. Yael Netz is the Head of the School of Exercise and Sport Sciences Wingate Institute, and Visiting Associate Professor at the University of Melbourne. Email her at neyael@wincol.ac.il. study selection criteria can be found in Netz, $\mathrm{Wu}$, Becker, and Tenenbaum (2005). This metaanalysis includes studies examining the treatment effects of exercise on mood change in the elderly, published between 1993 and 2001. Outcomes for participants in 15 treatment groups from these studies were measured by the Profile of Mood States (POMS; McNair, Lorr, \& Droppleman, 1971), before and after exercise. Six identifiable mood states are measured in the POMS: Tension-Anxiety, Depression-Dejection, Anger-Hostility, Vigor-Activity, Fatigue-Inertia, and Confusion-Bewilderment, however, not all 15 groups provide measures of all six outcomes. (Below each scale is referred to using the first word of its label.) The numbers of mood states measured range from 4 to 6 in these groups. The 15 independent treatment groups in this metaanalysis produced 71 effect sizes.

\section{Calculation of the Effect Size}

The effect size in this synthesis is the standardized mean-change measure (Becker, 1988), which represents the magnitude of the 
difference between the pretest and the posttest means for each outcome. It is defined as $g=\frac{\bar{Y}^{t r t}-\bar{X}^{t r t}}{S_{X}^{t r t}}$, where $\bar{Y}^{\text {trt }}$ denotes the posttest mean of the treatment group, $\bar{X}^{\text {trt }}$ denotes the pretest mean of the treatment group, and $S_{X}{ }^{t r t}$ denotes the standard deviation of the pretest in the treatment group. The effect size $g$ represents change from pretest to posttest in preteststandard-deviation units. A $g$ value of 1.0 for a treatment group indicates the participants' mean level of the outcome after exercise improved one standard deviation relative to their initial level.

All gs were corrected for bias due to small sample sizes. The unbiased effect size, denoted $d$, was obtained by correcting $g$ via $d=\left(1-\frac{3}{4 n-5}\right) g$, where $n$ is the sample size for which $\mathrm{g}$ is computed. The variance of $d$ is defined as $\operatorname{var}(d)=\frac{4(1-r)+d^{2}}{2 n}$, where $r$ is the pretest-posttest correlation. Because $r$ is not reported in any of the studies, it is assumed to be 0.7 in this meta-analysis.

The signs of effect sizes for all outcomes except vigor (which is scored positively) were reversed; therefore, all positive $d$ values in the dataset indicate improved mood status.

\section{Methodology}

Several approaches to synthesizing multivariate data in meta-analysis are discussed and summarized in Becker (2000). Here, two methods are presented: One commonly used approach creates independent subgroups for analysis; the other is more sophisticated, yet requires more assumptions. The latter approach uses generalized least squares (GLS) to take dependence among the outcomes into account while analyzing multivariate data (Raudenbush, Becker, \& Kalaian, 1988). The two methods are used to calculate the mean effects of exercise (and associated standard errors) for each of the six mood states.

Method I: Creating Independent Subgroups

The 71 effect sizes were first categorized into six subgroups based on what mood state was measured. Effect sizes within each of the six sets of outcomes are independent, because each treatment group had at most one effect size for each mood state.

The mean effect size for each mood status can then be calculated separately. Each mean effect size is calculated by weighting each individual effect by its associated variance. The variance used for weighting was estimated based on the random-effects model, in which betweensamples variation was accounted for, producing more conservative results. The variance of each effect was computed as $\operatorname{var}(d)+S_{\delta}^{2}$, where $\operatorname{var}(d)$ is defined above and $S_{\delta}^{2}$ is a method-ofmoments estimator of between-studies variation given in formula 18 of Shadish and Haddock (1994). More details on random-effects modeling can be found in Shadish and Haddock (1994, pp. 273-275).

Below is the SAS macro for calculating the mean effects and their standard errors for the six outcomes. The remarks in the right hand column document each of the steps. 


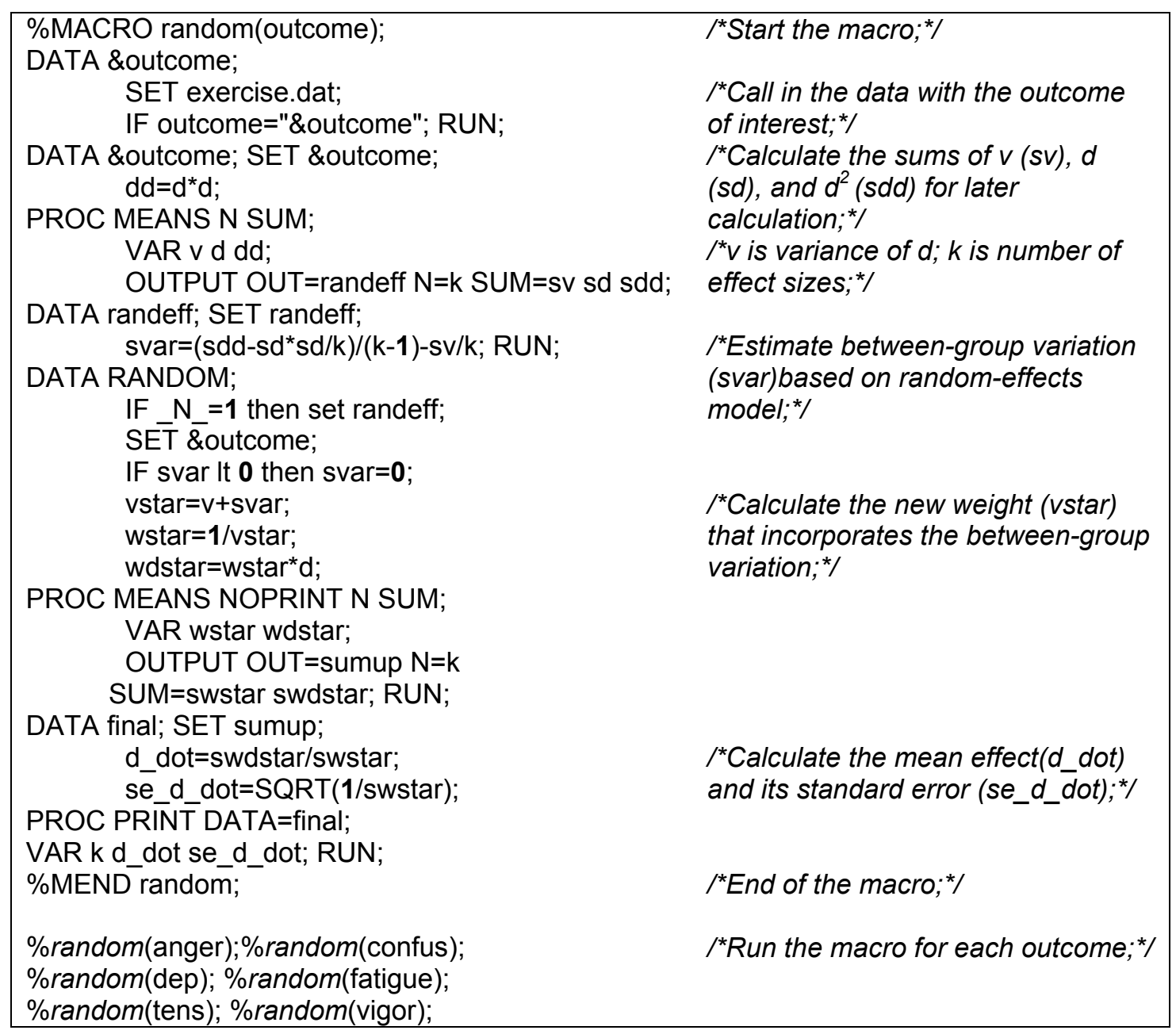

Method II: Accounting for Dependence among the Outcomes

To use the generalized least squares (GLS) method to model the dependence among outcomes and calculate the mean effects of exercise on each mood state, a vector $\mathbf{d}$ is created, containing all 71 effect sizes and a $71 * 71$ variance-covariance matrix among the effects is obtained.

Let $\mathbf{d}_{i}$ represent the vector effect size for group $i$ ( $i=1$ to 15$) ; d_{i j}$ in the vector represents the effect size from group $i$ on measure $j(j=1$ to
6 for tension, depression, anger, vigor, fatigue, confusion). As noted earlier, not every study measured all six outcomes. Therefore, for example, the first group had only measures of tension $(j=1)$, depression $(j=2)$, anger $(j=3)$, and vigor $(j=4)$, and $\mathbf{d}_{1}=\left(d_{11}, d_{12}, d_{13}, d_{14}\right)^{\prime}$; The fifteenth group measured all six outcomes and $\mathbf{d}_{15}=\left(d_{(15) 1}, d_{(15) 2}, d_{(15) 3}, d_{(15) 4}, d_{(15) 5}, d_{(15) 6}\right)$ '.

The linear model that can be used to represent variation in effect sizes is:

$$
\mathbf{d}=\mathbf{X} \times \boldsymbol{\delta}+\mathbf{e}
$$




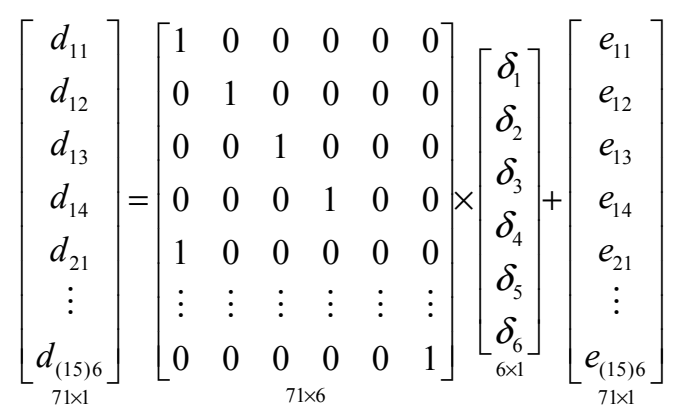

where the matrix $\mathbf{X}$ contains six columns to indicate which one of the six outcomes each effect size measured (e.g., a one in the first column means the effect was a measure of tension). The $\delta \mathrm{s}$, the regression coefficients for the dummy variables, represent the estimated mean effects, and $e_{i j}$ is the corresponding residual for outcome $j$ for group $i$.

The $\delta$ s can be estimated using GLS estimation, assuming the errors e have a mean vector of 0 and a known variance-covariance matrix $\boldsymbol{\Sigma}$. The matrix $\boldsymbol{\Sigma}$ can be estimated by $\mathbf{S}$, which is the $71 * 71$ variance-covariance matrix among the $d_{i j}$ values, with the variancecovariance for each treatment group $\left(\mathbf{S}_{i}\right)$ on the diagonal. Other elements in the matrix $\mathbf{S}$ are all 0 assuming the 15 treatment groups are independent of each other.

In each $\boldsymbol{S}_{i}$, the diagonal elements are the variances of each effect size, the $\operatorname{var}\left(d_{i j}\right)$ for $j=1$ through 6 as defined above, plus the between studies variance. The off-diagonal elements in $\mathbf{S}_{i}$ are the covariances between pairs of effect sizes $d_{i j}$ and $d_{i j}$ for study $i$, each of which is defined as $S\left(d_{i j}, d_{i j^{\prime}}\right)=r_{i j j^{\prime}} S\left(d_{i j}\right) S\left(d_{i j^{\prime}}\right)$. The $\mathrm{S}\left(d_{i j}\right)$ and
$\mathrm{S}\left(d_{i j^{\prime}}\right)$ are the square roots of $\operatorname{var}\left(d_{i j}\right)$ and $\operatorname{var}\left(d_{i j^{\prime}}\right)$. The $r_{i j j^{\prime}}$ are the correlations between outcomes $j$ and $j$, which unfortunately often are not reported and have to be assumed. In the current study, the correlations reported in the POMS manual (McNair et al., 1971) were used, which ranged from .13 between anger and vigor to .77 between tension and depression.

The mean effect for each outcome can be estimated by solving

$$
\hat{\boldsymbol{\delta}}=\left(\mathbf{X}^{\prime} \mathbf{S}^{-1} \mathbf{X}\right)^{-1} \mathbf{X}^{\prime} \mathbf{S}^{-1} \mathbf{d} .
$$

The variance-covariance matrix of the estimated mean effects is

$$
\operatorname{Var}(\hat{\boldsymbol{\delta}})=\left(\mathbf{X}^{\prime} \mathbf{S}^{-1} \mathbf{X}\right)^{-1}
$$

An easy way to obtain the estimates is to set up the values in $\mathbf{d}, \mathbf{X}$ and $\mathbf{S}$ in Excel, and then call them into SAS in the form of a vector (d) and matrices ( $\mathbf{X}$ and $\mathbf{S})$. That is, in the Excel spreadsheet the 71 effect sizes are in one column. Six more columns, each with 71 values, indicate the outcome(s) represented by each of the 71 effect sizes. Each column indexes one of the 6 outcomes, and each column contains a 1 in row $r$ if the effect size in row $r$ measured that specific outcome. The $71 * 71$ variancecovariance matrix can be computed and saved in 71 columns, each with 71 values. The SAS IML code used to retrieve the data from Excel and to compute the estimates of mean effect for the outcomes and their standard errors are shown below. 


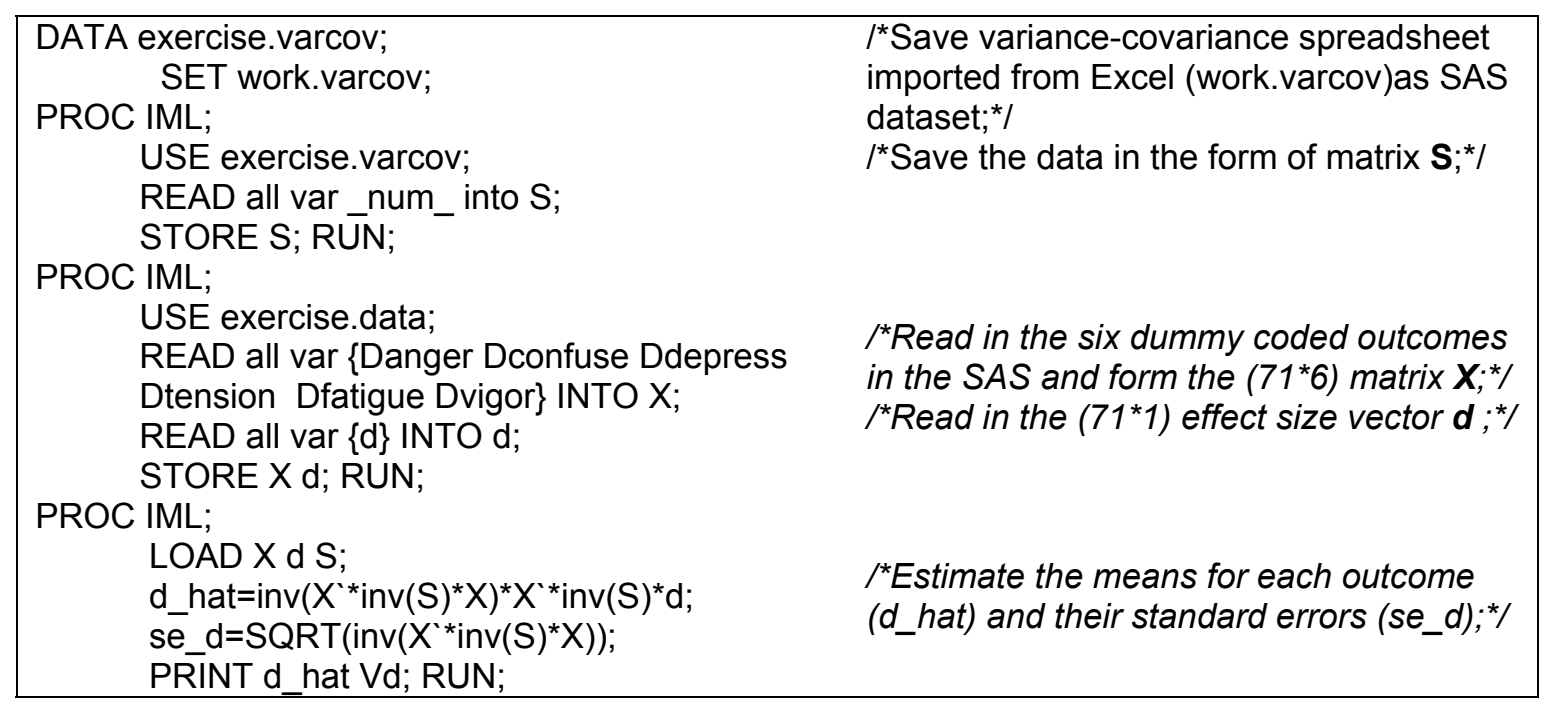

Results

The estimated mean effects of exercise on six mood states in the elderly are shown in Table 1. Under both methods, almost all mood states show significant improvement after the exercise intervention, except tension estimated using method I $(\bar{d}=0.12)$ and vigor under both methods. Using method I, fatigue $(\bar{d}=0.30)$, anger $(\bar{d}=0.29)$, and depression $(\bar{d}=0.29)$ improve the most; under method II, tension ( $\bar{d}=0.28)$, anger $(\bar{d}=0.27)$, and depression $(\bar{d}=0.26)$ improve the most.
The standard errors for outcomes based on method I are larger than those computed based on method II. This occurs in part because the intercorrelations among the outcomes allow estimates of each mean to borrow strength from other outcomes and thus precision is increased. Also, Table 1 shows the correlations among the group means. Although none of the entries is large, some are moderate in size suggesting that it would be wrong to treat the means as if they were independent, as one would if tests to compare means were conducted using method I. 
Table 1. Results

\begin{tabular}{|c|c|c|c|c|c|c|c|c|c|c|}
\hline \multirow{3}{*}{ Outcome } & \multirow{3}{*}{$k$} & \multicolumn{2}{|c|}{ Method I } & \multicolumn{7}{|c|}{ Method II } \\
\hline & & \multirow{2}{*}{$\bar{d}$} & \multirow{2}{*}{$S E$} & \multirow{2}{*}{$d$} & \multirow{2}{*}{$S E$} & \multicolumn{5}{|c|}{ Correlations } \\
\hline & & & & & & $\mathrm{C}$ & $\mathrm{D}$ & $\mathrm{T}$ & $\mathrm{F}$ & $\mathrm{V}$ \\
\hline Anger (A) & 13 & $0.29 *$ & 0.076 & $0.27 *$ & 0.068 & .34 & .30 & .28 & .20 & .02 \\
\hline Confusion (C) & 12 & $0.15^{*}$ & 0.065 & $0.14 *$ & 0.053 & & .44 & .40 & .38 & .08 \\
\hline Depression (D) & 13 & $0.29 *$ & 0.084 & $0.26^{*}$ & 0.075 & & & .35 & .28 & .07 \\
\hline Tension $(\mathrm{T})$ & 8 & 0.12 & 0.115 & $0.28 *$ & 0.065 & & & & .30 & .06 \\
\hline Fatigue (F) & 14 & $0.30^{*}$ & 0.072 & $0.13 *$ & 0.063 & & & & & .09 \\
\hline Vigor (V) & 11 & 0.20 & 0.243 & 0.17 & 0.204 & & & & & \\
\hline
\end{tabular}

Note. The "*” indicates the mean effect size is significantly different from 0 at the .05 level

\section{Conclusion}

Exercise has a positive impact on elders' mood change, though all changes are modest (at most three tenths of a standard deviation). However, the magnitudes of the impact on different mood states are varied. Additional analyses would examine this variation among effect sizes, which can be quantified using $Q$ statistics (Shadish $\&$ Haddock, 1994). Further investigation could focus on moderators such as type of exercise or participant age which might explain the variation in effects.

\section{References}

Becker, B. J. (1988). Synthesizing standardized mean-change measures. British Journal of Mathematical and Statistical Psychology, 41, 257-278.
Becker, B. J. (2000). Multivariate metaanalysis. In H. E. A. Tinsley \& S. D. Brown (Eds.), Handbook of applied multivariate statistics and mathematical modeling. New York: Academic Press.

McNair, D. M., Lorr, M., \& Droppleman, L. F. (1971). Manual for the Profile of Mood States. San Diego, CA: Educational and Industrial Testing Service.

Netz, Y., Wu, M., Becker, B. J., \& Tenenbaum, G. (2005). Physical activity and psychological well-being in advanced age: A meta-analysis of intervention studies. Psychology and Aging, 20(2), 272-284.

Raudenbush, S. W., Becker, B. J., \& Kalaian, H. (1988). Modeling multivariate effect sizes. Psychological Bulletin, 103(1), 111-120.

Shadish, W. R., \& Haddock, C. K. (1994). Combining estimates of effect size. In H. Cooper \& L. V. Hedges (Eds.), The handbook of research synthesis. New York: Russell Sage Foundation. 


\section{Bimodality Revisited}

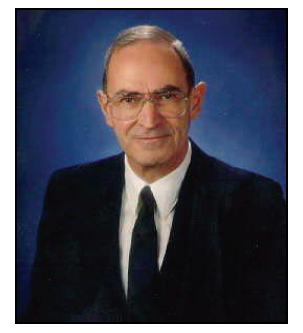

Thomas R. Knapp

University of Rochester and The Ohio State University

Degree of bimodality is an important feature of a frequency distribution, because it could suggest heterogeneity, such as polarization or two underlying distributions combined into one. The literature contains several measures of bimodality. This article attempts to summarize most of those measures, with their attendant advantages and disadvantages.

Key words: Bimodality, kurtosis, moments, polarization

\section{Introduction}

The bimodality of a frequency distribution is of considerable interest in a number of disciplines. A Google search on 'bimodality' returns almost 300,000 entries. Applications of bimodality considerations are found in substantive investigations in fields as diverse as agriculture (e.g., Doehlert, et al., 2004), economics (e.g., Esteban \& Ray, 1994), linguistics (e.g., Spivey, Grosjean, \& Knoblich, 2005), medicine (e.g., Lim, Bakri, Morad, \& Hamid, 2002; Grandi, et al., 2005), psychology (e.g., Lindner, 1997; Beach, Finchman, Amir, \& Leonard, 2005), and sociology (e.g., DiMaggio, Evans, \& Bryson, 1996; Greeley, 1997; Evans, Bryson, \&

Thomas R. Knapp, Ed.D. (Harvard, 1959), is Professor Emeritus of Education and Nursing, University of Rochester and The Ohio State University. He has contributed previous articles to JMASM and has a chapter on "Effective sample size" in Real data analysis (2007, Information Age Publishers). His website is www.tomswebpage.net; his e-mail address is tknapp5@juno.com.
DiMaggio, 2001; Evans, 2003; Mouw and Sobel, 2001).

Esteban and Ray (1984) were concerned with the concept of societal polarization. They argued that one of the indicators of polarization is the bimodality of a frequency distribution for any variable that is an operationalization of an opinion construct such as attitude toward abortion. DiMaggio, Evans, and Bryson (1996), Greeley (1997), Mouw and Sobel (2001) studied the bimodality of several attitude variables-mostly Likert-type scales in the National Election Study (NES) and General Social Survey (GSS) data sets.

\section{Purpose}

The purpose of this article is to trace the methodological foundations of bimodality, some of the attempts that have been made to measure it, and some of the contributions to statistical inferences regarding it.

Historical Review

Karl Pearson

In his first of a series of articles on the mathematical theory of evolution, Pearson (1894) devised a procedure for determining whether or not a frequency distribution could be 
resolved into two normal distributions. The procedure involved six equations in six unknowns (the mean, standard deviation, and membership proportion for each of the two underlying normal distributions), which in turn led to a ninth-degree polynomial equation. If the given distribution had two peaks that were rather far apart it could be described as bimodal. He used as an example some data collected by Professor W.F.R. Weldon on 1000 crabs in Naples.

In a later article (1929) he showed that $b_{2}-b_{1}$, where $b_{2}$ is the standardized fourth moment around the mean and $b_{1}$ is the square of the standardized third moment around the mean, must be greater than or equal to 1 , with the equality holding for the two-point Bernoulli distribution, which is the most extreme case of bimodality.

\section{Darlington to DeCarlo}

Darlington (1970) claimed that $b_{2}$ (he called it $\mathrm{k}$ ) is more a measure of unimodality vs. bimodality than a measure of peakedness vs. flatness as often discussed in statistics textbooks, i.e., it is a measure of the extent to which a distribution's $z$-scores cluster around +1 and -1 , with the two-point Bernoulli distribution being the most bimodal, having a $\mathrm{k}$ of 1 .

Chissom (1970) discussed various interpretations of the kurtosis statistic $\alpha_{4}=b_{2}-3$, which is equal to 0 for the normal distribution. He pointed out that $\alpha_{4}=-2$ for perfectly bimodal distributions.

In a brief note, Hildebrand (1971) expressed general agreement with Darlington, but gave examples of two bimodal distributions, for one of which k-3 was equal to -1.2 and for the other of which k-3 was equal to 3 .

Moors (1986) agreed that $\mathrm{k}$ should be interpreted as the extent to which scores cluster around one s.d. to the right of the mean and one s.d. to the left of the mean.

Ruppert (1987) provided a long discussion of the various interpretations that have been made of $b_{2}$, including peakedness and tail-thickness, and emphasized Hampel's (1974) influence function approach to the understanding of kurtosis.

Balandra and MacGillivray (1988) wrote a critical review of the literature on kurtosis and favored the viewing of kurtosis as "a vague concept" (p. 116) regarding the location of a distribution's shoulders vis-a-vis its center and its tails.

In a more recent review of the literature on kurtosis, DeCarlo (1997) clarified the role of measures of kurtosis in tests for normality, tests for bimodality, and other matters, in the context of several previously-cited examples.

Reschenhofer and Schilling, Watkins, \& Watkins

It has often been claimed that a mixture of two normal distributions is necessarily bimodal. Reschendofer (2001) showed that to be true only if the two modes differ by two or more standard deviation units. Schilling, Watkins, and Watkins (2002) made the same claim for the special case of the distribution of adult heights when men and women are included in the same distribution. Those results are consistent with the arguments made by Darlington (1970) and Moors (1986) regarding the clustering of data at $z$-scores of +1 and -1 (a difference of two $\sigma$ 's).

\section{Choonpradub \& McNeil}

Choonpradub and McNeil (2005) were concerned that traditional box plots don't provide any indication of bimodality for the distributions such plots are meant to summarize. They recommended an enhancement (thickening the ends of the box denoting the quartiles) that might reflect bimodality.

\section{Haldane to Frankland and Zumbo}

The previously-cited authors were concerned primarily with the description of bimodality. Haldane (1952), however, suggested a fairly simple test for statistically significant bimodality, based upon the successive discrepancies of frequencies for adjacent categories in a sample frequency distribution. He used as an example the distribution of differences in hair color for 162 pairs of siblings.

Shenton \& Bowman (1977) laid the groundwork for statistical inferences based upon the skewness coefficient $\sqrt{ } b_{1}$, the kurtosis coefficient $b_{2}$, their respective univariate sampling distributions, and their joint bivariate sampling distribution.

A truly bimodal distribution should have 
a reasonably deep dip between the two modes. Hartigan and Hartigan (1985) developed a dip test that could be used to distinguish between unimodality and bimodality.

Tokeshi's (1992) test of the bimodality of a sample frequency distribution is a type of randomization (permutation) test that compares an actual sample distribution with all of the possible ways the total frequency could have been allocated to the various categories that comprise the variable of interest.

The estimation of the number and location of underlying modes for a sample frequency distribution was investigated by Minnotte (1997).

Frankland and Zumbo (2002) provided an SPSS program for distinguishing between a single underlying normal distribution and a bimodal composite of two underlying normal distributions.

Other Methodological Contributions

There is a set of miscellaneous formulas for the CLUSTER procedure in the SAS User's Guide. One of those formulas, derived by Warren Sarle (Personal Communication, $5 / 10 / 06$ ), is a formula for the bimodality coefficient:

$$
b=\left[\left(m_{3}^{2}+1\right) /\left(m_{4}+\left[\left(3(n-1)^{2}\right) /((n-2)(n-3))\right]\right)\right]
$$

where $m_{3}$ is skewness and $m_{4}$ is kurtosis. Values of $b$ greater than 0.555 (the value for a uniform population) may indicate bimodal or multimodal marginal distributions. The maximum of 1.0 (obtained for the Bernoulli distribution) is obtained for a population with only two distinct values. Very heavy-tailed distributions have small values of $b$ regardless of the number of modes.

The notation is unconventional, because the m's usually represent the unstandardized moments about the mean (so just substitute $b_{1}$ for $\mathrm{m}_{3}{ }^{2}$ and $\mathrm{b}_{2}$ for $\mathrm{m}_{4}$ ). Slight variations of it (for large $\mathrm{n}$ the term inside the square brackets is often deleted if 3 has not been subtracted from $b_{2}$, or replaced by 3 if it has).
There is another statistic that is also called a bimodality coefficient; it is a function of the likelihood ratio for normal distributions vs. mixtures of normal distributions (see Ashman \& Bird, 1994 for an application to astronomy).

In his technical article about Lmoments, Hosking (1990) claimed that the ratio of two of them "could be interpreted as a measure of tendency to bimodality" (p. 111).

A Personal View of Bimodality

Bimodality should be thought of topologically. If you push down on the peak of a unimodal distribution the frequency curve gets flatter and flatter until it becomes a uniform distribution. If you keep pushing further the curve crawls upward to the left and to the right and ultimately ends up as a two-point distribution. How then to measure the degree of bimodality of an actual distribution? As Pearson (1929), Shenton and Bowman (1977), and others had pointed out, $b_{2}-b_{1}$ must be greater than or equal to 1 , so that $b_{2}-b_{1}$ should be a reasonable measure of bimodality, because it takes on its smallest value (1), for the two-point Bernoulli distribution, and it takes on its largest value (conceptually infinite) for a distribution with a single tall peak.

That approach was taken in Knapp (1959) and in a subsequent unpublished paper Knapp (1970) in which an attempt was made to derive the sampling distribution of $b_{2}-b_{1}$ for samples from a normal distribution. That attempt was only partially successful because only the first two moments could be derived mathematically (a Monte Carlo approach was used for the rest of the basis for statistical inference), and significant non-normality is not necessarily the same as significant bimodality.

\section{Some Examples of Descriptive Comparisons}

Consider the following hypothetical frequency distributions for a variable that ranges from 1 to 11 and for a sample size of 100 (see Figures 1 through 9). 


\begin{tabular}{cc}
$\mathrm{X}$ & $\mathrm{f}$ \\
1 & 6 \\
2 & 10 \\
3 & 11 \\
4 & 10 \\
5 & 9 \\
6 & 8 \\
7 & 9 \\
8 & 10 \\
9 & 11 \\
10 & 10 \\
11 & 6 \\
& \\
\hline
\end{tabular}

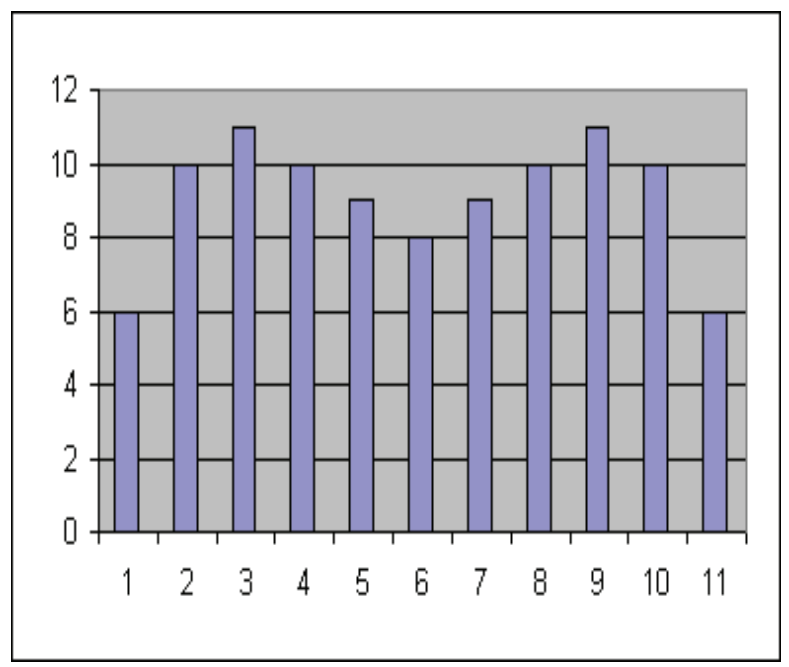

Figure 1

$\begin{array}{cc}\mathrm{X} & \mathrm{f} \\ 1 & 1 \\ 2 & 7 \\ 3 & 9 \\ 4 & 11 \\ 5 & 10 \\ 6 & 8 \\ 7 & 9 \\ 8 & 11 \\ 9 & 12 \\ 10 & 13 \\ 11 & 9\end{array}$

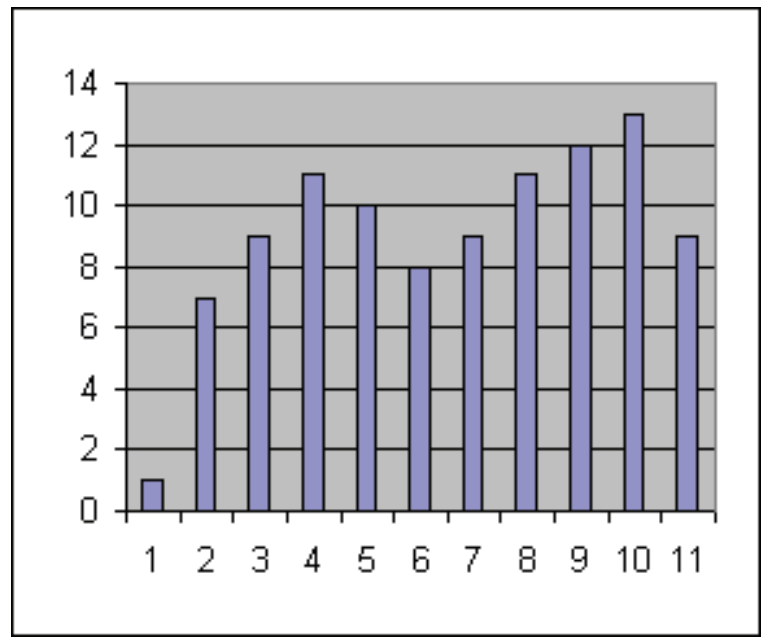

Figure 2 


$\begin{array}{cc}\mathrm{X} & \mathrm{f} \\ 1 & 2 \\ 2 & 8 \\ 3 & 10 \\ 4 & 11 \\ 5 & 12 \\ 6 & 14 \\ 7 & 12 \\ 8 & 11 \\ 9 & 10 \\ 10 & 8 \\ 11 & 2\end{array}$

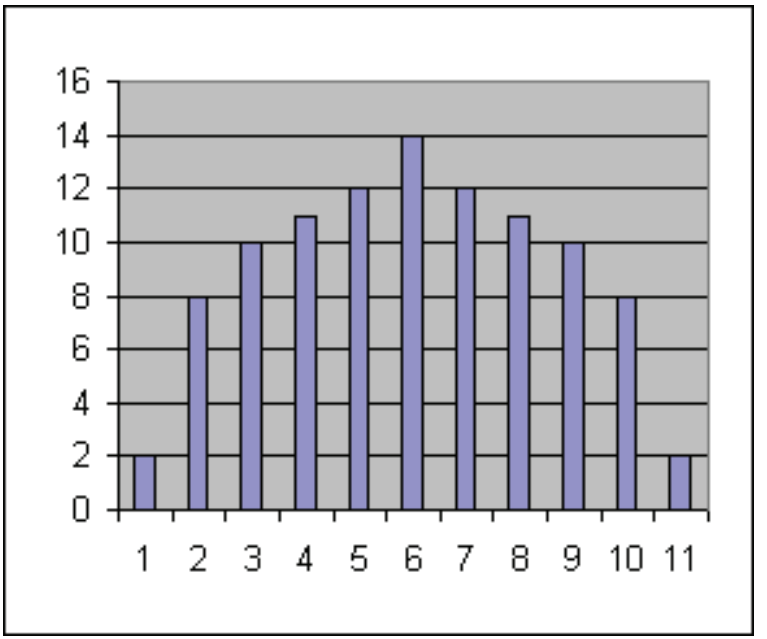

Figure 3

$\begin{array}{cc}\mathrm{X} & \mathrm{f} \\ 1 & 1 \\ 2 & 3 \\ 3 & 6 \\ 4 & 12 \\ 5 & 18 \\ 6 & 20 \\ 7 & 18 \\ 8 & 12 \\ 9 & 6 \\ 10 & 3 \\ 11 & 1\end{array}$

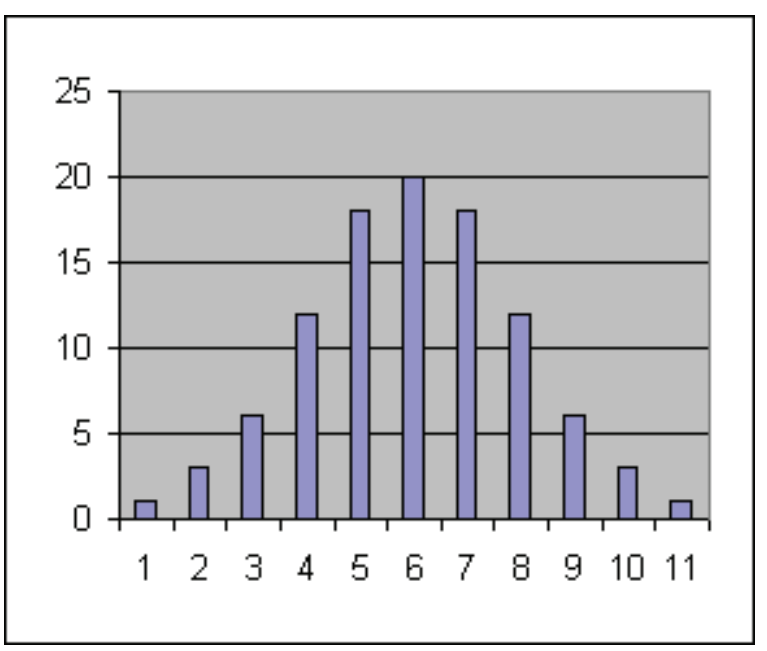

Figure 4 


$\begin{array}{cc}\mathrm{X} & \mathrm{f} \\ 1 & 1 \\ 2 & 2 \\ 3 & 4 \\ 4 & 6 \\ 5 & 12 \\ 6 & 16 \\ 7 & 21 \\ 8 & 23 \\ 9 & 14 \\ 10 & 1 \\ 11 & 0\end{array}$

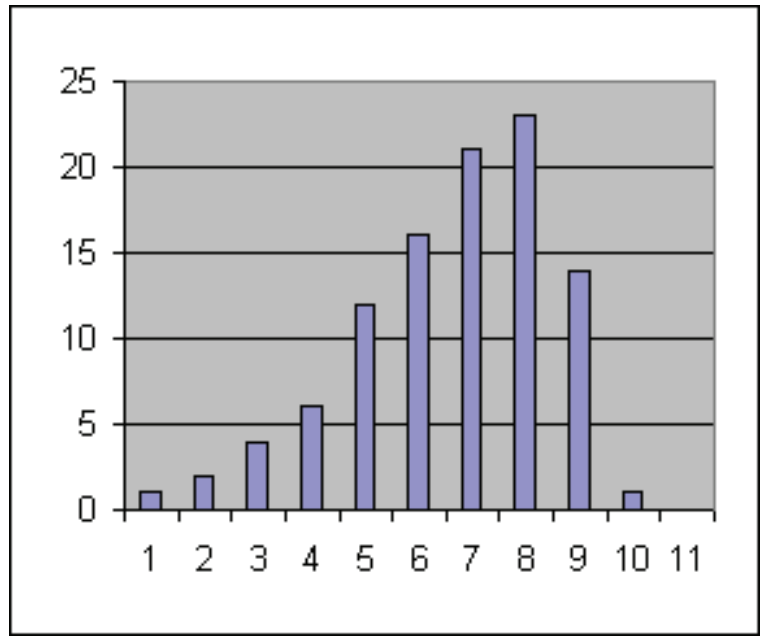

Figure 5

$\begin{array}{cc}\mathrm{X} & \mathrm{f} \\ 1 & 3 \\ 2 & 3 \\ 3 & 4 \\ 4 & 6 \\ 5 & 12 \\ 6 & 44 \\ 7 & 12 \\ 8 & 6 \\ 9 & 4 \\ 10 & 3 \\ 11 & 3\end{array}$

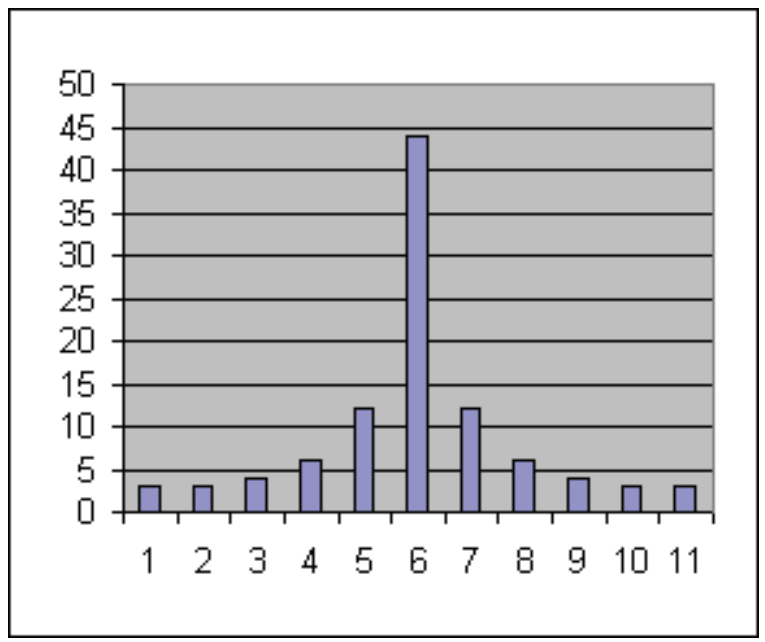

Figure 6 


$\begin{array}{cc}\mathrm{X} & \mathrm{f} \\ 1 & 2 \\ 2 & 3 \\ 3 & 3 \\ 4 & 3 \\ 5 & 3 \\ 6 & 4 \\ 7 & 5 \\ 8 & 11 \\ 9 & 21 \\ 10 & 41 \\ 11 & 4\end{array}$

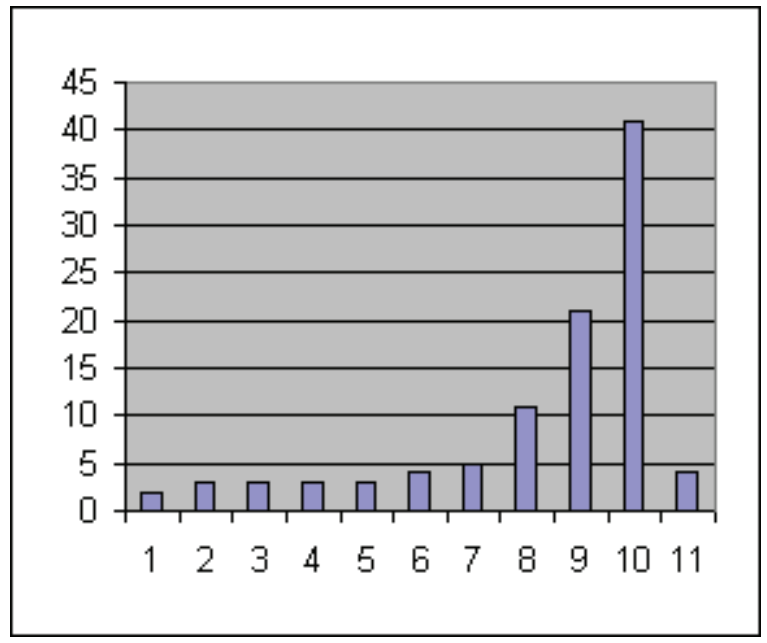

Figure 7

$\begin{array}{cc}X & f \\ 1 & 13 \\ 2 & 11 \\ 3 & 10 \\ 4 & 9 \\ 5 & 7 \\ 6 & 1 \\ 7 & 8 \\ 8 & 9 \\ 9 & 9 \\ 10 & 11 \\ 11 & 12\end{array}$

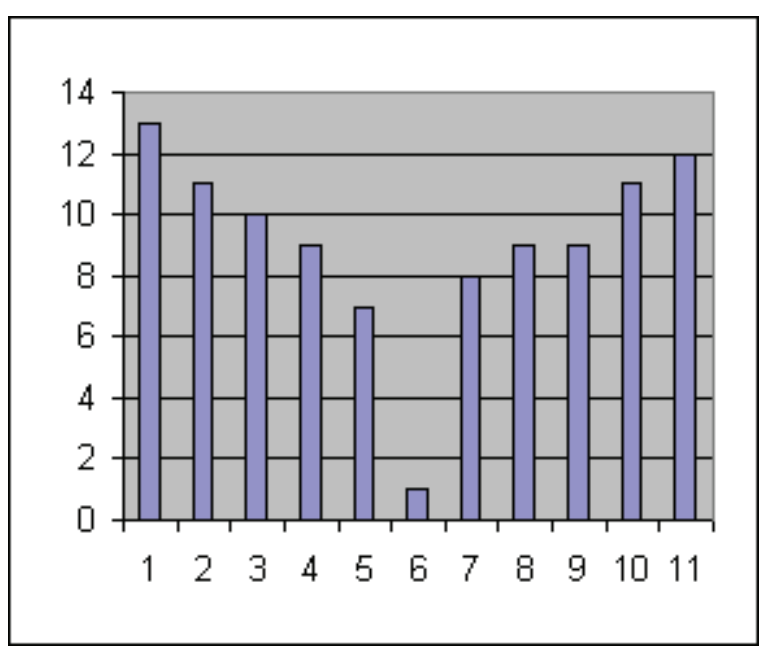

Figure 8 


$\begin{array}{cc}X & f \\ 1 & 10 \\ 2 & 10 \\ 3 & 10 \\ 4 & 10 \\ 5 & 10 \\ 6 & 10 \\ 7 & 10 \\ 8 & 10 \\ 9 & 10 \\ 10 & 10 \\ 11 & 0\end{array}$

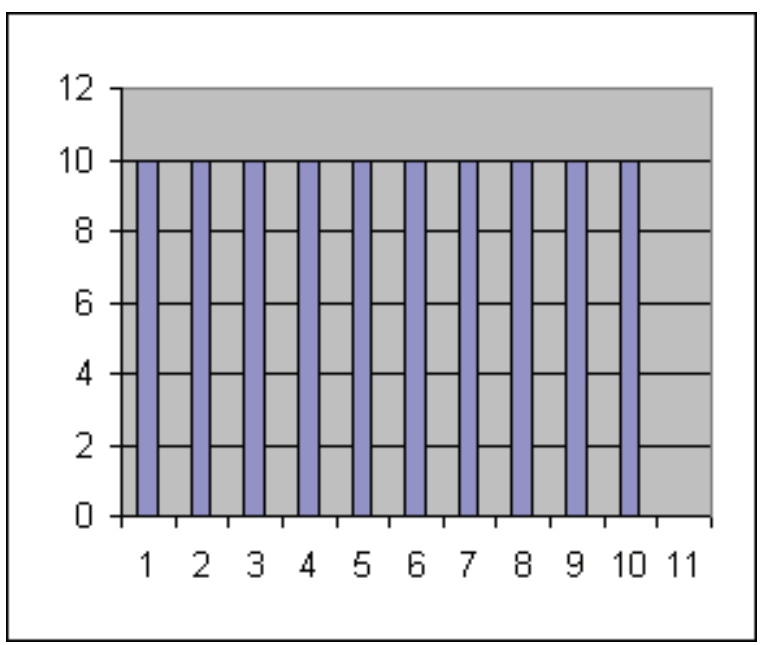

Figure 9

Table 1. Results from Figures 1 through 9

\begin{tabular}{|c|c|c|c|c|c|c|c|}
\hline Figure & $\mathbf{b}_{\mathbf{1}}$ & $\mathbf{b}_{\mathbf{2}}$ & rank* $^{*}$ & $\mathbf{b}_{\mathbf{2}}-\mathbf{b}_{\mathbf{1}}$ & rank* $^{*}$ & $\left.\mathbf{( b}_{\mathbf{1}}+\mathbf{1}\right) / \mathbf{b}_{\mathbf{2}}$ & rank* $^{*}$ \\
\hline 1 & .000 & 1.757 & 2 & 1.757 & 2 & .569 & 4 \\
\hline 2 & .021 & 1.786 & 4 & 1.765 & 3 & .572 & 3 \\
\hline 3 & .000 & 2.046 & 5 & 2.046 & 6 & .489 & 7 \\
\hline 4 & .000 & 2.804 & 6 & 2.804 & 8 & .357 & 8 \\
\hline 5 & .549 & 3.113 & 7 & 2.564 & 7 & .498 & 6 \\
\hline 6 & .000 & 4.043 & 8 & 4.043 & 9 & .247 & 9 \\
\hline 7 & 2.443 & 4.414 & 9 & 1.971 & 5 & .780 & 1 \\
\hline 8 & .001 & 1.515 & 1 & 1.514 & 1 & .660 & 2 \\
\hline 9 & .000 & 1.776 & 3 & 1.776 & 4 & .563 & 5 \\
\hline
\end{tabular}

Notes. $* 1=$ most bimodal; $9=$ least bimodal

For each distribution, $\mathrm{b}_{2}, \mathrm{~b}_{2}-\mathrm{b}_{1}$, and $\left(b_{1}+1\right) / b_{2}$ were calculated (see Table 1$)$. The relative agreement among the three measures of bimodality is fairly good except for Figure 7. That figure is clearly not bimodal, which would intuitively rule out $\left(b_{1}+1\right) / b_{2}$ as an indicator of its bimodality. $b_{2}$ alone would suggest that
Figure 7 is the least bimodal of the nine figures, but $b_{2}-b_{1}$ would suggest that four of the other distributions (Figures 3, 4, 5, and 6) are less bimodal, with Figure 6 being the least. Therefore, $b_{2}-b_{1}$ is the better indicator of bimodality because a flattening out in Figure 7 may be seen, followed by a second mode 
popping up at the low end of the scale, if you push down hard enough on the mode at an abscissa value of 10 . The appearance of two modes would take much longer with Figure 6 (Three modes would pop up there first--one at each end to go along with the one in the middle).

Statistical Inferences Using the Same Examples In addition to its simplicity, Haldane's test appears to be the most defensible, because it is appropriate for both interval and ordinal scales. It has been applied to the distributions in Figures 1-9, with the relatively surprising result that none of those distributions is significantly bimodal at the .05 level (see Figure 10). It is
Surprising, because Figure 8, for example, really looks bimodal and the sample size is reasonably large (100). But, Figure 10 is an example of one that is; note the deeper trough between the two peaks.

Two real-data examples

Sullivan (2005) found that the frequency distribution of Type 1 rates for age at first birth (with number of previously childless women of childbearing age in the denominator) exhibited a bimodal pattern in the $90 \mathrm{~s}$, with peaks at both 20 and 30 years of age. Figure 11 is the Sullivan graph which illustrates that phenomenon for the years 1991, 1995, and 1999:

$\begin{array}{cc}X & f \\ 1 & 5 \\ 2 & 5 \\ 3 & 30 \\ 4 & 5 \\ 5 & 5 \\ 6 & 0 \\ 7 & 5 \\ 8 & 5 \\ 9 & 30 \\ 10 & 5 \\ 11 & 5\end{array}$

Figure 10 
Birm adality in Type I Finst-Bürth Rotes, U.S. Womth, 1990*

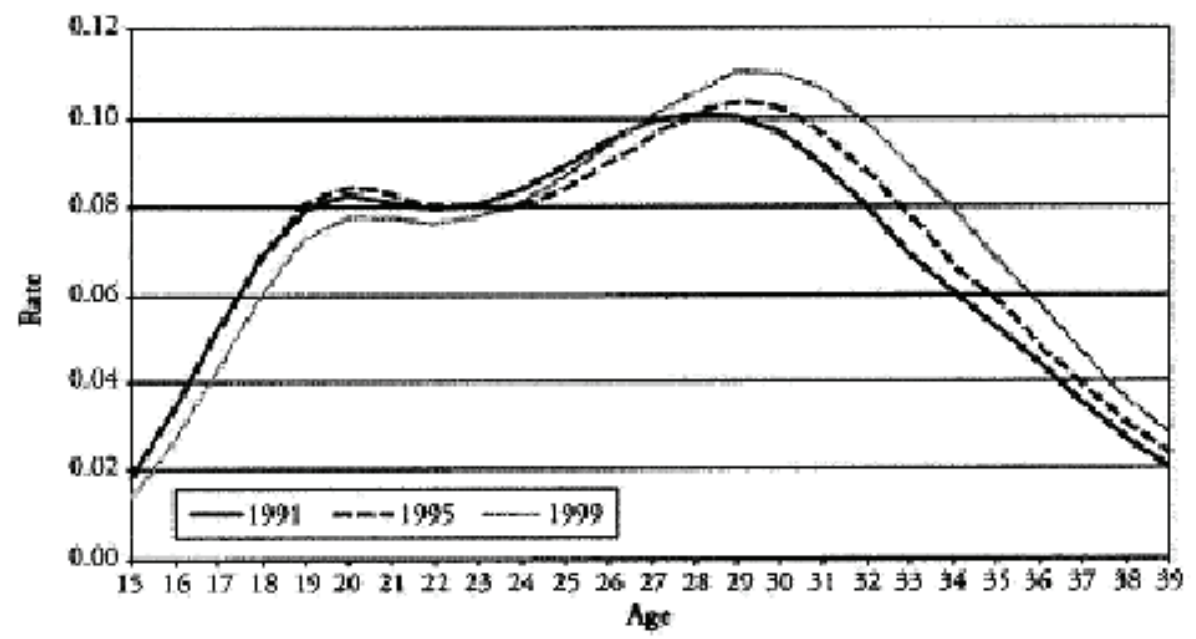

Soume: VCHS (1991. 1995. and 15995.

Reproduced with permission of the copyright Gwnar.

Figure 11. Sullivan Graph

Another interesting recent example of bimodality is discussed in the paper by Roller (2005) regarding the results of a questionnaire sent to U.S. members of the International Reading Association that elicited responses to questions about President George W. Bush's "No Child Left Behind" (NCLB) program. In that article, she said that several of the five-point frequency distributions were bimodal. Here is the example that she emphasized:

Item: "The educational benefits resulting from NCLB implementation in your school district will, on balance, outweigh any adverse impacts for students in the aggregate." 
Table 2

\begin{tabular}{|l|c|}
\hline \multicolumn{1}{|c|}{ Response } & Frequency \\
\hline Strongly Agree & 115 \\
\hline Agree & 396 \\
\hline Neither Agree Nor Disagree & 285 \\
\hline Disagree & 357 \\
\hline Strongly Disagree & 219 \\
\hline & \\
\hline Total & 1372 \\
\hline No Response & 178 \\
\hline Grand Total & 1550 \\
\hline
\end{tabular}

Roller (2005) called the attention of the reader to the modes at Agree and Disagree (see Table 2). $b_{2}$ for these data is $1.899 ; b_{1}$ is .000 (to three decimal places--the distribution is very close to symmetric); $b_{2}-b_{1}=1.899 ;$ and $\left(b_{1}+\right.$ $1) / b_{2}=.527$. Haldane's test supports the hypothesis of underlying bimodality. But the dip between those two modes at Neither Agree nor Disagree could be an artifact of a non-committal response rather than a valley between two peaks. (The large No Response percentage might be further evidence of such an artifact.)

There has been a considerable amount of empirical research regarding the middle category of a five-point Likert-type scale; see, for example, Guy \& Norvell (1977) and Armstrong (1987). Mouw and Sobel (2001) argued that DiMaggio et al. (1996) should not have applied their measure of bimodality $\left(b_{2}-3\right)$ to Likert-type scales, because it assumes interval-scale properties. The treating of ordinal scales as interval scales is one of the most controversial matters in statistical methodology. There appears to be no solution to the problem that would be acceptable to the warring factions.
Miscellany

Although all of the standard computer packages (SAS, SPSS, Minitab, Excel) include the calculation of one or more measures of skewness and kurtosis, the formulas used in those packages vary somewhat from one another. If you'd like to compute $b_{1}$ in Excel, for instance, you need to square SKEW and multiply that by $\left\{(n-1)^{2} /(n-2)^{2}\right\}$ in order to undo the sample adjustments. As well, in order to compute $b_{2}$ you need to add,

$$
\left\{3(\mathrm{n}-1)^{2} /(\mathrm{n}-2)(\mathrm{n}-3)\right\}
$$

to KURT and multiply that by

$$
\{(\mathrm{n}-2)(\mathrm{n}-3) /(\mathrm{n}+1)(\mathrm{n}-1)\} \text {. }
$$

Baretto, Borges, \& Guo (2003) pointed out that a typographical error in an article citing one of Tokeshi's (1992) formulas has led to several incorrect tests of the bimodality of distributions that are of interest to researchers concerned with the range-size of various animal species. (Even in their correct form his formulas are tricky, because they require very careful attention to summation operations and combinatorial notation.) 
In an interesting article many years ago, Baker (1930) hinted that one should not get too excited about bimodality because a bimodal distribution can often be changed into a unimodal distribution by means of an algebraic transformation. He gave as an example a continuous bimodal fourth-degree polynomial distribution of $\mathrm{X}$ that could be converted into a continuous unimodal distribution by replacing $\mathrm{X}$ with $\mathrm{e}^{\mathrm{X}}$.

\section{Conclusion}

There are several measures of the bimodality of a frequency distribution. There are also several tests of the statistical significance of sample bimodality. Hopefully, this article has provided at least a partial summary of such procedures.

\section{References}

Armstrong, R. I. (1987). The midpoint on a five-point Likert-type scale. Perceptual and Motor Skills, 64, 359-362.

Ashman, K. M., Bird, C. M., \& Zepf, S. E. (1994). Detecting bimodality in astronomical data sets. Astronomical Journal, 108 (6), 23482361.

Baker, G. A. (1930). Transformations of bimodal distributions. Annals of Mathematical Statistics, 1 (4), 334-344.

Balandra, K. P., \& MacGillivray, H. L. (1988). Kurtosis: A critical review. The American Statistician, 42 (2), 111-119.

Barreto, S., Borges, P. A. V., \& Guo, Q. (2003). A typing error in Tokeshi's test of bimodality. Global Ecology and Biogeography, 12, 173-174.

Beach, S. R. H., Fincham, S. D., Amir, N., \& Leonard, K. E. (2005). The taxometrics of marriage: Is marital discord categorical? Journal of Family Psychology, 19(2), 276-285.

Chissom, B. S. (1970). Interpretation of the kurtosis statistic. The American Statistician, 24(4), 19-22.
Choonpradub, C., \& McNeil, D. (2005). Can the box plot be improved? Songklanakarin Journal of Science and Technology, 27(3), 649657.

Darlington, R. B. (1970). Is kurtosis really "peakedness"? The American Statistician, 24(2), 19-22.

DeCarlo, L. T. (1997). On the meaning and use of kurtosis. Psychological Methods, 2 (3), 292-307.

DiMaggio, P., Evans, J., \& Bryson, B. (1996). Have Americans' social attitudes become more polarized? The American Journal of Sociology, 102 (3), 690-755.

Doehlert, D. C., McMullen, M. S., Jannink, J-L, Panigrahi, S., Gu, H., \& Riveland, N. R. (2004). Evaluation of oat kernel size uniformity. Crop Science, 44, 1178-1186.

Esteban, J. M, \& Ray, D. (1994). On the measurement of polarization. Econometrica, 62(4), 819-851.

Evans, J. H. (2003). Have Americans' attitudes become more polarized? An Update. Social Science Quarterly, 84(1), 71-90.

Evans, J. H., Bryson, B., \& DiMaggio, P. (2001). Opinion polarization: Important contributions, necessary limitations. The American Journal of Sociology, 106(4), 944960.

Frankland, B. W., \& Zumbo, B. D. (2002). Quantifying bimodality, Part I: An easily implemented method using SPSS. Journal of Modern Applied Statistical Methods, 1(1), 157166.

Grandi, C., Tapia, J. L., Marshall, G., and the NEOCOSUR Collaborative Group. (2005). An assessment of the severity, proportionality and risk of mortality of very low birth weight infants with fetal growth restriction: A multicenter South American analysis. Journal de Pedatria, 81(3), 198-204.

Greeley, A. M. (1997). Polarizing Catholics?: Don't believe your mail! America, 176(6), 11-15.

Guy, R. F., \& Norvell, M. (1977). The neutral point on a Likert scale. The Journal of Psychology, 95, 199-204. 
Haldane, J. B. S. (1952). Simple tests for bimodality and bitangentiality. Annals of Eugenics, 16, 359-364.

Hampel, F. R. (1974). The influence curve and its role in robust estimation. Journal of the American Statistical Association, 69, 383393.

Hartigan, J. A., \& Hartigan, P. M. (1985). The dip test of unimodality. The Annals of Statistics, 13(1), 70-84.

Hildebrand, D. K. (1971). Kurtosis measures bimodality? The American Statistician, 25(1), 42-43.

Hosking, J. R. M. (1990). L-moments: Analysis and estimation of distributions using linear combinations of order statistics. Journal of the Royal Statistical Society. Series B (Methodological), 52(1), 105-124.

Knapp, T. R. (1959). Two-group classification in the absence of a criterion. Unpublished Ed.D. dissertation, Harvard University.

Knapp, T.R. (1970). A measure of bimodality and its sampling distribution for samples from a normal population. Unpublished paper, University of Rochester.

Lim, T-O, Bakri, R., Morad, Z., \& Hamid, M. A. (2002). Bimodality in blood glucose distribution. Diabetes Care, 25, 22122217.

Lindner, M. D. (1997). Reliability, distribution, and validity of age-related cognitive deficits in the Morris Water Maze. Neurobiology of Learning and Memory, 68, 203-220.

Minnotte, M. C. (1997). Nonparametric testing of the existence of modes. The Annals of Statistics, 25 (4), 1646-1660.

Moors, J. J. A. (1986). The meaning of kurtosis: Darlington reexamined. The American Statistician, 40(4), 283-284.
Mouw, T., \& Sobel, M. E. (2001). Culture wars and opinion polarization: The case of abortion. The American Journal of Sociology, 106(4), 913-943.

Pearson, K. (1894). Contributions to the mathematical theory of evolution: On the dissection of asymmetrical frequency-curves. Philosophical Transactions of the Royal Society, Series A, Part 1, 185, 71-90.

Pearson, K. (1929). Editorial note. Biometrika, 21, 370-375.

Reschenhofer, E. (2001). The bimodality principle. Journal of Statistics Education, 9 (1). [Online]

Roller, C. M. (2005, February) No child left behind: A survey of its impact on IRA members. $8 \mathrm{pp}$. Retrieved from the International Reading Association website, www.reading.org.

Ruppert, D. (1987). What is kurtosis?: An influence function approach. The American Statistician, 41(1), 1-5.

Schilling, M. F., Watkins, M. E., \& Watkins, W. (2002). Is human height bimodal? The American Statistician, 56(3), 223-229.

Shenton, L. R., \& Bowman, K. O. (1977). A bivariate model for the distribution of $\sqrt{ }_{1}$ and $b_{2}$. Journal of the American Statistical Association, 72(357), 206-211.

Spivey, M. J., Grosjean, M., \& Knoblich, G. (2005). Continuous attraction toward phonological competitors. PNAS, 102 (29), 10393-10398.

Sullivan, R. (2005). The age pattern of first-birth rates among U.S. women: The bimodal 1990s. Demography, 42(2), 259-273.

Tokeshi, M. (1992). Dynamics and distribution in animal communities: Theory and analysis. Researches in Population Ecology, 34, 249-273. 


\section{Ordinal Versions of Coefficients Alpha and Theta for Likert Rating Scales}

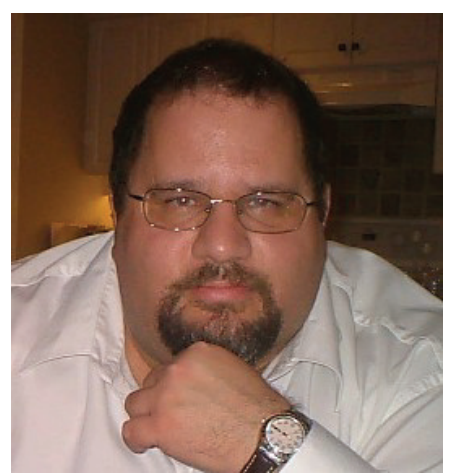

Bruno D. Zumbo

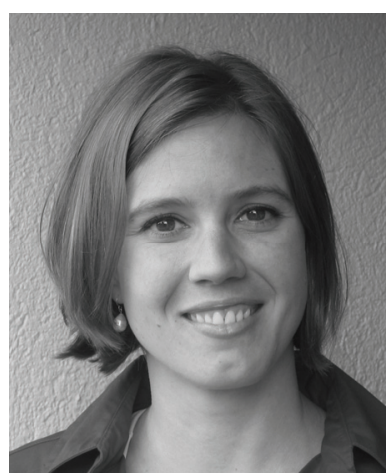
Anne M. Gadermann
University of British Columbia

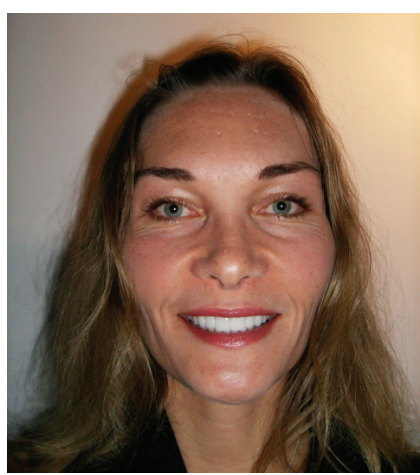

Cornelia Zeisser

Two new reliability indices, ordinal coefficient alpha and ordinal coefficient theta, are introduced. A simulation study was conducted in order to compare the new ordinal reliability estimates to each other and to coefficient alpha with Likert data. Results indicate that ordinal coefficients alpha and theta are consistently suitable estimates of the theoretical reliability, regardless of the magnitude of the theoretical reliability, the number of scale points, and the skewness of the scale point distributions. In contrast, coefficient alpha is in general a negatively biased estimate of reliability. The use of ordinal coefficients alpha and theta as alternatives to coefficient alpha when estimating the reliability based on Likert response items are recommended. The choice between the two ordinal coefficients depends on whether one is assuming a factor analysis model (ordinal coefficient alpha) or a principal components analysis model (ordinal coefficient theta).

Key words: Internal consistency, reliability, coefficient alpha, coefficient theta.

\section{Introduction}

Coefficient alpha is the most widely used index of reliability in the social sciences (Zumbo \& Rupp, 2004). There is, however, ongoing debate about the use of alpha for Likert type rating response scales because alpha assumes that the item responses are continuous. Using Likert type

Bruno D. Zumbo is Professor, Measurement, Evaluation, and Research Methodology (MERM) Program, and Department of Statistics and the Institute of Applied Mathematics at the University of British Columbia (UBC), Canada. Email address: bruno.zumbo@ubc.ca. Anne M. Gadermann and Cornelia Zeisser are completing their Ph.D.'s in the MERM Program at UBC. The authors would like to thank Professor Ed Diener for providing the data set that was part of the International College Student Data. response scales, it has been demonstrated that the magnitude of coefficient alpha can be spuriously deflated with less than five scale points. However, reliability was found to level off beyond six points (Gelin, Beasley, \& Zumbo, 2003). Likert type data are commonly utilized in psychological and educational settings to measure unobserved continuous variables. Yet, lack of clarity still prevails regarding the statistical impact of various numbers of response scale points on outcomes that are based on a continuous concept. Of course, a special case of coefficient alpha is KR-20, which is computed from binary data.

One can compute estimates of reliability from correlation (or, more generally, covariance) matrices. For example, the Pearson correlation matrix is commonly used to compute coefficient alpha. An important assumption for the use of the Pearson correlation matrix is the assumption of continuity. If this assumption is violated, the 
Pearson correlation matrix may be distorted (Rupp, Koh, \& Zumbo, 2003). If the data are ordinal, the correlation matrix of choice is the polychoric correlation matrix, which estimates the linear relationship for two unobserved continuous variables given only observed ordinal data (Flora \& Curran, 2004). Hence, for Likert type scales it may be useful to investigate reliability estimates based on the polychoric correlation matrix, thereby taking into account the ordinal nature of the data. A special case of the polychoric correlation matrix is the tetrachoric correlation matrix for binary data.

Rationale and theoretical framework

Coefficient alpha is used as a default for estimating the internal consistency based on the Pearson correlation matrix in widely available software packages such as SPSS and SAS; however, this is done ignoring the Likert response format of the items at hand. The purpose of this article was to introduce two new reliability indices, ordinal coefficient alpha and ordinal coefficient theta, and test their appropriateness as estimates of internal consistency for items with Likert response formats.

Considering only a Pearson correlation matrix and a factor analysis model, McDonald (1985, p. 217) describes how one can compute coefficient alpha from a factor analysis model. For a composite score based on $\mathrm{p}$ items coefficient alpha can be computed as

$$
\alpha=\frac{p}{p-1}\left\lfloor\frac{p(\bar{f})^{2}-\bar{f}^{2}}{p(\bar{f})^{2}+\bar{u}^{2}}\right\rfloor,
$$

where $\bar{f}$ is the average of the $\mathrm{p}$ factor loadings, $\bar{f}^{2}$ is the average of the squares of the $\mathrm{p}$ factor loadings, and $\bar{u}^{2}$ is the average of the $\mathrm{p}$ uniquenesses.

Armor (1974) introduced a reliability estimate, coefficient theta, which was developed to account for multidimensionality in a scale and is based on a principal components model. Coefficient theta for the single factor solution is computed with the following equation (Armor, p. 28):

$$
\Theta=[\mathrm{p} /(\mathrm{p}-1)]^{*}\left[1-\left(1 / \lambda_{1}\right)\right],
$$

where the only new symbol $\lambda_{1}$ denotes the largest eigenvalue from the principal component analysis of the correlation matrix of the items involved in the composite.

Ordinal coefficient alpha and ordinal coefficient theta are computed by applying equations (1) or (2), respectively, to the polychoric correlation matrix. These reliability estimates are ordinal in the sense that they take into account the ordinal nature of the Likert response data.

In the following, a computer simulation study is reported that investigated the population estimation bias of ordinal coefficients alpha and theta for response scales ranging from two to seven points, with symmetric as well as skewed Likert response distributions, and theoretical reliabilities of .4, .6, .8, and .9. Next, ordinal coefficients alpha and theta were demonstrated with real data. The article closes with discussion of the findings and recommendations.

$$
\text { Methodology }
$$

Simulation study

Simulation data were generated to reflect the conditions of theoretical alpha $(.4, .6$, .8 , and .9) as well as skewness conditions of zero and -2 of the item responses. The fundamental equations of factor analysis were used to create a population covariance matrix; this covariance matrix was then used to generate normally distributed item responses. That is, item response data were generated using a factor analysis model. As indicated by Jöreskog (1971) and Henrysson and Wedman (1972), the decomposition of an observed score $\mathrm{X}$ into a true score and an error score in classical test theory can be generalized to a factor analytic model with one common factor. The formula $\mathrm{X}$ $=\mathrm{T}+\mathrm{E}$ can be defined as

$$
\mathrm{X}_{i}=f_{i} \xi+u_{i} \quad i=1,2, \ldots, \mathrm{p},
$$

where $\mathrm{X}_{i}$ denotes the observed scores, $f_{i}$ denotes the factor loadings, $\xi$ the common factor that can also be regarded as true score, $u_{i}$, uniqueness of variables, denotes the error 
scores, and $i$ indexes the items running from one to $p$. In a factor model, the reliability of the observed score can be obtained by summing all true score variances and covariances in the matrix and then by dividing this sum by the total variance (Reuterberg \& Gustafsson, 1992). Novick and Lewis (1967) showed that coefficient alpha yields an unbiased estimate of reliability when the loadings of each variable on the common factor are equal. The formula for the reliability of a composite score is

$$
\rho_{x x}=\frac{\left(\sum_{i=1}^{p} f_{i}\right)^{2}}{\left(\sum_{i=1}^{p} f_{i}\right)^{2}+\sum_{i=1}^{p} \operatorname{var}(e)_{i i}}
$$

where $\operatorname{var}(e)_{i i}$ denotes the error variance in a factor analytical model and all the other symbols are defined above. To obtain the population reliabilities of $.4, .6, .8$, and .9 , factor loadings of $.213 .311, .471$, and .625 , respectively, were computed using the above formula. Therefore, in summary 14 items with continuous (normally distributed) distributions were generated using one common factor model with equal factor loadings across the 14 items. Fourteen items were chosen because it is a typical scale length in health and educational research (Slocum, 2005).

These (underlying) item response distributions were then transformed into Likert responses by applying the thresholds (for the symmetric as well as skewed item responses) as provided in the Appendix. The number of response options was simulated to range from 2 to 7; by including 2 response options, one is also able to investigate how the new reliability estimates perform in the presence of binary data.

As noted above, the unidimensionality and equal factor loadings provide a strict condition where empirical alpha should equal theoretical alpha. It was confirmed that the simulation methodology worked correctly because the theoretical alpha was obtained when analyzing the continuous data. It should be noted that, given the simulation design, there was no interest in the sample-to-sample variability in the estimates but rather the focus was on accuracy (bias) of the estimates. Therefore, population analogues of the empirical reliability estimates were computed with a sample size of 10,000 simulees in each cell of our simulation design.

The following steps were followed for the analysis. The data were simulated and coefficient alpha was obtained using SPSS. The simulated data were then read into PRELIS. In order to compute ordinal coefficient alpha the polychoric correlation matrix was factor analysed using the MINRES procedure. The resulting factor loadings and uniquenesses were then used to compute ordinal coefficient alpha. In addition, the eigenvalues of the polychoric correlation matrix among the items were computed from the principal components analysis and used to compute ordinal theta.

\section{Results}

The reliability estimates for the simulated data are displayed in Tables 1 to 4, for theoretical reliability of 0.4 to 0.9 , respectively. As can be seen from these tables, coefficient alpha is consistently a negatively biased estimate of the theoretical reliability. Note that in the case of equal factor loadings and unidimensionality coefficient alpha should equal the reliability; that is, it is not a lower bound. The negative bias of alpha was even more evident under the condition of negative skewness; for example, in the case of theoretical reliability of .6 and 3 response options alpha underestimates the theoretical reliability by .175 . These results highlight that coefficient alpha, likewise KR-20 for binary data, gives one a downwardly biased estimate of the theoretical reliability with Likert data. With regard to the number of scale points our finding is a replication of the finding of Gelin, Beasley, and Zumbo (2003) that showed that alpha computed from Likert item response data approaches its theoretical value as the number of scale points increases, and levels off at about 6 scale points. 
Table 1. Reliability Estimates for Theoretical Alpha of .4 (all factor loadings are .213)

\begin{tabular}{|c|c|c|c|c|c|c|}
\hline & \multicolumn{6}{|c|}{ Skewness } \\
\hline \multirow{2}{*}{$\begin{array}{c}\text { \# of } \\
\text { response } \\
\text { options }\end{array}$} & \multicolumn{3}{|c|}{0} & \multicolumn{3}{|c|}{-2} \\
\hline & Alpha & Ordinal Alpha & Ordinal Theta & Alpha & Ordinal Alpha & Ordinal Theta \\
\hline 2 & .288 & .393 & .395 & .211 & .389 & .391 \\
\hline 3 & .328 & .401 & .400 & .233 & .383 & .387 \\
\hline 4 & .356 & .399 & .400 & .258 & .379 & .382 \\
\hline 5 & .377 & .406 & .408 & .255 & .384 & .387 \\
\hline 6 & .378 & .398 & .400 & .291 & .382 & .387 \\
\hline 7 & .386 & .401 & .404 & .303 & .391 & .391 \\
\hline
\end{tabular}

Table 2. Reliability Estimates for Theoretical Alpha of .6 (all factor loadings are .311)

\begin{tabular}{|c|c|c|c|c|c|c|}
\hline \multirow{3}{*}{$\begin{array}{c}\text { \# of } \\
\text { response } \\
\text { options }\end{array}$} & \multicolumn{6}{|c|}{ Skewness } \\
\hline & \multicolumn{3}{|c|}{0} & \multicolumn{3}{|c|}{-2} \\
\hline & Alpha & Ordinal Alpha & Ordinal Theta & Alpha & Ordinal Alpha & Ordinal Theta \\
\hline 2 & .488 & .608 & .609 & .379 & .596 & .596 \\
\hline 3 & .527 & .609 & .609 & .425 & .603 & .603 \\
\hline 4 & .561 & .608 & .609 & .421 & .598 & .600 \\
\hline 5 & .576 & .607 & .609 & .452 & .597 & .598 \\
\hline 6 & .587 & .609 & .609 & .459 & .599 & .600 \\
\hline 7 & .589 & .606 & .607 & .477 & .598 & .598 \\
\hline
\end{tabular}

Table 3. Reliability Estimates for Theoretical Alpha of .8 (all factor loadings are .471)

\begin{tabular}{|c|c|c|c|c|c|c|}
\hline \multirow{3}{*}{$\begin{array}{c}\text { \# of } \\
\text { response } \\
\text { options }\end{array}$} & \multicolumn{6}{|c|}{ Skewness } \\
\hline & \multicolumn{3}{|c|}{0} & \multicolumn{3}{|c|}{-2} \\
\hline & Alpha & Ordinal Alpha & Ordinal Theta & Alpha & Ordinal Alpha & Ordinal Theta \\
\hline 2 & .702 & .802 & .802 & .629 & .806 & .806 \\
\hline 3 & .732 & .799 & .799 & .655 & .798 & .798 \\
\hline 4 & .762 & .800 & .800 & .668 & .803 & .804 \\
\hline 5 & .773 & .798 & .798 & .689 & .800 & .800 \\
\hline 6 & .783 & .801 & .801 & .709 & .803 & .804 \\
\hline 7 & .785 & .798 & .798 & .725 & .804 & .804 \\
\hline
\end{tabular}


Table 4. Reliability Estimates for Theoretical Alpha of .9 (all factor loadings are .625)

\begin{tabular}{|c|c|c|c|c|c|c|}
\hline & \multicolumn{6}{|c|}{ Skewness } \\
\hline $\begin{array}{c}\text { \# of } \\
\text { response } \\
\text { options }\end{array}$ & \multicolumn{3}{|c|}{0} & \multicolumn{2}{l|}{} & \\
\hline & Alpha & Ordinal Alpha & Ordinal Theta & Alpha & Ordinal Alpha & Ordinal Theta \\
\hline 2 & .826 & .897 & .897 & .778 & .899 & .899 \\
\hline 3 & .849 & .899 & .899 & .806 & .899 & .899 \\
\hline 4 & .872 & .897 & .897 & .810 & .898 & .898 \\
\hline 5 & .882 & .897 & .897 & .830 & .899 & .899 \\
\hline 6 & .886 & .898 & .898 & .840 & .900 & .900 \\
\hline 7 & .891 & .898 & .898 & .852 & .900 & .900 \\
\hline
\end{tabular}

In contrast to coefficient alpha, ordinal coefficients alpha and theta were consistently found to be suitable estimates of reliability regardless of the magnitude of the theoretical reliability and number of scale points. In addition, it should be noted that the skewness of the item response distribution affects coefficient alpha, whereas ordinal coefficients alpha and theta remain unaffected by skewness. Specifically, ordinal coefficients alpha and theta are still suitable in the presence of skewed data; however, coefficient alpha becomes more biased with skewness. A comparison between the two ordinal estimates shows that they are almost exactly identical. In the following, ordinal coefficients alpha and theta are compared to coefficient alpha in the context of real data.

\section{Real data examples}

The real data examples are based on two samples. The data of the first sample was collected between 1995-1996 by Professor Ed Diener and his collaborators worldwide with College students from 42 nations. The following scales were used. The Satisfaction with Life Scale (SWLS) (Diener, Emmons, Larsen, \& Griffin, 1985) is a 5-item instrument designed to measure global cognitive judgments of one's life using a 7-point Likert-type scale ranging from 1 (strongly disagree) to 7 (strongly agree). Diener's Affect Balance Scale (Veenhoven, 2004) is an 8-item instrument designed to measure positive and negative affect (each being one dimension with four items; this was supported in the present study by a principal component analysis of the polychoric correlation matrix) using a 7-point Likert-type response scale ranging from 1 (never) to 7 (always). The Positive and Negative Affect Schedule (PANAS) (Watson, Clark, \& Tellegen, 1988) consists of two 10-item scales with a 5-point Likert type response scale, ranging from 1 (very slightly) to 5 (extremely). In the present study only the Positive Affect Schedule (PAS) was used. Sample sizes for these questionnaires ranged between 6958 and 7014 .

The data of the second sample was collected in 1993 by the first author at a Canadian university. The Eysenck Personality Questionnaire (EPQ) (Eysenck \& Eysenck, 1975) was administered to 922 students. This questionnaire consists of four subscales with a binary response scale with 0 (no) and 1 (yes). For the present study only the neuroticism (23 items) and extraversion (21 items) subscales were used. 
Coefficient alpha was computed for the (sub)scales using SPSS. The data were entered into PRELIS to obtain the polychoric correlation matrix to compute ordinal coefficients alpha and theta as described above. The items of the SWLS exhibited a skewness ranging from -.56 to .18 , with an average skewness of -.27 . The positive items of the affect scale exhibited a skewness ranging from -.06 to .53 , with an average skewness of .17. The negative items of the affect scale exhibited a skewness ranging from .90 to 1.27 , with an average skewness of 1.04. The items of the PAS exhibited a skewness ranging from -.39 to .05 , with an average skewness of -.21 . The items of the extraversion scale exhibited a skewness ranging from -3.27 to .56 , with an average skewness of -1.02 . The items of the neuroticism scale exhibited a skewness of -1.88 to .89 , with an average skewness of -.32 .

The reliability estimates, coefficient alpha and ordinal coefficients alpha and theta, for the scales are provided in Table 5. Table 5 shows that ordinal coefficients alpha and theta display a larger reliability estimate than coefficient alpha for all scales. However, for the four scales with the 5- and 7-point Likert type response scales, the difference between coefficient alpha and ordinal coefficients alpha and theta is small. In contrast, for the scales with the binary response format the difference between coefficient alpha and ordinal coefficients alpha and theta is more prominent. This is in accordance with the findings of the simulation study, which showed that with increased number of response options, coefficient alpha and the ordinal estimates become closer. Based on the findings from the simulation study, where ordinal coefficients alpha and theta were consistently demonstrated to be more precise estimates, this finding can be interpreted as showing that ordinal coefficients alpha and theta are closer to the theoretical alpha of the scales.

Table 5. Reliability Estimates for Real Data with the SWLS, Positive and Negative Affect, PAS, Extraversion and Neuroticism Scales

\begin{tabular}{|l|c|c|c|}
\hline Scale & Alpha & Ordinal Alpha & Ordinal Theta \\
\hline SWLS & .814 & .835 & .836 \\
\hline Positive Affect & .709 & .735 & .738 \\
\hline Negative Affect & .667 & .684 & .686 \\
\hline PAS & .824 & .845 & .846 \\
\hline Extraversion & .819 & .908 & .916 \\
\hline Neuroticism & .830 & .905 & .910 \\
\hline
\end{tabular}




\section{Conclusion}

In summary, it was found that coefficient alpha computed from Likert response data results in a negatively biased estimate of the theoretical reliability. Because it is a special case of coefficient alpha, KR-20 also shows this bias when used with binary response data. It should be noted that coefficient alpha (and KR-20) are correlation-based statistics and hence assume continuous data. What is noteworthy about the coefficient alpha findings is that the measurement model used in the simulation involves all of the assumptions of coefficient alpha, so that alpha would equal the conceptual/theoretical reliability. However, it was found that coefficient alpha is rather drastically affected by Likert data - e.g., imagine a 14 item scale comprised of a 3-point Likert response format with a skewness of -2 ; the resulting coefficient is .66 when the theoretical reliability is .80 .

Ordinal coefficients alpha and theta, on the other hand, were found to be suitable alternatives to coefficient alpha when a researcher is confronted with having to compute a reliability estimate with Likert response data. It should be noted that with advances in statistical software, these ordinal coefficients are easy to calculate using the newly developed and freely available software FACTOR (LorenzoSeva \& Ferrando, in press) or with widely available software such as PRELIS that provide polychoric correlation matrices. Depending on how they are computed, polychoric correlation matrices can be non positive-definite - i.e., pairwise estimation of the elements of a polychoric correlation matrix is problematic because it can lead to non positive-definite correlation matrices; as opposed to estimating all the correlations in the matrix simultaneously.

The matter of how to estimate polychoric correlation matrices to avoid non positive-definiteness is an open area of research that needs further study but in the meantime a solution to this potential problem, when a non positive-definite matrix is found, is to use software, e.g., EQS, that estimates the polychoric correlations in a manner that reduces the concern for non positive-definite matrices.
In the present study, ordinal coefficients alpha and theta performed equally well. A direction for future research would be to compare ordinal coefficients alpha and theta in the presence of multidimensional items because theta was originally developed to account for multidimensionality in an item set.

Based on the present study, the following recommendations are presented:

1. Use either ordinal coefficient alpha or ordinal coefficient theta to correct for the negative bias in coefficient alpha, and of course KR-20, due to Likert or binary response data.

2. In terms of which of these two ordinal reliability coefficients to use, the decision should be based on whether one is assuming a factor analysis model (ordinal coefficient alpha) or a principal components model (ordinal coefficient theta). For a distinction between principal components analysis and factor analysis the reader is referred to Fabrigar, Wegener, MacCallum, \& Strahan (1999) or Zumbo (2007).

It should be noted that the strategy of using the polychoric correlation could be applied to any reliability estimate that can be computed from a correlation matrix. For example, although it is not described herein, one would have an ordinal version of the McDonald's coefficient omega, yet another reliability estimate, by applying the equation described by McDonald (1985, p. 217), or of Revelle's reliability coefficient beta (Zinbarg, Revelle, Yovel, \& Li, 2005). Future research should explore these other coefficients as well.

\section{References}

Armor, D. J. (1974). Theta reliability and factor scaling. In $H$. Costner (Ed.), Sociological methodology (pp. 17-50). San Francisco: Jossey-Bass.

Diener, E., Emmons, R. A., Larsen, R. J., \& Griffin, S. (1985). The satisfaction with life scale. Journal of Personality Assessment, 49, 71-75. 
Eysenck, H. J., \& Eysenck, S. B. G. (1975). Manual of the Eysenck personality questionnaire. London: Hodder \& Stoughton.

Fabrigar, L. R., Wegener, D. T., MacCallum, R. C., \& Strahan, E. J. (1999). Evaluating the use of exploratory factor analysis in psychological research. Psychological Methods, 4, 272-299.

Flora, D. B., \& Curran, P. J. (2004). An empirical evaluation of alternative methods of estimation for confirmatory factor analysis with ordinal data. Psychological Methods, 9, 466491.

Gelin, M. N., Beasley, T. M., \& Zumbo, B. D. (2003, April). What is the impact on scale reliability and exploratory factor analysis of a Pearson correlation matrix when some respondents are not able to follow the rating scale? Paper presented at the annual meeting of the American Educational Research Association (AERA) in Chicago, Illinois.

Henrysson, S., \& Wedman, I. (1972). Analysis of the inter-item covariance matrix. Scandinavian Journal of Educational Research, 16, 25-35.

Jöreskog, K. G. (1971). Statistical analysis of sets of congeneric tests. Psychometrika, 36, 109-133.

Lorenzo-Seva, U., \& Ferrando, P. J. (in press). FACTOR: A computer program to fit the exploratory factor analysis model. Behavior Research Methods.

McDonald, R. P. (1985). Factor analysis and related methods. Hillsdale NJ: Erlbaum.

Novick, M. R., \& Lewis, M. R. (1967). Coefficient alpha and the reliability of composite measurements. Psychometrika, 32, 113.

Reuterberg, S. E., \& Gustafsson, J. E. (1992). Confirmatory factor analysis and reliability: Testing measurement model assumptions. Educational and Psychological Measurement, 52, 795-811.
Rupp, A., Koh, K. \& Zumbo, B.D. (2003, April). What is the impact on exploratory factor analysis results of a polychoric correlation matrix from LISREL/PRELIS and EQS when some respondents are not able to follow the rating scale. Paper presented at the annual meeting of the American Educational Research Association (AERA) in Chicago, Illinois.

Slocum, S. L. (2005). Assessing unidimensionality of psychological scales: Using individual and integrative criteria from factor analysis. Unpublished Doctoral Dissertation, University of British Columbia, Canada.

Veenhoven (2004). World database of happiness. Retrieved September 14 from http://www2.eur.nl/fsw/research/happiness/hap_ quer/hqs_fp.htm

Watson, D., Clark, L. A., \& Tellegen, A. (1988). Development and validation of brief measures of positive and negative affect: The PANAS Scales. Journal of Personality and Social Psychology, 54, 1063-1070.

Zinbarg, R. E., Revelle, W., Yovel, I., \& Li, W. (2005). Cronbach's $\alpha$, Revelle's $\beta$, and McDonald's $\omega_{\mathrm{H}}$ : There relations with each other and two alternative conceptualizations of reliability. Psychometrika, 70, 123-133.

Zumbo, B. D., \& Rupp, A. A. (2004). Responsible modelling of measurement data for appropriate inferences: Important advances in reliability and validity theory. In D. Kaplan (Ed.), The SAGE Handbook of Quantitative Methodology for the Social Sciences (pp. 73-92). Thousand Oaks, CA: Sage Press.

Zumbo, B.D. (2007). Validity: Foundational Issues and Statistical Methodology. In C.R. Rao and S. Sinharay (Eds.) Handbook of Statistics, Vol. 26: Psychometrics (pp. 45-79). Elsevier Science B.V.: The Netherlands. 
Appendix: Thresholds for Symmetric and Skewed Likert Responses

Thresholds for symmetric scale point distribution:

1. Two-point scale: (Lowest thru $0=1$ ) $(\mathrm{ELSE}=2)$

2. Three-point scale: (Lowest thru $-1=1)(-$ .9999 thru 1=2) $(\mathrm{ELSE}=3)$

3. Four-point scale: (Lowest thru $-1.5=1)(-$ 1.4999 thru $0=2)(0.0001$ thru $1.5=3)$ $(\mathrm{ELSE}=4)$

4. Five-point scale: (Lowest thru $-1.8=1)(-$ 1.7999 thru $-0.6=2) \quad(-0.5999$ thru $0.6000=3) \quad(0.6001 \quad$ thru $\quad 1.8=4)$ $(\mathrm{ELSE}=5)$

5. Six-point scale: (Lowest thru $-2=1)(-$ 1.9999 thru $-1.0=2)(-0.9999$ thru $0=3)$ $(0.0001$ thru $1=4)(1.0001$ thru $2=5)$ $(\mathrm{ELSE}=6)$

6. Seven-point scale: (Lowest thru $2.14286=1)(-2.14285$ thru $-1.28571=2)$ $(-1.28570$ thru $-0.42857=3)(-0.42857$ thru $\quad 0.428571=4) \quad(0.428572 \quad$ thru $1.28571=5)(1.28571$ thru $2.14286=6)$ (else $=7$ )
Thresholds for scale point distribution with skewness of -2 :

1. Two-point scale: (Lowest thru $1.06251930227=1)(\mathrm{ELSE}=2)$

2. Three-point scale: (Lowest thru $0.9002=3) \quad(0.9003 \quad$ thru $1.29883663264=2)(\mathrm{ELSE}=1)$

3. Four-point scale: (Lowest thru $0.8508=4)(0.8509$ thru $1.086=3)(1.087$ thru $1.2816=2)(\mathrm{ELSE}=1)$

4. Five-point scale: (Lowest thru $0.6808=5)(0.6809$ thru $1.036=4)(1.037$ thru $1.2816=3)(1.2817$ thru $1.6546=2)$ $(\mathrm{ELSE}=1)$

5. Six-point scale (Lowest thru $0.5008=6$ ) $(0.5009$ thru $1.036=5) \quad(1.037$ thru $1.0816=4)(1.0817$ thru $1.4546=3)$ $(1.4547$ thru $1.8002=2)(E L S E=1)$

6. Seven-point scale: (Lowest thru $0.4008=7) \quad(0.4009$ thru $0.8360=6)$ (0.8361 thru $1.1816=5)(1.1817$ thru $1.4546=4) \quad(1.4547$ thru $1.8002=3)$ $(1.8003$ thru $2.1002=2)(\mathrm{ELSE}=1)$ 


\section{On Flexible Tests of Independence and Homoscedasticity}

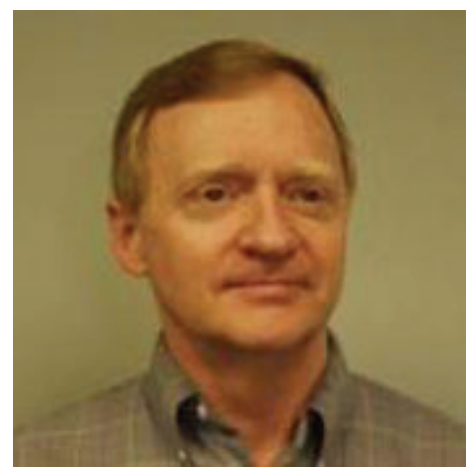

Rand R. Wilcox

University of Southern California

Consider the nonparametric regression model $Y=m(X)+\tau(X) \mathcal{\varepsilon}$, where $X$ and $\varepsilon$ are independent random variables, $\varepsilon$ has a mean of zero and variance $\sigma^{2}, \tau$ is some unknown function used to model heteroscedasticity, and $m(X)$ is an unknown function reflecting some conditional measure of location associated with $Y$, given $X$. Detecting dependence, by testing the hypothesis that $m(X)$ does not vary with $X$, has the potential of being more sensitive to a wider range of associations compared to using Pearson's correlation. This note has two goals. The first is to point out situations where a certain variation of an extant test of this hypothesis fails to control the probability of a Type I error, but another variation avoids this problem. The successful variation provides a new test of $H_{0}: \tau(X) \equiv 1$, the hypothesis that the error term is homoscedastic, which has the potential of higher power versus a method recently studied by Wilcox (2006). The second goal is to report some simulation results on how this method performs.

Key words: Heteroscedasticity, smoothers, wild bootstrap, Winsorized correlations.

\section{Introduction}

Consider the nonparametric regression model $Y=m(X)+\tau(X) \mathcal{\varepsilon}$, where $X$ and $\varepsilon$ are independent random variables, $\varepsilon$ has a mean of zero and variance $\sigma^{2}, \tau$ is some unknown

Rand R. Wilcox (rwilcox@usc.edu) is Professor of Psychology at the University of Southern California. He is the author of seven textbooks on statistics, the most recent of which is Introduction to Robust Estimation and Hypothesis Testing (2005, $2^{\text {nd }}$ Ed., San Diego, CA: Academic Press).
Pearson's correlation between $X$ and $Y$. A concern about this approach is that it limits the function used to model heteroscedasticity, and $m(X)$ is an unknown function reflecting some conditional measure of location associated with $Y$, given $X$. Typically, it is assumed that $m(X)=\beta_{0}+\beta_{1} X$, where $\beta_{0}$ and $\beta_{1}$ are the unknown slope and intercept, and of course a common approach toward establishing an association is testing $H_{0}: \rho=0$, where $\rho$ is types of associations between $X$ and $Y$ that can be detected. For example, there are many types of curvilinear associations between $X$ and $Y$ for which $\rho$ will be close to zero, which in turn can mean relatively low power when 
testing $H_{0}: \rho=0$. Also, heteroscedasticity can affect power when using the usual Student's T test of this hypothesis (e.g., Wilcox, 2003), roughly because the wrong standard error is being used. In some cases, heteroscedasticity might increase power, but the reverse can happen as well. Perhaps more importantly, when Student's T rejects, there is uncertainty whether the main reason is due to heteroscedasticity or because $m(X)$ varies with $X$.

A test of $H_{0}: \rho=0$ that allows heteroscedasticity is given in Wilcox (2003, section 7.3.2), but again, curvilinear associations might be missed. Of course, if it is assumed that curvature can be represented by a particular parametric model, curvilinear associations can be addressed. For example, it might be assumed that $Y=\beta_{0}+\beta_{1} X+\beta_{2} X^{2}+\varepsilon$.

However, experience with smoothers (e.g., Hastie \& Tibshirani, 1990) suggest that it is not always evident which parametric model provides a good reflection of the data.

Using a special case of a wild bootstrap method derived by Stute, Manteiga and Quindimil (1998), it is possible to test

$$
H_{0}: m(X)=\theta \text {, }
$$

where $\theta$ is some unknown measure of location. That is, the hypothesis is that the regression line is a straight, horizontal line having intercept $\theta$. Wilcox (2001) reported simulation results indicating that good control over the probability of a Type I error is achieved when $\theta$ is taken to be the population mean of the $Y$ values, $\mu_{y}$. A seemingly natural way of robustifying this method is to replace the mean with some robust estimator, and a 20\% trimmed mean was suggested by (Wilcox, 2003). One goal here is to describe situations where using a $20 \%$ trimmed mean, control over the probability of a Type I error is very poor, but when using the usual mean, satisfactory control is maintained. (Using the median of the $Y$ values can also result in poor control over the probability of a Type I error.)

Wilcox (2006) suggested a flexible method for testing

$$
H_{0}: \tau \equiv 1
$$

the hypothesis that the error term is homoscedastic. The success of the wild bootstrap test of (1) suggests an alternative approach to testing (2). The second goal in this paper is to report simulation results on this alternative approach and to describe situations where it has more power than the approach studied by Wilcox (2006).

Description of the Methods

Testing (1)

The wild bootstrap test of (1) is applied as follows. Let $\left(X_{1}, Y_{1}\right), \ldots,\left(X_{n}, Y_{n}\right)$ be a random sample of $n$ points from some unknown bivariate distribution. Let $\bar{Y}$ be the usual sample mean based on the $Y$ values. Fix $j$ and set $I_{i}=1$ if $X_{i} \leq X_{j}$, otherwise $I_{i}=0$, and let

$$
R_{j}=\frac{1}{\sqrt{n}} \sum I_{i} r_{i}
$$

where $r_{i}=Y_{i}-\bar{Y}$. The test statistic is the maximum absolute value of all the $R_{j}$ values:

$$
D=\max \left|R_{j}\right|
$$

where the maximum is over all $j$. The critical value is computed as follows. Generate $n$ observations from a uniform distribution and label the results $U_{1}, \ldots, U_{n}$. For $i=1, \ldots, n$, set $V_{i}=\sqrt{12}\left(U_{i}-.5\right), \quad r_{i}^{*}=r_{i} V_{i}$, and $Y_{i}^{*}=\bar{Y}+r_{i}^{*}$. Then based on the $n$ pairs of points $\left(X_{1}, Y_{1}^{*}\right), \ldots,\left(X_{n}, Y_{n}^{*}\right)$, compute the test statistic as described in the previous paragraph and label it $D^{*}$. Repeat this process $B$ times and label the resulting (bootstrap) test statistics $D_{1}^{*}, \ldots, D_{B}^{*}$. Finally, put these $B$ values in ascending order, which are labeled $D_{(1)}^{*} \leq \ldots \leq D_{(B)}^{*}$. Then, the $\alpha$ level critical value is $D_{(u)}^{*}$, where $u=(1-\alpha) B$ rounded to the nearest integer. That is, reject if $D \geq D_{(u)}^{*}$. 
The corresponding $\mathrm{p}$-value is the proportion of $D_{b}^{*}$ values, among the $B$ bootstrap samples, for which $D<D_{b}^{*}$.

Let $Y_{(1)} \leq \ldots \leq Y_{(n)}$ be the $Y_{i}$ values written in ascending order and let $g$ be equal to $.2 \mathrm{n}$ rounded to the nearest integer. Then the $20 \%$ trimmed mean of the $Y$ values is

$$
\bar{Y}_{t}=\frac{1}{n-2 g} \sum_{i=g+1}^{n-g} Y_{(i)} .
$$

An alternative test of (1) is obtained simply by replacing the usual sample mean, $\bar{Y}$, with $\bar{Y}_{t}$.

Testing (2).

Now a test of (2) is described that is based on a simple modification of the method in Wilcox (2006). Let $m(X)$ be the conditional median of $Y$, given $X$. The first step is to approximate $m(X)$ using what is called a running interval smoother, which is applied follows. Let $f$, called a span, be some constant to be chosen and let $M$ be the median of the values $X_{1}, \ldots, X_{n}$. The median absolute deviation (MAD) measure of dispersion is the median of the values $\left|X_{1}-M\right|, \ldots,\left|X_{n}-M\right|$. The point $X$ is said to be close to $X_{i}$ if

$$
\left|X_{i}-X\right| \leq f \frac{M A D}{.6745}
$$

Under normality, MAD/.6745 estimates the standard deviation, in which case $X$ is close to $X_{i}$ if $X$ is within $f$ standard deviations of $X_{i}$. Let

$$
N\left(X_{i}\right)=\left\{j:\left|X_{j}-X_{i}\right| \leq f \times M A D N\right\},
$$

where for convenience, MADN is MAD/.6745. That is, $\mathrm{N}\left(X_{i}\right)$ indexes the set of all $X_{j}$ values that are close to $X_{i}$. Then $m\left(X_{i}\right)$ is taken to be the median of the $Y_{j}$ values such that $j \in N\left(X_{i}\right)$. Generally, a good choice for the span is $f=.8$ (Wilcox, 2005), and this value is used here exclusively. Let $v_{i}=\left|Y_{i}-m\left(X_{i}\right)\right|$ $(i=1, \ldots, n)$ be the absolute residuals. When (2) is true (there is homoscedasticity), the regression line between $X$ and $v$ should be a straight, horizontal line, which can be tested with the method.

Wilcox (2006) suggested two alternative methods for testing (2). Let $\beta$ be the slope of the regression line between the $X_{i}$ and $v_{i}$ values. Then, a test of (2) corresponds to testing

$$
H_{0}: \beta=0 \text {. }
$$

Alternatively, if $\rho$ is some correlation between $X_{i}$ and $v_{i}$, then $H_{0}: \rho=0$ should be true. A natural strategy is to use least squares regression or Pearson's correlation, but this was found to be unsatisfactory. What was found to perform well in simulations was a test of (5) using the Theil (1950) and Sen (1968) regression estimator in conjunction with a percentile bootstrap method, or an approach based on a so-called Winsorized correlation coefficient.

Consider first the regression method. The goal is to test the hypothesis that the (population) regression line between $v$ and $X$ is horizontal. To elaborate on the Theil-Sen estimator, for any $i<i^{\prime}$, for which $X_{i} \neq X_{i^{\prime}}$, let

$$
S_{i i^{\prime}}=\frac{v_{i}-v_{i^{\prime}}}{X_{i}-X_{i^{\prime}}}
$$

The Theil-Sen estimate of the slope is $\hat{\beta}_{t s}$, the median of all the slopes represented by $S_{i i^{\prime}}$. Let $\beta_{t s}$ be the population slope estimated by $\hat{\beta}_{t s}$. To test $H_{0}: \beta_{t s}=0$, it currently seems that a basic percentile bootstrap method performs relatively well. In particular, a bootstrap sample is obtained by randomly sampling, with replacement, $\mathrm{n}$ pairs of points from $\left(v_{1}, X_{1}\right), \ldots,\left(v_{n} X_{n}\right)$. Let $\hat{\beta}^{*}$ be the Theil-Sen 
estimate of $\beta_{t s}$ based on this bootstrap sample. Repeat this bootstrap process $\mathrm{B}$ times yielding $\hat{\beta}_{1}^{*}, \ldots, \hat{\beta}_{B}^{*}$. Let $\hat{\beta}_{(1)}^{*} \leq \ldots \leq \hat{\beta}_{(B)}^{*}$ be the bootstrap estimates written in ascending order. Let $l=\alpha / 2$, rounded to the nearest integer, and $u=B-l$. Then,

$$
\left(\hat{\beta}_{(l+1)}^{*}, \hat{\beta}_{(u)}^{*}\right)
$$

is an approximate $1-\alpha$ confidence interval for $\beta_{t s}$. Let $\hat{p}$ be the proportion of bootstrap estimates less than zero. Then, a p-value is $2 \min (\hat{p}, 1-\hat{p})$.

As for the Winsorized correlation approach, set $Y_{i 1}=v_{i}$ and $Y_{i 2}=X_{i}(i=1, \ldots, n)$. Next, Winsorize the $Y$ values. That is, for fixed $j$, let $Y_{(1) j} \leq \ldots \leq Y_{(n) j}$ be the $\mathrm{n}$ values written in ascending order, and let

$$
\begin{aligned}
& W_{i j}=Y_{(g+1) j} \text { if } Y_{i j} \leq Y_{(g+1) j} \\
& W_{i j}=Y_{i j} \text { if } Y_{(g+1) j}<Y_{i j}<Y_{(n-g) j} \\
& W_{i j}=Y_{(n-g) j} \text { if } Y_{i j} \leq Y_{(n-g) j}
\end{aligned}
$$

where $\mathrm{g}=[\gamma \mathrm{n}], \gamma(0 \leq \gamma<.5)$ is the amount of Winsorizing to be done and [.] is the greatest integer function. Here, $\gamma=.2$ is used. Then the estimate of $\rho_{w}$, the sample Winsorized correlation between R and X, is just Pearson's correlation based on the Winsorized values. That is, estimate $\rho_{w}$ with

$$
r_{w}=\frac{\sum\left(W_{i 1}-\bar{W}_{1}\right)\left(W_{i 2}-\bar{W}_{2}\right)}{\sqrt{\sum\left(W_{i 1}-\bar{W}_{1}\right)^{2} \sum\left(W_{i 2}-\bar{W}\right)^{2}}}
$$

To test $H_{0}: \rho_{w}=0$, compute

$$
T_{w}=r_{w} \sqrt{\frac{n-2}{1-r_{w}^{2}}},
$$

and reject if $\left|T_{w}\right| \geq t_{1-\alpha / 2}$, the $1-\alpha / 2$ quantile of Student's $\mathrm{t}$ distribution with $v=h-2$ degrees of freedom, where $h=n-2 g$.

A Simulation Study

Wilcox (2006) studied the small-sample properties of the method using simulations where both the $X$ and $Y$ values were generated from one of four g-and-h distributions (Hoaglin, 1985), one of which was normal. If $Z$ has a standard normal distribution, then

$$
\begin{gathered}
W=\frac{\exp (g Z)-1}{g} \exp \left(h Z^{2} / 2\right) \quad \text { if } g>0 \\
W=\exp \left(h Z^{2} / 2\right) \text { if } g=0
\end{gathered}
$$

has a g-and-h distribution where $\mathrm{g}$ and $\mathrm{h}$ are parameters that determine the first four moments. The four distributions used were the standard normal $(\mathrm{g}=\mathrm{h}=0.0)$, a symmetric heavytailed distribution $(\mathrm{h}=0.2, \mathrm{~g}=0.0)$, an asymmetric distribution with relatively light tails $(\mathrm{h}=0.0$, $\mathrm{g}=0.2$ ), and an asymmetric distribution with heavy tails $(\mathrm{g}=\mathrm{h}=0.2)$. Table 1 shows the skewness $\left(\kappa_{1}\right)$ and kurtosis $\left(\kappa_{2}\right)$ for each distribution considered. Additional properties of the g-and-h distribution are summarized by Hoaglin (1985).

Table 1. Some Properties of the g-and-h Distribution.

\begin{tabular}{|c|c|c|c|}
\hline $\mathrm{g}$ & $\mathrm{h}$ & $K_{1}$ & $K_{2}$ \\
\hline 0.00 & 0.00 & 0.00 & 3.00 \\
\hline 0.00 & 0.2 & 0.00 & 21.46 \\
\hline 0.2 & 0.0 & 0.61 & 3.68 \\
\hline 0.2 & 0.2 & 2.81 & 155.98 \\
\hline
\end{tabular}


Although skewed distributions were considered, it turns out that when $Y$ has other skewed distributions, not considered by Wilcox (2001), control over the probability of a Type I error is poor when using a $20 \%$ trimmed mean, but control remains good when using the mean instead. Suppose, for example, $Y$ has a chisquared distribution with 1 degree of freedom. Then, when $X$ has a standard normal distribution and $n=30$, the actual Type I error probability is approximately .54 when testing at the .05 level (based on simulations with 1,000 replications). In contrast, when using the mean of $Y$, the Type I error probability is approximately .034. Problems remain with 3 degrees of freedom but they become negligible when the degrees of freedom are increased to 5 . If $Y$ has a lognormal distribution, the Type I error probability is .25 and .035 using a $20 \%$ trimmed mean and mean, respectively. So, it is evident that when $Y$ has a sufficiently skewed distribution, using a $20 \%$ trimmed mean can be disastrous.

Now consider the problem of testing (2) with the wild bootstrap method First consider the exact same conditions considered by Wilcox (2001), where observations were generated with either $m(X)=X$ or $m(X)=X^{2}$. Table 2 shows the estimated probability of a Type I error when testing at the .05 level with $n=30$. Again the estimates are based on 1,000 replications with $\mathrm{B}=500$. (From Robey and Barcikowski, 1992, 1,000 replications is sufficient from a power point of view. More specifically, if the hypothesis that the actual Type I error rate is .05 is tested and if one wants power to be .9 when testing at the .05 level and the true $\alpha$ value differs from .05 by .025 , then 976 replications are required.)
Table 2. Type I Error Rates, $\mathrm{n}=30, \alpha=.05$

\begin{tabular}{|c|c|c|c|c|c|}
\hline \multicolumn{2}{|c|}{$\mathrm{X}$} & \multicolumn{2}{c|}{$\mathcal{E}$} & \multicolumn{1}{c}{} \\
\cline { 1 - 1 } $\mathrm{g}$ & $\mathrm{h}$ & $\mathrm{g}$ & $\mathrm{h}$ & $m(X)=X$ & $m(X)=X^{2}$ \\
\hline 0.0 & 0.0 & 0.0 & 0.0 & .036 & .038 \\
\hline 0.0 & 0.0 & 0.0 & 0.2 & .040 & .032 \\
\hline 0.0 & 0.0 & 0.2 & 0.0 & .046 & .028 \\
\hline 0.0 & 0.0 & 0.2 & 0.2 & .041 & .028 \\
\hline 0.0 & 0.2 & 0.0 & 0.0 & .039 & .042 \\
\hline 0.0 & 0.2 & 0.0 & 0.2 & .039 & .034 \\
\hline 0.0 & 0.2 & 0.2 & 0.0 & .043 & .038 \\
\hline 0.0 & 0.2 & 0.2 & 0.2 & .043 & .032 \\
\hline 0.2 & 0.0 & 0.0 & 0.0 & .040 & .041 \\
\hline 0.2 & 0.0 & 0.0 & 0.2 & .037 & .025 \\
\hline 0.2 & 0.0 & 0.2 & 0.0 & .044 & .038 \\
\hline 0.2 & 0.0 & 0.2 & 0.2 & .033 & .027 \\
\hline 0.2 & 0.2 & 0.0 & 0.0 & .034 & .039 \\
\hline 0.2 & 0.2 & 0.0 & 0.2 & .033 & .035 \\
\hline 0.2 & 0.2 & 0.2 & 0.0 & .043 & .036 \\
\hline 0.2 & 0.2 & 0.2 & 0.2 & .040 & .034 \\
\hline
\end{tabular}

As indicated in Table 2, among all situations considered, the estimated probability of a Type I error ranged between .027 and .044 . From Wilcox (2006), when using the Theil-Sen estimator, the estimates ranged between .030 and .067. As for the method based on the Winsorized correlation, the estimates ranged between .021 and .050. Because generating observations from a chi-squared distribution with 1 degree of freedom, or a lognormal distribution, caused problems when using the wild bootstrap method with a $20 \%$ trimmed mean, these two distributions were also 
considered when using the wild bootstrap to test (2). The estimated probability of a Type I error for these two cases were .037 and .036, respectively.

A practical issue is how the power of the method compares to the power of the methods studied by Wilcox (2006). Checks revealed that the method can have more or less power depending on the nature of the heteroscedasticity. For example, if $Y=X^{2}+|.2 X| \varepsilon$, with both $\mathrm{X}$ and $\varepsilon$ having standard normal distributions, the wild bootstrap method has power .34, versus .09 and .04 when using the Winsorized correlation or the TheilSen estimator, respectively. But if $Y=X^{2}+|X+1| \varepsilon$, the estimated power for these three methods is $.39, .51$ and .35 . Currently, it is unclear how best to characterize the situations where the wild bootstrap method will have more or less power. All that can be said is that given some data, the choice of method can make a practical difference.

\section{Conclusion}

For a wide range of situations, inferences based on a $20 \%$ trimmed mean, rather than a mean, can have considerable practical value in terms of both Type I errors and power (Wilcox, 2003, 2005). But, it is evident that when testing (1) with a wild bootstrap, using a $20 \%$ trimmed mean can be disastrous. Perhaps there is some modification of the wild bootstrap that both corrects this problem and has some practical advantage over using means, but this remains to be seen.

\section{References}

Hastie, T. J. \& Tibshirani, R. J. (1990). Generalized additive models. New York: Chapman and Hall.

Hoaglin, D. C. (1985) Summarizing shape numerically: The g-and distributions. In D. Hoaglin, F. Mosteller and J. Tukey (Eds.) Exploring data tables, trends, and shapes. New York: Wiley.

Robey, R. R. \& Barcikowski, R. S. (1992). Type I error and the number of iterations in Monte Carlo studies of robustness. British Journal of Mathematical and Statistical Psychology, 45, 283-288.

Sen, P. K. (1968). Estimate of the regression coefficient based on Kendall's tau. Journal of the American Statistical Association, 63, 1379-1389.

Stute, W., Manteiga, W. G. \& Quindimil, M. P. (1998). Bootstrap approximations in model checks for regression. Journal of the American Statistical Association, 93, 141-149.

Theil, H. (1950). A rank-invariant method of linear and polynomial regression analysis. Indagationes Mathematicae, 12, 8591.

Wilcox, R. R. (2001). Detecting nonlinear associations plus comments on testing hypotheses about the correlation coefficient. Journal of Educational and Behavioral Statistics, 26, 73-84.

Wilcox, R. R. (2003). Applying contemporary statistical techniques testing. San Diego CA: Academic Press.

Wilcox, R. R. (2005). Introduction to robust estimation and hypothesis testing, (2nd Ed.) San Diego CA: Academic Press.

Wilcox, R. R. (2006). Testing the hypothesis of a homoscedastic error term in simple, nonparametric regression. Educational and Psychological Measurement, 66, 85-92. 


\title{
Regular Articles \\ Application of a New Procedure for Power Analysis and Comparison of the Adjusted Univariate and Multivariate Tests in Repeated Measures Designs
}

\author{
Sean W. Mulvenon \\ University of Arkansas \\ Kening Wang \\ University of Arkansas
}

\author{
M. Austin Betz \\ Arizona State University \\ Bruno Zumbo \\ University of British Columbia
}

A relationship between the multivariate and univariate noncentrality parameters in repeated measures designs was developed for the purpose of assessing the relative power of the univariate and multivariate approaches. An application is provided examining the use of repeated measures designs to evaluate student achievement in a $\mathrm{K}-12$ school system.

Key words: Repeated measures designs, adjusted degrees of freedom test, noncentrality parameter, sphericity.

\section{Introduction}

Repeated measures designs are used frequently by social and behavioral science researchers (Maxwell \& Delaney, 2004; Keselman, H. J., Huberty, Lix, Olejnik, Cribbie, Donahue, Kowalchuk, Lowman, Petoskey, Keselman, J. C., \& Levin, 1998). A major advantage of repeated measures designs is that subjects serve as their own controls, thus variability among the subjects due to individual differences is removed, and test results are more powerful. Various procedures can be used to do variance analysis in repeated measures designs. In

Sean W. Mulvenon is Professor of Educational Statistics and the Billingsley Chair for Educational Research and Policy Studies. Email him at seanm@uark.edu. M. Austin Betz is Professor Emeritus at Arizona Statue University. Kening Wang is a Research Associate in statistics and SAS programmer at the National Office for Research on Measurement and Evaluation Systems. Bruno D. Zumbo is a Professor of Educational Statistics at the University of British Columbia and a research fellow at the Assessment and Testing Research Unit at Carleton University. Email him at bruno.zumbo@ubc.ca addition to the traditional approaches, univariate and multivariate analyses, some methods such as Improved General Approximate method (Huynh, 1978), multivariate Welch (1951)/James (1951)type test ( $W J$ test), mixed model approach (Littell, Milliken, Stroup, \& Wolfinger, 1996), and empirical Bayes method (Boik, 1997) have also been studied and recommended. Guidelines for choosing an analysis strategy are generally based on whether the design is balanced or not (Keselman, 1998; Keselman, Algina, \& Kowalchuk, 2002). If group sizes are equal and there is no missing data, univariate and multivariate methods are frequently used by researchers and are recommended as appropriate statistical methods (Kirk, 1995; Morrison, 1990; Maxwell \& Delaney, 2004).

Both of the univariate and multivariate methods require the data satisfy certain assumptions: independent observations, multivariate normality, and homogeneous variance/covariance across groups. In addition to the above assumptions, the univariate analysis has the additional assumption of sphericity (Huynh \& Feldt, 1970; Rouanet \& Lépine, 1970). Sphericity refers to differences between any pair of repeated measures are equally variable. If sphericity is met, the univariate analysis has greater power than the multivariate analysis (due to a clear degrees of freedom advantage in the denominator), and it allows the 
use of fewer subjects than the multivariate analysis for equal power (Morrison, 1990). Unfortunately, the assumption of sphericity is not often met in the behavioral and social research (Davidson, 1972; McCall \& Appelbaum, 1973; Rogan, Keselman, \& Mendoza, 1979; Keselman, Huberty et al., 1998). If sphericity is not satisfied, the univariate analysis produces biased tests of significance (Box, 1954), and an adjusted degrees of freedom test, such as Greenhouse \& Geisser (1959) or Huynh \& Feldt (1976) test is suggested. The adjusted univariate analyses modify the $d f$ of the traditional $F$ statistic using a sample estimate of the sphericity parameter epsilon $(\epsilon)$. The $\epsilon$ is a measure of the degree of violation of the sphericity assumption, with perfect conformity to sphericity producing a $\in$ of 1.0 (Huynh \& Feldt, 1970).

Because the empirical evidence indicates that if the design is balanced, both the adjusted univariate and the multivariate approaches give the necessary control of Type I error (Davidson, 1972; Maxwell \& Arvey, 1982; Muller \& Barton, 1989; Keselman, J., Lix, \& Keselman, H., 1996), power becomes a critical factor in the selection between the adjusted univariate analysis and the multivariate analysis. Prospective power analysis will help researchers to determine an appropriate sample size to obtain the desired level of power to detect the meaningful differences that are hypothesized. Selecting an insufficient sample size will increase the risk of failing to detect an important difference when it may exist (Type II error). Conversely, selecting an excessive sample size may produce a statistically significant result, but one with limited meaningfulness due to small differences.

Sample size also affects the relative power of the adjusted univariate and multivariate tests. Without sphericity, multivariate tests may be more powerful than the adjusted univariate tests (Davidson, 1972). However, if sample sizes are small, the adjusted univariate analysis may still be more powerful than the multivariate analysis, because the estimators of the covariance parameters lack precision, and as a result, the power of the multivariate analysis is low (Boik, 1981). As sample size increases, the power of the multivariate test improves and can be greater than the power of the adjusted univariate test (Boik, 1997).

Power analysis and minimum sample size calculations are needed for choosing the most suitable method under different conditions. Using the expansions of Fujikoshi (1973), Sugiura (1973), or Vander Merwe and Crowther (1984), power of the multivariate tests can be computed. Muller and Peterson (1984) provided power approximations of the multivariate tests. For the adjusted univariate tests, Muller and Barton (1989, 1991) provided power approximations based on the expected value approximations for the epsilon $(\epsilon)$ estimator. Vonesh and Schork (1986) presented a statistical methodology for determining the minimum sample size for the within-subjects repeatedmeasures design.

They developed a formulae for calculating the multivariate noncentrality parameter, subject to constraint $\Delta=\left|\mu_{\mathrm{j}}-\mu_{\mathrm{k}}\right|$, which represents a minimal difference between any pair of treatment means. Rochon (1991) extend the procedures of Vonesh and Schork to the between-subjects repeated-measures design when there are only two treatment groups under consideration. All of the above researches provide strong basis for the purpose of the current paper, that is, to develop a relationship between the multivariate and univariate noncentrality parameters for assessing the relative power of the univariate and multivariate approaches in repeated measures designs. A major goal of this article is to compare the statistical power of the univariate and multivariate procedures and provide a method for selecting an appropriate sample size, given a desired effect size and level of power, when researchers are developing a study.

Theoretical Foundations and Statistics

The Model and Hypothesis

The usual general linear model with $g$ betweensubject groups and one within-subject repeated measures factor having $p$ levels can be written as follows:

$$
\mathrm{Y}=\mathrm{XM}+\mathrm{E}
$$


Where $\mathrm{Y}$ is an $N \times p$ matrix from $N$ subjects; $\mathrm{X}$ is an $N \times g$ between-subject design matrix; $\mathrm{M}$ is an $g \times p$ parameters matrix; and $\mathrm{E}$ is an $N \times p$ matrix of random errors. The rows of $E$ are assumed to be independently and identically distributed as $N_{p}(0, \Sigma)$, where $\Sigma$ is a $p \times p$ positive definite covariance matrix.

The general linear multivariate model hypothesis has the usual form:

$$
H_{0}: \Theta=C M U=\Theta_{0}
$$

where $C$ is an $a \times g$ between-subject contrasts; $M$ is an $g \times p$ parameters matrix; $U$ is an $p \times b$ within-subject contrasts; and $\Theta$ is an $a \times b$ secondary parameters matrix. Without loss of generality, assume $\Theta_{0}=0$. Define $\Sigma_{*}=\mathrm{U}^{\prime} \Sigma \mathrm{U}$, which is a covariance matrix of $\operatorname{row}_{\mathrm{i}}(E U)^{\prime}$. Also define $\omega=\left(\Theta-\Theta_{0}\right)^{\prime}\left[C\left(X^{\prime} X\right)^{-1} C^{\prime}\right]^{-1}\left(\Theta-\Theta_{0}\right)$, which is an unscaled noncentrality matrix. Then, the scaled noncentrality matrix $(\Omega)$ and its trace $\left(\delta_{\mathrm{m}}\right)$ are given by $\Omega=\omega \Sigma^{-1}$ and $\delta_{\mathrm{m}}=\operatorname{tr}(\Omega)$ respectively. Using two theorems (Theorem 2, p30; and Theorem 3, p31) from Magnus and Neudecker (1988), the general form of the noncentrality parameter for the F-distribution can be written as:

$$
\begin{aligned}
\delta_{\mathrm{m}}= & \left(\operatorname{vec} \mathrm{M}^{\prime}\right)^{\prime}\left(\mathrm{C}^{\prime} \otimes U\right) \\
& {\left[\left[\mathrm{C}\left(\mathrm{X}^{\prime} \mathrm{X}\right)^{-1} \mathrm{C}^{\prime}\right]^{-1} \otimes \Sigma_{*}^{-1}\right] } \\
& \left(\mathrm{C} \otimes \mathrm{U}^{\prime}\right)\left(\mathrm{vec} \mathrm{M}^{\prime}\right)
\end{aligned}
$$

The hypothesis in (2) can be tested using the multivariate test. If using the HotellingLawley trace statistic, the noncentrality parameter has the form (Muller \& Peterson, 1984; Muller, LaVange, Ramey, \& Ramey, 1992):

$$
\begin{aligned}
\delta_{\mathrm{HLT}} & =(\mathrm{ab}) \cdot \mathrm{F}_{\mathrm{A}}(\mathrm{HLT}) \\
& =\frac{\mathrm{HLT}_{\mathrm{A}} / \mathrm{S}}{1 / \mathrm{df}_{\mathrm{d}}(\mathrm{HLT})}
\end{aligned}
$$

where $a=g-1, b=p-1, S=\min (a, b)$, and $d f$ ${ }_{d}(H L T)=S[(N-g)-b-1]+2$.

The hypotheses in (2) also can be tested using the adjusted univariate test. Additionally, for repeated measures designs, the univariate analysis is a simply by-product of the multivariate analysis (Wang, 1983). A Univariate noncentrality parameter can be derived from (3) and be expressed as:

$$
\delta_{u}=b \mathcal{E} \frac{\operatorname{tr}(\omega)}{\operatorname{tr}\left(\Sigma_{*}\right)}=\left(\frac{\operatorname{tr}\left(\Sigma_{*}\right)}{\operatorname{tr}\left(\Sigma_{*}^{2}\right)}\right) \cdot \operatorname{tr}(\omega)
$$

where

$$
\varepsilon=\frac{\operatorname{tr}^{2}\left(\Sigma_{*}\right)}{b \cdot \operatorname{tr}\left(\Sigma_{*}^{2}\right)}(\mathrm{Box}, 1954)
$$

The sphericity parameter $\epsilon(1 / b \leq \epsilon \leq 1)$ reflects a discrepancy from sphericity. If sphericity is not met $(\epsilon \neq 1), T_{\text {univariate }}^{2} \approx F(a b \in, b(N-g) \epsilon$, $\delta_{\mathrm{u}}$ ). The Greenhouse \& Geisser test uses the maximum likelihood estimator (MLE) $\hat{\varepsilon}$ to adjust the degrees of freedom of the univariate test.

Lower Bounds of Noncentrality Parameters

The noncentral $F$-distribution can be used for power and sample size calculations. The power associated with the $F$-test is a monotonically increasing function of the noncentrality parameter. In this subsection, minimizing of the noncentrality parameters, developing the lower bounds of the multivariate and univariate noncentrality parameters using the same constraints, and establishing a relationship between them are described.

As shown and demonstrated in the Appendix, for fixed $\epsilon>0$, subject to the constraint

$$
\Delta=\frac{\left|c^{\prime} M d\right|}{\sqrt{c^{\prime} c} \sqrt{d^{\prime} d}} \text { and } \bar{\theta}=\frac{\operatorname{tr}\left(\Sigma_{*}\right)}{b}
$$

the lower bounds of multivariate and univariate noncentrality parameters can be expressed as:

$$
\delta_{m}^{*}=\frac{N \Delta^{2}}{d^{\prime} \Sigma d} \quad \text { and } \quad \delta_{u}^{*}=\frac{\varepsilon N \Delta^{2}}{\bar{\theta}}
$$


where $c$ and $d$ are arbitrary vectors of contrast coefficients. $\delta_{m} \geq \delta_{m}^{*}$, and $\delta_{u} \geq \delta_{u}^{*}$. The value of $\bar{\theta}$ is represented as:

$$
\bar{\theta}=\frac{\operatorname{tr}\left(\Sigma_{*}\right)}{b}=\frac{\sum_{i=1}^{b} \lambda_{i}}{b}
$$

which is the mean eigenvalue of the error matrix $\Sigma_{*}$.

From (7) and (8), the relationship between the lower bounds of multivariate and univariate noncentrality parameters can be expressed as:

$$
\delta_{u}^{*}=\varepsilon^{*} \delta_{m}^{*}
$$

where $\varepsilon^{*}=\varepsilon \phi, \phi=\frac{\sigma_{0}^{2}}{\bar{\theta}}$, and $\phi$ represents the bias ratio.

As shown by Boik (1981), $\sigma_{0}^{2}=d^{\prime} \Sigma d$ is the experimental error of contrast among the $p$-repeated measures, and $\bar{\theta}$ is the average experimental error of any set of $b=p-1$ orthonormal contrasts. Further, when the sphericity assumption is met $(\epsilon=1.0), \quad \phi$ will always equal unity (Boik, 1981). For fixed $\epsilon$, the bias ratio has a range of values, $\phi_{\min }<\phi<\phi$ max. The upper and lower limits of $\phi$ are given by Boik (1981):

$$
\begin{gathered}
\phi_{\max }=1+\mathrm{B}, \text { when } 1 / \mathrm{b} \leq \epsilon \leq 1, \\
1-B \quad \text { when } \frac{b-1}{b} \leq \varepsilon \leq 1.0 \\
0 \text { when } \frac{1}{b} \leq \varepsilon \leq \frac{b-1}{b}
\end{gathered}
$$

where $B=\left[\frac{(b-1)(1-\varepsilon)}{\varepsilon}\right]^{1 / 2}$. If $\epsilon=1$, then $B=$ 0 , and $\phi_{\min }=\phi_{\max }=1$.

$$
\text { Because } \varepsilon^{*}=\varepsilon \phi \text { and } \phi_{\min }<\phi<\phi_{\max },
$$

the maximum and minimum values of multiplier $\mathcal{E}^{*}$ can be obtained as:

$$
\varepsilon_{\max }^{*}=\varepsilon(1+B)=\varepsilon+\sqrt{(b-1)(1-\varepsilon) \varepsilon}
$$

and

$$
\varepsilon_{\min }^{*}=\varepsilon(1-B)=\varepsilon-\sqrt{(b-1)(1-\varepsilon) \varepsilon}
$$

$\varepsilon_{\text {max }}^{*}$ varies between a minimum of 1 , when $\epsilon=$ $1 / b$ or $\epsilon=1$; and a maximum of $\frac{1}{2}(1+\sqrt{b})$, when $\varepsilon=\frac{1}{2}\left(1+\sqrt{\frac{1}{b}}\right) . \quad \varepsilon_{\min }^{*}$ varies between a minimum of 0 , when $\epsilon=(b-1) / b$; and a maximum of 1 , when $\epsilon=1$. For example, let

$$
\begin{aligned}
& \mathcal{E}=\frac{1}{2}\left(1+\sqrt{\frac{1}{b}}\right) \text { and }(b-1) / b<\epsilon \leq 1, \text { then } \\
& \varepsilon_{\max }^{*}=\frac{1}{2}(1+\sqrt{\mathrm{b}}) \text {, and } \varepsilon_{\min }^{*}=\frac{1}{2}\left[1-(\mathrm{b}-2) \sqrt{\frac{1}{\mathrm{~b}}}\right] . \text { Let }
\end{aligned}
$$
$\varepsilon=\frac{1}{2}\left(1+\sqrt{\frac{1}{b}}\right)$, but if $\epsilon$ is in the interval of $[1 /$ $b,(b-1) / b]$, then $\varepsilon_{\max }^{*}=\frac{1}{2}(1+\sqrt{b})$, but $\varepsilon_{\min }^{*}=0$, because of the restrictive nature of the bias ratio $\phi_{\min }=0$, when $1 / b \leq \epsilon \leq(b-1) / b$.

Best and Worst Case Scenarios for the Univariate Test

An examination of (9), (11), and (12) allows the determination of best and worst case scenarios for the lower bound of univariate noncentrality parameter $\left(\delta_{u}^{*}\right)$ by substituting the maximum and minimum values of $\mathcal{E}^{*}$ in (9). The best case scenario for $\delta_{u}^{*}$ is 


$$
\delta_{u_{-}}^{*} \text { best }=[\varepsilon+\sqrt{(b-1)(1-\varepsilon) \varepsilon}] \cdot \delta_{m}^{*}
$$

\section{Because}

$$
1 \leq \varepsilon_{\max }^{*}=[\varepsilon+\sqrt{(b-1)(1-\varepsilon) \varepsilon}] \leq \frac{1}{2}(1+\sqrt{b})
$$

this suggests the best case scenario for the univariate case, which means the minimum power of the univariate test will generally exceed the minimum power of the multivariate test.

However, substituting $\varepsilon_{\min }^{*}$ in (9) yields the worst case scenario:

$$
\delta_{u_{-}}^{*} \text { worst }=[\varepsilon-\sqrt{(b-1)(1-\varepsilon) \varepsilon}] \cdot \delta_{m}^{*}
$$

Because

$$
0 \leq \varepsilon_{\min }^{*}=[\varepsilon-\sqrt{(b-1)(1-\varepsilon) \varepsilon}] \leq 1,
$$

this suggests the worst case scenario for the univariate case, which means the minimum power of the univariate test will be generally lower than the minimum power of the multivariate test.

\section{Univariate versus Multivariate}

Power Analysis and Minimum Sample Size Calculation

For computing the minimum necessary sample size to obtain a desired level of power in the multivariate case, Vonesh and Schork (1986) presented a statistic method, and Rochon (1991) extended it to the between-subjects repeatedmeasures design. If let $\Sigma$ to be a positive covariance matrix, which means $\rho_{\mathrm{jk}} \geq 0$ for $\mathrm{j}<\mathrm{k}$; and let $\sigma_{\max }^{2}$ represents the largest variance, then the lower bound of $\delta_{\mathrm{m}}$ can be approximated:

$$
\delta_{m}^{*}=\frac{N \Delta^{2}}{2 \sigma_{\max }^{2}\left(1-\rho_{\min }\right)} \leq \delta_{m}
$$

where $\rho_{\min }=\min _{\mathrm{t}}\left\{\rho_{\mathrm{t}}\right\}$. This would guarantee power greater than the normal level. Using the above approximation, the minimum sample size for the multivariate case can be determined by utilizing

$$
1-\beta_{m}=P\left[F\left(d f_{n}, d f_{d} ; \delta_{m}\right)>F_{\alpha}\left(d f_{n}, d f_{d}\right)\right]
$$

To determine sample sizes in the univariate case when the assumption of sphericity is untenable, the following is used

$$
\begin{aligned}
& 1-\beta_{\mathrm{u}}= \\
& \mathrm{P}\left[\begin{array}{l}
\mathrm{F}\left(\mathrm{ab} \varepsilon, \mathrm{b}(\mathrm{N}-\mathrm{g}) \varepsilon ; \delta_{\mathrm{u}}\right) \\
>\mathrm{F}_{\alpha}(\mathrm{ab} \varepsilon, \mathrm{b}(\mathrm{N}-\mathrm{g}) \varepsilon)
\end{array}\right]
\end{aligned}
$$

where $\delta_{u}$ and $\epsilon$ are given in (5).

In order to determine the minimum sample sizes in the univariate case, applying (13) and (14), the upper (best case) and lower (worst case) limits of the $\delta_{u}^{*}$ can be obtained, if $\epsilon$ and $\delta_{m}^{*}$ are known. $\delta_{m}^{*}$ can be approximated by (15). In general, however, it will not be known. In the present context, suppose $\varepsilon=\frac{1}{2}\left(1+\sqrt{\frac{1}{b}}\right)$, then if $\epsilon$ is in the interval $[(b-1) / b, 1]$, the upper (best case scenario) limit of the $\delta_{u}^{*}$ can be obtained as $\delta_{u}^{*}=\frac{1}{2}(1+\sqrt{b}) \delta_{m}^{*}$; and the lower (worst case) limit of the $\delta_{u}^{*}$ can be obtained as $\delta_{u}^{*}=\frac{1}{2}\left[1-(b-2) \sqrt{\frac{1}{b}}\right] \delta_{m}^{*} . \quad$ This enables determination of the upper and lower limits of the $\delta_{u}^{*}$ for simulation study of the best and the worst case scenarios for the univariate case.

Simulation Procedure

The simulation was conducted in SAS/IML and SAS program is available from the author on request. The process of minimum sample size determination, or statistical power 
analysis, involves the following four components: Type I error $(\alpha)$, power $(1-\beta)$, effect size $\Delta$ or standardized effect size $\Delta_{*}$, and the minimum correlation $\rho_{\text {min }}$. Desired statistical power is set to be 0.80 in this study. The $80 \%$ level of power is based on Cohen's wellinformed conjecture that the rate of Type II error should be about fourfold that of Type I error (Cohen, 1992). Detailed procedures were given as the following steps:

1) Specify the desired power $(1-\beta)$ to be 0.8 , and $\alpha=0.05$. Set all possible combinations of the following values: $p=3,4 ; g=2,3,4 ; \rho_{\min }=$ $.1, .2, \ldots, .9$ by .1 ; and $\Delta_{*}=.2, .3, \ldots, 1.5$ by .1 .

2) The necessary sample size $\left(\mathrm{N}_{\mathrm{m}}\right)$ was computed for all the above combinations using the multivariate procedure.

3) Using the upper limit of the $\delta_{u}^{*}$ $\left(\delta_{u}^{*}=\frac{1}{2}(1+\sqrt{b}) \delta_{m}^{*}\right)$ to calculate the necessary sample size $\left(\mathrm{N}_{\mathrm{u}}\right)$ for the best case scenario of the univariate procedure.

4) Using the lower limit of the $\delta_{u}^{*}$ $\left(\delta_{u}^{*}=\frac{1}{2}\left[1-(b-2) \sqrt{\frac{1}{b}}\right] \delta_{m}^{*}\right)$ to calculate the necessary sample size $\left(\mathrm{N}_{1}\right)$ for the worst case scenario of the univariate procedure.

\section{Monte Carlo}

Table 1 contains a selection of the results from the univariate and multivariate simulations. A comparison of the minimum sample size estimates between the multivariate procedure and the univariate

procedure for the best and the worst case scenarios indicates some clear trends.

First, when the effect size is small, for example, $\Delta_{*} \leq 0.4$, and if minimum correlation is also small, then the minimum sample sizes of the multivariate procedure $\left(\mathrm{N}_{\mathrm{m}}\right)$ are much larger than the univariate procedure for the best case scenario $\left(\mathrm{N}_{\mathrm{u}}\right)$. This trend indicates that when the above conditions hold, researchers need to consider using the univariate procedure, especially when sample sizes anticipated for the study may be small. This result is consistent with Boik's (1997) conclusion that if sample sizes are small, the adjusted univariate analysis may still be more powerful than the multivariate analysis. When the design becomes more complex, this trend is more obvious, because the minimum sample sizes generally increase as the number of groups and repeated trials increases (due to space considerations, results of other combinations of groups and trials are not included in the table).

Second, when the effect size is large, for example, $\Delta_{*} \geq 0.8$, the multivariate procedure could generally be recommended due to small minimum sample sizes. Simulation results indicate that there is small degree of divergence of the minimum sample sizes between the multivariate procedure $\left(\mathrm{N}_{\mathrm{m}}\right)$ and the univariate procedure for the best case scenario $\left(\mathrm{N}_{\mathrm{u}}\right)$.

Third, when the effect size is moderate, for example, $0.4<\Delta_{*}<0.8$, the minimum correlation $\left(\rho_{\min }\right)$ will provide valuable information in selecting between the univariate and multivariate procedures. If $\rho_{\min }$ is large, for example, $\rho_{\min } \geq .80$, then the univariate procedure is recommended; otherwise, researchers need to consider using the multivariate procedure.

Upon inspection of this table, a pattern was also found for the relationship between the minimum sample size and the effect size. For fixed power, the minimum sample size generally decreases as the effect size increases. Thus, if sample size is fixed, larger treatment differences will provide greater power. The same pattern can be observed for the relationship between the minimum sample size and the minimum correlation. 
Table 1 . Necessary sample size estimates by groups, trials, standardized effect size $\left(\Delta_{*}\right)$, and minimum correlation $\left(\rho_{\min }\right)$ for desired power $=.80$ at $\alpha=.05$

\begin{tabular}{|c|c|c|c|c|c|c|}
\hline Groups & trials & $\Delta *$ & $\rho_{\min }$ & $\mathrm{N}_{\mathrm{m}}$ & $\mathrm{N}_{\mathrm{u}}$ & $\mathrm{N}_{1}$ \\
\hline 2 & 3 & 0.2 & 0.1 & 227 & 9 & $>500$ \\
\hline 2 & 3 & 0.2 & 0.2 & 202 & 9 & $>500$ \\
\hline 2 & 3 & 0.2 & 0.3 & 177 & 9 & $>500$ \\
\hline 2 & 3 & 0.2 & 0.4 & 152 & 9 & $>500$ \\
\hline 2 & 3 & 0.2 & 0.5 & 127 & 9 & $>500$ \\
\hline 2 & 3 & 0.2 & 0.6 & 103 & 8 & $>500$ \\
\hline 2 & 3 & 0.2 & 0.7 & 78 & 8 & $>500$ \\
\hline 2 & 3 & 0.2 & 0.8 & 53 & 8 & $>500$ \\
\hline 2 & 3 & 0.2 & 0.9 & 28 & 7 & $>500$ \\
\hline 2 & 3 & 0.3 & 0.1 & 103 & 8 & $>500$ \\
\hline 2 & 3 & 0.3 & 0.2 & 92 & 8 & $>500$ \\
\hline 2 & 3 & 0.3 & 0.3 & 81 & 8 & $>500$ \\
\hline 2 & 3 & 0.3 & 0.4 & 70 & 8 & $>500$ \\
\hline 2 & 3 & 0.3 & 0.5 & 59 & 8 & $>500$ \\
\hline 2 & 3 & 0.3 & 0.6 & 48 & 7 & $>500$ \\
\hline 2 & 3 & 0.3 & 0.7 & 37 & 7 & $>500$ \\
\hline 2 & 3 & 0.3 & 0.8 & 26 & 7 & $>500$ \\
\hline 2 & 3 & 0.3 & 0.9 & 15 & 6 & $>500$ \\
\hline 2 & 3 & 0.4 & 0.1 & 59 & 8 & $>500$ \\
\hline 2 & 3 & 0.4 & 0.2 & 53 & 8 & $>500$ \\
\hline 2 & 3 & 0.4 & 0.3 & 47 & 8 & $>500$ \\
\hline 2 & 3 & 0.4 & 0.4 & 41 & 7 & $>500$ \\
\hline 2 & 3 & 0.4 & 0.5 & 35 & 7 & $>500$ \\
\hline 2 & 3 & 0.4 & 0.6 & 28 & 7 & $>500$ \\
\hline 2 & 3 & 0.4 & 0.7 & 22 & 6 & $>500$ \\
\hline 2 & 3 & 0.4 & 0.8 & 16 & 6 & $>500$ \\
\hline 2 & 3 & 0.4 & 0.9 & 10 & 5 & $>500$ \\
\hline 2 & 3 & 0.5 & 0.1 & 39 & 7 & $>500$ \\
\hline 2 & 3 & 0.5 & 0.2 & 35 & 7 & $>500$ \\
\hline 2 & 3 & 0.5 & 0.3 & 31 & 7 & $>500$ \\
\hline 2 & 3 & 0.5 & 0.4 & 27 & 7 & $>500$ \\
\hline 2 & 3 & 0.5 & 0.5 & 24 & 6 & $>500$ \\
\hline 2 & 3 & 0.5 & 0.6 & 20 & 6 & $>500$ \\
\hline 2 & 3 & 0.5 & 0.7 & 16 & 6 & $>500$ \\
\hline 2 & 3 & 0.5 & 0.8 & 12 & 5 & $>500$ \\
\hline 2 & 3 & 0.5 & 0.9 & 8 & 5 & 26 \\
\hline 2 & 3 & 0.6 & 0.1 & 28 & 7 & $>500$ \\
\hline 2 & 3 & 0.6 & 0.2 & 26 & 7 & $>500$ \\
\hline 2 & 3 & 0.6 & 0.3 & 23 & 6 & $>500$ \\
\hline 2 & 3 & 0.6 & 0.4 & 20 & 6 & $>500$ \\
\hline 2 & 3 & 0.6 & 0.5 & 18 & 6 & $>500$ \\
\hline
\end{tabular}


MULVENON, WANG, ZUMBO, \& BETZ

\begin{tabular}{|c|c|c|c|c|c|c|}
\hline 2 & 3 & 0.6 & 0.6 & 15 & 6 & $>500$ \\
\hline 2 & 3 & 0.6 & 0.7 & 12 & 5 & $>500$ \\
\hline 2 & 3 & 0.6 & 0.8 & 10 & 5 & $>500$ \\
\hline 2 & 3 & 0.6 & 0.9 & 7 & 5 & 8 \\
\hline 2 & 3 & 0.7 & 0.1 & 22 & 6 & $>500$ \\
\hline 2 & 3 & 0.7 & 0.2 & 20 & 6 & $>500$ \\
\hline 2 & 3 & 0.7 & 0.3 & 18 & 6 & $>500$ \\
\hline 2 & 3 & 0.7 & 0.4 & 16 & 6 & $>500$ \\
\hline 2 & 3 & 0.7 & 0.5 & 14 & 5 & $>500$ \\
\hline 2 & 3 & 0.7 & 0.6 & 12 & 5 & $>500$ \\
\hline 2 & 3 & 0.7 & 0.7 & 10 & 5 & $>500$ \\
\hline 2 & 3 & 0.7 & 0.8 & 8 & 5 & 37 \\
\hline 2 & 3 & 0.7 & 0.9 & 6 & 5 & 6 \\
\hline 2 & 3 & 0.8 & 0.1 & 18 & 6 & $>500$ \\
\hline 2 & 3 & 0.8 & 0.2 & 16 & 6 & $>500$ \\
\hline 2 & 3 & 0.8 & 0.3 & 15 & 5 & $>500$ \\
\hline 2 & 3 & 0.8 & 0.4 & 13 & 5 & $>500$ \\
\hline 2 & 3 & 0.8 & 0.5 & 12 & 5 & $>500$ \\
\hline 2 & 3 & 0.8 & 0.6 & 10 & 5 & $>500$ \\
\hline 2 & 3 & 0.8 & 0.7 & 9 & 5 & 63 \\
\hline 2 & 3 & 0.8 & 0.8 & 7 & 5 & 11 \\
\hline 2 & 3 & 0.8 & 0.9 & 6 & 5 & 5 \\
\hline 2 & 3 & 0.9 & 0.1 & 15 & 6 & $>500$ \\
\hline 2 & 3 & 0.9 & 0.2 & 14 & 5 & $>500$ \\
\hline 2 & 3 & 0.9 & 0.3 & 13 & 5 & $>500$ \\
\hline 2 & 3 & 0.9 & 0.4 & 11 & 5 & $>500$ \\
\hline 2 & 3 & 0.9 & 0.5 & 10 & 5 & $>500$ \\
\hline 2 & 3 & 0.9 & 0.6 & 9 & 5 & $>500$ \\
\hline 2 & 3 & 0.9 & 0.7 & 8 & 5 & 14 \\
\hline 2 & 3 & 0.9 & 0.8 & 7 & 5 & 6 \\
\hline 2 & 3 & 0.9 & 0.9 & 6 & 5 & 5 \\
\hline 2 & 3 & 1.0 & 0.1 & 13 & 5 & $>500$ \\
\hline 2 & 3 & 1.0 & 0.2 & 12 & 5 & $>500$ \\
\hline 2 & 3 & 1.0 & 0.3 & 11 & 5 & $>500$ \\
\hline 2 & 3 & 1.0 & 0.4 & 10 & 5 & $>500$ \\
\hline 2 & 3 & 1.0 & 0.5 & 9 & 5 & $>500$ \\
\hline 2 & 3 & 1.0 & 0.6 & 8 & 5 & 26 \\
\hline 2 & 3 & 1.0 & 0.7 & 7 & 5 & 10 \\
\hline 2 & 3 & 1.0 & 0.8 & 6 & 5 & 6 \\
\hline 2 & 3 & 1.0 & 0.9 & 6 & 5 & 5 \\
\hline 2 & 3 & 1.1 & 0.1 & 11 & 5 & $>500$ \\
\hline 2 & 3 & 1.1 & 0.2 & 11 & 5 & $>500$ \\
\hline 2 & 3 & 1.1 & 0.3 & 10 & 5 & $>500$ \\
\hline 2 & 3 & 1.1 & 0.4 & 9 & 5 & $>500$ \\
\hline 2 & 3 & 1.1 & 0.5 & 8 & 5 & 49 \\
\hline 2 & 3 & 1.1 & 0.6 & 8 & 5 & 9 \\
\hline 2 & 3 & 1.1 & 0.7 & 7 & 5 & 7 \\
\hline 2 & 3 & 1.1 & 0.8 & 6 & 5 & 5 \\
\hline 2 & 3 & 1.1 & 0.9 & 5 & 5 & 5 \\
\hline
\end{tabular}




\begin{tabular}{|c|c|c|c|c|c|c|}
\hline 2 & 3 & 1.2 & 0.1 & 10 & 5 & $>500$ \\
\hline 2 & 3 & 1.2 & 0.2 & 10 & 5 & $>500$ \\
\hline 2 & 3 & 1.2 & 0.3 & 9 & 5 & $>500$ \\
\hline 2 & 3 & 1.2 & 0.4 & 8 & 5 & 63 \\
\hline 2 & 3 & 1.2 & 0.5 & 8 & 5 & 10 \\
\hline 2 & 3 & 1.2 & 0.6 & 7 & 5 & 8 \\
\hline 2 & 3 & 1.2 & 0.7 & 6 & 5 & 6 \\
\hline 2 & 3 & 1.2 & 0.8 & 6 & 5 & 5 \\
\hline 2 & 3 & 1.2 & 0.9 & 5 & 5 & 5 \\
\hline 2 & 3 & 1.3 & 0.1 & 9 & 5 & $>500$ \\
\hline 2 & 3 & 1.3 & 0.2 & 9 & 5 & 100 \\
\hline 2 & 3 & 1.3 & 0.3 & 8 & 5 & 52 \\
\hline 2 & 3 & 1.3 & 0.4 & 8 & 5 & 11 \\
\hline 2 & 3 & 1.3 & 0.5 & 7 & 5 & 9 \\
\hline 2 & 3 & 1.3 & 0.6 & 7 & 5 & 6 \\
\hline 2 & 3 & 1.3 & 0.7 & 6 & 5 & 5 \\
\hline 2 & 3 & 1.3 & 0.8 & 6 & 5 & 5 \\
\hline 2 & 3 & 1.3 & 0.9 & 5 & 5 & 5 \\
\hline 2 & 3 & 1.4 & 0.1 & 9 & 5 & 37 \\
\hline 2 & 3 & 1.4 & 0.2 & 8 & 5 & 37 \\
\hline 2 & 3 & 1.4 & 0.3 & 8 & 5 & 11 \\
\hline 2 & 3 & 1.4 & 0.4 & 7 & 5 & 11 \\
\hline 2 & 3 & 1.4 & 0.5 & 7 & 5 & 7 \\
\hline 2 & 3 & 1.4 & 0.6 & 6 & 5 & 6 \\
\hline 2 & 3 & 1.4 & 0.7 & 6 & 5 & 5 \\
\hline 2 & 3 & 1.4 & 0.8 & 6 & 5 & 5 \\
\hline 2 & 3 & 1.4 & 0.9 & 5 & 5 & 5 \\
\hline 2 & 3 & 1.5 & 0.1 & 8 & 5 & 26 \\
\hline 2 & 3 & 1.5 & 0.2 & 8 & 5 & 11 \\
\hline 2 & 3 & 1.5 & 0.3 & 7 & 5 & 11 \\
\hline 2 & 3 & 1.5 & 0.4 & 7 & 5 & 7 \\
\hline 2 & 3 & 1.5 & 0.5 & 7 & 5 & 6 \\
\hline 2 & 3 & 1.5 & 0.6 & 6 & 5 & 5 \\
\hline 2 & 3 & 1.5 & 0.7 & 6 & 5 & 5 \\
\hline 2 & 3 & 1.5 & 0.8 & 5 & 5 & 5 \\
\hline 2 & 3 & 1.5 & 0.9 & 5 & 5 & 5 \\
\hline
\end{tabular}

Notes: ${ }^{*}$ Due to space considerations, not all of the simulation results are included in the table, but they are available from the author on request.

${ }^{1} \mathrm{~N}_{\mathrm{m}}$ represents the necessary sample size computed using the multivariate procedure.

${ }^{2} \mathrm{~N}_{\mathrm{u}}$ represents the necessary sample size computed for the "best case scenario" of the univariate procedure.

${ }^{3} \mathrm{~N}_{1}$ represents the necessary sample size computed for the "worst case scenario" of the univariate procedure. 
A Case Study: Examination of Student Achievement Models

The most effective method to evaluate student achievement is to monitor change in performance between two or more points, or more specifically a repeated measures design. Recent "No Child Left Behind" (NCLB) legislation has contributed to a proliferation of growth models advocated as best methods to examine student achievement. A major concern with the use of most of these growth models is they assume large samples. However, within most traditional educational settings, sample sizes are relatively small. The use of the more traditional repeated measures designs, univariate or multivariate, may be more appropriate than hierarchical linear models or latent growth analyses.

\section{Case Study}

A recent and growing concern in $\mathrm{K}-12$ education has been the preparation of students to be successful in college. To address this issue, numerous studies have been completed that examine a student's high school record of achievement. However, education is a linear system, with students in theory, starting at grade one and progressing through the system to grade twelve. Additionally, in large school districts, a significant amount of concern is directed at the preparation of student's prior to high school. This case study examines three elementary schools and the difference in performance of students as they progress through this K-12 school system.

Each elementary school has grades kindergarten through fifth grade. Students were administered standardized reading tests in fifth, seventh (while at a middle school within the same district), and tenth grade. The primary research question, does elementary school you attended makes a difference in determining your starting point $\left(10^{\text {th }}\right.$ grade $)$ at the local high school? Table 2 provides a means and standard deviations of scaled scores for students from each of the three elementary schools. The small sample sizes reflect the issue of mobility of students, and in particular from School A, where annually 30 percent of students are identified as highly mobile.

A total of four analyses were completed: (1) School A versus School B, (2) School A versus School C, (3) School B versus School C, and (4) School A, School B, and School C. Table 3 provides the multivariate and univariate results in addition to retrospective and prospective power estimation values. The result demonstrated the importance of the univariate procedure with large effect sizes and a limited number of observations. Additionally, it is expected that standardized tests will have a strong correlation from year to year, which also contributes to the strength of the univariate procedure.

The case study was done as a study of convenience with data that represented the most common type of educational data used to complete school evaluations. In practice, analyses will be completed at the classroom, grade or school level in efforts to evaluate the impact of instructional practices or new educational interventions. The present case study does an excellent job of replicating the sample size and type of outcome variables (standardized test) that will be employed and demonstrated, in practice, why greater consideration needs to be given to use of the univariate method in repeated measures designs.

Table 2. Case Study: School Test Scores

\begin{tabular}{lllll}
\hline School & N & Score 1 & Score 2 & Score 3 \\
\hline & & & & \\
A & 12 & $614.3(37.6)$ & $653.5(34.6)$ & $685.2(29.5)$ \\
B & 27 & $666.1(35.1)$ & $680.6(26.1)$ & $713.0(27.6)$ \\
C & 25 & $653.4(34.3)$ & $678.8(27.9)$ & $704.8(29.7)$ \\
\hline
\end{tabular}


Table 3. Power Results for Univariate and Multivariate Comparisons

\begin{tabular}{|c|c|c|c|c|c|c|c|c|c|c|c|c|}
\hline \multirow{3}{*}{$\begin{array}{l}\text { Comparison } \\
\text { Schools } \\
\text { A vs. B }\end{array}$} & \multirow{3}{*}{$\begin{array}{l}\mathrm{N} \\
\\
\\
39\end{array}$} & \multirow{2}{*}{\multicolumn{3}{|c|}{$\begin{array}{c}\text { Retrospective Power } \\
\text { Delta GG_PWR M_PWR }\end{array}$}} & \multirow{3}{*}{$\begin{array}{c}\text { Univ_F } \\
7.51(.0015)\end{array}$} & \multirow{3}{*}{$\begin{array}{l}\text { Mult_F } \\
5.67(.0072)\end{array}$} & \multirow{3}{*}{$\begin{array}{l}\mathrm{N} \\
\\
\\
60\end{array}$} & \multicolumn{5}{|c|}{ Prospective Power } \\
\hline & & & & & & & & Delta & PM & PU & LU & UU \\
\hline & & 2.74 & 0.88 & 0.23 & & & & 0.5 & 0.81 & 0.81 & 0.53 & 0.90 \\
\hline A vs. C & 37 & 1.99 & 0.60 & 0.14 & $3.95(.0291)$ & $2.82(.0738)$ & 75 & 0.5 & 0.81 & 0.81 & 0.53 & 0.90 \\
\hline B vs. C & 52 & 1.40 & 0.36 & 0.17 & $1.95(>.05)$ & $1.79(>.05)$ & 78 & 0.5 & 0.81 & 0.81 & 0.53 & 0.90 \\
\hline A vs. B vs. C & 64 & 2.94 & 0.82 & 0.13 & $4.31(.0037)$ & $3.46(0.114)$ & 117 & 0.5 & 0.80 & 0.80 & 0.49 & 0.89 \\
\hline \multicolumn{8}{|c|}{$\begin{array}{l}\mathrm{N}=\text { sample size } \\
\text { Delta = effect size } \\
\text { GG_PWR = Univariate Power } \\
\text { M_PWR = Multivariate Power } \\
\text { Univ_F = Univariate F-test and alpha } \\
\text { Mult_F = Hotelling-Lawley Trace F-test and Alpha }\end{array}$} & \multicolumn{5}{|c|}{$\begin{array}{l}\mathrm{N}=\text { sample Size } \\
\text { Delta = Effect size } \\
\text { PM = Prospective Multivariate } \\
\text { Power } \\
\text { PU = Prospective Univariate } \\
\text { Power } \\
\text { LU = Lower Bound Univariate } \\
\text { Power } \\
\text { UU = Upper Bound Univariate } \\
\text { Power }\end{array}$} \\
\hline
\end{tabular}

\section{Conclusion}

The relationship between $\delta_{\mathrm{u}}{ }^{*}$ and $\delta_{\mathrm{m}}{ }^{*}$, which was developed in this study, provides a theoretical foundation for calculation of prospective power estimates for the univariate case in repeated measures designs. The relationship $\delta_{\mathrm{u}}^{*}=\epsilon^{*} \delta_{\mathrm{m}}^{*}$ can be employed to compute the univariate noncentrality parameter when the multivariate noncentrality parameter has been computed. This permits calculation of minimum sample size estimates and power analysis for the univariate procedure; and it provides a basis to address the question of which procedure to propose, univariate or multivariate, when designing a study which involves repeated measures.
Some researchers have compared the benefits of using either a multivariate or univariate procedure. Barcikowski and Robey (1984) and Stevens (2002) suggested that when conducting an exploratory analysis, both the adjusted univariate and multivariate procedures should be employed because each analysis could possibly reveal different treatment effects. O'Brien and Kaiser (1985) reported after a thorough review of the literature, under no conditions is one procedure uniformly more powerful. Results from this study indicate that generally, a researcher can use the multivariate procedure in most cases, as it does provide adequate power protection. However, the univariate procedure clearly provides greater 
protection under some specific conditions, indicated as best case scenarios, and therefore can be recommended for these conditions.

Maxwell and Delaney (1990) provided an empirical guideline that if the sample size $(N)$ is less than $p+10$ ( $p$ representing the number of repeated trials), the univariate procedure is recommended; otherwise, if $N \geq p+10$, the multivariate procedure is recommended. In the $2^{\text {nd }}$ edition, Maxwell \& Delaney (2004) modified the empirical guideline, and it is that the multivariate approach probably should be used if (1) $p \leq 4, \varepsilon \leq .90$, and $n \geq p+15$, or if (2) $5 \leq p$ $\leq 8, \varepsilon \leq .85$, and $n \geq p+20$. Results from this study indicate that the suggested guideline by Maxwell and Delaney works well, but only when the effect size and the minimal correlation are large.

In closing, this study effectively validates many of the recommendations of Boik, Maxwell \& Delaney, and others; additionally, it expands the window where univariate repeated measures designs should be employed.

\section{References}

Barcikowski, R. S., \& Robey, R. R. (1984). Decisions in single group repeated measures analysis: Statistical tests and three computer packages. The American Statistician, $38,148-150$.

Boik, R. (1981). A priori tests in repeated measures designs: Effects of nonsphericity. Psychological Bulletin, 86, 10841089.

Boik, R. J. (1997). Analysis of repeated measures under second-stage sphericity: An empirical Bayes approach. Journal of Educational and Behavioral Statistics, 22,155192.

Box, G.E.P. (1954). Some theorems on quadratic forms applied in the study of analysis of variance problems, II: Effects of inequality of variance and of correlation between errors in the two-way classification. Annals of Mathematical Statistics, 25, 484-498.

Cohen, J. (1992). A power primer. Psychological Bulletin, 112, 155-159.
Davidson, M. L. (1972). Univariate versus multivariate tests in repeated-measures experiments. Psychological Bulletin, 77, 446452.

Fujikoshi, Y. (1973). Asymptotic formulas for the distribution of three statistics for multivariate linear hypothesis. Annals of the Institute of Statistical Mathematics, 25, 423-437.

Greenhouse, S. W., \& Geisser, S. (1959). On methods in the analysis of profile data. Psychometrika, 24, 95-112.

Huynh, H. \& Feldt, L. (1970). Conditions under which mean square ratios in repeated measurements designs have exact $\mathrm{F}$ distributions. Journal of the American Statistical Association, 65, 1582-1589.

Huynh, H., \& Feldt, L. S. (1976). Estimation of the Box correction for degrees of freedom from sample data in randomized block and split plot designs. Journal of Educational Statistics, 1, 69-82.

Huynh, H. (1978). Some approximate tests for repeated measurement designs. Psychometrika, 43, 161-175.

James, G. S. (1951). The comparison of several groups of observations when the ratios of the population variances are unknown. Biometrika, 38, 324-329.

Kirk, R. E. (1995). Experimental design: Procedures for the behavioral sciences ( ${ }^{\text {rd }}$ ed.). Belmont, CA: Brooks/Cole.

Keselman, J., Lix, L., \& Keselman, H. (1996). The analysis of repeated measurement: A quantitative research synthesis. British Journal of Mathematical and Statistical Psychology, 49, 275-298.

Keselman, H. J. (1998). Testing treatment effects in repeated measures designs: An update for psychophysiological researchers. Psychophysiology, 35, 470-478.

Keselman, H. J., Huberty, C. J., Lix, L. M., Olejnik, S., Cribbie, R. A., Donahue, B., Kowalchuk, R. K., Lowman, L. L., Petoskey, M. D., Keselman, J. C., \& Levin, J. R. (1998). Statistical practices of educational researchers: An analysis of their ANOVA, MANOVA and ANCOVA analyses. Review of Educational Research, 68, 350-386. 
Keselman, H. J., Algina, J., Kowalchuk, R. K. (2002). A Comparison of Data Analysis Strategies for Testing Omnibus Effects in Higher-Order Repeated Measures Designs. Multivariate Behavioral Research, 37, 331-357.

Littell, R. C., Milliken, G. A., Stroup, W. W., \& Wolfinger, R. D. (1996). SAS system for mixed models. Cary, NC: SAS Institute.

Magnus, J. R., \& Neudecker, H. (1988). Matrix differential calculus. New York: Wiley.

Maxwell, S. E., \& Arvey, R. D. (1982).

Small sample profile analysis with many variables. Psychological Bulletin, 92, 778-785.

Maxwell, S. E., \& Delaney, H. D. (1990). Designing experiments and analyzing data. Belmont, CA: Wadsworth.

Maxwell, S. E., \& Delaney, H. D. (2004). Designing experiments and analyzing data $\left(2^{\text {nd }}\right.$ ed.). Mahwah, NJ: Lawrence Erlbaum Associates.

McCall, R. B. \& Appelbaum, M. I. (1973). Bias in the analysis of repeatedmeasures designs: Some alternative approaches. Child Development, 44, 401-415.

Morrison, D. F. (1990). Multivariate statistical methods ( $3^{\text {rd }}$ ed.). New York: McGraw-Hill.

Muller, K. E., \& Peterson, B. L. (1984). Practical methods for computing power in testing the multivariate general linear hypothesis. Computational Statistics and Data Analysis, 2, 143-158.

Muller, K. E., \& Barton, C. N. (1989). Approximate power for repeated measures ANOVA lacking sphericity. Journal of the American Statistical Association, 84, 549-555.

Muller, K. E., \& Barton, C. N. (1991). Correction of power formula for reported measures ANOVA lacking sphericity. Journal of the American Statistical Association, 86, 255256.

Muller, K. E, LaVange, L. M, Ramey, S. L, Ramey, C. T. (1992). Power calculations for general linear multivariate models including repeated measures applications. Journal of the American Statistical Association, 87:1209 1226.
O’Brian, R. G., \& Kaiser, M. K. (1985). MANOVA method for analyzing repeated measures designs: An extensive primer. Psychological Bulletin, 97, 316-333.

Rao, C. R. (1973). Linear statistical inference and its application ( $2^{\text {nd }}$ ed.). New York: Wiley.

Rochon, J. (1991). Sample size calculations for two-group repeated measures experiments. Biometrics, 47, 1383-1393.

Rogan, J. C., Keselman, H. J., \& Mendoza, J. L. (1979). Analysis of repeated measurements. British Journal of Mathematical and Statistical Psychology, 32, 269-286.

Rouanet, H. \& Lepine, D. (1970). Comparison between treatments in a repeated measures design: ANOVA and multivariate methods. British Journal of Mathematical and Statistical Psychology, 23, 147-163.

SAS Institute Inc. (1999). SAS Manual for Linear Models (Version 8). SAS Institute Inc. Cary, NC.

Stevens, J. P. (2002). Applied multivariate statistics for the social sciences $\left(4^{\text {th }}\right.$ ed.). Mahwah, NJ: Lawrence Erlbaum Associates.

Sugiura, N. (1973). Further asymptotic formulas for the non-null distributions of three statistics for multivariate linear hypothesis. Annals of the Institute of Statistical Mathematics, 25, 153-163.

Van der Merwe, L., \& Crowther, N. A. S. (1984). An approximation to the distribution of Hotelling's $T^{2}$-statistic. South African Statistical Journal, 18, 68-90.

Vonesh, E. F., \& Schork, M. A. (1986). Sample sizes in the multivariate analysis of repeated measurements. Biometrics, 42, 601610.

Wang, C. M. (1983). On the analysis of multivariate repeated measures designs. Communications in Statistics Part A- Theory and Methods, 12, 1647-1659.

Welch, B. (1951). On the comparison of several mean values: An alternative approach. Biometrika, 38, 330-336. 


\section{Appendix A}

Proof of Rationale for Lower Bounds of Noncentrality Parameters

In (3), for matrix $C(g-1 \times g), C C^{\prime}=\mathrm{I}_{\mathrm{g}-1}$; and for matrix $U(p-1 \times p), U U^{\prime}=\mathrm{I}_{\mathrm{p}-1}$. Define vectors of contrast coefficients $c(g \times 1)$ and $d(p \times 1)$ as $a^{\prime} C=c$ and $b U=d$, where $a$ is a vector $(g-1 \times 1)$ and $b$ is a vector $(p-1 \times 1)$. Thus, $\mathrm{a}^{\prime} \mathrm{C} \mathrm{M} \mathrm{U} \mathrm{b}=\mathrm{c}^{\prime} \mathrm{M} \mathrm{d}=\Delta$. Because $\Delta$ is a scaler, it can be expressed as the form: $b^{\prime}\left(U^{\prime} M^{\prime} C^{\prime}\right) a=\Delta$. Using the vec operator, we obtain:

$$
v e c\left[b^{\prime}\left(U^{\prime} M^{\prime} C^{\prime}\right) a\right]=\left(a^{\prime} C \otimes b^{\prime} U^{\prime}\right) v e c M^{\prime}
$$

Applying the constraints in (6), and using (1f.1.3) of Rao (1973, p. 60) to (3), the lower bound of $\delta_{m}$ is obtained by evaluating:

$$
\min _{a, b}\left\{\begin{array}{c}
\inf \delta_{m} \\
{\left[a^{\prime} C \otimes b^{\prime} U^{\prime}\right] \text { vec } M^{\prime}}
\end{array}\right\}=\frac{n \Delta^{2}}{d^{\prime} \Sigma d}=\delta_{m}^{*}
$$

For the lower bound of $\delta_{\mathrm{u}}$, subject to the same constraint as used in the multivariate case, the minimum of $\operatorname{tr}(\omega)$ is $n \Delta^{2}$, then replacing $\operatorname{tr}(\omega)$ with $n \Delta^{2}$, the lower bound of $\delta_{\mathrm{u}}$ is obtained as:

$$
\delta_{u}^{*}=b \varepsilon \cdot \frac{n \Delta^{2}}{\operatorname{tr}\left(\Sigma_{*}\right)}=\frac{\varepsilon n \Delta^{2}}{\bar{\theta}},
$$

where

$$
\bar{\theta}=\frac{\operatorname{tr}\left(\Sigma_{*}\right)}{b} .
$$




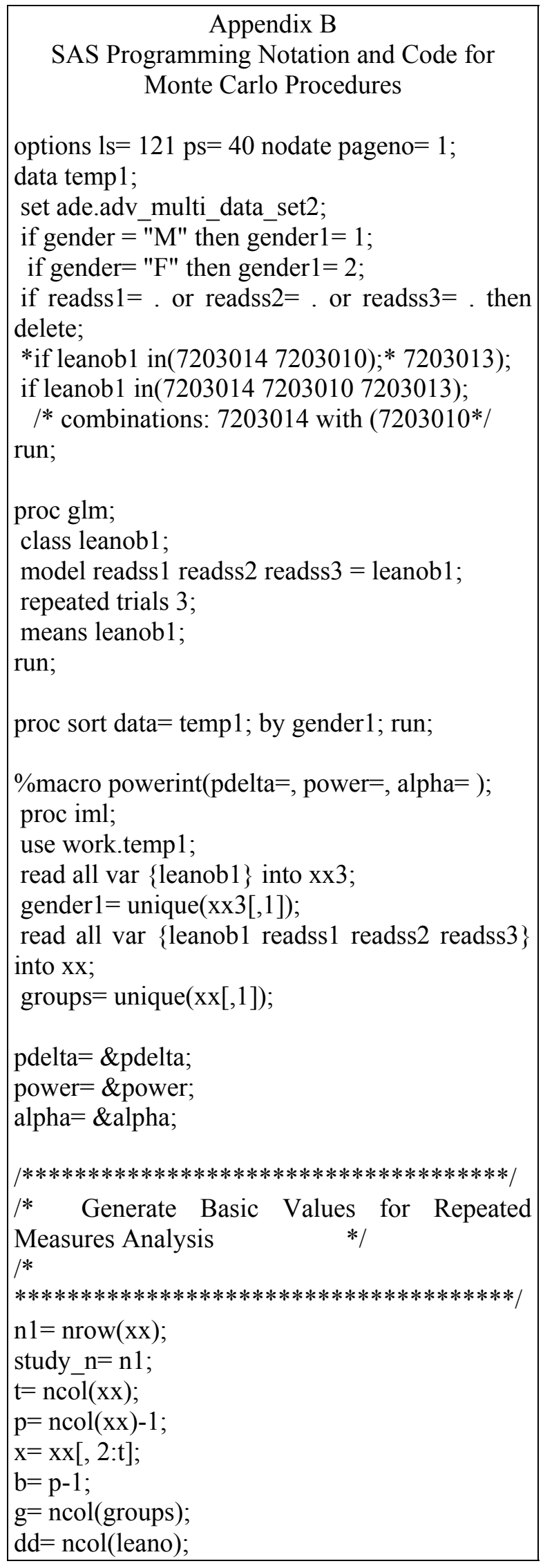

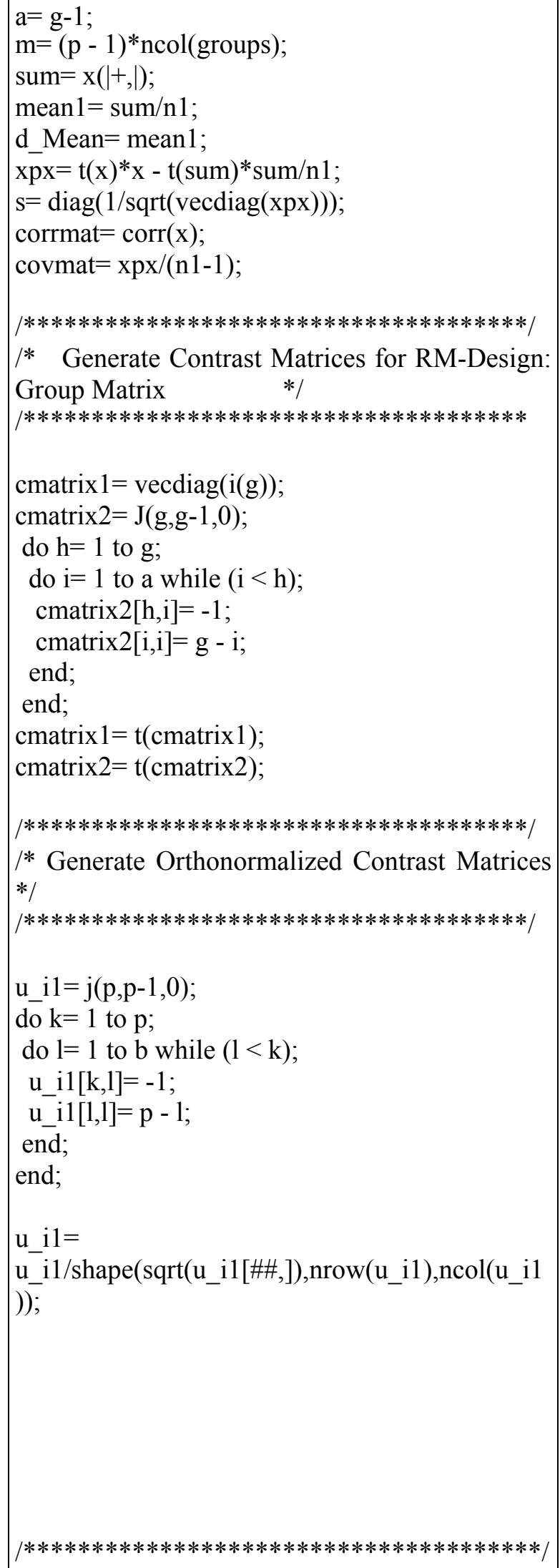




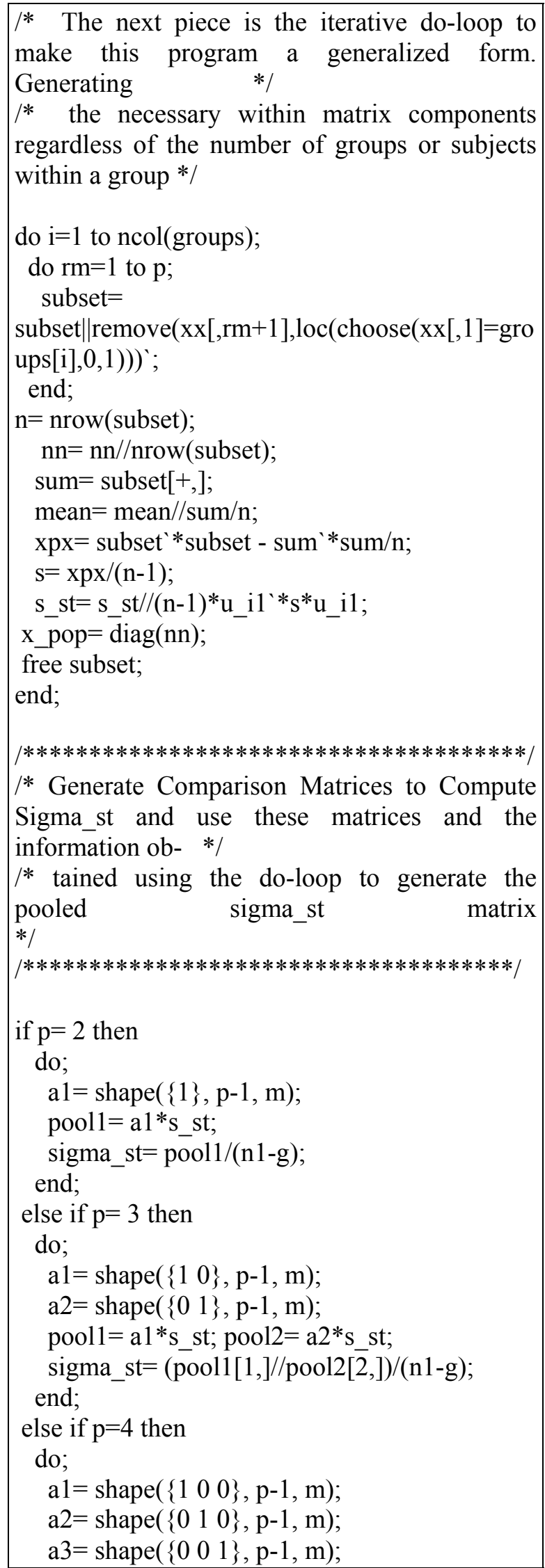

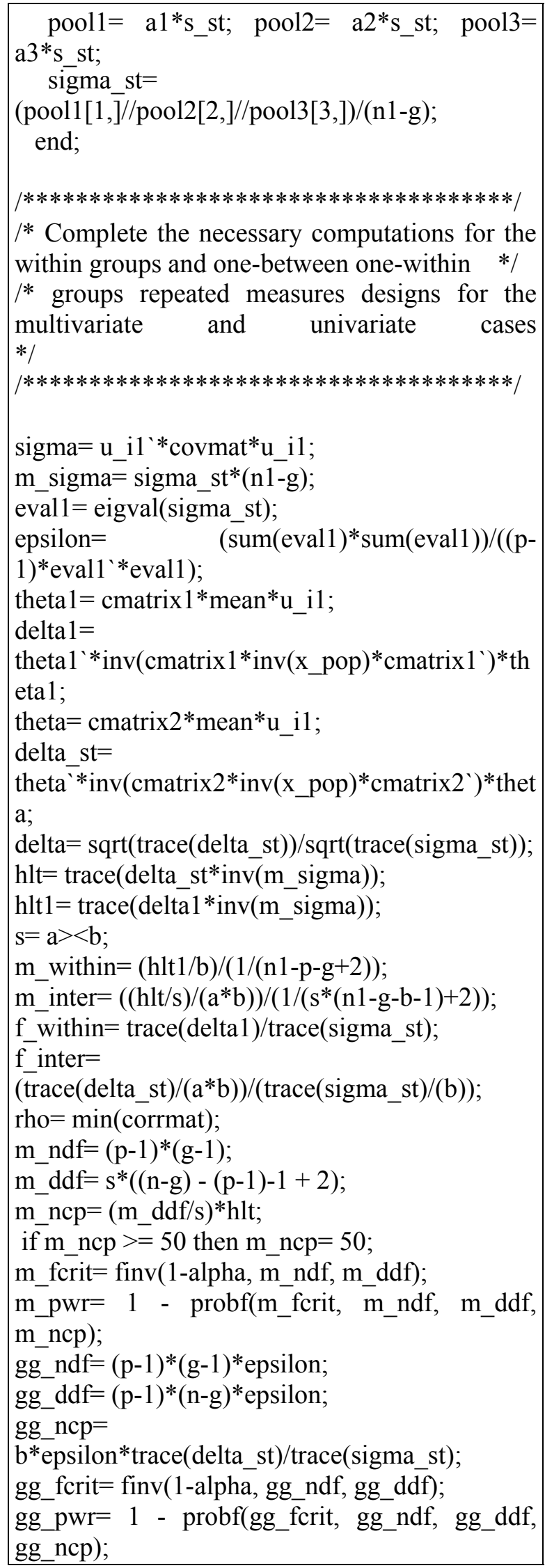
within groups and one-between one-within */ /* groups repeated measures designs for the multivariate and univariate cases 
do $\mathrm{n} 2=12$ to 1000 by 3 until (rm pwr $>$ power); $\mathrm{rm} \_\mathrm{ndf}=(\mathrm{p}-1) *(\mathrm{~g}-1)$;

$\mathrm{rm} \_\mathrm{ddf}=\mathrm{s}^{*}(\mathrm{n} 2-(\mathrm{p}-1)-1)+2$;

rm_ncp $=\left((\right.$ n $2 / g) *\left(\right.$ delta $\left.\left.^{* * 2}\right) / 2\right) /(2 *(1-$ rho $))$;

rm_fcrit $=$ finv $(1-$ alpha, rm_ndf, rm_ddf $)$;

rm_pwr $=1$ - probf(rm_fcrit, rm_ndf, rm_ddf, rm_ncp);

end;

lb_eps $=1 /(p-1)$;

do eps1_str $=1$ b_eps to 0.999 by .001 until (rgg_pwr $>=$ rm_pwr);

rgg_ndf $=(p-1)^{*}(g-1) *$ eps $1 \_s t r ;$

rgg_ddf $=(\mathrm{p}-1) *(\mathrm{n} 2-\mathrm{g}) *$ eps $1 \_$str;

rgg ncp $=$ rm ncp*eps 1 str;

rg_f ${ }_{\text {f }}$ it $=$ finv $(1-$ alpha, $\operatorname{rgg}$ _ndf, rgg_ddf $)$;

rgg_pwr= 1 - probf(rg_fcrit, rgg_ndf, rgg_ddf, rgg_ncp);

end;

do $n 3=12$ to 1000 by 3 until (pm pwr $>$ power); $\mathrm{pm} \_\mathrm{ndf}=(\mathrm{p}-1) *(\mathrm{~g}-1)$;

pm_ddf $=\mathrm{s}^{*}(\mathrm{n} 3-(\mathrm{p}-1)-1)+2$;

$\mathrm{pm} \_\mathrm{ncp}=((\mathrm{n} 3 / \mathrm{g}) *($ pdelta $* * 2) / 2) /(2 *(1-$ rho $))$;

pm_fcrit $=$ finv(1-alpha, pm_ndf, pm_ddf);

pm_pwr= 1-probf(pm_fcrit, pm_ndf, pm_ddf, pm_ncp);

end;

total $\mathrm{n}=\mathrm{n} 3$;

grp_size $=$ total_n $/ \mathrm{g}$;

do eps_star $=1$ lb_eps to 1.0 by .001 until

(pgg_pwr $>=$ pm_pwr);

pgg_ndf $=(\mathrm{p}-1) *(\mathrm{~g}-1) *$ eps_star;

pgg_ddf $=(\mathrm{p}-1) *(\mathrm{n} 3-\mathrm{g}) *$ eps_star;

pgg_ncp $=$ pm_ncp*eps_star;

pg_f crit $=$ finv(1-alpha, $\overline{p g g}$ _ndf, pgg_ddf);

pgg_pwr=1 - probf(pg_fcrit, pgg_ndf, pgg_ddf, pgg_ncp);

if (pm_pwr $>$ power) \& (pgg_pwr $>=$ pm_pwr) then do;

end;

end;

/* Generate E_Max and E_Min for Bias Ratio */

$\mathrm{B}=\mathrm{P}-1$

$\mathrm{Q}=\mathrm{P}+1$

$\mathrm{E} 1=1 / \mathrm{B}$

$\mathrm{E} 2=(\mathrm{B}-1) / \mathrm{B}$

$\mathrm{E} 3=1 / 2 *(1+\operatorname{SQRT}(1 / \mathrm{B}))$

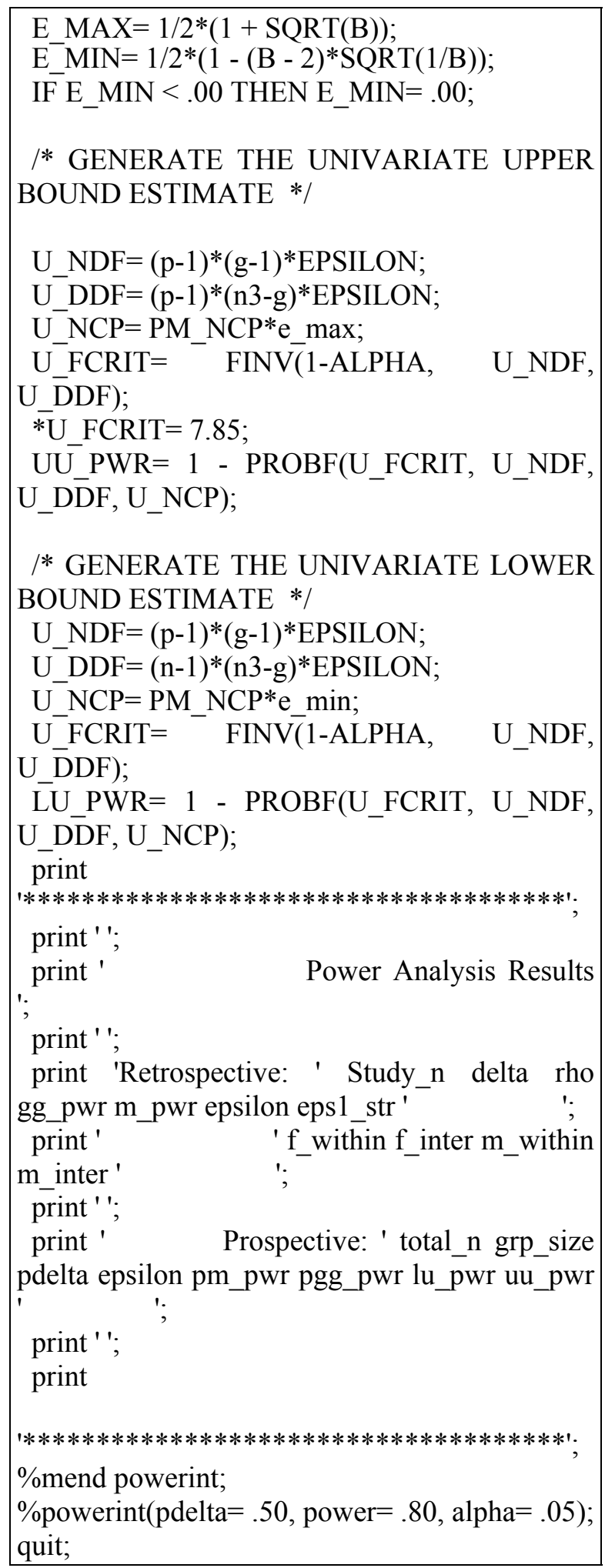




\section{Analyses of Unbalanced Groups-Versus-Individual Research Designs Using Three Alternative Approximate Degrees of Freedom Tests: Test Development and Type I Error Rates}

\author{
Stephanie Wehry \\ University of North Florida
}

\author{
James Algina \\ University of Florida
}

Three approximate degrees of freedom quasi-F tests of treatment effectiveness were developed for use in research designs when one treatment is individually delivered and the other is delivered to individuals nested in groups of unequal size. Imbalance in the data was studied from the prospective of subject attrition. The results indicated the test that best controls the Type I error rate depends on the number of groups in the group-administered treatment but does not depend on the subject attrition rates included in the study.

Key words: Groups versus-individuals, approximate degrees of freedom, unbalanced designs, Type I error rate

\section{Introduction}

In the simplest groups-versus-individuals research design, two treatments are compared, one of which is administered to $J$ groups. The $j$ th group $(j=1, \ldots, J)$ has $n_{j}$ participants, for a total of $N_{G}=\sum_{j=1}^{J} n_{j}$ such participants. The other treatment is administered individually to $N_{I}$ participants. For example, psychotherapy researchers investigating the efficacy of group therapy often use a wait-list control group (Burlingame, Kircher, \& Taylor, 1994). The therapy is provided to participants in groups because the researcher believes group processes will enhance the effectiveness of the therapy. Group processes do not affect the participants in

Stephanie Wehry is Assistant Director for Research and Evaluation at the Florida Institute for Education. Her research interests are evaluating early childhood education programs, applied statistics, and psychometrics. Email her at swehry@unf.edu. James Algina is Professor of Educational Psychology. His interests are in psychometric theory and applied statistics. Email him at algina@ufl.edu. the wait-list control group because they do not receive a treatment. In comparative studies, the effectiveness of an active treatment delivered to groups is compared to the effectiveness of an active treatment delivered individually. For example, Bates, Thompson, and Flanagan (1999) compared the effectiveness of a mood induction procedure administered to groups to the effectiveness of the same procedure administered to individuals. Using a more complex groups-versus-individuals research design, Boling and Robinson (1999) investigated the effects of three types of study environment on a measure of knowledge following a distance-learning lecture. The three types of study environment included a printed study guide accessed by individuals, an interactive multi-media study guide accessed by individuals, and a printed study guide accessed by cooperative study groups.

Burlingame, Kircher, and Taylor (1994) reported that independent samples $t$ tests, ANOVAs, and ANCOVAs were the most commonly used methods for analyzing data in group psychotherapy research. It is well known that the independent samples $t$ test requires scores be independently distributed both between and within treatments - an assumption that is most likely violated in the groups-versusindividual research design. This lack of 
independence is indicated by a non-zero intraclass correlation coefficient for participants who receive the group-administered treatment. Myers, Dicecco, and Lorch (1981), using simulated data, showed that the Type I error rates for independent samples $t$ test is greater than nominal alpha when the intraclass correlation is positive. Burlingame, Kircher, and Honts (1994) reported similar results.

The Myers, Dicecco, \& Lorch (1981) Quasi-F Test Statistic

Myers et al. (1981) developed a quasi-F statistic that takes into account the lack of independence of data collected from the participants in the same group in a groupsversus-individuals research design. The Myers et al. test statistic is based on the two models for the data. The model for the $i$ th $\left(i=1, \ldots, N_{I}\right)$ participant within the individually administered treatment $\left(T_{I}\right)$ is

$$
Y_{i / T_{I}}=\mu_{I}+\varepsilon_{i / T_{I}}
$$

and the model for the $i$ th participant $\left(i=1, \ldots, n_{j}\right) \quad$ within the $j$ th group $(j=1, \ldots, J)$ within the group-administered treatment $\left(T_{G}\right)$ is

$$
Y_{i / j / T_{G}}=\mu_{G}+\alpha_{j / T_{G}}+\varepsilon_{i / j / T_{G}} .
$$

Myers et al. assumed that $\varepsilon_{I / T_{I}} \sim N\left(0, \sigma_{S / T_{I}}^{2}\right)$, $\alpha_{j / T_{G}} \sim N\left(0, \tau^{2}\right)$, and $\varepsilon_{i / j / T_{G}} \sim N\left(0, \sigma_{S / G / T_{G}}^{2}\right)$. The assumption about the $\alpha_{j / T_{G}}$ implies that the groups in the group-administered treatment are considered to be representative of an infinitely large number of groups. Therefore, the Myers et al. method permits generalization of the result to this larger number of groups. In addition, Myers et al. assumed that the groups within the groupadministered treatments were $\operatorname{balanced}\left(n_{1}=, \cdots,=n_{J}\right)$.
Formulated as an approximate degrees of freedom (APDF) $t$ statistic, the Myers et al. test statistic is

$$
t_{A P D F}=\frac{\bar{Y}_{I}-\bar{Y}_{G}}{\sqrt{a_{1} M S_{S / T_{I}}+a_{2} M S_{G / T_{G}}}}
$$

where $a_{1}$ is $\left(1 / N_{I}\right)$ and $a_{2}$ is $\left(1 / N_{G}\right)$. The mean

$$
\bar{Y}_{I}=\frac{1}{N_{I}} \sum_{i=1}^{N_{I}} Y_{i / T_{I}}
$$

is the mean of the criterion scores for the participants in the individually administered treatment $\left(T_{I}\right)$,

$$
M S_{S / T_{I}}=\frac{\sum_{i=1}^{N_{I}}\left(Y_{i / T_{I}}-\bar{Y}_{I}\right)^{2}}{N_{I}-1}
$$

is the variance for participants who received the individually-administered treatment,

$$
\bar{Y}_{G}=\frac{1}{N_{G}} \sum_{j=1}^{J} \sum_{i=1}^{n_{j}} Y_{i / j / T_{G}}
$$

is the mean of the criterion scores of participants who received the group-administered treatment, and

$$
M S_{G / T_{G}}=\frac{\sum_{j=1}^{J} n_{j}\left(\bar{Y}_{j / T_{G}}-\bar{Y}_{G}\right)^{2}}{J-1}
$$

is the between-group mean square for these participants. It can be shown that the squared denominator of the $t$ statistic estimates the sampling variance of the numerator given the assumptions made by Myers et al. about the random effect and residuals. The estimated Satterthwaite (1941) approximate degrees of freedom are 


$$
\hat{f}_{2}=\frac{\left(a_{1} M S_{S / T_{I}}+a_{2} M S_{G / T_{G}}\right)^{2}}{\frac{\left(a_{1} M S_{S / T_{I}}\right)^{2}}{N_{I}-1}+\frac{\left(a_{2} M S_{G / T_{G}}\right)^{2}}{J-1}} .
$$

An Alternative Approximation for the Degrees of Freedom

The Satterthwaite (1941) approximation of the distribution of the linear combination of mean squares in the denominator of the $t$ statistic is based on the assumptions that $M S_{S / T_{I}}$ and $M S_{G / T_{G}}$ are independent random variables that are distributed as multiples of chi-square distributions. The distribution of the sum is approximated as chi-square with degrees of freedom estimated by equating the first two moments of the sample and the approximating chi-square distribution.

The discussion in Satterthwaite (1941) implied that this approximation of the distribution of the denominator improves as $J-1$ or $N_{I}-1$ increases and as

$$
\frac{\left(N_{I}-1\right)\left(n \tau^{2}+\sigma_{S / G / T_{G}}^{2}\right)}{(J-1) \sigma_{S / T_{I}}^{2}}
$$

becomes closer to 1.0. When there are two groups in the group-administered treatment level, $J$ is as small as possible and the ratio of equation (3) is typically larger than 1.0 and increases as the number of participants in the two groups increases and as the intraclass correlation increases. Scarino and Davenport (1986) studied the Type I error rate of the Welch APDF $t$ test and found it could be seriously inflated when (a) there is a negative relationship between the sampling variances of the means and the degrees of freedom for the estimated sampling variances and (b) the smaller of the two degrees of freedom is small. Wehry and Algina (2003) applied the work of Scarino and Davenport to the Myers et al. (1981) quasi-F test and showed that when $J$ equals two or three and $\tau>0$, the Satterthwaite approximation of the denominator degrees of freedom also resulted in a quasi-F test that does not control the Type I error rate at nominal alpha.
Scarino and Davenport (1986) developed a four-moment approximation of the degrees of freedom for use with the Welch $t$ when the ratio of the sampling variances is large and the corresponding ratio of degrees of freedom is small. Wehry and Algina (2003) adapted the four-moment approximation for use with the groups-versus-individual research design. The four-moment approximation to the degrees of freedom is

$$
\hat{f}_{4}=\frac{\left\{\frac{u^{2}}{m_{1}}+\frac{1}{m_{2}}\right\}^{3}}{\left(\frac{u^{3}}{m_{1}^{2}}+\frac{1}{m_{2}^{2}}\right)^{2}}
$$

where $u=a_{2} M S_{G / T_{G}} / a_{1} M S_{S / T_{I}}, m_{1}=J-1$, and $m_{2}=N_{I}-1$. Like the Satterthwaite approximation employed by Myers et al. (1981), the four-moment degrees of freedom is based on the assumption of a balanced design.

Scarino and Davenport (1986) reported that the four-moment APDF test is conservative under some conditions and suggested using an average of the two-moment and four-moment approximations of the degrees of freedom. Wehry and Algina (2003) conducted a study of the APDF quasi-F test with the two-moment, four-moment, and an arithmetic average of the two- and four-moment approximations of the degrees of freedom using both analytical results and simulated data. They concluded that when the group-administered treatment is delivered to two groups, the four-moment APDF quasi-F test should be used and when the group-administered treatment is delivered to three or more groups, the average-moment APDF quasi-F test should be used. However, the two-moment APDF quasi-F test is only slightly liberal in conditions involving more than three groups.

Quasi-F Statistics For Use When Data Are Not Balanced Across Groups In The GroupAdministered Treatment Level

The purpose of the present study is to extend the work of Myers et al. (1981) and Wehry and Algina (2003) to include groupsversus-individuals research designs that are not 
balanced across either treatment levels (i.e., $N_{I} \neq N_{G}$ ) or the groups in the groupadministered treatment level (i.e., $n_{j} \neq n_{j^{\prime}}$ for at least one pair of $j$ and $\left.j^{\prime}\right)$. Usually in experimental research an equal number of participants are randomly assigned to each treatment level; however, $N_{I}$ and $N_{G}$, as well as the $n_{j}$ can be affected by attrition of participants. Burlingame, Kircher, and Taylor (1994) found $18 \%$ subject attrition was the median reported attrition rate of subjects in a survey of psychotherapy literature. Clarke (1998) suggested that the attrition rate in waitlist control groups could even be higher than that of the active treatment level.

Imbalance can also result from studying naturally occurring groups such as family units and classrooms. Methods that accommodate imbalance across groups in the groupadministered treatment level have not been developed. A possible solution to the imbalance across groups in the group-administered treatment level is to randomly eliminate participants until balance is achieved. However, eliminating data results in a loss of statistical power.

APDF Quasi-F Test for Unbalanced Data

$$
\text { As is well known, if the variances of } \bar{Y}_{I}
$$
and $\bar{Y}_{G}$ were known, the hypothesis $H_{O}: \mu_{I}-\mu_{G}=0$ could be tested by

$$
\chi^{2}=\frac{\left(\bar{Y}_{I}-\bar{Y}_{G}\right)^{2}}{\operatorname{Var}\left(\bar{Y}_{I}-\bar{Y}_{G}\right)} .
$$

Because observations are independent across treatment levels, substituting the variances of $\bar{Y}_{I}$ and $\bar{Y}_{G}$ into equation (5) results in

$$
\chi^{2}=\frac{\left(\bar{Y}_{I}-\bar{Y}_{G}\right)^{2}}{\frac{\sigma_{S: T_{I}}^{2}}{N_{I}}+\frac{\tau^{2} \sum_{j=1}^{J} n_{j}^{2}}{N_{G}^{2}}+\frac{\sigma_{S / G / T_{G}}^{2}}{N_{G}}} .
$$

However, the variances are not known, and, in order to develop a test statistic that can be used in practice, two steps must be completed: Develop estimators of the variance components in equation (6) and approximate the distribution of the resulting test statistic. Approximating the distribution of the denominator by a chi-square distribution and the distribution of the test statistic by an $F$ distribution is a common practice in statistics.

\section{Variance Component Estimates}

There are numerous methods for estimating the variance components. Perhaps the most commonly used method is the method of moments, also called the ANOVA estimation of variance components (Milliken \& Johnson, 1992). Meyers et al. (1981) used the method of moments variance component estimators in formulating the quasi-F test statistic. The method of moments procedure is based on equating the expected values of the sums of squares to their respective observed values.

Other estimation methods include maximum likelihood (ML), restricted maximum likelihood (REML), minimum norm quadratic unbiased (MINQUE), and minimum variance quadratic unbiased (MIVQUE) estimators. ML estimators are values of the parameter space that maximize the likelihood function. In REML, the likelihood equations are partitioned into two parts, one part that is free of fixed effects. REML maximizes the part that has no fixed effects. MINQUE and MIVQUE are iterative and the researcher must provide initial values of the components. All methods produce the same results when the design is balanced (Milliken \& Johnson, 1992; Swallow \& Monahan, 1984).

Swallow and Monahan (1984) conducted a Monte Carlo study of ANOVA, ML, REML, MIVQUE and MINQUE methods of estimating the variance components of a oneway unbalanced, random effects design. All simulated data were normal, and the variables manipulated were the degree of imbalance, the number of groups, and the ratio of $\tau^{2} / \sigma_{S / G / T_{G}}^{2}$. In terms of bias of the estimates, the results indicated, except in cases of extreme patterns of imbalance, $\quad n_{j}=(1,1,1,1,13$, and 13) and $n_{j}=(1,1,1,1,1,1,1,19$, and 19), ANOVA, REML, and MINQUE estimators showed little difference. However, the results indicated that 
ML methods were the best estimators of $\tau^{2}$ when $\tau^{2} / \sigma_{S / G / T_{G}}^{2} \leq .5$ because of the small bias and the low mean square error of the estimate. When $\tau^{2} / \sigma_{S / G / T_{G}}^{2}$ is large, Swallow and Monahan indicated there may be a substantial downward bias and that ML methods have no superiority over the other methods. There was little difference among the methods studied when estimating $\sigma_{S / G / T_{G}}^{2}$. Milliken and Johnson (1992) suggested that ANOVA estimates should have good properties for nearly balanced data, and Swallow and Monahan concluded that unless the data are severely unbalanced and $\tau / \sigma_{G}^{2}>1$, ANOVA estimates are adequate.

The results of the Swallow and Monahan (1984) study and the recommendations of Milliken and Johnson (1992) suggested that ANOVA estimates of the variance components are likely to be adequate for the groups-versusindividuals research design. Data as extreme as that simulated in the Swallow and Monahan study seems likely to be rare in group research; therefore, method of moments estimators of the variance components are used for the quasi-F test for comparing the effectiveness of two treatment levels when data are unbalanced.

The expected values for the mean squares for groups (henceforth when the term groups is used, it will refer to the groups within the group-administered treatments) are

$$
E M S_{G / T_{G}}=\sigma_{S / G / T_{G}}^{2}+n_{o} \tau^{2},
$$

where

$$
n_{o}=\frac{1}{J-1}\left(N_{G}-\frac{\sum_{j=1}^{J} n_{j}^{2}}{N_{G}}\right)
$$

(Snedecor \& Cochran, 1956). The other two expected values are

$$
E M S_{S / G / T_{G}}=\sigma_{S / G / T_{G}}^{2}
$$

and

$$
E M S_{S / T_{I}}=\sigma_{S / T_{I}}^{2}
$$

The mean squares are equated with their respective expected values of equations (7), (8), and (9) are the resulting equations are solved for the ANOVA variance component estimates. The variance component estimates are then substituted into equation (6) to obtain the quasi$F$ test statistic for comparing weighted treatment level means.

The Quasi-F Test Statistic

Using the estimated variance components the quasi-F test statistic is

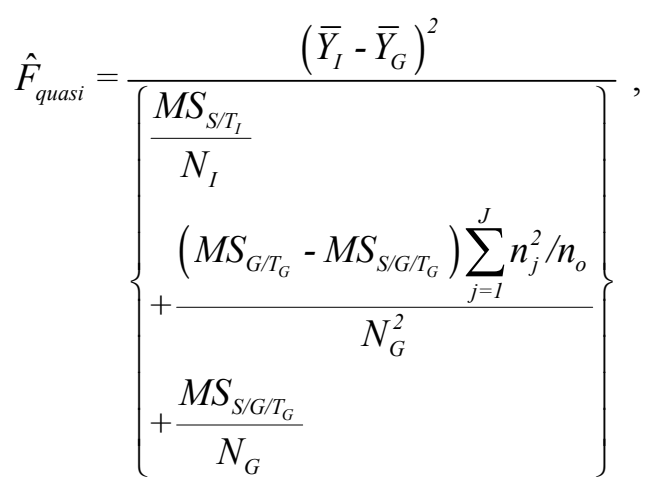

which simplifies to

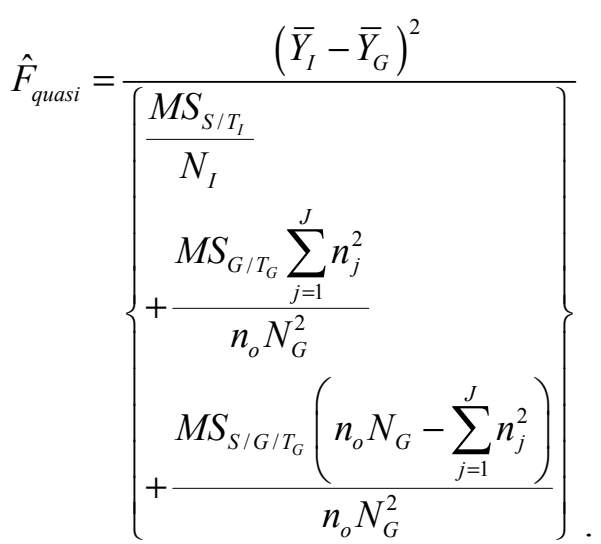

The denominator of the quasi-F statistic is a synthetic mean square in the form of

$$
M S=a_{1} M S_{S / T_{I}}+a_{2} M S_{G / T_{G}}+a_{3} M S_{S / G / T_{G}},
$$


where

$$
\begin{gathered}
a_{1}=\frac{1}{N_{I}}, \\
a_{2}=\frac{\sum_{j=1}^{J} n_{j}^{2}}{n_{o} N_{G}^{2}},
\end{gathered}
$$

and

$$
a_{3}=\left(\frac{n_{o} N_{G}-\sum_{j=1}^{J} n_{j}^{2}}{n_{o} N_{G}^{2}}\right) .
$$

Approximating Chi-Square Distribution

The model for the group-administered treatment is a random effects ANOVA model [see equation (2)]. For a design that is balanced across classes, Searle (1992) showed the mean squares between and within classes are independent and are distributed as multiples of chi-square distributions. When the data are not balanced across classes, the mean squares within and between are still independent; however, the mean square between classes is not distributed as a multiple of a chi-square distribution. Nevertheless, Burdick, and Graybill (1988) indicated as long as $\tau$ is not too large, approximating the mean square between as a multiple of a chi-square distribution does not result in a large error.

Two-Moment Approximation of the Degrees of Freedom

The Satterthwaite (1941) approximation for the degrees of freedom for the linear combination in equation (10) is

$$
\hat{f}_{2}=\frac{\left(a_{1} M S_{S / T_{I}}+a_{2} M S_{G / T_{G}}+a_{3} M S_{S / G / T_{G}}\right)^{2}}{\frac{\left(a_{1} M S_{S / T_{I}}\right)^{2}}{N_{I}-1}+\frac{\left(a_{2} M S_{G / T_{G}}\right)^{2}}{J-1}+\frac{\left(a_{3} M S_{S / G / T_{G}}\right)^{2}}{N_{G}-J}}
$$

It should be noted that $a_{3} \leq 0$, with equality holding only when $n_{o}=n$. Therefore, when data are not balanced across groups in the groupadministered treatment level, it is possible for the denominator of the quasi-F statistic to be less than or equal to zero when the estimate of $\tau^{2}$ is substantially smaller than zero. In these cases, as suggested by Searle (1992), it is reasonable to assume $\tau^{2}$ is zero and replace the quasi-F statistic by the Welch $t$-test where

$$
t_{W_{A P D F}}=\frac{\left(\bar{Y}_{I}-\bar{Y}_{G}\right)}{\sqrt{\frac{M S_{S / T_{I}}}{N_{I}}+\frac{M S_{S / T_{G}}}{N_{G}}}}
$$

and

$$
M S_{S / T_{G}}=\frac{\sum_{j=1}^{J} \sum_{i=1}^{n_{j}}\left(Y_{i / j / T_{G}}-\bar{Y}_{G}\right)^{2}}{\left(N_{G}-1\right)}
$$

with two-moment degrees of freedom

$$
\hat{d f}=\frac{\left(\frac{M S_{S / T_{I}}}{N_{I}}+\frac{M S_{S / T_{G}}}{N_{G}}\right)^{2}}{\frac{\left(M S_{S / T_{I}}\right)^{2}}{N_{I}^{2}\left(N_{I}-1\right)}+\frac{\left(M S_{S / T_{G}}\right)^{2}}{N_{G}^{2}\left(N_{G}-1\right)}}
$$

(Welch, 1938).

Modified Four-Moment Approximation of the Degrees of Freedom

Because the coefficients of the variance component terms in the synthetic error term for unbalanced data are not all positive and because of the occurrence of conditions in which the ratio of the degrees of freedom is less than one when the ratio of the corresponding sampling variances is greater than one, the two-moment quasi-F test may not control the Type I error rate at the nominal level. The four-moment approximation was developed by Scariano and Davenport (1986) for a synthetic mean square that is the sum of two positive terms. Rather than expanding the four-moment approach to three terms including one that is negative, a simpler approach that combines the two-moment and four-moment approximations was used in this study. 
In order to compute the modified fourmoment approximation, the degrees of freedom for $\quad a_{2} M S_{G / T_{G}}+a_{3} M S_{S / G / T_{G}}$ are first approximated using the two-moment approach. As noted previously, Searle (1992) showed $M S_{G / T_{G}}$ and $M S_{S / G / T_{G}}$ are independent when data are unbalanced, Burdick and Graybill (1988) indicated as long as $\tau^{2}$ is not too large $M S_{G / T_{G}}$ can be approximated as a multiple of chi-square distribution, and Swallow and Monahan (1984) showed that method of moments estimation works well in one-way, random effects, unbalanced ANOVA designs as long as $\tau^{2} / \sigma_{S / G / T_{G}}^{2} \leq 1$. The two-moment degrees of freedom for

$$
M S_{{\text {error } r_{G}}}=a_{2} M S_{G / T_{G}}+a_{3} M S_{S / G / T_{G}}
$$

are

$$
\hat{f}_{2_{G}}=\frac{\left(M S_{\text {error }_{T_{G}}}\right)^{2}}{\left[\frac{\left(a_{2} M S_{G / T_{G}}\right)^{2}}{(J-1)}+\frac{\left(a_{3} M S_{S / G / T_{G}}\right)^{2}}{\left(N_{G}-J\right)}\right]} .
$$

This value of $\hat{f}_{2_{G}}$ along with $M S_{\text {error }_{T_{G}}}$ and the estimate of the individual treatment level variance, $M S_{S / T_{I}}$, are used in the four-moment approximation of equation (4). In the modified four-moment $u=M S_{\text {error }_{G}} / a_{1} M S_{S / T_{I}}, \quad m_{1}=\hat{f}_{2_{G}}$, and $m_{2}=\left(N_{I}-1\right)$. When $M S_{\text {error } r_{G}} \leq 0$, the quasi-F statistic is replaced by the Welch $t$-test.

Modified Averaged Degrees of Freedom Approximation of the Degrees of Freedom

Scariano and Davenport (1986) reported that, with completely balanced data, the fourmoment quasi-F test is conservative under some conditions. Therefore, an arithmetic average of the two-moment and the modified four-moment approximations was also included in the present study. When $M S_{\text {error }} \leq 0$, data were analyzed using the Welch $t$ test; otherwise, the twomoment approximation and the modified four- moment approximation to the degrees of freedom were arithmetically averaged resulting in an averaged degrees of freedom quasi-F test.

Example 1

Participants were randomly assigned to two conditions and completed three trials of the prisoner's dilemma. The data are the number of competitive choices across the three trials. In one condition, participants completed the three trials independently. In the second condition, participants worked in teams and discussed how to respond to each trial. However, participants within a team responded individually. For participants in the individual treatment the relevant results are $N_{I}=32, \bar{Y}_{I}=.469$, $M S_{S / T_{I}}=.773$. For participants in the groupadministered treatment, the results are $N_{G}=48, J=15, \quad \sum_{j=1}^{J} n_{j}^{2}=141, \quad \bar{Y}_{G}=.905$, $M S_{G / T_{G}}=1.896$, and $M S_{S / G / T_{G}}=.833$. The calculated $t$ statistic is -1.86 . The degrees of freedom are $\hat{f}_{2}=56.37, \hat{f}_{4}=56.04, \quad$ and $\hat{f}_{a}=54.70$. For all three degrees of freedom, $($ Prob $>|t|)=.068$. Because the theory predicts more competitive response following group discussion, the results are in support of the theory.

\section{Example 2}

In an evaluation of a pre-school literacy program, the evaluators were interested in whether reading achievement was different in single-classroom sites and multiple-classroom sites. The available data are mean end-of year reading achievement for each of the classrooms. For single-classroom sites the relevant results are $N_{I}=38, \bar{Y}_{I}=88.85, M S_{S / T_{I}}=57.84$. For participants in the multiple-classroom sites, the results are $N_{G}=63, J=29, \sum_{j=1}^{J} n_{j}^{2}=216$, $\bar{Y}_{G}=87.52, \quad M S_{G / T_{G}}=69.09, \quad$ and $M S_{S / G / T_{G}}=22.22$. The calculated $t$ statistic is 
0.71. The degrees of freedom are $\hat{f}_{2}=30.87$, $\hat{f}_{4}=17.76$, and $\hat{f}_{a}=24.31$. For all three degrees of freedom, $($ Prob $>|t|)=.76$. The results do not support the belief that mean reading achievement is different in singleclassroom and multiple classroom sites.

\section{Methodology}

Variables Manipulated in the Monte Carlo Study The design of the Monte Carlo study had five between-subjects factors and one within-subjects factor. There were a total of 2700 conditions. The design included the three approaches to the approximation of the error term degrees of freedom as levels of the withinsubjects factor. The number of groups, planned size of the groups, level of the intraclass correlation, ratio of the group to individual treatment level variances, and the rate of subject attrition were the five between-subjects factors. There were five levels of the number of groups, $J=2,3,4,5$, and 6 ; five levels of planned group size, $n=4,8,12,16$, and 20 subjects nested in the groups; three levels of intraclass correlation, $\tau^{2} /\left(\tau^{2}+\sigma_{S / G / T_{G}}^{2}\right)=.0, .2$, and .4; three levels of the ratio of group to individual treatment level variances, $\left(\tau^{2}+\sigma_{S / G / T_{G}}^{2}\right) / \sigma_{S / I}^{2}=0.75,1.00$, and 1.25; and four combinations of individual and group treatment level attrition rates, .15 and $.15, .15$ and $.25, .25$ and .15 , and .25 and .25 .

\section{Data Generation}

The simulation in the study was carried out using the random number generation functions of SAS, Release 6.12. Scores for simulated participants in the individually administered treatment level were generated using the equation

$$
Y_{i / I}=\mu_{I}+\varepsilon_{i / T_{I}}
$$

where $\mu_{I}$ was arbitrarily set at 100 and the $\varepsilon_{i: T_{I}}$ swere pseudorandom standard normal deviates generated using RANNOR. The variable $Y_{\dot{r} T_{I}}$ was set to the missing data indicator if $U_{i / T_{I}}<p_{T_{I}}$ where $p_{T_{I}}$ is the individually administered treatment level attrition rate and $U_{i / T_{I}}$ was a pseudorandom uniform deviate generated using RANUNI. However, $N_{I}$ was not permitted to be smaller than two.

Scores for simulated participants in the group-administered treatment level were generated using the equation

$$
Y_{i / j / T_{G}}=\mu_{G}+\alpha_{j / T_{G}}+\varepsilon_{i / j / T_{G}}
$$

where $\mu_{G}$ was arbitrarily set at $100, \alpha_{j / T_{G}}$ was a pseudorandom normal deviate with mean zero and variance $\tau^{2}$, and $\varepsilon_{i / j / T_{G}}$ was a pseudorandom normal deviate with mean zero and variance $\sigma_{S / G / T_{G}}^{2}$. The variable $Y_{i / j / T_{G}}$ was set to a missing value indicator if $U_{i / j / T_{G}}<p_{T_{G}}$, where $p_{T_{G}}$ is the group-administered treatment level attrition rate and $U_{i / j / T_{G}}$ was a pseudorandom uniform deviate generated using RANUNI. However, in all cases $n_{j}$ was not permitted to be smaller than two.

Each of the conditions was replicated 10,000 times, and the Type I errors of the three tests were counted over the replications of each condition. All tests were conduted at $\alpha=.05$.

\section{Results}

A Number of Groups $(5) \times$ Planned Group Size (5) $\times$ Intraclass Correlation (3) $\times$ Ratio of Variance (3) $\times$ Attrition Rate (4) $\times$ Degrees of Freedom Approximation (3), with repeated measures on the last factor, ANOVA was used to analyze the Type I error rate data. Because there was only one data point for each combination of the six factors, the five-way interaction of the first five factors was used as the error term for between-replications effects and the six-way interaction was used as the error term for all within-replications effects. For each effect omega squared was used to express the size of the effect as a proportion of the total variance. An effect was considered important if 
it was significant at $\alpha=.05$ and accounted for more than $1 \%$ of the total variance in the Type I error rate. Table 1 presents the omega squares for all significant effects. The sum of the omega squares for all of the important effects was 0.929. All factors except subject attrition rate were involved in an effect that met our criterion for an important influence on the Type I error rate.

Averaged over all factors, other than number of groups in the group-administered treatment, the average Type I error rate of the two-moment test was greater than that for the averaged degrees of freedom test. Also the average Type I error rate of the averaged degrees of freedom test was greater than that for the modified four-moment test. When there were two groups only the modified four-moment test controlled the Type I error rate near nominal alpha; however, the modified four-moment test resulted in a conservative quasi-F test with three or more groups. In all conditions involving two groups, increasing the planned size of the groups, the ratio of treatment level variances, or the intraclass correlation increased the Type I error rate. Under conditions involving three or more groups, increasing the intraclass correlation increased the Type I error rate of all three tests and increasing the ratio of treatment level variances and the planned size of the groups increased the Type I error rate of the two-moment and averaged degrees of freedom tests. As the number of groups increased the effect of increasing the ICC or the planned size of the groups declined. However, under conditions of three groups or more groups, increasing the ratio of the treatment level variances and the planned size of the groups decreased the Type I error rate of the modified four-moment quasi-F test.

Table 2 contains the minimum and maximum Type I error rate averaged over subject attrition by number of groups, approximate degrees of freedom approach, and intraclass correlation. Minima and maxima were computed over planned size of groups and ratio of treatment level variances. In Table 2 bold and italicized figures indicate the degrees of freedom approach that resulted in better control of Type I error rate for a particular number of groups and ICC. When both bold figures and italicized figures are presented, the italicized figures indicate the degrees of freedom approximation that tended to result in a higher Type I error rate. Tests are considered unacceptable if the maximum Type I error rate is above .075 , the upper limit of Bradley's (1978) liberal criterion for a robust test or if the minimum Type I error rate is below .025 the lower limit of Bradley's (1978) liberal criterion.

Inspection of Table 2 indicates that when there are two groups, the modified fourmoment test should be used at the risk of a conservative test when the ICC is near zero. The averaged degrees of freedom test may be more attractive with a low ICC, but the fact that it has a strong liberal tendency when the ICC is 0.20 raises the question of how the two tests function for ICCs between 0.00 and 0.20 . Supplementary results are shown in Table 3 for ICCs of 0.05 , 0.10 , and 0.15 . In the simulations conducted to obtain these results, all other conditions were the same as in the original study. The findings that the averaged degree of freedom test has a liberal tendency for an ICC of 0.10 and that the conservative tendency of the modified fourmoment test is less marked with an ICC of 0.05 than with an ICC of 0.00 suggest the modified four-moment test should be used when there are two groups in the group-administered treatment.

When there are three groups, the results in Tables 2 and 3 suggest the averaged degree of freedom test should be used at the risk of a slightly conservative test when the ICC is near zero. Then the two-moment test may be more attractive. However, it is not clear how valid an estimated ICC will be in selecting between the two tests. Given the very mild conservative tendency for the averaged degrees of freedom test, it is recommended when there are three groups.

When there are four or more groups either the two-moment test or the averaged degrees of freedom test might be used. The former can be somewhat liberal, with the tendency increasing as the ICC increased, but decreasing as the number of groups increased. The averaged degrees of freedom test can be somewhat conservative, with the tendency decreasing as the ICC increased and as the number of groups decreased. 
Table 1. Mean Square Components and $\hat{\omega}_{j}^{2}$ for the Important Effects $\left(\hat{\omega}_{j}^{2}>.01\right)$

\begin{tabular}{lc}
\hline Source of MS & $\hat{\omega}_{j}^{2}$ \\
\hline Between Replications Effects & 0.239 \\
Number of Groups $-g$ & 0.024 \\
Planned Size of Groups - $n$ & 0.073 \\
Intraclass Correlation - icc & 0.056 \\
$g \times n$ & 0.079 \\
$g \times$ icc & \\
Within-Replication Effects & 0.390 \\
Approximation $-t$ & 0.020 \\
$t \times g$ & 0.031 \\
$t \times n$ & 0.017 \\
$t \times$ ratio of treatment level variance & \\
\hline
\end{tabular}


Table 2. Minimum and Maximum Average Type I Error Rate by Number of Groups, Test, and ICC

\begin{tabular}{|c|c|c|c|c|}
\hline \multirow[b]{2}{*}{$\begin{array}{c}\text { Number of } \\
\text { Groups }\end{array}$} & \multirow[b]{2}{*}{ Test } & \multicolumn{3}{|c|}{ ICC } \\
\hline & & 0.00 & 0.20 & 0.40 \\
\hline \multirow[t]{3}{*}{2} & $\hat{f_{2}}$ & $.0470, .0759$ & $.0532, .1118$ & $.0571, .1204$ \\
\hline & $\hat{f}_{\text {ave }}$ & $.0390, .0572$ & $.0459, .0907$ & $.0496, .1005$ \\
\hline & $\hat{f}_{4}$ & $.0338, .0401$ & $.0412, .0580$ & $.0437, .0663$ \\
\hline \multirow[t]{3}{*}{3} & $\hat{f}_{2}$ & $.0471, .0589$ & $.0514, .0770$ & $.0537, .0776$ \\
\hline & $\hat{f}_{\text {ave }}$ & $.0411, .0488$ & $.0450, .0634$ & $.0476, .0637$ \\
\hline & $\hat{f_{4}}$ & $.0299, .0362$ & $.0322, .0404$ & $.0319, .0403$ \\
\hline \multirow[t]{3}{*}{4} & $\hat{f}_{2}$ & $.0488, .0560$ & $.0506, .0631$ & $.0520, .0637$ \\
\hline & $\hat{f}_{\text {ave }}$ & $.0422, .0481$ & $.0459, .0528$ & $.0458, .0539$ \\
\hline & $\hat{f}_{4}$ & $.0281, .0400$ & $.0283, .0393$ & $.0298, .0390$ \\
\hline \multirow[t]{3}{*}{5} & $\hat{f}_{2}$ & $.0473, .0533$ & $.0513, .0585$ & $.0491, .0603$ \\
\hline & $\hat{f}_{\text {ave }}$ & $.0436, .0467$ & $.0469, .0499$ & $.0451, .0509$ \\
\hline & $\hat{f_{4}}$ & $.0282, .0417$ & $.0303, .0411$ & $.0326, .0410$ \\
\hline \multirow[t]{3}{*}{6} & $\hat{f}_{2}$ & $.0480, .0557$ & $.0488, .0568$ & $.0507, .0557$ \\
\hline & $\hat{f}_{\text {ave }}$ & $.0442, .0491$ & $.0451, .0500$ & $.0464, .0505$ \\
\hline & $\hat{f}_{4}$ & $.0299, .0436$ & $.0317, .0423$ & $.0326, .0405$ \\
\hline
\end{tabular}

Table 3. Minimum and Maximum Average Type I Error Rate by Number of Groups, Test, and ICC: Supplemental Conditions

\begin{tabular}{ccccc}
\hline \multirow{2}{*}{$\begin{array}{c}\text { Number of } \\
\text { Groups }\end{array}$} & & \multicolumn{3}{c}{ ICC } \\
\cline { 3 - 5 } & Test & 0.05 & 0.10 & 0.15 \\
\hline 2 & $\hat{f}_{2}$ & $.0482, .0908$ & $.0489, .0990$ & $.0508, .1066$ \\
& $\hat{f}_{\text {ave }}$ & $.0396, .0705$ & $.0404, .0784$ & $.0430, .0870$ \\
& $\hat{f}_{4}$ & $\mathbf{. 0 3 5 2 , . 0 4 8 1}$ & $\mathbf{. 0 3 6 0 , . 0 5 1 3}$ & $\mathbf{. 0 3 8 3 , . 0 5 6 2}$ \\
& $\hat{f}_{2}$ & $.0492, .0660$ & $.0472, .0711$ & $.0495, .0733$ \\
& $\hat{f}_{\text {ave }}$ & $\mathbf{. 0 4 1 8 , . 0 5 3 8}$ & $\mathbf{. 0 4 1 6 , . 0 5 8 5}$ & $\mathbf{. 0 4 3 6 , . 0 6 0 4}$ \\
& $\hat{f}_{4}$ & $.0296, .0377$ & $.0310, .0373$ & $.0307, .0389$ \\
\hline
\end{tabular}




\section{Conclusion}

Myers et al. (1981) presented a two-moment, quasi-F test for use when one treatment is delivered to individuals and one is delivered to groups of participants and the data are balanced for the groups in the group-administered treatment. Wehry and Algina (2003) extended that quasi-F test to include a four-moment and an averaged degrees of freedom quasi-F test for use when data are balanced across the groupadministered treatment level.

In this study, the two-moment approach developed by Myers et al. (1981) and the fourmoment and averaged degrees of freedom approaches developed by Wehry and Algina (2003) were extended to include groups versus individual research designs in which data are not necessarily balanced across treatment levels or across groups in the group-administered treatment level. In addition, Type I error rates of the resulting tests were estimated. The results indicated the modified four-moment test should be used when the group-administered treatment is delivered to two groups and the averaged degrees of freedom approach should be used when the group-administered treatment is delivered to three groups. When there are four or more groups, either test could be used-the averaged degrees of freedom test is has a slightly conservative tendency and the twomoment test has a slightly liberal tendency. When there are four or five groups the Type I error rate for the averaged degrees of freedom test is between .040 and .055 . The Type I error for two-moment test can be larger than .06. When there are six groups, the averaged degrees of freedom test controls the Type I error rate between .044 and .051 ; the two-moment test controls it between .048 and .057 .

Although, it is recommended to use the four-moment test when there are two groups, researchers should be very cautious about using a group-versus-individuals design with only a few groups. For a balanced design, Wehry and Algina (2003) showed that power is likely to be very low when there are just two groups and there is no reason for the design to be more powerful when the design is unbalanced. More generally, Myers et al. (1981) have shown that the number of groups can have a larger effect on power than the number of participants per groups and therefore recommended designs with as large a number of groups as possible.

At least four lines of additional research are attractive. Comparison of the three approximate degrees of freedom tests to mixed model tests using Satterthwaite or KenwardRogers degrees of freedom might be investigated. One difference between the current approaches and the mixed-model approach is the estimate of the mean for the group-administered treatment. In the present approach the estimated mean is computed by weighting the group means by the group sample sizes. In the mixed model approach, the mean for the group-administered treatment would be estimated by generalized least squares and would have a sampling variance that is not larger than the sampling variance of the mean used in the present approach. This may make the mixed model approach more powerful. However, Wehry and Algina (2003) found that with balanced designs, the mixed model approach had poor control of the Type I error rate in some situations and this problem may generalize to unbalanced designs.

The performance of the three tests when data are not normal is important. Micceri (1987) reported that a wide variety of psychometric distributions may not be normal and that random-effects ANOVA tests may not be robust to departures from normality, especially when conditions involve unbalanced designs or small sample sizes. Developing robust versions of the tests is important. Finally extension of the tests to more than two groups and to multivariate designs would be useful.

\section{References}

Bates, G. W., Thompson, J. C., \& Flanagan, C. (1999). The effectiveness of individual versus group induction of depressed mood. The Journal of Psychology, 33, 245-252.

Boling, N. C., \& Robinson, D. H. (1999). Individual study, interactive multimedia, or cooperative learning: Which activity best supplements lecture-based distance education? Journal of Educational Psychology, 91, 169174. 
Bradley, J. V, (1978). Robustness? British Journal of Mathematical and Statistical Psychology, 31, 144-152.

Burdick, R. K., \& Graybill, F. A. (1988). The present status of confidence interval estimations on variance components in balanced and unbalanced random models. Communications in statistics: theory and methods, 17, 1165-1195.

Burlingame, G. M., Kircher, J. C., \& Honts, C. R. (1994). Analysis of variance versus bootstrap procedures for analyzing dependent observations in small group research. Small Group Research, 25, 486-501.

Burlingame, G. M., Kircher, J. C., \& Taylor, S. (1994). Methodological considerations in group psychotherapy research: Past, present, and future practices. In A. Fuhriman \& G. Burlingame (Eds.), Handbook of group psychotherapy and counseling: An empirical and clinical synthesis. (pp. 41-80). New York: Wiley.

Clarke, G. N. (1998). Improving the transition from basic efficacy research to effectiveness studies: Methodological issues and procedures. In A. E. Kazdin (Ed.), Methodological issues and strategies in clinical research, $\left(2^{\text {nd }}\right.$ ed.) (pp. 541-559). New York: Wiley.

Micceri, T. (1989). The unicorn, the normal curve, and other improbable creatures. Psychological Bulletin, 105, 156-166.
Myers, J., Dicecco, J., \& Lorch, Jr., J. (1981). Group dynamics and individual performances: Pseudogroup and Quasi-F analyses. Journal of Personality and Social Psychology, 40, 86-98.

Milliken, G. A., \& Johnson, D. E. (1992). Analysis of messy data volume 1; Designed experiments. Boca Raton, FL: Chapman \& Hall.

Satterthwaite, F. W. (1941). Synthesis of variance. Psychometrika, 6, 309-316.

Scariano, S. M., \& Davenport, J. M. (1986). A four-moment approach and other practical solutions to the Behrens-Fisher problem. Communications in statistics: theory and methods, 15, 1467-1504.

Searle, S. R. (1992). Variance components. New York: Wiley.

Snedecor, G. W., \& Cochran, W. G. (1956). Statistical methods applied to experiments in agriculture and biology ( $5^{\text {th }} \mathrm{Ed}$.). Ames, IA: Iowa State Coll. Press.

Swallow, W. H., \& Monahan, J. F. (1984). Monte Carlo comparisons of ANOVA, MIVQUE, REML, and ML estimators of variance components. Technometrics, 26, 47-57.

Wehry, S. \& Algina, J. (2003). Type I Error Rates of Four Methods for Analyzing Data Collected in a Groups-Versus-Individuals Design. Journal of Modern Applied Statistical Methods, 2, 400-413

Welch, B. L. (1938). On the comparison of several mean values: An alternative approach. Biometrika, 38, 330-336. 


\section{Type I Error Rates of the Kenward-Roger Adjusted Degree of Freedom $F$-test for a Split-Plot Design with Missing Values}

\author{
Miguel A. Padilla \\ University of Alabama at Birmingham
}

\author{
James Algina \\ University of Florida
}

The Type I error rate of the Kenward-Roger (KR) test, implemented by PROC MIXED in SAS, was assessed through a simulation study for a one between- and one within-subjects factor split-plot design with ignorable missing values and covariance heterogeneity. The KR test controlled the Type I error well under all of the simulation factors, with all estimated Type I error rates between .040 and .075 . The best control was for testing the between-subjects main effect (error rates between .041 and .057) and the worst control was for the between-by-within interaction (.040 to .075). The simulated factors had very small effects on the Type I error rates, with simple effects in two-way tables no larger than .01.

Key words: Missing values, Kenward-Roger $F$-test, robustness, mixed models, split-plot design.

Introduction

According to Keselman et al. (1998), one of the most commonly used designs in educational and psychological research is the split-plot design, a design which includes both between-subjects and within-subjects factors. Responses on the within-subjects factor are obtained by repeatedly measuring each participant in the study. The repeated measures might be obtained at different points in time or under different treatments. Unfortunately, data collected in split-plot designs can be incomplete for a variety of reasons. Consider participants who drop out of a longitudinal study because of illness or death, refuse to answer questions on a survey because of its length or the sensitivity of the questions, or are unable to answer questions on a performance assessment test because of time constraints or lack of ability. Each results in missing values.

Miguel A. Padilla is a postdoctoral fellow in the School of Public at the University of Alabama at Birmingham. His research interests are in applied statistics. Email him at mpadilla@uab.edu. James Algina is a Professor of Educational Psychology at the University of Florida. His research interests are in applied statistics and psychometrics. Email him at algina@ufl.edu.

Little and Rubin (2002, p. 12) and Rubin
(1976) defined three types of missing data mechanisms. The missing data mechanisms, ordered from most restrictive to least restrictive in terms of assumptions made about the process that leads to the missing data, are missing completely at random (MCAR), missing at random (MAR), and not missing at random (NMAR). Generally, the NMAR missing data condition constitutes any missing data condition that is not MCAR or MAR. Let $f\left(\boldsymbol{r}_{i} \mid \boldsymbol{y}_{i}, \boldsymbol{X}_{i}, \boldsymbol{\psi}\right)$ denote the distribution of the missing data indicators for participant $i$, where $\boldsymbol{r}_{i}$ is a $K \times 1$ vector whose elements are zero for missing and one for observed in the corresponding elements of the $K \times 1$ vector of repeated variables $\boldsymbol{y}_{i}, \boldsymbol{X}_{i}$ is the design matrix for the factors, and $\psi$ contains the parameters for the relationship of $\boldsymbol{r}_{i}$ to $\boldsymbol{y}_{i}$ and $\boldsymbol{X}_{i}$.

$f\left(\boldsymbol{r}_{i} \mid \boldsymbol{y}_{i}, \boldsymbol{X}_{i}, \boldsymbol{\psi}\right)=f\left(\boldsymbol{r}_{i} \mid \boldsymbol{X}_{i}, \boldsymbol{\psi}\right)$, that is, if the distribution of the missing data indicators does not depend on the repeated measures. The $\boldsymbol{y}_{i}$ vector can be partitioned as $\boldsymbol{y}_{i}=\left[\begin{array}{ll}\boldsymbol{y}_{i o}^{\prime} & \boldsymbol{y}_{i m}^{\prime}\end{array}\right]^{\prime}$ where $\boldsymbol{y}_{i o}$ contains the repeated measures variables on which participant $i$ has observed scores and $\boldsymbol{y}_{i m}$ contains the repeated measures variables on which participant $i$ has missing scores. If $f\left(\boldsymbol{r}_{i} \mid \boldsymbol{y}_{i}, \boldsymbol{X}_{i}, \boldsymbol{\psi}\right)=f\left(\boldsymbol{r}_{i} \mid \boldsymbol{y}_{i o}, \boldsymbol{X}_{i}, \boldsymbol{\psi}\right)$, that is, the missing data indicator does not depend on the variables of which participant $i$ 
has missing scores, then the data are MAR.

The distribution of $\boldsymbol{y}_{i}$ can be written as $f\left(\boldsymbol{y}_{i} \mid \boldsymbol{X}_{i}, \boldsymbol{\theta}\right)$, where $\boldsymbol{\theta}$ contains the main effect and interaction parameters as well as the parameters for the covariance matrix for the repeated measures. A general method for consistent maximum likelihood (ML) estimation of $\boldsymbol{\theta}$ is obtained by including both the observed scores on the repeated measures and the missing data indicators, as well as $\boldsymbol{\theta}$ and $\boldsymbol{\psi}$, in the likelihood. However, Rubin (1976) showed that if the missing data mechanism is MCAR or MAR and if the parameters $\boldsymbol{\psi}$ and $\boldsymbol{\theta}$ are disjoint, ML estimators of the $\boldsymbol{\theta}$ parameters are consistent when the missing data indicators and $\psi$ are excluded from the data analysis.

Excluding the missing data indicators and $\psi$ is referred to as ignoring the missing data mechanism. Thus, the MCAR or MAR missing data mechanisms are ignorable for purposes of ML estimation. If the data are MCAR, both listwise deletion and ML ignoring the missing data mechanism will produce consistent estimators, but the ML estimators will be more accurate because they use all of the available data. Rubin (1976) also showed that the MCAR missing data mechanism is ignorable for sampling distribution based inference procedures such as hypothesis tests and confidence intervals. So, if the data are MCAR, either listwise deletion or ML ignoring the missing data mechanism can be used for inference, but ML will result in more powerful tests and narrower confidence intervals because it does not delete the observed data for participants with some missing values.

When ML estimation is used, whether the MAR missing data mechanism is ignorable for sampling distribution based inference depends on how the sampling covariance matrix is calculated. The MAR missing data mechanism is ignorable for sampling distribution based inferences on the means if the sampling covariance matrix is estimated from the observed information matrix for the means and the covariance parameter estimates, but not if the matrix is estimated from the portion of the observed information matrix that pertains only to the means (Kenward \& Molenberghs, 1998).
The MAR mechanism may not be ignorable for sampling distribution based inferences if the sampling covariance matrix is estimated from the expected information matrix. If the expected information matrix is used, it must take into account the actual sampling process implied by the MAR mechanism (Kenward \& Molenberghs, 1998). Kenward and Molenberghs (1998) referred to using this type of expected information matrix as using the unconditional sampling framework; whereas using the information matrix that ignores this sampling process is referred to as using the naïve sampling framework.

If the missing data mechanism is NMAR, the missing data mechanism is non-ignorable for purposes of ML estimation, and the pattern of missing values must be taken into account to obtain consistent ML estimates. This can be accomplished by using a selection model that incorporates a model for the missing values indicator or by using a pattern mixture model, which stratifies the data on the basis of the pattern of missing values (Albert \& Follmann, 2000; Algina \& Keselman, 2004a, 2004b; Diggle \& Kenward, 1994; Fitzmaurice, Laird, \& Shneyer, 2001; Kenward, 1998; Little, 1995; Troxel, 1998). Little (1995) provided details about these two approaches.

Unfortunately, traditional methods for analyzing data from a split-plot design such as ANOVA, adjusted degrees of freedom ANOVA, and MANOVA use listwise deletion and therefore are not likely to yield valid inferences except when the missing data mechanism is MCAR, an often unrealistic assumption in applied settings. Furthermore, these tests also assume that the covariance matrices $\left(\boldsymbol{\Sigma}_{j}, j=1, \ldots\right.$ . $J$ ) are homogenous across the $J$ levels of the between-subjects factor, another oftenunrealistic assumption. The tests will often fail to control the Type I error when the homogeneity assumption is violated (Keselman \& Keselman, 1990; Keselman, Keselman, \& Lix, 1995; Keselman, Lix, \& Keselman, 1996). For further details about these tests, see Greenhouse-Geisser (1959), Huynh and Feldt (1976), Huynh and Feldt (1970), Keselman and Keselman (1993), Mendoza (1980), and Looney and Stanley (1989). 
As a response to the unsatisfactory results created by violating the homogeneity of covariance assumption required by the standard $F$-tests, the multivariate Welch-James (WJ) test, which does not require the sphericity assumption or the homogeneity of covariance assumption, has been proposed for use in split-plot designs (Algina \& Keselman, 1997, 1998; Keselman, Algina, Wilcox, \& Kowalchuk, 2000; Keselman, Carriere, \& Lix, 1993). The WJ test tends to control the Type I error rates for the withinsubjects main effect and the between- by within-subjects interaction whether or not the dispersion matrices are heterogeneous. However, the WJ test also utilizes listwise deletion when there are missing values and would be expected to yield valid inferences only when the missing data are MCAR.

The Kenward-Roger (KR) adjusted degrees of freedom $F$-test is similar to the WJ test, but uses all available data in parameter estimation when there are missing values. Because parameter estimation is carried out by ML, the estimated parameters are consistent when data are MCAR or MAR. Additionally, the $\mathrm{KR}$ test is computed through a mixed-effects linear model so multisample sphericity is not required and heterogeneity of covariance can be modeled. Furthermore, the KR test uses a more accurate estimator of the sampling covariance matrix than the standard mixed model $F$-test.

When the mixed-effects linear model is used to analyze data, likelihood ratio, score, or Wald hypothesis tests can be used. Wald tests seem to be the most common. For example, when PROC MIXED in SAS is used, the default procedure for tests on the fixed effects is the Wald test. Let $\mathbf{L}$ be a $r \times J K$ contrast matrix of full row rank and let $\boldsymbol{\mu}=\left[\begin{array}{llll}\boldsymbol{\mu}_{1}^{\prime} & \boldsymbol{\mu}_{2}^{\prime} & \cdots & \boldsymbol{\mu}_{J}^{\prime}\end{array}\right]^{\prime}$. Each $\boldsymbol{\mu}_{j}$ is a $K \times 1$ vector of population means for the $K$ levels of the within-subjects factor in the split-plot design. The main effect and interaction hypotheses about the between- and within-subjects factors can be expressed as

$$
H_{0}: \mathbf{L} \boldsymbol{\mu}=\mathbf{0}
$$

where $\mathbf{0}$ is a $r \times 1$ vector with all elements equal to zero. Let $\Sigma_{j}$ denote the $K \times K$ population covariance matrix of the repeated measures for the $j^{\text {th }}$ level of the between-subjects factor, $\mathbf{S}_{j}$ the $K \times K$ restricted ML (REML) estimate of the covariance matrix and $\boldsymbol{\Sigma}_{i j}$ and $\mathbf{S}_{i j}$ the $K_{i} \times K_{i}$ sections $\left(i=1,2, \ldots, n_{j}\right)$ of the population and sample covariance matrices, respectively that pertain to the dependent variables on which the $i^{\text {th }}$ participant in the $j^{\text {th }}$ group has observed scores. In addition let $\mathbf{A}_{\mathrm{i}}$ denote a $K_{i} \times K$ indicator matrix obtained by eliminating the $k^{\text {th }}$ $(k=1,2, \ldots, K)$ row from the $K \times K$ identity matrix if the data for the $i^{\text {th }}$ participant is missing on the $k^{\text {th }}$ level of the within-subjects factor. The PROC MIXED default test statistic for testing the null hypothesis is

$$
F=\frac{\overline{\mathbf{y}}^{\prime} \mathbf{L}^{\prime}\left(\mathbf{L} \hat{\mathbf{M}}^{-1} \mathbf{L}^{\prime}\right)^{-1} \mathbf{L} \overline{\mathbf{y}}}{r}
$$

where $\quad \overline{\mathbf{y}}=\left[\begin{array}{llll}\overline{\mathbf{y}}_{1}^{\prime} & \overline{\mathbf{y}}_{2}^{\prime} & \cdots & \overline{\mathbf{y}}_{J}^{\prime}\end{array}\right]^{\prime} \quad$ is the $\mathrm{ML}$ estimate of $\boldsymbol{\mu}, r=\operatorname{rank}(\mathbf{L})$, and $\hat{\mathbf{M}}$ is a block diagonal matrix in which the $j^{\text {th }}$ block is $\sum_{i} \mathbf{A}_{i}^{\prime} \mathbf{S}_{i j}^{-1} \mathbf{A}_{i}$. The vector

$$
\overline{\mathbf{y}}_{j}=\left(\sum_{i} \mathbf{A}_{i}^{\prime} \mathbf{S}_{i j}^{-1} \mathbf{A}_{i}\right)^{-}\left(\sum_{i} \mathbf{A}_{i}^{\prime} \mathbf{S}_{i j}^{-1} \mathbf{y}_{i}\right) .
$$

The matrix $\hat{\mathbf{M}}^{-1}$ is the estimated sampling covariance matrix of the mean vector $\overline{\mathbf{y}}$ and is based on the expected information matrix calculated under the naïve sampling framework. Even when data are MCAR or there are no missing data, using $\hat{\mathbf{M}}^{-1}$ has two drawbacks:

1. $\hat{\mathbf{M}}^{-1}$ tends to be too small because it fails to take into account the uncertainty in $\overline{\mathbf{y}}$ introduced by substituting $\mathbf{S}_{i j}$ for $\boldsymbol{\Sigma}_{i j}$ when $\overline{\mathbf{y}}$ is obtained (Kackar \& Harville, 1984).

2. $\hat{\mathbf{M}}^{-1}$ is a biased estimate of $\mathbf{M}^{-1}$ (Prasad \& Rao, 1990; Booth \& Hobert 1998). Harville and Jeske (1992) developed a better estimator of $\mathbf{M}^{-1}$, denoted by $\hat{\boldsymbol{m}}^{\circledR}$. Kenward and Roger (1997) then developed an alternative estimator of $\mathbf{M}^{-1}$, denoted by $\hat{\boldsymbol{\Phi}}_{A}$. 
Kenward and Roger (1997) also developed the test statistic

$$
F^{*} \sim \lambda \frac{\overline{\mathbf{y}}^{\prime} \mathbf{L}^{\prime}\left(\mathbf{L} \hat{\boldsymbol{\Phi}}_{A} \mathbf{L}^{\prime}\right)^{-1} \mathbf{L} \overline{\mathbf{y}}}{r}
$$

where $\lambda$ is a scaling factor and $F_{r, d}$, is the critical value where $d$ is the approximate degrees of freedom. Both $\lambda$ and $d$ are estimated from the data. The Kenward-Roger procedure is implemented in SAS's PROC MIXED, but uses $\hat{\boldsymbol{m}}^{\circledR}$ in place of $\hat{\boldsymbol{\Phi}}_{A}$.

Keselman et al. (1993) and Algina and Keselman (1997) investigated the performance of the WJ test at controlling the Type I error rate in a split-plot design under several simulation conditions. In the former study the authors investigated (a) the number of levels of the within-subjects factor $(K=4,8)$, (b) the ratio of total sample size $N$ to $K-1$ (i.e., $N /(K-1)$ ), (c) the ratio of the smallest $n_{j}$ to $K-1$ (i.e., $n_{\min } /(K-$ 1)), (d) sample size inequality, (e) pairing of $n_{j}$ with covariance matrices, and (f) the shape of the distribution of the data. In all conditions the number of levels of the between-subjects factor was three $(J=3)$ and heterogeneity of covariance matrices was held constant at a ratio of $1: 3: 5$.

The latter study added $J=6$, degree of departure from sphericity measured by epsilon $(\varepsilon)$, and heterogeneity of covariance matrices with a ratio of 1:5:9. The authors were interested in the sample sizes required to control the Type I error rate when testing the within-subjects main effect and the between- by within-subjects interaction. In the first study, the sample sizes ranged from 30 to 171 and in the second study they ranged from 20 to 714 . From these two studies the authors provided sample size guidelines for the $\mathrm{WJ}$ test to control the Type I error under normal and non-normal data. The final sample size recommendations are summarized in Table 1.

Fai and Cornelius (1996) developed and compared four alternative test statistics $\left(F_{1}\right.$ to $\left.F_{4}\right)$ that can be used to test linear hypotheses on means in multivariate studies. They showed how to use the data to estimate the denominator degrees of freedom for the four statistics and the scaling factors $\lambda_{2}$ and $\lambda_{4}$ for the $F_{2}$ and $F_{4}$ statistics. The $F_{1}$ and $F_{2}$ statistics use $\hat{\mathbf{M}}^{-1}$ to estimate the covariance matrix of the mean vector whereas $F_{3}$ and $F_{4}$ use $\hat{\boldsymbol{m}}^{\circledR}$. The $F_{4}$ (3) statistic is similar to the statistic obtained by using the KR option in PROC MIXED, but with a different formula for the scaling factor and the degrees of freedom. The $F_{1}$ test is available in SAS when the Satterthwaite option is used in PROC MIXED. For further details on $F_{1}$ through $F_{2}$ see Fai and Cornelius.

Fai and Cornelius (1996) applied their tests to split-plot designs with a three-level between-subjects factor $(J)$ and a four-level within-subjects factor $(K)$. The covariance structure was compound symmetric. The design was unbalanced in that the number of subjects varied across levels of the between-subjects factor and data were not generated for some combinations of subjects and the within-subjects factor. Because the missing data were never generated, the missing data mechanism was effectively MCAR. Estimated Type I error rates and power were reported for the main effect of the between-subjects factor. All four tests provided reasonable control of the Type I error rate. The performance of $F_{1}$ and $F_{3}$, which do not include a scaling factor were very similar. Type I error rates and power for $F_{4}$ was always larger than for $F_{3}$.

Schaalje, McBride, and Fellingham (2002), reporting on a study conducted by McBride (2002), reported Type I error rates for $F_{1}$ and the test obtained using the KR option in PROC MIXED. McBride investigated the performance of these tests in a split-plot design. The following provides a social science example of the design investigated by McBride. Suppose three methods for structuring interactions among students in a mathematics classroom are to be compared; $n$ schools are randomly assigned to each method, where $n$ was three in half of the conditions studied by McBride and five in the other half. The methods will be implemented for three, six, or nine weeks. Each school contributes $K$ classes. Each class is assigned a single interaction quality score. In half of the conditions studied by McBride, $K=3$ and the 
Table 1. Final $n_{\min } /(K-1)$ Recommendations for Distribution by Between-Subjects Factor $(J)$ by Test by Within-Subjects Factor $(K)$

\begin{tabular}{|c|c|c|c|c|}
\hline \multirow[b]{2}{*}{ Distribution } & \multirow[b]{2}{*}{$\mathrm{J}$} & \multirow[b]{2}{*}{ Test } & \multicolumn{2}{|c|}{$n_{\min } /(K-1)$} \\
\hline & & & $K=4$ & $K=8$ \\
\hline \multirow[t]{4}{*}{ Normal } & 3 & $K$ & 2.00 & 3.00 \\
\hline & & $J \times K$ & 3.00 & 4.00 \\
\hline & 6 & K & 1.33 & 1.43 \\
\hline & & $J \times K$ & 4.75 & 5.00 \\
\hline \multirow[t]{4}{*}{ Non-normal } & 3 & $K$ & 3.00 & 4.00 \\
\hline & & $J \times K$ & 8.00 & 6.00 \\
\hline & 6 & $K$ & 1.33 & 1.71 \\
\hline & & $J \times K$ & 14.00 & 10.14 \\
\hline
\end{tabular}

Note. Based on Keselman et al. (1993) and Algina and Keselman (1997)

design was balanced. In the other half, $K=5$ so that within each school two classes would be assigned to two of the implementation periods and one class would be assigned to the remaining implementation period. In these conditions the design was unbalanced, but no data were missing.

McBride also investigated the effect of the covariance structure, which included the following five structures: compound symmetric (equal correlations and equal variance for the repeated measures), heterogeneous compound symmetric (equal correlations, but unequal variances for the repeated measures), Toeplitz, heterogeneous first-order autoregressive (correlations conform to a first-order autoregressive pattern, but the variances for the repeated measures are unequal), and first-order ante-dependence (see Wolfinger, 1996, for examples of these covariance structures). The results indicated that employing the KR option provided better control than did employing the
Satterthwaite option in PROC MIXED. Type I error rates were closer to the nominal level for balanced designs than for unbalanced designs. For unbalanced designs, Type I error rates improved as $n$ increased.

Kenward and Roger (1997) investigated how well the original KR procedure controlled Type I error rates in four situations: (a) a fourtreatment, two-period cross-over design, (b) a row-column- $\alpha$ design, (c) a random coefficients regression model for repeated measures data, and (d) a split-plot design. In (c) and (d) there were missing data. In (c) the missing data mechanism was MCAR. The missing data mechanism in (d) was not specified. In all situations, the KR test controlled the Type I error rate well.

Kowalchuk, Keselman, Algina, and Wolfinger (2004) compared the performance of the KR and the WJ procedures at controlling the Type I error rate under several simulation conditions for a $(J=3) \times(K=4)$ split-plot design. The simulation conditions they 
investigated were (a) type of population covariance structure, (b) degree of group size inequality, (c) positive and negative pairings of covariance matrices and group sample sizes, (d) shape of the data, and (e) type of covariance structure fit to the data. All simulation conditions had heterogeneous covariance matrices across the levels of the betweensubjects factor $(J)$ with a ratio of $1: 3: 5$. Data with missing values were not investigated. The KR test coupled with modeling the true covariance structure of the data performed better than did the WJ test under all conditions with small sample sizes. Also, the authors showed that always assuming an unstructured covariance structure performed comparably to modeling the true covariance structure when using the KR test.

Based on the previous results, the KR test and similar tests like the $F_{4}$ test (Fai \& Cornelius, 1996) can control the Type I error rate for a variety of repeated measures designs when there are either missing data but no covariance heterogeneity or covariance heterogeneity but no missing data. The purpose of this study is to investigate control of the Type I error rate by the KR test as it is implemented in PROC MIXED when there are both missing data and covariance heterogeneity. Because of the similarities between the KR test and the WJ test and because Type I error rates for the WJ test have been extensively evaluated by Algina and Keselman and their colleagues, the KR test will be evaluated under conditions similar to those used by these authors to evaluate the WJ test, with the addition of missing value conditions.

\section{Methodology}

\section{Study Variables}

Eight variables were manipulated in this simulation. The variables of interest are (a) the number of levels of the between-subjects factor (A), (b) the number of levels of the within-subjects factor (B), (c) $n_{\min } /(K-1)$ where $K$ is the number of levels of the within-subjects factor, (d) sample size inequality across the between-subjects factor (SSI), (e) degree of sphericity as quantified with Box's (1954) epsilon $(\varepsilon)$, (f) nature of pairing of group sizes with covariance matrices (NPSC), (g) type of missing data mechanism (TMDM), and (h) percent of missing data (PM). For each combination of levels of the factors, five thousand replications were generated.

Both the number of levels of the between-subjects and within-subjects factors were investigated in the study. Each of these factors had two levels with $J=3,6$ and $K=3,6$. In the initial planning, the study was going to investigate $J=3,6$ and $K=4,8$, but preliminary simulations indicated that using PROC MIXED took an inordinate amount of time when $K=8$.

The sample sizes investigated were $n_{\min } /(K-1)=4,6$ for $J=3$ and $n_{\min } /(K-1)=5$, 7.7 for $J=6$. Within each pair of $n_{\min } /(K-1)$ ratios, the smaller ratio corresponds to sample size recommendations in Table 1 for the between- by within-subjects interaction with normal data, $K=8$, and $J=3,6$. The larger $n_{\min } /(K-1)$ values were based on the recommendations from Table 1 and the higher demands missing values will place on the data analysis.

Keselman et al. (1998) found that unequal sample sizes in split-plot designs were common, occurring in a little over $50 \%$ of the split-plot designs. For this reason unequal sample sizes were investigated. In particular, moderate and severe group size inequalities were investigated as defined by Keselman et al. (1993) through the coefficient of variation:

$$
C=(\bar{n} \sqrt{J})^{-1} \sqrt{\sum_{j=1}^{J}\left(n_{j}-\bar{n}\right)^{2}},
$$

where $C \simeq .16, .33$ describe moderate and severe group size inequality, respectively.

Departures from sphericity quantified by Box's (1954) epsilon $(\varepsilon)$, were also investigated with $\varepsilon=.60, .75, .90$, where $\varepsilon=.60$ and $\varepsilon=.75$ represent relatively severe and moderate violations of sphericity, respectively. In past studies $\varepsilon=.40, .57, .75$ were investigated (Algina \& Keselman, 1997; Keselman, Keselman, \& Shaffer, 1991; Algina \& Oshima, 1994). However, $\varepsilon$ has a lower bound of $\varepsilon=1 /(K-1)$, so for $K=3$ the lower bound is $\varepsilon=.50$ and so $\varepsilon=.40$ cannot be investigated. Also, according to Huynh and Feldt (1976) $\varepsilon=$ 
.75 represents the lower limit of $\varepsilon$ found in educational and psychological data. The epsilon values in this simulation study were chosen based on this contention. In particular, note that $\varepsilon=.75$ is the mid value and the other values are $\varepsilon \pm .15$. The actual covariance matrices are shown in Table 2. ratio of sample size to heterogeneity of covariance matrices was set at 1:3:5 for $J=3$ and 1:3:5:1:3:5 for $J=6$ (Algina \& Keselman, 1997; Keselman et al., 1993; Keselman, Algina, Kowalchuk, \& Wolfinger, 1999). Furthermore,

Table 2. Pooled Covariance Matrices

$$
\begin{aligned}
& K=3 \quad K=6 \\
& \varepsilon=.90\left[\begin{array}{ccc}
18.0 & 5.0 & 6.0 \\
& 8.0 & 5.0 \\
& & 7.0
\end{array}\right]\left[\begin{array}{cccccc}
18.0 & 5.0 & 7.0 & 7.0 & 6.0 & 5.0 \\
& 12.0 & 8.0 & 7.0 & 6.0 & 5.0 \\
& & 10.0 & 6.0 & 6.0 & 5.0 \\
& & & 10.0 & 5.0 & 5.0 \\
& & & & 9.0 & 5.0 \\
& & & & & 8.0
\end{array}\right] \\
& \varepsilon=.75\left[\begin{array}{ccc}
23.2 & 4.5 & 7.4 \\
& 10.3 & 5.3 \\
& & 4.3
\end{array}\right]\left[\begin{array}{cccccc}
29.6 & 12.7 & 7.5 & 7.0 & 5.9 & 5.9 \\
& 15.1 & 7.9 & 6.0 & 6.4 & 4.9 \\
& & 13.2 & 6.9 & 6.0 & 5.4 \\
& & & 9.4 & 6.0 & 4.8 \\
& & & & 8.0 & 5.0 \\
& & & & & 5.9
\end{array}\right] \\
& \varepsilon=.60\left[\begin{array}{ccc}
23.8 & 1.9 & 9.3 \\
& 9.5 & 5.7 \\
& & 3.9
\end{array}\right]\left[\begin{array}{cccccc}
28.8 & 4.8 & 10.1 & 9.8 & 8.3 & 7.3 \\
& 17.4 & 8.1 & 7.4 & 6.9 & 4.1 \\
& & 9.9 & 7.7 & 6.5 & 5.7 \\
& & & 8.3 & 5.6 & 4.3 \\
& & & & 5.6 & 4.4 \\
& & & & & 4.3
\end{array}\right]
\end{aligned}
$$

The pairing direction, positive or negative, between the unequal group sizes and the heterogeneous covariance matrices were also investigated. A pairing is positive when the largest $n_{j}$ is paired with the covariance matrix with the largest elements and negative when the largest $n_{j}$ is paired with the covariance matrix with the smallest elements. In order to have comparability with previous research results, the previous studies have shown that this ratio and pairing can have a strong impact on the Type I error rate for approximate univariate $F$-tests, such as the Huynh-Feldt $F$-test (1976), and multivariate tests, particularly when the sample size is small (Keselman \& Keselman, 1990). Specifically, positive pairings produce conservative Type I errors and negative pairing produce liberal Type I errors. 
The MCAR and MAR missing data mechanisms were investigated in connection with $5 \%$, and $15 \%$ probability of missing data at each level of the within-subjects factor except the first level; there were no missing data in the first level (see the Data Generation section for an explanation). Only the MCAR and MAR missing data mechanisms were investigated because Padilla and Algina (2004) demonstrated that the NMAR missing data mechanism negatively impacts the Type I error rate of the KR test statistic in a repeated measured design with no between-subjects factors.

\section{Data Generation} model

The data were generated by using the

$$
y_{i j k}=\mu+e_{i j k} .
$$

The mean vector $\boldsymbol{\mu}_{j}=\left[\begin{array}{llll}\mu_{1} & \mu_{2} & \ldots & \mu_{K}\end{array}\right]^{\prime}$ was the same for all $J$ groups and the elements $\mu_{k}$ were equal because the focus of the study was on control of the Type I error rate by the KR test. The common elements were arbitrarily set to zero. The e vector was a $K \times 1$ random vector such that $\mathbf{e} \sim N I D\left(\mathbf{0}, \Sigma_{j}\right)$.

All data simulations and analyses were conducted using SAS version 9.0. For each combination of levels of the simulation factors, the following steps were used to simulate the data in the $j^{\text {th }}$ level of the between-subjects factor.

1. Simulate $\mathbf{Z}$, a $n_{j} \times K$ matrix of pseudorandom standard normal variables where $n_{j}$ is the sample size for the $j^{\text {th }}$ level of the between-subjects split-plot design.

2. Calculate $\mathbf{T}$ a $K \times K$ upper triangular Cholesky factor of the covariance matrix $\Sigma$.

3. Calculate $\boldsymbol{y}=d_{j} \mathbf{Z T}$, where $d_{j}$ is a constant selected to create the required degree of covariance heterogeneity.

4. In all conditions there were no missing values on $y_{i 1}$ :

a. For MCAR, eliminate $y_{i k}(k=2$, ..., $K)$ if $U_{i k}<\pi$ where $\pi$ is the expected proportion of the missing data on $y_{k}$ and $U_{i k}$ is a uniform random variable.

b. $\quad$ For MAR, eliminate $y_{i k}$ if $U_{i k}<$ $\Phi\left(m y_{i 1}+c\right)$, where $\Phi$ is the cumulative standard normal distribution function and the parameters $m$ and $c$ will be described below.

In selecting data points for elimination, the parameter $m$ controls how dependent the missing data are on $y_{1}$ in the MAR condition and was set to one. Let

$$
r_{i k}=\left\{\begin{array}{ll}
1 & \text { if } y_{i k} \text { is missing } \\
0 & \text { otherwise }
\end{array} .\right.
$$

With $m=1$, the biserial correlation between $r_{i k}$ and $y_{1}$ was .5 in the MAR condition. Hence, the missing data indicators depend fairly heavily on $y_{1}$. With $m=1$, the expected proportion of missing data on $y_{k}$ is dependent on $c$. In the procedure described in the preceding paragraphs, the probability that $r_{i k}=1$ is related to $y_{1}$ is modeled by a normal ogive (probit) model. Using well-known facts about the normal ogive model (see, for example, Lord \& Novick, 1968, equations 16.9.3 and 16.94), it can be shown that

$$
c=\left\{\Phi^{-1}(\pi)\right\} \sqrt{1+m^{2}} .
$$

Thus, for $m=1$, and for $5 \%$ and $15 \%$ missing data conditions, the expression becomes $c=-1.645 \sqrt{2}$, and $c=-1.036 \sqrt{2}$, respectively.

Data Analysis

The SAS PROC MIXED program used in this simulation is

\section{proc mixed;}

class Person A B;

model score $=$ A B A*B/ ddfm=kenwardroger; repeated $\mathrm{B} /$ subject $=$ Person group $=\mathrm{A}$ type $=\mathrm{un}$;

run;

The following list describes various aspects of the code.

- Person is a variable that identifies simulated subjects. 
- Score is the variable containing scores on the dependent variable.

- $\quad$ A is a variable that identifies the levels of the between-subjects factor.

- $\quad$ B is a variable that identifies the levels of the within-subjects factor.

- ddfm = kenwardroger instructs SAS to use the KR statistic to test the main effects and the interaction.

Repeated is a key word that tells SAS that B is a repeated measures (within-subjects) factor and is necessary when there are missing data.

- $\quad$ Group $=\mathbf{A}$ tells SAS to model the covariance matrix for each level of A. That is, it specifies modeling heterogeneity of covariance matrices across the levels of A.

- $\quad$ Subject $=$ Person tells SAS that the score values are correlated within each person.

- $\quad$ Type $=$ un instructs SAS to estimate an unstructured covariance matrix with $K$ estimated variances and $K(K-1) / 2$ estimated covariances.

Although there are several covariance structures that can be used to model the covariance matrix (Wolfinger, 1996), only the unstructured between-subjects heterogeneous structure $(\mathrm{UN}-\mathrm{H})$ covariance matrix was used in this simulation. Although using a UN-H covariance structure comes at the cost of estimating $K(K+1) / 2$ parameters, Kowalchuk et al. (2004) showed that under similar simulation conditions assuming an unstructured covariance structure performed comparably to modeling the true covariance structure when using the KR test.

The corresponding $p$-values of applying the KR test to 5,000 replications were available for each combination of the investigated conditions. The result of each test was summarized by a dichotomous variable, defined in the following manner:

$$
\text { Type I Error }=\left\{\begin{array}{l}
0 \text { if the } p-\text { value }<.05 \\
1 \text { otherwise }
\end{array} .\right.
$$

For each of the between-subjects, within-subjects, and between- by within-subjects KR tests the Type I error variable was analyzed by using logistic regression with the study variables as factors. A forward selection approach was used to select appropriate models. The models used were an intercept-only model, a model with main effects only, a model with main effects and two-way interactions, and so forth. A model was considered adequate for the data if the $\chi^{2}$ goodness of fit test was nonsignificant or if Bentler's (1990) Comparative Fit Index $(C F I) \geq 95$. An index of fit was used because, due to the large number of replications, the $\chi^{2}$ goodness of fit statistic for the logistic model could be very sensitive to small effects of the factors. The CFI in this context was calculated as follows:

$$
C F I=1-\left(\lambda / \lambda_{i}\right)
$$

where $\lambda=\max \left(\chi^{2}-d f, 0\right), \chi^{2}$ and $d f$ are the chi-squared goodness of fit statistic for the fitted model and the corresponding degrees of freedom, $\lambda_{i}=\max \left(\chi_{i}^{2}-d f_{i}, \chi^{2}-d f, 0\right)$, and $\chi_{i}^{2}$ and $d f_{i}$ is the chi-squared goodness of fit statistic for the intercept-only model and its corresponding degrees of freedom.

Assessment of the Type I error rates were based on Bradley's (1978) liberal criterion for identifying conditions in which hypothesis testing procedures work adequately. His liberal criterion is $.5 \alpha \leq \tau \leq 1.5 \alpha$ where $\alpha$ is the nominal Type I error and $\tau$ is the actual Type I error. Using $\alpha=.05$, the liberal criterion is $.025 \leq \tau \leq .075$.

\section{Results}

Analysis of Type I Error Rates for the BetweenSubjects Main Effect

The distribution of Type I error rates for the between-subjects main effect is shown in Figure 1 and has $M=.050$ and $S D=.003$. The range of the Type I error rate is [.041, .057]. The goodness of fit test for the intercept-only model was not significant, $\chi^{2}(383)=398.64, p=.28$, suggesting that the effects of the factors were quite small. Because the Type I errors rates for the between-subjects main effect were predominately within Bradley's liberal criterion and because the intercept only model could not be rejected, it appears that the KR between-subjects omnibus test controls the Type 
I error well at all levels of the investigated factors in this study.

The distribution of Type I error rates for the within-subjects main effect is shown in Figure 2 and has $M=.052$ and $S D=.005$. The range of the Type I error rate is $[.041, .070]$. Hence, in all conditions the Type I error rate was well within Bradley's (1978) liberal criterion interval. CFI for the model with main effects and two-way interactions was .98. In addition the goodness of fit test was non-significant, $\chi^{2}(339)$ $=354.24, p=.27$. Thus, the two-way interaction model was selected for further analysis. Wald tests indicated that all factors that had significant main effects also entered into significant twoway interactions. As might be expected from Figure 2, all effects were small. Mean Type I Error rates were between .048 and .061 in all two-way tables and no simple effect was as large as .01. Type I error rates tended to be larger when $J, K$, and percent missing data were larger. Type I error rates also tended to be larger for MAR data ${ }^{1}$.

Analysis of Type I Error Rates for the WithinSubjects Main Effect

Because a major focus of this study is the effect of sample size on Type I error rates, two-way tables of means for the only interactions with sample size are presented in Table 3. These results indicate that control of the Type I error rate was good regardless of the sample size and that the effect of sample size on the Type I error rate was quite small.
Analysis of Type I Error Rates for the Betweenby Within-Subjects Interaction

The distribution of Type I error rates for the interaction effect is presented in Figure 3 and has $M=.054$ and $S D=.007$. The range of the Type I error rate is $[.040, .075]$. Consequently, in all conditions the Type I error rate was once again within Bradley's liberal criterion interval. CFI for the model with main effects and two-way interactions was 1.00 . In addition, the goodness of fit test was non-significant, $\chi^{2}(339)$ $=368.79, p=.23$. Wald tests indicated that all factors that had significant main effects also entered into significant two-way interactions. As might be expected from Figure 3, all effects of factor were small. Mean Type I Error rates were between.049 and .058 in all two-way tables and no simple effect was as large as .01. Type I error rates tended to be larger when $K$, sample size inequality, and percent missing were larger. Type I error rates also tended to be larger when the sample size-covariance pairing was negative. The effect of $J$ was miniscule. The effect of type of missing data tended to be small and to vary in direction over levels of the factors with which it interacted.

Because a major focus of this study is the effect of sample size on Type I error rates, two-way tables of means for the interactions only with sample size are presented in Table 4. These results indicate that control of the Type I error rate was good regardless of the sample size and that the effect of sample size on the Type I error rate was quite small. 


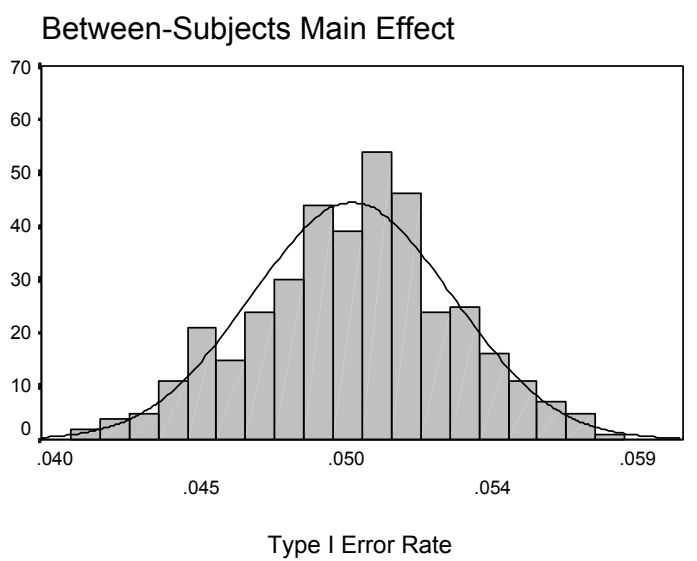

Figure 1. Distribution of Type I Error Rates: Between-Subjects KR F-Test

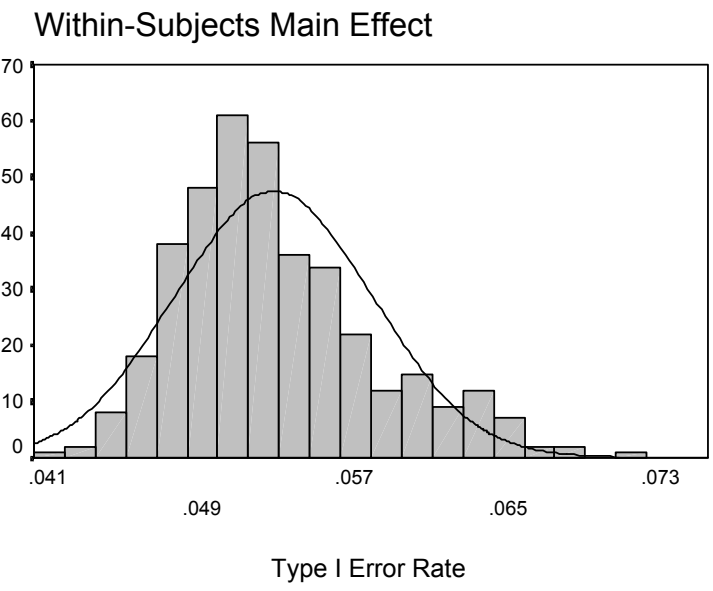

Figure 2. Distribution of Type I Error Rates: Within-Subjects KR F-Test

Table 3. Effect of $n_{\min } /(K-1)$ on Type I Error Rates for the Within-Subjects Main Effect

\begin{tabular}{lccc}
\hline & & \multicolumn{2}{c}{$\mathrm{n}_{\min } /(K-1)$} \\
\cline { 3 - 4 } Factor & Factor levels & Small & Large \\
\hline \multirow{2}{*}{$\mathrm{K}$} & 3 & .0503 & .0514 \\
& 6 & .0541 & .0539 \\
$\mathrm{PM}$ & $5 \%$ & & .0509 \\
& $15 \%$ & .0594 & .0545 \\
\hline
\end{tabular}

Note. Each proportion is out of 480,000 hypothesis tests. 
Between- by Within-Subjects Interaction

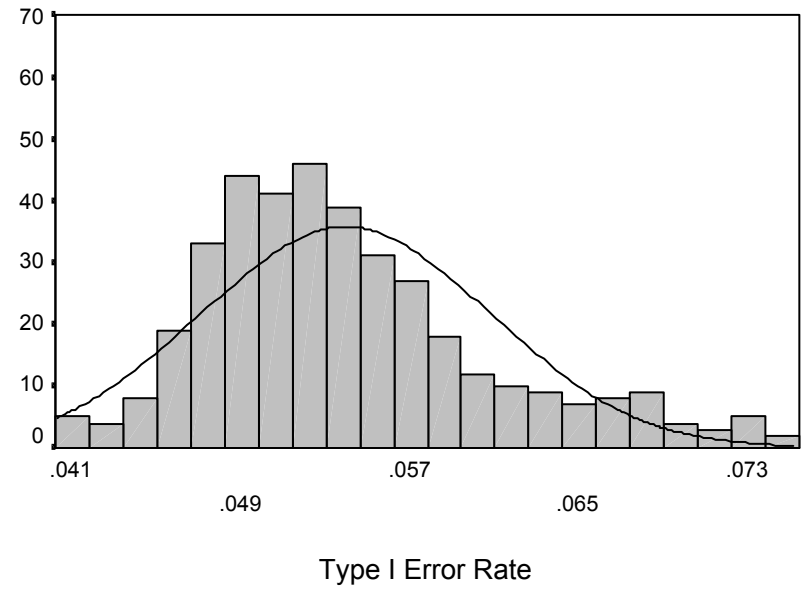

Figure 3. Distribution of Type I Error Rates: Interaction KR F-Test

Table 4. Effect of $n_{\min } /(K-1)$ on Type I Error Rates for the Between- by Within-Subjects Interaction

\begin{tabular}{cccc}
\hline & & \multicolumn{2}{c}{$\mathrm{n}_{\min } /(K-1)$} \\
\cline { 3 - 4 } Factor & Factor levels & Small & Large \\
\hline \multirow{2}{*}{$\mathrm{K}$} & 3 & .0509 & .0507 \\
& 6 & .0577 & .0553 \\
$\mathrm{NSI}$ & .16 & .0527 & .0524 \\
& .33 & .0559 & .0537 \\
NPSC & & & .0517 \\
& Positive & .0509 & .0543 \\
TMDM & Negative & .0577 & .0519 \\
& MCAR & .0552 & .0542 \\
& MAR & .0534 & .0504 \\
PM & $5 \%$ & .0508 & .0557 \\
& $15 \%$ & .0578 & \\
\hline
\end{tabular}

Note. Each proportion is out of 480,000 hypothesis tests. 


\section{Conclusion}

The results of this study support the conclusion that sampling distribution based inferences on the means using ML estimates can control the Type I error rate under MCAR missing data mechanisms. Additionally, sampling distribution based inferences using ML estimates can control the Type I error rate when the missing data mechanism is MAR (Little \& Rubin, 2002; Rubin, 1976) Most important this control can be obtained with relatively modest sample size requirements.

With respect to the between-subjects main effect, the KR test statistic controlled the Type I error rate well across all levels of the simulation factors. Most Type I error rates were within both Bradley's conservative criterion and all were well within the liberal criterion. None of the simulation factors affected the Type I error rate of the between-subjects main effect. In regard to the within-subjects main effect and the within- by between-subjects interaction, although a number of factors affected Type I error rates, all effects were very small and all Type I error rates were within Bradley's liberal criterion.

The effects of the factors on Type I error rates were generally quite small. Nevertheless it is clear that the effects of the factors on the on Type I error rates must be due to their effects on the accuracy of the $F$-distribution as an approximation to the sampling distribution of the test statistic. The KR test statistic was selected because it uses a better estimator of the covariance matrix for small sample sizes and Satterthwaite (1946) type degrees of freedom based on the better estimate of the covariance matrix. However, when the data are incomplete in addition to being relatively small and paired with a MAR missing data mechanism, the accuracy of the approximation may be worse than when the data are complete.

Although the design investigated in this study was a popular split-plot design with one between- and one within-subjects factor, the positive findings open the door for further simulation work on using ML to directly estimate model parameters from split-plot designs with missing values. One condition that can be investigated is a non-normal distribution of the dependent variable. In the present study, the data were generated under a multivariate normal distribution and since data from educational or psychological research cannot be presumed to be normal, investigation of a nonnormal data condition can provide applied researchers with valuable information as to whether the KR test is robust to the normality assumption. In other words, can the KR test control the Type I error when the normality assumption is violated?

Even though all of the Type I error rates of the KR test were within Bradley's (1978) liberal criterion, it is not clear at what percent of missing data the KR test will begin to breakdown. Additionally, it is not clear how small the sample sizes can be and still have the KR test provide reasonable control of the Type I error. Consequently, future work could focus on what are the percent of missing data and sample size requirements needed for the KR test to provide reasonable control of the Type I error.

An alternative to the estimator of the sampling covariance matrix used in the KR test is the sandwich estimator (White, 1980, Liang \& Zeger, 1986). The sandwich estimator provides a consistent estimator of the covariance matrix given that the model for the means is correct. That is the model for the covariance structure need not be correct. Hence, it may be fruitful to compare the performance of the $F$-test using the sandwich estimator to the KR test at controlling the Type I error in a simulation study with ignorable missing data.

\section{References}

Albert, P. S., \& Follmann, D. A. (2000). Modeling repeated count data subject to information dropout. Biometrics, 56, 667-677.

Algina, J., \& Keselman, H. J. (1997). Testing repeated measure hypotheses when covariance matrices are heterogeneous: Revisiting the robustness of the Welch-James test. Multivariate Behavioral Research, 32, 255274.

Algina, J., \& Keselman, H. J. (1998). A power comparison of the Welch-James and Improved General Approximation tests in the split-plot design. Journal of Educational and Behavioral Statistics, 23, 152-169. 
Algina, J., \& Keselman, H. J. (2004a). A comparison of methods for longitudinal analysis with missing data. Journal of Modern Applied Statistical Methods, 3, 13-26.

Algina, J., \& Keselman, H. J. (2004b). Assessing treatment effects in randomized longitudinal two-group designs with missing observations. Journal of Modern Applied Statistical Methods, 3, 271-287.

Algina, J., \& Oshima, T. C. (1994). Type I error rates for Huynh's general approximation and improved general approximation tests. British Journal of Mathematical and Statistical Psychology, 47, 151-165.

Bentler, P. M. (1990). Comparative fit indexes in structural models. Psychological Bulletin, 107, 238-246.

Booth, J. G., \& Hobert, J. P. (1998). Standard errors of prediction in generalized linear mixed models. Journal of the American Statistical Association, 96, 262-272.

Box, G. E. P. (1954). Some theorem on quadratic forms applied in the study of analysis of variance problems, I. Effect of inequality of variance and correlations between errors in the two-way classification. Annals of Mathematical Statistics, 25, 484-498.

Bradley, J. (1978). Robustness? British Journal of Mathematical and Statistical Psychology, 31, 144-152.

Diggle, P. D., \& Kenward, M. G. (1994). Informative dropout in longitudinal data analysis. Applied Statistics, 43, 49-93.

Fai, H. T., \& Cornelius, P. L. (1996). Approximate $F$-tests for multiple degree of freedom hypotheses in generalized least squares analyses of unbalanced split-plot experiments. Journal of Statistical Computation and Simulation, 54, 363-378.

Fitzmaurice, G. M., Laird, N. M., \& Shneyer, L. (2001). An alternative parameterization of the general linear mixture model for longitudinal data with non-ignorable drop-outs. Statistics in Medicine, 20, 1009-1021.

Greenhouse, S. W., \& Geisser, S. (1959). On methods in the analysis of profile data. Psychometrika, 24, 95-112.

Harville, D. A., \& Jeske, D. R. (1992). Mean squared error of estimation or prediction under a general linear model. Journal of the American Statistical Association, 87, 724-731.

Huynh, H., \& Feldt, L. S. (1970). Conditions under which mean square ratios in repeated measures designs have exact $F$ distributions. Journal of the American Statistical Association, 65, 1582-1589.

Huynh, H. \& Feldt, L. S. (1976). Estimation of the Box correction for degree of freedom from sample data in randomized block and split-plot designs. Journal of Educational Statistics, 1, 69-82.

Kackar, R. N., \& Harville, D. A. (1984). Approximations for standard errors of estimators of fixed and random effects in mixed linear models. Journal of the American Statistical Association, 79, 853-862.

Kenward, M. G. (1998). Selection models for repeated measurements with nonrandom dropout: An illustration of sensitivity. Statistics in Medicine, 17, 2723-2732.

Kenward, M. G., \& Molenberghs, G. (1998). Likelihood based frequentist inference when data are missing at random. Statistical Science, 13, 236-247.

Kenward, M. G., \& Roger, J. H. (1997). Small sample inference for fixed effects from restricted maximum likelihood. Biometrics, 53, 983-997.

Keselman, H. J., Algina, J., Kowalchuk, R. K., \& Wolfinger, R. D. (1999). The analysis of repeated measurements: A comparison of mixed-model Satterthwaite $\mathrm{F}$ tests and a nonpooled adjusted degrees of freedom multivariate test. Communications in Statistics Theory and Methods, 28, 2967-2999.

Keselman, H. J., Algina, J., Wilcox, R. R., \& Kowalchuk (2000). Testing repeated measures hypothesis when covariance matrices are heterogeneous: Revisiting the robustness of the Welch-James test again. Educational and Psychological Measurement, 60, 925-938.

Keselman, H. J., Carriere, K. C., \& Lix, L. M. (1993). Testing repeated measure hypotheses when covariance matrices are heterogeneous. Journal of Educational Statistics, 18, 305-319.

Keselman, H. J., Huberty, C. J., Lix, L. M., Olejnik, S., Cribbie, R. A., Donohue, B., Kowalchuk, R. K., Lowman, L. L., Petosky, M. D., Keselman, J. C., \& Levin, J. R. (1998). 
Statistical practices of educational researchers: An analysis of their ANOVA, MANOVA, and ANCOVA analyses. Review of Educational Research, 68, 350-386.

Keselman, H. J., \& Keselman, J. C. (1990). Analysing unbalanced repeated measures designs. British Journal of Mathematical and Statistical Psychology, 43, 265-282.

Keselman, H. J., \& Keselman, J. C. (1993). Analysis of repeated measurement. In L. K. Edwards (Eds.), Applied analysis of variance in behavioral science (pp. 105-145). New York: Marcel Dekker.

Keselman, H. J., Keselman, J. C., \& Lix, L. M. (1995). The analysis of repeated measurements: Univariate tests, multivariate tests, or both? British Journal of Mathematical and Statistical Psychology, 48, 319-338.

Keselman, H. J., Keselman, J. C., \& Shaffer, J. P. (1991). Multiple pairwise comparisons of repeated measures means under violation of multisample sphericity. Psychological Bulletin, 110, 162-170.

Keselman, J. C., Lix, L. M., \& Keselman, H. J. (1996). The analysis of repeated measurements: A quantitative research synthesis. British Journal of Mathematical and Statistical Psychology, 49, 275-298.

Kowalchuk, R. K., Keselman, H. J., Algina, J., \& Wolfinger, R. D. (2004). The analysis of repeated measurements with mixedmodel adjusted $F$-tests. Educational and Psychological Measurement, 64, 224-242.

Liang, K-Y., \& Zeger, S. L. (1986). Longitudinal analysis using generalized linear models. Biometrika, 73, 13-22.

Little, R. J. A. (1995). Modeling the drop-out mechanism in repeated-measures studies. Journal of the American Statistical Association, 90, 1112-1121.

Little, R. J. A., \& Rubin, D. B. (2002). Statistical analysis with missing data ( $2^{\text {th }}$ ed.). New York: John Wiley \& Sons.

Looney, S. W., \& Stanley, W. B. (1989). Exploratory repeated measures analysis for two or more groups: Review and update. American Statistician, 43, 220-225.
Lord, F. M., \& Novick, M. R. (1968). Statistical theories of mental test score, with contributions by Alan Birnbaum. Reading, MA: Addison-Wesley.

McBride, G. B. (2002). Statistical methods helping and hindering environmental science and management. Journal of Agricultural, Biological, and Environmental Statistics, 7, 300-305.

Mendoza, J. L. (1980). A significance test for multisample sphericity. Psychometrika, 45, 495-498.

Padilla, M. A., \& Algina, J. (2004). Type I error rates for a one factor withinsubjects design with missing values. Journal of Modern Applied Statistical Methods, 3, 406-416.

Prasad, N. G. N, \& Rao, J. N. K. (1990).

The estimation of mean squared error of small-area estimators. Journal of the American Statistical Association, 85, 163-171.

Rubin, D. B. (1976). Inference and missing data. Biometrika, 63, 581-592.

Satterthwaite, F. E. (1946). An approximation distribution of estimates of variance components. Biometrics Bulletin, 2, 110-114.

Schaalje, G. B., McBride, J. B., \& Fellingham, G. W. (2002). Adequacy of approximations to distributions of test statistics in complex mixed linear models. Journal of Agricultural, Biological, and Environmental Statistics, 7, 512-524.

Troxel, A. B. (1998). Analysis of longitudinal data with non-ignorable nonmonotone missing values. Applied Statistics, 47, 425-438.

White, H. (1980). A hetersckedasticity-consistent covariance matrix and a direct test for hetersckedasticity. Econometrica, 48, 817-838.

Wolfinger, R. D. (1996). Heterogeneous variance-covariance structures for repeated measures. Journal of Agricultural, Biological, and Environmental Statistics, 1, 205-230. 


\section{Reliability and Statistical Power: How Measurement Fallibility Affects Power and Required Sample Sizes for Several Parametric and Nonparametric Statistics}

\author{
Gibbs Y. Kanyongo \\ Duquesne University
}

\author{
Gordon P. Brook \\ Lydia Kyei-Blankson Gulsah Gocmen
Ohio University
}

The relationship between reliability and statistical power is considered, and tables that account for reduced reliability are presented. A series of Monte Carlo experiments were conducted to determine the effect of changes in reliability on parametric and nonparametric statistical methods, including the paired samples dependent $t$ test, pooled-variance independent $t$ test, one-way analysis of variance with three levels, Wilcoxon signed-rank test for paired samples, and Mann-Whitney-Wilcoxon test for independent groups. Power tables were created that illustrate the reduction in statistical power from decreased reliability for given sample sizes. Sample size tables were created to provide the approximate sample sizes required to achieve given levels of statistical power based for several levels of reliability.

Key words: Pseudorandom generation, effect size, Monte Carlo simulations.

\section{Introduction}

Students of statistics usually become familiar with the factors that affect statistical power. For example, most students learn that sample size, level of significance, and estimated effect size all determine the a priori power of a statistical analysis. Some know that how effectively a particular design reduces error variance affects power, as does the directionality of the alternative hypothesis. However, many students do not realize that the reliability of measurements may also affect the statistical power (Hopkins \& Hopkins, 1979). Light, Singer, and Willett (1990) provided tables to illustrate the point. Unfortunately, their tables provide only a very few situations and are therefore limited in their usefulness. It is not

Gibbs Y. Kanyongo is an Assistant Professor of Education. He teaches educational statistics and research. Email: kanyongog@duq.edu. Gordon Brooks is an Associate Professor in the College of Education at Ohio University. He teaches educational statistics and research, and Monte Carlo simulations. He is also an author of several computer simulation programs. Lydia Kyei-Blankson and Gulsah Gocmen are graduate student in the College of Education. clear how the Light et al. tables were developed. The present study extends their tables and provides such information for additional statistical methods.

Using the information provided in these tables, researchers can account for different levels of reliability as they determine sample sizes for their studies. Perhaps the converse approach is even more useful; however, that is, researchers might be encouraged to improve the reliability of their instruments in order to need fewer participants in their studies. These tables can also be useful tools in teaching students the relationship between reliability of a survey instrument and statistical power.

\section{Background}

One of the chief concerns of research design is to ensure that a study has adequate statistical power to detect meaningful differences, if indeed they exist. There is a very good reason researchers should worry about power a priori: If researchers are going to invest a great amount of money and time in carrying out a study, then they would certainly want to have a reasonable chance, perhaps $70 \%$ or $80 \%$, to find a difference between groups if it does exist. Thus, a priori power (the probability of rejecting a null hypothesis that is false) will inform researchers how many subjects per group 
will be needed for adequate power. Several factors affect statistical power. That is, once the statistical method and the alternative hypothesis have been set, the power of a statistical test is directly dependent on the sample size, level of significance, and effect size (Stevens, 2002). Often overlooked, however, is the relationship that variance has with power. Specifically, variance influences power through the effect size. For example, Cohen (1988) defined the effect for the $t$ statistic as

$$
\delta=\left(\mu_{1}-\mu_{0}\right) / \sigma_{X}
$$

If variance can be reduced, effect size increases. Variance reduction techniques include using a more homogeneous population and improving the reliability of measurements (Aron \& Aron, 1997; Zimmerman, Williams, \& Zumbo, 1993). Similarly, because variance is reduced, analysis of covariance is more powerful than analysis of variance when a useful covariate is incorporated into the design.

\section{Reliability and Effect Size}

Cleary and Linn (1969) reported that "in the derivation and interpretation of statistical tests, the observations are generally considered to be free of error of measurement" (p. 50). From a classical test theory perspective, an individual's observed score (X) is the sum of true score (T) and error score $(\mathrm{E})$; that is, $X=T$ $+E$. Thus, if there is no error of measurement, then the observations are the true scores; implicitly, statistical hypotheses are proposed in terms of true scores. For a set of scores, however, measurements made without error occur only when the instruments provide perfectly reliable scores. Observed score variance, $\sigma_{X}{ }^{2}$, is defined as the sum of true score variance, $\sigma_{\mathrm{T}}{ }^{2}$, and measurement error variance. Because reliability, $\rho_{\mathrm{xx}}$, is defined as the ratio of true score variance to observed score variance,

$$
\rho_{\mathrm{XX}}{ }^{\prime}=\sigma_{\mathrm{T}}^{2} / \sigma_{\mathrm{X}}^{2}=1-{\sigma_{\mathrm{E}}^{2}}^{2} / \sigma_{\mathrm{X}}^{2},
$$

reliability can only be perfect (i.e., ) when there is no measurement error (Lord \& Novick, 1968). Because $\sigma_{\mathrm{X}}$ can be written as

$$
\sigma_{\mathrm{T}} / \sqrt{ } \sigma_{\mathrm{XX}}
$$

the standardized effect size for the $t$ test can be written as

$$
\delta=\left(\mu_{1}-\mu_{0}\right)\left(\sqrt{ } \sigma_{\mathrm{XX}},\right) / \sigma_{\mathrm{T}}
$$

(Levin \& Subkoviak, 1977; Williams \& Zimmerman, 1989). Consequently, reliability affects statistical power indirectly through effect sizes. Cohen (1988) reported that reduced reliability results in reduced effect sizes in observed data (ES), which therefore reduces power. That is, observed effect sizes,

$$
\mathrm{ES}=\mathrm{ESP} * ل_{\mathrm{r}_{\mathrm{XX}}}
$$

where ESP is the population effect size. Therefore, when reliability is perfect, observed ES equals ESP; but when reliability is less than perfect, ES is a value smaller than the true ESP. Some introductory statistics textbooks discuss this problem in reference to attenuation in correlation due to unreliability of measures (e.g., Glass \& Hopkins, 1996).

\section{Reliability and Power \\ Controversy surrounds the relationship} between power and reliability (Williams \& Zimmerman, 1989). Good statistical power can exist with poor reliability and a change in variance unrelated to reliability can change power. However, there are persuasive reasons to consider reliability as an important factor in determining statistical power. For example, statistical power is a function of level of significance, sample size, and effect size only under the assumption of no measurement error, but measures in the social sciences are typically not measured perfectly (Cleary \& Linn, 1969; Levin \& Subkoviak, 1977). Indeed, the implicit assumption that our measures are perfectly reliable is not justified in practice and therefore measurement error should be considered a priori for sample size (Crocker \& Algina, 1986; Subkoviak \& Levin, 1977; Sutcliffe, 1958).

There is no controversy that statistical power depends on observed variance. Zimmerman and Williams (1986) noted that when speaking of statistical power it is irrelevant whether the observed variance is all true score 
variance or contains some amount of measurement error; that is, "the greater the observed variability of a dependent variable, whatever its source, the less is the power of a statistical test" (p. 123). However, because reliability is defined by observed variance in conjunction with either true or error variance, one cannot be certain which source of variance is changed when reliability improves. That is, if observed variance increases, one cannot be certain whether the increase is due to an increase in true score variance or a increase in error variance, or both. Or as Zimmerman et al. (1993) reported, power changes as reliability changes only if observed score variance changes simultaneously.

Knowing that improved reliability results in less measurement error, if it is assumed that true variance is a fixed value for the given population, it follows that a change in reliability will result in a change in observed score variance. Indeed, statistical power is a mathematical function of reliability only if either true score variance or error variance is a constant; otherwise power and reliability are simply related (Cohen, 1988; Williams \& Zimmerman, 1989). But, improvement in reliability is usually interpreted as a reduction in the measurement error variance that occurs from a more precise measurement (Zimmerman \& Williams, 1986). Therefore, a reduction in reliability that is accompanied by an increase in observed score variance will indeed reduce statistical power (Zimmerman et al., 1993). That is, if true score variance remains constant but lower reliability leads to increased error variance, then statistical power will be reduced because of the increased observed score variance ( Humphreys, 1993).

Based on such an assumption, Light et al. (1990) advised that when measurements are less than perfectly reliable, improving the power of statistical tests involves a decision either to increase sample size or to increase reliabilitythe researcher must compare the costs associated with instrument improvement to the costs of adding study participants (see also Cleary \& Linn, 1969; Feldt \& Brennan, 1993). Researchers may encounter such a situation if an instrument does not perform as reliably in a given study as it has elsewhere, leading to increased variance in the current project. Assuming that the increased variance is not due to more heterogeneity in the population and that the true score variance of the population hasn't changed, the observed score variance will change as a consequence of the change in reliability.

Unfortunately, there are few easy ways to account for reliability when determining sample sizes. The tables found in Cohen (1988) do not provide the option to vary reliability. Computer programs such as Sample Power and PASS 2000 also assume perfect reliability. This article will report on the impact of reliability on power as well as provide tables to assist researchers in finding sample sizes necessary with fallible measures.

\section{Methodology}

Two Monte Carlo programs, MC2G (Brooks, 2002) and MC3G (Brooks, 2002) written in Borland Delphi Professional version 6.0, were used to create normally distributed but unreliable data and perform analyses for several statistical methods, namely: (a) paired samples dependent $t$ test, (b) pooled-variance independent $t$ test, (c) one-way analysis of variance with three levels, (d) Spearman rank correlation, (e) Wilcoxon signed-rank test for paired samples, and (f) Mann-WhitneyWilcoxon test for independent groups. The program output was used to create power and sample size tables for these tests.

Reliability was varied from .70 to 1.0 in increments of 0.05 . For power tables, power rates varied from .70 to .90 by .10 . Population effect sizes were varied from small to large using Cohen's (1988) conventional standards. Specifically, for $t$ tests and their nonparametric alternatives, a small standardized effect size was set at $\mathrm{d}=.20$, medium was $\mathrm{d}=.50$, and a large effect was set to be $d=.80$; for correlations, a small effect was set at $\mathrm{r}=.10$, medium was $\mathrm{r}=$ .30 , and a large effect was set to be $r=.50$; for ANOVA, a small standardized difference effect was set at $\mathrm{f}=.10$, medium was $\mathrm{f}=.25$, and a large effect was set to be $\mathrm{f}=.40$. For the power tables, the sample sizes were obtained under the assumption of perfect reliability. That is, the sample sizes were fixed at the values needed to 
achieve power levels of $.70, .80$ and .90 , respectively, when reliability was 1.0. The remaining values in the power tables were determined by systematically varying the reliability with that given sample size. For the sample sizes tables, power was fixed, reliability was varied, and sample sizes were tried repeatedly until the desired power was achieved.

\section{Data Generation}

The two Monte Carlo programs generate uniformly distributed pseudorandom numbers that are used as input to the procedure that converts them into normally distributed data. All data were generated to follow the standard normal distribution. For each sample, the appropriate statistical analysis was performed. The number of correct rejections of the null hypothesis was stored and reported by the program. These procedures were repeated as necessary for each sample condition created. The programs use the L'Ecuyer (1988) uniform random number generator. Specifically, the Fortran code of Press, Teukolsky, Vetterling, and Flannery (1992), was translated into Delphi Pascal. The L'Ecuyer generator was chosen because of its large period and because combined generators are recommended for use with the Box-Muller method for generating random normal deviates (Park \& Miller, 1988).

The computer algorithm for the BoxMuller method used by the MC2G and MC3G programs was adapted for Delphi Pascal from the standard Pascal code provided by Press, Flannery, Teukolsky, and Vetterling (1989). The programs generate normally distributed data of varying reliability based on classical test theory. That is, reliability is not defined using a particular measure of reliability (e.g., splithalf or internal consistency); rather it is defined as the proportion of raw score variance explained by true score variance (Equation 2). Each raw score generated is taken to be a standardized total score.

In order to generate data with less-thanperfect reliability, scores were generated using the true-score standard deviations provided by the researchers; then for each score, the programs added a random error component. Consequently, as reliability decreased, the variation of the random error component increased, resulting in increased raw score variance. For correlation analyses, the same reliability was used for both measures; for independent sample analyses, the same reliability was used each for each group.

\section{Monte Carlo}

The number of iterations for the study is based on the procedures provided by Robey and Barcikowski (1992). Significance levels for both tests on which Robey and Barcikowski's method is based were set at .05 with a power level of .90; the magnitude of departure was chosen to be $\alpha \pm .2$, which falls between their intermediate and stringent criteria for accuracy. The magnitude of departure is justified by the fact that at $\pm .2 \alpha$, the accuracy range for $\alpha=.05$ is $.04 \leq \alpha \leq .06$.

Based on the calculations for these parameters (this set of values was not tabled), 5422 iterations would be required to "confidently detect departures from robustness in Monte Carlo results" (Robey \& Barcikowski, 1992 , p. 283), but applies to power studies also (Brooks, Barcikowski, \& Robey, 1999). However, to assure even greater stability in the results, a larger number of simulations was chosen for each type of analysis. Specifically, 10,000 samples were used for the power tables. The sample size algorithm used by the programs runs repeated analyses beginning with 100 samples per analysis, gradually increasing to 10,000 samples per analysis. Sometimes, however, the algorithm aborts before the 10,000 sample level is reached when the desired power level is approximated closely enough earlier in the process (at least 1000 samples were run in every case).

\section{Results}

Tables 1 through 5 show the relationship between statistical power and reliability for the dependent $\mathrm{t}$ test, independent $\mathrm{t}$ test, one-way ANOVA with three groups, Wilcoxon signed ranks, and Mann-Whitney-Wilcoxon tests, respectively. The tables clearly show that, as reliability is reduced while true score variance remains constant, statistical power is reduced. There is a relatively linear relationship between statistical power and reliability when sample 
size is fixed. For example, Table 1 shows that when statistical power is chosen to be .71 for the dependent $t$ test, 12 cases are required when perfect reliability is assumed and a large effect size $(\mathrm{d}=.8)$ is expected. When reliability was changed to .90 with 12 cases, the actual statistical power was observed to be .63 . Reliability set at .80 resulted in observed statistical power of .54. Finally, actual power for 12 cases was .46 when reliability was set at .70 . Such depreciation of power occurs for all other tests examined in the study.

Table 1. Actual statistical power for paired-samples dependent $t$ tests resulting from different reliability values for given sample sizes at two-tailed $\alpha=.05$

\begin{tabular}{|c|c|c|c|c|c|c|c|c|}
\hline \multirow[b]{2}{*}{$\begin{array}{l}\text { Effect } \\
\text { Size }\end{array}$} & \multirow{2}{*}{$\begin{array}{l}\mathrm{N} \\
\text { per } \\
\text { group }\end{array}$} & \multicolumn{6}{|c|}{ Reliability } & \multirow[b]{2}{*}{.70} \\
\hline & & 1.0 & .95 & .90 & .85 & .80 & .75 & \\
\hline & 12 & $\begin{array}{c}.71 \\
\end{array}$ & $\begin{array}{c}.67 \\
.67\end{array}$ & 2.63 & 2.59 & 2.54 & 2.50 & 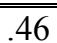 \\
\hline \multirow{2}{*}{$(\mathrm{d}=.8)$} & 15 & .82 & .78 & .74 & .70 & .65 & .61 & .56 \\
\hline & 19 & .91 & .88 & .85 & .81 & .77 & .73 & .68 \\
\hline Medium & 27 & .71 & .66 & .62 & .58 & .53 & .49 & .45 \\
\hline \multirow[t]{2}{*}{$(\mathrm{d}=.5)$} & 34 & .81 & .77 & .73 & .68 & .64 & .59 & .55 \\
\hline & 44 & .90 & .87 & .83 & .80 & .75 & .71 & .66 \\
\hline Small & 157 & .70 & .66 & .62 & .57 & .53 & .49 & .45 \\
\hline \multirow{2}{*}{$(\mathrm{d}=.2)$} & 199 & .80 & .76 & .72 & .67 & .63 & .59 & .54 \\
\hline & 264 & .90 & .87 & .83 & .80 & .75 & .71 & .66 \\
\hline
\end{tabular}

Table 2.Actual statistical power for pooled-variance independent $t$ tests resulting from different reliability values for given sample sizes at two-tailed $\alpha=.05$

\begin{tabular}{|c|c|c|c|c|c|c|c|c|}
\hline \multirow[b]{2}{*}{$\begin{array}{l}\text { Effect } \\
\text { Size }\end{array}$} & \multicolumn{3}{|l|}{$\overline{\mathrm{N}}$} & \multicolumn{5}{|c|}{ Reliability } \\
\hline & $\begin{array}{l}\text { per } \\
\text { group }\end{array}$ & 1.0 & .95 & .90 & .85 & .80 & .75 & .70 \\
\hline \multirow{3}{*}{$\begin{array}{l}\text { Large } \\
(\mathrm{d}=.8)\end{array}$} & 21 & .72 & 7.70 & .67 & .65 & .62 & .59 & .56 \\
\hline & 26 & .81 & .79 & .77 & .74 & .72 & .69 & .66 \\
\hline & 34 & .90 & .89 & .87 & .85 & .83 & .81 & .78 \\
\hline \multirow{3}{*}{$\begin{array}{l}\text { Medium } \\
(\mathrm{d}=.5)\end{array}$} & 51 & .70 & .68 & .66 & .64 & .61 & .58 & .55 \\
\hline & 64 & .80 & .78 & .76 & .74 & .71 & .68 & .65 \\
\hline & 86 & .90 & .89 & .87 & .85 & .83 & .81 & .78 \\
\hline \multirow{3}{*}{$\begin{array}{l}\text { Small } \\
(\mathrm{d}=.2)\end{array}$} & 309 & .70 & .68 & .65 & .63 & .60 & .58 & .54 \\
\hline & 393 & .80 & .78 & .76 & .73 & .71 & .68 & .65 \\
\hline & 526 & .90 & .89 & .87 & .85 & .83 & .80 & .77 \\
\hline
\end{tabular}


Table 3. Actual statistical power for one-way analysis of variance with three groups resulting from different reliability values for given sample sizes at two-tailed $\alpha=.05$

\begin{tabular}{|c|c|c|c|c|c|c|c|c|}
\hline \multirow[b]{2}{*}{$\begin{array}{l}\text { Effect } \\
\text { Size }\end{array}$} & \multicolumn{3}{|l|}{$\overline{\mathrm{N}}$} & \multicolumn{5}{|c|}{ Reliability } \\
\hline & $\begin{array}{l}\text { per } \\
\text { group }\end{array}$ & 1.0 & .95 & 90 & .85 & .80 & .75 & .70 \\
\hline \multirow{3}{*}{$\begin{array}{l}\text { Large } \\
(\mathrm{f}=.40)\end{array}$} & 17 & .70 & .67 & .65 & .63 & .60 & .56 & .53 \\
\hline & 21 & .80 & .78 & .75 & .73 & .71 & .67 & .64 \\
\hline & 28 & .91 & .89 & .87 & .85 & .83 & .80 & .77 \\
\hline \multirow{3}{*}{$\begin{array}{l}\text { Medium } \\
(\mathrm{f}=.25)\end{array}$} & 41 & .70 & .67 & .65 & .62 & .60 & .57 & .54 \\
\hline & 51 & .80 & .78 & .75 & .73 & .70 & .67 & .64 \\
\hline & 66 & .90 & .88 & .86 & .84 & .82 & .79 & .76 \\
\hline \multirow{3}{*}{$\begin{array}{l}\text { Small } \\
(\mathrm{f}=.10)\end{array}$} & 269 & .71 & .68 & .65 & .62 & .60 & .57 & .54 \\
\hline & 333 & .80 & .78 & .75 & .73 & .70 & .67 & .64 \\
\hline & 441 & .90 & .89 & .87 & .85 & .82 & .80 & .77 \\
\hline
\end{tabular}

Table 4. Actual statistical power for Wilcoxon signed-rank tests resulting from different reliability values for given sample sizes at two-tailed $\alpha=.05$

\begin{tabular}{lllllllll}
\hline \hline & $\mathrm{N}$ & \multicolumn{7}{c}{ Reliability } \\
\cline { 2 - 9 } $\begin{array}{l}\text { Effect } \\
\text { Size }\end{array}$ & $\begin{array}{l}\text { per } \\
\text { group }\end{array}$ & 1.0 & .95 & .90 & .85 & .80 & .75 & .70 \\
\hline \hline Large & 12 & .70 & .66 & .62 & .58 & .54 & .50 & .45 \\
$(\mathrm{~d}=.8)$ & 15 & .80 & .76 & .71 & .67 & .63 & .58 & .54 \\
& 19 & .90 & .87 & .83 & .79 & .75 & .71 & .66 \\
Medium & 28 & .70 & .65 & .61 & .57 & .53 & .48 & .44 \\
$(\mathrm{~d}=.5)$ & 35 & .80 & .76 & .72 & .68 & .63 & .58 & .54 \\
& 46 & .90 & .87 & .84 & .80 & .75 & .71 & .66 \\
Small & 164 & .70 & .66 & .62 & .57 & .53 & .49 & .45 \\
$(\mathrm{~d}=.2)$ & 208 & .80 & .76 & .72 & .68 & .63 & .59 & .54 \\
& 276 & .90 & .87 & .83 & .80 & .75 & .71 & .66 \\
\hline \hline
\end{tabular}

Table 5. Actual statistical power for Mann-Whitney-Wilcoxon tests resulting from different reliability values for given sample sizes at two-tailed $\alpha=.05$

\begin{tabular}{|c|c|c|c|c|c|c|c|c|}
\hline \multirow[b]{2}{*}{$\begin{array}{l}\text { Effect } \\
\text { Size }\end{array}$} & \multicolumn{3}{|l|}{$\mathrm{N}$} & \multicolumn{5}{|c|}{ Reliability } \\
\hline & $\begin{array}{l}\text { per } \\
\text { group }\end{array}$ & 1.0 & .95 & .90 & .85 & .80 & .75 & .70 \\
\hline Large & 21 & .69 & .67 & .64 & .62 & .59 & .57 & .53 \\
\hline \multirow[t]{2}{*}{$(\mathrm{d}=.8)$} & 27 & .80 & .78 & .76 & .74 & .71 & .68 & .65 \\
\hline & 35 & .90 & .88 & .86 & .84 & .82 & .80 & .77 \\
\hline Medium & 53 & .70 & .68 & .66 & .63 & .61 & .60 & .55 \\
\hline \multirow[t]{2}{*}{$(\mathrm{d}=.5)$} & 67 & .80 & .78 & .76 & .73 & .71 & .68 & .65 \\
\hline & 90 & .90 & .89 & .87 & .85 & .83 & .81 & .78 \\
\hline Small & 323 & .70 & .67 & .65 & .62 & .59 & .57 & .54 \\
\hline \multirow[t]{2}{*}{$(\mathrm{d}=.2)$} & 411 & .80 & .78 & .76 & .73 & .71 & .68 & .65 \\
\hline & 550 & .89 & .88 & .87 & .85 & .82 & .80 & .77 \\
\hline
\end{tabular}


Tables 6 through 10 show the sample sizes required to maintain a given power level when reliability is less than perfect. Again, there are relatively linear relationships for all tests at all power levels. For example, Table 6 shows that when the desired statistical power level is set at .80 and a large effect size $(\mathrm{d}=.8)$ is expected, the use of 15 cases results in power of .80 when reliability is 1.0 ; but when reliability is reduced to $.90,17$ cases are required. If reliability is .80 , then the study needs 21 participants. Finally, 25 cases must be used to achieve power of .80 when reliability is .70 .

Table 6. Sample sizes required for paired-samples dependent $t$ tests in order to achieve the given statistical power values under different reliability conditions at two-tailed $\alpha=.05$

\begin{tabular}{lllllllll}
\hline \hline & \multicolumn{7}{c}{ Reliability } \\
\cline { 2 - 9 } $\begin{array}{l}\text { Effect } \\
\text { Size }\end{array}$ & Power & 1.0 & .95 & .90 & .85 & .80 & .75 & .70 \\
\hline \hline Large & .70 & 12 & 13 & 14 & 15 & 17 & 18 & 20 \\
$(\mathrm{~d}=.8)$ & .80 & 15 & 16 & 17 & 19 & 21 & 23 & 25 \\
& .90 & 19 & 20 & 22 & 24 & 27 & 29 & 33 \\
Medium & .70 & 27 & 29 & 32 & 35 & 39 & 43 & 48 \\
$(\mathrm{~d}=.5)$ & .80 & 34 & 37 & 40 & 44 & 49 & 54 & 60 \\
& .90 & 44 & 49 & 53 & 59 & 65 & 72 & 80 \\
Small & .70 & 157 & 172 & 192 & 214 & 234 & 258 & 287 \\
$(\mathrm{~d}=.2)$ & .80 & 199 & 220 & 243 & 266 & 289 & 329 & 369 \\
& .90 & 264 & 286 & 328 & 364 & 400 & 440 & 492 \\
\hline \hline
\end{tabular}

Table 7. Sample sizes required for pooled-variance independent $t$ tests in order to achieve the given statistical power values under different reliability conditions at two-tailed $\alpha=.05$

\section{Reliability}

\begin{tabular}{lllllllll}
$\begin{array}{l}\text { Effect } \\
\text { Size }\end{array}$ & Power & 1.0 & .95 & .90 & .85 & .80 & .75 & .70 \\
\hline \hline Large & .70 & 21 & 22 & 23 & 24 & 25 & 27 & 29 \\
$(\mathrm{~d}=.8)$ & .80 & 26 & 27 & 28 & 30 & 32 & 34 & 37 \\
& .90 & 34 & 36 & 38 & 40 & 42 & 45 & 48 \\
Medium & .70 & 51 & 53 & 56 & 59 & 63 & 67 & 72 \\
$(\mathrm{~d}=.5)$ & .80 & 64 & 67 & 71 & 75 & 79 & 85 & 91 \\
& .90 & 86 & 89 & 95 & 102 & 107 & 114 & 121 \\
Small & .70 & 309 & 327 & 345 & 365 & 387 & 415 & 443 \\
$(\mathrm{~d}=.2)$ & .80 & 393 & 415 & 438 & 466 & 492 & 527 & 566 \\
& .90 & 526 & 557 & 583 & 618 & 658 & 702 & 755 \\
\hline \hline
\end{tabular}


Table 8. Sample sizes required for one-way analysis of variance with three groups in order to achieve the given statistical power values under different reliability conditions at two-tailed $\alpha=.05$ Reliability

\begin{tabular}{lllllllll}
$\begin{array}{l}\text { Effect } \\
\text { Size }\end{array}$ & Power & 1.0 & .95 & .90 & .85 & .80 & .75 & .70 \\
\hline \hline Large & .70 & 17 & 18 & 19 & 20 & 21 & 22 & 24 \\
$(\mathrm{f}=.40)$ & .80 & 21 & 22 & 23 & 25 & 26 & 28 & 30 \\
& .90 & 28 & 29 & 30 & 32 & 34 & 36 & 39 \\
Medium & .70 & 41 & 44 & 45 & 48 & 50 & 54 & 58 \\
$(\mathrm{f}=.25)$ & .80 & 51 & 54 & 56 & 61 & 65 & 68 & 73 \\
& .90 & 66 & 70 & 75 & 78 & 83 & 88 & 95 \\
Small & .70 & 269 & 288 & 300 & 314 & 332 & 356 & 382 \\
$(\mathrm{f}=.10)$ & .80 & 333 & 353 & 374 & 395 & 419 & 451 & 482 \\
& .90 & 441 & 464 & 488 & 516 & 551 & 583 & 619 \\
\hline \hline
\end{tabular}

Table 9. Sample sizes required for Wilcoxon signed-ranks tests in order to achieve the given statistical power values under different reliability conditions at two-tailed $\alpha=.05$ Reliability

\begin{tabular}{lllllllll}
$\begin{array}{l}\text { Effect } \\
\text { Size }\end{array}$ & Power & 1.0 & .95 & .90 & .85 & .80 & .75 & .70 \\
\hline \hline Large & .70 & 12 & 13 & 14 & 16 & 17 & 19 & 20 \\
$(\mathrm{~d}=.8)$ & .80 & 15 & 17 & 18 & 20 & 21 & 24 & 26 \\
& .90 & 19 & 21 & 23 & 25 & 28 & 31 & 34 \\
Medium & .70 & 28 & 31 & 34 & 37 & 40 & 45 & 50 \\
$(\mathrm{~d}=.5)$ & .80 & 35 & 39 & 42 & 46 & 51 & 57 & 63 \\
& .90 & 46 & 51 & 56 & 62 & 68 & 75 & 85 \\
Small & .70 & 164 & 181 & 201 & 222 & 246 & 273 & 304 \\
$(\mathrm{~d}=.2)$ & .80 & 208 & 225 & 253 & 282 & 314 & 346 & 387 \\
& .90 & 276 & 307 & 338 & 376 & 417 & 462 & 511 \\
\hline \hline
\end{tabular}

Table 10. Sample sizes required for Mann-Whitney-Wilcoxon tests in order to achieve the given statistical power values under different reliability conditions at two-tailed $\alpha=.05$ Reliability

\begin{tabular}{|c|c|c|c|c|c|c|c|c|}
\hline $\begin{array}{l}\text { Effect } \\
\text { Size }\end{array}$ & Power & 1.0 & .95 & .90 & .85 & .80 & .75 & .70 \\
\hline \multirow{3}{*}{$\begin{array}{l}\text { Large } \\
(\mathrm{f}=.40)\end{array}$} & .70 & 21 & 22 & 23 & 25 & 27 & 28 & 30 \\
\hline & .80 & 27 & 28 & 30 & 32 & 34 & 36 & 39 \\
\hline & .90 & 35 & 37 & 40 & 42 & 44 & 47 & 51 \\
\hline \multirow{3}{*}{$\begin{array}{l}\text { Medium } \\
(\mathrm{f}=.25)\end{array}$} & .70 & 53 & 56 & 58 & 62 & 67 & 69 & 75 \\
\hline & .80 & 67 & 70 & 74 & 79 & 84 & 89 & 96 \\
\hline & .90 & 90 & 93 & 97 & 105 & 113 & 117 & 127 \\
\hline \multirow{3}{*}{$\begin{array}{l}\text { Small } \\
(\mathrm{f}=.10)\end{array}$} & .70 & 323 & 339 & 358 & 386 & 405 & 437 & 463 \\
\hline & .80 & 411 & 430 & 458 & 484 & 517 & 551 & 593 \\
\hline & .90 & 550 & 575 & 611 & 653 & 692 & 733 & 796 \\
\hline
\end{tabular}




\section{Conclusion}

In social sciences, few things are measured perfectly (Subkoviak \& Levin, 1977). Researchers should therefore make an effort to minimize the effects of measurement error. Although some authors suggest that lower reliability is acceptable for group studies of attitudes or personality variables (e.g., Fink \& Kosecoff, 1998; McMillan \& Schumacher, 2001), it becomes obvious based on the tables provided here that improving reliability will increase power and therefore fewer members of these groups will be needed to participate in the study.

For example, in a two group study using a dependent measure that produces scores with a reliability of .70,91 participants are required for a medium effect size at a power of .80 ; if reliability is improved to .85 , the number of participants can be reduced to 75 (see Table 7). Perhaps for some studies, the additional effort required to improve the instrument is not justifiable; but for research with high per-subject costs, investment to improve the instrument may be very worthwhile. As well, the effect of measurement fallibility is even more dramatic for small effect sizes. In the same example as above, but for a small effect size, an improvement from reliability of .70 to .85 will result in a sample size reduction of around 100 (see Table 7).

Perhaps the most advantageous way for researchers to use the sample size information provided here is to make informed decisions about the trade-off between sample size and reliability. That is, researchers can make informed decisions about the costs and benefits of spending time and effort to improve an instrument. The issue really isn't how many more people do we need because our instrument is not perfectly reliable? Researchers would already have an estimate of variance based on that level of unreliability from pilot studies or previous research-after all, the effect size would be based on that observed variance-not true score variance. Rather, the implication intended from this work is more emphasis on the development of reliable and valid instruments. As instruments and reliability improve, because the true score variance of participants would (presumably) remain the same, observed score variance will decrease and would provide additional power. There are several strategies that have been developed for minimizing the effects of measurement error and increasing reliability. These include revising items, increasing the number of items, lengthening item scales, administering the instrument systematically, timing of data collection and use of multiple raters or scores (Light et al., 1990).

Before choosing a final sample size, the possibility of measurement error should be considered. To determine sample sizes "without simultaneously considering errors of measurement is to live in a 'fool's paradise"" (Levin \& Subkoviak, 1977, p. 337). If one suspects that measurement error exists and there is no viable means to reduce it, sample size should be increased accordingly. Researchers can identify potential problems with measurement error through pilot studies or previous research. Where reliability information is lacking, the researcher should use cautious estimates, with a preference toward more conservative values, when deciding sample sizes (Levin \& Subkoviak, 1977).

\section{References}

Aron, A., \& Aron, E. N. (1997). Statistics for the behavioral and social sciences: A brief course. Upper Saddle River, NJ: Prentice Hall.

Brooks, G. P. (2002). MC2G: Monte Carlo Analyses for 1 or 2 Groups (Version 3.0.7) [Computer software]. Retrieved from http://oak.cats.ohiou.edu/ brooksg/mc2g.htm

Brooks, G. P. (2002). MC3G: Monte Carlo Analyses for 3 Groups. (Version 1.1.1) [Computer software]. Retrieved from http://oak.cats.ohiou.edu/ brooksg/mc3g.htm

Brooks, G. P., Barcikowski, R. S., \& Robey, R. R. (1999, April). Monte Carlo simulation for perusal and practice. Paper presented at the meeting of the American Educational Research Association, Montreal, Quebec, Canada.

Cleary, T. A., \& Linn, R. L. (1969). Error of measurement and the power of a statistical test. British Journal of Mathematical and Statistical Psychology, 22, 49-55. 
Cohen, J. (1988). Statistical power analysis for the behavioral sciences (2nd ed.). Hillsdale, NJ: Lawrence Erlbaum Associates.

Crocker, L. \& Algina, J. (1986). Introduction to classical and modern test theory. Fort Worth, TX: Holt, Rinehart, \& Winston.

Feldt, L. S., \& Brennan, R. L. (1993). Reliability. In R. L. Linn (Ed.). Educational measurement (pp. 105-146). Phoenix, AZ: Oryx.

Fink, A., \& Kosecoff, J. (1998). How to conduct surveys: A step-by-step guide (2nd. ed.). Thousand Oaks, CA: Sage.

Glass, G. V., \& Hopkins, K. D. (1996). Statistical methods in education and psychology ( $3^{\text {rd }}$ ed.). Boston: Allyn \& Bacon.

Hopkins, K. D., \& Hopkins, B. R. (1979). The effect of the reliability of the dependent variable on power. Journal of Special Education, 13, 463-466.

Humphreys, L. G. (1993). Further comments on reliability and power of significance tests. Applied Psychological Measurement, 17, 11-14.

L'Ecuyer, P. (1988). Efficient and portable combined random number generators. Communications of the ACM, 31, 742-749, 774.

Levin, J. R., \& Subkoviak, M. J. (1977). Planning an experiment in the company of measurement error. Applied Psychological Measurement, 1, 331-338.

Light, R. J., Singer, J. D., \& Willett, J. B. (1990). By design: Planning research on higher education. Cambridge, MA: Harvard University.

Lord, F. M., \& Novick, M. R. (1968). Statistical theories of mental test scores. Reading, MA: Addison-Wesley.

McMillan, J. H., \& Schumacher, S. (2001). Research in education: A conceptual introduction (5th ed.). New York: Longman.

Nicewander, W. A. \& Price J. M. (1983). Reliability of measurement and the power of statistical tests: Some new results. Psychological Bulletin, 94, 524-533.
Park, S. K., \& Miller, K. W. (1988). Random number generators: Good ones are hard to find. Communications of the ACM, 31, 11921201.

Press, W. H., Flannery, B. P., Teukolsky, S. A., \& Vetterling, W. T. (1989). Numerical recipes in Pascal: The art of scientific computing. New York: Cambridge University.

Press, W. H., Teukolsky, S. A., Vetterling, W. T., \& Flannery, B. P. (1992). Numerical recipes in FORTRAN: The art of scientific computing (2nd ed.). New York: Cambridge University.

Robey, R. R., \& Barcikowski, R. S. (1992). Type I error and the number of iterations in Monte Carlo studies of robustness. British Journal of Mathematical and Statistical Psychology, 45, 283-288.

Stevens, J. (2002). Applied multivariate statistics for the social sciences (4th ed.). Mahwah, NJ: Lawrence Erlbaum Associates.

Subkoviak, M. J., \& Levin, J. R. (1977). Fallibility of measurement and the power of a statistical test. Journal of Educational Measurement, 14, 47-52.

Sutcliffe, J. P. (1958). Error of measurement and the sensitivity of a test of significance. Psychometrika, 23, 9-17.

Williams, R. H., \& Zimmerman, D. W. (1989). Statistical power analysis and reliability of measurement. Journal of General Psychology, 116, 359-369.

Zimmerman, D. W., \& Williams, R. H. (1986). Note on the reliability of experimental measures and the poser of significance tests. Psychological Bulletin, 100, 123-124.

Zimmerman, D. W., Williams, R. H., \& Zumbo, B. D. (1993). Reliability of measurement and power of significance tests based on differences. Applied Psychological Measurement, 17, 1-9. 


\section{Comparison of the $t$ vs. Wilcoxon Signed-Rank Test for Likert Scale Data and Small Samples}

\author{
Gary E. Meek \\ Black Hills State University
}

\author{
Ceyhun Ozgur \\ Valparaiso University
}

Kenneth Dunning

The University of Akron

The one sample t-test is compared with the Wilcoxon Signed-Rank test for identical data sets representing various Likert scales. An empirical approach is used with simulated data. Comparisons are based on observed error rates for 27,850 data sets. Recommendations are provided.

Key words: Nonparametric, Wilcoxon's signed-rank test, one sample t-test, Likert scales, Type I and Type II error rates.

\section{Introduction}

There has been disagreement since the 1940s concerning the use of the t-test versus its nonparametric equivalents when the assumptions of the t-test may not be valid, particularly those of normality. Similarly, controversies have raged at various times over the past 60 years about the use of classical or parametric procedures versus distribution-free or nonparametric procedures when the level of measurement is less than interval. The discussions in the literature began with Stevens (1946) and Siegel (1956) who stated that the level of measurement attained in the data should be a major factor in test selection. Siegel (1956) took a definite stance that nonparametric procedures should be utilized whenever the level is no more informative than ordinal.

Gary Meek is retired after $40+$ years in academia. $\mathrm{He}$ has $40+$ peer-reviewed publications. His doctorate in statistics is from Case Western Reserve University. Email address: meek@mato.com. Ceyhun Ozgur is Professor of Information/Decision sciences, and has published about two dozen refereed journal articles. Email address: Ceyhun.Ozgur@valpo.edu. Kenneth A. Dunning is Professor of Management / Information systems. He specializes in six-sigma applications. Email address: dunning@uakron.edu
In the behavioral sciences, particularly in psychology, Baggaley (1960) and Binder (1984) fueled the fire started by Stevens (1946). The extensive use of Likert scales in the behavioral sciences continues to make test selection a debatable issue. The debate is not restricted to the social sciences, because Likert scales also are widely used in opinion-based research in marketing, human resource management and other areas of business as well as in education and nursing. The liveliness of the discussions surrounding this issue in presentations at various conferences provided the motivation for this investigation.

Comparisons of distribution-free and parametric procedures initially were based upon theoretical considerations involving asymptotic relative efficiency (ARE), which is a large sample property. It pertains to the limit of the ratio of the sample sizes required to attain a specified power as the alternative, or true value, approaches the value under the null hypothesis and the sample size goes to infinity. Although the ARE is theoretically appealing, infinite sample sizes are difficult to obtain in practice.

According to Conover (1999) and Siegel (1956), the ARE of the one sample Wilcoxon signed-rank (WSR) test for location as compared with the one sample t-test for a normal population is 0.955. Conover (1999) stated that if the underlying population is uniformly distributed the ARE is 1.0 and for most nonnormal populations exceeds 1.0 , but is never less than 0.864 . 
The ARE is an important theoretical consideration for comparing the theoretical power of two different statistical procedures. However, it is considered to be of limited value when working with small samples. Sawilowsky (1990) stated that at best, Monte Carlo studies have shown that ARE may be indicative of the promise of relative power of non-parametric procedures versus their parametric counterparts for small samples.

Conover (1999) pointed out that the ttest is more powerful than its rank-sum alternatives when populations are normally distributed. However, as most statisticians would agree, normality is a very difficult property to obtain. Sawilowsky and Blair (1992) demonstrated that when populations are not normally distributed, the Wilcoxn rank-sum procedure is more powerful than the t-test.

For the correlated layout, Siegel (1956, p. 83) stated that, for small sample sizes, the efficiency of the Wilcoxon signed-rank test to the t-test is near 95 percent. Most textbooks that include the Wilcoxon signed-rank procedure and discuss its assumptions versus the t-test recommend using the Wilcoxon signed-rank test in small sample situations whenever there is any question about normality or an interval level of measurement, but symmetry is reasonable and the differences are ordinal. Therefore, the purpose of this article is to compare the performance of these two procedures with Likert scale data in small sample situations where the assumptions of normality and interval measurement, are not satisfied.

Some Literature Review for Independent and Dependent Tests with Ordinal Scaled Data

There is limited evidence of practical comparisons of parametric versus nonparametric procedures based on the actual scale of measurement available in the data. The term practical is used because discussion in the literature, Stevens (1946), Siegel (1956), Baggaley (1960), Binder (1984) and Conover (1999), has historically been predicated on philosophical issues or asymptotic properties. Sawilowsky (1991) presented an excellent summary of the level of measurement issue and the weak measurement versus strong statistics controversy.
Some studies that have considered scale of measurement in comparing parametric vs. nonparametric tests are Blair and Higgins (1985), Nanna and Sawilowsky (1998), Nanna (2002), and two preliminary studies done by Meek, et al., (2000) and (2001).

Blair and Higgins (1985) used Monte Carlo methods with ten theoretical distributions to compare power of the paired samples t-test and the Wilcoxon signed ranks test for paired data, utilizing samples of 10,25 and 50 . They found the paired t-test to have a slight power advantage over the Wilcoxon procedure under normal and uniform distributions but little or no advantage under the other distributions for $n=$ 10 and none at the larger sample sizes.

The first study by Meek, et al., (2000) used an identical approach to that utilized in this paper but compared the two independent samples t-test to the Mann-Whitney procedure under various combinations of Likert scales and sample sizes. Their findings indicated that, for small samples, there appeared to be little difference in precision between the t-test and the Mann-Whitney for data collected on a Likert scale. More germane to this article, the second article by Meek, et al., (2001) used a similar approach to compare the performances of the Wilcoxon signed-rank test and the t-test with Likert scales but was limited by having only slightly more than 2400 cases, and therefore, the results are not discussed further here.

Nanna and Sawilowsky

compared the power of the independent samples t-test to that of the Wilcoxon rank-sum procedure with actual data sets measured on an ordinal scale. Their data were based on Functional Independence Measure (FIM) scores in medical rehabilitation. FIM scores used a 7point Likert scale and often are highly skewed. Nanna and Sawilowsky (1998) found that the Wilcoxon rank-sum procedure had higher power than the t-test test for almost all combinations of sample size and alpha level examined. Nanna (2002) found that the rank transformation procedure provided an increase in power over Hotelling's $\mathrm{T}^{2}$ when testing for equality of centroids using Likert scale data. Nanna (2002) used essentially the same FIM data sets as Nanna and Sawilowsky (1998). 
Many of the current textbooks that include coverage of the Wilcoxon signed-rank procedure are quite limited in their discussions of its assumptions and make no specific recommendations for its use compared to the $t$ test other than to indicate it should be used if the assumptions of normality and interval measurement are questionable, particularly in small sample situations. Although robustness of the $\mathrm{t}$-test is often cited as a reason for choosing $\mathrm{t}$ over the Wilcoxon signed rank and other nonparametric procedures, Bradley (1980) found that both the $Z$ test and t-test were very nonrobust for L-shaped distributions when comparing average p-values to nominal alphas.

Sawilowsky (1990) stated that the concept of robustness relates to both Type I and Type II errors and that choosing a test procedure requires one to consider other issues and properties too. Sawilowsky (1991) pointed out that there are no hard and fast rules for choosing between parametric and nonparametric procedures and Sawilowsky (2005) presented a summary of misconceptions regarding such choices. Heeren and D'Agostino (1987) found the independent samples t-test to be robust with ordinal data while Sawilowsky and Blair (1992) found that the t-test was reasonably robust when sample sizes were equal and at least of size $n=$ 30 per group.

Several current statistics texts in the business field were reviewed to determine how they presented nonparametric versus parametric procedures. Anderson, et al., (2005), Moore, et al., (2003) and Newbold (1995) did not mention the assumptions underlying the Wilcoxon signed-rank test and made no recommendations regarding its use. Bowerman, et al., (2007) stated that when $\mathrm{n}$ is small, the distribution is non-normal and the measurement is ordinal the t-test is not valid and the Wilcoxon signed-rank test should be used. Keller (2005), Berenson, et al., (2004) and Chou (1989) made statements similar to those of Bowerman, et al., (2007). Keller (2005, p.738) further stated that the t-test cannot be used if the data are ordinal, thus eliminating its use with Likert scales. Doane and Seward (2007) recommended the use of the Wilcoxon signed-rank test in small sample situations because it is free of the normality assumption, uses ordinal data, is robust to outliers and has fairly good power over a range of non-normal population shapes. Conover (1999) differed, and stated that, as does the ttest, the Wilcoxon signed-rank test requires an interval scale of measurement that also should eliminate its use with a Likert scale. Siegel (1956) specified that the Wilcoxon signed-rank test requires a level of measurement that is between ordinal and interval, called an ordered metric scale.

All of the textbooks cited above stress that the basis for their recommendations is to be able to calculate an exact probability of making a Type I error. If the assumptions underlying any procedure are questionable then it is not possible to do so. However, it is seldom possible to completely verify that all assumptions of any procedure are totally satisfied and, sometimes, it is of more interest to protect against a Type II error than against a Type I error.

Simulation of the Data

In order to generate data that would be typical of Likert scale responses from distributions with specified means, the simulations were obtained using the method detailed in the study by Meek, et al., (2000). That is, binomial distributions were used to generate integer results from a population whose range was 0 to k-1 and had a mean of $\mu-1$. These distributions, and the resulting data, were then shifted one unit to the right to obtain a range of observed values from 1 to $\mathrm{k}$ with a population mean of $\mu$. Data were generated to represent five-point and seven-point Likert scales. A total of 27,850 simulations were conducted with $8,750(31.4 \%)$ of them representing symmetric distributions. Because the one sample Wilcoxon signed-rank procedure is a test of the population median the symmetric cases are the only ones where it is truly appropriate, assuming that a Likert scale truly generates ordinal data. Based on Doane and Seward's (2007) statement that the Wilcoxon signed-rank procedure is fairly robust to non-normal or asymmetric shapes it should be reasonable for use on the majority of the remaining cases, too.

In addition to the level of measurement's being ordinal, at best, the underlying distributions used to generate observations were discrete, though infinite, and 
the actual distributions were skewed in slightly more than two-thirds of the cases rather than symmetric. Thus, in all cases, the basic assumptions of the t-test were violated while in approximately $69 \%$ of the cases at least one assumption for the Wilcoxon signed-rank test was violated. Of the 27,850 data sets on which comparisons were made 11,350 represent a fivepoint Likert scale and 16,500 a seven-point Likert scale with varying sample sizes of 5,10 and 15 for both scales.

\section{Experimental Design}

Comparisons of the one sample t-test and the Wilcoxon signed-rank test for location were based on corresponding p-values and the number of incorrect decisions that resulted from each. The p-values were calculated for each test procedure's results using Minitab ${ }^{\circledR}$ and the numbers of rejections and non-rejections at various nominal significance levels were tabulated for combinations of scale size, sample size, hypothesized mean and actual mean. The numbers of rejections for each test procedure were determined by comparing the $p$-values to nominal significance levels of $0.01,0.05$ and 0.10 . The absolute differences between the hypothesized and actual means that were evaluated were $0.0,0.5$ and 1.0. Differences greater than 1.0 were not considered because both tests were rejecting $\mathrm{H}_{\mathrm{o}}$ with sample sizes of 10 and 15 approximately $90 \%$ of the time at that difference at the 0.10 level using a 5-point scale. A similar percentage of rejections occurred for the 7-point scale when the sample size was 15 .

Two-way contingency tables were constructed for each combination by numbers of rejections and non-rejections versus the test procedure used. The Chi-square test of association was used to test for a relationship between the statistical decision and the procedure used. It is recognized that the use of the Chi-square test is questionable since both the t-test and the Wilcoxon signed-rank test were run on the same samples. The Chi-square test results do help to highlight disparities in the numbers of rejections between the two procedures. Tables were constructed identifying for which combinations significant differences occurred and at what level. It should be noted that Chi-square tests could not be run for combinations having alphas of 0.01 and 0.05 when $\mathrm{n}$ is five because the theoretical (expected) number of rejections by the Wilcoxon signedrank test is zero in those cases.

Another, and possibly more informative, way of comparing the two procedures is to look at their corresponding error rates. Thus, tables were constructed to compare the error rates of the two test procedures for all of the various combinations indicated above. Because the majority of samples simulated were from asymmetric distributions a separate table was constructed showing error rates for the procedures when the actual distributions were symmetric. In a very limited number of cases (eighteen) the Wilcoxon signed-rank procedure had more rejections than the t-test. These are tabulated.

\section{Results}

One of the assumptions underlying the Wilcoxon signed-rank procedure is that the distribution is symmetric. Several comparisons were made for which this assumption is violated; for example, data on the 7-point scale when the actual mean is 2.0 or when it is 6.0. These simulations and corresponding tests were conducted to see what happens in that situation. It is recognized that any results under those conditions are questionable but, in terms of actual errors, are useful because Doane and Seward (2007) indicated that the Wilcoxon signed-rank test is robust to non-normal, and somewhat asymmetrical, population shapes. In fact, the assumptions underlying the t-test are violated in every situation because there is neither an underlying normal distribution nor an interval level of measurement. Even so, the results indicate that, in almost every case when the null hypothesis was false, the t-test performed as well or better than the Wilcoxon signed-rank test. There were a total of 13 cases in which the Wilcoxon signed-rank test rejected more times than the t-test when $\mathrm{H}_{\mathrm{o}}$ was false and in only one of those was the difference significant, and that was at the 0.10 level.

The results of comparing the numbers of rejections for the two procedures using contingency tables are presented in Tables 1 through 6. In each of those tables, the first two 
columns represent the values for the hypothesized mean and the actual mean, respectively. Columns 3 and 4 identify the sample size and the number of samples generated for that combination of hypothesized and actual means. The alpha values listed at the tops of columns 5, 6 and 7 represent the nominal significance levels at which the $t$ and Wilcoxon signed-rank tests were run. Except for Tables 1 and 4 , the last three columns in each table give the levels at which the Chi-square tests comparing corresponding results of the $t$ and Wilcoxon signed-rank procedures were significant. In Tables 1 and 4, columns 5 and 6 have asterisks entered because the Wilcoxon signed-rank test cannot reject at alphas of 0.05 and 0.01 when $\mathrm{n}=5$.

A brief explanation of the entries in columns 5, 6 and 7 follows. For example, an entry of NS in the column headed by $\alpha=0.10$ indicates that the numbers of rejections by $t$ and the Wilcoxon signed-rank test were not significantly different at a nominal alpha of 0.10 for the combination of hypothesized and actual means listed for that row. Similarly, an entry of 0.05 under the column headed by $\alpha=0.01$ indicates that the numbers of rejections by the two tests were significantly different at the 0.05 level of significance for the set of means in that row.

As an example, in the second row of Table 3, below, where the hypothesized mean is 1.5 and the actual mean is 2.0 the t-test rejected 139 times (not presented) out of 200 runs at 0.10 while the Wilcoxon signed-rank test rejected 125 times (not presented) at 0.10 . Thus, $t$ and Wilcoxon signed-rank test each failed to reject at that level 61 and 75 times, respectively. Casting those values into a contingency table using tests as columns and decisions as rows results in a calculated Chi-square value of 2.18 which is not significant at $\alpha=0.10$. This is the significance level, NS, entered in row 2 and column 7 of Table 3. Other than the asterisks in Tables 1 and 4 , already explained above, all other entries in columns 5, 6 and 7 of Tables 1 through 6 were obtained similarly. It should be noted that corrections for 1 degree of freedom for the Chisquare test are not incorporated in Minitab ${ }^{\circledR}$.
Tables 1, 2 and 3 correspond to data generated for a 5-point Likert scale while Tables 4,5 and 6 , given below, are for data generated on a 7-point Likert scale. All of the significant differences between the numbers of rejections for the two procedures for the 5-point scale correspond to more rejections by the t-test than by the Wilcoxon signed-rank test. From Table 1, it is seen that the t-test rejected the null hypothesis significantly more times than the Wilcoxon signed-rank test in 18 of the 25 comparisons, or 16 of 23 if we ignore the cases where $\mathrm{H}_{\mathrm{o}}$ corresponds to a boundary value.

As the sample size increases, the cases where the numbers of rejections for the two procedures differ significantly drops correspondingly for significance levels of 0.10 and 0.05 , to 8 and 12 , respectively, out of 23 for $\mathrm{n}=10$ and 3 and 5, respectively, out of 23 for $n$ $=15$. However, they stay about the same for $0.01,18$ and 17 for $\mathrm{n}=10$ and $\mathrm{n}=15$, respectively. These numbers ignore boundary value cases.

As with the 5-point scale, ignoring boundary values, in the 7-point scale we see that at $\alpha=0.10$ the significant differences decrease from 25 to 4 as the sample size increases from 5 to 15 . Correspondingly, at 0.05 and 0.01 , the significant differences decrease from 13 to 3 and 27 to 21 , respectively, as $n$ increases from 10 to 15.

A better way to compare the two test procedures, rather than looking at significant differences between the numbers of rejections, is to look at their estimated Type I and Type II error rates. These are presented below for all distributions in Table 7.

In Table 7, it is obvious that the Wilcoxon signed-rank procedure protects better against a Type I error because its average Type I error rate was always less than that of the t-test, whose Type I error rate exceeded the nominal significance level five times with the 7-point scale. It should be noted though that, except for $\mathrm{n}=15$ with the 7 -point scale, the actual Type I error rate for the t-test was closer to the nominal level in all other comparisons. The average Type 
Table 1: Five-point scale comparison of $t$ test and Wilcoxon signed-rank test for a sample size of 5

\begin{tabular}{|c|c|c|c|c|c|c|}
\hline $\begin{array}{l}\text { Hypothesized } \\
\text { Mean }\end{array}$ & Actual Mean & $\mathrm{N}$ & $\begin{array}{l}\# \text { of } \\
\text { runs }\end{array}$ & $\alpha=.01$ & $\alpha=.05$ & $\alpha=.1$ \\
\hline $1.0^{(1)}$ & 2.0 & 5 & 100 & * & * & 2.01 \\
\hline 1.5 & 2.0 & 5 & 100 & * & * & NS \\
\hline 2.0 & 2.0 & 5 & 100 & * & * & .01 \\
\hline 2.5 & 2.0 & 5 & 100 & $*$ & * & NS \\
\hline 3.0 & 2.0 & 5 & 100 & $*$ & * & .01 \\
\hline 1.5 & 2.5 & 5 & 100 & $*$ & $*$ & NS \\
\hline 2.0 & 2.5 & 5 & 100 & $*$ & $*$ & .01 \\
\hline 2.5 & 2.5 & 5 & 100 & $*$ & $*$ & NS \\
\hline 3.0 & 2.5 & 5 & 100 & $*$ & $*$ & .01 \\
\hline 3.5 & 2.5 & 5 & 100 & $*$ & * & .10 \\
\hline 2.0 & 3.0 & 5 & 300 & $*$ & * & .01 \\
\hline 2.5 & 3.0 & 5 & 300 & $*$ & * & .10 \\
\hline 3.0 & 3.0 & 5 & 300 & $*$ & $*$ & .01 \\
\hline 3.5 & 3.0 & 5 & 300 & $*$ & $*$ & .05 \\
\hline 4.0 & 3.0 & 5 & 300 & $*$ & $*$ & .01 \\
\hline 2.5 & 3.5 & 5 & 100 & $*$ & $*$ & .05 \\
\hline 3.0 & 3.5 & 5 & 100 & $*$ & * & .01 \\
\hline 3.5 & 3.5 & 5 & 100 & * & * & NS \\
\hline 4.0 & 3.5 & 5 & 100 & $*$ & $*$ & .01 \\
\hline 4.5 & 3.5 & 5 & 100 & $*$ & $*$ & NS \\
\hline 3.0 & 4.0 & 5 & 100 & $*$ & $*$ & .01 \\
\hline 3.5 & 4.0 & 5 & 100 & $*$ & $*$ & .10 \\
\hline 4.0 & 4.0 & 5 & 100 & $*$ & $*$ & .01 \\
\hline 4.5 & 4.0 & 5 & 100 & * & $*$ & NS \\
\hline $5.0^{(1)}$ & 4.0 & 5 & 100 & * & * & .01 \\
\hline
\end{tabular}

(1) When the hypothesized value equals a boundary value a better test would be to reject $\mathrm{H}_{\mathrm{o}}$ if any other value occurs in the sample.

* $\mathrm{H}_{\mathrm{o}}$ cannot be rejected at a significance level of 0.05 or 0.01 for samples of size 5 using Wilcoxon signed-rank test.

Table 2: Five-point scale comparison of $\mathrm{t}$ test and Wilcoxon signed-rank test for a sample size of 10

\begin{tabular}{|c|c|c|c|c|c|c|}
\hline $\begin{array}{l}\text { Hypothesized } \\
\text { Mean }\end{array}$ & Actual Mean & $\mathrm{n}$ & $\begin{array}{l}\text { \# of } \\
\text { runs }\end{array}$ & $\alpha=.01$ & $\alpha=.05$ & $\alpha=.1$ \\
\hline $1.0^{(1)}$ & 2.0 & 10 & 100 & .01 & 2.01 & 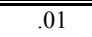 \\
\hline 1.5 & 2.0 & 10 & 100 & .05 & .05 & NS \\
\hline 2.0 & 2.0 & 10 & 100 & NS & .10 & NS \\
\hline 2.5 & 2.0 & 10 & 100 & .01 & NS & NS \\
\hline 3.0 & 2.0 & 10 & 100 & .01 & .10 & NS \\
\hline 1.5 & 2.5 & 10 & 100 & .01 & NS & NS \\
\hline 2.0 & 2.5 & 10 & 100 & .05 & NS & .10 \\
\hline 2.5 & 2.5 & 10 & 100 & NS & NS & NS \\
\hline 3.0 & 2.5 & 10 & 100 & .01 & .10 & .10 \\
\hline 3.5 & 2.5 & 10 & 100 & .01 & NS & NS \\
\hline 2.0 & 3.0 & 10 & 300 & .01 & .01 & NS \\
\hline 2.5 & 3.0 & 10 & 300 & .01 & NS & NS \\
\hline 3.0 & 3.0 & 10 & 450 & .05 & .05 & .05 \\
\hline 3.5 & 3.0 & 10 & 300 & .01 & .10 & .10 \\
\hline 4.0 & 3.0 & 10 & 300 & .01 . & .01 & .05 \\
\hline 2.5 & 3.5 & 10 & 100 & NS & NS & NS \\
\hline 3.0 & 3.5 & 10 & 100 & .01 & .05 & NS \\
\hline 3.5 & 3.5 & 10 & 100 & NS & NS & NS \\
\hline 4.0 & 3.5 & 10 & 100 & .10 & .05 & .10 \\
\hline 4.5 & 3.5 & 10 & 100 & .01 & NS & NS \\
\hline 3.0 & 4.0 & 10 & 100 & .01 & .01 & .05 \\
\hline 3.5 & 4.0 & 10 & 100 & .05 & NS & NS \\
\hline 4.0 & 4.0 & 10 & 100 & NS & .10 & .10 \\
\hline 4.5 & 4.0 & 10 & 100 & .05 & NS & NS \\
\hline $5.0^{(1)}$ & 4.0 & 10 & 100 & .01 & .05 & .05 \\
\hline
\end{tabular}

(1) When the hypothesized value equals a boundary value a better test would be to reject $\mathrm{H}_{\mathrm{o}}$ if any other value occurs in the sample. 
Table 3: Five-point scale comparison of $\mathrm{t}$ test and Wilcoxon signed-rank test for a sample size of 15

\begin{tabular}{|c|c|c|c|c|c|c|}
\hline $\begin{array}{c}\text { Hypothesized } \\
\text { Mean }\end{array}$ & Actual Mean & $\mathrm{n}$ & $\begin{array}{l}\# \text { of } \\
\text { runs }\end{array}$ & $\alpha=.01$ & $\alpha=.05$ & $\alpha=.1$ \\
\hline $1.0^{(1)}$ & 2.0 & $\overline{15}$ & 200 & .01 & NS & NS \\
\hline 1.5 & 2.0 & 15 & 200 & .10 & .10 & NS \\
\hline 2.0 & 2.0 & 15 & 200 & NS & NS & NS \\
\hline 2.5 & 2.0 & 15 & 200 & .10 & NS & NS \\
\hline 3.0 & 2.0 & 15 & 300 & .01 & NS & NS \\
\hline 1.5 & 2.5 & 15 & 100 & .05 & NS & NS \\
\hline 2.0 & 2.5 & 15 & 100 & .01 & NS & NS \\
\hline 2.5 & 2.5 & 15 & 100 & NS & NS & NS \\
\hline 3.0 & 2.5 & 15 & 100 & .01 & NS & NS \\
\hline 3.5 & 2.5 & 15 & 100 & .05 & NS & NS \\
\hline 2.0 & 3.0 & 15 & 300 & .01 & NS & NS \\
\hline 2.5 & 3.0 & 15 & 300 & .01 & NS & NS \\
\hline 3.0 & 3.0 & 15 & 400 & NS & .05 & NS \\
\hline 3.5 & 3.0 & 15 & 300 & .01 & .05 & .05 \\
\hline 4.0 & 3.0 & 15 & 300 & .01 & NS & NS \\
\hline 2.5 & 3.5 & 15 & 100 & .01 & NS & NS \\
\hline 3.0 & 3.5 & 15 & 100 & .01 & NS & NS \\
\hline 3.5 & 3.5 & 15 & 100 & NS & NS & NS \\
\hline 4.0 & 3.5 & 15 & 100 & .01 & .10 & NS \\
\hline 4.5 & 3.5 & 15 & 100 & .05 & NS & NS \\
\hline 3.0 & 4.0 & 15 & 100 & .01 & .10 & NS \\
\hline 3.5 & 4.0 & 15 & 100 & .05 & NS & NS \\
\hline 4.0 & 4.0 & 15 & 100 & NS & NS & .05 \\
\hline 4.5 & 4.0 & 15 & 100 & NS & NS & .10 \\
\hline $5.0^{(1)}$ & 4.0 & 15 & 100 & .01 & NS & NS \\
\hline
\end{tabular}

(1) When the hypothesized value equals a boundary value a better test would be to reject Ho if any other value occurs in the sample. 
Table 4: Seven-point scale comparison of $t$ test and Wilcoxon signed-rank test for a sample size of 5

\begin{tabular}{|c|c|c|c|c|c|c|}
\hline $\begin{array}{c}\text { Hypothesized } \\
\text { Mean }\end{array}$ & Actual Mean & $\mathrm{N}$ & $\begin{array}{l}\text { \# of } \\
\text { runs }\end{array}$ & $\alpha=.01$ & $\alpha=.05$ & $\alpha=.1$ \\
\hline $\bar{~} 1.0^{(1)}$ & 2.0 & $\overline{5}$ & 100 & * & * & 01 \\
\hline 1.5 & 2.0 & 5 & 100 & $*$ & $*$ & NS \\
\hline 2.0 & 2.0 & 5 & 100 & $*$ & $*$ & .01 \\
\hline 2.5 & 2.0 & 5 & 100 & $*$ & $*$ & NS \\
\hline 3.0 & 2.0 & 5 & 100 & $*$ & $*$ & .01 \\
\hline 1.5 & 2.5 & 5 & 100 & $*$ & $*$ & NS \\
\hline 2.0 & 2.5 & 5 & 100 & $*$ & $*$ & .01 \\
\hline 2.5 & 2.5 & 5 & 100 & $*$ & $*$ & NS \\
\hline 3.0 & 2.5 & 5 & 100 & $*$ & $*$ & .01 \\
\hline 3.5 & 2.5 & 5 & 100 & $*$ & $*$ & NS \\
\hline 2.0 & 3.0 & 5 & 100 & $*$ & $*$ & .01 \\
\hline 2.5 & 3.0 & 5 & 100 & $*$ & $*$ & NS \\
\hline 3.0 & 3.0 & 5 & 100 & $*$ & $*$ & .01 \\
\hline 3.5 & 3.0 & 5 & 100 & $*$ & $*$ & NS \\
\hline 4.0 & 3.0 & 5 & 100 & $*$ & $*$ & .01 \\
\hline 2.5 & 3.5 & 5 & 100 & $*$ & $*$ & .10 \\
\hline 3.0 & 3.5 & 5 & 100 & $*$ & $*$ & .01 \\
\hline 3.5 & 3.5 & 5 & 100 & $*$ & $*$ & NS \\
\hline 4.0 & 3.5 & 5 & 100 & $*$ & * & .01 \\
\hline 4.5 & 3.5 & 5 & 100 & $*$ & $*$ & .05 \\
\hline 3.0 & 4.0 & 5 & 300 & $*$ & $*$ & .01 \\
\hline 3.5 & 4.0 & 5 & 300 & $*$ & $*$ & .05 \\
\hline 4.0 & 4.0 & 5 & 300 & $*$ & $*$ & .01 \\
\hline 4.5 & 4.0 & 5 & 300 & $*$ & $*$ & .05 \\
\hline 5.0 & 4.0 & 5 & 300 & $*$ & $*$ & .01 \\
\hline 3.5 & 4.5 & 5 & 100 & $*$ & * & NS \\
\hline 4.0 & 4.5 & 5 & 100 & $*$ & $*$ & .01 \\
\hline 4.5 & 4.5 & 5 & 100 & $*$ & $*$ & NS \\
\hline 5.0 & 4.5 & 5 & 100 & $*$ & $*$ & .01 \\
\hline 5.5 & 4.5 & 5 & 100 & $*$ & $*$ & NS \\
\hline 4.0 & 5.0 & 5 & 100 & $*$ & * & .01 \\
\hline 4.5 & 5.0 & 5 & 100 & $*$ & $*$ & NS \\
\hline 5.0 & 5.0 & 5 & 100 & $*$ & * & NS \\
\hline 5.5 & 5.0 & 5 & 100 & $*$ & $*$ & NS \\
\hline 6.0 & 5.0 & 5 & 100 & $*$ & $*$ & .01 \\
\hline 4.5 & 5.5 & 5 & 100 & $*$ & $*$ & .05 \\
\hline 5.0 & 5.5 & 5 & 100 & $*$ & $*$ & .01 \\
\hline 5.5 & 5.5 & 5 & 100 & $*$ & $*$ & NS \\
\hline 6.0 & 5.5 & 5 & 100 & $*$ & $*$ & .01 \\
\hline 6.5 & 5.5 & 5 & 100 & $*$ & $*$ & NS \\
\hline 5.0 & 6.0 & 5 & 100 & $*$ & $*$ & .01 \\
\hline 5.5 & 6.0 & 5 & 100 & $*$ & $*$ & NS \\
\hline 6.0 & 6.0 & 5 & 100 & $*$ & $*$ & .01 \\
\hline 6.5 & 6.0 & 5 & 100 & $*$ & $*$ & NS \\
\hline $7.0^{(1)}$ & 6.0 & 5 & 100 & $*$ & $*$ & .01 \\
\hline
\end{tabular}

${ }^{(1)}$ When the hypothesized value equals a boundary value a better test would be to reject $\mathrm{H}_{0}$ if any other value occurs in the sample.

* Ho cannot be rejected at a significance level of 0.05 or 0.01 for samples of size 5 using Wilcoxon signed-rank test. 
Table 5: Seven-point scale comparison of t test and Wilcoxon signed-rank test

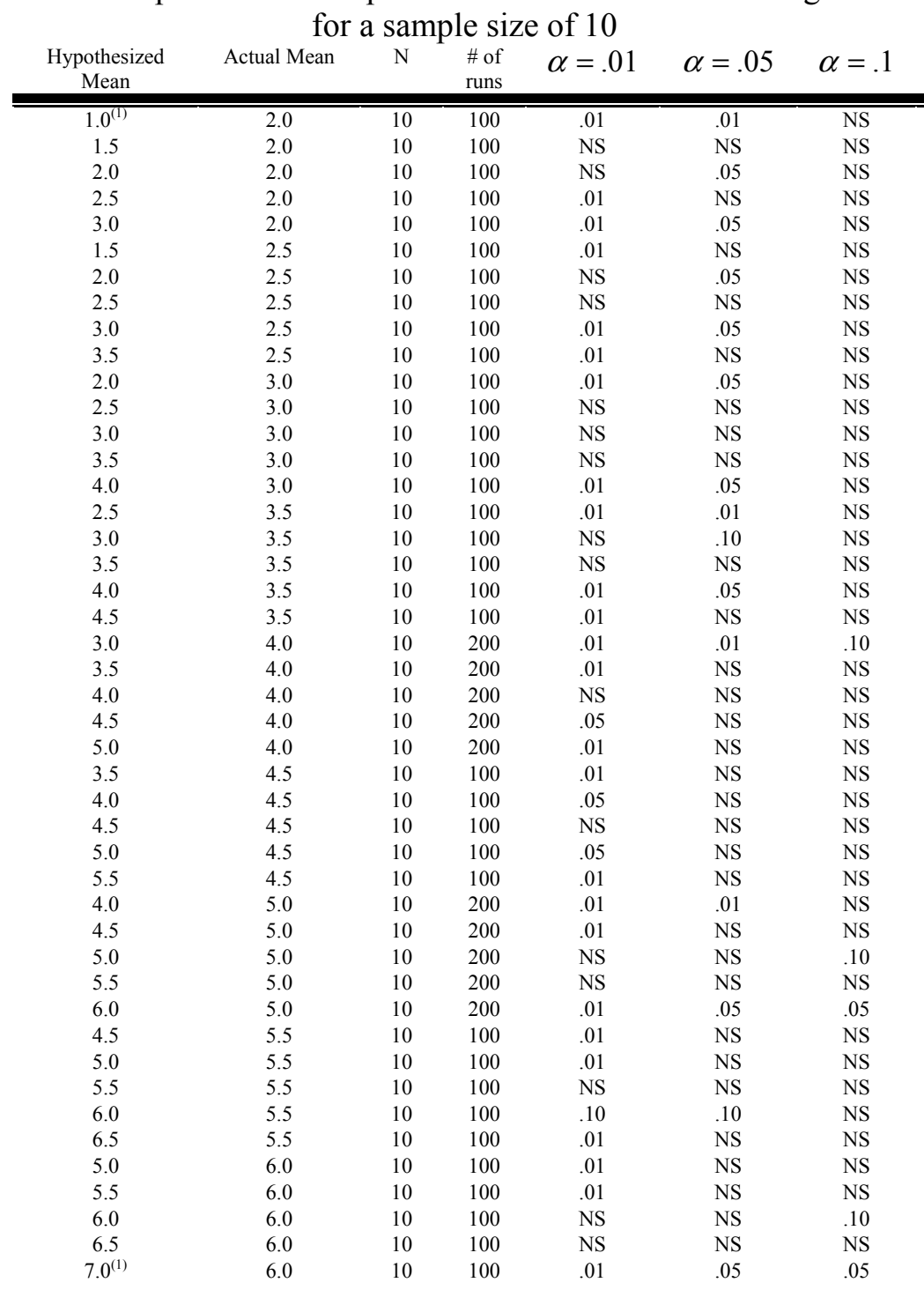

(1) When the hypothesized value equals a boundary value a better test would be to reject $\mathrm{H}_{\mathrm{o}}$ if any other value occurs in the sample. 
Table 6: Seven-point scale comparison of t test and Wilcoxon signed-rank test for a sample size of 15

\begin{tabular}{|c|c|c|c|c|c|c|}
\hline $\begin{array}{c}\text { Hypothesized } \\
\text { Mean }\end{array}$ & Actual Mean & $\mathrm{N}$ & $\begin{array}{l}\# \text { of } \\
\text { runs }\end{array}$ & $\alpha=.01$ & $\alpha=.05$ & $\alpha=.1$ \\
\hline $1.0^{(1)}$ & 2.0 & 15 & 100 & .01 & NS & NS \\
\hline 1.5 & 2.0 & 15 & 100 & NS & NS & NS \\
\hline 2.0 & 2.0 & 15 & 100 & NS & NS & NS \\
\hline 2.5 & 2.0 & 15 & 100 & NS & NS & .10 \\
\hline 3.0 & 2.0 & 15 & 100 & .10 & NS & NS \\
\hline 1.5 & 2.5 & 15 & 100 & NS & NS & .10 \\
\hline 2.0 & 2.5 & 15 & 100 & .05 & NS & NS \\
\hline 2.5 & 2.5 & 15 & 100 & NS & NS & NS \\
\hline 3.0 & 2.5 & 15 & 100 & NS & NS & NS \\
\hline 3.5 & 2.5 & 15 & 100 & .10 & NS & NS \\
\hline 2.0 & 3.0 & 15 & 100 & .01 & NS & .10 \\
\hline 2.5 & 3.0 & 15 & 100 & NS & NS & NS \\
\hline 3.0 & 3.0 & 15 & 100 & NS & NS & NS \\
\hline 3.5 & 3.0 & 15 & 100 & NS & NS & NS \\
\hline 4.0 & 3.0 & 15 & 100 & .01 & NS & NS \\
\hline 2.5 & 3.5 & 15 & 100 & .05 & NS & NS \\
\hline 3.0 & 3.5 & 15 & 100 & .05 & NS & NS \\
\hline 3.5 & 3.5 & 15 & 100 & NS & NS & NS \\
\hline 4.0 & 3.5 & 15 & 100 & NS & NS & NS \\
\hline 4.5 & 3.5 & 15 & 100 & .05 & NS & NS \\
\hline 3.0 & 4.0 & 15 & 300 & .01 & NS & NS \\
\hline 3.5 & 4.0 & 15 & 300 & .05 & NS & NS \\
\hline 4.0 & 4.0 & 15 & 300 & NS & .10 & NS \\
\hline 4.5 & 4.0 & 15 & 300 & .05 & NS & .10 \\
\hline 5.0 & 4.0 & 15 & 300 & .01 & .05 & NS \\
\hline 3.5 & 4.5 & 15 & 100 & NS & NS & NS \\
\hline 4.0 & 4.5 & 15 & 100 & .10 & NS & NS \\
\hline 4.5 & 4.5 & 15 & 100 & NS & NS & NS \\
\hline 5.0 & 4.5 & 15 & 100 & .05 & NS & NS \\
\hline 5.5 & 4.5 & 15 & 100 & .10 & .01 & NS \\
\hline 4.0 & 5.0 & 15 & 100 & .05 & NS & NS \\
\hline 4.5 & 5.0 & 15 & 100 & NS & NS & NS \\
\hline 5.0 & 5.0 & 15 & 100 & NS & NS & NS \\
\hline 5.5 & 5.0 & 15 & 100 & NS & NS & NS \\
\hline 6.0 & 5.0 & 15 & 100 & .01 & NS & NS \\
\hline 4.5 & 5.5 & 15 & 100 & .10 & NS & NS \\
\hline 5.0 & 5.5 & 15 & 100 & NS & NS & NS \\
\hline 5.5 & 5.5 & 15 & 100 & NS & NS & NS \\
\hline 6.0 & 5.5 & 15 & 100 & NS & NS & NS \\
\hline 6.5 & 5.5 & 15 & 100 & NS & NS & NS \\
\hline 5.0 & 6.0 & 15 & 100 & .05 & NS & NS \\
\hline 5.5 & 6.0 & 15 & 100 & .10 & NS & NS \\
\hline 6.0 & 6.0 & 15 & 100 & .05 & NS & NS \\
\hline 6.5 & 6.0 & 15 & 100 & NS & NS & NS \\
\hline $7.0^{(1)}$ & 6.0 & 15 & 100 & NS & NS & NS \\
\hline
\end{tabular}

${ }^{(1)}$ When the hypothesized value equals a boundary value a better test would be to reject $\mathrm{H}_{\mathrm{o}}$ if any other value occurs in the sample. 
Table 7: Average error rates $(\%)$

WSR test average @ $\alpha$ of t-test average $@ \alpha$ of

\begin{tabular}{|c|c|c|c|c|c|c|c|c|c|}
\hline $\begin{array}{l}\Delta=\mu_{\mathrm{o}^{-}} \\
\mu_{\mathrm{a}}\end{array}$ & $\mathrm{n}$ & $\begin{array}{l}\text { Error } \\
\text { Type }\end{array}$ & Runs & 0.01 & 0.05 & 0.10 & 0.01 & 0.05 & 0.10 \\
\hline Five & Point & Scale & & & & & & & \\
\hline$-1.0^{(1)}$ & 5 & II & 600 & $*$ & $*$ & 74.2 & 89.0 & 59.0 & 38.2 \\
\hline-0.5 & 5 & II & 700 & * & * & 88.4 & 98.4 & 88.1 & 76.4 \\
\hline 0.0 & 5 & I & 700 & $*$ & $*$ & 1.9 & 0.1 & 3.7 & 9.0 \\
\hline+0.5 & 5 & II & 700 & * & * & 87.6 & 98.4 & 87.0 & 76.4 \\
\hline$+1.0^{(1)}$ & 5 & II & 600 & * & * & 73.2 & 87.3 & 59.0 & 36.5 \\
\hline$-1.0^{(1)}$ & 10 & II & 600 & 84.7 & 32.2 & 14.7 & 47.5 & 17.5 & 9.0 \\
\hline-0.5 & 10 & II & 700 & 99.1 & 83.6 & 68.4 & 92.3 & 75.4 & 63.7 \\
\hline 0.0 & 10 & I & 850 & 0.0 & 1.9 & 5.1 & 1.2 & 4.1 & 9.5 \\
\hline+0.5 & 10 & II & 700 & 96.9 & 78.4 & 65.0 & 88.0 & 70.1 & 57.6 \\
\hline$+1.0^{(1)}$ & 10 & II & 600 & 82.5 & 30.5 & 11.8 & 48.0 & 19.2 & 7.3 \\
\hline$-1.0^{(1)}$ & 15 & II & 600 & 39.3 & 7.0 & 2.2 & 20.0 & 4.2 & 1.0 \\
\hline-0.5 & 15 & II & 800 & 89.8 & 62.0 & 46.6 & 79.9 & 54.8 & 41.9 \\
\hline 0.0 & 15 & I & 900 & 0.3 & 2.1 & 6.0 & 0.9 & 4.6 & 8.9 \\
\hline+0.5 & 15 & II & 800 & 87.6 & 60.5 & 44.8 & 76.4 & 53.4 & 40.0 \\
\hline$+1.0^{(1)}$ & 15 & II & 700 & 38.1 & 7.3 & 3.3 & 16.9 & 4.6 & 1.7 \\
\hline Seven & Point & Scale & & & & & & & \\
\hline$-1.0^{(1)}$ & 5 & II & 1000 & * & $*$ & 76.0 & 89.1 & 70.3 & 51.5 \\
\hline-0.5 & 5 & II & 1100 & $*$ & * & 88.7 & 97.7 & 87.9 & 78.6 \\
\hline 0.0 & 5 & I & 1100 & $*$ & * & 3.5 & 0.6 & 5.4 & 11.5 \\
\hline+0.5 & 5 & II & 1100 & $*$ & * & 89.5 & 97.8 & 88.9 & 79.8 \\
\hline$+1.0^{(1)}$ & 5 & II & 1000 & * & * & 77.2 & 90.3 & 70.4 & 53.8 \\
\hline$-1.0_{(1)}$ & 10 & II & 1000 & 88.2 & 44.2 & 28.1 & 61.7 & 35.4 & 23.1 \\
\hline-0.5 & 10 & II & 1100 & 98.8 & 83.2 & 69.6 & 91.6 & 76.8 & 63.7 \\
\hline 0.0 & 10 & I & 1100 & 0.0 & 3.0 & 6.5 & 0.9 & 4.7 & 10.2 \\
\hline+0.5 & 10 & II & 1100 & 98.4 & 84.9 & 72.9 & 92.6 & 79.2 & 68.6 \\
\hline$+1.0^{(1)}$ & 10 & II & 1000 & 89.7 & 47.1 & 29.4 & 66.9 & 39.0 & 23.9 \\
\hline$-1.0^{(1)}$ & 15 & II & 1000 & 58.0 & 21.9 & 12.3 & 42.8 & 17.3 & 10.4 \\
\hline-0.5 & 15 & II & 1100 & 93.1 & 72.5 & 58.5 & 86.5 & 67.1 & 53.0 \\
\hline 0.0 & 15 & I & 1100 & 0.5 & 4.8 & 9.3 & 1.8 & 6.4 & 11.4 \\
\hline+0.5 & 15 & II & 1100 & 91.8 & 73.3 & 58.7 & 87.5 & 69.4 & 54.8 \\
\hline$+1.0^{(1)}$ & 15 & II & 1000 & 60.1 & 22.3 & 11.4 & 43.7 & 17.3 & 9.6 \\
\hline
\end{tabular}

(1) If the hypothesized value equals a boundary value the result was deleted since a better test is to reject $\mathrm{H}_{\mathrm{o}}$ if any value other than $\mu_{\mathrm{o}}$ occurs in the sample.

*Ho cannot be rejected at a nominal $\alpha$ value of either 0.01 or 0.05 for a sample of size 5 .

Bold-faced entries indicate cases where the nominal $\alpha$ was exceeded. 
II error rates using the t-test were lower than those using the Wilcoxon signed-rank test for every set of mean differences. Of the total of 630 combinations of mean comparisons and significance levels, the Wilcoxon signed-rank test rejected more times than the t-test when $\mathrm{H}_{\mathrm{o}}$ was false in only 13 combinations and the difference was significant, at 0.10 , in only one of those. Because many of these cases involve distributions that are not symmetric and means are compared rather than medians it may not be fair to compare the Wilcoxon signed-rank test's Type II error rates to those of the t-test, even though at least two of the t-test's assumptions are violated in every case. Therefore, error rates for cases involving only symmetric distributions, where means and medians are the same, are presented in Table 8 below.

As can be seen in Table 8 the pattern of error rates is very similar to that shown in Table 7 for all distributions. That is, even though the Wilcoxon signed-rank test's assumptions are satisfied in all cases, assuming data generated on a Likert scale can be considered ordinal, and the t-test's assumptions are not satisfied in any cases the average Type II error for the t-test is smaller than that of the Wilcoxon signed-rank test. As before, the Wilcoxon signed-rank test protects better against a Type I error with smaller average error rates. Surprisingly, and contrary to popular belief, the t-test, even though its assumption are violated, appears to protect substantially better against Type II errors for sample sizes of 5 and for larger mean differences at a significance level of 0.01 . This phenomenon occurred for both symmetric and non-symmetric distributions. For the cases involving distributions that were not symmetric there did not seem to be any substantial differences between distributions that were skewed to the left from those that were skewed to the right.

In all, 630 combinations of means, sample sizes, Likert scales and nominal $\alpha$-levels were run. The Wilcoxon signed-rank test rejected more times than the t-test in only eighteen of those combinations and only once did it do so significantly. The combination for which the Wilcoxon signed-rank test rejected significantly more times than the t-test at a level of 0.10 was: $n=15,7$-point scale, hypothesized mean of 2.5 vs. actual mean of 2.0 and a nominal $\alpha$ of 0.10 . Cases for which Wilcoxon signed-rank test rejections exceeded t rejections are given in Table 9. Of the 18 cases in Table 9, five correspond to cases where $\mathrm{H}_{\mathrm{o}}$ was true and 13 to cases where $H_{o}$ was false. 
Table 8: Average error rates (in \%) for symmetric distributions

\begin{tabular}{|c|c|c|c|c|c|c|c|c|c|c|}
\hline \multirow[b]{2}{*}{ Scale } & \multirow[b]{2}{*}{$\mathrm{N}$} & \multirow[b]{2}{*}{ Runs } & \multirow[b]{2}{*}{$\Delta=\mu_{0}-\mu_{\mathrm{a}}$} & \multicolumn{4}{|c|}{ WSR test error @ $\alpha=$} & \multicolumn{3}{|c|}{ t-test error @ $\alpha=$} \\
\hline & & & & Error & 0.01 & 0.05 & 0.10 & 0.01 & 0.05 & 0.10 \\
\hline$\overline{5}$ & $\overline{5}$ & 300 & -1.0 & II & * & * & 87 & 92 & 62 & 441 \\
\hline 5 & 5 & 300 & -0.5 & II & $*$ & * & 87 & 99 & 92 & 82 \\
\hline 5 & 5 & 300 & 0.0 & I & $*$ & $*$ & 0 & 0 & 4 & 10 \\
\hline 5 & 5 & 300 & 0.5 & II & $*$ & $*$ & 84 & 98 & 87 & 78 \\
\hline 5 & 5 & 300 & 1.0 & II & $*$ & $*$ & 84 & 87 & 59 & 35 \\
\hline 5 & 10 & 300 & -1.0 & II & 89 & 35 & 16 & 49 & 20 & 12 \\
\hline 5 & 10 & 300 & -0.5 & II & 99 & 81 & 67 & 92 & 76 & 70 \\
\hline 5 & 10 & 450 & 0.0 & I & 0 & 2 & 5 & 1 & 4 & 9 \\
\hline 5 & 10 & 300 & 0.5 & II & 97 & 78 & 67 & 90 & 72 & 61 \\
\hline 5 & 10 & 300 & 1.0 & II & 88 & 33 & 12 & 53 & 19 & 7 \\
\hline 5 & 15 & 300 & -1.0 & II & 45 & 7 & 2 & 23 & 4 & 1 \\
\hline 5 & 15 & 300 & -0.5 & II & 92 & 67 & 52 & 84 & 61 & 48 \\
\hline 5 & 15 & 400 & 0.0 & I & 0 & 2 & 6 & 1 & 5 & 8 \\
\hline 5 & 15 & 300 & 0.5 & II & 90 & 64 & 49 & 78 & 56 & 42 \\
\hline 5 & 15 & 300 & 1.0 & II & 42 & 7 & 3 & 18 & 5 & 2 \\
\hline 7 & 5 & 300 & -1.0 & II & $*$ & $*$ & 86 & 91 & 71 & 51 \\
\hline 7 & 5 & 300 & -0.5 & II & $*$ & $*$ & 85 & 98 & 89 & 77 \\
\hline 7 & 5 & 300 & 0.0 & I & * & * & 1 & 1 & 5 & $11^{1}$ \\
\hline 7 & 5 & 300 & 0.5 & II & $*$ & $*$ & 90 & 98 & 94 & 84 \\
\hline 7 & 5 & 300 & 1.0 & II & $*$ & $*$ & 91 & 95 & 75 & 62 \\
\hline 7 & 10 & 200 & -1.0 & II & 92 & 49 & 28 & 64 & 36 & 20 \\
\hline 7 & 10 & 200 & -0.5 & II & 99 & 85 & 72 & 91 & 79 & 66 \\
\hline 7 & 10 & 200 & 0.0 & I & 0 & 4 & 7 & 1 & $6^{1}$ & 10 \\
\hline 7 & 10 & 200 & 0.5 & II & 98 & 85 & 75 & 94 & 82 & 69 \\
\hline 7 & 10 & 200 & 1.0 & II & 93 & 38 & 27 & 66 & 35 & 21 \\
\hline 7 & 15 & 300 & -1.0 & II & 65 & 23 & 15 & 44 & 18 & 12 \\
\hline 7 & 15 & 300 & -0.5 & II & 94 & 75 & 62 & 89 & 71 & 56 \\
\hline 7 & 15 & 300 & 0.0 & I & 0 & 3 & 9 & 1 & 5 & $11^{1}$ \\
\hline 7 & 15 & 300 & 0.5 & II & 93 & 73 & 61 & 88 & 69 & 54 \\
\hline 7 & 15 & 300 & 1.0 & II & 65 & 23 & 11 & 45 & 16 & 8 \\
\hline
\end{tabular}

${ }^{1}$ Identifies cases where the nominal $\alpha$ was exceeded.

* Indicates cases where $\mathrm{n}$ is too small for Wilcoxon signed-rank test to reject at the nominal significance level. 
the study. Contrary to what was expected, based on the literature, the t-test was much better at

Table 9: Cases where Wilcoxon signed-rank rejections exceed t rejections

\begin{tabular}{ccccccccc} 
Scale & $\mathrm{N}$ & Runs & Actual $\mu$ & Hypoth. $\mu$ & Nom. $\alpha$ & WSR rej. & t-test rej. & Sig. \\
\hline \hline 5 & 5 & 100 & 2.5 & 2.5 & 0.10 & 6 & 2 & NS \\
5 & 10 & 300 & 3.0 & 2.5 & 0.10 & 99 & 91 & NS \\
5 & 15 & 200 & 2.0 & 2.5 & 0.10 & 115 & 114 & NS \\
5 & 15 & 100 & 2.5 & 2.5 & 0.10 & 6 & 4 & NS \\
5 & 15 & 100 & 4.0 & 3.5 & 0.10 & 66 & 65 & NS \\
7 & 5 & 100 & 2.5 & 2.5 & 0.10 & 9 & 8 & NS \\
7 & 5 & 100 & 5.5 & 5.5 & 0.10 & 9 & 8 & NS \\
7 & 5 & 100 & 6.0 & 6.5 & 0.10 & 14 & 11 & NS \\
7 & 10 & 100 & 2.0 & 2.5 & 0.10 & 55 & 50 & NS \\
7 & 10 & 100 & 5.5 & 4.5 & 0.10 & 64 & 63 & NS \\
7 & 10 & 100 & 6.0 & 5.5 & 0.10 & 49 & 48 & NS \\
7 & 15 & 100 & 2.0 & 2.5 & 0.10 & 67 & 55 & 0.10 \\
7 & 15 & 100 & 2.0 & 2.5 & 0.05 & 51 & 47 & NS \\
7 & 15 & 100 & 2.0 & 3.0 & 0.05 & 84 & 81 & NS \\
7 & 15 & 100 & 2.5 & 2.5 & 0.10 & 17 & 13 & NS \\
7 & 15 & 100 & 5.5 & 4.5 & 0.10 & 88 & 87 & NS \\
7 & 15 & 100 & 5.5 & 4.5 & 0.05 & 81 & 79 & NS \\
7 & 15 & 100 & 6.0 & 5.5 & 0.05 & 42 & 39 & NS
\end{tabular}

\section{Conclusion}

Based on the 27,850 simulations conducted in this study, of which 8,750 involved symmetric distributions, it appears that the t-test may be preferred over the signed-rank procedure, even for very small sample sizes, unless it is imperative that one be able to calculate the exact probability of committing a Type I error. As in the Meek, et al., (2000) and (2001) studies, the Blair and Higgins (1985) study, and the Nanna (2002) study the level of measurement does not appear to be an important factor in test selection, at least in the case of a Likert scale. A more important consideration, at least with respect to the one sample test of location, is which error is more critical to guard against. The limitations of this study are that all data were generated from binomial distributions, the assumptions for the $\mathrm{t}$ test are violated in all cases and the symmetry assumption of the signed-rank test is violated in $69 \%$ of the cases. Even with these limitations the t-test showed a lower average Type II error rate across all of the sample sizes that were used in

protecting against a Type II error for the sample size of five than was the signed-rank test, even when the Wilcoxon signed-rank test's assumptions were all satisfied. As the sample size increased the number of significant differences between the two procedures decreased dramatically for the 0.10 and 0.05 significance levels, to the point that the tests had similar error rates for those significance levels when $n=15$. Although the results of this study seem to be in conflict with those of Blair and Higgins (1985), Nanna and Sawilowsky (1998), and Nanna (2002) their studies involved testing either two populations or the multivariate case and used different underlying distributions.

In summary, the results of these simulations indicated:

1. Except for a sample size of 5, the numbers of significant differences were fewest at a nominal $\alpha$ of 0.10 while significant differences decreased for both 0.10 and 0.05 as the sample size increased, but not for 0.01 ; 
2. The t-test tended to have a higher Type I error rate, but closer to the nominal value, on average, while the Wilcoxon signed-rank test had a higher Type II error rate;

3. There did not appear to be any dramatic differences between error rates when the distributions were symmetric as opposed to being asymmetric;

4. The t-test actually appears to reject false hypotheses better; i.e., to have higher power, than the Wilcoxon signed-rank test when the sample sizes are small, even though its assumptions are violated in every case; and,

5. This study appears to contradict statements and recommendations about the use of the t-test vs. the Wilcoxon signed-rank test in small sample applications involving these particular non-normal distributions and ordinal data.

Further study needs to be done using different types of underlying distributions to generate the data to determine if these results might be attributed to having used a binomial generator. Additional points that might be considered in the future are other Likert scales, such as a 9-point, and ordinal measurements that do not correspond to Likert scale data. Regardless of this study's limitations it is quite surprising to find that all of the recommendations in the literature for using Wilcoxon's signed-rank procedure over the ttest, particularly with small sample sizes and Likert scale data, appear to be groundless, even when the t-test's assumptions are violated. Under conditions similar to the ones in this study it seems the only justification for using the Wilcoxon signed-rank procedure over the t-test is that it be imperative that an exact Type I error be able to be calculated.

\section{References}

Anderson, D., Sweeney, D. \& Williams, T. (2005) Statistics for business and economics $\left(9^{\text {th }}\right.$ ed.). Mason, OH: Southwestern Publishing Co.
Baggaley, Andrew R. (1960). Some remarks on scales of measurement and related topics. The Journal of General Psychology, 62, 141-145.

Berenson, M., Levine, D. \& Kreihbel, T. (2004). Basic business statistics: concepts and application, $\left(9^{\text {th }}\right.$ ed.). Englewood Cliffs, NJ: Prentice-Hall Publishing Co.

Blair, R. C., \& Higgins, J. J. (1985). Comparison of the power of the paired samples $t$ test to that of Wilcoxon's signed-ranks test under various population shapes. Psychological Bulletin, 97(1), 119-128.

Bowerman, B. \& O'Connell, R. (2007). Business statistics in practice. New York: McGraw-Hill Irwin Publishing Co.

Bradley, J. V. (1968). Distribution free statistical tests. Englewood-Cliffs, NJ: PrenticeHall Publishing Co.

Bradley, J. V. (1980). Nonrobustness in one-sample $\mathrm{Z}$ and t tests: A large-scale sampling study. Bulletin of the Psychonomic Society, 15, 29-32.

Chou, Y. L. (1989). Statistical analysis for business and economics. New York: Elsevier Science Publishing Co.

Conover, W. J. (1971). Practical nonparametric statistics. New York: John Wiley \& Sons.

Doane, D. \& Seward, L. (2007) Applied statistics in business and economics. New York: McGraw-Hill Irwin Publishing Co.

Harris, R. J. (1975). A primer of multivariate statistics. New York: Academic Press.

Heeren, T.\& D'Agostino, R. (1987). Robustness of the two independent samples when applied to ordinal scale data. Statistics in Medicine, 6, 79-90

Keller, G. (2005). Statistics for management and economic ( $7^{\text {th }}$ ed.). Belmont, CA: Thomson Publishing Co.

Meek, G., Ozgur, C. \& Dunning, K. (2000). Does scale of measurement really make a difference in test selection: An empirical comparison of $\mathrm{t}$ test vs. Mann Whitney. Proceedings of the 2000 National Annual Meeting of the Decision Sciences Institute, 951953. 
Meek, G., Ozgur, C. \& Dunning, K. (2001). Does scale of measurement really make a difference in test selection: The one-sample case, $\mathrm{t}$ vs. Wilcoxon signed-rank test. Proceedings of the 2001 National Annual Meeting of the Decision Sciences Institute, 1280 (abstract).

Moore, D., McCabe, G., Duckworth, W. \& Sclove, S. (2003) The practice of business statistics: using data for decisions. New York, NY: W. H. Freeman and Co.

Nanna, M. J. (2002). Hotellings $\mathrm{T}^{2}$ vs the rank transform with real Likert data. Journal of Modern Applied Statistical Methods, 1(1) 8399.

Nanna, M. J., \& Sawilowsky, S. S. (1998). Analysis of Likert scale data in disability and medical rehabilitation research. Psychological Methods, 3(1), 55-67.

Newbold, P. (1995). Statistics for business and economics ( $4^{\text {th }}$ ed.). Englewood Cliffs, NJ: Prentice-Hall, Inc.

Russel, C. J. \& Bobko, P. (1992). Moderated regression analysis and Likert scales: Too coarse. Journal of Applied Psychology, 77, (3), 336-342.
Sawilowsky, S. S. (1991). Comments on using alternatives to normal theory statistics in social and behavioral sciences. Canadian Psychology, 34(4), 432-433.

Sawilowsky, S. S. (2005). Misconceptions leading to choosing the $t$ test over the Wilcoxon Mann-Whitney test for shift in location parameter. Journal of Modern Applied Statistical Methods, 4(2), 598-600.

Sawilowsky, S. S. (1990). Nonparametric tests of interaction in experimental design. Review of Educational Research, 60(1), 91-126.

Sawilowsky, S. S. \& Blair, R. C. (1992). A more realistic look at the robustness and type II error properties of the $t$ test to departures from population normality. Psychological Bulletin, $111,352-360$

Siegel, S. (1956) Nonparametric Statistics for the Behavioral Sciences. New York, NY: McGraw-Hill.

Stevens, S. S. (1946). On the theory of scales of measurement. Science, 103(2684), 677680 . 


\title{
Another Look at Confidence Intervals for the Noncentral $T$ Distribution
}

\author{
Bruno Lecoutre
}

Centre National de la Recherche Scientifique and Université de Rouen, France

An alternative approach to the computation of confidence intervals for the noncentrality parameter of the Noncentral $t$ distribution is proposed. It involves the percent points of a statistical distribution. This conceptual improvement renders the technical process for deriving the limits more comprehensible. Accurate approximations can be derived and easily used.

Key words: Confidence intervals, noncentral $\mathrm{t}$ distribution, lambda-prime distribution, Bayesian inference.

\section{Introduction}

In spite of several recent presentations (see especially, Fidler \& Thompson, 2001; Bird, 2002), many potential users, as well as statistical instructors, consider computing or teaching confidence intervals for the noncentrality parameter of the Noncentral $t$ distribution to be very complex tasks. One of the conceptual difficulties is the lack of explicit formula. Although the considerable advances in computing techniques are supposed to render the task easy, they do not solve the conceptual difficulties.

The latter state is all the more deceptive in that when the number of degrees of freedom is large enough so that the Normal approximation holds the solution is very simple: the confidence limits are given by the percent points of a Normal distribution, as for the familiar case of an unstandardized difference between means. Thus, it can be expected that in the general case the limits would also be

Bruno Lecoutre is Research Director, CNRS. His research interests are in experimental data analysis, Bayesian methods, foundations of statistics, and the null hypothesis significance tests controversy. Contact him at ERIS, Laboratoire de Mathématiques Raphaël Salem, UMR 6085, C.N.R.S. et Université de Rouen Avenue de l'Université, BP 12, 76801 SaintEtienne-du-Rouvray, France. Email address: bruno.lecoutre@univ-rouen.fr computed as the percent points of a statistical distribution. Unfortunately, this is not the case with the usual presentations.

Moreover, warnings about the accuracy of some computer programs of the Noncentral $t$ distribution (typically, the Noncentral $t$ algorithm fails for large sample size or effect size) cast doubt on some numerical results. Consequently, there remains the need for accurate approximations that are not currently easily available. Even when an exact computation is wanted, it needs an iterative algorithm, for which an accurate approximation constitutes a good starting point.

An alternative approach is proposed in this article that results in computing the confidence limits as the percent points of a statistical distribution as in the most familiar situations. An interesting consequence of this conceptual improvement is that standard techniques to approximate statistical distributions can be used in order to find easy to use very accurate approximations. In conclusion, the question of the justification and interpretation of confidence intervals will be briefly examined.

Considerations and discussions regarding how and when to use confidence intervals for the Noncentral $t$ distribution, may be found elsewhere. Therefore, this article is not methodological. In this perspective, it will be sufficient, with no loss of generality, to consider the elementary case of the inference about a standardized difference between two means. 
Computing confidence intervals from the Noncentral $t$ distribution

When comparing two means, the $t$ test statistic is the ratio $\left(\bar{Y}_{1}-\bar{Y}_{2}\right) / E$ of the two statistics, $\bar{Y}_{1}-\bar{Y}_{2}$ that is an estimate of the population difference $\mu_{1}-\mu_{2}$ and the standard error $E$ of that estimate (see e.g., Fidler \& Thomson, 2001, p. 587). In other words, $E$ is an estimate of the standard deviation $\varepsilon$ of the sampling distribution for $\bar{Y}_{1}-\bar{Y}_{2}$. For instance, in the particular case of two independent groups, assuming a common variance $\sigma^{2}$, one has $\varepsilon=\sigma \sqrt{1 / n_{1}+1 / n_{2}}$.

The sampling distribution of the ratio $\left(\bar{Y}_{1}-\bar{Y}_{2}\right) / E$ is a Noncentral $t$ distribution with $d f$ degrees of freedom and a noncentrality parameter $\lambda$, equal to $\left(\mu_{1}-\mu_{2}\right) / \varepsilon$. This distribution is usually written $t_{d f}^{\prime}(\lambda)$. The noncentrality parameter is termed $\lambda$, as in Algina and Keselman (2003), in order to avoid confusion with the population effect size. Formally, the Noncentral $t$ distribution is the noncentrality parameter $\lambda$ plus the standard Normal $z$ distribution, all divided by the square root of the usual Chi-square distribution divided by the degrees of freedom (see e.g., Fidler \& Thomson, 2001, p. 589):

$$
t_{d f}^{\prime}(\lambda)=(\lambda+z) / \sqrt{\chi_{d f}^{2} / d f} .
$$

The traditional approach for finding the lower (for instance) limit $\lambda_{L}$ of the noncentrality parameter $\lambda$ uses the probability $p_{\lambda}$ that $t^{\prime}{ }_{d f}(\lambda)$ exceeds the value $t_{C A L C}$ observed in the data in hand:

$$
p_{\lambda}=\operatorname{Pr}\left(t_{d f}^{\prime}(\lambda)>t_{C A L C}\right)
$$

Then, one must vary the $\lambda$ value in order to find, by successive approximations, the particular value $\lambda_{L}$ such that $p_{\lambda_{L}}=\alpha / 2$ :

$$
p_{\lambda}=\operatorname{Pr}\left(t_{d f}^{\prime}(\lambda)>t_{C A L C}\right)=\alpha / 2 .
$$

The conceptual difficulties come from the fact that finding the limit $\lambda_{L}$ involves as many different distributions as considered $\lambda$ values. A practical consequence is that it is a highly difficult task to derive accurate approximations.

An alternative approach: computing confidence intervals as percent points of the Lambda-prime distribution

An alternative solution consists in computing the confidence limits for the noncentrality parameter as percent points of a statistical distribution. When $d f$ is large enough so that the normal approximation holds, $\lambda_{L}$ is simply the $100 \alpha / 2$ percent point of the standardized Normal distribution with mean $t_{C A L C}$. This can be generalized by introducing an appropriate statistical distribution. Even if it has not been made explicit in the usual presentations, this distribution is in fact not unfamiliar (without mentioning the fiducial and Bayesian presentations discussed in the conclusion).

Indeed, it is usual to plot $p_{\lambda}$ (or its complement $1-p_{\lambda}$ ) as a function of $\lambda$. An illustration is given in Figure 1 for $t_{C A L C}=$ +1.0076 with $d f=22$ (hence a $p$-value $p=0.3246$, two-sided), which corresponds to the two-group A way data example given by Fidler $\&$ Thomson (2001, p. 586). The $p_{\lambda}$ value increasingly varies from zero (when $\lambda$ tends to $\infty)$ to one (when $\lambda$ tends to $+\infty$ ), so that the corresponding curve is nothing else than the cumulative distribution function of a probability distribution. Such a graphical representation is commonly proposed to get a graphical solution for the confidence limits (see, for instance, Steiger \& Fouladi, 1997, pp. 240), but the proponents fail to recognize that, in doing this, they implicitly define the confidence limits as the percent points of this probability distribution. 


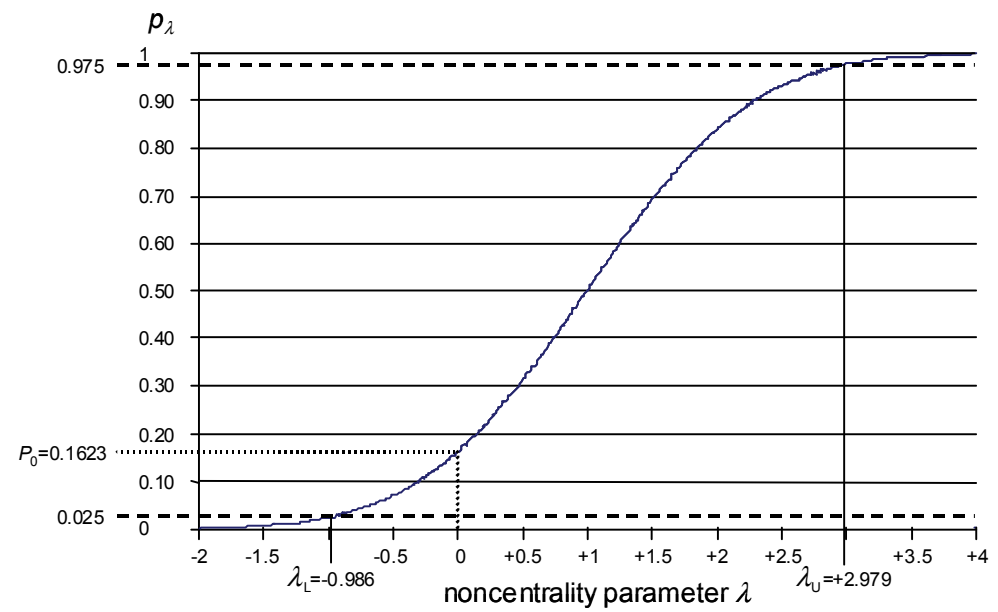

Figure 1 - Plot of $p_{\lambda}$ as a function of $\lambda$ for $t_{C A L C}=+1.0076$ and $d f=22$ and graphical solution for the $95 \%$ confidence interval for $\lambda$. The curve is the cumulative distribution function of the $\Lambda_{22}^{\prime}(+1.0076)$ distribution.

As for the Noncentral $t$, this distribution can be easily defined from the Normal and Chisquare distributions, but the result has not been so popularized. $\left(\bar{Y}_{1}-\bar{Y}_{2}\right) / E>t_{C A L C}$ can be equivalently written as $\bar{Y}_{1}-\bar{Y}_{2}-t_{C A L C} E>0$. Consequently, $p_{\lambda}$ is the probability that $\bar{Y}_{1}-\bar{Y}_{2}-t_{C A L C} E$ exceeds zero.

$\bar{Y}_{1}-\bar{Y}_{2}-t_{C}$ sampling distribution of independent standard Normal and Chi-square distributions as:

$$
\varepsilon\left(\lambda+z-t_{C A L C} \sqrt{\chi_{d f}^{2} / d f}\right) .
$$

so that

$$
\begin{aligned}
& p_{\lambda} \\
& =\operatorname{Pr}\left(\bar{Y}_{1}-\bar{Y}_{2}-t_{C A L C} E>0\right) \\
& =\operatorname{Pr}\left(-z+t_{C A L C} \sqrt{\chi_{d f}^{2} / d f}<\lambda\right) \\
& =\operatorname{Pr}\left(z+t_{C A L C} \sqrt{\chi_{d f}^{2} / d f}<\lambda\right),
\end{aligned}
$$

because the Normal distribution is symmetric around zero.
Thus, $p_{\lambda}$ can be computed from the distribution characterized by $z+t_{C A L C} \sqrt{\chi_{d f}^{2} / d f}$. This distribution, which was considered (with no name) by Fisher (1990/1973, pp. 126-127) in the fiducial framework, was called Lambda-prime in Lecoutre (1999). It is also a noncentral distribution, again with $d f$ degrees of freedom, but with noncentrality $t_{C A L C}$. Formally:

$$
\Lambda_{d f}^{\prime}\left(t_{C A L C}\right)=z+t_{C A L C} \sqrt{\chi_{d f}^{2} / d f} .
$$

Consequently, it is possible to inverse in some sense the problem in (1) and compute $p_{\lambda}$ as the probability that the Lambda-prime distribution with noncentrality $t_{C A L C}$ is smaller than $\lambda$ :

$$
p_{\lambda}=\operatorname{Pr}\left(\Lambda_{d f}^{\prime}\left(t_{C A L C}\right)<\lambda\right) \text {. }
$$

Thus, the curve in Figure 1 is the cumulative distribution function of the Lambda-prime distribution with 22 degrees of freedom and noncentrality +1.0076 .

In order to find the limit, solve

$$
p_{\lambda}=\operatorname{Pr}\left(\Lambda_{d f}^{\prime}\left(t_{C A L C}\right)<\lambda_{L}\right)=\alpha / 2
$$


(2) is technically equivalent to (1) and requires a similar iterative process, but it has a conceptual advantage. Indeed, it involves a unique distribution, so that $\lambda_{L}$ is the $100 \alpha / 2$ percent point of the $\Lambda_{d f}^{\prime}\left(t_{C A L C}\right)$ distribution. In the same way, the upper limit $\lambda_{U}$ is its $100(1-\alpha / 2)$ percent point. For instance, in Figure 1, the limits $\lambda_{L}=$ 0.986 and $\lambda_{U}=+2.979$ of the $95 \%$ confidence interval are respectively the 2.5 and 97.5 percent points of the $\Lambda_{22}^{\prime}(+1.0076)$ distribution.

Note again that the statistic $\bar{Y}_{1}-\bar{Y}_{2}-t_{C A L C} E$ should not be regarded as less natural than the $t$ test statistic. Indeed, it is similar to the familiar limits $\bar{Y}_{1}-\bar{Y}_{2} \pm t_{1-\alpha / 2} E$ of the $100(1-\alpha) \%$ confidence interval for a raw difference. This analogy will be discussed in the conclusion.

Approximations of the $100 \pi$ percent point of the $\Lambda_{d f}^{\prime}(t)$ distribution

Beyond its conceptual simplification, the alternative approach allows to derive accurate approximations. In this Section, in order to simplify the notations, $t_{C A L C}$ will be written $t$.

Numerical example

Consider Bird's first example (Bird, 2002, p. 206), which is also considered in
Algina and Keselman (2003). There were three independent groups of size 30 each, with means $\bar{Y}_{1}=22.467, \bar{Y}_{2}=24.933$, and $\bar{Y}_{3}=32.000$ and within group standard deviation 7.435. Bird reported the $97.5 \%$ confidence intervals of two standardized contrasts $\left(\bar{Y}_{1}+\bar{Y}_{2}\right) / 2-\bar{Y}_{3}$ and $\bar{Y}_{1}-\bar{Y}_{2}$. The computations for the first contrast will be detailed to illustrate the approximation methods. For this contrast, the $t$ test statistic is $t=-4.9924(d f=87)$. The exact confidence interval of $\lambda$ is: [-7.3766, -2.5844].

It can be computed by the usual method based on the Noncentral $t$ distribution using the available programs (for instance the Noncentral Distribution Calculator of Steiger, 2004), or alternatively as the 1.25 and 98.75 percent points of the Lambda-prime distribution with 87 degrees of freedom and eccentricity -4.9924 .

Three approximation methods will be considered. The results for the two contrasts of interest are presented in Table 1. The limits for the standardized contrast $\left(\bar{Y}_{1}+\bar{Y}_{2}\right) / 2-\bar{Y}_{3}$ in Table 1 are obtained by multiplying the limits for $\lambda$ by the appropriate constant $(0.223607)$ referred as SE in Bird's table, page 208. Note that this constant can be simply computed as the ratio of the observed standardized contrast to the $t$ test value: $-1.1163 /-4.9924=0.2236$.

Table 1. Bird's example: comparison of the three approximation methods

\begin{tabular}{|c|c|c|c|c|c|c|c|}
\hline \multirow[b]{2}{*}{ Contrast } & \multirow[b]{2}{*}{ Value } & \multirow[b]{2}{*}{$T$} & \multirow[b]{2}{*}{$97.5 \% \mathrm{CI}$} & \multirow[b]{2}{*}{ Exact } & \multicolumn{3}{|c|}{ Approximation } \\
\hline & & & & & Bird & Normal & Chi-square \\
\hline \multirow{2}{*}{$\left(\bar{Y}_{1}+\bar{Y}_{2}\right) / 2-\bar{Y}_{3}$} & \multirow{2}{*}{-1.1163} & \multirow{2}{*}{-4.9924} & LowerLimit & -1.6495 & -1.6264 & -1.6489 & -1.6495 \\
\hline & & & UpperLimit & -0.5779 & -0.6063 & -0.5773 & -0.5779 \\
\hline \multirow{2}{*}{$\bar{Y}_{1}-\bar{Y}_{2}$} & \multirow{2}{*}{-0.3318} & \multirow{2}{*}{-1.2849} & LowerLimit & -0.9123 & -0.9207 & -0.9123 & $-0.9123^{*}$ \\
\hline & & & UpperLimit & +0.2506 & +0.2572 & +0.2506 & $+0.2506^{*}$ \\
\hline
\end{tabular}

$\overline{\text { Note. }{ }^{*} \text { Normal approximation }}$ 
Three Approximation Methods - Bird's Approximation

Reconsidered in the new approach, the Bird (2002, p. 203) approximation of the $100 \pi$ percent point of the Lambda-prime consists in adding $t$ and the $100 \pi$ percent point of the standard (central) $t$ distribution with the same degrees of freedom:

$$
\Lambda_{d f, \pi}^{\prime}(t) \approx t+t_{d f, \pi}
$$

The approximate $100(1-\alpha) \%$ confidence interval for $\lambda$ is obtained:

$$
t \pm t_{d f, 1-\alpha \prime 2}
$$

hence here for $t_{87,0.9875}=+2.2809$ the approximate confidence interval: $-4.9924 \pm$ $2.2809 \rightarrow[-7.2733,-2.7116]$.

Algina and Keseleman (2003) found that the accuracy of this approximation does vary with the magnitude of the parameter, which can be verified in Table 1 .

\section{A simple normal approximation}

The Lambda-prime distribution is generally asymmetric. However, when $t=0$ it reduces to the standard Normal distribution, and when $d f$ is large it tends to the $N(t, 1)$ distribution. So we can expect that a Normal approximation with the same mean and variance is appropriate, at least for small $t$ and for large $d f$. where

The $\Lambda_{d f}^{\prime}(t)$ distribution has mean $M=k t$

$$
k=\sqrt{\frac{2}{d f}} \frac{\Gamma\left(\frac{d f+1}{2}\right)}{\Gamma\left(\frac{d f}{2}\right)},
$$

and variance

$$
V=1+t^{2}\left(1-k^{2}\right)=1+t^{2}-M^{2},
$$

so, that it can be approximated by the $N(M, V)$ distribution:

$$
\Lambda_{d f, \pi}^{\prime}(t) \approx k t+z_{\pi} \sqrt{1+t^{2}\left(1-k^{2}\right)} .
$$

The approximate $100(1-\alpha) \%$ confidence interval for $\lambda$ is obtained:

$$
k t \pm z_{1-\alpha / 2} \sqrt{1+t^{2}\left(1-k^{2}\right)} .
$$
logarithm:

In order to find $k$, one can compute its

$\log (k)$

$=(\log (2)-\log (d f)) / 2+\log \operatorname{Gamma}((d f+1) / 2)$

- $\log \operatorname{Gamma}(d f / 2)$,

and then take the exponential of $\log (k)$. $\log \operatorname{Gamma}(x)$ is the logarithm of the Gamma function $\Gamma(x)$, that generalizes factorials to numbers beyond the integers. It is standard and for instance available in Excel. $k$ can also be computed using the series expansion (Johnson \& Welch, 1939):

$$
k=\begin{aligned}
& 1-\frac{1}{4 \mathrm{df}}+\frac{1}{32 \mathrm{df}^{2}}+\frac{5}{128 \mathrm{df}^{3}} \\
& -\frac{21}{2048 \mathrm{df}^{4}}-\frac{399}{8192 \mathrm{df}^{5}}+\frac{869}{65536 \mathrm{df}^{6}}+\cdots
\end{aligned} .
$$

Alternatively, Table 2 can be used for finding the wanted value. 
Table 2 - $k$ values for $d f$ ranking from 1 to 100 . For $k>100$ the approximation $k \approx 1-1 /(4 d f)$ gives an error less than $10^{-5}$.

\begin{tabular}{rcrcrcrrrc}
\hline \hline$d f$ & $k$ & $d f$ & $k$ & $d f$ & $k$ & $d f$ & $k$ & $d f$ & $k$ \\
\hline 1 & 0.797885 & 2 & 0.886227 & 3 & 0.921318 & 4 & 0.939986 & 5 & 0.951533 \\
6 & 0.959369 & 7 & 0.965030 & 8 & 0.969311 & 9 & 0.972659 & 10 & 0.975350 \\
11 & 0.977559 & 12 & 0.979406 & 13 & 0.980971 & 14 & 0.982316 & 15 & 0.983484 \\
16 & 0.984506 & 17 & 0.985410 & 18 & 0.986214 & 19 & 0.986934 & 20 & 0.987583 \\
21 & 0.988170 & 22 & 0.988705 & 23 & 0.989193 & 24 & 0.989640 & 25 & 0.990052 \\
26 & 0.990433 & 27 & 0.990786 & 28 & 0.991113 & 29 & 0.991418 & 30 & 0.991703 \\
31 & 0.991969 & 32 & 0.992219 & 33 & 0.992454 & 34 & 0.992675 & 35 & 0.992884 \\
36 & 0.993080 & 37 & 0.993267 & 38 & 0.993443 & 39 & 0.993611 & 40 & 0.993770 \\
41 & 0.993922 & 42 & 0.994066 & 43 & 0.994203 & 44 & 0.994335 & 45 & 0.994460 \\
46 & 0.994580 & 47 & 0.994695 & 48 & 0.994806 & 49 & 0.994911 & 50 & 0.995013 \\
51 & 0.995110 & 52 & 0.995204 & 53 & 0.995294 & 54 & 0.995381 & 55 & 0.995465 \\
56 & 0.995546 & 57 & 0.995624 & 58 & 0.995699 & 59 & 0.995772 & 60 & 0.995842 \\
61 & 0.995910 & 62 & 0.995976 & 63 & 0.996040 & 64 & 0.996102 & 65 & 0.996161 \\
66 & 0.996219 & 67 & 0.996276 & 68 & 0.996330 & 69 & 0.996383 & 70 & 0.996435 \\
71 & 0.996485 & 72 & 0.996534 & 73 & 0.996581 & 74 & 0.996627 & 75 & 0.996672 \\
76 & 0.996716 & 77 & 0.996759 & 78 & 0.996800 & 79 & 0.996841 & 80 & 0.996880 \\
81 & 0.996918 & 82 & 0.996956 & 83 & 0.996993 & 84 & 0.997028 & 85 & 0.997063 \\
86 & 0.997097 & 87 & 0.997131 & 88 & 0.997163 & 89 & 0.997195 & 90 & 0.997226 \\
91 & 0.997257 & 92 & 0.997286 & 93 & 0.997315 & 94 & 0.997344 & 95 & 0.997372 \\
96 & 0.997399 & 97 & 0.997426 & 98 & 0.997452 & 99 & 0.997478 & 100 & 0.997503 \\
\hline \hline
\end{tabular}

In Bird's example, it is found that $k=0.997131, \quad M=-4.9781$, and $V=1.1428$, hence for $z_{0.9875}=+2.2414$ the approximate confidence interval for $\lambda$ : $-4.9781 \pm$ $2.2414 \sqrt{1.1428} \rightarrow[-7.3742,-2.5820]$ that is close to the exact interval.

A Chi-square approximation

For large $t$ values, a better approximation can be found that takes into account the asymmetry of the distribution. This needs to consider the third central moment that can be deduced from the mean:

$$
W=2 k^{2}-\frac{2 d f-1}{d f} k t^{3}=2 M^{3}-\frac{2 d f-1}{d f} t^{2} M
$$

Next, compute the skewness of the distribution as the ratio of $W$ to the third power of the square root of the variance $V$ (i.e. $W / V^{(3 / 2)}$ ). The skewness is a measure of the degree of asymmetry of the distribution. When it is small, one can use the Normal approximation $N(M, V)$ above. For practical applications, it was empirically found that a more sophisticated approximation is not necessary when the skewness is smaller than 0.001. Otherwise, the following Chi-square approximation that fits the skewness can be used. It involves again reasonably simple computations. Let

$$
c=\frac{W}{4 V}, q=\frac{V}{2 c^{2}} \text { and } a=M-q c .
$$

Then the approximation is given by percent points of the Chi-square distribution with $q$ degrees of freedom:

$$
\begin{array}{cc}
\Lambda_{d f, \pi}^{\prime}(t) \approx a+c \chi_{q, \pi}^{2} & \text { if } c>0, \\
\Lambda_{d f, \pi}^{\prime}(t) \approx a+c \chi_{q, 1-\pi}^{2} & \text { if } c<0 .
\end{array}
$$


If $t>0$ (which is equivalent to $c>0$ ), we get the approximate $100(1-\alpha) \%$ confidence interval for $\lambda:\left[a+c \chi_{q, \alpha / 2}^{2}, a+c \chi_{q, 1-\alpha / 2}^{2}\right]$. If $t<0$, the limits are exchanged.

In practice $q$ is generally very large and the Wilson and Hilferty (1931) approximation can be used (this is needed if your computer program does not work for high degrees of freedom values):

$$
\chi_{q, \pi}^{2} \approx q\left(\sqrt{\frac{2}{9 q}} z_{\pi}+1-\frac{2}{9 q}\right)^{3}
$$

Some programs for the Chi-square distribution accepts only integer degrees of freedom. In this case, the $100 \pi$ percent point of the Gamma distribution with parameter $q / 2$ can be used alternatively:

$$
\chi_{q, \pi}^{2}=2 \operatorname{Gamma}_{\pi}(q / 2) .
$$

In Bird's example, it is found that $W=-0.0041$, $c=-0.0009016, \quad q=702948.01 \quad$ and $a=628.7998$, hence the approximate confidence interval (computations have been performed with the maximum number of decimals for intermediate values): $[628.7998-0.0009016 \times$
$705608.34,628.7998-0.0009016 \times 700293.06]$

$\rightarrow[-7.3766,-2.5844]$, where $\chi_{702948.01,0.0125}^{2}=$ 700293.06 and $\chi_{702948.01,0.09875}^{2}=705608.34$ are computed using the Wilson-Hilferty approximation. This interval coincides with the exact interval with four decimal place accuracy.

A Comparison of the Three Methods

Table 3 gives a more systematic comparison of the three approximation methods. The exact probability levels associated with the different approximations of the $100 \pi$ percent point of the $\Lambda_{d f}^{\prime}(t)$ distribution are reported for $100 \pi=2.5$ and $100 \pi=97.5$ (which gives the limits of the $95 \%$ confidence interval), and for $100 \pi=0.5$ and $100 \pi=99.5$ (which gives the limits of the $99 \%$ confidence interval). In the two cases, results are given for 10 and 50 degrees of freedom. They are reported only for positive values of $t$; the results for negative values can be deduced by symmetry.

Bird's approximation is very inaccurate for small $d f$ or large $t$ and can hardly be recommended. By contrast, the simple Normal approximation works very well. The Chi-square approximation is quasi exact for most practical applications. 
Table 3. Exact probability levels associated with the three approximations of the $100 \pi$ percent point of the $\Lambda_{d f}^{\prime}(t)$ distribution

$2.50 \%$ and $97.50 \%$ percent points

\begin{tabular}{|c|c|c|c|c|c|c|c|c|c|c|c|c|}
\hline \multirow[b]{3}{*}{$t$} & \multirow{2}{*}{\multicolumn{4}{|c|}{ Bird's approximation }} & \multirow{2}{*}{\multicolumn{4}{|c|}{$\begin{array}{c}\text { Normal approximation } \\
d f=10 \quad d f=50\end{array}$}} & \multicolumn{4}{|c|}{ Chi square approximation } \\
\hline & & & & & & & & & & $=10$ & & \\
\hline & 2.50 & 97.50 & 2.50 & 97.50 & 2.50 & 97.50 & 2.50 & 97.50 & 2.50 & 97.50 & 2.50 & 97.50 \\
\hline 0 & 1.29 & 98.71 & 2.23 & 97.77 & 2.50 & 97.50 & 2.50 & 97.50 & $2.50^{*}$ & $97.50^{*}$ & $2.50^{*}$ & $97.50^{*}$ \\
\hline 0.25 & 1.33 & 98.72 & 2.24 & 97.77 & 2.50 & 97.50 & 2.50 & 97.50 & $2.50^{*}$ & $97.50^{*}$ & $2.50^{*}$ & $97.50^{*}$ \\
\hline 0.50 & 1.38 & 98.70 & 2.26 & 97.77 & 2.50 & 97.50 & 2.50 & 97.50 & $2.50^{*}$ & $97.50^{*}$ & $2.50^{*}$ & $97.50^{*}$ \\
\hline 0.75 & 1.46 & 98.66 & 2.28 & 97.76 & 2.50 & 97.50 & 2.50 & 97.50 & 2.50 & 97.50 & $2.50^{*}$ & $97.50^{*}$ \\
\hline 1.00 & 1.57 & 98.60 & 2.31 & 97.74 & 2.49 & 97.49 & 2.50 & 97.50 & 2.50 & 97.50 & $2.50^{*}$ & $97.50^{*}$ \\
\hline 1.50 & 1.86 & 98.41 & 2.39 & 97.69 & 2.48 & 97.48 & 2.50 & 97.50 & 2.50 & 97.50 & $2.50^{*}$ & $97.50^{*}$ \\
\hline 2.00 & 2.27 & 98.10 & 2.50 & 97.61 & 2.46 & 97.46 & 2.50 & 97.50 & 2.50 & 97.50 & $2.50^{*}$ & $97.50^{*}$ \\
\hline 2.50 & 2.81 & 97.68 & 2.63 & 97.50 & 2.42 & 97.43 & 2.50 & 97.50 & .50 & 97.50 & 2.50 & 97.50 \\
\hline 3.00 & 3.50 & 97.14 & 2.80 & 97. & 2.39 & 97.39 & 2.49 & 97.49 & 2.50 & 97.50 & 2.50 & 97.50 \\
\hline 4.00 & 5.31 & 95.75 & 3.22 & 97. & 2.30 & 97.31 & 2.49 & 97.49 & 2.50 & 97.50 & 2.50 & 97.50 \\
\hline 5.00 & 7.63 & 94.01 & 3.77 & 96.53 & 2.22 & 97.24 & 2.47 & 97.48 & 2.49 & 97.50 & 2.50 & 97.50 \\
\hline 10.00 & 21.04 & 84.57 & 8.19 & 92.66 & 1.95 & 97.04 & 2.40 & 97.40 & 2.47 & 97.51 & 2.50 & 97.50 \\
\hline 15.00 & 30.54 & 77.72 & 14.10 & 87.58 & 1.84 & 96.97 & 2.33 & 97.34 & 2.46 & 97.52 & 2.50 & 97.50 \\
\hline 20.00 & 36.36 & 73.27 & 19.72 & 82.78 & 1.80 & 96.94 & 2.29 & 97.31 & 2.45 & 97.52 & 2.50 & 97.50 \\
\hline 25.00 & 40.12 & 70.25 & 24.39 & 78.77 & 1.78 & 96.93 & 2.27 & 97.28 & 2.45 & 97.52 & 2.50 & 97.50 \\
\hline
\end{tabular}

Note. ${ }^{*}$ Normal approximation (exact for $t=0$ )

$0.50 \%$ and $99.50 \%$ percent points

\begin{tabular}{|c|c|c|c|c|c|c|c|c|c|c|c|c|}
\hline \multirow[b]{3}{*}{$t$} & \multirow{2}{*}{\multicolumn{4}{|c|}{$\begin{array}{l}\text { Bird's approximation } \\
d f=10 \quad d f=50\end{array}$}} & \multirow{2}{*}{\multicolumn{4}{|c|}{$\begin{array}{l}\text { Normal approximation } \\
d f=10 \quad d f=50\end{array}$}} & \multirow{2}{*}{\multicolumn{4}{|c|}{$\begin{array}{c}\text { Chi square approximation } \\
d f=10 \quad d f=50\end{array}$}} \\
\hline & & & & & & & & & & & & \\
\hline & 0.50 & 99.50 & 0.50 & 99.50 & 0.50 & 99.50 & 0.50 & 99.50 & 0.50 & 99.50 & 0.50 & 99.50 \\
\hline 0 & 0.08 & 99.92 & 0.37 & $\begin{array}{l}99.63 \\
\end{array}$ & $\overline{0.50}$ & 99.50 & 0.50 & 99.50 & $0.50^{\prime}$ & $99.50^{*}$ & 0.50 & $99.50^{*}$ \\
\hline 0.25 & 0.08 & 99.92 & 0.37 & 99.63 & 0.50 & 99.50 & 0.50 & 99.50 & $0.50^{\prime}$ & $99.50^{*}$ & $0.50^{\circ}$ & $99.50^{*}$ \\
\hline 0.50 & 0.08 & 99.92 & 0.38 & 99.63 & 0.50 & 99.50 & 0.50 & 99.50 & $0.50^{*}$ & $99.50^{*}$ & 0.50 & $99.50^{*}$ \\
\hline 0.75 & 0.09 & 99.92 & 0.38 & 99.63 & 0.50 & 99.50 & 0.50 & 99.50 & 0.50 & 99.50 & 0.50 & $99.50^{*}$ \\
\hline 1.00 & 0.11 & 99.91 & 0.39 & 99.62 & 0.50 & 99.50 & 0.50 & 99.50 & 0.50 & 99.50 & 0.50 & $99.50^{*}$ \\
\hline 1.50 & 0.14 & 99.88 & 0.41 & 99.60 & 0.49 & 99.49 & 0.50 & 99.50 & 0.50 & 99.50 & 0.50 & $99.50^{*}$ \\
\hline 2.00 & 0.20 & 99.83 & 0.44 & 99.58 & 0.48 & 99.48 & 0.50 & 99.50 & 0.50 & 99.50 & 0.50 & $99.50^{*}$ \\
\hline 2.50 & 0.30 & 99.74 & 0.48 & 99.55 & 0.46 & 99.46 & 0.50 & 99.50 & 0.50 & 99.50 & 0.50 & 99.50 \\
\hline 3.00 & 0.44 & 99.61 & 0.53 & 99.50 & 0.45 & 99.45 & 0.50 & 99.50 & 0.50 & 99.50 & 0.50 & 99.50 \\
\hline 4.00 & 0.92 & 99.17 & 0.67 & 99.38 & 0.41 & 99.41 & 0.49 & 99.49 & 0.50 & 99.50 & 0.50 & 99.50 \\
\hline 5.00 & 1.77 & 98.44 & 0.86 & 99.21 & 0.37 & 99.37 & 0.49 & 99.49 & 0.49 & 99.51 & 0.50 & 99.50 \\
\hline 10.00 & 11.13 & 91.64 & 3.03 & 97.23 & 0.25 & 99.27 & 0.45 & 99.45 & 0.47 & 99.52 & 0.50 & 99.50 \\
\hline 15.00 & 21.38 & 84.59 & 7.25 & 93.53 & 0.21 & 99.23 & 0.42 & 99.42 & 0.45 & 99.53 & 0.50 & 99.50 \\
\hline 20.00 & 28.67 & 79.33 & 12.32 & 89.21 & 0.19 & 99.22 & 0.40 & 99.40 & 0.44 & 99.53 & 0.49 & 99.50 \\
\hline 25.00 & 33.67 & 75.54 & 17.15 & 85.12 & 0.18 & 99.21 & 0.39 & 99.39 & 0.44 & 99.53 & 0.49 & 99.51 \\
\hline
\end{tabular}

Note. ${ }^{*}$ Normal approximation (exact for $t=0$ ) 


\section{Conclusion}

Returning to the analogy between the statistic $\bar{Y}_{1}-\bar{Y}_{2}-\mathrm{t}_{\mathrm{CALC}} \mathrm{E}$ and the familiar limits $\bar{Y}_{1}-\bar{Y}_{2} \pm \mathrm{t}_{1-\alpha / 2} \mathrm{E}$ of the $100(1-\alpha) \%$ confidence interval for a raw difference, it can be tempting to consider the interval $\bar{Y}_{1}-\bar{Y}_{2} \pm \mathrm{t}_{\mathrm{CALC}} \mathrm{E}$. Assume for instance that $t_{\text {CALC }}$ is positive, then one can remark that $t_{C A L C}$ is the $100(1-p / 2)$ percent point $t_{1-p / 2}$ of the $t$ distribution, where $p$ is the two-sided p-value of the usual $t$ test. Thus, the analogy seems again more compelling.

By the definition of $t_{\mathrm{CALC}}$, for the data in hand the bounds of this interval are zero, the traditional null hypothesis value, and two times the observed difference, what Rosnow and Rosenthal (1996) called the counter-null value. In their methodological article, Rosnow and Rosenthal (page 336) considered such an interval. Taking the example of an observed difference between two means +0.266 and a $\mathrm{p}$-value $\mathrm{p}=0.23$, they interpreted the specific null counter-null interval $[0,+0.532]$ as a $77 \%$ confidence interval, that is as a $100(1-p) \%$ confidence interval. This interpretation reveals a typical confusion between Frequentist and Bayesian probabilities.

In the Frequentist conception of confidence intervals, the confidence level is the proportion of repeated intervals that contain the (fixed) parameter; it is usually termed the coverage probability. The procedure, and in particular the confidence level, must be determined before knowing the data. In the case of the Rosnow and Rosenthal interval $[0,+0.532]$, two possibilities can be envisaged to define the procedure and thus to compute the coverage probability.

Nevertheless, the procedure can proceed by computing the interval $\bar{Y}_{1}-\bar{Y}_{2} \pm \mathrm{t}_{\mathrm{CALC}} \mathrm{E}$, with the data dependent value $t_{C A L C}$. For each repeated sample the bounds of this interval are zero and the particular counter-null value for this sample. Of course, the coverage probability of this interval varies with the parameters and it is not equal to 0.77 (except for two particular values of the ratio $\left(\mu_{1}-\mu_{2}\right) / \varepsilon$ symmetrical around zero).
The procedure can also proceed by computing the interval $\bar{Y}_{1}-\bar{Y}_{2} \pm \mathrm{t}_{1-\mathrm{p} / 2} \mathrm{E}$, with the fixed value $p=0.23$ for each repeated sample. The coverage probability of this interval is 0.77 . However, this is not a Frequentist approach, because 0.77 has been determined by the data in hand. Clearly, 0.77 is a data dependent probability, which needs a Bayesian approach to be correctly interpreted. The Bayesian inference associates to the interval $[0$, $+0.532]$ the posterior probability that this interval contains the parameter, given the data.

Although confidence intervals refer to a Frequentist justification, they are often (mis)interpreted in Bayesian terms. The distinction between the Frequentist coverage probability and the Bayesian posterior probability is all the more subtle in the present situation that it turns out that it is correct from a Bayesian viewpoint to say that there is a $77 \%$ chance that the interval $[0,+0.532]$ contains $\left(\mu_{1}-\mu_{2}\right) / \varepsilon$, or again in the example in Figure 1 to say that there is a $95 \%$ chance that the interval $[-0.986,+2.979]$ contains the noncentrality parameter $\lambda$. This simply assumes a prior distribution that does not favor any particular value of the parameters, what Bayesian called a non-informative prior.

This distribution is revised by the data and the corresponding posterior distribution for the noncentrality parameter $\lambda$ is just the $\Lambda_{\mathrm{df}}^{\prime}\left(\mathrm{t}_{\mathrm{CALC}}\right)$ distribution. Consequently, the Lambda-prime distribution, in addition to its status of sampling distribution gains the status of a probability distribution that expresses the uncertainty about the unknown parameter $\lambda$. One can use the confidence interval for $\lambda$ with the benefits of both the Frequentist and Bayesian interpretations and without worrying about the correct justification (not to speak of Fisher's fiducial argument).

The fact that even experts in statistics are not immune to conceptual confusions and interpret Frequentist intervals in Bayesian terms should not be regarded as an error. Rather this means that, as most statistical inference users, they are also, perhaps without knowing it, interested in the Bayesian probability. This should invite us not to radicalize the opposition between the Bayesian and Frequentist inferences 
but rather to consider their interplay. This is a difficult challenge, but it is already well advanced in the statistical literature (see Bayarri \& Berger, 2004).

\section{References}

Algina, J., \& Keselman, H.J. (2003). Approximate confidence intervals for effect sizes. Educational and Psychological Measurement, 63, 537-553.

Bayarri, M.J., \& Berger, J. O. (2004). The interplay of Bayesian and frequentist analysis. Statistical Science, 19, 58-80

Bird, K. D. (2002). Confidence intervals for effect sizes in analysis of variance. Educational and Psychological Measurement, 62, 197-226.

Fidler, F., \& Thompson, B. (2001). Computing correct confidence intervals for ANOVA fixed and random-effects effect sizes. Educational and Psychological Measurement, 61, 575-605.

Fisher, R. A. (1990/1973). Statistical methods and scientific inference (3rd edition 1973 reprinted). Oxford: Oxford University Press.
Johnson, N. L., \& Welch, B. L. (1939). On the calculation of the cumulants of the $\chi$ distribution. Biometrika, 31, 216-218.

Lecoutre, B. (1999). Two useful distributions for Bayesian predictive procedures under normal models. Journal of Statistical Planning and Inference, 77, 93-105.

Rosnow, R.L., \& Rosenthal, R. (1996). Computing contrasts, effect sizes, and counternulls on other people's published data: General procedures for research consumers. Psychological Methods, 1, 331-340.

Steiger, J. H. (2004). Beyond the F-test: Effect size confidence intervals and tests of close fit in the analysis of variance and contrast analysis. Psychological Methods, 9, 164-182.

Steiger, J. H., \& Fouladi, R. T. (1997). Noncentrality interval estimation and the evaluation of statistical models. In L. L. Harlow, S. A. Mulaik, \& J.H. Steiger (Eds.), What if there were no significance tests? (pp. 221-257). Mahwah, NJ: Lawrence Erlbaum.

Wilson, E. B., \& Hilferty, M. M. (1931). The distribution of chi-square. Proceedings of the National Academy of Sciences of the United States of America, 17, 684-688. 


\section{Tests for Treatment Group Equality When Data are Nonnormal and Heteroscedastic}

\author{
Robert A. Cribbie \\ York University \\ Carmen Bewell \\ York University
}

\author{
Rand R. Wilcox \\ University of Southern California \\ H. J. Keselman \\ University of Manitoba
}

Several tests for group mean equality have been suggested for analyzing nonnormal and heteroscedastic data. A Monte Carlo study compared the Welch tests on ranked data and heterogeneous, nonparametric statistics with previously recommended procedures. Type I error rates for the Welch tests on ranks and the heterogeneous, nonparametric statistics were well controlled with a slight power advantage for the Welch tests on ranks.

Key words: Welch test on ranks, nonparametric statistics, nonnormality, heteroscedasticity, mean equality

\section{Introduction}

Researchers in the behavioral sciences are often interested in comparing the typical performance of subjects across independent groups, and they often select traditional test statistics (e.g., twosample t, ANOVA F) without regard for their underlying assumptions, even though it has been pointed out that these assumptions may frequently be violated (e.g., Micceri, 1989; Keselman et al., 1998; Wilcox, 1988). Many authors have highlighted available procedures for analyzing data that violate either the assumption of normality or the assumption of variance homogeneity. Brown and Forsythe (1974), Kohr and Games (1974), and many

Robert A. Cribbie is Associate Professor of Psychology, Department of Psychology, Toronto, Ontario, M3J 1P3 (cribbie@yorku.ca), specializing in robust test statistics and multiplicity control. Rand. R. Wilcox is Professor of Psychology, Department of Psychology, (rwilcox@usc.edu). Carmen Bewell (cbewell@yorku.ca) is a Ph. D. student in Clinical Psychology. H. J. Keselman, Department of Psychology, is Professor of Psychology (hj_keselman@umanitoba.ca). others demonstrated the general effectiveness (i.e., Type I error control) of Welch's (1938, 1951) two-sample and omnibus test statistics with heterogeneous variances. In addition, Keselman, Cribbie and Zumbo (1997), Wilcox (1995; 1997), Yuen and Dixon (1973), and Zimmerman and Zumbo (1993a), among many others, have demonstrated the effectiveness of several alternatives to traditional parametric tests that can be used with nonnormal data, including nonparametric test statistics and tests with robust estimators (e.g., trimmed means).

However, there has been little success in discovering a test that is robust (with respect to Type I and Type II errors) to the simultaneous violations of both assumptions. That is, although procedures have been proposed for analyzing data that violate both the normality and variance heterogeneity assumptions concurrently (described below), there has not been a thorough investigation and comparison of the Type I error and power properties of these procedures. Therefore, the current article compares potential strategies for analyzing nonnormal and heteroscedastic data, with the goal of being able to recommend a procedure that provides a good balance between Type I error control and power.

One possibility for analyzing nonnormal and heteroscedastic data is to utilize the Welch two-sample and omnibus tests, which have been found to provide excellent Type I error control 
and power for some patterns of nonnormality (with unequal variances); however, for other patterns the Type I error rates can deviate considerably from the nominal rate (e.g., Cressie \& Whitford, 1986; Keselman, Lix \& Kowalchuk, 1998). Another potential solution when variances are heterogeneous and distribution shapes are nonnormal is to use a heteroscedastic statistic, such as Welch's (1938, 1951) tests, with sample estimators that are intended to be robust to the biasing effects of nonnormality, e.g., trimmed means and Winsorized variances (see Yuen \& Dixon, 1973; Wilcox, 1995, 1997). By minimizing the effects of extreme observations the trimmed mean can provide a more accurate representation of the central tendency of the majority of the distribution. An increase in power may also be experienced if eliminating the extreme observations reduces the standard error of the mean. However, Keselman, Lix, et al. (1998) reported that under some patterns of nonnormality power could be depressed relative to utilizing the usual means and variances.

Nonparametric test statistics (e.g., Wilcoxon, Mann-Whitney, Kruskal-Wallis) have been studied for unequal variances and nonnormal data. Zimmerman $(1987 ; 1996)$ and Zimmerman and Zumbo (1993a), among others, showed that nonparametric test statistics are not robust to unequal variances, regardless of whether the data are normal or nonnormal.

Zimmerman and Zumbo (1993a) explained that, "an attractive hypothesis is that both problems [nonnormality and variance heterogeneity] can be solved at once by the Welch $t$ test performed on the ranks of measures instead of the measures themselves" (p. 507). Thus, with this approach, researchers would convert nonnormal, heteroscedastic data to ranks, and analyze the data with the Welch twosample or omnibus tests. Zimmerman and Zumbo (1993a; 1993b) conducted simulation studies with several patterns of nonnormality and variance heterogeneity and report that the Welch test on ranks "counteracts effects of nonnormality and unequal variances at the same time" (p. 535). More specifically, for many patterns of nonnormality and variance homogeneity, the Welch test on ranks provided better overall Type I and Type II error control relative to the two-sample $t$ and Welch $t$ on unranked data or the two-sample $t$ on ranks. However, it should be noted that for some patterns of nonnormality (e.g., lognormal) Type I error rates were not controlled within Bradley's (1978) liberal criterion (+/-.5 $\alpha)$.

Another potential solution is the heteroscedastic rank-based test statistics proposed by Brunner and Munzel (2000) and Brunner, Dette and Munk (1997). Specifically these authors presented two-sample and omnibus, respectively, heteroscedastic rankbased test statistics that, unlike the traditional Kruskal-Wallis nonparametric statistic, consider the variance heterogeneity of the group distributions in the computational procedure. Munzel and Hothorn (2001) presented findings on the Type I error and power properties of the Brunner and Munzel two-sample procedure for nonnnormal distributions with unequal variances, indicating that Type I error and power rates were considerably better than those of the parametric and nonparametric competitors. However, results were only reported for a manyto-one multiple comparisons setting for the discretized normal distribution.

The purpose of this article is to compare the Type I error control and power of the above strategies under several conditions of nonnormality and/or heteroscedasticity. It extends the conditions investigated by Zimmerman and Zumbo (1993a; 1993b) and Munzel and Hothorn (2001) to independent groups designs with more than two levels of the independent variable and, with respect to nonnormality, investigates skewed distributions not previously investigated and that have been reported to be representative of many behavioral science variables (Micceri, 1989; Wilcox, 1995). The Type I error control and power of the procedures in a multiple comparisons setting is also examined.

\section{Test Statistics}

Five omnibus test statistic and data configuration combinations were evaluated and compared in this study. These included: a) Welch's (1951) test statistic on unranked data (Welch); b) Welch's test statistic on trimmed means and Winsorized variances (20\% symmetric trimming) (Welch-t); c) Welch's test 
statistic on ranked data (Welch-r); d) the Kruskal-Wallis (Kruskal \& Wallis, 1952) omnibus nonparametric test statistic (which utilizes ranked data) (KW); and e) the Brunner, Dette and Munk (1997) heterogeneous nonparametric test statistic (BDM).

Welch

Welch's (1938) two-sample test statistic can be expressed as:

$$
t_{w}=\frac{\overline{X_{j}}-\overline{X_{j^{\prime}}}}{\sqrt{\frac{s_{j}^{2}}{n_{j}}+\frac{s_{j^{\prime}}^{2}}{n_{j^{\prime}}}}}
$$

which is distributed as a t variable with degrees of freedom due to Satterthwaite (1946),

$$
v_{w}=\frac{\left(\frac{s_{j}^{2}}{n_{j}}+\frac{s_{j^{\prime}}^{2}}{n_{j^{\prime}}}\right)^{2}}{\left[\frac{s_{j}^{4}}{n_{j}^{2}\left(n_{j}-1\right)}+\frac{s_{j^{\prime}}^{4}}{n_{j^{\prime}}^{2}\left(n_{j^{\prime}}-1\right)}\right]},
$$

where $\bar{X}_{\mathrm{j}}, \mathrm{s}_{\mathrm{j}}^{2}$ and $\mathrm{n}_{\mathrm{j}}$ represent the sample means, variances, and sample sizes, respectively, for the jth group $\left(j \neq j^{\prime}, j=1, \ldots, J\right)$.

Welch's (1951) omnibus test can be expressed as:

$$
\begin{aligned}
& \sum_{j} w_{j}\left(\overline{X_{j}}-\overline{X_{j}^{*}}\right)^{2} \\
& F_{w}=\frac{J-1}{1+\left(\frac{2(J-2)}{J^{2}-1}\right)\left(\frac{\sum_{j}\left(1-w_{j} / \sum_{j} w_{j}^{2}\right)^{2}}{n_{j}-1}\right)},
\end{aligned}
$$

which is distributed as an F variable with $\mathrm{J}-1$ and $v_{\mathrm{w}}$ degrees of freedom, where

$$
w_{j}=\frac{n_{j}}{s_{j}^{2}},
$$

$$
\overline{X_{j}^{*}}=\frac{\sum_{j} w_{j} \overline{X_{j}}}{\sum_{j} w_{j}},
$$

and

$$
v_{w}=\frac{J^{2}-1}{3 \sum_{j} \frac{\left(1-w_{j} / \sum_{j} w_{j}\right)^{2}}{n_{j}-1}} .
$$

\section{Kruskal-Wallis}

The Kruskal-Wallis nonparametric procedure begins by ranking the observations in the combined sample. Let the rank of the ith observation in the jth group be represented by $r_{i j}$ and the sum of the ranks for the jth group be represented by $a_{j}=\Sigma_{i} r_{i j}$. The statistic tests the null hypothesis $\mathrm{H}_{\mathrm{o}}: \lambda_{1}=\ldots=\lambda_{\mathrm{J}}$ (where $\lambda$ represents the population mean only under the assumption that the population shapes are identical) and rejects $\mathrm{H}_{\mathrm{o}}$ if $\mathrm{KW} \geq \chi_{(\mathrm{J}-1)}^{2}$ where: 


$$
K W=12\left(\frac{\sum_{j} a_{j}^{2} / n_{j}}{N(N+1)}\right)-3(N+3)
$$

and $\mathrm{N}=\sum_{\mathrm{j}} \mathrm{n}_{\mathrm{j}}$. Multiple comparisons are performed with a modified two-sample version of the omnibus Kruskal-Wallis test (see Sprent \& Smeeton, 1993). The null hypothesis $H_{0}: \lambda_{j}=$ $\lambda_{j}$, is rejected if $\left|t_{K W}\right| \geq t_{\alpha, N-J}$, where:

$$
\begin{gathered}
t_{K W}=\frac{\bar{X}_{1}-\bar{X}_{2}}{\sqrt{\frac{\left(S_{r}-C\right)(N-1-K W)\left(n_{j}+n_{j^{\prime}}\right)}{n_{j} n_{j^{\prime}}(N-J)(N-1)}}}, \\
S_{r}=\sum_{i} \sum_{j} r_{i j}^{2}
\end{gathered}
$$

and

$$
C=\frac{N(N+1)^{2}}{4}
$$

Welch- $\mathrm{t}$

Trimmed means are computed by removing a percentage of observations from each of the tails of a distribution. Let $g_{j}=\left[\gamma n_{j}\right]$, where $\gamma$ represents the proportion of observations to be trimmed from each tail of the distribution and $[\mathrm{x}]$ is the largest integer less than or equal to $x$. Further, let $h_{j}$ represent the remaining (effective) sample size following removal of the trimmed observations. Recommendations have been made in the literature for $15 \%$ symmetric trimming (Mudholkar, Mudholkar \& Srivastava, 1991) and $20 \%$ symmetric trimming (Wilcox, 1995). The jth sample trimmed mean can be represented as:

$$
\overline{X_{t j}}=\frac{1}{h_{j}} \sum_{i=g_{j}+1}^{n_{j}-g_{j}} X_{i j}
$$

and the jth sample Winsorized mean as

$$
\overline{X_{w j}}=\frac{1}{n_{j}} \sum_{i=1}^{n_{j}} Y_{i j}
$$

where:

$$
\begin{aligned}
Y_{i j} & =X_{\left(g_{j}+1\right) j} \text { if } X_{i j} \leq X_{\left(g_{j}+1\right) j}, \\
& =X_{i j} \text { if } X_{\left(g_{j}+1\right) j}<X_{i j}<X_{\left(n_{j}-g_{j}\right) j}, \\
& =X_{\left(n_{j}-g_{j}\right) j} \text { if } X_{i j} \geq X_{\left(n_{j}-g_{j}\right) j} .
\end{aligned}
$$

An associated Winsorized variance is computed by replacing the censored observations from the lower tail with the lowest uncensored observation and the censored observations from the upper tail with the highest uncensored observation. The Winsorized variance is:

$$
s_{w j}^{2}=\frac{1}{h_{j}-1} \sum_{i=1}^{n_{j}}\left(Y_{i j}-\overline{X_{w j}}\right)^{2} .
$$

The sample trimmed means and Winsorized variances can then be substituted into Welch's (1938; 1951) two-sample and omnibus test statistics. For example, substituting the trimmed means and Winsorized variances into the Welch (1938) two-sample test yields the statistic:

$$
t_{w}=\frac{\overline{X_{t j}}-\overline{X_{t j^{\prime}}}}{\sqrt{\frac{s_{w j}^{2}}{h_{j}}+\frac{s_{w j^{\prime}}^{2}}{h_{j^{\prime}}}}}
$$


with error degrees of freedom,

$$
v_{w}=\frac{\left(\frac{s_{w j}^{2}}{h_{j}}+\frac{s_{w j^{\prime}}^{2}}{h_{j^{\prime}}}\right)^{2}}{\left[\frac{s_{w j}^{4}}{h_{j}^{2}\left(h_{j}-1\right)}+\frac{s_{w j^{\prime}}^{4}}{h_{j^{\prime}}^{2}\left(h_{\left.j^{\prime}-1\right)}\right.}\right]} .
$$

Welch-r.

The Welch test can be performed on the ranked data, where ranks are established regardless of group membership. The null hypothesis, $\mathrm{H}_{0}: \lambda_{1}=\ldots=\lambda_{\mathrm{J}}$, is rejected if $\mathrm{F}_{\mathrm{w}} \geq \mathrm{F}_{\alpha, \mathrm{J}-1, \mathrm{vw}}$.

\section{Brunner, Dette, and Munk}

Brunner, Dette and Munk (1997) proposed the following heterogeneous, rankbased F statistic:

$$
F_{B}=\frac{N}{\operatorname{tr}\left(M_{11} V\right)} Q M Q^{\prime}
$$

where

$$
M=\underline{I}-\frac{1}{J} \underline{J}, \underline{I}=\text { identity matrix of rank } J,
$$

$\underline{J}=J$ by $J$ matrix of $1 s$,

$$
V=N * \operatorname{diag}\left(\frac{s_{1}^{2}}{n_{1}}, \ldots, \frac{s_{J}^{2}}{n_{J}}\right)
$$$$
s_{j}^{2}=\frac{1}{N^{2}\left(n_{j}-1\right)} \sum_{j}\left(R_{i j}-\overline{R_{j}}\right)^{2}
$$$$
Q=\frac{1}{N}\left(\overline{R_{1}}-\frac{1}{2}, \ldots, \overline{R_{j}}-\frac{1}{2}\right) \text {, }
$$

$$
\overline{R_{j}}=\frac{1}{n_{j}} \sum_{i=1}^{n_{j}} R_{i j}
$$

and $R_{i j}$ is the rank of $\mathrm{X}_{\mathrm{ij}}$ after the data are pooled. The null hypothesis, $\mathrm{H}_{0}: \lambda_{1}=\ldots=\lambda_{\mathrm{J}}$ is rejected if $\mathrm{F}_{\mathrm{B}} \geq \mathrm{F}_{\alpha, v 1, v 2}$ where:

$$
\begin{gathered}
v_{1}=\frac{M_{11}[\operatorname{tr}(V)]^{2}}{\operatorname{tr}(M V M V)} \\
v_{2}=\frac{[\operatorname{tr}(V)]^{2}}{\operatorname{tr}\left(V^{2} \Lambda\right)}
\end{gathered}
$$

and $\Lambda=\operatorname{diag}\left\{\left(\mathrm{n}_{1}-1\right)^{-1}, \ldots,\left(\mathrm{n}_{\mathrm{J}}-1\right)^{-1}\right\}$. Multiple comparisons are performed with the two-sample version of the Brunner, Dette and Munk (1997) procedure (see Brunner \& Munzel, 2000).

It is important to note that the null hypotheses associated with the above tests differ based on the characteristic(s) of the data that each test is sensitive to. The Welch test evaluates the null hypothesis that all population means are equal (i.e., $\mathrm{H}_{0}: \mu_{1}=\ldots=\mu_{\mathrm{J}}$ ). The Welch-t evaluates the null hypothesis that all population trimmed means are equal (i.e., $\mathrm{H}_{\mathrm{o}}: \mu_{\mathrm{t} 1}$ $\left.=\ldots=\mu_{\mathrm{tJ}}\right)$. The $\mathrm{K}-\mathrm{W}$, Welch-r and BDM procedures evaluate the null hypothesis that all distribution functions are equal (i.e., $\mathrm{H}_{\mathrm{o}}: \lambda_{1}=\ldots$ $\left.=\lambda_{\mathrm{J}}\right)$. It is important to note that with the $\mathrm{K}-\mathrm{W}$, Welch-r and BDM procedures that the null hypotheses only relate to a test of location when population distribution shapes and variances are equal, where the procedures are sensitive to differences in the mean ranks (see Brunner, Dette \& Munk, 1997, p. 1498; Kruskal \& Wallis, 1952; Sprent \& Smeeton, 2001). Hence, an important component of this article is to evaluate the rates of rejection for these procedures when variances are unequal.

Pairwise Multiple Comparison Procedures (MCPs)

Tukey

The Tukey (1953) procedure rejects $\mathrm{H}_{0}$ : $\mu_{j}=\mu_{j},\left(j \neq j^{\prime}\right)$ if $|t| \geq q(\alpha, J, v) /(2)^{1 / 2}$, where $q$ is a value from the Studentized range distribution with $\mathrm{J}$ groups and $v$ degrees of 
freedom, and $t$ and $v$ represents the appropriate two-sample t-distributed test statistic and associated degrees of freedom, respectively.

\section{REGWQ}

Ryan (1960) proposed a modification to the Newman-Keuls (Newman, 1939; Keuls, 1952) procedure that ensures that the familywise (overall) Type I error rate is maintained at $\alpha$, even in the presence of partial null hypotheses. Ryan's original procedure became known as the REGWQ after modifications to the procedure proposed by Einot and Gabriel (1975) and Welsch (1977). The REGWQ MCP sequentially tests all ordered mean differences for stretch sizes (inclusive ranges between rank-ordered means) $\mathrm{p}=\mathrm{J}, \mathrm{J}-1, \ldots, 2$, and rejects $\mathrm{H}_{\mathrm{o}}: \mu_{\mathrm{j}}=\mu_{\mathrm{j}}$, $\left(j \neq j^{\prime}\right)$ if an associated omnibus test has been rejected and:

$$
|t| \geq q\left(\alpha_{p}, p, v\right) /(2)^{1 / 2},
$$

where $\alpha_{\mathrm{p}}=\alpha$ for $\mathrm{p}=\mathrm{J}, \mathrm{J}-1, \quad$ and $\alpha_{p}=1-(1-\alpha)^{p / J}$, for $p=J-2, \ldots, 2$. If any $H_{o} s$ are retained for $\mathrm{p}=\mathrm{p}^{\prime}$ then all $\mathrm{H}_{\mathrm{o}} \mathrm{s}$ contained in that stretch are retained and not tested at later stages (i.e., $\mathrm{p}<\mathrm{p}^{\prime}$ ). If all $\mathrm{H}_{\mathrm{o}} \mathrm{s}$ are retained for $\mathrm{p}=$ $\mathrm{p}^{\prime}$ then all $\mathrm{H}_{\mathrm{o}} \mathrm{s}$ with $\mathrm{p} \leq \mathrm{p}^{\prime}$ are retained.

\section{Methodology}

A Monte Carlo study was used to compare the Type I error and power rates of the Welch test on ranks and the Brunner heteroscedastic rankbased statistics with that of the Welch test on unranked data, the Welch test with trimmed means and Winsorized variances and the Kruskal-Wallis (Kruskal \& Wallis, 1952) nonparametric test in a one-way independent groups design. In addition, the procedures were compared in a pairwise multiple comparison framework, with the Tukey (1953) and REGWQ (Ryan, 1960; Einot \& Gabriel, 1975; Welsch, 1977) procedures.

Seven variables were manipulated in this study: a) number of levels of the independent variable; b) total sample size; c) degree of sample size imbalance; d) degree of variance inequality; e) pairings of group sizes and variances; f) configuration of population means; and g) population distribution shape.
The number of levels of the independent variable was set at $J=4$ and $J=7$, resulting in 6 and 21 pairwise comparisons, respectively. This permits evaluation of the effect of the number of pairwise comparisons computed on Type I error control and power.

In order to investigate the effects of sample size, the total sample size $(\mathrm{N})$ was manipulated by setting the average $n_{j}=10,15$, and 20 resulting in $\mathrm{N}=40,60$ and 80 for $\mathrm{J}=4$, and $\mathrm{N}=70,105$ and 140 for $\mathrm{J}=7$. The sample sizes were selected to be similar to those used by Zimmerman and Zumbo (1993a, b) in their investigations of the two-sample Welch (1938) test on ranked data. For the nonnull mean configurations used in this study, the group sizes 10,15 and 20 result in a priori omnibus (ANOVA $F$ statistic) power estimates of approximately .80, .95, and .98, respectively (assuming equal group sizes and variances).

Sample size balance or imbalance was also manipulated. Keselman et al. (1998) reported that unbalanced designs were more common than balanced designs in a review of studies published in educational and psychological journals. In addition, the effects of variance heterogeneity can be exacerbated when paired with unequal sample sizes. Therefore, three sample size conditions were examined (equal, moderately unequal and extremely unequal). The sample sizes used are enumerated in Table 1.

Degree of variance heterogeneity was also manipulated. According to Keselman et al. (1998), ratios of largest to smallest variances of 8:1 are not uncommon in educational and psychological studies and can have deleterious effects on the performance of many test statistics, especially when paired with unequal sample sizes. Therefore, three levels of variance equality/inequality were examined in this study: a) equal variances; b) largest to smallest variance ratio of $4: 1$; and c) largest to smallest variance ratio of $8: 1$. See Table 1 for group variances.

Pairings of variances and sample sizes can have differing effects on the Type I error and power rates of many test statistics. Specifically, when variances and sample sizes are directly (positively) paired Type I error estimates for the usual $t / F$ tests can be 
Table 1. Sample Sizes and Population Variances Used in the Simulation Study.

\begin{tabular}{lll}
\hline $\mathrm{J}$ & Sample Sizes & Population Variances \\
\hline 4 & $10,10,10,10$ & $1,1,1,1$ \\
& $9,10,10,11$ & $1,2,4,4$ \\
& $5,8,12,15$ & $1,3,5,8$ \\
& $15,15,15,15$ & \\
& $13,15,15,17$ & \\
$7,12,18,23$ & \\
& $20,20,20,20$ & \\
$17,20,20,23$ & $1,1,1,1,1,1,1$ \\
& $9,16,24,31$ & $1,1,2,2,3,3,4$ \\
& & \\
& $10,10,10,10,10,10,10$ & \\
$9,9,10,10,10,11,11$ & \\
$5,6,8,10,12,14,15$ & \\
$15,15,15,15,15,15,15$ & \\
$13,14,15,15,15,16,17$ & \\
$7,9,12,15,18,21,23$ & \\
& $20,20,20,20,20,20,20$ & \\
$17,18,20,20,20,22,24$ & \\
& $9,12,16,20,24,28,31$ &
\end{tabular}

conservative (with correspondingly deflated power). On the other hand, when variances and sample sizes are inversely (negatively) paired Type I error estimates for the usual $\mathrm{t} / \mathrm{F}$ tests can be liberal (with correspondingly inflated power). Therefore, both positive and negative pairings were examined.

Several configurations of nonnull population means were investigated, in addition to the complete null case. Following Toothaker's (1991) definitions of mean configuration, equally spaced, minimum variability and maximum variability configurations were utilized. See Table 2 for a listing of the mean configurations.

Another factor examined in this study was population distribution shape. The three distribution shapes investigated were: 1) normally distributed data; 2) moderately skewed data from the g- and h- distribution (Hoaglin, 1985), where $\mathrm{g}=.5$ and $\mathrm{h}=0$ (Skewness $=1.75$, Kurtosis $=8.90$ ); and 3) substantially skewed data from the $\mathrm{g}$ - and $\mathrm{h}$ - distribution, where $\mathrm{g}=1$ and $\mathrm{h}=0($ Skewness $=6.20$, Kurtosis $=114)$.

Empirical Type I error rates were recorded for all procedures, with familywise error rates reported for the MCPs. In this paper, the robustness of a procedure, with respect to Type I error control, will be determined using Bradley's (1978) liberal criterion. That is, a procedure is deemed robust with respect to Type I errors if the empirical rate of Type I error falls within the range $+/-.5 \alpha$. Power rates were also recorded for all the procedures, with power rates for the MCPs quantified with respect to average per-pair power (where per-pair power is the probability of rejecting a false pairwise null 
Table 2. Population Mean Configurations Used in the Simulation Study.

\section{Population Means}

$\begin{array}{lllllll}\mu_{1} & \mu_{2} & \mu_{3} & \mu_{4} & \mu_{5} & \mu_{6} & \mu_{7} \\ & & & & & & \\ \mathrm{~J}=4 & & & & & & \\ & & & & & & \\ 0.00 & 0.00 & 0.00 & 0.00 & & & \\ 0.00 & 0.00 & 0.00 & 1.28 & & & \\ 0.00 & 0.00 & 0.66 & 1.32 & & & \\ 0.00 & 0.00 & 1.09 & 1.09 & & & \\ 0.00 & 0.50 & 1.00 & 1.50 & & & \\ & & & & & & \\ \mathrm{~J}=7 & & & & & & \\ & & & & & & \\ 0.00 & 0.00 & 0.00 & 0.00 & 0.00 & 0.00 & 0.00 \\ 0.00 & 0.00 & 0.00 & 0.00 & 0.00 & 0.00 & 1.30 \\ 0.00 & 0.00 & 0.00 & 0.00 & 0.00 & 1.04 & 1.04 \\ 0.00 & 0.00 & 0.00 & 0.51 & 1.02 & 1.02 & 1.02 \\ 0.00 & 0.00 & 0.62 & 0.62 & 0.62 & 1.24 & 1.24 \\ 0.00 & 0.23 & 0.46 & 0.69 & 0.92 & 1.15 & 1.38\end{array}$

hypothesis) and all-pairs power (the probability of rejecting all false pairwise null hypothesis).

The simulation program was written in SAS/IML (SAS Institute, Inc., 1999). Pseudorandom normal variates were generated with the SAS generator RANNOR. If $Z_{i j}$ is a standard normal deviate, then $X_{\mathrm{ij}}=\mu_{\mathrm{j}}+\left(\sigma_{\mathrm{j}} Z_{\mathrm{ij}}\right)$ is a normal variate with mean $\mu_{j}$ and variance $\sigma_{j}^{2}$. To generate data from the g- and hdistributions, standard unit normal variables were converted to the random variable:

$$
X_{i j}=\left[\frac{\exp \left(g Z_{i j}\right)}{g}\right]\left[\exp \left(\frac{h Z_{i j}^{2}}{2}\right)\right] .
$$

To obtain a distribution with standard deviation $\sigma_{\mathrm{j}}$, each $\mathrm{X}_{\mathrm{ij}}$ was multiplied by a value of $\sigma_{\mathrm{j}}$. When $\mathrm{g}>0$ the $\mathrm{g}$ - and $\mathrm{h}$ - distribution population mean is not 0 and therefore the population mean was subtracted from $\mathrm{X}_{\mathrm{ij}}$ before being multiplied by $\sigma_{j}$. When working with trimmed means, the population trimmed mean for the jth group was also subtracted from the variate before multiplying by $\sigma_{j}$. In order to ensure that the null hypothesis associated with the rank-based procedures was true when distribution shapes were nonnormal and variances were unequal, the Nelder and Mead (1965) minimization function was implemented through an S-Plus version of the FORTRAN code in Olsson (1974. See also Olsson \& Nelson, 1975).

Distributions were shifted accordingly. Specifically, the S-Plus function 'nelder' was used, which is available in the library of R and S-Plus functions described in Wilcox (2005). Five thousand replications were performed for each condition, using a nominal significance level of .05. 


\section{Results}

The pattern of Type I error and power results were consistent across sample size inequality, variance inequality, and nonnull mean configurations, and were therefore averaged over these conditions. Further, the pattern of results was similar across sample size conditions and therefore only the results for the largest sample size condition are presented and discussed (except when noted otherwise). For the pairwise MCPs, partial null familywise error rates were controlled within Bradley's limits in all cases where complete null Type I error rates were controlled, and therefore are not reported.

Omnibus Tests

Type I error Control

Type $\mathrm{I}$ error rates ( $\%$ ) for $\mathrm{J}=4$ and $\mathrm{J}=7$ are presented in Table 3. When the distribution shapes were normal, Type I error rates were maintained within Bradley's liberal bounds $(2.5 \%-7.5 \%)$ by all, but one, procedure for $\mathrm{J}=4$ and $\mathrm{J}=7$; the Kruskal-Wallis procedure was liberal $(7.8 \%)$ for $J=4$ when sample sizes and variances were negatively paired. When the distribution shapes were skewed, the Welch and Kruskal-Wallis tests did not always maintain Type I error rates within Bradley's bounds when $\mathrm{J}=4$ and sample sizes and variances were negatively paired. The Welch test in particular became very liberal (e.g., 16.9\%), whereas the Kruskal-Wallis test exhibited some inflation (e.g., 7.9\%). The remaining procedures were able to maintain Type I error rates within Bradley's bounds under all conditions.

\section{Power}

Power rates $(\%)$ for $\mathrm{J}=4$ and $\mathrm{J}=7$ are presented in Table 4 . When the variances were equal there was very little difference between the procedures, with the exception that the Welch test had reduced power for the $g=1, h=0$ distribution. In general, the power for the Welch test on ranks, the Brunner heteroscedastic nonparametric procedure and the Kruskal-Wallis procedure was slightly larger than that for either of the other Welch statistics. With unequal variances, the usual Welch test and the Welch test with trimmed means had deflated power relative to the remaining procedures for both nonnormal distributions, although the Brunner heteroscedastic nonparametric procedure had especially low power with negatively paired sample sizes and variances, particularly for $\mathrm{J}=$ 7. There was very little difference between the power rates of the Kruskal-Wallis and the Welch test on ranks. Caution, however, should be taken in interpreting the power rates of the Welch and Kruskal-Wallis procedures with negatively paired sample sizes and variances given that the Type I error rates were not adequately controlled in some of these conditions.

Pairwise MCPs

The pattern of familywise error and average per-pair power results for the MCPs were consistent across $\mathrm{J}=4$ and $\mathrm{J}=7$ and therefore only results for $\mathrm{J}=7$ are displayed and discussed. The all-pairs power rates for $\mathrm{J}=4$ are displayed and discussed. (The $\mathrm{J}=7$ rates were too low for meaningful comparisons.)

\section{Type I error Control}

Complete null familywise error rates (\%) for the REGWQ and Tukey pairwise MCPs are presented in Table 5. The REGWQ procedure maintained rates within Bradley's bounds under all conditions, with the exception that the procedure became conservative (i.e., empirical familywise error rates less than $2.5 \%$ ) when it was used with either the Welch test or the Welch test on trimmed means and the data were $\mathrm{g}=1, \mathrm{~h}=0$ distributed. The Tukey procedure maintained rates within Bradley's limits when applied with Welch's statistic on trimmed means, the Welch on ranks, or the BrunnerMunzel heteroscedastic statistic, although the Type I error rates became liberal when the Tukey procedure was applied with the usual Welch test or the Kruskal-Wallis test when sample sizes and variances were negatively paired.

\section{Power}

Average per-pair and all-pairs power rates for the REGWQ and Tukey pairwise MCPs are presented in Tables 6 and 7, respectively. Power rates overall were very low given the strict familywise error control and the inflated variances in the heteroscedastic conditions. There was very little difference in the overall 
pattern of results for the Tukey and REGWQ procedures so given that the power was generally slightly larger for the REGWQ procedure (especially all-pairs power) only its' results will be discussed. When the variances were equal, there was very little difference between the procedures in terms of per-pair or all-pairs power across all distributions, although the REGWQ procedure when applied with the
Kruskal-Wallis statistic was generally the most powerful. When variances were unequal, the Welch test with trimmed means had less power than the Welch test on ranks or the BrunnerMunzel procedure across all distributions, with a slight advantage going to the Welch test on ranks (the usual Welch and Kruskal-Wallis procedures are not discussed because, when the variances were not equal, the Type I error rates were not controlled).

Table 3. Type I Error Percentages for $n=20$ for the Welch test (Welch), the Welch test with trimmed means and Winsorized variances (Welch-t), the Welch test with ranked data (Welch-r), the KruskalWallis nonparametric test (K-W) and the Brunner, Dette and Munk (1997) heteroscedastic nonparametric test (BDM).

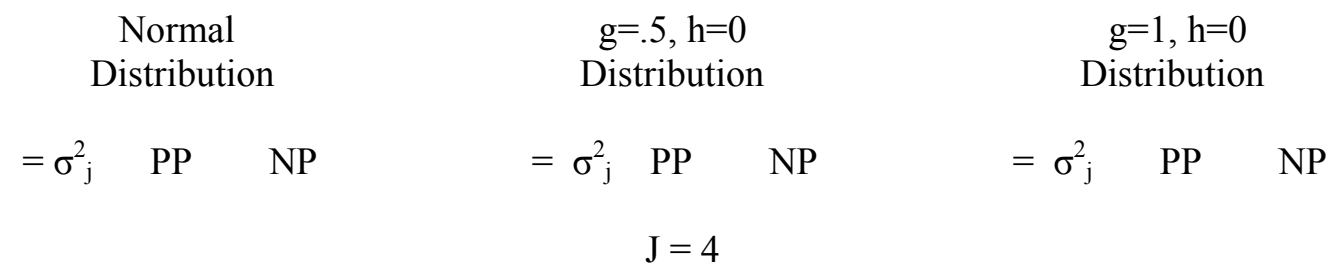

$\begin{array}{llllllllll}\text { Welch } & 5.3 & 5.1 & 5.3 & 5.9 & 5.7 & 7.2 & 6.5 & 7.4 & \mathbf{1 3 . 5} \\ \text { Welch-t } & 5.7 & 5.5 & 5.8 & 5.6 & 5.3 & 6.2 & 4.9 & 4.9 & 6.7 \\ \text { Welch-r } & 5.7 & 6.4 & 6.6 & 5.7 & 5.9 & 6.4 & 5.7 & 6.0 & 6.4 \\ \text { K-W } & 4.8 & 4.0 & \mathbf{7 . 8} & 4.8 & 3.9 & \mathbf{7 . 9} & 4.8 & 4.1 & \mathbf{7 . 9} \\ \text { BDM } & 6.8 & 7.0 & 7.0 & 6.8 & 7.2 & 7.1 & 6.8 & 7.3 & 7.2 \\ & & & & & & & & & \end{array}$

$\begin{array}{llllllllll}\text { Welch } & 5.0 & 4.9 & 5.0 & 6.7 & 6.5 & \mathbf{8 . 4} & \mathbf{9 . 6} & \mathbf{1 0 . 0} & \mathbf{1 6 . 9} \\ \text { Welch-t } & 6.2 & 5.7 & 6.4 & 6.0 & 5.8 & 6.7 & 6.0 & 6.1 & 7.5 \\ \text { Welch-r } & 5.6 & 5.7 & 6.2 & 5.6 & 5.8 & 6.4 & 5.7 & 5.8 & 6.6 \\ \text { K-W } & 4.3 & 3.6 & 7.0 & 4.3 & 3.7 & 7.2 & 4.3 & 3.8 & 7.4 \\ \text { BDM } & 5.7 & 5.6 & 6.5 & 5.7 & 5.6 & 6.5 & 5.7 & 6.0 & 6.6\end{array}$

Note: $=\sigma^{2}{ }_{j}=$ equal population variances; $\mathrm{PP}$ and $\mathrm{NP}=$ positive and negative pairings of sample sizes and variances, respectively. Values exceeding Bradley's liberal limits $(2.5 \%-7.5 \%)$ are presented in bold. 
Table 4. Power percentages for $\mathrm{n}=20$ for the Welch test (Welch), the Welch test with trimmed means and Winsorized variances (Welch-t), the Welch test with ranked data (Welch-r), the KruskalWallis nonparametric test (K-W) and the Brunner, Dette and Munk (1997) heteroscedastic nonparametric test (BDM).

\begin{tabular}{cccccccccc} 
& $\begin{array}{c}\text { Normal } \\
\text { Distribution }\end{array}$ & \multicolumn{2}{c}{$\begin{array}{c}\mathrm{g}=.5, \mathrm{~h}=0 \\
\text { Distribution }\end{array}$} & \multicolumn{3}{c}{$\begin{array}{c}\mathrm{g}=1, \mathrm{~h}=0 \\
\text { Distribution }\end{array}$} \\
$=\sigma_{\mathrm{j}}{ }_{\mathrm{j}}$ & $\mathrm{PP}$ & $\mathrm{NP}$ & $=\sigma_{\mathrm{j}}^{2}$ & $\mathrm{PP}$ & $\mathrm{NP}$ & $=\sigma^{2}{ }_{\mathrm{j}}$ & $\mathrm{PP}$ & $\mathrm{NP}$ \\
& & & & & & & & & \\
& & & & $\mathrm{J}=4$ & & & & \\
98.3 & 57.8 & 66.8 & 93.5 & 45.4 & 62.8 & 69.8 & 22.0 & $\mathbf{5 2 . 4}$ \\
95.8 & 54.2 & 60.0 & 94.2 & 47.8 & 60.0 & 88.7 & 40.3 & 56.4 \\
98.0 & 57.5 & 66.5 & 97.7 & 63.0 & 65.3 & 96.9 & 73.4 & 65.0 \\
98.2 & 49.5 & $\mathbf{6 5 . 8}$ & 98.2 & 57.4 & $\mathbf{6 4 . 1}$ & 97.4 & 71.3 & $\mathbf{6 3 . 5}$ \\
97.8 & 60.7 & 51.3 & 95.8 & 63.8 & 47.1 & 93.1 & 70.8 & 45.3 \\
& & & & & & & & \\
& & & & $\mathrm{~J}=7$ & & & & \\
98.6 & 54.4 & 69.5 & 95.3 & 43.3 & $\mathbf{6 8 . 4}$ & $\mathbf{7 5 . 1}$ & $\mathbf{2 4 . 9}$ & $\mathbf{5 9 . 7}$ \\
96.3 & 47.7 & 63.4 & 95.5 & 44.9 & 65.1 & 91.7 & 38.9 & 63.2 \\
98.5 & 54.5 & 70.0 & 98.7 & 59.6 & 71.8 & 98.7 & 69.5 & 74.4 \\
98.6 & 46.7 & 66.8 & 98.9 & 53.1 & 68.5 & 98.9 & 65.1 & 70.5 \\
98.1 & 54.8 & 49.4 & 97.1 & 56.9 & 48.0 & 95.6 & 63.3 & 48.1
\end{tabular}

Note: $=\sigma^{2}=$ equal population variances; PP and NP = positive and negative pairings of sample sizes and variances, respectively. Conditions for which values exceeding Bradley's liberal limits $(2.5 \%$ $7.5 \%)$ are presented in bold. 
Table 5. Type I Error Percentages for $\mathrm{J}=7$ and $\mathrm{n}=20$ for the for the Tukey and REGW MCPs with the Welch test (W), the Welch test with trimmed means and Winsorized variances (WT), the Welch test with ranked data (WR), the Kruskal-Wallis nonparametric test (KW) and the Brunner and Munzel (2000) heteroscedastic nonparametric test (BM).

\begin{tabular}{lrcccccccrr} 
& \multicolumn{3}{c}{$\begin{array}{c}\text { Normal } \\
\text { Distribution }\end{array}$} & \multicolumn{3}{c}{$\begin{array}{c}\mathrm{g}=.5, \mathrm{~h}=0 \\
\text { Distribution }\end{array}$} & \multicolumn{3}{c}{$\begin{array}{c}\mathrm{g}=1, \mathrm{~h}=0 \\
\text { Distribution }\end{array}$} \\
& $=\sigma^{2}{ }_{\mathrm{j}}$ & $\mathrm{PP}$ & $\mathrm{NP}$ & $=\sigma^{2}{ }_{\mathrm{j}}$ & $\mathrm{PP}$ & $\mathrm{NP}$ & $=\sigma^{2}{ }_{j}$ & $\mathrm{PP}$ & $\mathrm{NP}$ \\
Tukey-W & 5.3 & 5.0 & 5.0 & 4.2 & 4.5 & 7.2 & & 2.5 & 4.8 & $\mathbf{1 3 . 4}$ \\
REGW-W & 3.6 & 3.2 & 2.6 & 3.2 & 2.9 & 3.3 & 1.3 & 1.4 & 3.6 \\
Tukey-WT & 6.2 & 6.0 & 6.3 & 5.1 & 5.1 & 6.4 & 3.0 & 3.5 & 6.5 \\
REGW-WT & 4.3 & 3.5 & 3.3 & 3.4 & 3.0 & 2.9 & 1.9 & 1.8 & 2.1 \\
Tukey-WR & 5.9 & 6.0 & 6.6 & 6.0 & 6.1 & 6.5 & 6.0 & 6.3 & 6.6 \\
REGW-WR & 4.5 & 4.5 & 4.2 & 4.5 & 4.5 & 4.3 & 4.5 & 4.6 & 4.5 \\
Tukey-KW & 4.5 & 4.2 & $\mathbf{8 . 1}$ & 4.5 & 4.2 & $\mathbf{8 . 0}$ & 4.5 & 4.3 & $\mathbf{8 . 4}$ \\
REGW-KW & 3.3 & 3.0 & 5.6 & 3.3 & 3.0 & 5.7 & 3.3 & 3.1 & 6.0 \\
Tukey-BM & 5.7 & 5.2 & 5.9 & 5.7 & 5.2 & 6.1 & 5.7 & 5.5 & 6.2 \\
REGW-BM & 3.6 & 3.3 & 3.2 & 3.6 & 3.4 & 3.2 & 3.6 & 3.6 & 3.4
\end{tabular}

$\underline{\text { Note: }}=\sigma_{j}^{2}=$ equal population variances; PP and NP = positive and negative pairings of sample sizes and variances, respectively. Values exceeding Bradley's liberal limits $(2.5 \%-7.5 \%)$ are presented in bold. 
Table 6. Per-Pair Power Percentages for $\mathrm{J}=7$ and $\mathrm{n}=20$ for the Tukey and REGW MCPs with the Welch test $(\mathrm{W})$, the Welch test with trimmed means and Winsorized variances (WT), the Welch test with ranked data (WR), the Kruskal-Wallis nonparametric test (KW), and the Brunner and Munzel (2000) heteroscedastic nonparametric test $(\mathrm{BM})$.

\begin{tabular}{lllllllllll} 
& \multicolumn{3}{c}{$\begin{array}{c}\text { Normal } \\
\text { Distribution }\end{array}$} & \multicolumn{3}{c}{$\begin{array}{c}\mathrm{g}=.5, \mathrm{~h}=0 \\
\text { Distribution }\end{array}$} & & \multicolumn{3}{c}{$\begin{array}{c}\mathrm{g}=1, \mathrm{~h}=0 \\
\text { Distribution }\end{array}$} \\
& $=\sigma^{2}{ }_{\mathrm{j}}$ & $\mathrm{PP}$ & $\mathrm{NP}$ & $=\sigma^{2}{ }_{\mathrm{j}}$ & $\mathrm{PP}$ & $\mathrm{NP}$ & $=\sigma^{2}$ & $\mathrm{PP}$ & $\mathrm{NP}$ \\
Tukey-W & 44.3 & 10.4 & 14.3 & & 34.5 & 6.2 & 14.7 & 17.0 & 1.6 & $\mathbf{1 1 . 6}$ \\
REGW-W & 45.8 & 10.4 & 9.7 & 34.7 & 5.8 & 10.1 & 13.2 & 1.1 & 5.7 \\
Tukey-WT & 37.1 & 8.5 & 12.1 & 35.1 & 7.1 & 13.5 & 29.7 & 4.9 & 13.4 \\
REGW-WT & 36.4 & 7.8 & 7.4 & 34.5 & 6.4 & 8.8 & 31.9 & 4.0 & 7.9 \\
Tukey-WR & 44.1 & 10.0 & 16.8 & 44.8 & 11.5 & 16.3 & 43.8 & 14.8 & 16.2 \\
REGW-WR & 46.5 & 10.5 & 14.8 & 46.8 & 12.0 & 14.2 & 45.6 & 15.5 & 14.0 \\
Tukey-KW & 46.6 & 8.5 & $\mathbf{1 7 . 0}$ & 47.7 & 10.0 & $\mathbf{1 6 . 7}$ & 46.8 & 13.9 & $\mathbf{1 6 . 7}$ \\
REGW-KW & 50.9 & 9.1 & 18.3 & 52.4 & 10.7 & 18.0 & 51.7 & 15.1 & 18.0 \\
Tukey-BM & 43.7 & 9.1 & 13.9 & 40.2 & 10.4 & 12.2 & 35.4 & 12.9 & 11.3 \\
REGW-BM & 44.8 & 9.1 & 8.8 & 39.5 & 9.8 & 7.4 & 33.5 & 11.9 & 6.7
\end{tabular}

Note: $=\sigma_{j}^{2}=$ equal population variances; PP and $\mathrm{NP}=$ positive and negative pairings of sample sizes and variances, respectively. Conditions for which values exceeding Bradley's liberal limits $(2.5 \%-7.5 \%)$ are presented in bold .

Table 7. All-Pairs Power Percentages for $J=4$ and $n=20$ for the Tukey and REGW MCPs with the Welch test (W), the Welch test with trimmed means and Winsorized variances (WT), the Welch test with ranked data (WR), the Kruskal-Wallis nonparametric test (KW), and the Brunner and Munzel (2000) heteroscedastic nonparametric test (BM).

\begin{tabular}{|c|c|c|c|c|c|c|c|c|c|}
\hline & & $\begin{array}{l}\text { Jorma } \\
\text { stribu }\end{array}$ & & & $\begin{array}{l}.5, \mathrm{~h}= \\
\text { tribut }\end{array}$ & & & $\begin{array}{l}=1, \mathrm{~h}= \\
\text { ributi }\end{array}$ & \\
\hline & $=\sigma_{j}^{2}$ & PP & NP & $=\sigma_{j}^{2}$ & PP & $\mathrm{NP}$ & $=\sigma_{j}^{2}$ & PP & NP \\
\hline Tukey-W & 28.8 & 4.2 & 2.3 & 17.2 & 1.6 & 2.1 & 4.3 & 0.2 & 1.2 \\
\hline REGW-W & 36.0 & 7.4 & 4.6 & 23.8 & 3.2 & 3.7 & 7.1 & 0.4 & 2.1 \\
\hline Tukey-WT & 22.4 & 3.1 & 1.5 & 18.4 & 2.0 & 1.7 & 12.6 & 1.0 & 1.5 \\
\hline REGW-WT & 29.9 & 5.5 & 3.1 & 25.3 & 3.9 & 3.2 & 18.0 & 2.2 & 2.7 \\
\hline Tukey-WR & 27.9 & 3.3 & 3.8 & 26.7 & 3.7 & 3.1 & 23.8 & 5.5 & 2.7 \\
\hline REGW-WR & 35.1 & 6.1 & 6.5 & 33.7 & 6.8 & 5.6 & 30.9 & 9.5 & 5.1 \\
\hline Tukey-KW & 31.4 & 3.1 & 6.0 & 31.2 & 3.8 & 5.1 & 28.3 & 6.1 & 4.6 \\
\hline REGW-KW & 38.4 & 5.7 & 9.7 & 38.2 & 6.8 & 8.5 & 35.7 & 10.4 & 7.9 \\
\hline Tukey-BM & 26.8 & 3.2 & 1.8 & 21.6 & 3.0 & 1.1 & 15.8 & 3.7 & 0.8 \\
\hline REGW-BM & 33.5 & 5.6 & 3.4 & 28.5 & 5.5 & 2.3 & 22.7 & 6.7 & 1.8 \\
\hline
\end{tabular}

Note: $=\sigma^{2}{ }_{j}=$ equal population variances; $\mathrm{PP}$ and $\mathrm{NP}=$ positive and negative pairings of sample sizes and variances, respectively. Conditions for which values exceeding Bradley's liberal limits (2.5\% - 7.5\%) are presented in bold. 


\section{Conclusion}

This article addressed the problem of testing for differences in the central tendency of independent groups with nonnormal (skewed) data and heterogeneous variances. This is an especially important issue for researchers in the behavioral sciences because these assumptions are rarely satisfied (e.g., Micceri, 1989; Keselman et al., 1998; Wilcox, 1988).

Of the omnibus tests evaluated in this paper, the Welch (1951) test with trimmed means and Winsorized variances, the Welch (1951) test on ranked data (Zimmerman \& Zumbo, 1993a), and the Brunner heteroscedastic rank-based procedures (Brunner, Dette \& Munk, 1997; Brunner \& Munzel, 2000) provided superior Type I error control relative to the remaining procedures. The Type I error rates of the omnibus Welch test became liberal when distributions were skewed, and the KruskalWallis test had liberal Type I error rates when variances were unequal (specifically when sample sizes and variances were negatively paired). These results concerning the liberal Type I error control of the Welch test with skewed and heteroscedastic data, and the Kruskal-Wallis procedure with unequal variances are consistent with previous reports (e.g., Algina, Oshima \& Lin, 1994; Zimmerman \& Zumbo, 1993a, 199b). With respect to power, there was very little difference between the procedures when the distributions were normal, although the power rates of the Welch test on ranks, the Brunner heteroscedastic nonparametric procedure, and the KruskalWallis procedure were generally the largest.

These tests were also contrasted when they were applied to the set of all possible pairwise comparisons. In this case, the REGWQ MCP was able to maintain Type I error rates below Bradley's upper liberal bound (7.5\%) with all of the tests investigated. The test statistics with a Tukey critical value also maintained their empirical Type I error rates below Bradley's upper liberal bound under most conditions; however, the Kruskal-Wallis statistic became slightly liberal when sample sizes and variances were negatively paired. These results are not unexpected given that the omnibus Kruskal-Wallis procedure also became liberal under these conditions. Adopting an REGWQ critical value generally resulted in more powerful tests than adopting a Tukey critical value, especially with respect to all-pairs power. Further, when the distributions were nonnormal, adopting an REGWQ critical value resulted in the largest power when used with one of the ranked data procedures (Welch on ranks, Kruskal-Wallis, or the Brunner \& Munzel heteroscedastic nonparametric procedure).

In summary, when treatment distributions were skewed and variances heterogeneous, both the Welch $(1938 ; 1951)$ tests with ranked data and the heteroscedastic, nonparametric procedures proposed by Brunner and colleagues (Brunner, Dette \& Munk, 1997; Brunner \& Munzel, 2000) provided good Type I error control (in both omnibus and pairwise multiple comparison settings). However, the Welch tests on ranked data are recommended as they were generally more powerful than the Brunner procedures. Further, the Welch tests on ranked data can easily be implemented in any software program that allows the user to rank the observations and run the Welch heteroscedastic procedures (e.g., SAS, SPSS, R).

\section{References}

Algina, J., Oshima, T. C., \& Lin, W.-Y. (1994). Type I error rates for Welch's test and James's second-order test under nonnormality and inequality of variance when there are two groups. Journal of Educational and Behavioral Statistics, 19, 275-291.

Bradley, J. V. (1978). Robustness? British Journal of Mathematical and Statistical Psychology, 31, 144-152.

Brown, M. B. \& Forsythe, A. B. (1974). The small sample behavior of some statistics which test the equality of several means. Technometrics, 16, 129-132.

Brunner, E., Dette, H. \& Munk, A. (1997). Box type approximations in nonparametric factorial designs. Journal of the American Statistical Association, 92, 14941502.

Brunner, E. \& Munzel, U. (2000). The nonparametric Behrens-Fisher problem: Asymptotic theory and a small sample approximation. Biometrical Journal, 42, 17-25. 
Cressie, N. A. C. \& Whitford, H. J. (1986). How to use the two sample $t$-test. Biometrical Journal, 28, 131-148.

Einot, I. \& Gabriel, K. R. (1975). A study of the powers of several methods of multiple comparisons. Journal of the American Statistical Association, 70, 574-583.

Hoaglin, D. C. (1985). Summarizing shape numerically: The g- and h-distributions. In D. Hoaglin, F. Mosteller \& J. Tukey (Eds.), Exploring data tables, trends, and shapes (pp. 461-513). New York: Wiley.

Keselman, H. J., Cribbie, R. A. \& Zumbo, B. D. (1997). Specialized tests for detecting treatment effects in the two-sample problem. Journal of Experimental Education, 65, 355-366.

Keselman, H. J., Huberty, C. J., Lix, L. M., Olejnik, S., Cribbie, R., Donahue, B., Kowalchuk, R. K., Lowman, L. L., Petoskey, M. D., Keselman, J. C. \& Levin, J. R. (1998). Statistical practices of educational researchers: An analysis of their ANOVA, MANOVA, and ANCOVA analyses. Review of Educational Research, 68, 350-386.

Keselman, H. J., Lix, L. M. \& Kowalchuk, R. K. (1998). Multiple comparison procedures for trimmed means. Psychological Methods, 3, 123-141.

Kohr, R. L. \& Games, P. A. (1974). Robustness of the analysis of variance, the Welch procedure and a Box procedure to heterogeneous variances. Journal of Experimental Education, 43, 61-69.

Kruskal, W. H. \& Wallis, W. A. (1952). Use of ranks in one-criterion variance analysis. Journal of the American Statistical Association, 47, 583-621.

Micceri, T. (1989). The unicorn, the normal curve, and other improbable creatures. Psychological Bulletin, 105, 156-166.

Mudholkar, A., Mudholkar, G. S. \& Srivastava, D. K. (1991). A construction and appraisal of pooled trimmed-t statistics. Communications in Statistics: Theory and Methods, 20, 1345-1359.

Munzel, U. \& Hothorn, L. A. (2001). A unified approach to simultaneous rank test procedures in the unbalanced one-way layout. Biometrical Journal, 43, 553-569.
Nelder, J. A., \& Mead, R. (1965). A simplex method for function minimization. Computer Journal, 71, 308-313.

Olsson, D. M. (1974). A sequential simplex program for solving minimization problems. Journal of Quality Control, 6, 53-57.

Olsson, D. M. \& Nelson, L. S. (1975). The Nelder-Mead simplex procedure for function minimization. Technometrics, 17, 4551.

Ryan, T. A. (1960). Significance tests for multiple comparison of proportions, variances, and other statistics. Psychological Bulletin, 57, 318-328.

SAS Institute, Inc. (1999). SAS/IML user's guide, Version 8. Cary, NC: SAS Institute Inc..

Satterthwaite, F. E. (1946). An approximate distribution of estimates of variance components. Biometrics, 2, 110-114.

Sprent, P. (1993). Applied nonparametric statistical methods (2nd ed.). London: Chapman \& Hall.

Toothaker, L. E. (1991). Multiple comparisons for researchers. Newbury Park, CA: Sage Publications Inc.

Welch, B. L. (1938). The significance of the difference between two means when population variances are unequal. Biometrika, 29, 350-362.

Welch, B. L. (1951). On the comparison of several mean values: An alternative approach. Biometrika, 38, 330-336.

Welsch, R. E. (1977). Stepwise multiple comparison procedures. Journal of the American Statistical Association, 72, 566-575.

Wilcox, R. R. (1988). A new alternative to the ANOVA $F$ and new results on James' second order method. British Journal of Mathematical and Statistical Psychology, 41, 109-117.

Wilcox, R. R. (1995). ANOVA: The practical importance of heteroscedastic methods, using trimmed means versus means, and designing simulation studies. British Journal of Mathematical and Statistical Psychology, 48, 99-114.

Wilcox, R. R. (1997). Three multiple comparison procedures for trimmed means. Biometrical Journal, 37, 643-656. 
Wilcox, R. R. (2005). Introduction to robust estimation and hypothesis testing. New York: Elsevier Academic Press.

Yuen, K. K. \& Dixon, W. J. (1973). The approximate behavior of the two-sample trimmed $t$. Biometrika, 60, 369-374.

Zimmerman, D. W. (1996). A note on homogeneity of variance of scores and ranks. Journal of Experimental Education, 64, 351362.

Zimmerman, D. W. (1987). Comparative power of the Student $t$ test and Mann-Whitney U-test for unequal sample sizes and variances. Journal of Experimental Education, 55, 171-174.
Zimmerman, D. W. \& Zumbo, B. D. (1993a). The relative power of parametric and nonparametric statistical methods. In G. Keren \& C. Lewis (Eds.), A handbook for data analysis in the behavioral sciences: Methodological issues. New Jersey: Lawrence Erlbaum Associates.

Zimmerman, D. W. \& Zumbo, B. D. (1993b). Rank transformations and the power of the Student $t$ test and Welch t' test for nonnormal populations with unequal variances. Canadian Journal of Experimental Psychology, 47, 523-529. 


\title{
The Effects of Heteroscedasticity on Tests of Equivalence
}

\author{
Jamie A. Gruman \\ University of Guelph \\ Robert A. Cribbie Chantal A. Arpin-Cribbie \\ York University
}

Tests of equivalence, which are designed to assess the similarity of group means, are becoming more popular, yet very little is known about the statistical properties of these tests. Monte Carlo methods are used to compare the test of equivalence proposed by Schuirmann with modified tests of equivalence that incorporate a heteroscedastic error term. It was found that the latter were more accurate than the Schuirmann test in detecting equivalence when sample sizes and variances were unequal.

Key words: Null hypothesis testing, heteroscedasticity, tests of equivalence.

\section{Introduction}

Over a half century ago, Hotelling, Bartky, Deming, Friedman \& Hoel (1948) wrote that "Unfortunately, too many people like to do their statistical work as they say their prayers merely substitute in a formula found in a highly respected book written a long time ago" ( $p$. 103). This quote, which can be found cited in The Task Force on Statistical Inference in Psychology's report outlining recommendations for the effective use of statistics (Wilkinson, 1999), underscores the fact that many researchers apply statistical methods thoughtlessly, without considering the methods' appropriateness to the research questions under consideration.

Many empirical questions in behavioral research involve testing the null hypothesis of no difference between groups on a specific dependent variable. In fact, formulating research

Jamie A. Gruman is Assistant Professor of Organizational Behavior. E-mail: jgruman@uoguelph.ca Robert A. Cribbie is an Associate Professor of Psychology at York University in Toronto, Ontario. He specializes in robust test statistics and multiplicity control. Chantal A. Arpin-Cribbie is a doctoral student in the Department of Psychology at York University in Toronto, Ontario. She specializes in clinical health psychology. questions involving two groups as tests of this null hypothesis is almost a conditioned reflex among scholars, even though such an hypothesis is frequently irrelevant to the research question (Westlake, 1976). Testing the null hypothesis of no difference is inappropriate for studies in which the primary objective is to demonstrate that two groups are equivalent, rather than different, on a particular measure. More specifically, when the research question deals with the equivalence of groups on a dependent measure, an equivalence test is the appropriate (and necessary) statistical method to be used. The present article will highlight the importance of equivalence tests in behavioral research and use a Monte Carlo study to compare tests of equivalence when the variances of the groups are not equal.

Researchers frequently conduct studies in which assessing the equivalence of two groups is the main purpose. For example, consider an investigation of two therapies for dealing with perfectionism. One therapy is lengthy and expensive; the other short and inexpensive. The pertinent research question may be to determine whether the therapies are equivalent in terms of their effectiveness. If they are equivalent, then the shorter, less expensive method can be implemented with considerable cost and time savings. Traditional statistical procedures such as t-tests and ANOVAs are illsuited to answering these questions because they focus, conceptually and statistically, on assessing group differences. For research 
questions pertaining to the equivalence of conditions, researchers require a statistical technique designed specifically to test the degree to which different conditions produce similar results. Tests of equivalence serve this purpose.

When employing tests of equivalence the goal is not to show that treatment conditions are perfectly identical, but only that the differences between the treatments are too small to be considered meaningful. Consider, for example, an investigation in which an attempt is made to demonstrate that scores on a computerbased test are equivalent to those from a paper and pencil based test (e.g., Epstein, Klinkenberg, Wiley \& McKinley, 2001). In this example, the researchers may not need to show that the test scores are exactly equivalent (as with the traditional null hypothesis $\mathrm{H}_{0}: \mu_{1}=\mu_{2}$, but only that differences in test scores are inconsequential (i.e., $\left|\mu_{1}-\mu_{2}\right|<D$, where $\mathrm{D}$ represents an a priori critical difference for determining equivalence).

A specific example may elucidate this issue more clearly. Alkhadher, Clarke \& Anderson (1998) conducted an investigation designed to assess the equivalence of the paperand-pencil version and a computer adaptive version of three subtests from the Differential Aptitude Tests (DAT), namely numerical ability (NA), abstract reasoning (AR) and mechanical reasoning (MR). It is noteworthy that the title of their article specifically underscores the equivalence of these subtests and that in their introduction they highlight that "their equivalence must be established empirically" (p.206). However, as a means of demonstrating the equivalence of the measures, Alkhader et al. proceeded to conduct ANOVAs, which are expressly designed to detect statistically significant group differences. Based on their analyses they claimed to have demonstrated the equivalence of two of the three subtests (AR and MR). However, what Alkhader et al. in fact demonstrated was merely that scores on the NA subtest on the computer adapted version of the DAT were statistically significantly different from the paper and pencil method as traditionally defined.

The question of the equivalence of the different administration methods on subtest scores remains a mystery. As Cribbie, Gruman \& Arpin-Cribbie (2004) and Rogers, Howard \&
Vessey (1993) note, the rejection or nonrejection of the null hypothesis of traditional tests tells us very little about the potential equivalence of the groups in question. Effectively establishing whether the computer adapted version of the DAT produced subtest scores that were equivalent to the paper and pencil version would have required the use of a statistical technique that could assess the degree to which these measures produced similar results. This can be accomplished through the use of equivalence testing, the purpose of which is to demonstrate that two (or more) conditions are functionally the same (Stegner, Bostrom \& Greenfield, 1996).

This approach to statistical analysis has been popular for many years in biology, where researchers interested in the interchangeability of genetically equivalent drugs have used the technique to determine drugs' comparative bioavailability, or bioequivalence (Westlake, 1976). However, researchers outside of biology have been slow to recognize the utility of this procedure and continue to use inappropriate statistics when conducting studies that consider the similarity of alternative conditions, tests, treatments, or procedures.

One of the more commonly discussed tests of equivalence was developed by Schuirmann (1987). Schuirmann's test of equivalence has been introduced to the behavioral sciences through influential articles by Rogers et al. (1993), Seaman \& Serlin (1998) and others. The first step in applying Schuirmann's test of equivalence is to establish a critical mean difference for declaring two population means equivalent (D). Any mean difference smaller than D would be considered meaningless within the framework of the experiment. The selection of an equivalency interval (D) is an important aspect of equivalence testing that is primarily dependent on a subjective level of confidence with which to declare two (or more) populations equivalent. This level of confidence can take on many different forms including a raw value (e.g., mean test scores different by 10 points), a percentage difference (e.g., +/- 10\%), a percentage of the pooled standard deviation difference, etc.

Researchers debating an appropriate value of $D$ should consider the nature of the 
research. For example, if the paper-and-pencil test discussed above was ten times more expensive to administer than the computer-based test, even a very significant difference in outcomes (e.g., $+20 \%$ ) might be acceptable for concluding that the tests are equivalent; Whereas if the paper-and-pencil test was only twice as expensive to administer as the computer based test a difference in outcomes of no more than $5 \%$ might be required for concluding that the tests are equivalent. For a further discussion on establishing D readers are referred to Greene, Concato \& Feinstein (2000).

When using this procedure it is assumed that the two samples are randomly and independently selected from normally distributed populations with equal variances. Two one-sided hypothesis tests can be used to establish equivalence, where the null hypothesis relates to the nonequivalence of the population means and can be expressed as two separate composite hypotheses:

$$
\mathrm{H}_{\mathrm{o} 1}: \mu_{1}-\mu_{2} \geq \mathrm{D} ; \mathrm{H}_{\mathrm{o} 2}: \mu_{1}-\mu_{2} \leq-\mathrm{D} \text {. }
$$

Rejection of $\mathrm{H}_{\mathrm{o} 1}$ implies that $\mu_{1}-\mu_{2}<\mathrm{D}$, and rejection of $\mathrm{H}_{\mathrm{o} 2}$ implies that $\mu_{1}-\mu_{2}>-\mathrm{D}$. Further, rejection of both hypotheses implies that $\mu_{1}-\mu_{2}$ falls within the bounds of (-D, D) and the means are deemed equivalent.

$$
\mathrm{H}_{\mathrm{o} 1} \text { is rejected if } \mathrm{t}_{1} \leq-\mathrm{t}_{v}^{\alpha} \text { where: }
$$

$$
t_{1}=\frac{\left(\bar{X}_{1}-\bar{X}_{2}\right)-D}{\sqrt{\frac{\left(n_{1}+n_{2}\right)\left[\left(n_{1}-1\right) s_{1}^{2}+\left(n_{2}-1\right) s_{2}^{2}\right]}{n_{1} n_{2}\left(n_{1}+n_{2}-2\right)}}}
$$

and $\mathrm{H}_{\mathrm{o} 2}$ is rejected if $\mathrm{t}_{2} \geq \mathrm{t}_{\alpha, \mathrm{df}}$ where:

$$
t_{2}=\frac{\left(\bar{X}_{1}-\bar{X}_{2}\right)-(-D)}{\sqrt{\frac{\left(n_{1}+n_{2}\right)\left[\left(n_{1}-1\right) s_{1}^{2}+\left(n_{2}-1\right) s_{2}^{2}\right]}{n_{1} n_{2}\left(n_{1}+n_{2}-2\right)}}} .
$$

$\bar{x}_{1}$ and $\bar{x}_{2}$ are the group means, $\mathrm{n}_{1}$ and $\mathrm{n}_{2}$ are the group sample sizes, $s_{1}$ and $s_{2}$ are the group standard deviations and $t_{\alpha, \mathrm{df}}$ is the upper-tailed $\alpha$-level $t$ critical value with $d f=n_{1}+n_{2}-2$ degrees of freedom.
One concern with the adoption of Schuirmann's test of equivalence is the potential effects of variance heterogeneity on the standard error of the statistic. This is an important consideration given that unequal variances (heteroscedasticity) appear to be the norm, rather than the exception in behavioral research (Keselman et al., 1998; Grissom, 2000). Keselman et al. have noted that researchers often report largest to smallest variance ratios as large as four to one, and largest to smallest variance ratios as large as eight to one are not uncommon. The standard error used with the Schuirmann test is identical to that used in the two independent samples t-test, and problems with this error term have a long history, termed the Behrens-Fisher problem (see, e.g., Scheffe, 1970).

One potential option is to use the heteroscedastic solution developed by Welch (1938) and Satterthwaite (1946). This idea was originally presented by Dannenberg, Dette \& Munk (1994), although the procedure has received little attention given that in biopharmaceutical equivalence trials independent groups designs (where these methods would be appropriate) are rare relative to crossover designs (see Hauschke, Steinijans \& Hothorn, 1996). However, independent groups designs are extremely common in behavioural research areas such as education, psychology, and management. Combining the numerator of Schuirmann's test with the error term of Welch's (1938) heteroscedastic test may provide an equivalence test that is robust to sample size and variance heterogeneity. For the SchuirmannWelch test of equivalence $\mathrm{H}_{01}$ is rejected if $t_{\mathrm{W} 1} \leq$ $-\mathrm{t}_{\alpha, \mathrm{dfw}}$ and $\mathrm{H}_{02}$ is rejected if $\mathrm{t}_{\mathrm{w} 2} \geq \mathrm{t}_{\alpha, \mathrm{dfw}}$ where :

$$
t_{W 1}=\frac{\left(\bar{X}_{1}-\bar{X}_{2}\right)-D}{\sqrt{\frac{s_{1}^{2}}{n_{1}}+\frac{s_{2}^{2}}{n_{2}}}},
$$

$$
t_{W 2}=\frac{\left(\bar{X}_{1}-\bar{X}_{2}\right)-(-D)}{\sqrt{\frac{s_{1}^{2}}{n_{1}}+\frac{s_{2}^{2}}{n_{2}}}},
$$


and

$$
d f_{w}=\frac{\left(\frac{s_{1}^{2}}{n_{1}}+\frac{s_{2}^{2}}{n_{2}}\right)^{2}}{\frac{s_{1}^{4}}{n_{1}^{2}\left(n_{1}-1\right)}+\frac{s_{2}^{4}}{n_{2}^{2}\left(n_{2}-1\right)}} .
$$

Recently, Tryon (2001) proposed a novel approach to equivalence testing that uses inferential confidence intervals to make decisions regarding the equivalence of two groups. Specifically, with Tyron's equivalence test two groups are declared equivalent if $\mathrm{Rg} . \leq$ D, where:

$$
\operatorname{Rg}=\left|\begin{array}{l}
{\left[\overline{\mathrm{X}}_{1}-\left(\mathrm{t}_{1-\alpha}\right)(\mathrm{E})\left(\mathrm{s}_{\overline{\mathrm{x}_{1}}}\right)\right]} \\
-\left[\overline{\mathrm{X}}_{2}+\left(\mathrm{t}_{1-\alpha}\right)(\mathrm{E})\left(\mathrm{s}_{\overline{\mathrm{x}_{2}}}\right)\right]
\end{array}\right|
$$

and

$$
E=\frac{\sqrt{s_{\bar{x}_{1}}^{2}+s_{\overline{x_{2}}}^{2}}}{s_{\overline{x_{1}}}+s_{\overline{x_{2}}}} .
$$

$\mathrm{s}_{\overline{\times}}$ represents the usual standard error of the mean (i.e., $s_{x} / \sqrt{n}$ ) and $t{ }_{1-\alpha}$ represents the (positive) two-tailed critical $t$ value with $\mathrm{df}=\mathrm{n}$ 1. A heteroscedastic version of the Tryon test is available by substituting the original degrees of freedom ( $\mathrm{df}=\mathrm{n}-1)$ by the Welch-Satterthwaite df divided by two (i.e., $\mathrm{df}_{\mathrm{w}} / 2$ ).

\section{Methodology}

\section{Monte Carlo Study}

A simulation study was used to compare the probability of detecting equivalence by: 1) Student t; 2) Welch t; 3) Schuirmann's equivalence test; 4) Schuirmann-Welch equivalence test; 5) Tryon equivalence test; and 6) Tryon-Welch equivalence test. Several variables were manipulated in this study including: a) sample size; b) population variances; and c) population mean configuration. Total sample sizes were set at $\mathrm{N}=20$ and $\mathrm{N}=$ 60. Sample sizes for $N=20$ were: 1) $n_{1}=10$, $\mathrm{n}_{2}=10$; 2) $\mathrm{n}_{1}=8, \mathrm{n}_{2}=12$; and 3) $\mathrm{n}_{1}=5, \mathrm{n}_{2}=15$. Sample sizes for $N=60$ were: 1) $n_{1}=30, n_{2}=30$; 2) $\mathrm{n}_{1}=25, \mathrm{n}_{2}=35 ;$ and 3) $\mathrm{n}_{1}=20, \mathrm{n}_{2}=40$.
Population variances were set at: 1) 1,1 ; 2) .5, 1.5 ; 3) $1.5, .5$; 4) .2, 1.8; and 5) 1.8, .2. These conditions were crossed resulting in: 1) equal $\mathrm{n}$ or $\sigma^{2} ; 2$ ) positively paired $n$ and $\sigma^{2}$ (largest $n$ with largest $\sigma^{2}$, smallest $\mathrm{n}$ with smallest $\sigma^{2}$ ); and 3) negatively paired $n$ and $\sigma^{2}$ (largest $n$ with smallest $\sigma^{2}$, smallest $n$ with largest $\sigma^{2}$ ).

Six mean configurations were evaluated in this study, including equivalent population means $\left(\mu_{1}=\mu_{2}\right)$ and five nonequivalent population means $\left(\mu_{2}=\mu_{1}+.4, \mu_{2}=\mu_{1}+.8, \mu_{2}=\right.$ $\mu_{1}+1, \mu_{2}=\mu_{1}+1.2$ and $\left.\mu_{2}=\mu_{1}+1.6\right)$. The critical mean difference for establishing population equivalence (D) was maintained at 1 throughout all conditions. Given that $\mathrm{D}$ is set at 1 , the equivalent mean configuration and nonequivalent configurations with $\mu_{2}-\mu_{1}<1$ fall under the alternate hypothesis of the Schuirmann and Tryon tests of equivalence (i.e., the population mean difference does not exceed the critical mean difference and thus the means are expected to be declared equivalent), and nonnull configurations with $\mu_{2}-\mu_{1}>1$ fall under the null hypothesis of the Schuirmann and Tryon tests of equivalence (i.e., the population mean difference exceeds the critical mean difference and thus the means are expected to be declared nonequivalent). For the case where $\mu_{2}-\mu_{1}=1=$ $\mathrm{D}$, the expected probability of declaring the two populations equivalent is $\alpha$.

Five thousand simulations were conducted for each condition using a nominal significance level of $\alpha=0.05$.

\section{Results}

The probability of declaring the two independent populations equivalent for $\mathrm{N}=20$ and $\mathrm{N}=60$ are presented in Tables 1 and 2, respectively.

$$
\begin{aligned}
& \mu 2-\mu 1=1=\mathrm{D} \\
& \text { The Schuirmann-Welch maintained }
\end{aligned}
$$
rejection (i.e., rejecting $\mathrm{H}_{\mathrm{o} 1}$ and $\mathrm{H}_{02}$ ) rates at approximately $\alpha(.039-.048)$ for $\mathrm{N}=20$ and exactly at $\alpha$ for $\mathrm{N}=60$ when $\mu 2-\mu 1=1$ [recall that $\mathrm{D}=1$ so $\mathrm{E}\left(\mathrm{t}_{\mathrm{w} 1}\right)=0$ ], regardless of the pattern of sample sizes and variances. However, the Schuirmann test had rejection rates ranging from .019 to .092 under positively and negatively 
Table 1. Probability of declaring the two populations equivalent for $\mathrm{N}=20$

under each of the testing conditions.

\begin{tabular}{|c|c|c|c|c|c|c|c|}
\hline Pairing & $\mu_{2}-\mu_{1}$ & $\mathrm{t}$ & $t-w$ & sch & sch-w & try & try-w \\
\hline Equal $\mathrm{n}$ or $\sigma$ & $\begin{array}{l}0 \\
.4 \\
.8 \\
1 \\
1.2 \\
1.6\end{array}$ & $\begin{array}{l}.948 \\
.867 \\
.621 \\
.462 \\
.309 \\
.099\end{array}$ & $\begin{array}{l}.949 \\
.873 \\
.641 \\
.487 \\
.338 \\
.123\end{array}$ & $\begin{array}{l}.352 \\
.251 \\
.094 \\
.045 \\
.018 \\
.002\end{array}$ & $\begin{array}{l}.340 \\
.243 \\
.091 \\
.044 \\
.018 \\
.002\end{array}$ & $\begin{array}{l}.289 \\
.217 \\
.091 \\
.048 \\
.022 \\
.003\end{array}$ & $\begin{array}{l}.261 \\
.195 \\
.081 \\
.043 \\
.020 \\
.003\end{array}$ \\
\hline Positive & $\begin{array}{l}0 \\
.4 \\
.8 \\
1 \\
1.2 \\
1.6\end{array}$ & $\begin{array}{l}.980 \\
.932 \\
.749 \\
.600 \\
.437 \\
.166\end{array}$ & $\begin{array}{l}.951 \\
.858 \\
.582 \\
.406 \\
.252 \\
.065\end{array}$ & $\begin{array}{l}.212 \\
.145 \\
.047 \\
.019 \\
.007 \\
.001\end{array}$ & $\begin{array}{l}.454 \\
.318 \\
.107 \\
.048 \\
.018 \\
.001\end{array}$ & $\begin{array}{l}.318 \\
.228 \\
.085 \\
.040 \\
.016 \\
.002\end{array}$ & $\begin{array}{l}.299 \\
.213 \\
.079 \\
.037 \\
.015 \\
.001\end{array}$ \\
\hline Negative & $\begin{array}{l}0 \\
.4 \\
.8 \\
1 \\
1.2 \\
1.6\end{array}$ & $\begin{array}{l}.865 \\
.773 \\
.536 \\
.399 \\
.273 \\
.096\end{array}$ & $\begin{array}{l}.947 \\
.894 \\
.738 \\
.632 \\
.516 \\
.300\end{array}$ & $\begin{array}{l}.403 \\
.317 \\
.156 \\
.092 \\
.051 \\
.011\end{array}$ & $\begin{array}{l}.189 \\
.146 \\
.067 \\
.039 \\
.019 \\
.004\end{array}$ & $\begin{array}{l}.218 \\
.175 \\
.092 \\
.057 \\
.033 \\
.008\end{array}$ & $\begin{array}{l}.161 \\
.130 \\
.067 \\
.041 \\
.023 \\
.006\end{array}$ \\
\hline
\end{tabular}

Note. $\mathrm{t}=$ independent samples $\mathrm{t} ; \mathrm{t}-\mathrm{w}=$ Welch $\mathrm{t} ; \mathrm{sch}=$ Schuirmann test of equivalence; $\mathrm{sch}-\mathrm{w}=$ Schuirmann-Welch test of equivalence; try $=$ Tyron test of equivalence; try- $w=$ Tyron-Welch test of equivlance.

paired sample sizes and variances respectively, for $\mathrm{N}=20$, and rates ranging from .028 to .084 under positively and negatively paired sample sizes and variances respectively, for $\mathrm{N}=60$. Both the Tryon and Tryon-Welch equivalence tests had reasonably accurate rejection rates for $\mu 2-\mu 1=1$ when $\mathrm{N}=20$, although rates were consistently mildly deflated under the unequal sample size and variance conditions when $\mathrm{N}=$ $60(.032-.036)$.

Rejection rates for the two independent samples $\mathrm{t}$ and Welch $\mathrm{t}$ for $\mu 2-\mu 1=1$ reflect the power of these tests for detecting a true difference in means (see Table 1). 
Table 2. Probability of declaring the two populations equivalent for $\mathrm{N}=60$ under each of the testing conditions.

\begin{tabular}{llllllll}
\hline Pairing & $\mu_{2}-\mu_{1}$ & $\mathrm{t}$ & $\mathrm{t}-\mathrm{w}$ & $\mathrm{sch}$ & $\mathrm{sch}-\mathrm{w}$ & try & try-w \\
\hline Equal n or $\sigma$ & 0 & .949 & .950 & .965 & .964 & .924 & .918 \\
& .4 & .676 & .680 & .732 & .729 & .657 & .650 \\
& .8 & .149 & .153 & .186 & .185 & .161 & .157 \\
& 1 & .037 & .038 & .050 & .050 & .044 & .043 \\
& 1.2 & .006 & .006 & .009 & .009 & .008 & .008 \\
& 1.6 & .000 & .000 & .000 & .000 & .000 & .000 \\
& & & & & & & \\
\hline Positive 0 & & .975 & .949 & .961 & .983 & .944 & .943 \\
& .4 & .757 & .639 & .682 & .781 & .671 & .668 \\
& .8 & .189 & .107 & .131 & .200 & .144 & .143 \\
& 1 & .048 & .021 & .028 & .050 & .035 & .035 \\
& 1.2 & .007 & .002 & .003 & .007 & .005 & .005 \\
& 1.6 & .000 & .000 & .000 & .000 & .000 & .000 \\
\hline \multirow{2}{*}{ Negative } & 0 & & & & & & \\
& .4 & .900 & .950 & .958 & .913 & .758 & .725 \\
& .8 & .612 & .732 & .747 & .647 & .495 & .466 \\
& 1 & .141 & .239 & .241 & .165 & .116 & .106 \\
& 1.2 & .041 & .087 & .084 & .050 & .036 & .032 \\
& 1.6 & .008 & .023 & .020 & .010 & .007 & .007 \\
& .000 & .001 & .000 & .000 & .000 & .000 \\
\hline
\end{tabular}

Note. $\mathrm{t}=$ independent samples $\mathrm{t}$; wel- $\mathrm{t}=$ Welch $\mathrm{t} ; \mathrm{sch}=$ Schuirmann test of equivalence; $\mathrm{sch}-\mathrm{w}=$ Schuirmann-Welch test of equivalence; try $=$ Tyron test of equivalence; try-w $=$ Tyron-Welch test of equivlance.

A Priori Equivalence $(\mu 2-\mu 1<D)$

When a priori population mean differences were less than the critical mean difference $(\mathrm{D}=1)$, and either the sample sizes or variances were equal, the probability of declaring the two populations equivalent was almost identical for the Schuirmann, Schuirmann-Welch, Tyron and Tyron-Welch test statistics. The rates for the equivalence tests were significantly less than the rates for the Student $\mathrm{t}$ and Welch $\mathrm{t}$ when the total sample size was small $(\mathrm{N}=20)$, although the rates were larger than those for the Student $\mathrm{t}$ and Welch $\mathrm{t}$ when the total sample size was large $(\mathrm{N}=60)$.
The probability of declaring the two populations equivalent was greater for the SchuirmannWelch test than the Schuirmann test when the sample sizes and variances were positively paired, whereas the probability of declaring the two populations equivalent was greater for the Schuirmann test than the Schuirmann-Welch test when the sample sizes and variances were negatively paired. This is due to the known bias in the non-heteroscedastic standard error, which becomes inflated when sample sizes and variances are positively paired and deflated when sample sizes and variances are negatively paired. 
This bias can also be seen in the results for the traditional tests as the probability of declaring the two populations equivalent (i.e., a statistically non significant effect) was greater for the Student $t$ than the Welch when the sample sizes and variances were positively paired, and the probability of declaring the two populations equivalent was greater for the Welch than the Student $t$ when sample sizes and variances were negatively paired. The rates for the Tryon and Tryon-Welch tests were very similar across all conditions (primarily because the original Tryon test does not use the pooled standard error like the Schuirmann test) but consistently less than those of the SchuirmannWelch test.

A Priori Nonequivalence $(\mu 2-\mu 1>D)$

When a priori population mean differences were greater than the critical difference $(D=1)$, and either the sample sizes or variances were equal, the probability of declaring the two populations equivalent was identical (and very low) for the Schuirmann and Schuirmann-Welch test statistics under all conditions and demonstrates an excellent ability to detect differences greater than D. This is due to the fact that the numerators of $t_{1}$ and $t_{w 1}$ have an expected positive value, whereas a rejection would only occur if $t_{1}$ and $t_{\mathrm{w} 1}$ are LESS THAN $\mathrm{t}_{\alpha, \mathrm{df}}$.

One way to think of this effect would be to relate it to traditional null hypothesis testing when testing a one-tailed alternative hypothesis (i.e., $\mathrm{H}_{1}: \mu_{1}-\mu_{2}>0$ ). We expect the Type I error rates to be approximately $\alpha$ when $\mu_{1}-\mu_{2}=0$, but when $\mu_{1}-\mu_{2}<0$ (i.e., an effect in the wrong direction) the Type I error rates will approach zero. The rates for the Schuirmann and Schuirmann-Welch tests were significantly less than the rates for the Student $t$ and Welch $t$ when the total sample size was small $(\mathrm{N}=20)$, reflecting the fact that the Student $\mathrm{t}$ and Welch $\mathrm{t}$ have less power when $\mathrm{N}=20$, although the rates were very similar for all tests when the total sample size was large $(N=60)$. Similar to the results for a priori equivalence, the probability of declaring the two populations equivalent was greater for the Schuirmann-Welch test than the Schuirmann test when the sample sizes and variances were positively paired, whereas the probability of declaring the two populations equivalent was greater for the Schuirmann test than the Schuirmann-Welch test when the sample sizes and variances were negatively paired. The rates for the Tryon and Tryon-Welch tests were very similar across all conditions, and were also very similar to rates for the Schuirmann-Welch procedure.

\section{Conclusion}

Behavioral researchers reliably use traditional statistical procedures such as Student's t-test when comparing groups even when the primary objective is to demonstrate that groups are equivalent, rather than different, on a particular measure. The present article highlights the need for tests of equivalence and compared alternatives to the original Schuirmann (1987) and Tryon (2001) tests of equivalence for situations in which treatment group variances are unequal. The Schuirmann-Welch test incorporated a heteroscedastic error term and error degrees of freedom, while the TryonWelch test incorporated heteroscedastic degrees of freedom. It was expected that these modifications would improve the performance of the test statistics when sample sizes and variances were unequal. The results of this study support the hypothesis that equivalence rates for the Schuirmann-Welch were more accurate than for the Schuirmann test, correcting for a bias in the standard error of the Schuirmann test that dates back to Fisher and Behrens in the 1930s. Equivalence rates were also more accurate (and more powerful) for the Schuirmann test than for either of the Tryon or Tryon-Welch statistics.

The results also highlight the fact that traditional test statistics such as the Student $t$ and Welch $t$ are not appropriate for testing research hypotheses that relate to the equivalence of two populations. The traditional null hypothesis testing procedures have an extreme bias towards declaring equivalence when sample sizes are small (i.e., a lack of power for detecting small treatment group differences), and are less likely to be able to detect equivalence relative to the Schuirmann or Schuirmann-Welch tests when sample sizes become large.

Tests of equivalence are popular in biopharmaceutical studies for demonstrating that 
the effects of two drugs are practically equivalent. It is expected that as the number of studies outlining the methodologies of equivalence tests grow, the popularity of tests of equivalence will increase in behavioral fields such as education, psychology, and management. Thus, methodologists should provide recommendations for applying these tests. The findings of this study emphasize the need for robust tests of equivalence (such as the Schuirmann-Welch test investigated in this paper) for situations in which data conditions are not optimal. Empirical data rarely meet all of the underlying assumptions of test statistics (Keselman et al., 1998; Micceri, 1989; Welch; 1988), and instead one should be cognizant of assumption violations and apply appropriate test statistics that minimize the likelihood that incorrect inferences are drawn regarding the results.

\section{References}

Alkhadher, O., Clarke, D. D., \& Anderson, N. (1998). Equivalance and predictive validity of paper-and-pencil and computerized adaptive formats of the differential aptitude tests. Journal of Occupational and Organizational Psychology, 71, 205-217.

Cribbie, R. A., Gruman, J. A., \& ArpinCribbie, C. A. (2004). Recommendations for applying tests of equivalence. Journal of Clinical Psychology, 60, 1-10.

Dannenberg, O., Dett, H., \& Munk, A. (1994). An extension of Welch's approximate tsolution to comparative bioequivalence trials. Biometrika, 81, 91-101.

Epstein, J., Klinkenberg, W. D., Wiley, D., \& McKinley, L. (2001). Insuring sample equivalence across internet and paper-and-pencil assessments. Computers in Human Behavior, 17, 339-346.

Greene, W. L., Concato, J., \& Feinstein, A. R. (2000). Claims of equivalence in medical research: Are they supported by the evidence? Annals of Internal Medicine, 132, 715-722.

Grissom, R. J. (2000). Heterogeneity of variance in clinical data. Journal of Consulting and Clinical Psychology, 68, 155-165.

Hauschke, D., Steinijans, V. W., \& Hothorn, L. A. (1996). A note on Welch's approximate ' $t$ 'solution to bioequivalence assessment. Biometrika, 83, 236-237.
Hotelling, H., Bartky, W., Deming, W. E., Friedman, M., \& Hoel, P. (1948). The teaching of statistics. Annals of Mathematical Statistics, 19, 95115.

Keselman, H. J., Huberty, C. J., Lix, L. M., Olejnik, S., Cribbie, R. A., Donahue, B., Kowalchuk, R. K., Lowman, L. L., Petoskey, M. D., Keselman, J. C. \& Levin, J. R. (1998). Statistical practices of educational researchers: An analysis of their ANOVA, MANOVA, and ANCOVA analyses. Review of Educational Research, 68, 350-386.

Micceri, T. (1989). The unicorn, the normal curve, and other improbable creatures. Psychological Bulletin, 105, 156-166.

Rogers, J. L., Howard, K. I. \& Vessey, J. T. (1993). Using significance tests to evaluate equivalence between two experimental groups. Psychological Bulletin, 113, 553-565.

Satterthwaite, F. E. (1946). An approximate distribution of estimates of variance components. Biometrics Bulletin, 2, 110-114.

Schuirmann, D. J. (1987). A comparison of the two one-sided tests procedure and the power approach for assessing equivalence of average bioavailability. Journal of Pharmacokinetics and Biopharmaceutics, 15, 657-680.

Seaman, M. A. \& Serlin, R. C. (1998). Equivalence confidence intervals for two-group comparisons of means. Psychological Methods, 3, 403-411.

Scheffe, H. (1970). Practical solutions of the Behrens-Fisher problem. Journal of the American Statistical Association, 65, 1501-1508.

Stegner, B. L., Bostrom, A. G., \& Greenfield, T. K. (1996). Equivalence testing for use in psychosocial and services research: An introduction with examples. Education and Program Planning, 19(3), 193-198.

Tyron, W. W. (2001). Evaluating statistical difference, equivalence, and indeterminacy using inferential confidence intervals: An integrated alternative method of conducting null hypothesis statistical tests. Psychological Methods, 6, 371-386.

Welch, B. L. (1938). The significance of the difference between two means when population variances are unequal. Biometrika, 29, 350-362.

Westlake, W. J. (1976). Symmetrical confidence intervals for bioequivalence trials. Biometrics, 37, 589-594.

Wilkinson, L., and the Task Force on Statistical Inference. (1999). Statistical methods in psychology journals: Guidelines and explanations. American Psychologist, 54(8), 594-604. 


\title{
Approximate Bayesian Confidence Intervals for the Mean of an Exponential Distribution Versus Fisher Matrix Bounds Models
}

\author{
Vincent A. R. Camara \\ University of South Florida
}

The aim of this article is to obtain and compare confidence intervals for the mean of an exponential distribution. Considering respectively the square error and the Higgins-Tsokos loss functions, approximate Bayesian confidence intervals for parameters of exponential population are derived. Using exponential data, the obtained approximate Bayesian confidence intervals will then be compared to the ones obtained with Fisher Matrix bounds method. It is shown that the proposed approximate Bayesian approach relies only on the observations. The Fisher Matrix bounds method, that uses the z-table, does not always yield the best confidence intervals, and the proposed approach often performs better.

Key words: Estimation, loss functions, Monte Carlo simulation, statistical analysis.

\section{Introduction}

There is a significant amount of research in Bayesian analysis and modeling which has been published the last thirty-five years Harris B. 1976, Higgins J. J. Tsokos 1976, Shafer R. E. 1973. A Bayesian analysis implies the exploitation of suitable prior information and the choice of a loss function in association with Bayes' Theorem. It rests on the notion that a parameter within a model is not merely an unknown quantity, but rather behaves as a random variable, which follows some distribution. In the area of life testing, it is indeed realistic to assume that a life parameter is stochastically dynamic. This assertion is supported by the fact that the complexity of electronic and structural systems is likely to

This work has been sponsored by the Research Center for Fayesian Applications Inc. Vincent A. R. Camara is a Mathematics and Statistics educator and researcher. His research interests include the theory and applications of Bayesian and empirical Bayes analyses with emphasis on the computational aspect of modeling. $\mathrm{He}$ is featured in Marquis Who's Who in America, in American Education, in Engineering and Science, and in the World. Email: gvcamara@ij.net. cause undetected component interactions resulting in an unpredictable fluctuation of the life parameter. Drake (1966) provided an account for the use of Bayesian statistics in reliability problems. He stated,

$\mathrm{He}$ [a Bayesian] realizes... that his selection of a prior (distribution) to express his present state of knowledge will necessarily be somewhat arbitrary. But he greatly appreciates this opportunity to make his entire assumptive structure clear to the world... Why should an engineer not use his engineering judgment and prior knowledge about a parameter in the classical distribution he has picked? For example, if it is the mean time between failures (MTBF) of an exponential distribution that must be evaluated from some tests, he undoubtedly has some idea of what the value will turn out to be". (315-320)

Consider the exponential underlying model characterized by

$$
f(x)=\theta e^{-\theta x} ; x \geq 0, \theta \succ 0
$$

It is well known that once the underlying model is found to have an exponential distribution, 
Fisher Matrix bounds method (Nelson, 1982) uses the Z-table and considers the following confidence interval [] for $\theta$.

$$
L_{\theta}=\frac{\Lambda}{e^{\frac{K_{\alpha} \sqrt{\operatorname{Var}(\theta)}}{\hat{\theta}}}}
$$

and

$$
U_{\theta}=\hat{\theta} e^{\frac{K_{\alpha} \sqrt{\operatorname{Var}(\theta)}}{\hat{\theta}}}
$$

where $K_{\alpha}$ is defined by

$$
\alpha=\frac{1}{\sqrt{2 \pi}} \int_{K_{\alpha}}^{\infty} e^{-\frac{t^{2}}{2}} d t=1-\Phi\left(K_{\alpha}\right)
$$

and

$$
\stackrel{\Lambda}{\operatorname{Var}}(\theta)=\left(\frac{\partial^{2} \Lambda}{\partial \theta^{2}}\right)^{-1}
$$

$\Lambda$ is the log-likelihood function of the exponential distribution (1).

Fisher Matrix bounds method considers large samples to ensure the use of the Z-table.. With some studies that have been conducted with small samples it has been found that the assumption of normal approximations for estimates based on small sample sizes reduces the accuracy of confidence bounds (Hartley, 2004).

For the above model (1), approximate Bayesian confidence bounds for the parameter $\theta$ and the population mean $\frac{1}{\theta}$ will be derived to challenge Fisher bounds method (2).

Although there is no specific analytical procedure that allows us to identify the appropriate loss function to be used, the most commonly used is the square error loss function. One of the reasons for selecting this loss function is because of its analytical tractability in Bayesian analysis. As it will be shown, selecting the square error loss does not always lead to the best approximate Bayesian confidence intervals. However, the obtained approximate Bayesian confidence intervals corresponding to the square error and the Higgins-Tsokos loss functions will be respectively used to challenge Fisher bounds method (2). The loss functions that will be used are given below, along with a statement of their key characteristics.

\section{Square Error Loss Function}

The popular square error loss function places a small weight on estimates near the true value and proportionately more weight on extreme deviation from the true value of the parameter. Its popularity is due to its analytical tractability in Bayesian modeling. The square error loss is defined as follows:

$$
L_{S E}(\stackrel{\Lambda}{\theta}, \theta)=(\stackrel{\Lambda}{\theta}-\theta)^{2}
$$

Higgins-Tsokos Loss Function:

The Higgins-Tsokos loss function places a heavy penalty on extreme over- or underestimation. That is, it places an exponential weight on extreme errors. The Higgins-Tsokos loss function is defined as follows:

$$
L_{H T}(\hat{\theta}, \theta)=\frac{f_{1} e^{f_{2}(\hat{\theta}-\theta)}+f_{2} e^{-f_{1}(\hat{A}-\theta)}}{f_{1}+f_{2}}-1, f_{1}, f_{2} \succ 0 .
$$

Assume that $\theta$ behaves as a random variable that is being characterized by the Pareto probability density function given by

$$
f_{1}(\theta)=\frac{a}{b}\left(\frac{b}{\theta}\right)^{a+1} ; \theta \geq b \succ 0, a \succ 0 .
$$

The Pareto prior has been selected because of its mathematical tractability. Using observations from exponential distributions, the Pareto will approximate prior (5) in such a way that good approximate Bayesian estimates of $\theta$ are obtained. 


\section{Preliminaries}

Let $x_{1}, x_{2}, \ldots \ldots ., x_{n}$ denote the observations of a given system that are being characterized by the exponential distribution (1). The following posterior distribution is obtained:

$$
h(\theta \backslash x) \frac{\theta^{n-a-1} e^{-\theta \sum_{1}^{n} x_{i}}}{\int_{b}^{\infty} \theta^{n-a-1} e^{-\theta \sum_{1}^{n} x_{i}} d \theta}, \theta \succ b . .
$$

\section{Methodology}

Approximate confidence bounds for $\theta$

With respectively the following approximate priors for the square error and the Higgins-Tsokos loss functions, good approximate Bayesian estimates of $\theta$ are obtained.

Approximate prior for the square error loss:

$$
\begin{gathered}
f_{1}(\theta)=\frac{a_{0}}{b_{0}}\left(\frac{b_{0}}{\theta}\right)^{a_{0}+1} ; \theta \geq b \succ 0, a \succ 0 . \\
\mathrm{a}_{0}=\mathrm{n}, \mathrm{b}_{0}=\frac{\mathrm{n}-1}{\sum_{1}^{\mathrm{n}} \mathrm{x}_{\mathrm{i}}}
\end{gathered}
$$

Approximate prior for the Higgins-Tsokos loss:

$$
\begin{gathered}
f_{1}(\theta)=\frac{a_{1}}{b_{1}}\left(\frac{b_{1}}{\theta}\right)^{a_{1}+1} ; \theta \geq b_{1} \succ 0, a_{1} \succ 0 . \\
\mathrm{a}_{1}=\mathrm{n}, \mathrm{b}_{1}=\frac{\mathrm{n}}{\sum_{1}^{\mathrm{n}} \mathrm{x}_{\mathrm{i}}}-\frac{1}{\mathrm{f}_{1}+\mathrm{f}_{2}} \operatorname{Ln}\left(\frac{\sum_{1}^{\mathrm{n}} \mathrm{x}_{\mathrm{i}}+\mathrm{f}_{2}}{\sum_{1}^{\mathrm{n}} \mathrm{x}_{\mathrm{i}}-\mathrm{f} 1}\right)
\end{gathered}
$$

with

$$
\mathrm{f}_{1} \prec \sum_{\mathrm{i}=1}^{\mathrm{n}} \mathrm{x}_{\mathrm{i}}
$$

It's easily shown that the approximate Bayesian estimate of the parameter $\theta$, subject to the square error loss; is the same as the Bayesian estimate of $\theta$ under the Higgins-Tsokos loss. They are equal to

$$
\frac{n}{\sum_{i=1}^{n} x_{i}}
$$

Using respectively the approximate posterior distributions that correspond to (7) and (8), along with the equalities $P(\theta \succ L \mid x)=1-\alpha / 2$ and $P(\theta \succ U \mid x)=\alpha / 2$, the following lower and upper confidence bounds for $\theta$ are obtained:

Approximate Bayesian confidence bounds of $\theta$ corresponding to the square error:

$$
L_{\theta(S E)}=\frac{n-1-\ln (1-\alpha / 2)}{\sum_{1}^{n} x_{i}}
$$

and

$$
U_{\theta(S E)}=\frac{n-1-\ln (\alpha / 2)}{\sum_{1}^{n} x_{i}}
$$

Approximate Bayesian confidence bounds of $\theta$ corresponding to the Higgins-Tsokos:

$$
\begin{gathered}
\mathrm{L}_{\theta(\mathrm{HT})} \frac{\mathrm{n}-\operatorname{Ln}(1-\alpha / 2)}{\sum_{\mathrm{i}=1}^{\mathrm{n}} \mathrm{x}_{\mathrm{i}}}-\frac{1}{\mathrm{f}_{1}+\mathrm{f}_{2}} \operatorname{Ln}\left(\frac{\sum_{\mathrm{i}=1}^{\mathrm{n}} \mathrm{x}_{\mathrm{i}}+\mathrm{f}_{2}}{\sum_{\mathrm{i}=1}^{\mathrm{n}} \mathrm{x}_{\mathrm{i}}-\mathrm{f}_{1}}\right) \\
\mathrm{U}_{\theta(\mathrm{HT})} \frac{\mathrm{n}-\operatorname{Ln}(\alpha / 2)}{\sum_{\mathrm{i}=1}^{\mathrm{n}} \mathrm{x}_{\mathrm{i}}}-\frac{1}{\mathrm{f}_{1}+\mathrm{f}_{2}} \operatorname{Ln}\left(\frac{\sum_{\mathrm{i}=1}^{\mathrm{n}} \mathrm{x}_{\mathrm{i}}+\mathrm{f}_{2}}{\sum_{\mathrm{i}=1}^{\mathrm{n}} \mathrm{x}_{\mathrm{i}}-\mathrm{f}_{1}}\right)
\end{gathered}
$$

Approximate Bayesian confidence bounds for the exponential population mean 
Thus, we respectively obtain the following $100(1-\alpha) \%$ empirical Bayes confidence bounds for the mean $b$ of the exponential failure model, when the squared error and the Higgins-Tsokos loss functions are considered:

$$
L_{b(S E)}=\frac{\sum_{i=1}^{n} x_{i}}{n-1-\ln (\alpha / 2)}
$$

and

$$
U_{b(S E)}=\frac{\sum_{i=1}^{n} x_{i}}{n-1-\ln (1-\alpha / 2)},
$$

and

$$
\begin{aligned}
& L_{b(H T)}=\frac{1}{\frac{n-\operatorname{Ln}(\alpha / 2)}{\sum_{i=1}^{n} x_{i}}-\frac{1}{f_{1}+f_{2}} \operatorname{Ln}\left(\frac{\sum_{i=1}^{n} x_{i}+f_{2}}{\sum_{i=1}^{n} x_{i}-f_{1}}\right)}(11) \\
& U_{b(H T)}=\frac{1}{\frac{n-\operatorname{Ln}(1-\alpha / 2)}{\sum_{i=1}^{n} x_{i}}-\frac{1}{f_{1}+f_{2}} \operatorname{Ln}\left(\frac{\sum_{i=1}^{n} x_{i}+f_{2}}{\sum_{i=1}^{n} x_{i}-f_{1}}\right)} .
\end{aligned}
$$

Numerical Results

In order to compare the proposed approximate Bayesian approach to the Fisher Matrix bounds method, samples that have been obtained from exponentially distributed populations will be considered. For the HigginsTsokos loss function, consider $f_{1}=1, f_{2}=1$. The lengths of the Fisher Matrix bounds and approximate Bayesian confidence intervals are respectively denoted by $l_{F}, l_{S E}$ and $l_{H T}$. 
Example 1

Monte Carlo simulation has been used to generate the following 30 observations from the exponential distribution with mean equal to 1 .

\begin{tabular}{|c|c|c|}
\hline 0.9549716 & 0.09670773 & 0.09107758, \\
\hline 2.6951610 & 1.47495800 & 0.56762340 \\
\hline 1.2636410, & 1.60653000 & 094337030 , \\
\hline 0.5499995 & 0.64000010 & 0.62536590 \\
\hline 1.4492260 & 0.78403890 & 1.08172600 \\
\hline 0.3108478 & 1.47283200, & 0.47580980 \\
\hline 3.1378870 & 0.11715670 & 0.92341850 \\
\hline 0.5124997 & 0.22012280 & 3.81572700 \\
\hline 0.5791140, & 0.50421350 & 0.14532570 \\
\hline 0.7749708 & 1.07792000 & 1.08156300 \\
\hline
\end{tabular}

Table 1: Fisher Matrix Bounds and Approximate Bayesian Confidence Intervals of the Exponential Population Mean When the Population Mean is Equal to 1.

\begin{tabular}{|c|c|c|c|}
\hline Confidence level & Fisher Matrix bounds & $\begin{array}{c}\text { Approx.Bayesian bounds } \\
(S E)\end{array}$ & $\begin{array}{c}\text { Approx.Bayesian } \\
\text { bounds (HT) }\end{array}$ \\
\hline $80 \%$ & $0.7909-1.2621$ & $0.9575-1.0298$ & $0.9575-1.0298$ \\
\hline $90 \%$ & $0.7392-1.3503$ & $0.9368-1.0317$ & $0.9368-1.0317$ \\
\hline $95 \%$ & $0.6985-1.4289$ & $0.9169-1.0326$ & $0.9169-1.0326$ \\
\hline $99 \%$ & $0.6238-1.6002$ & $0.8739-1.0334$ & $0.8739-1.0334$ \\
\hline
\end{tabular}

\begin{tabular}{|l|l|l|}
\hline Confidence level & $\left(l_{F}\right) \div\left(l_{S E}\right)$ & $\left(l_{F}\right) \div\left(l_{H T}\right)$ \\
\hline $80 \%$ & 6.5172 & 6.5172 \\
\hline $90 \%$ & 6.4394 & 6.4394 \\
\hline $95 \%$ & 6.3128 & 6.3128 \\
\hline $99 \%$ & 6.1216 & 6.1216 \\
\hline
\end{tabular}


Example 2

Monte Carlo simulation has been used to generate the following 30 observations from the exponential distribution with mean equal to 9

\begin{tabular}{|lllll|}
\hline & & & & \\
2.0270, & 4.0103, & 30.0421, & 0.1189, & 2.7558. \\
13.7441, & 13.3840, & 27.0930, & 7.3750, & 3.7323, \\
23.4171, & 0.06310. & 5.6839, & 8.7473, & 10.2778, \\
25.2331, & 10.1903, & 0.3761, & 3.3068, & 3.4954, \\
6.9136, & 1.8234, & 16.3160, & 2.4359, & 19.9108, \\
2.5285, & 3.9314, & 3.4645, & 6.9229, & 10.4509. \\
\hline
\end{tabular}

Table 2: Fisher Matrix Bounds and Approximate Bayesian Confidence Intervals of the Exponential Population Mean When the Population Mean is Equal to 9.

\begin{tabular}{|c|c|c|c|}
\hline Confidence level & Fisher Matrix bounds & $\begin{array}{c}\text { Approx.Bayesian bounds } \\
(\mathrm{SE})\end{array}$ & $\begin{array}{l}\text { Approx.Bayesian } \\
\text { bounds (HT) }\end{array}$ \\
\hline $80 \%$ & $7.1184-11.3598$ & $8.6182-9.2688$ & $8.6182-9.2688$ \\
\hline $90 \%$ & $6.6534-12.1537$ & $8.4315-9.2861$ & $8.4315-9.2861$ \\
\hline $95 \%$ & $6.2873-12.8614$ & $8.2527-9.2944$ & $8.2527-9.2944$ \\
\hline $99 \%$ & $5.6144-14.4028$ & $7.8655-9.3009$ & $7.8655-9.3009$ \\
\hline
\end{tabular}

\begin{tabular}{|l|l|l|}
\hline Confidence level & $\left(l_{F}\right) \div\left(l_{S E}\right)$ & $\left(l_{F}\right) \div\left(l_{H T}\right)$ \\
\hline $80 \%$ & 6.5192 & 6.5192 \\
\hline $90 \%$ & 6.4361 & 6.4361 \\
\hline $95 \%$ & 6.3109 & 6.3109 \\
\hline $99 \%$ & 6.1226 & 6.1226 \\
\hline
\end{tabular}


Example 3

Monte Carlo simulation has been used to generate the following 40 observations from the exponential distribution with mean equal to 20

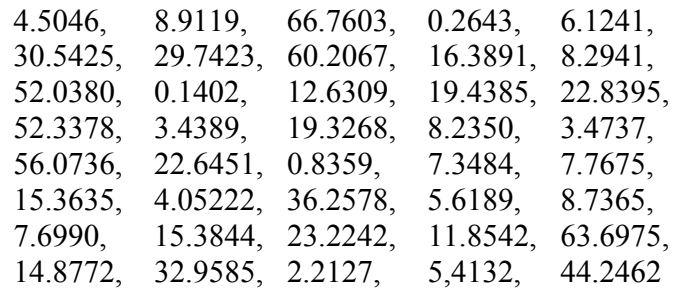

Table 3: Fisher Matrix Bounds and Approximate Bayesian Confidence Intervals of the Exponential Population Mean When the Population Mean is Equal to 20.

\begin{tabular}{|c|c|c|c|}
\hline Confidence level & Fisher Matrix bounds & $\begin{array}{c}\text { Approx.Bayesian bounds } \\
\text { (SE) }\end{array}$ & Approx.Bayesian \\
& & $19.6574-20.7619$ & $19.6574-20.7619$ \\
\hline $80 \%$ & $16.5786-24.8507$ & $19.3330-20.7907$ & $19.3330-20.7907$ \\
\hline $90 \%$ & $15.6366-26.3479$ & $19.0191-20.8045$ & $19.0191-20.8045$ \\
\hline $95 \%$ & $14.8886-27.6715$ & $18.3281-20.8153$ & $18.3281-20.8153$ \\
\hline $99 \%$ & $13.4983-30.5216$ & & \\
\hline
\end{tabular}

\begin{tabular}{|l|l|l|}
\hline Confidence level & $\left(l_{F}\right) \div\left(l_{S E}\right)$ & $\left(l_{F}\right) \div\left(l_{H T}\right)$ \\
\hline $80 \%$ & 7.4894 & 7.4894 \\
\hline $90 \%$ & 7.3480 & 7.3480 \\
\hline $95 \%$ & 7.1596 & 7.1596 \\
\hline $99 \%$ & 6.8443 & 6.8443 \\
\hline
\end{tabular}


Example 4

The following exponential data and results were obtained by Washington State
Department of Ecology while conducting research on the amount of lead concentration in certain types of fish found in the Spokane River.

\begin{tabular}{|c|c|c|c|}
\hline \multicolumn{3}{|c|}{$\begin{array}{c}\text { Lead (Pb) Concentrations in 1999 Spokane River Fish } \\
\text { Source: WA State Dept. of Ecology report } \\
\text { concentrations in parts per million (ppm) }\end{array}$} \\
\hline Filets & trout & whitefish & sucker \\
\cline { 2 - 4 } & 0.480 & 0.020 & 0.088 \\
& 0.071 & 0.020 & 0.210 \\
& 0.110 & 0.020 & 0.280 \\
& 0.320 & 0.020 & 0.030 \\
& 0.120 & 0.020 & 0.036 \\
& 0.220 & 0.065 & 0.047 \\
& 0.055 & 0.020 & 0.077 \\
& 0.320 & 0.037 & 0.069 \\
& 0.077 & 0.020 & 0.160 \\
& 0.081 & 0.036 & 0.088 \\
& 0.170 & & 0.120 \\
& 0.130 & & 0.054 \\
& 0.110 & & 0.080 \\
& 0.081 & & 0.059 \\
& 0.098 & & 0.094 \\
& 0.180 & & 0.059 \\
& 0.230 & & 0.068 \\
& 0.082 & & 0.020 \\
& 0.210 & & 0.090 \\
& 0.200 & & 0.046 \\
& 0.025 & & \\
\hline Mean & 0.038 & & $\mathbf{0 . 0 8 9}$ \\
\hline & $\mathbf{0 . 1 5 5}$ & $\mathbf{0 . 0 2 8}$ & \\
\hline & $\mathbf{0 . 1 1 0}$ & $\mathbf{0 . 0 1 5}$ & \\
\hline
\end{tabular}

Table 4: Fisher Matrix Bounds and Approximate Bayesian Confidence Intervals of the Mean Lead Concentration in Trout.

\begin{tabular}{|c|c|c|c|}
\hline Confidence level & Fisher Matrix bounds & $\begin{array}{c}\text { Approx.Bayesian bounds } \\
(\mathrm{SE})\end{array}$ & $\begin{array}{c}\text { Approx.Bayesian } \\
\text { bounds (HT) }\end{array}$ \\
\hline $80 \%$ & $0.11791-0.20351$ & $0.15280-0.169507$ & $0.15301-0.16976$ \\
\hline $90 \%$ & $0.10896-0.22021$ & $0.14820-0.16996$ & $0.14839-0.17022$ \\
\hline $95 \%$ & $0.10199-0.23526$ & $0.14386-0.17018$ & $0.14404-0.17044$ \\
\hline $99 \%$ & $0.08936-0.26851$ & $0.13471-0.17035$ & $0.13487-0.17061$ \\
\hline
\end{tabular}

\begin{tabular}{|l|l|l|}
\hline Confidence level & $\left(l_{F}\right) \div\left(l_{S E}\right)$ & $\left(l_{F}\right) \div\left(l_{H T}\right)$ \\
\hline $80 \%$ & 5.1236 & 5.1104 \\
\hline $90 \%$ & 5.1125 & 5.0961 \\
\hline $95 \%$ & 5.0634 & 5.0481 \\
\hline $99 \%$ & 5.0266 & 5.0125 \\
\hline
\end{tabular}


Table 5. Fisher Matrix Bounds and Approximate Bayesian Confidence Intervals of the Mean Lead Concentration in Whitefish.

\begin{tabular}{|c|c|c|c|}
\hline Confidence level & Fisher Matrix bounds & $\begin{array}{c}\text { Approx.Bayesian bounds } \\
(\mathrm{SE})\end{array}$ & $\begin{array}{c}\text { Approx.Bayesian } \\
\text { bounds (HT) }\end{array}$ \\
\hline $80 \%$ & $0.01854-0.04167$ & $0.02698-0.03429$ & $0.02556-0.03204$ \\
\hline $90 \%$ & $0.01649-0.04684$ & $0.02528-0.03452$ & $0.02403-0.03224$ \\
\hline $95 \%$ & $0.01495-0.05166$ & $0.02378-0.03464$ & $0.02267-0.03234$ \\
\hline $99 \%$ & $0.01229-0.06285$ & $0.02090-0.03472$ & $0.02004-0.03241$ \\
\hline
\end{tabular}

\begin{tabular}{|l|l|l|}
\hline Confidence level & $\left(l_{F}\right) \div\left(l_{S E}\right)$ & $\left(l_{F}\right) \div\left(l_{H T}\right)$ \\
\hline $80 \%$ & 3.1641 & 3.5694 \\
\hline $90 \%$ & 3.2846 & 3.6967 \\
\hline $95 \%$ & 3.3802 & 3.7962 \\
\hline $99 \%$ & 3.6584 & 4.0873 \\
\hline
\end{tabular}

Table 6. Fisher Matrix Bounds and Approximate Bayesian Confidence Intervals of the Mean Lead Concentration in Sucker.

\begin{tabular}{|c|c|c|c|}
\hline Confidence level & Fisher Matrix bounds & $\begin{array}{c}\text { Approx.Bayesian bounds } \\
\text { (SE) }\end{array}$ & $\begin{array}{c}\text { Approx.Bayesian } \\
\text { bounds (HT) }\end{array}$ \\
\hline $80 \%$ & $0.06666-0.11816$ & $0.08742-0.09803$ & $0.08799-0.09875$ \\
\hline $90 \%$ & $0.06136-0.12835$ & $0.08454-0.09833$ & $0.08507-0.09905$ \\
\hline $95 \%$ & $0.05725-0.13756$ & $0.08183-0.09847$ & $0.08234-0.09919$ \\
\hline $99 \%$ & $0.04984-0.15802$ & $0.07618-0.09858$ & $0.07662-0.09931$ \\
\hline
\end{tabular}

\begin{tabular}{|l|l|l|}
\hline Confidence level & $\left(l_{F}\right) \div\left(l_{S E}\right)$ & $\left(l_{F}\right) \div\left(l_{H T}\right)$ \\
\hline $80 \%$ & 4.8539 & 4.7862 \\
\hline $90 \%$ & 4.8578 & 4.7918 \\
\hline $95 \%$ & 4.8263 & 4.7661 \\
\hline $99 \%$ & 4.8294 & 4.7677 \\
\hline
\end{tabular}


Example 5

The following exponential data represent a random sample of cycles to failure in ten-thousands for twenty heater switches subject to an overload voltage.

$$
\begin{array}{lllll}
0.01, & 0.034, & 0.194, & 0.567, & 0.601, \\
0.712, & 1.291, & 1.367, & 1.949, & 2.37 \\
2.411, & 2.875, & 3.162, & 3.28, & 3.491 \\
3.686, & 3.854, & 4.211, & 4.397, & 6.473
\end{array}
$$

Elfessi and Raineke (2001) conducted some studies on the above data and obtained the following the following maximum likelihood estimate and $95 \%$ confidence interval for the parameter $\theta: 0.4261$ and $(0.2603,0.6322)$.
Table 1, Table 2 and Table 3 show that, in the first three examples, the proposed approximate Bayesian confidence intervals perform better than confidence interval obtained with Fisher Matrix bounds method. All seven Tables show that the proposed approximate Bayesian confidences intervals perform well.

\section{Conclusion}

Approximate Bayesian confidence intervals for parameters of exponential populations under two different loss functions have been derived. The loss functions that are employed are the square error and the Higgins-Tsokos loss functions. Based on the above numerical results, the following may be concluded:

Table 7: Fisher Matrix Bounds and Approximate Bayesian Confidence Intervals of $\theta$

\begin{tabular}{|c|c|c|c|}
\hline Confidence level & Fisher Matrix bounds & $\begin{array}{c}\text { Approx.Bayesian bounds } \\
(\mathrm{SE})\end{array}$ & $\begin{array}{c}\text { Approx.Bayesian } \\
\text { bounds (HT) }\end{array}$ \\
\hline $80 \%$ & $0.32005-0.56732$ & $0.38575-0.43256$ & $0.38575-0.43256$ \\
\hline $90 \%$ & $0.29464-0.61626$ & $0.38460-0.44733$ & $0.38459-0.44733$ \\
\hline $95 \%$ & $0.27491-0.66049$ & $0.38404-0.46210$ & $0.38404-0.46210$ \\
\hline $99 \%$ & $0.23932-0.75871$ & $0.38361-0.49639$ & $0.38361-0.49639$ \\
\hline
\end{tabular}

\begin{tabular}{|l|l|l|}
\hline Confidence level & $\left(l_{F}\right) \div\left(l_{S E}\right)$ & $\left(l_{F}\right) \div\left(l_{H T}\right)$ \\
\hline $80 \%$ & 5.2824 & 5.2824 \\
\hline $90 \%$ & 5.1270 & 5.1262 \\
\hline $95 \%$ & 4.9353 & 4.9395 \\
\hline $99 \%$ & 4.6053 & 4.6053 \\
\hline
\end{tabular}


1. When representative samples are considered, the Fisher Matrix bounds method used to construct confidence intervals for exponential parameters does not always yield the best coverage accuracy.

2. The Fisher Matrix bounds method used to construct confidence intervals for the mean of an exponential population does not always yield the best coverage accuracy. In fact, in Table 1, Table 2 and Table 3, each of the obtained approximate Bayesian confidence intervals contains the population mean and is strictly included in the corresponding confidence interval obtained with Fisher Matrix bounds method.

3. Contrary to Fisher Matrix bounds method that uses the Z-table, the proposed approach relies only on the observations.

4. With the proposed approach, approximate Bayesian confidence intervals for exponential population means are easily computed for any level of significance.

5. Bayesian analysis contributes to reinforcing well-known statistical theories such as the estimation theory.

\section{References}

Nelson, W. (1982). Applied life data analysis. New York: John Wiley \& Sons, Inc..

Hartley, M. A. (2004). A simulation study of the error induced in one-sided reliability confidence bounds for the Weibull distribution using a small sample size with heavily censored data. Storming Media.

Higgins, J. J. \& Tsokos. (1980). A study of the effect of the loss function on Bayes estimates of failure intensity, MTBF, and reliability. Applied Mathematics and Computation, 6, 145-166.
Britney, R. R. \& Winkler, R. L. (1968). Bayesian III point estimation under various loss functions. American Statistical Association, 356-364.

Elfessi, A. \& Raineke, D. M. (2001). Journal of Statistics Education, 9(1).

Canfield, A (1970). Bayesian approach to reliability estimation using a loss function. IEEE Trans. Reliability R-19(1), 13-16.

Camara, V. A. R. \& Tsokos, C. P. (1999). Sensitivity behavior of bayesian reliability analysis for different loss functions. International Journal of Applied Mathematics.

Harris, B. (1976). A survey of statistical methods in system reliability using Bernoulli sampling of components. Proceedings of the conference on the theory and applications of Reliability with emphasis on Bayesian and Nonparametric Methods. New York.

Higgins, J. J. \& Tsokos, C. P. (1976). Comparison of Bayes estimates of failure intensity for fitted priors of life data. Proceedings of the Conference on the Theory an Applications of Reliability with Emphasis on Bayesian and Nonparametric Methods. New York.

Higgins, J. J. \& Tsokos, C. P. (1976). On the behavior of some quantities used in Bayesian reliability demonstration tests. IEEE Trans. Reliability R-25(4), 261-264.

Camara, V. A. R. \& Tsokos, C. P. (1999). The effect of loss functions on empirical Bayes reliability analysis. Journal of Engineering Problems.

Schafer, R. E. et al (1970). Bayesian reliability demonstration: Phase I- data for the a prior distribution. Rome Air Development Center, Griffis AFBNY RADC-TR-69-389.

Schafer, R. E., et al. (1971). Bayesian reliability: Phase II - development of a priori distribution. Rome Air Development Center, Griffis AFR, NY RADC-YR-71-209.

Schafer, R. E., et al (1973). Bayesian reliability demonstration: Phase III development of test plans. Rome Air development Center, Griffs AFB, NY RADCTR-73-39.

Shafer, R. E. \& Feduccia, A. J. (1972). Prior distribution fitted to observed reliability data. IEEE Trans. Reliability R-21 (3), 148-154. 
Tsokos, C. P. \& Shimi, I. (1976). Proceedings of the Conference on the theory and Applications of Reliability with Emphasis on Bayesian and Nonparametric Methods. Methods, 1-2, New York.

Bhattacharya, S. K. (1967). Bayesian approach to life testing and reliability estimation. Journal of the American Statistical Association, 62, 48-62.

Camara, V. A. R. \& Tsokos, C. P. (1999). Bayesian estimate of a parameter and choice of the loss function. Nonlinear Studies Journal.
Drake, A. W. (1966). Bayesian statistics for the reliability engineer. Proceeding of Annual Symposium On Reliability, 315-320.

Camara, V. A. R. \& Tsokos, C. P. (1996). Effect of loss functions on Bayesian reliability analysis. Proceedings of International Conference On Nonlinear Problems in Aviation and Aerospace, 75-90.

Winkler, R. L. (1972). Introduction to Bayesian inference and decision making. 174179, 395-397. 


\title{
Better Binomial Confidence Intervals
}

\author{
James F. Reed III \\ Lehigh Valley Hospital \& Health Network
}

The construction of a confidence interval for a binomial parameter is a basic analysis in statistical inference. Most introductory statistics textbook authors present the binomial confidence interval based on the asymptotic normality of the sample proportion and estimating the standard error - the Wald method. For the one sample binomial confidence interval the Clopper-Pearson exact method has been regarded as definitive as it eliminates both overshoot and zero width intervals. The Clopper-Pearson exact method is the most conservative and is unquestionably a better alternative to the Wald method. Other viable alternatives include Wilson's Score, the Agresti-Coull method, and the Borkowf SAIFS-z.

Key words: Binomial distribution, confidence intervals, coverage probability, Wald method, ClopperPearson Method, Score Method, Agresti-Coull method.

\section{Introduction}

The International Committee of Medical Journal editors indicated that confidence intervals are preferred over simple point estimates and pvalues. This applies to over 300 international medical/scientific journals. Most introductory statistics textbook authors present the binomial confidence interval based on the asymptotic normality of the sample proportion and estimating the standard error. This approximate method is referred to as the Wald interval. In order to avoid approximation, some advanced statistics textbooks recommend the ClopperPearson exact binomial confidence interval. Other methods, asymptotic as well as exact, have been proposed and appear sporadically in introductory textbooks. There is a rather large

James Reed III is the Interim Chief of Health Studies and Director of Research at Lehigh Valley Hospital and Health Network. He has published over 100 journal articles and book chapters. His interests include applied statistical analyses, medical education, and statistical methods in simulation studies. Email: James_F.Reed@1vh.com set of articles, primarily in the statistics literature, about these and other less common methods of constructing binomial confidence intervals.

The purpose of this article is to provide a review of alternatives to the Wald method for computing a binomial confidence interval and provide a set of tractable and better methods of constructing binomial confidence intervals for a single proportion.

\section{Methodology}

When a binomial confidence interval is reported, the computational method is rarely given. This may imply that there is only one standard method for computing a binomial confidence interval - the Wald method (W). The W binomial confidence interval, either with or without a continuity correction, is found in every introductory statistics text. Typically, a warning or rule of thumb for determining when not to use $\mathrm{W}$ is included, but usually ignored. Occasionally, the Wald with a continuity correction (WCC) is included. For a single proportion the W and WCC lower bound (LB) and upper bound (UB) are defined as: 


$$
\begin{gathered}
\text { W LB }=p-z_{\alpha / 2} \sqrt{[p q / n]} \\
\text { W UB }=p+z_{\alpha / 2} \sqrt{[p q / n]}, \\
\text { WCC LB }=p-\left(z_{\alpha / 2} \sqrt{[p q / n]}+1 /(2 n)\right) \\
\text { WCC UB }=p+\left(z_{\alpha / 2} \sqrt{[p q / n]}+1 /(2 n)\right)
\end{gathered}
$$

where $\mathrm{p}=\mathrm{r} / \mathrm{n}, \mathrm{q}=1-\mathrm{p}, \mathrm{r}=$ number of successes, and $\mathrm{n}$ is the total sample size.

Even though these two confidence interval methods are similar to large-sample formulas for means, both the W and WCC confidence intervals behave poorly in terms of zero width intervals and overshoot (Beal, 1987; Vollset, 1993; Newcombe, 1998; Pires, 2002; Rieczigel, 2003; Agresti, 2003). For instance, when $\mathrm{r}=0$ or $\mathrm{n}, \mathrm{W}$ and WCC have zero width or degenerate confidence intervals. Despite the known poor performance of the $\mathrm{W}$ and $\mathrm{WCC}$ confidence intervals, they continue to dominate in statistics textbooks, typically accompanied by warnings that when $\mathrm{np}$ is small, usually less than 5 or 10, exact or score methods should be used. A slightly different version of the rule of thumb requires that npq should be greater than or equal to 5 . A better rule is to not compute confidence bounds for a proportion using the $\mathrm{W}$ method but rather to use one of the better methods. For small proportions the calculated lower bound can be below zero. Conversely, when a proportion approaches one, such as in the sensitivity and specificity of diagnostic or screening tests, and the upper bound may exceed one. This overshoot is avoided by truncating the interval to lie within $[0,1]$. Overshoot and zero width confidence intervals may be avoided by a variety of better methods.

One of the standard measures of binomial confidence interval performance is the coverage probability, $C(\pi \mid n, \alpha)$. Given $X=k, n$, and $\alpha$, let $\delta(\pi \mid k, n, \alpha)=1$ if $\pi \in[\operatorname{LB}(k, n, \alpha)$, $\mathrm{UB}(\mathrm{k}, \mathrm{n}, \alpha)]$, and $\delta(\pi \mid \mathrm{k}, \mathrm{n}, \alpha)=0$ otherwise. Then, $\mathrm{C}(\pi \mid \mathrm{n}, \alpha)$ for a given $\pi$ is:

$$
\mathrm{C}(\pi \mid \mathrm{n}, \alpha)=\Sigma \mathrm{P}(\mathrm{X}=\mathrm{k} \mid \mathrm{n}, \pi) \delta(\pi \mid \mathrm{k}, \mathrm{n}, \alpha)
$$

Figure 1 shows the $95 \%$ confidence interval coverage probability of the standard Wald methods $\{\mathrm{W}, \mathrm{WCC}\}$ as a function of $\pi, \pi$ $\in[0,1]$, for $n=20$. The coverage probability curves demonstrate the subnomial coverage for values of $\pi$ near 0 and 1 .
The Clopper-Pearson (CP) binomial confidence interval is the best-known exact method for interval estimation and is considered by most to be the gold standard (Clopper \& Pearson, 1934). The CP confidence interval eliminates overshoot and zero width intervals and is strictly conservative. The CP lower and upper limits are defined by inverting the exact binomial tests with equal-tailed acceptance regions.

$$
\begin{aligned}
& \text { CP } \quad L B=0 \text { if } x=0,(\alpha / 2)^{1 / n} \text { if } x=n \text {. } \\
& \mathrm{LB}=\left[1+(\mathrm{n}-\mathrm{r}+1) /\left(\mathrm{r} \times \mathrm{F}_{2 \mathrm{r}, 2(\mathrm{n}-\mathrm{r}+1), 1-\alpha / 2)]^{-1}}\right.\right. \\
& \mathrm{CP} \quad \mathrm{UB}=1-(\alpha / 2)^{1 / \mathrm{n}} \text { if } \mathrm{x}=0,1 \text { if } \mathrm{x}=\mathrm{n} \text {. }
\end{aligned}
$$

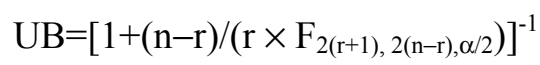

Fleiss (1981) preferred a more computationally intense binomial confidence interval with a continuity correction (SCC) attributed to Wilson (Wilson, 1927). For a single proportion, Wilson's Score (S) and Wilson's Score with continuity correction (SCC) LB and $\mathrm{UB}$ are defined as:

$$
\begin{aligned}
& S \mathrm{LB}=\left(2 n p+z^{2}-z \sqrt{ }\left\{z^{2}+4 n p q\right\}\right) / 2\left(n+z^{2}\right) \\
& S \text { UB }=\left(2 n p+z^{2}+z \sqrt{ }\left\{z^{2}+4 n p q\right\}\right) / 2\left(n+z^{2}\right)
\end{aligned}
$$

SCC LB $=$

$\left[2 n p+z^{2}-1-z \sqrt{ }\left\{z^{2}-2-1 / n+4 p(n q+1)\right\}\right] /\left(2 n+2 z^{2}\right)$

SCC UB $=$

$\left[2 n p+z^{2}+1+z \sqrt{ }\left\{z^{2}+2-1 / n+4 p(n q-1)\right\}\right] /\left(2 n+2 z^{2}\right)$

Blyth and Still (1983) investigated the performance of W, WCC, CP, Sterne's binomial confidence interval method (Sterne, 1954), and Pratt's (P) approximate confidence interval method (Pratt, 1968). Their results demonstrate the need for a continuity correction even when $n$ is large. Blythe and Still then suggested a modification to W (WBS). While the WBS was an improvement over W and WCC, they concluded that it still was not 

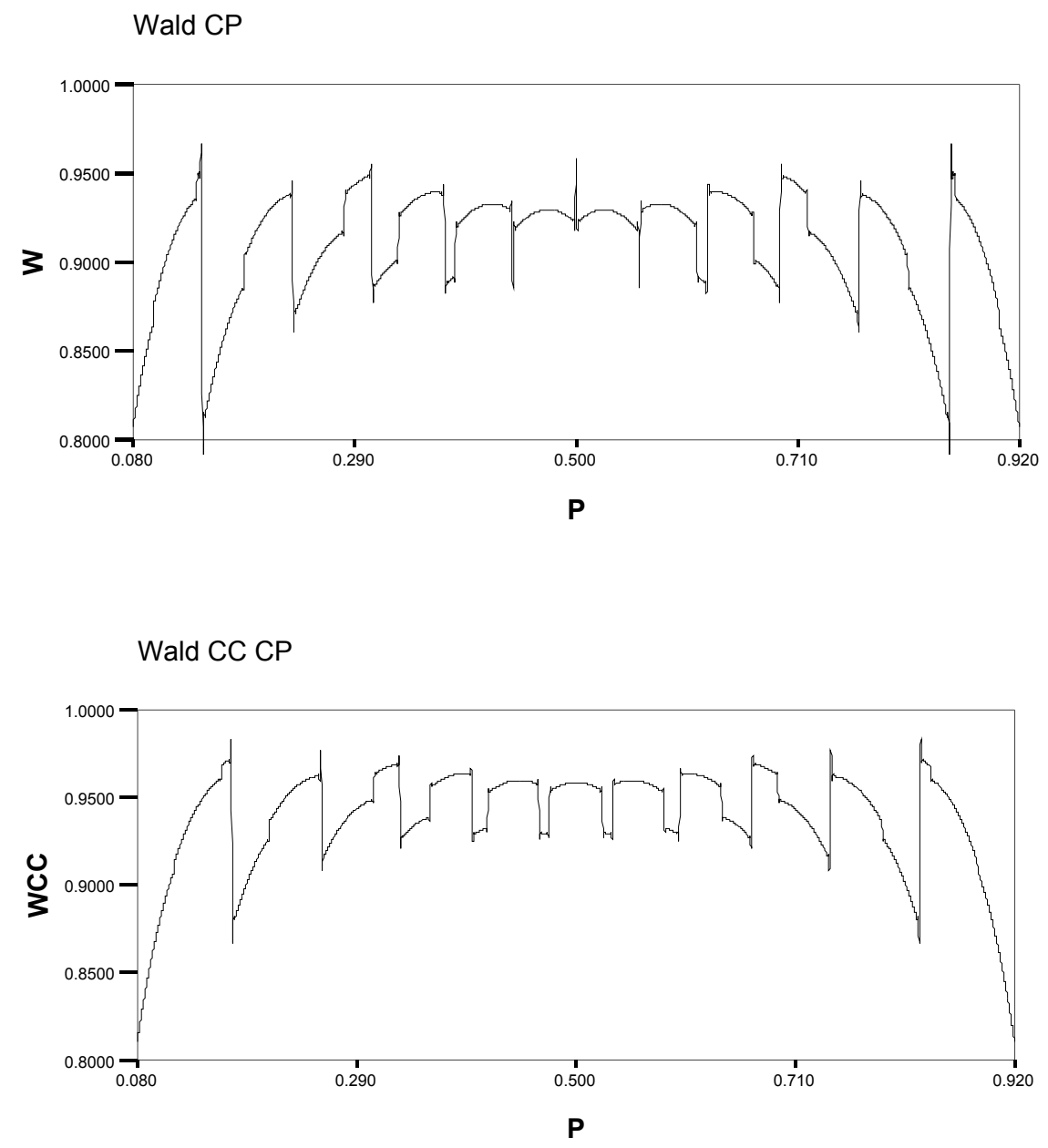

Figure 1. Coverage Probabilities $(n=20)$ for the Wald and Wald CC Binomial Confidence Interval Methods.

satisfactory. The LB and UB for WBS are defined as:

$$
L B=p-\left[z / \sqrt{ }\left(n-z^{2}-2 z / \sqrt{ } n-1 / n\right][\sqrt{ }(p q)+1 / 2 n],\right.
$$

except $\mathrm{LB}=0$ when $\mathrm{r}=0$.

$$
\mathrm{UB}=\mathrm{p}+\left[\mathrm{z} / \sqrt{ }\left(\mathrm{n}-\mathrm{z}^{2}-2 \mathrm{z} / \sqrt{ } \mathrm{n}-1 / \mathrm{n}\right][\sqrt{ }(\mathrm{pq})+1 / 2 \mathrm{n}],\right.
$$

except $\mathrm{UB}=1$ for $\mathrm{r}=\mathrm{n}$.

Vollset (Vollset, 1993) compared thirteen methods for computing binomial confidence intervals using evaluative criteria of $\mathrm{C}(\mathrm{P})$, interval width, and errors relative to limits. Vollset proposed a mean Pratt (MP), a modification of $\mathrm{P}$ that is a closed form approximation to the mid-P exact interval. Define the UB of $P$ as:

$$
\left.\mathrm{P} \mathrm{UB}=[1+(\mathrm{r}+1) /(\mathrm{n}-\mathrm{r}))^{2}((\mathrm{~A}-\mathrm{b}) / \mathrm{c})^{3}\right]^{-1}
$$

with

$$
\begin{gathered}
A=81(r+1)(n-r)-9 n-8, \\
B=3 z \sqrt{ }\left[9(r+1)(n-r)\left(9 n+5 z^{2}\right)+n+1\right],
\end{gathered}
$$


and

$$
\mathrm{C}=81(\mathrm{r}+1) 2-9(\mathrm{r}+1)\left(2+\mathrm{z}^{2}\right)+1 .
$$

For P LB, replace $\mathrm{r}$ with $\mathrm{r}-1$ and $\mathrm{z}$ with $-\mathrm{z}$.

The Vollset MP lower and upper bound are then defined as:

$$
\begin{aligned}
& \text { MP LB }=\left\{\mathrm{P}_{1}(\mathrm{r})+\mathrm{P}_{1}(\mathrm{r}+1)\right\} / 2, \\
& \mathrm{MP} \mathrm{UB}=\left\{\mathrm{P}_{\mathrm{u}}(\mathrm{r})+\mathrm{P}_{\mathrm{u}}(\mathrm{r}-1)\right\} / 2
\end{aligned}
$$

Vollset argued that $\mathrm{W}$ and WCC were unsatisfactory and the Clopper-Pearson, Pratt's approximation, SCC, MP, S and SCC are methods that may be safely used in all applications.

Newcombe (1998) compared seven methods for constructing two-sided binomial confidence intervals (W, WCC, S, SCC, Clopper-Pearson, mid-P and a likelihood-based method). The W and WCC were quickly judged as being inadequate, highly anti-conservative, asymmetrical in coverage, and incurred a higher risk of unacceptable boundary limits. Newcombe argued that neither W nor WCC should be acceptable methods for the scientific literature since other methods are tractable and all perform much better. Newcombe further argued that the use of the simple asymptotic standard error of a proportion should be restricted to sample size planning and introductory teaching purposes. Newcombe preferred three methods: the Clopper-Pearson method, the Score method and mid-P binomial based method.

Agresti and Coull, in noting the poor performance of the Wald interval and conservativeness of the Clopper-Pearson interval, proposed a straightforward adjustment the add 4 to Wald. They suggested that by simply adding two successes and two failures and then use the Wald formula. Alternatively, one could add $\mathrm{z}^{2} / 2$ successes and $\mathrm{z}^{2} / 2$ failures before computing the Wald confidence interval.
The latter is preferred. The Agresti-Coull adjusted Wald (AC) lower and upper bounds are:

$$
\begin{gathered}
L B=p^{\prime}-z \sqrt{ }\left[p^{\prime} q^{\prime} / n^{\prime}\right], \\
U B=p^{\prime}+z \sqrt{ } \sqrt{\left.p^{\prime} q^{\prime} / n^{\prime}\right], \text { where }} \\
\mathrm{p}^{\prime}=\left(2 \mathrm{r}+\mathrm{z}^{2}\right) /\left(2 \mathrm{n}+\mathrm{z}^{2}\right), \text { and } \mathrm{n}^{\prime}=\mathrm{n}+\mathrm{z}^{2}
\end{gathered}
$$

Pires (2002) compared twelve methods for constructing confidence intervals for a binomial proportion and concluded that a clear classification of conservative methods included the Clopper-Pearson, the Score, and two arcsine transformation methods. A second tier of recommended confidence interval construction methods included a Bayesian method and the SCC.

Agresti (2003) argued for reducing the effects of discreteness in binomial confidence intervals by inverting two-sided tests rather than two one-sided tests. In most statistical practice, for interval estimation of a proportion or a difference or ratio of proportions, the inversion of the asymptotic score test is the best choice. If one wants to be a bit more conservative, mid-P adaptations or the Clopper-Pearson are recommended. For teaching purposes, the Waldtype interval plus and minus a normal-score multiple of a standard error is simplest.

Reiczigel compared four methods for constructing binomial confidence intervals: Wilson's Score, Agresti and Coull Adjusted Wald, the Clopper-Pearson, the mid-P, and Sterne's interval (Rieczigel, 2003). Unique to this study is the recommendation of using the Sterne interval and the Agresti-Coull adjusted Wald interval for binomial confidence intervals.

Tobi et al. (2005) compared the performance of seven approximate methods and the exact Copper-Pearson exact confidence intervals for small proportions. Three criteria were used to evaluate the performance of confidence intervals; coverage, confidence interval width, and aberrant confidence intervals. They concluded that: (1) one should 
compute confidence intervals for small proportions even when the number of events equals zero, (2) report what method has been used for confidence interval calculation, (3) the W method should be discarded, and (4) the Clopper-Pearson and the SCC are the best choices to calculate confidence intervals for small proportions.

Borkowf (2005) argued that even though the Agresti-Coull method binomial confidence intervals are substantially better than the Wald method, it can yield sub nominal coverage for some values of $\pi$ for moderate sample sizes. A binomial confidence interval, which results in near nominal coverage and is easy to calculate by first augmenting the original data with a single imaginary failure to compute the lower confidence bound and a single imaginary success to compute the upper confidence bound is proposed - a single augmentation with an imaginary failure or success (SAIFS) method. The lower and upper SAIFS confidence bounds are then:

$$
\text { SAIFS LB }=p_{1}-\xi_{1-\alpha / 2} \sqrt{ }\left[p_{1} q_{1} / n\right]
$$

and

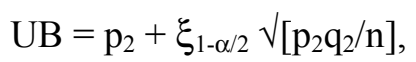

with

$$
\mathrm{p}_{1}=(\mathrm{r}+0) /(\mathrm{n}+1) \text { and } \mathrm{p}_{2}=(\mathrm{r}+1) /(\mathrm{n}+1)
$$

Borkowf (2005) evaluated two forms of the SAIFS. The first uses the z-quantiles $\left(\xi_{1-\alpha / 2}\right)$ and the second used the t-quantiles $\left(\tau_{n-1,1-\alpha / 2}\right)$. Compared to the Clopper-Pearson method, the SAIFS method using either the $\mathrm{z}$ or $\mathrm{t}$ quantiles results in confidence intervals with mean widths that are narrower for proportion parameters near 0 or 1 and whose coverage probabilities are marginally better over all values of $\pi$. The SAIFS-Z is preferred.
Figure 2 shows the $95 \%$ confidence interval coverage probability as a function of $\pi$, $\pi \in[0,1]$, for $\mathrm{n}=20$ for CP, WBS, S, SCC, AC, and SAIFS-Z. Note that the sawtooth appearance of the coverage functions is due to the discontinuities for values of $p$ corresponding to any lower or upper limits in the set of $n+1$ confidence intervals. The Clopper-Pearson and Borkowf SAIFS-z methods give at least nominal coverage for all values of $\pi \in[0,1]$, with severe over coverage near 0 and 1 . The Score CC method gives at least nominal coverage for all values of $\pi \in[0,1]$ and avoids the over coverage of either the Clopper-Pearson or Score methods. The Score and Agresti-Coull methods yield nearly nominal coverage for all values of $\pi \in$ $[0,1]$.

\section{Conclusion}

For the one sample binomial confidence interval, a new generation of introductory and medical statistics textbooks should emphasize the poor performance properties of W, WCC and include better binomial confidence methods. At least one from the set of Clopper-Pearson, S, SCC, Agresti-Coull, or the SAIFS-z methods should be mentioned. With the widespread use of laptop computers and access to computing resources on the internet, the complexity of computing binomial confidence intervals should not be an issue. The question remains as to which method to use. The Clopper-Pearson exact method has been regarded as definitive as it eliminates both overshoot and zero width intervals. The Clopper-Pearson exact method is the most conservative and is unquestionably a better alternative to the $\mathrm{W}$ when constructing and reporting binomial confidence intervals. In terms of programming ease, the Clopper-Pearson is easily programmed as are the Blythe \& Still, Wilson's Score, Score with a continuity correction, the Agresti-Coull method, and the Borkowf SAIFS-z. 

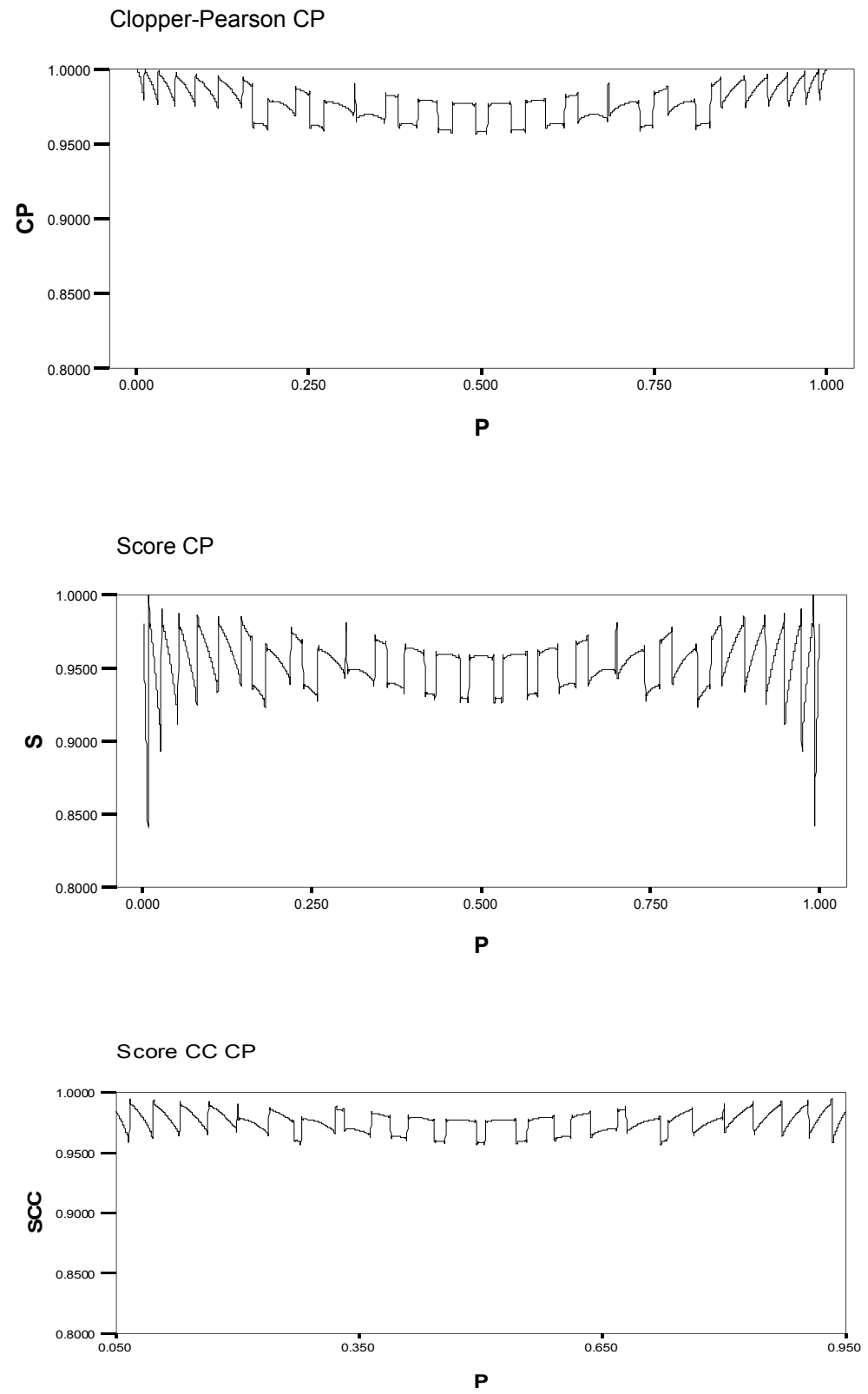

Figure 2. Coverage Probabilities $(\mathrm{n}=20)$ for the Clopper-Pearson, Score, Score CC, Agresti-Coull, and Borkowf SAIFS-z Binomial Confidence Interval Methods. 

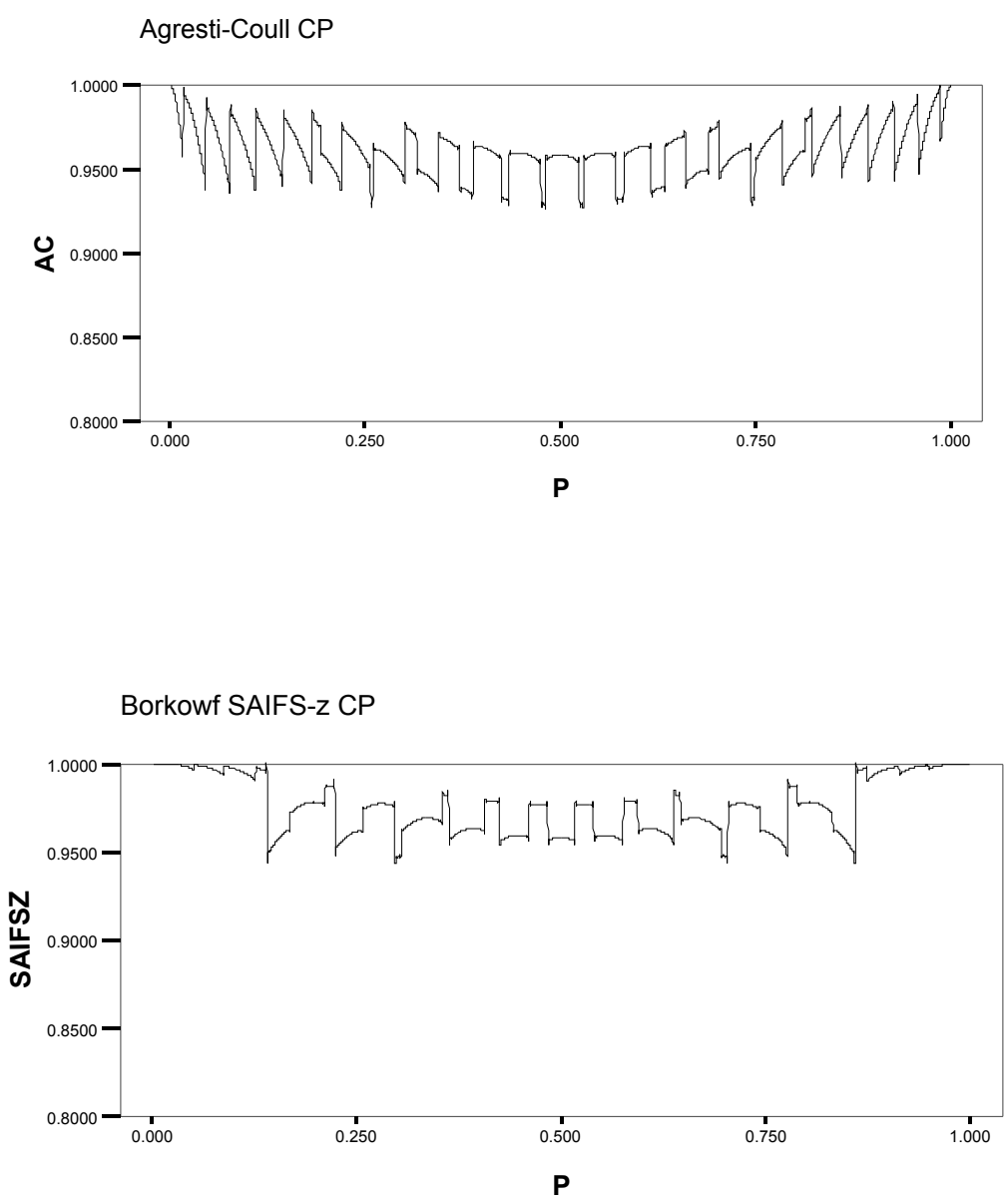

Figure 2 (Continued). Coverage Probabilities $(\mathrm{n}=20)$ for the Clopper-Pearson, Score, Score CC, Agresti-Coull, and Borkowf SAIFS-z Binomial Confidence Interval Methods. 
Table 1. Methods for Calculation of Confidence Intervals for a Single Proportion

Method Formula

Clopper-Pearson

$\mathrm{CP}$

$\mathrm{LB}=0$ if $\mathrm{x}=0,(\alpha / 2)^{1 / \mathrm{n}}$ if $\mathrm{x}=\mathrm{n}$.

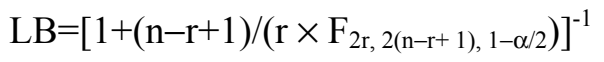

$U B=1-(\alpha / 2)^{1 / n}$ if $x=0,1$ if $x=n$.

$\mathrm{UB}=\left[1+(\mathrm{n}-\mathrm{r}) /\left(\mathrm{r} \times \mathrm{F}_{2(\mathrm{r}+1), 2(\mathrm{n}-\mathrm{r}), \alpha / 2)}\right]^{-1}\right.$

Score (Wilson)

$\mathrm{S}$

$\mathrm{LB}=\left(2 \mathrm{np}+\mathrm{z}^{2}-\mathrm{z} \sqrt{ }\left\{\mathrm{z}^{2}+4 n p q\right\}\right) / 2\left(\mathrm{n}+\mathrm{z}^{2}\right)$

$\mathrm{UB}=\left(2 \mathrm{np}+\mathrm{z}^{2}+\mathrm{z} \sqrt{ }\left\{\mathrm{z}^{2}+4 \mathrm{npq}\right\}\right) / 2\left(\mathrm{n}+\mathrm{z}^{2}\right)$

Score $(\mathrm{w} / \mathrm{CC})$

SCC

$\mathrm{LB}=\left[2 \mathrm{np}+\mathrm{z}^{2}-1-\mathrm{z} \sqrt{ }\left\{\mathrm{z}^{2}-2-1 / \mathrm{n}+4 \mathrm{p}(\mathrm{nq}+1)\right\}\right] /\left(2 \mathrm{n}+2 \mathrm{z}^{2}\right)$

$\mathrm{UB}=\left[2 \mathrm{np}+\mathrm{z}^{2}+1+\mathrm{z} \sqrt{ }\left\{\mathrm{z}^{2}+2-1 / \mathrm{n}+4 \mathrm{p}(\mathrm{nq}-1)\right\}\right] /\left(2 \mathrm{n}+2 \mathrm{z}^{2}\right)$

Agresti-Coull

$\mathrm{AC}$

$L B=p^{\prime}-z \sqrt{ }\left[p^{\prime} q^{\prime} / n^{\prime}\right]$

$\mathrm{UB}=\mathrm{p}^{\prime}+\mathrm{z} \sqrt{ }\left[\mathrm{p}^{\prime} \mathrm{q}^{\prime} / \mathrm{n}^{\prime}\right]$, where

$\mathrm{p}^{\prime}=\left(2 \mathrm{r}+\mathrm{z}^{2}\right) /\left(2 \mathrm{n}+\mathrm{z}^{2}\right)$, and $\mathrm{n}^{\prime}=\mathrm{n}+\mathrm{z}^{2}$.

Borkowf

SAIFS

$\mathrm{LB}=\mathrm{p}_{1}-\xi_{1-\alpha / 2} \sqrt{\left[\mathrm{p}_{1} \mathrm{q}_{1} / \mathrm{n}\right]}$

$\mathrm{UB}=\mathrm{p}_{2}+\xi_{1-\alpha / 2} \sqrt{ }\left[\mathrm{p}_{2} \mathrm{q}_{2} / \mathrm{n}\right]$, with

$\mathrm{p}_{1}=(\mathrm{r}+0) /(\mathrm{n}+1)$ and $\mathrm{p}_{2}=(\mathrm{r}+1) /(\mathrm{n}+1)$, where

$\xi_{1-\alpha / 2}$ are z-quantiles or $\tau_{\mathrm{n}-1,1-\alpha / 2}$ the t-quantiles 


\section{References}

Agresti A. \& Coull B. A. (1998). Approximate is better than 'exact' for interval estimation of binomial proportions. The American Statistician, 52, 119-126.

Agresti, A. \& Min, Y. (2001). On smallsample confidence intervals for parameters in discrete distributions. Biometrics, 57, 963-71.

Agresti, A. (2003). Dealing with discreteness: Making 'exact' confidence intervals for proportions, differences of proportions, and odds ratios more exact. Statistical Methods Medical Research, 12, 3-21.

Blyth, C. R. \& Still, H. A. (1983). Binomial confidence intervals. Journal of the American Statistical Association, 78, 108-116.

Bonett, D. G. \& Price, R. M. (2005). Confidence intervals for a ratio of binomial proportions based on paired data. Statistical Methods Medical Research,15.

Borkowf, C. B. (2005). Constructing binomial confidence intervals with near nominal coverage by adding a single imaginary failure or success. Statistical Methods Medical Research, 25 .

Clopper, C. J. \& Pearson, E. S. (1934). The use of confidence or fiducial limits illustrated in the case of the binomial. Biometrika, 26, 404-413.

Fleiss, J. H. (1981). Statistical methods for rates and proportions ( $2^{\text {nd }}$ Ed.). New York: John Wiley \& Sons.

Newcombe, R. G. (1998). Two-sided confidence intervals for the single proportion: Comparison of seven methods. Statistical Methods Medical Research, 17, 857-72.
Pires, A. M. (2002). Confidence intervals for a binomial proportion: Comparison of methods and software evaluation. Proceedings of the Conference ComStat 2002. http://www.math.ist.utl.pt/ apires.

Pratt, J. W. (1968). A normal approximation for binomial, F, Beta, and other common, related tail probabilities. Journal of the American Statistical Association, 63, 14571483.

Radhakrishna, S., Murthy, B. N., Nair, N. G. K., Jayabal, P., \& Jayasri, R. (1992). Confidence intervals in medical research. Indian Journal of Medical Research [B], 96, 199-205.

Reiczigel, J. (2003). Confidence intervals for the binomial parameter: Some new considerations. Statistical Methods Medical Research, 22, 611-21.

Sterne, T. E. (1954). Some remarks on confidence or fiducial limits'. Biometrika, 41, 275-278.

Tobi, H., van den Berg, P. B., \& deJongvan den Berg, L. T. W. (2005). Small proportions: What to report for confidence intervals. Pharmacoepidemiology and Drug Safety, 14, 239-247.

Vollset, S. E. (1993). Confidence intervals for a binomial proportion. Statistical Methods Medical Research, 12, 809-24.

Wilson, E. B. (1927). Probable inference, the law of succession, and statistical inference. Journal of the American Statistical Association, 22, 209-212. 


\title{
A Comparison of Eight Shrinkage Formulas under Extreme Conditions
}

\author{
David A. Walker \\ Northern Illinois University
}

The performance of various shrinkage formulas for estimating the population squared multiple correlation coefficient $\left(\rho^{2}\right)$ were compared under extreme conditions often found in educational research with small sample sizes of $10,15,20,25,30$ and regressor variates ranging from 2 to 4 . A new formula for estimating $\rho^{2}$, Adj $R^{2}{ }_{D W}$, was examined in terms of its performance under various conditions of $\mathrm{N}, \mathrm{p}, \rho^{2}$, along with its bias properties and standard error estimates. The two shrinkage formulas that performed most consistently were the Claudy ( $\operatorname{Adj~}^{2}{ }_{C}$ ) and Walker (Adj $\left.R^{2}{ }_{D W}\right)$.

Key Words: Adjusted $\mathrm{R}^{2}$, shrinkage, population squared multiple correlation

\section{Introduction}

Various shrinkage formulas for estimating the population squared multiple correlation coefficient $\left(\rho^{2}\right)$ has been the topic of interest (cf. Carter, 1979; Claudy, 1978; Huberty \& Mourad, 1980; Lucke \& Embretson, 1984). The purpose of this article is to compare the performance of eight shrinkage formulas for estimating the population multiple correlation coefficient with small sample sizes of 10, 15, 20, 25, 30 and with regressor variates ranging from 2 to 4 . Small sample sizes were used because in applied research fields, such as educational research, these sample conditions often are encountered (Claudy, 1972; Huberty \& Mourad, 1980). Also, regressor variates were chosen to be between 2 and 4 for the same reason cited formerly with sample size; typicality of conditions frequently encountered in educational research.

David Walker is Associate Professor of Educational Research and Assessment at Northern Illinois University. His research interests include structural equation modeling, effect sizes, factor analyses, predictive discriminant analysis, predictive validity, weighting, and bootstrapping. Email him at P60DAW1@wpo.cso.niu.edu
The sample squared multiple correlation coefficient, or $\mathrm{R}^{2}$, indicates the percentage of variance in the dependent variable explained by the linear combination of the independent variables. $\mathrm{R}^{2}$ has been found to overestimate the population multiple correlation $\left(\rho^{2}\right)$ and, hence, is seen as an upwardly biased approximation of $\rho^{2}$ with limited accuracy (Agresti \& Finlay, 1997; Pedhazur, 1997). This overestimation has been linked to the problem of error, often either measurement or sampling error, connected to the variability found in random independent variables (Claudy, 1972), related to sample size, and associated with the number of $X$ variables in a model (Huberty \& Mourad, 1980; Shumacker, Mount, \& Monahan, 2002). The population multiple correlation can be expressed as (Browne, 1975):

$$
\rho^{2}=\operatorname{corr}^{2}\left\{\mathrm{Y}, \sim \mathrm{Y}\left(\mathrm{X} \mid \beta_{0}, \beta\right)\right\}
$$

where,

$\mathrm{Y}=$ Dependent variable

$\mathrm{X}=$ Set of regressors

$\beta=$ Population regression weights

Due to amending for this overestimation, the adjusted $R^{2}$ (adj $R^{2}$ ) has been used as a more accurate method than $\mathrm{R}^{2}$ for estimating $\rho^{2}$. That is, the adj $R^{2}$ is more exact than $\mathrm{R}^{2}$ due to its correction for shrinkage and its ability to produce an accurate estimate of the population value for $\rho^{2}$. Adjusted $R^{2}$ can be 
expressed as (Agresti \& Finlay, 1997):

$$
\mathrm{R}_{\text {adj }}^{2}=\mathrm{R}^{2}-\frac{\mathrm{p}-1}{\mathrm{~N}-\mathrm{p}} *\left(1-\mathrm{R}^{2}\right)
$$

Other shrinkage formulas for estimating the population multiple correlation coefficient have been presented with the goal of reducing the positive bias of $\mathrm{R}^{2}$. As noted by Carter (1979), many of the subsequent formulas are decidedly related algebraically and/or are hybrids of one another.

Formulas 3 to 6 and 9 are reproduced in Huberty and Mourad (1980). According to Huberty and Mourad, Smith proposed, but presented by Ezekiel (1929), the first adjusted $\mathrm{R}^{2}$ shrinkage formula, $\mathrm{R}_{\mathrm{S}}^{2}$, where:

$\mathrm{R}_{\mathrm{S}}^{2}=1-\frac{\mathrm{N}}{\mathrm{N}-\mathrm{p}-1} \quad * \quad\left(1-\mathrm{R}^{2}\right)$

Ezekiel (1930) proposed $\mathrm{R}_{\mathrm{E}}^{2}$, where:

$\mathrm{R}_{\mathrm{E}}^{2}=1-\frac{\mathrm{N}-1}{\mathrm{~N}-\mathrm{p}-1} *\left(1-\mathrm{R}^{2}\right)$

Wherry (1931) proposed $\mathrm{R}^{2}$, where:

$\mathrm{R}^{2}{ }_{\mathrm{W}}=1-\frac{\mathrm{N}-1}{\mathrm{~N}-\mathrm{p}} *\left(1-\mathrm{R}^{2}\right)$

Olkin and Pratt (1958) proposed $\mathrm{R}_{\mathrm{OP}}^{2}$, where:

$$
\begin{aligned}
& \mathrm{R}^{2}{ }_{\mathrm{OP}}=1-\frac{\mathrm{N}-3}{\mathrm{~N}-\mathrm{p}-1} *\left(1-\mathrm{R}^{2}\right)- \\
& \frac{2(\mathrm{~N}-3)}{(\mathrm{N}-\mathrm{p}-1)(\mathrm{N}-\mathrm{p}+1)} \quad *\left(1-\mathrm{R}^{2}\right)^{2}
\end{aligned}
$$

Pratt (1964 as cited in Claudy, 1978) proposed $\mathrm{R}_{\mathrm{P}}^{2}$, where:

$$
\begin{aligned}
& \mathrm{R}_{\mathrm{P}}^{2}=1-\frac{(\mathrm{N}-3) *\left(1-\mathrm{R}^{2}\right)}{\mathrm{N}-\mathrm{p}-1} * \\
& 1+\frac{2\left(1-\mathrm{R}^{2}\right)}{(\mathrm{N}-\mathrm{p}-2.3)}
\end{aligned}
$$

Herzberg (1969 as cited in Claudy, 1978) proposed $\mathrm{R}_{\mathrm{H}}^{2}$, where:

$$
\begin{aligned}
& \mathrm{R}_{\mathrm{H}}^{2}=1-\frac{(\mathrm{N}-3) *\left(1-\mathrm{R}^{2}\right)}{\mathrm{N}-\mathrm{p}-1} * \\
& 1+\frac{2\left(1-\mathrm{R}^{2}\right)}{(\mathrm{N}-\mathrm{p}+1)}
\end{aligned}
$$

Claudy (1978) proposed $\mathrm{R}_{\mathrm{C}}^{2}$, where:

$$
\begin{aligned}
& \mathrm{R}_{\mathrm{C}}^{2}=1-\frac{\mathrm{N}-4}{\mathrm{~N}-\mathrm{p}-1} *\left(1-\mathrm{R}^{2}\right)- \\
& \frac{2(\mathrm{~N}-4)}{(\mathrm{N}-\mathrm{p}-1)(\mathrm{N}-\mathrm{p}+1)} *\left(1-\mathrm{R}^{2}\right)^{2}
\end{aligned}
$$

Walker (2006) proposed $\mathrm{R}^{2}{ }_{\mathrm{DW}}$, which is an algebraic alteration of $\mathrm{R}_{\mathrm{C}}^{2}$ and, hence, $\mathrm{N}-4.15$ was a more optimal empirical modification of $\mathrm{N}$ -4 than $\mathrm{N}-5$, where:

$$
\begin{gathered}
\mathrm{R}^{2}{ }_{\mathrm{DW}}=1-\frac{\mathrm{N}-4.15}{\mathrm{~N}-\mathrm{p}-1} *\left(1-\mathrm{R}^{2}\right)- \\
\frac{2(\mathrm{~N}-4.15)}{(\mathrm{N}-\mathrm{p}-1)(\mathrm{N}-\mathrm{p}+1)}
\end{gathered}
$$

where,

$\mathrm{N}=$ Sample size

$p=$ Number of $X$ variables

$\mathrm{R}^{2}=$ Multiple correlation coefficient

\section{Methodology}

Via a simulation program written in SPSS (Statistical Package for the Social Sciences) v. 12.0, the following study reviewed the shrinkage performance of the eight multiple correlation estimators noted previously when $\rho^{2}$ is known at $.15, .30, .45, .60, .75, .90, \mathrm{~N}=10,15,20,25,30$, $\mathrm{p}=2,3,4$, under normal distributional assumptions, and where the number of iterations within the simulation was 500 .

\section{Results}

Overall, the study's findings indicated that all of the eight shrinkage formulas utilized under the research's specified conditions did succumb to bias, as was expected, either via under or overestimation of the population multiple 
correlation. Table 1 indicates that the two most consistently accurate formulas were Claudy and Walker. When looking at small sample sizes with few predictors with a $\rho^{2} \leq .45$, Table 1 shows that the Smith, Ezekiel, Wherry, and Olkin and Pratt formulas typically underestimated, often times greatly, $\rho^{2}$ in comparison to the Pratt, Herzberg, Claudy, and Walker formulas. However, the Pratt and Herzberg formulas tended to overestimate the population multiple correlation at $.60, .75$., and .90 , respectively, regardless of the sample size and especially when $\mathrm{p}=2$ and 3 . The Claudy and Walker formulas were consistently accurate in these same conditions, with only a small portion of overestimation when $\mathrm{p}=2$.

Table 1. Values for Eight Shrinkage Formulas when $N=10$ to $30, p=2$ to 4

\begin{tabular}{|c|c|c|c|c|c|c|c|c|}
\hline \multicolumn{9}{|c|}{$10, \mathrm{p}$} \\
\hline$\rho^{2}$ & Smith & Ezekiel & Wherry & \multicolumn{2}{|c|}{ Olkin-Pratt Claudy } & Pratt & Herzberg & Walker \\
\hline .150 & -.214 & -.093 & .044 & -.011 & .134 & . 199 & .181 & .155 \\
\hline .300 & .000 & .100 & .213 & .191 & .307 & .389 & .357 & .324 \\
\hline .450 & .214 & .293 & .381 & .383 & .471 & .572 & .528 & .484 \\
\hline .600 & .429 & .486 & .550 & .564 & .627 & .747 & .693 & .636 \\
\hline .750 & .643 & .679 & .719 & .736 & .774 & .914 & .854 & .779 \\
\hline .900 & .857 & .871 & .888 & .898 & .912 & 1.000 & 1.000 & .915 \\
\hline \multicolumn{9}{|c|}{$\mathrm{N}=15, \mathrm{p}=2$} \\
\hline$\rho^{2}$ & Smith & Ezekiel & Wherry & Olkin-Pratt & Claudy & Pratt & Herzberg & Walker \\
\hline .150 & -.063 & .008 & .085 & .047 & .126 & .176 & $.170^{\circ}$ & .138 \\
\hline .300 & .125 & .183 & .246 & .230 & .294 & .348 & .336 & .304 \\
\hline .450 & .313 & .358 & .408 & .407 & .456 & .515 & .500 & .464 \\
\hline .600 & .500 & .533 & .569 & .577 & .612 & .679 & .660 & .618 \\
\hline .750 & .688 & .708 & .731 & .741 & .763 & .838 & .817 & .766 \\
\hline .900 & .875 & .883 & .892 & .899 & .907 & .993 & .971 & .908 \\
\hline \multicolumn{9}{|c|}{$\mathrm{N}=20, \mathrm{p}=2$} \\
\hline$\rho^{2}$ & Smith & Ezekiel & Wherry & Olkin-Pratt & Claudy & Pratt & Herzberg & Walker \\
\hline .150 & .000 & .050 & .103 & .074 & .128 & .168 & $.165^{\circ}$ & .137 \\
\hline .300 & .176 & .218 & .261 & .248 & .293 & .332 & .327 & .299 \\
\hline .450 & .353 & .385 & .419 & .418 & .452 & .494 & .487 & .458 \\
\hline .600 & .529 & .553 & .578 & .583 & .608 & .654 & .644 & .611 \\
\hline .750 & .706 & .721 & .736 & .743 & .759 & .810 & .799 & .761 \\
\hline .900 & .882 & .888 & .894 & .899 & .905 & .963 & .952 & .906 \\
\hline \multicolumn{9}{|c|}{$\mathrm{N}=25, \mathrm{p}=2$} \\
\hline$\rho^{2}$ & Smith & Ezekiel & Wherry & Olkin-Pratt & Claudy & Pratt & Herzberg & Walker \\
\hline .150 & .034 & .073 & .113 & .090 & .131 & .163 & .162 & .137 \\
\hline .300 & .205 & .236 & .270 & .259 & .293 & .325 & .321 & .298 \\
\hline .450 & .375 & .400 & .426 & .425 & .451 & .484 & .479 & .455 \\
\hline .600 & .545 & .564 & .583 & .587 & .605 & .641 & .635 & .608 \\
\hline .750 & .716 & .727 & .739 & .745 & .756 & .795 & .789 & .758 \\
\hline .900 & .886 & .891 & .896 & .899 & .904 & .948 & .941 & .904 \\
\hline
\end{tabular}


Table 1. Continued

\begin{tabular}{|c|c|c|c|c|c|c|c|c|}
\hline \multirow[b]{2}{*}{$\rho^{2}$} & \multicolumn{8}{|c|}{$\mathrm{N}=30, \mathrm{p}=2$} \\
\hline & Smith & Ezekiel & Wherry & Olkin-Pratt & Claudy & Pratt & Herzberg & Walker \\
\hline .150 & .056 & .087 & .120 & .100 & .133 & .161 & .160 & .138 \\
\hline .300 & .222 & .248 & .275 & .266 & .293 & .320 & .318 & .297 \\
\hline .450 & .389 & .409 & .430 & .429 & .450 & .477 & .474 & .453 \\
\hline .600 & .556 & .570 & .586 & .589 & .604 & .633 & .629 & .606 \\
\hline .750 & .722 & .731 & .741 & .746 & .755 & .786 & .782 & .757 \\
\hline .900 & .889 & .893 & .896 & .899 & .903 & .939 & .934 & .904 \\
\hline \multicolumn{9}{|c|}{$\mathrm{N}=10, \mathrm{p}=3$} \\
\hline$\rho^{2}$ & Smith & Ezekiel & Wherry & Olkin-Pratt & Claudy & Pratt & Herzberg & Walker \\
\hline .150 & -.417 & -.275 & -.093 & -.202 & -.031 & .012 & .010 & -.005 \\
\hline .300 & -.167 & -.050 & .100 & .040 & .178 & .250 & .222 & .198 \\
\hline .450 & .083 & .175 & .293 & .270 & .374 & .477 & .428 & .390 \\
\hline .600 & .333 & .400 & .486 & .487 & .560 & .692 & .627 & .571 \\
\hline .750 & .583 & .625 & .679 & .690 & .734 & .897 & .819 & .741 \\
\hline .900 & .833 & .850 & .871 & .880 & .898 & 1.000 & 1.000 & .900 \\
\hline \multicolumn{9}{|c|}{$\mathrm{N}=15, \mathrm{p}=3$} \\
\hline$\rho^{2}$ & Smith & Ezekiel & Wherry & Olkin-Pratt & Claudy & Pratt & Herzberg & Walker \\
\hline .150 & -.159 & -.082 & .008 & -.049 & .039 & .087 & .083 & .052 \\
\hline .300 & .045 & .109 & .183 & .154 & .225 & .278 & .267 & .235 \\
\hline .450 & .250 & .300 & .358 & .349 & .403 & .464 & .448 & .412 \\
\hline .600 & .455 & .491 & .533 & .537 & .575 & .645 & .624 & .581 \\
\hline .750 & .659 & .682 & .708 & .717 & .740 & .821 & .797 & .744 \\
\hline .900 & .864 & .873 & .883 & .889 & .898 & .992 & .966 & .900 \\
\hline \multicolumn{9}{|c|}{$\mathrm{N}=20, \mathrm{p}=3$} \\
\hline$\rho^{2}$ & Smith & Ezekiel & Wherry & Olkin-Pratt & Claudy & Pratt & Herzberg & Walker \\
\hline .150 & -.063 & -.009 & .050 & .012 & .070 & .109 & .107 & .078 \\
\hline .300 & .125 & .169 & .218 & .198 & .246 & .286 & .280 & .253 \\
\hline .450 & .313 & .347 & .385 & .380 & .416 & .459 & .451 & .422 \\
\hline .600 & .500 & .525 & .553 & .556 & .582 & .630 & .620 & .586 \\
\hline .750 & .688 & .703 & .721 & .727 & .743 & .797 & .785 & .745 \\
\hline .900 & .875 & .881 & .888 & .893 & .899 & .961 & .948 & .900 \\
\hline \multicolumn{9}{|c|}{$\mathrm{N}=25, \mathrm{p}=3$} \\
\hline$\rho^{2}$ & Smith & Ezekiel & Wherry & Olkin-Pratt & Claudy & Pratt & Herzberg & Walker \\
\hline .150 & -.012 & .029 & .073 & .044 & .087 & .120 & $.118^{\circ}$ & .094 \\
\hline .300 & .167 & .200 & .236 & .222 & .257 & .290 & .286 & .263 \\
\hline .450 & .345 & .371 & .400 & .396 & .424 & .457 & .452 & .428 \\
\hline .600 & .524 & .543 & .564 & .566 & .586 & .622 & .616 & .589 \\
\hline .750 & .702 & .714 & .727 & .732 & .745 & .785 & .778 & .746 \\
\hline .900 & .881 & .886 & .891 & .894 & .899 & .945 & .938 & .900 \\
\hline
\end{tabular}


Table 1. Continued

\begin{tabular}{|c|c|c|c|c|c|c|c|c|}
\hline \multirow[b]{2}{*}{$\rho^{2}$} & \multicolumn{8}{|c|}{$\mathrm{N}=30, \mathrm{p}=3$} \\
\hline & Smith & Ezekiel & Wherry & Olkin-Pratt & Claudy & Pratt & Herzberg & Walker \\
\hline .150 & .019 & .052 & .087 & .064 & .098 & .126 & $125^{\circ}$ & .104 \\
\hline .300 & .192 & .219 & .248 & .237 & .265 & .292 & .290 & .269 \\
\hline .450 & .365 & .387 & .409 & .406 & .428 & .456 & .453 & .432 \\
\hline .600 & .538 & .554 & .570 & .573 & .589 & .618 & .614 & .591 \\
\hline .750 & .712 & .721 & .731 & .736 & .746 & .778 & .773 & .747 \\
\hline .900 & .885 & .888 & .893 & .895 & .899 & .936 & .931 & .900 \\
\hline \multicolumn{9}{|c|}{$\mathrm{N}=10, \mathrm{p}=4$} \\
\hline$\rho^{2}$ & Smith & Ezekiel & Wherry & Olkin-Pratt & Claudy & Pratt & Herzberg & Walker \\
\hline .150 & -.700 & -.530 & -.275 & -.479 & -.268 & -.285 & -.240 & -.236 \\
\hline .300 & -.400 & -.260 & -.050 & -.176 & -.008 & .029 & .025 & .017 \\
\hline .450 & -.100 & .010 & .175 & .109 & .236 & .326 & .281 & .255 \\
\hline .600 & .200 & .280 & .400 & .376 & .465 & .606 & .528 & .479 \\
\hline .750 & .500 & .550 & .625 & .625 & .679 & .870 & .766 & .687 \\
\hline .900 & .800 & .820 & .850 & .856 & .877 & 1.000 & .995 & .880 \\
\hline \multicolumn{9}{|c|}{$\mathrm{N}=15, \mathrm{p}=4$} \\
\hline$\rho^{2}$ & Smith & Ezekiel & Wherry & Olkin-Pratt & Claudy & Pratt & Herzberg & Walker \\
\hline .150 & -.275 & -.190 & -.082 & -.165 & -.067 & -.024 & -.023 & -.053 \\
\hline .300 & -.050 & .020 & .109 & .062 & .140 & .191 & .183 & .152 \\
\hline .450 & .175 & .230 & .300 & .279 & .340 & .401 & .384 & .349 \\
\hline .600 & .400 & .440 & .491 & .488 & .531 & .604 & .581 & .537 \\
\hline .750 & .625 & .650 & .682 & .688 & .714 & .801 & .773 & .717 \\
\hline .900 & .850 & .860 & .873 & .878 & .888 & .991 & .961 & .890 \\
\hline \multicolumn{9}{|c|}{$\mathrm{N}=20, \mathrm{p}=4$} \\
\hline$\rho^{2}$ & Smith & Ezekiel & Wherry & Olkin-Pratt & Claudy & Pratt & Herzberg & Walker \\
\hline .150 & -.133 & -.077 & -.009 & -.060 & .003 & .042 & .041 & .012 \\
\hline .300 & .067 & .113 & .169 & .141 & .192 & .232 & .227 & .199 \\
\hline .450 & .267 & .303 & .347 & .336 & .375 & .419 & .411 & .381 \\
\hline .600 & .467 & .493 & .525 & .525 & .553 & .603 & .592 & .557 \\
\hline .750 & .667 & .683 & .703 & .708 & .725 & .782 & .769 & .728 \\
\hline .900 & .867 & .873 & .881 & .885 & .892 & .958 & .944 & .893 \\
\hline \multicolumn{9}{|c|}{$\mathrm{N}=25, \mathrm{p}=4$} \\
\hline$\rho^{2}$ & Smith & Ezekiel & Wherry & Olkin-Pratt & Claudy & Pratt & Herzberg & Walker \\
\hline .150 & -.063 & -.020 & .029 & -.007 & .039 & .071 & .070 & .045 \\
\hline .300 & .125 & .160 & .200 & .181 & .218 & .251 & .248 & .224 \\
\hline .450 & .313 & .340 & .371 & .365 & .394 & .428 & .423 & .398 \\
\hline .600 & .500 & .520 & .543 & .544 & .565 & .602 & .596 & .568 \\
\hline .750 & .688 & .700 & .714 & .719 & .732 & .773 & .766 & .733 \\
\hline .900 & .875 & .880 & .886 & .889 & .894 & .942 & .935 & .895 \\
\hline
\end{tabular}


Table 1. Continued

\begin{tabular}{lcrrrrrrc}
\multicolumn{10}{c}{$\mathrm{N}=30, \mathrm{p}=4$} \\
$\rho^{2}$ & \multicolumn{10}{c}{ Smith } & Ezekiel & Wherry & Olkin-Pratt & Claudy & Pratt & Herzberg & Walker \\
.150 & -.020 & .014 & .052 & .024 & .060 & .088 & .088 & .066 \\
.300 & .160 & .188 & .219 & .205 & .234 & .262 & .259 & .239 \\
.450 & .340 & .362 & .387 & .382 & .405 & .433 & .429 & .408 \\
.600 & .520 & .536 & .554 & .555 & .572 & .602 & .597 & .574 \\
.750 & .700 & .710 & .721 & .725 & .735 & .769 & .764 & .737 \\
.900 & .880 & .884 & .888 & .891 & .895 & .933 & .928 & .896
\end{tabular}

Table 2 depicts adjusted $\mathrm{R}^{2}$ Walker's bias properties or the error that results when estimating $\rho^{2}$. Because Walker has similar properties as the Olkin and Pratt formula, the following bias formula presented by Lucke and Embretson (1984) was modified:

$$
\begin{aligned}
& \operatorname{Bias} \mathrm{R}_{\mathrm{DW}}^{2}=1-\frac{\mathrm{N}-4.15}{\mathrm{~N}+1} * \mathrm{R}^{2} * \\
& \frac{2\left(1-\mathrm{R}^{2}\right)}{(\mathrm{N}-1)}
\end{aligned}
$$

The bias properties for this shrinkage formula show that it is a function of sample size. As would be anticipated, when the sample increases, the bias in this estimator decreases. This formula's bias properties are similar in comparison to other estimators found by Lucke and Embretson (1984). 
Table 2. Bias Properties for Adjusted $\mathrm{R}^{2}$ Walker, $\mathrm{N}=10$ to 30

$\begin{array}{lcl}\rho^{2} & \mathrm{~N} & \text { Bias } \\ .150 & 10 & .174 \\ .300 & 10 & .131 \\ .450 & 10 & .093 \\ .600 & 10 & .061 \\ .750 & 10 & .033 \\ .900 & 10 & .012 \\ .150 & 15 & .109 \\ .300 & 15 & .080 \\ .450 & 15 & .055 \\ .600 & 15 & .034 \\ .750 & 15 & .018 \\ .900 & 15 & .006 \\ .150 & 20 & .079 \\ .300 & 20 & .057 \\ .450 & 20 & .038 \\ .600 & 20 & .023 \\ .750 & 20 & .011 \\ .900 & 20 & .003 \\ .150 & 25 & .062 \\ .300 & 25 & .044 \\ .450 & 25 & .029 \\ .600 & 25 & .017 \\ .750 & 25 & .008 \\ .900 & 25 & .002 \\ .150 & 30 & .051 \\ .300 & 30 & .036 \\ .450 & 30 & .024 \\ .600 & 30 & .014 \\ .750 & 30 & .006 \\ .900 & 30 & .002\end{array}$

Table 3 illustrates Walker's accurateness via standard error estimates for every situation presented in the research. A bootstrapping program conducted 500 resamples to derive the standard error estimate terms presented. Replications of 500 were chosen because the standard error estimates converged quickly at this level and there were relatively no precision differences above this value. As would be expected, bias was greatest under conditions of small $\mathrm{N}$, specifically when $\mathrm{N}=10$ and 15 , where error ranged from $1 \%$ to $1.5 \%$ in these two situations regardless of $\mathrm{p}$. When $\mathrm{N}=$ 20, 25, and 30, standard errors were all $<1 \%$. For instance, Figure 1 shows that the Walker formula produced almost no bias under the extreme case of $\mathrm{N}=10, \mathrm{p}=2$, and $\rho^{2}=.15$, and became more accurate in this same situation when the sample size increased to $\mathrm{N}=15$. Further, Figure 2 illustrates this same small bias propensity with the Walker formula, and also the Claudy formula, when $\mathrm{p}=2$ and $\rho^{2}=.45$, and shows that both the Pratt and Herzberg formulas in this same situation produced overestimations of the $\rho^{2}$ value. 
Table 3. Standard Error Estimates for Adj. $\mathrm{R}^{2}$ Walker

$\mathrm{p}=2$

\begin{tabular}{|c|c|c|}
\hline $\mathrm{N}$ & SE & SE Range (Min/Max) \\
\hline 10 & .015 & $(.000, .026)$ \\
\hline 15 & .010 & $(.000, .017)$ \\
\hline 20 & .008 & $(.000, .013)$ \\
\hline 25 & .006 & $(.000, .011)$ \\
\hline 30 & .005 & $(.000, .009)$ \\
\hline
\end{tabular}

\begin{tabular}{|c|c|c|}
\hline \multicolumn{3}{|c|}{} \\
\hline $\mathrm{p}=3$ & $\mathrm{SE}$ & SE Range (Min/Max) \\
\hline 10 & .014 & $(.000, .024)$ \\
\hline 15 & .010 & $(.000, .017)$ \\
\hline 20 & .007 & $(.000, .013)$ \\
\hline 25 & .006 & $(.000, .011)$ \\
\hline 30 & .005 & $(.000, .009)$ \\
\hline
\end{tabular}

\begin{tabular}{|c|c|c|}
\hline $\mathrm{p}=4$ & \multicolumn{3}{|c|}{} \\
\hline $\mathrm{N}$ & $\mathrm{SE}$ & SE Range (Min/Max) \\
\hline 10 & .015 & $(.000, .026)$ \\
\hline 15 & .010 & $(.000, .016)$ \\
\hline 20 & .007 & $(.000, .013)$ \\
\hline 25 & .006 & $(.000, .011)$ \\
\hline 30 & .005 & $(.000, .009)$ \\
\hline
\end{tabular}

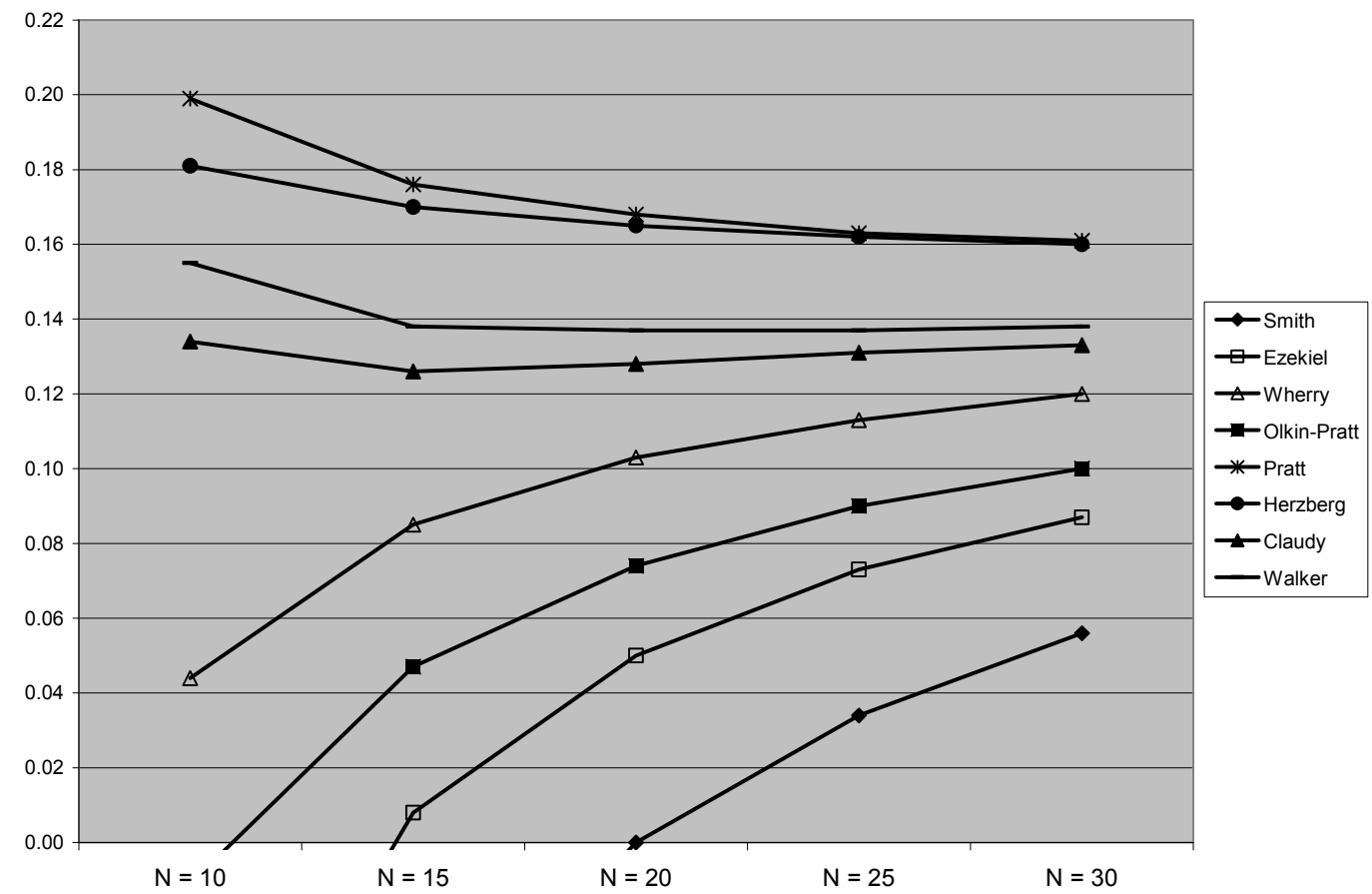

Figure 1. A Comparison of Shrinkage Formulas when $\rho^{2}=.15, p=2$ 


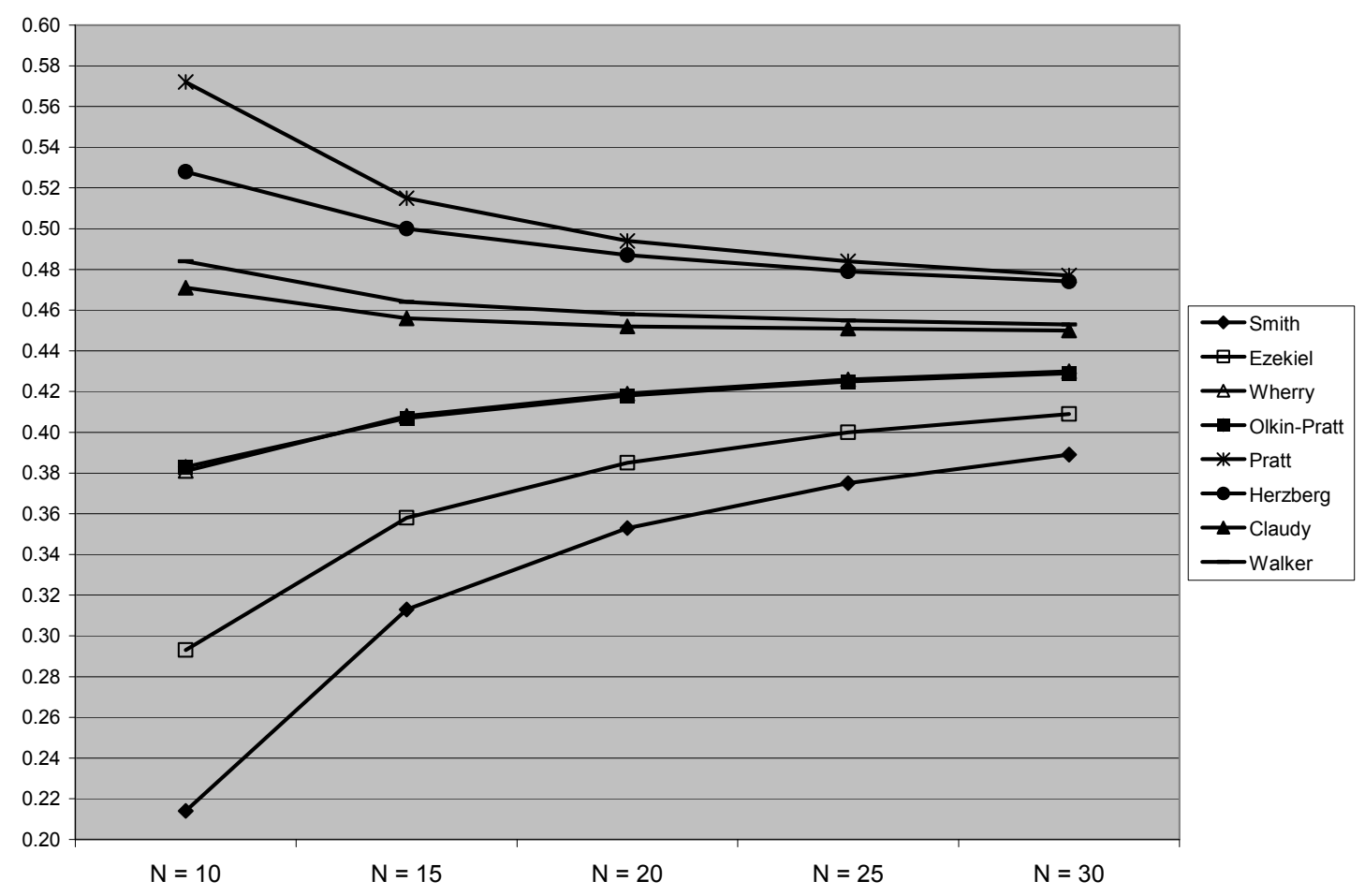

Figure 2. A Comparison of Shrinkage Formulas when $\rho^{2}=.45, \mathrm{p}=2$

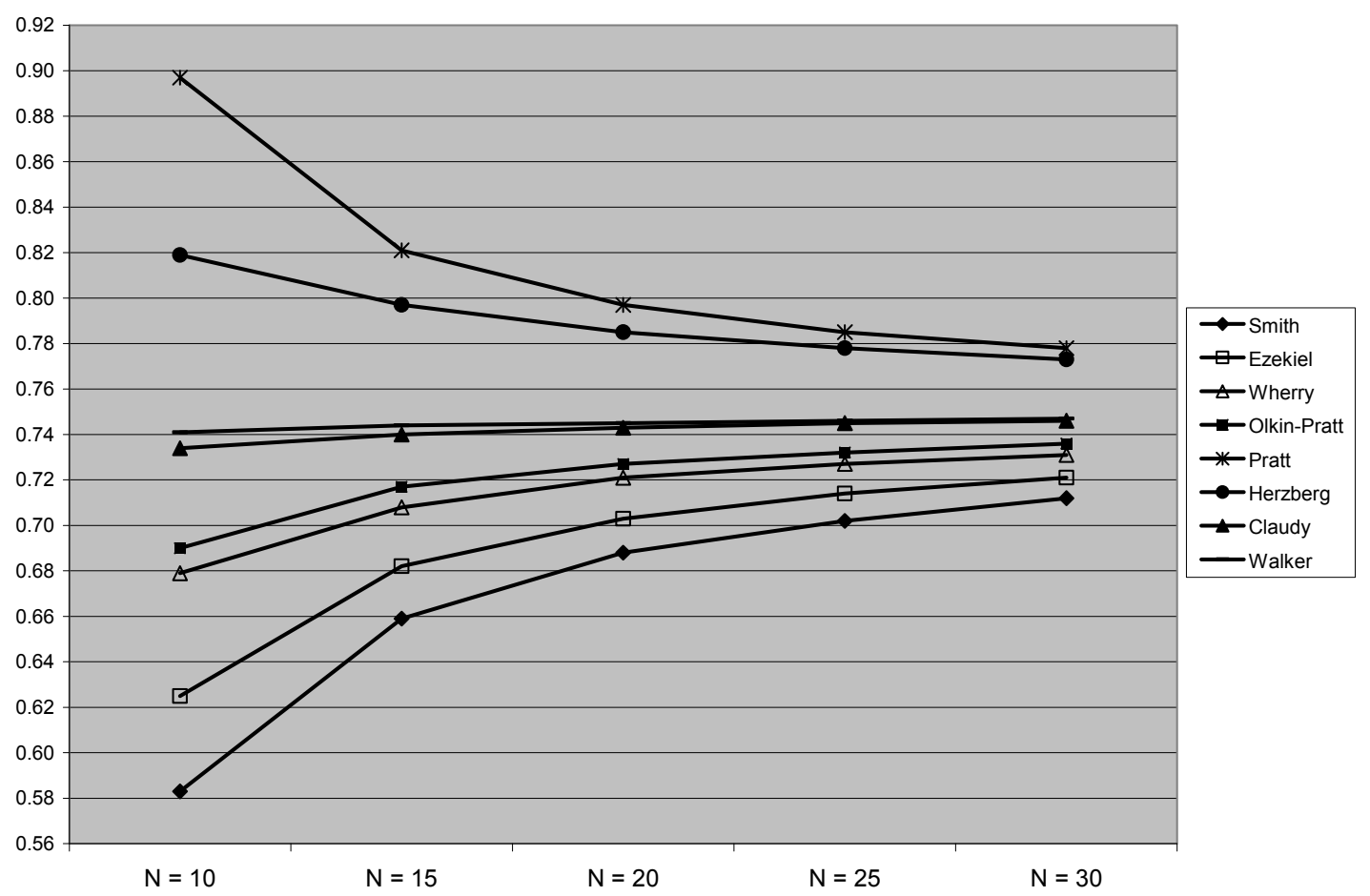

Figure 3. A Comparison of Shrinkage Formulas when $\rho^{2}=.75, p=3$ 


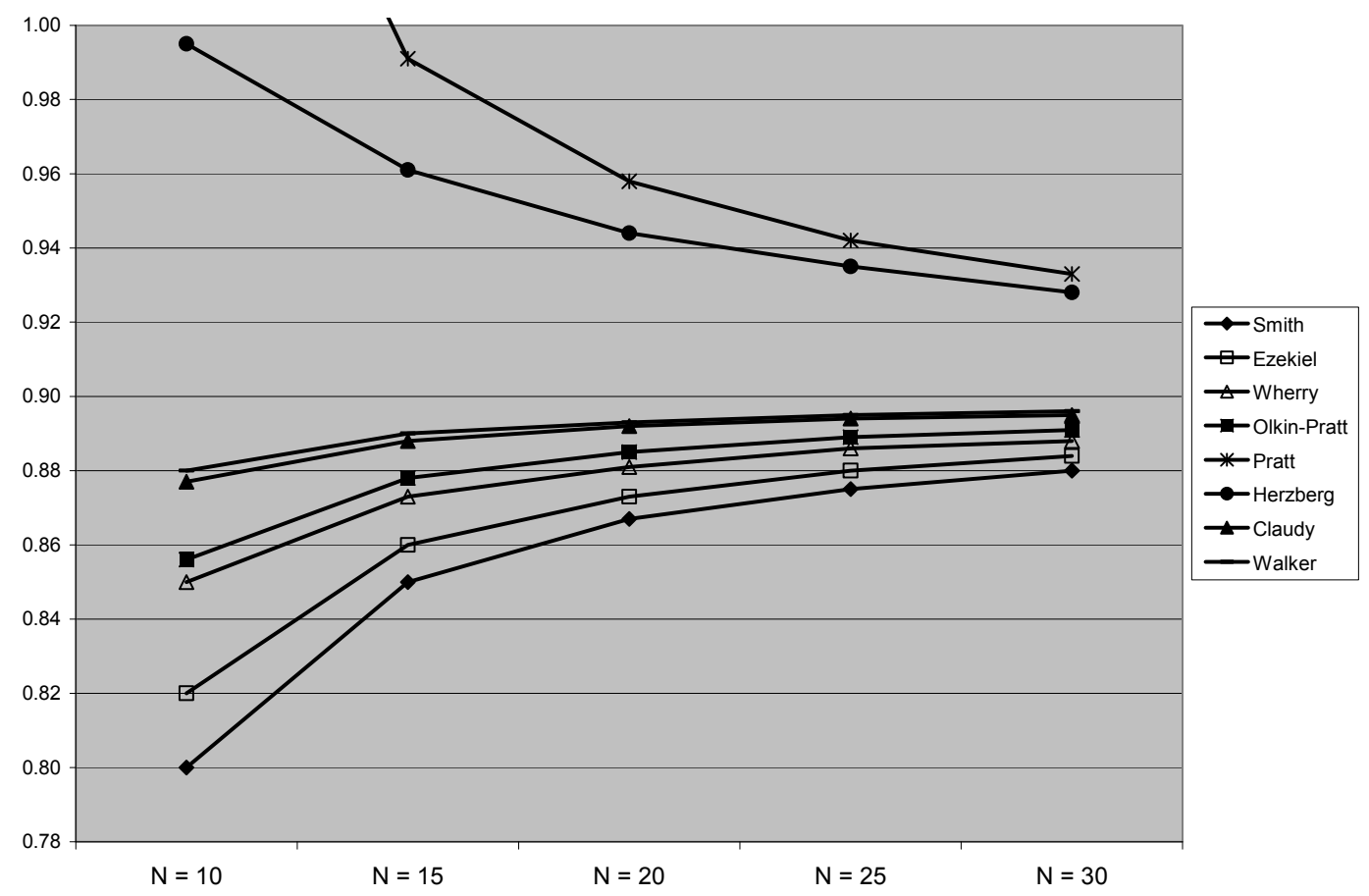

Figure 4. A Comparison of Shrinkage Formulas when $\rho^{2}=.90, p=4$

Considering data depicted in Figures 3 and 4 , it is recommended that when $\mathrm{N}=10$ to 30 with either $\mathrm{p}=3$ or 4 , use the Walker formula, which was more accurate in every instance than Claudy, and the majority of the time more exact than either Pratt or Herzberg due to their overestimations typically at $\rho^{2}$ values of .60 , .75 , and .90. When $\mathrm{N}=10$ to 30 and $\mathrm{p}=2$, the Claudy formula was more accurate than Walker, except in the case where $\rho^{2}=.15$. It is not recommended, however, to use either Smith or Ezekiel in any of the presented situations when $\rho^{2} \leq .60$. Wherry and Olkin and Pratt may be regarded in some instances when $\rho^{2}=.60$, but tend to be more accurate in all cases at the .75 and .90 levels.

Lastly, extreme research situations can produce adjusted $\mathrm{R}^{2}$ values that are nonsensical. For example, the negative values depicted in Table 1 and Figure 1 have been noted before in previous research associated with shrinkage formulas by Huberty and Mourad (1980), where it was found that, "Negative values will result from using a small $\mathrm{R}^{2}$ value and/or a small $\mathrm{N} / \mathrm{p}$ ratio" (p. 108). Thus, these negative figures should be considered to take on the value of zero.

\section{Conclusion}

When estimating the population multiple correlation coefficient, reducing the positive bias found in $\mathrm{R}^{2}$, the coefficient of determination, is approached via an unbiased estimator called the adjusted $\mathrm{R}^{2}$. However, a caveat with adjusted $\mathrm{R}^{2}$ is that not all unbiased estimators of $\rho^{2}$ function the same under varying research situations. The goal of this research was to look at this issue and determine which of the eight estimators chosen performed the most consistently under biased research conditions often found within the field of educational research, where $\mathrm{N}$ was small and the number of $\mathrm{X}$ variables ranged from 2 to 4 . 
The results of this study yielded no definitive answers pertaining to the best estimators in every situation examined, but it did ascertain that the two most consistently accurate formulas in the many conditions studied were Claudy and Walker. The tabled data derived from this research should provide researchers and students with information to understand when to use various adjusted $\mathrm{R}^{2}$ estimators pertaining to a given research situation. Also, this research introduced a new shrinkage formula, Adj. $\mathrm{R}_{\mathrm{DW}}^{2}$, and provided a complete error profile and comparison analysis under extreme research conditions for the user's consideration. Future research affiliated with shrinkage formulas should include the performance of these eight estimators under the same extreme conditions, but when operating in very biased distributional situations such as with outlier data points and/or under non-normal conditions of various skew.

\section{References}

Agresti, A., \& Finlay, B. (1997). Statistical methods for the social sciences (3rd ed). Upper Saddle River, NJ: Prentice Hall.

Browne, M. W. (1975). Predictive validity of a linear regression equation. British Journalof Mathematical and Statistical Psychology, 28, 79-87.

Carter, D. S. (1979). Comparison of different shrinkage formulas in estimating population multiple correlation coefficients. Educational and Psychological Measurement, 39, 261-266.

Claudy, J. G. (1972). A comparison of five variable weighting procedures. Educational and Psychological Measurement, 32, 311-322.

Claudy, J. G. (1978). Multiple regression and validity estimation in one sample. AppliedPsychological Measurement, 2, 595-607.
Ezekiel, M. (1929). The application of the theory of error to multiple and curvilinear correlation. American Statistical Association Journal, 24, 99-104.

Ezekiel, M. (1930). Methods of correlational analysis. New York: Wiley.

Herzberg, P. A. (1969). The parameters of cross-validation. Psychometric Monograph, 16.

Huberty, C. J., \& Mourad, S. A. (1980). Estimation in multiple correlation/prediction. Educational and Psychological Measurement, 40, 101-112.

Lucke, J. F., \& Embretson, S. (1984). The biases and mean squared errors of estimators of multinormal squared multiple correlation. Journal of Educational Statistics, 9, 183-192.

Olkin, I., \& Pratt, J. (1958). Unbiased estimation of certain correlation coefficients. Annals of Mathematical Statistics, 29, 201-211.

Pedhazur, E. J. (1997). Multiple regression in behavioral research: Explanation and prediction (3rd ed.). Fort Worth, TX: Harcourt Brace College Publishers.

Schumacker, R. E., Mount, R. E., \& Monahan, M. P. (2002). Factors affecting multiple regression and discriminant analysis with a dichotomous dependent variable: Prediction, explanation, and classification. Multiple Linear Regression Viewpoints, 28, 3239.

Walker, D. A. (2006, April). A comparison of eight shrinkage formulas under extreme conditions. Paper presented at the annual meeting of the American Educational Research Association, San Francisco, CA.

Wherry, R. J. (1931). A new formula for predicting the shrinkage of the coefficient of multiple correlation. Annals of Mathematical Statistics, 2, 440-451. 


\title{
Beta-Weibull Distribution: Some Properties and Applications to Censored Data
}

\author{
Carl Lee Felix Famoye Olugbenga Olumolade \\ Central Michigan University
}

Some properties of a four-parameter beta-Weibull distribution are discussed. The beta-Weibull distribution is shown to have bathtub, unimodal, increasing, and decreasing hazard functions. The distribution is applied to censored data sets on bus-motor failures and a censored data set on head-andneck-cancer clinical trial. A simulation is conducted to compare the beta-Weibull distribution with the exponentiated Weibull distribution.

Key words: Bathtub, unimodal, censored data, bootstrap.

\section{Introduction}

Let $F(x)$ be the cumulative distribution function of a Weibull random variable $X$. Famoye, Lee, and Olumolade (2005) defined the cumulative distribution function for betaWeibull random variable as

$$
G(x)=\frac{\Gamma(\alpha+\beta)}{\Gamma(\alpha) \Gamma(\beta)} \int_{0}^{F(x)} t^{\alpha-1}(1-t)^{\beta-1} d t, 0<\alpha, \beta<\infty .
$$

From (1), the corresponding probability density function for the beta-Weibull distribution is given by

$$
g(x)=\frac{\Gamma(\alpha+\beta)}{\Gamma(\alpha) \Gamma(\beta)} \frac{c}{\gamma}\left(\frac{x}{\gamma}\right)^{c-1}\left[1-e^{-(x / \gamma)^{c}}\right]^{\alpha-1} e^{-\beta(x / \gamma)^{c}}
$$

Carl Lee is Professor of Mathematics. His research interests are in generalized distributions and data mining. Email him at carl.lee@cmich.edu. Felix Famoye is Professor of Mathematics. His research interest is in generalized distributions. Email him at felix.famoye@cmich.edu. Olugbenga Olumolade is a graduate student in the Department of Mathematics. Email him at olumo10o@cmich.edu. The first two authors gratefully acknowledge support from the President Research Investment Fund at Central Michigan University under the grant \#65139. for $x>0, \alpha, \beta, c, \gamma>0$. One may introduce a location parameter $\xi$ in the density in (2) by replacing $x$ with $x-\xi$ where $-\infty<\xi<\infty$. In the rest of this article, take $\xi$ to be zero.

The Weibull distribution has wide applications in many disciplines. See, e.g., Hallinan (1993), Johnson, Kotz, and Balakrishnan (1994). Various extensions have appeared in the literature. For instance, 1 Zacks (1984) introduced the Weibull-exponential distribution. Mudholkar and Kollia (1994) defined a generalized Weibull distribution by introducing an additional shape parameter. Mudholkar, Srivastava, and Kollia (1996) applied the generalized Weibull distribution to model survival data. They showed that the distribution has increasing, decreasing, bathtub, and unimodal hazard functions.

Mudholkar, Srivastava, and Freimer (1995), Mudholkar and Hutson (1996) and Nassar and Eissa (2003) studied various properties of the exponentiated Weibull distribution. Mudholkar et al. (1995) applied exponentiated Weibull distribution to model failure data. Mudholkar and Hutson (1996) applied exponentiated Weibull distribution to extreme value data. They showed that exponentiated Weibull distribution has increasing, decreasing, bathtub, and unimodal hazard rates. The exponentiated exponential distribution proposed by Gupta and Kundu $(1999,2001)$ is a special case of the exponentiated Weibull family. 
Recently, Famoye et al. (2005) introduced a four-parameter beta-Weibull distribution. They showed that the beta-Weibull distribution is unimodal and obtained some results on the non-central moments. The maximum likelihood technique was used for parameter estimation and a likelihood ratio test was derived for the beta-Weibull distribution. The exponentiated Weibull distribution, Rayleigh distribution (Johnson et al., 1994, p. $686)$, the Type 2 extreme value distribution (Johnson, Kotz and Balakrishnan, 1995, p. 3), Burr Type (X) distribution (Johnson et al., 1994, p. 54), and the distribution of the order statistic from a Weibull population are special cases of the beta-Weibull distribution (Famoye et al., 2005).

In this article, the hazard function and entropy of the beta-Weibull distribution is examined. It is applied to several failure rate data and survival data. Some properties of the beta-Weibull model are discussed and the shapes of the hazard function are provided. Application of the beta-Weibull distribution to censored data sets is presented. Finally, the results of a simulation study are presented. The simulation study compares the beta-Weibull distribution with the exponentiated Weibull distribution.

Some Properties of Beta-Weibull Distribution

The survival function is given by $S(x)=$ $1-G(x)$. The hazard function (or failure rate) of beta-Weibull distribution is given by

$$
h(x)=\frac{g(x)}{1-G(x)}=\frac{g(x)}{S(x)}
$$

where $G(x)$ and $g(x)$ are given by (1) and (2) respectively and $S(x)$ is the survival function.

Theorem 1: The limit of beta-Weibull hazard function as $x \rightarrow 0$ is

$$
\lim _{x \rightarrow 0} h(x)= \begin{cases}\infty, & \text { when } \alpha c<1 \\ \frac{c \Gamma(\alpha+\beta)}{\gamma \Gamma(\alpha) \Gamma(\beta)}, & \text { when } \alpha c=1 \\ 0, & \text { when } \alpha c>1 .\end{cases}
$$

and the limit of beta-Weibull hazard function as $x \rightarrow \infty$ is given by

$$
\lim _{x \rightarrow \infty} h(x)= \begin{cases}\infty, & \text { when } c>1 \\ \frac{\beta}{\gamma}, & \text { when } c=1 \\ 0, & \text { when } c<1 .\end{cases}
$$

Proof: When $x \rightarrow 0$, the limit of $h(x)$ is the same as the limit of $g(x)$. Famoye et al. (2005) obtained the limit in (4) for $g(x)$. When $x \rightarrow \infty$, the beta-Weibull hazard function in (3) is indeterminate as both numerator and denominator become 0. By using L'Hôpital's rule, the limit of $h(x)$ as $x \rightarrow \infty$ is given by (5). This completes the proof.

Theorem 2: The beta-Weibull distribution has

(a) a constant $(=\beta / \gamma)$ failure rate when $\alpha=c$ $=1$,

(b) a decreasing failure rate when $\alpha c \leq 1$ and $c \leq 1$,

(c) an increasing failure rate when $\alpha c \geq 1$ and $c \geq 1$,

(d) a bathtub failure rate when $\alpha c<1$ and $c>$ 1 , and

(e) upside down bathtub (or unimodal) failure rate when $\alpha c>1$ and $c<1$.

Proof: It follows from Theorem 1.

Glaser (1980) gave sufficient conditions to characterize a given failure rate distribution as being bathtub shaped (BT), increasing failure rate (IFR), upside-down bathtub (UBT), or decreasing failure rate (DFR). Glaser defined the quantity $\eta(t)=-g^{\prime}(t) / g(t)$ where $g(t)$ is the probability density function and gave a list of conditions to characterize a given failure rate based on $\eta^{\prime}(t)$. It is not difficult to show that the beta-Weibull distribution satisfies all the conditions given by Glasser (1980). In Figure 1, the various shapes for the beta-Weibull hazard functions are provided. 


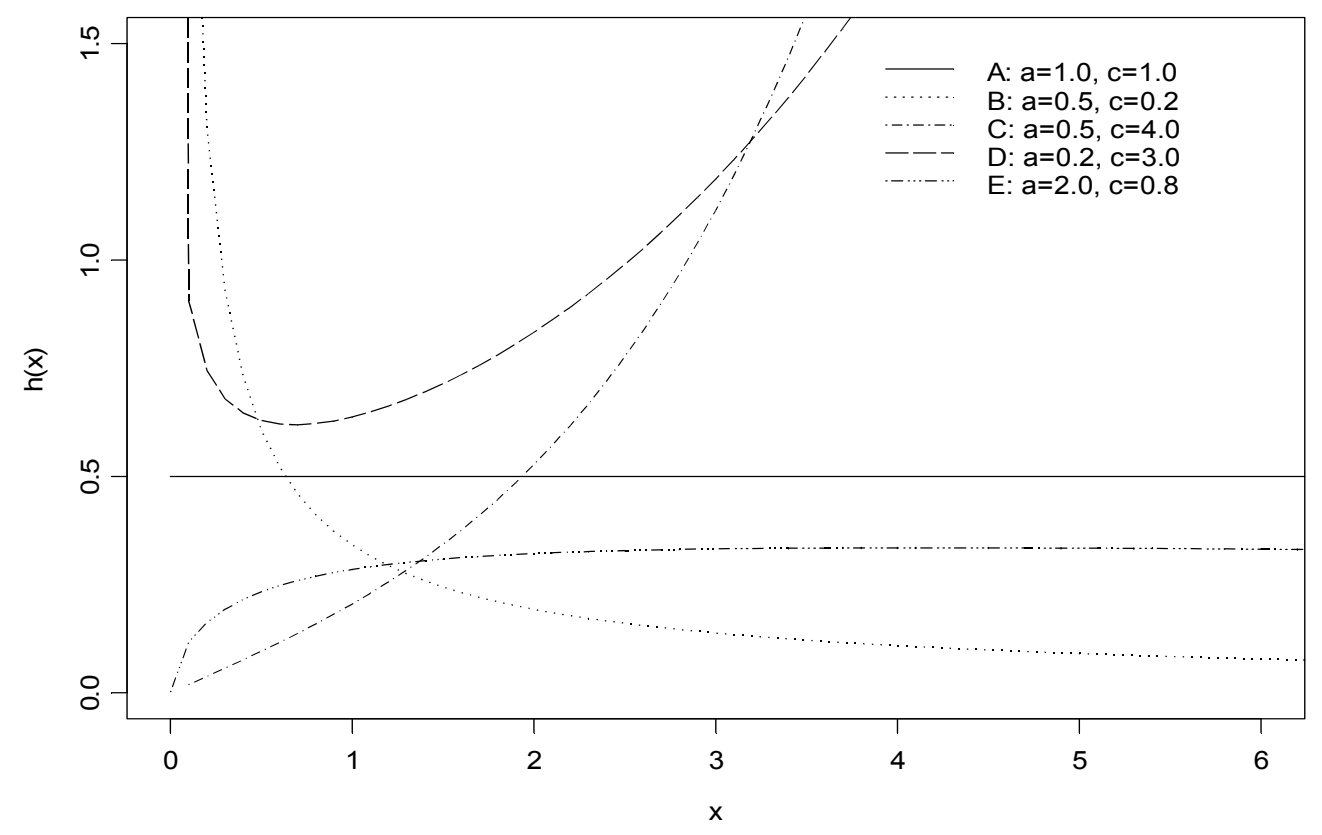

Figure 1: Beta-Weibull hazard functions for $\beta=2.0, \gamma=4.0$ and various values of $\alpha=$ a and $c$

Entropies

Entropy has been used in various situations in science and engineering. Numerous entropy measures have been studied and compared in the literature. See the recent work of Nadarajah and Zografos (2005) and the references therein. Nadarajah and Zografos (2003) derived formulas for Renyi and Shannon entropies for 26 continuous univariate distributions.
The entropy of a random variable $X$ with density $g(x)$ is a measure of variation of the uncertainty. Renyi entropy is defined by

$$
I_{R}(\rho)=\frac{1}{1-\rho} \log \left\{\int g^{\rho}(x) d x\right\}
$$

where $\rho>0$ and $\rho \neq 1$, Renyi (1961). For the beta-Weibull density see equation 7 . By using the substitution $t=(x / \gamma)^{c}$ and simplifying the resulting quantity, equation 8 is obtained. Hence, 
$\int_{0}^{\infty} g^{\rho}(x) d x=\left[\frac{\Gamma(\alpha+\beta)}{\Gamma(\alpha) \Gamma(\beta)} \frac{c}{\gamma}\right]^{\rho} \int_{0}^{\infty}(x / \gamma)^{\rho(c-1)}\left[1-\exp \left[-(x / \gamma)^{c}\right]\right]^{\rho(\alpha-1)} \exp \left[-\rho \beta(x / \gamma)^{c}\right] d x$

$\int_{0}^{\infty} g^{\rho}(x) d x=\left[\frac{\Gamma(\alpha+\beta)}{\Gamma(\alpha) \Gamma(\beta)}\right]^{\rho}\left(\frac{c}{\gamma}\right)^{\rho-1} \sum_{k=0}^{\infty} \frac{(-1)^{k} \Gamma(\rho(\alpha-1)+1) \Gamma(\rho(1-1 / c)+1 / c)}{k ! \Gamma(\rho(\alpha-1)-k+1)(k+\beta \rho)^{\rho(1-1 / c)+1 / c}}$

$I_{R}(\rho)=-\log (c / \gamma)+\frac{\rho}{1-\rho} \log \left(\frac{\Gamma(\alpha+\beta)}{\Gamma(\alpha) \Gamma(\beta)}\right)+\frac{1}{1-\rho} \log \left\{\sum_{k=0}^{\infty} \frac{(-1)^{k} \Gamma(\rho(\alpha-1)+1) \Gamma(\rho(1-1 / c)+1 / c)}{k ! \Gamma(\rho(\alpha-1)-k+1)(k+\beta \rho)^{\rho(1-1 / c)+1 / c}}\right\}$

$$
\begin{aligned}
& \mathrm{E}[-\log (g(x))]=-\log \left(\frac{\Gamma(\alpha+\beta)}{\Gamma(\alpha) \Gamma(\beta)}\right)-\left[(\alpha-1) \psi(\alpha)+(1-1 / c) \Gamma^{\prime}(1)\right] \\
& +\frac{\Gamma(\alpha+\beta)}{\Gamma(\alpha) \Gamma(\beta)} \sum_{k=0}^{\infty}\left(\begin{array}{c}
\alpha-1 \\
k
\end{array}\right) \frac{(-1)^{k}}{k+\beta}[(\alpha-1) \psi(\alpha-k)+(1-1 / c) \log (k+\beta)+\beta /(k+\beta)]
\end{aligned}
$$

Renyi entropy for the beta-Weibull density is given by equation 9 .

The Shannon's (1948) entropy is defined as $\mathrm{E}[-\log (g(x))]$. This is a special case of (6) when $\rho \rightarrow 1$. Hence, the Shannon entropy is obtained by taking the limit of (9) as $\rho \rightarrow 1$. On taking the limit of (9) as $\rho \rightarrow 1,0 / 0$ is obtained and hence, the L'Hopital's rule is applied. After using this rule and simplifying, equation 10 is obtained, where $\psi(\cdot)$ is the digamma function and $\Gamma^{\prime}(\cdot)$ is the derivative of the gamma function.
Applications of beta-Weibull distribution to censored data

In survival analysis, the data may be in grouped form or in ungrouped form and quite often, the data involve censoring. In the case of grouped data, the right censoring is in the form of a last open interval as provided in Tables 1 and 2. Suppose a grouped data consisting of $k$ intervals and the $j^{\text {th }}$ interval $\left(I_{j-1}, I_{j}\right)$ contains $n_{j}$ observations for $j=1,2,3, \ldots, k-1$. The boundary $I_{0}$ is equal to 0 and the $k^{\text {th }}$ interval 
$\left(I_{j-1}, \infty\right)$ has $n_{k}$ observations. The total number of observations is $n=\sum_{j=1}^{k} n_{j}$. By using the result in Lawless (1982), the log-likelihood function for the grouped data is

$$
\begin{aligned}
& \ell(\alpha, \beta, c, \gamma) \\
& =\sum_{j=1}^{k-1} n_{j} \log \left[S\left(I_{j-1}\right)-S\left(I_{j}\right)\right], \\
& +n_{k} \log \left[S\left(I_{k-1}\right)\right]
\end{aligned}
$$

where $S($.$) is the beta-Weibull survival function.$ Estimates of the parameters are obtained by maximizing (11), the logarithm of the censored likelihood function.

The log-likelihood function for the ungrouped data $x_{j}, j=1,2,3, \ldots, n$ is given by

$$
\ell(\alpha, \beta, c, \gamma)=\sum_{u} \log \left[h\left(x_{j}\right)\right]+\sum_{j=1}^{n} \log \left[S\left(x_{j}\right)\right],
$$

where $h($.$) is the beta-Weibull hazard function$ given by (3) and $\Sigma_{u}$ denotes the summation over the uncensored observations. Estimates of the parameters are obtained by maximizing (12), the log-likelihood function. Both the loglikelihood functions in (11) and (12) are maximized directly by using $n \operatorname{lminb}$, an SPLUS non-linear optimization routine with bounds. Taking the first and second partial derivatives of (11) and (12) with respect to the model parameters are quite involving. Hence, the Bootstrap method is used, Efron (1981), to estimate the standard errors of the parameter estimates for the beta-Weibull distribution.
Mudholkar et al. (1995) re-analyzed the classical bus-motor-failure data, first considered by Davis (1952), for a fleet of 191 buses. Mudholkar et al. (1995) re-analyzed the first, second, third, fourth, and fifth motor failures. They found that only the exponentiated Weibull provides a good fit to the first two data sets. However, the exponential, the Weibull, and the exponentiated Weibull provide good fits to the last three data sets. In this article, the beta Weibull is applied to all data sets and it provides excellent fits to all. However, the result for the first and the second motor failures are presented in Tables 1 and 2.

The beta-Weibull parameter estimates (standard errors in parentheses) in Table 1 are as follows: $\hat{\alpha}=0.3707(.0610), \quad \hat{\beta}=$ $0.1256(.0189), \quad \hat{c}=4.5753(.1853), \quad \hat{\gamma}=$ 76.2155(1.5219). The beta-Weibull model has an increasing hazard rate for these parameter estimates because $\hat{\alpha} \hat{c}>1$ and $\hat{c}>1$.

The beta-Weibull maximum likelihood estimates (standard errors in parentheses) in Table 2 are as follows: $\hat{\alpha}=0.1479(0.0634), \hat{\beta}$ $=0.1757(0.0821), \hat{c}=5.5104(1.3385), \hat{\gamma}=$ 81.4003(5.6775). The beta-Weibull model has a bathtub hazard rate for these parameter estimates because $\hat{\alpha} \hat{c}<1$ and $\hat{c}>1$.

The exponentiated Weibull and betaWeibull distributions provided adequate fits to the two data sets, but the fit from beta-Weibull distribution is better by using the chi-square goodness of fit measure. Also, the expected frequencies from the beta-Weibull model are much closer to the observed frequencies than the corresponding results from exponentiated Weibull model. In particular, it is noticed that only Beta-Weibull identifies that the failure rate has a bathtub shape, which logically fits the failure rate of motors well as shown in the above data. The last class $(120,000$ miles and up) had lower occurrence because the data is rightcensored. 
Table 1. Re-analysis of the First Bus-Motor Failure

\begin{tabular}{ccccc}
\hline $\begin{array}{c}\text { Class interval } \\
(1,000 \text { miles })\end{array}$ & $\begin{array}{c}\text { Observed } \\
\text { frequency }\end{array}$ & Weibull & $\begin{array}{c}\text { Expected frequency } \\
\text { Exponentiated } \\
\text { Weibull }\end{array}$ & $\begin{array}{c}\text { Beta } \\
\text { Weibull }\end{array}$ \\
\hline $0-20$ & 6 & 1.4066 & 3.8965 & 5.2925 \\
$20-40$ & 11 & 8.9031 & 11.7722 & 11.8987 \\
$40-60$ & 16 & 21.2228 & 19.6848 & 17.4895 \\
$60-80$ & 25 & 33.5374 & 27.4955 & 24.2573 \\
$80-100$ & 34 & 39.8566 & 34.5251 & 34.1451 \\
$100-120$ & 46 & 36.7799 & 38.3690 & 42.5039 \\
$120-140$ & 33 & 26.3822 & 33.8352 & 35.5682 \\
$140-160$ & 16 & 14.5357 & 18.0184 & 16.2516 \\
$160-$ up & 4 & 8.3757 & 3.4034 & 3.5932 \\
Total & 191 & 191.0 & 191.0 & 191.0 \\
& & & & \\
Pearson $\chi^{2}$ & & 26.218 & 3.979 & 0.836 \\
df & & 6 & 5 & 4 \\
$p$-value & & -389.936 & -381.811 & -380.3336 \\
Log-likelihood & & & & \\
\hline
\end{tabular}

Table 2. Re-analysis of the Second Bus-Motor Failure

\begin{tabular}{ccccc}
\hline $\begin{array}{c}\text { Class interval } \\
(1,000 \text { miles })\end{array}$ & $\begin{array}{c}\text { Observed } \\
\text { frequency }\end{array}$ & Weibull & $\begin{array}{c}\text { Expected frequency } \\
\text { Exponentiated } \\
\text { Weibull }\end{array}$ & $\begin{array}{c}\text { Beta } \\
\text { Weibull }\end{array}$ \\
\hline $0-20$ & 19 & 13.3474 & 16.7866 & 18.6316 \\
$20-40$ & 13 & 19.4117 & 15.8037 & 14.1624 \\
$40-60$ & 13 & 18.7796 & 15.4234 & 13.0820 \\
$60-80$ & 15 & 15.7765 & 15.1924 & 13.4357 \\
$80-100$ & 15 & 12.1399 & 15.0160 & 16.0268 \\
$100-120$ & 18 & 8.7520 & 14.6341 & 17.5898 \\
$120-$ up & 11 & 15.7929 & 11.1438 & 11.0717 \\
Total & 104 & 104.0 & 104.0 & 104.0 \\
& & & & \\
Pearson $\chi^{2}$ & & 18.2291 & 1.9485 & 0.3611 \\
df & & 4 & 3 & 0.8348 \\
$p$-value & & 0.0011 & 0.5832 & -200.918 \\
Log-likelihood & & -208.872 & -201.707 & \\
\hline
\end{tabular}


Mudholkar et al. (1995) applied the exponentiated Weibull distribution to model Efron's (1988) Arm A data on the survival times of 51 head-and-neck cancer patients given in Table 3. The beta-Weibull model was applied to fit the data in Table 3 and the result were grouped into 13 classes as in Table 12 of Mudholkar et al. (1995). For more details about the data, see Mudholkar et al. (1995). The results of the analysis and that of Mudholkar et al.
(1995) are presented in Table 4. The fits from both exponentiated Weibull and beta-Weibull distributions are very similar for the data. It appears the exponentiated Weibull distribution is slightly better because it has only three parameters compared to the beta-Weibull distribution with four parameters. A likelihood ratio test can be applied to test the adequacy of beta-Weibull distribution against a reduced special case (Famoye et al. 2005).

Table 3. Survival Times (in days) for the Patients in Arm A of the Head-and-Neck-Cancer Trial 7, 34, 42, 63, 64, 74+, 83, 84, 91, 108, 112, 129, 133, 133, 139, 140, 140, 146, 149, 154, 157, $160,160,165,173,176,185+, 218,225,241,248,273,277,279+, 297,319+, 405,417,420$, $440,523,523+, 583,594,1101,1116+, 1146,1226+, 1349+, 1412+, 1417$.

Note. Data is from Efron (1988); + indicates observations lost to follow-up.

Table 4. Re-analysis of Arm A of the Head-and-Neck-Cancer Trial

\begin{tabular}{|c|c|c|c|c|c|}
\hline $\begin{array}{l}j^{\text {th }} \text { class interval } \\
\text { (in months) }\end{array}$ & $N_{j}$ & $S_{j}$ & Weibull & $\begin{array}{l}\text { Expeded Deaths ( } \\
\text { Exponentiated } \\
\text { Weibull }\end{array}$ & $\begin{array}{c}\text { Beta } \\
\text { Weibull }\end{array}$ \\
\hline $0-1$ & 51 & 1 & 4.2739 & 1.8814 & 1.8374 \\
\hline $1-2$ & 50 & 2 & 3.8787 & 4.2669 & 4.2335 \\
\hline $2-3$ & 48 & 5 & 3.5922 & 4.6938 & 4.6845 \\
\hline $3-4$ & 42 & 2 & 3.0697 & 4.1702 & 4.1676 \\
\hline $4-6$ & 72 & 15 & 5.1380 & 6.8828 & 6.8742 \\
\hline $6-8$ & 49 & 3 & 4.4120 & 4.3158 & 4.3023 \\
\hline $8-11$ & 56 & 4 & 3.8190 & 4.4572 & 4.4353 \\
\hline $11-14$ & 45 & 3 & 3.0079 & 3.1773 & 3.1583 \\
\hline $14-18$ & 45 & 2 & 2.9567 & 2.8248 & 2.8091 \\
\hline $18-24$ & 46 & 2 & 2.9666 & 2.5099 & 2.5019 \\
\hline $24-31$ & 49 & 0 & 3.0988 & 2.2784 & 2.2833 \\
\hline $31-38$ & 47 & 2 & 2.9258 & 1.9072 & 1.9241 \\
\hline $38-47$ & 28 & 1 & 1.7189 & 1.0029 & 1.0197 \\
\hline$\sum_{j=1}^{13} R_{j}^{2}$ & & & 27.930 & 17.490 & 17.410 \\
\hline Approx. df & & & 11 & 10 & 9 \\
\hline$p$-value & & & 0.0033 & 0.0642 & 0.0427 \\
\hline
\end{tabular}

Note. $R_{j}=\sqrt{2} \operatorname{sign}\left(S_{j}-E_{j}\right)\left[S_{j} \log \left(S_{j} / E_{j}\right)+\left(N_{j}-S_{j}\right) \log \left(\frac{N_{j}-S_{j}}{N_{j}-E_{j}}\right)\right]^{1 / 2}$. 
The parameter estimates (standard errors in parentheses) from beta-Weibull model are as follows: $\hat{\alpha}=11.2139(3.3705), \quad \hat{\beta}=$ $0.5874(0.1791), \quad \hat{c}=0.3859(0.0622), \quad \hat{\gamma}=$ $0.2947(0.1451)$. The estimates show that the model has a unimodal hazard function because $\hat{\alpha} \hat{c}>1$ and $\hat{c}<1$.

Comparison between beta-Weibull and exponentiated Weibull distributions

In the previous section, the fits from both beta-Weibull and exponentiated Weibull distributions are very close. In this section, a simulation is conducted to compare these two distributions. The parameters are estimated by the method of maximum likelihood. Samples of sizes $n=250,500$, and 1000 were generated from beta-Weibull and exponentiated Weibull distributions. The parameter sets for which the beta-Weibull hazard function is bathtub (Table 5), unimodal (Table 6), increasing (Table 7), and decreasing (Table 8) are simulated. For each simulated sample, the likelihood ratio test proposed by Famoye et al. (2005) is applied to compare the beta-Weibull and exponentiated Weibull distributions. In each case, there is no significance difference between the two models. The biases were examined (actual parameter value minus the estimated value) and the standard errors of the maximum likelihood estimates. These biases and the standard errors tell a different story.

For each sample size, 100 different samples were generated in order to obtain 100 parameter estimates which are used to compute the biases and the standard errors. The biases and the standard errors of the maximum likelihood estimates (mle) are reported in Tables 5 through 8 . When the parameter $\beta=1$, the simulated data is considered to be from the exponentiated Weibull distribution. The following are some observations from the simulation study.

a. For the parameter set of a bathtub hazard function (Table 5):

a.1 When $\beta<1$, the biases of the mle from beta-Weibull estimates are smaller than the corresponding biases from the exponentiated
Weibull distribution. The standard errors of the mle of $\alpha$ and $c$ for the two distributions are comparable, while the standard errors of the mle of $\gamma$ are larger for beta-Weibull distribution.

a.2 When $\beta=1$, the biases and standard errors of the mle of $\alpha$ and $c$ for the two distributions are comparable. When comparing the mle of $\gamma$, the beta-Weibull distribution seems to have larger bias and standard error.

a.3 When $\beta>1$, the biases and standard errors of the mle for beta-Weibull distribution seem to be larger than the biases and standard errors of the mle for exponentiated Weibull distribution.

b. For the parameter set of a unimodal hazard function (Table 6):

b.1 When $\beta<1$, similar results as in (a.1) are observed.

b. 2 When $\beta=1$, similar results as in (a.2) are observed.

b.3 When $\beta>1$, the biases of the mle of $\alpha$ and $c$ are larger for beta-Weibull, while the standard errors of the mle of $\alpha$ and $c$ for the two distributions are comparable. The mle of $\gamma$ have comparable biases for the two distributions. The mle of $\gamma$ have larger standard errors for the betaWeibull distribution.

c. For the parameter set of an increasing hazard function (Table 7):

c. 1 When $\beta<1$, similar results as in (a.1) are observed.

c. 2 When $\beta=1$, the biases and standard errors of the mle of $\alpha$ for beta-Weibull are smaller than the biases and standard errors of the mle from exponentiated Weibull. The biases of the mle of $c$ are larger for beta-Weibull but the standard errors are comparable for the two distributions. Both biases and standard errors of the mle of $\gamma$ are larger for beta-Weibull.

c. 3 When $\beta>1$, the biases and standard errors of the mle of $\alpha$ for the two distributions are 
comparable. The biases of the mle of $c$ are slightly larger for beta-Weibull but the standard errors are comparable for the two distributions. The estimates of $\gamma$ have larger biases and standard errors for the beta-Weibull.

d. For the parameter set of a decreasing hazard function (Table 8):

d.1 When $\beta<1$, both biases and standard errors of the mle of $\alpha, c$ and $\gamma$ are smaller for beta-Weibull.

d.2 When $\beta=1$, similar results as in (c.2) are observed.

d.3 When $\beta>1$, the biases and standard errors of the mle of $\alpha$ and $c$ for the two distributions are comparable. The estimates of $\gamma$ have comparable biases with larger standard errors for the beta-Weibull.

\section{Conclusion}

The biases of the mle from beta-Weibull distribution are smaller than the biases of the mle from exponentiated Weibull model with comparable standard errors when $\beta<1$. The biases and standard errors are, in general, smaller for the exponentiated Weibull distribution when $\beta \geq 1$. In all the three examples in previous section, the estimates for parameter $\beta$ are less than 1.0 and thus, this simulation study supports the use of the betaWeibull distribution for describing the data sets. In addition, another implication of the simulation results is that one can take the advantage of the Beta-Weibull distribution and the exponentiated Weibull distribution by using the Beta-Weibull distribution and setting up the upper bound of parameter estimate of $\beta$ to be one. 
Table 5: Bias (standard error) of parameter estimate for $\alpha=0.5, c=1.5$ and various values of $\beta$ and $\gamma$ (bathtub hazard function)

\begin{tabular}{|c|c|c|c|c|c|c|}
\hline \multicolumn{3}{|c|}{ Actual values } & \multicolumn{3}{|c|}{ Exponentiated Weibull distribution } & \\
\hline$\beta$ & $\gamma$ & $n$ & $\hat{\alpha}$ & $\hat{c}$ & $\hat{\gamma}$ & \\
\hline \multirow[t]{3}{*}{0.5} & 2 & 250 & $.006(.097)$ & $-.093(.224)$ & $-1.457(0.397)$ & \\
\hline & & 500 & $.036(.067)$ & $-.151(.164)$ & $-1.577(0.262)$ & \\
\hline & & 1000 & $.036(.048)$ & $-.143(.118)$ & $-1.597(0.217)$ & \\
\hline \multirow[t]{3}{*}{0.5} & 4 & 250 & $.005(.097)$ & $-.093(.224)$ & $-2.909(0.793)$ & \\
\hline & & 500 & $.037(.067)$ & $-.155(.168)$ & $-3.158(0.528)$ & \\
\hline & & 1000 & $.036(.047)$ & $-.144(.119)$ & $-3.192(0.431)$ & \\
\hline \multirow[t]{3}{*}{1.0} & 2 & 250 & $-.030(.147)$ & $-.036(.317)$ & $.021(0.364)$ & \\
\hline & & 500 & $-.009(.105)$ & $-.035(.229)$ & $-.003(0.270)$ & \\
\hline & & 1000 & $.000(.058)$ & $-.023(.133)$ & $-.010(0.157)$ & \\
\hline \multirow[t]{3}{*}{1.0} & 4 & 250 & $-.031(.147)$ & $-.038(.318)$ & $.046(0.722)$ & \\
\hline & & 500 & $-.011(.105)$ & $-.030(.228)$ & $.004(0.537)$ & \\
\hline & & 1000 & $.000(.058)$ & $-.023(.133)$ & $-.021(0.314)$ & \\
\hline \multirow[t]{3}{*}{2.0} & 2 & 250 & $-.017(.137)$ & $-.024(.289)$ & $.821(0.200)$ & \\
\hline & & 500 & $-.012(.101)$ & $.000(.200)$ & $.815(0.149)$ & \\
\hline & & 1000 & $-.023(.070)$ & $.042(.133)$ & $.839(0.102)$ & \\
\hline \multirow[t]{3}{*}{2.0} & 4 & 250 & $-.020(.134)$ & $-.016(.278)$ & $1.658(0.378)$ & \\
\hline & & 500 & $-.012(.100)$ & $.001(.198)$ & $1.631(0.294)$ & \\
\hline & & 1000 & $-.023(.070)$ & $.042(.133)$ & $1.677(0.204)$ & \\
\hline \multicolumn{3}{|c|}{ Actual values } & \multicolumn{4}{|c|}{ Beta-Weibull distribution } \\
\hline$\beta$ & $\gamma$ & $n$ & $\hat{\alpha}$ & $\hat{\beta}$ & $\hat{c}$ & $\hat{\gamma}$ \\
\hline \multirow[t]{3}{*}{0.5} & 2 & 250 & $-.030(.094)$ & $-.104(.253)$ & $.032(.210)$ & $-.171(0.897)$ \\
\hline & & 500 & $-.005(.068)$ & $-.066(.237)$ & $-.002(.163)$ & $-.143(0.853)$ \\
\hline & & 1000 & $-.013(.051)$ & $-.018(.232)$ & $.026(.123)$ & $.024(0.825)$ \\
\hline \multirow[t]{3}{*}{0.5} & 4 & 250 & $-.030(.094)$ & $-.106(.250)$ & $.030(.210)$ & $-.360(1.778)$ \\
\hline & & 500 & $-.004(.069)$ & $-.069(.239)$ & $-.007(.170)$ & $.311(1.728)$ \\
\hline & & 1000 & $-.013(.052)$ & $-.018(.231)$ & $.025(.123)$ & $.041(1.641)$ \\
\hline \multirow[t]{3}{*}{1.0} & 2 & 250 & $-.049(.145)$ & $.185(.395)$ & $.033(.291)$ & $.392(0.731)$ \\
\hline & & 500 & $-.035(.107)$ & $.261(.385)$ & $.053(.227)$ & $.508(0.749)$ \\
\hline & & 1000 & $-.027(.065)$ & $.245(.378)$ & $.061(.157)$ & $.489(0.710)$ \\
\hline \multirow[t]{3}{*}{1.0} & 4 & 250 & $-.049(.143)$ & $.163(.409)$ & $.028(.292)$ & $.717(1.481)$ \\
\hline & & 500 & $-.037(.106)$ & $.253(.405)$ & $.058(.224)$ & $1.003(1.539)$ \\
\hline & & 1000 & $-.027(.065)$ & $.245(.378)$ & $.061(.157)$ & $.977(1.421)$ \\
\hline \multirow[t]{3}{*}{2.0} & 2 & 250 & $-.036(.132)$ & $1.126(.497)$ & $.047(.255)$ & $.999(0.496)$ \\
\hline & & 500 & $-.040(.100)$ & $1.261(.384)$ & $.086(.198)$ & $1.127(0.429)$ \\
\hline & & 1000 & $-.051(.075)$ & $1.203(.509)$ & $.119(.154)$ & $1.098(0.516)$ \\
\hline \multirow[t]{3}{*}{2.0} & 4 & 250 & $-.038(.133)$ & $1.124(.490)$ & $.047(.259)$ & $1.994(0.992)$ \\
\hline & & 500 & $-.039(.099)$ & $1.240(.415)$ & $.085(.197)$ & $2.217(0.902)$ \\
\hline & & 1000 & $-.052(.073)$ & $1.224(.486)$ & $.123(.148)$ & $2.237(0.990)$ \\
\hline
\end{tabular}


Table 6: Bias (standard error) of parameter estimate for $\alpha=1.5, c=0.75$ and various values of $\beta$ and $\gamma$ (unimodal hazard function)

\begin{tabular}{|c|c|c|c|c|c|c|}
\hline \multicolumn{3}{|c|}{ Actual values } & \multicolumn{3}{|c|}{ Exponentiated Weibull distribution } & \\
\hline$\beta$ & $\gamma$ & $n$ & $\hat{\alpha}$ & $\hat{c}$ & $\hat{\gamma}$ & \\
\hline \multirow[t]{3}{*}{0.5} & 2 & 250 & $-.562(.449)$ & $.119(.056)$ & $-1.080(0.399)$ & \\
\hline & & 500 & $-.450(.303)$ & $.103(.043)$ & $-1.277(0.555)$ & \\
\hline & & 1000 & $-.343(.197)$ & $.090(.029)$ & $-1.496(0.402)$ & \\
\hline \multirow[t]{3}{*}{0.5} & 4 & 250 & $-.594(.511)$ & $.121(.059)$ & $-2.090(1.457)$ & \\
\hline & & 500 & $-.446(.303)$ & $.103(.043)$ & $-2.572(1.110)$ & \\
\hline & & 1000 & $-.337(.198)$ & $.089(.029)$ & $-3.016(0.800)$ & \\
\hline \multirow[t]{3}{*}{1.0} & 2 & 250 & $-.052(.458)$ & $-.022(.120)$ & $-.077(0.680)$ & \\
\hline & & 500 & $-.028(.367)$ & $-.010(.088)$ & $-.046(0.509)$ & \\
\hline & & 1000 & $-.045(.266)$ & $.002(.066)$ & $.019(0.388)$ & \\
\hline \multirow[t]{3}{*}{1.0} & 4 & 250 & $-.018(.431)$ & $-.028(.116)$ & $-.243(1.310)$ & \\
\hline & & 500 & $-.022(.363)$ & $-.012(.087)$ & $-.108(1.006)$ & \\
\hline & & 1000 & $-.022(.250)$ & $-.003(.064)$ & $-.018(0.747)$ & \\
\hline \multirow[t]{3}{*}{2.0} & 2 & 250 & $-.058(.532)$ & $-.035(.137)$ & $1.085(0.328)$ & \\
\hline & & 500 & $.004(.338)$ & $-.032(.099)$ & $1.090(0.238)$ & \\
\hline & & 1000 & $.061(.213)$ & $-.035(.066)$ & $1.077(0.159)$ & \\
\hline \multirow[t]{3}{*}{2.0} & 4 & 250 & $-.055(.523)$ & $-.030(.131)$ & $2.172(0.633)$ & \\
\hline & & 500 & $.015(.335)$ & $-.035(.098)$ & $2.164(0.472)$ & \\
\hline & & 1000 & $.061(.204)$ & $-.035(.065)$ & $2.151(0.308)$ & \\
\hline \multicolumn{3}{|c|}{ Actual values } & \multicolumn{4}{|c|}{ Beta-Weibull distribution } \\
\hline$\beta$ & $\gamma$ & $n$ & $\hat{\alpha}$ & $\hat{\beta}$ & $\hat{c}$ & $\hat{\gamma}$ \\
\hline \multirow[t]{3}{*}{0.5} & 2 & 250 & $-.412(.399)$ & $-.107(.221)$ & $.072(.065)$ & $.153(0.765)$ \\
\hline & & 500 & $-.305(.269)$ & $-.071(.225)$ & $-.052(.052)$ & $.147(0.791)$ \\
\hline & & 1000 & $-.209(.177)$ & $-.047(.222)$ & $.039(.042)$ & $.116(0.782)$ \\
\hline \multirow[t]{3}{*}{0.5} & 4 & 250 & $-.439(.436)$ & $-.115(.216)$ & $.075(.066)$ & $.291(1.519)$ \\
\hline & & 500 & $-.306(.268)$ & $-.086(.221)$ & $.055(.051)$ & $.191(1.562)$ \\
\hline & & 1000 & $-.207(.180)$ & $-.056(.222)$ & $.040(.043)$ & $.168(1.565)$ \\
\hline \multirow[t]{3}{*}{1.0} & 2 & 250 & $-.019(.439)$ & $.147(.395)$ & $-.038(.116)$ & $.227(0.940)$ \\
\hline & & 500 & $-.001(.373)$ & $.156(.388)$ & $-.026(.088)$ & $.281(0.877)$ \\
\hline & & 1000 & $-.006(.247)$ & $.163(.409)$ & $-.018(.066)$ & $.320(0.887)$ \\
\hline \multirow[t]{3}{*}{1.0} & 4 & 250 & $.005(.426)$ & $.139(.393)$ & $-.041(.115)$ & $.392(1.833)$ \\
\hline & & 500 & $.004(.373)$ & $.162(.394)$ & $-.027(.089)$ & $.580(1.769)$ \\
\hline & & 1000 & $.009(.244)$ & $.148(.402)$ & $-.020(.067)$ & $.561(1.744)$ \\
\hline \multirow[t]{3}{*}{2.0} & 2 & 250 & $-.041(.558)$ & $1.013(.553)$ & $-.047(.135)$ & $1.074(0.644)$ \\
\hline & & 500 & $.024(.342)$ & $.975(.678)$ & $-.045(.098)$ & $1.027(0.749)$ \\
\hline & & 1000 & $.071(.224)$ & $.995(.531)$ & $-.043(.070)$ & $1.055(0.522)$ \\
\hline \multirow[t]{3}{*}{2.0} & 4 & 250 & $-.048(.575)$ & $1.011(.623)$ & $-.041(.133)$ & $2.179(1.321)$ \\
\hline & & 500 & $.032(.343)$ & $.974(.678)$ & $-.047(.099)$ & $2.046(1.495)$ \\
\hline & & 1000 & $.076(.214)$ & $1.025(.538)$ & $-.046(.068)$ & $2.159(1.066)$ \\
\hline
\end{tabular}


Table 7: Bias (standard error) of parameter estimate for $\alpha=1.5, c=1.5$ and various values of $\beta$ and $\gamma$ (increasing hazard function)

\begin{tabular}{|c|c|c|c|c|c|c|}
\hline \multicolumn{3}{|c|}{ Actual values } & \multicolumn{3}{|c|}{ Exponentiated Weibull distribution } & \\
\hline$\beta$ & $\gamma$ & $n$ & $\hat{\alpha}$ & $\hat{c}$ & $\hat{\gamma}$ & \\
\hline \multirow[t]{3}{*}{0.5} & 2 & 250 & $-.149(.451)$ & $.042(.219)$ & $-.926(0.494)$ & \\
\hline & & 500 & $-.146(.449)$ & $.067(.190)$ & $-.908(0.444)$ & \\
\hline & & 1000 & $-.120(.274)$ & $.076(.120)$ & $-.897(0.293)$ & \\
\hline \multirow[t]{3}{*}{0.5} & 4 & 250 & $-.160(.507)$ & $.039(.225)$ & $-1.861(1.037)$ & \\
\hline & & 500 & $-.087(.421)$ & $.038(.185)$ & $-1.942(0.849)$ & \\
\hline & & 1000 & $-.083(.261)$ & $.063(.114)$ & $-1.861(0.562)$ & \\
\hline \multirow[t]{3}{*}{1.0} & 2 & 250 & $-.054(.621)$ & $-.064(.284)$ & $-.034(0.386)$ & \\
\hline & & 500 & $-.046(.401)$ & $-.017(.187)$ & $.003(0.274)$ & \\
\hline & & 1000 & $-.034(.274)$ & $-.005(.137)$ & $.009(0.206)$ & \\
\hline \multirow[t]{3}{*}{1.0} & 4 & 250 & $-.047(.620)$ & $-.068(.284)$ & $-.081(0.770)$ & \\
\hline & & 500 & $-.007(.375)$ & $-.037(.181)$ & $-.047(0.521)$ & \\
\hline & & 1000 & $-.006(.261)$ & $-.017(.134)$ & $-.024(0.396)$ & \\
\hline \multirow[t]{3}{*}{2.0} & 2 & 250 & $-.054(.530)$ & $-.065(.266)$ & $.667(0.243)$ & \\
\hline & & 500 & $.011(.330)$ & $-.066(.194)$ & $659(0.170)$ & \\
\hline & & 1000 & $.058(.206)$ & $-.068(.130)$ & $.646(0.112)$ & \\
\hline \multirow[t]{3}{*}{2.0} & 4 & 250 & $-.038(.526)$ & $-.074(.271)$ & $1.321(0.488)$ & \\
\hline & & 500 & $.026(.330)$ & $-.074(.194)$ & $1.303(0.343)$ & \\
\hline & & 1000 & $.068(.208)$ & $-.075(.130)$ & $1.281(0.226)$ & \\
\hline \multicolumn{3}{|c|}{ Actual values } & \multicolumn{4}{|c|}{ Beta-Weibull distribution } \\
\hline$\beta$ & $\gamma$ & $n$ & $\hat{\alpha}$ & $\hat{\beta}$ & $\hat{c}$ & $\hat{\gamma}$ \\
\hline \multirow[t]{3}{*}{0.5} & 2 & 250 & $-.056(.385)$ & $-.066(.220)$ & $-.031(.197)$ & $-.125(0.542)$ \\
\hline & & 500 & $-.045(.378)$ & $-.030(.214)$ & $-.013(.165)$ & $-.032(0.506)$ \\
\hline & & 1000 & $-.029(.229)$ & $-.081(.224)$ & $-.002(.103)$ & $-.145(0.507)$ \\
\hline \multirow[t]{3}{*}{0.5} & 4 & 250 & $-.055(.413)$ & $-.045(.222)$ & $-.038(.196)$ & $-.178(1.129)$ \\
\hline & & 500 & $-.002(.368)$ & $-.031(.215)$ & $-.035(.168)$ & $-.135(0.983)$ \\
\hline & & 1000 & $-.001(.228)$ & $-.072(.235)$ & $-.013(.107)$ & $-.265(1.028)$ \\
\hline \multirow[t]{3}{*}{1.0} & 2 & 250 & $-.013(.514)$ & $.113(.415)$ & $-.090(.266)$ & $.127(0.577)$ \\
\hline & & 500 & $-.012(.386)$ & $.141(.408)$ & $-.049(.179)$ & $.197(0.518)$ \\
\hline & & 1000 & $.003(.251)$ & $.126(.437)$ & $-.041(.135)$ & $.184(0.564)$ \\
\hline \multirow[t]{3}{*}{1.0} & 4 & 250 & $-.009(.515)$ & $.114(.416)$ & $-.092(.267)$ & $.250(1.150)$ \\
\hline & & 500 & $.013(.381)$ & $.108(.387)$ & $-.059(.181)$ & $.272(0.956)$ \\
\hline & & 1000 & $.013(.253)$ & $.056(.415)$ & $-.037(.139)$ & $.170(1.038)$ \\
\hline \multirow[t]{3}{*}{2.0} & 2 & 250 & $-.036(.558)$ & $1.012(.588)$ & $-.091(.262)$ & $.714(0.473)$ \\
\hline & & 500 & $.030(.337)$ & $.983(.683)$ & $-.092(.194)$ & $.695(0.482)$ \\
\hline & & 1000 & $.078(.207)$ & $1.053(.531)$ & $-.094(.132)$ & $.721(0.398)$ \\
\hline \multirow[t]{3}{*}{2.0} & 4 & 250 & $-.037(.581)$ & $.962(.624)$ & $-.091(.275)$ & $1.356(0.921)$ \\
\hline & & 500 & $.038(.342)$ & $.948(.671)$ & $-.093(.197)$ & $1.321(0.940)$ \\
\hline & & 1000 & $.075(.221)$ & $.977(.523)$ & $-.089(.139)$ & $1.317(0.739)$ \\
\hline
\end{tabular}


Table 8: Bias (standard error) of parameter estimate for $\alpha=1.5, c=0.5$ and various values of $\beta$ and $\gamma$ (decreasing hazard function)

\begin{tabular}{|c|c|c|c|c|c|c|}
\hline \multicolumn{3}{|c|}{ Actual values } & \multicolumn{3}{|c|}{ Exponentiated Weibull distribution } & \\
\hline$\beta$ & $\gamma$ & $n$ & $\hat{\alpha}$ & $\hat{c}$ & $\hat{\gamma}$ & \\
\hline \multirow[t]{3}{*}{0.5} & 2 & 250 & $-1.018(.627)$ & $.111(.035)$ & $-.644(0.914)$ & \\
\hline & & 500 & $-.742(.270)$ & $.097(.022)$ & $-1.145(0.634)$ & \\
\hline & & 1000 & $-.633(.180)$ & $.090(.016)$ & $-1.433(0.469)$ & \\
\hline \multirow[t]{3}{*}{0.5} & 4 & 250 & $-1.017(.627)$ & $.112(.035)$ & $-1.286(1.829)$ & \\
\hline & & 500 & $-.746(.272)$ & $.097(.022)$ & $-2.280(1.269)$ & \\
\hline & & 1000 & $-.633(.180)$ & $.090(.016)$ & $-2.867(0.937)$ & \\
\hline \multirow[t]{3}{*}{1.0} & 2 & 250 & $-.114(.478)$ & $-.002(.071)$ & $-.028(0.896)$ & \\
\hline & & 500 & $-.043(.363)$ & $-.004(.056)$ & $-.067(0.723)$ & \\
\hline & & 1000 & $.034(.248)$ & $.001(.042)$ & $-.004(0.540)$ & \\
\hline \multirow[t]{3}{*}{1.0} & 4 & 250 & $-.095(.467)$ & $-.005(.072)$ & $-.130(1.794)$ & \\
\hline & & 500 & $-.043(.364)$ & $-.004(.056)$ & $-.136(1.446)$ & \\
\hline & & 1000 & $-.029(.246)$ & $-.000(.042)$ & $-.028(1.076)$ & \\
\hline \multirow[t]{3}{*}{2.0} & 2 & 250 & $-.065(.524)$ & $-.020(.089)$ & $1.361(0.333)$ & \\
\hline & & 500 & $.005(.326)$ & $-.020(.062)$ & $1.374(0.232)$ & \\
\hline & & 1000 & $.063(.206)$ & $-.024(.043)$ & $1.366(0.161)$ & \\
\hline \multirow[t]{3}{*}{2.0} & 4 & 250 & $-.054(.522)$ & $-.022(.090)$ & $2.711(0.667)$ & \\
\hline & & 500 & $.019(.318)$ & $-.022(.060)$ & $2.737(0.455)$ & \\
\hline & & 1000 & $.061(.204)$ & $-.023(.043)$ & $2.729(0.320)$ & \\
\hline \multicolumn{3}{|c|}{ Actual values } & \multicolumn{4}{|c|}{ Beta-Weibull distribution } \\
\hline$\beta$ & $\gamma$ & $n$ & $\hat{\alpha}$ & $\hat{\beta}$ & $\hat{c}$ & $\hat{\gamma}$ \\
\hline \multirow[t]{3}{*}{0.5} & 2 & 250 & $-.777(.482)$ & $-.125(.226)$ & $.076(.039)$ & $.564(0.883)$ \\
\hline & & 500 & $-.549(.247)$ & $-.093(.203)$ & $-.061(.030)$ & $.473(0.795)$ \\
\hline & & 1000 & $-.472(.165)$ & $-.122(.207)$ & $.059(.024)$ & $.226(0.879)$ \\
\hline \multirow[t]{3}{*}{0.5} & 4 & 250 & $-.773(.481)$ & $-.120(.227)$ & $.076(.039)$ & $1.154(1.774)$ \\
\hline & & 500 & $-.555(.251)$ & $-.097(.205)$ & $.062(.031)$ & $.924(1.598)$ \\
\hline & & 1000 & $-.472(.165)$ & $-.122(.207)$ & $.059(.024)$ & $.452(1.758)$ \\
\hline \multirow[t]{3}{*}{1.0} & 2 & 250 & $-.086(.483)$ & $.164(.370)$ & $-.012(.072)$ & $.420(1.088)$ \\
\hline & & 500 & $-.008(.368)$ & $.205(.357)$ & $-.017(.057)$ & $.467(1.055)$ \\
\hline & & 1000 & $-.000(.241)$ & $.166(.400)$ & $-.012(.044)$ & $.345(1.142)$ \\
\hline \multirow[t]{3}{*}{1.0} & 4 & 250 & $-.071(.478)$ & $-.159(.368)$ & $-.015(.073)$ & $.772(2.178)$ \\
\hline & & 500 & -.009 (.369) & $.205(.356)$ & $-.017(.057)$ & $.933(2.108)$ \\
\hline & & 1000 & $.001(.243)$ & $.155(.402)$ & $-.011(.045)$ & $.633(2.287)$ \\
\hline \multirow[t]{3}{*}{2.0} & 2 & 250 & $-.047(.552)$ & $1.061(.529)$ & $-.029(.088)$ & $1.366(0.607)$ \\
\hline & & 500 & $.029(.330)$ & $1.059(.563)$ & $-.030(.062)$ & $1.342(0.636)$ \\
\hline & & 1000 & $.078(.215)$ & $1.028(.541)$ & $-.031(.046)$ & $1.302(0.595)$ \\
\hline \multirow[t]{3}{*}{2.0} & 4 & 250 & $-.051(.576)$ & $1.023(.578)$ & $-.028(.091)$ & $2.682(1.236)$ \\
\hline & & 500 & $.037(.329)$ & $1.040(.566)$ & $-.030(.061)$ & $2.631(1.313)$ \\
\hline & & 1000 & $.075(.214)$ & $1.023(.536)$ & $-.030(.045)$ & $2.597(1.185)$ \\
\hline
\end{tabular}




\section{References}

Davis, D. J. (1952). An analysis of some failure data. Journal of American Statistical Association, 47, 113-150.

Efron, B. (1981). Nonparametric estimates of standard error: the jackknife, the bootstrap, and other resampling methods. Biometrika, 68, 589-599.

Efron, B. (1988). Logistic regression, survival analysis, and the Kaplan-Meier curve. Journal of American Statistical Association, 83, 414-425.

Famoye, F., Lee, C., \& Olumolade, O. (2005). The beta-Weibull distribution. Journal of Statistical Theory and Applications, 4(2), 121-136.

Glaser, R. E. (1980). Bathtub and related failure rate characterizations. Journal of American Statistical Association, 75 (371), 667672.

Gupta, R.D. \& Kundu, D. (1999). Generalized exponential distributions, Australian \& New Zealand Journal of Statistics 41(2), 173-188.

Gupta, R. D. \& Kundu, D. (2001). Exponetiated exponential family: an alternative to gamma and Weibull distributions, Biometrical Journal, 43, 117-130.

Hallinan, A. J. Jr. (1993). A review of the Weibull distribution. Journal of Quality Technology, 25, 85-93.

Johnson, N.L., Kotz, S. and Balakrishnan, N. (1994). Continuous univariate distributions, Volume 1 ( $2^{\text {nd }}$ Ed.). John Wiley and Sons, New York.

Johnson, N. L., Kotz, S., \& Balakrishnan, N. (1995). Continuous univariate distributions, Volume 2 ( $2^{\text {nd }}$ Ed). John Wiley and Sons, New York.

Lawless, J. F. (1982). Statistical Models and Methods for Lifetime Data. John Wiley and Sons, New York.
Mudholkar, G. S. \& Hutson, A. D. (1996). The exponentiated Weibull family: Some properties and a flood data application. Communications in Statistics-Theory and Methods, 25(12), 3059-3083.

Mudholkar, G. S. \& Kollia, G. D. (1994). Generalized Weibull family: A structural analysis. Communications in Statistics-Theory and Methods, 23(4), 1149-1171.

Mudholkar, G. S., Srivastava, D. K., \& Freimer, M. (1995). The exponentiated Weibull family: A reanalysis of the bus-motor-failure data. Technometrics, 37(4), 436-445.

Mudholkar, G. S., Srivastava, D. K., \& Kollia, G. D. (1996). A generalization of the Weibull distribution with application to the analysis of survival data. Journal of American Statistical Association, 91(436), 1575-1583.

Nadarajah, S. \& Zografos, K. (2003). Formulas for Renyi information and related measures for univariate distributions. Information Sciences, 155, 119-138.

Nadarajah, S. \& Zografos, K. (2005). Expressions for Renyi and Shannon entropies for bivariate distributions. Information Sciences 170, 173-189.

Nassar, M. M. \& Eissa, F. H. (2003). On the exponentiated Weibull distribution. Communications in Statistics-Theory and Methods, 32(7), 1317-1336.

Renyi, A. (1961) On measures of entropy and information, in: Proceedings of the $4^{\text {th }}$ Berkeley Symposium on Mathematical Statistics and Probability, Vol. I, University of California Press, Berkeley, 547-561.

Shannon, C. E. (1948). A mathematical theory of communication. Bell System Technical Journal, 27, 379-432.

Zacks, S. (1984). Estimating the shift to wear-out systems having exponential-Weibull life distributions. Operations Research, 32, 741749 . 


\section{On the Properties of Beta-Gamma Distribution}

\author{
Lingji Kong \\ Union College
}

\author{
Carl Lee J. H. Sepanski \\ Central Michigan University
}

A class of generalized gamma distribution called the beta-gamma distribution is proposed. Some of its properties are examined. Its shape can be reversed J-shaped, unimodal, or bimodal. Reliability and hazard functions are also derived, and applications are discussed.

Key words: Gamma distribution, Beta distribution, reliability function, hazard function, MLE, application.

\section{Introduction}

Let $f(\cdot)$ and $F(\cdot)$ be the probability density function and the cumulative distribution function (cdf) of a random variable, respectively. Eugene, Lee, and Famoye (2002) first introduced a generalized distribution based on the logit of the beta random variable with a cumulative distribution function given by

$$
G(x)=\frac{1}{B(\alpha, \beta)} \int_{0}^{F(x)} t^{\alpha-1}(1-t)^{\beta-1} d t, 0<\alpha, \beta<\infty,
$$

and the corresponding probability density function is

$$
g(x)=\frac{1}{B(\alpha, \beta)} F(x)^{\alpha-1}[1-F(x)]^{\beta-1} f(x), 0<\alpha, \beta<\infty
$$

Lingji Kong is an Assistant Professor of Mathematics. His email is 1kong@unionky.edu. Carl Lee is Professor of Statistics and senior research fellow at the Center for Applied Research and Technology, Central Michigan University. His research area is in generalized distributions and data mining. His email is lee1c@cmich.edu. Jungsywan Sepanski is an Associate Professor of Statistics in the Mathematics Department at Central Michigan University. Her research area is in measurement error and estimation. Her email is sepan1jh@cmich.edu.
Eugene, et al. (2002) studied properties of $g(x)$ when $F(\cdot)$ is the cdf of a normal distribution. Maynard (2003) examined the case when $F(\cdot)$ is the cdf of an exponential distribution.

Gamma distribution and its generalized distributions (e.g. McDonald, 1984) have been applied widely to the analyses of income distributions, life testing, and many physical and economical phenomena (e.g. Farewell, 1977, Lawless, 1980). In this article, the case when $F(\cdot)$ is the cdf of the gamma distribution is studied.

A random variable $X$ is said to have a beta-gamma distribution, $B G(\alpha, \beta, \rho, \lambda)$, if its probability density function is given by

$$
\begin{aligned}
& g(x)=\frac{x^{\rho-1} e^{-x / \lambda}}{B(\alpha, \beta) \Gamma(\rho) \lambda^{\rho}} F(x)^{\alpha-1}[1-F(x)]^{\beta-1}, \\
& 0<\alpha, \beta, \rho, \lambda<\infty, x>0
\end{aligned}
$$

where $F(x)$ is the cdf of the gamma distribution with parameters $\rho$ and $\lambda$. One can also introduce a location parameter $\xi$ in the density in (1) by replacing $x$ with $x-\xi$ where $-\infty<\xi<\infty$. In the rest of this article, it is assumed that $\xi$ is zero. When both $\alpha$ and $\beta$ are integers with $\alpha+\beta$ being a bounded integer, the beta-gamma density function in (1) is the marginal probability density function of 
the $\alpha^{\text {th }}$ order statistic in a random sample of size $\alpha+\beta$ from the gamma distribution with parameters $\rho$ and $\lambda$. When $\alpha=\beta=1$, the beta-gamma distribution yields the gamma distribution. When $\rho=1$, the beta-gamma distribution is beta-exponential distribution introduced in Maynard (2003).

Properties

The limit of $g(x)$ as $x$ goes to 0 and the mode of the probability density function $g(x)$ in (1) is given in Lemma 1 . The modes for cases when $\rho \alpha \leq 1$ and $\rho \alpha>1$ are studied respectively. Although some cases can be shown mathematically, plotting the function $g(x)$ using Maple computer programs are employed to examine shapes and modalities for other cases. Illustrative graphs of $g(x)$ based on observations from numerous plots are presented. Numerical percentiles are presented in Table 7 to Table 9 .

Limits

Lemma 1: The limit as $x$ goes to 0 of the beta-gamma probability density function $g(x)$ in (1) is

$$
\begin{aligned}
& \lim _{x \rightarrow 0} g(x)= \\
& \begin{cases}\infty & \text { if } \alpha \rho<1 \\
\frac{1}{\Gamma^{\alpha}(\rho) B(\alpha, \beta) \rho^{\alpha-1} \lambda} & \text { if } \alpha \rho=1 \\
0 & \text { if } \alpha \rho>1\end{cases}
\end{aligned}
$$

The proof is given in Appendix.

Modes of $g(x)$ When $\alpha \rho \leq 1$

Note that the derivative $d f / d x=f(\rho-1-x / \lambda) / x$. The first derivative of the logarithm of the probability density function $g(x)$ is given by

$$
\frac{\rho-1-x / \lambda}{x}+\frac{\alpha-1}{F} f+\frac{1-\beta}{1-F} f .
$$

The mode(s) $x_{m}$ of $g(x)$ if exists is the solution to the equation by setting (3) to be zero.

It is shown below that $g(x)$ has a reversed-J shape when $\rho \alpha \leq 1$ and $\beta \geq 1$. The derivative in (3) is equal to

$$
(1-\beta) \frac{\mathrm{f}}{1-\mathrm{F}}+\frac{1}{\mathrm{xF}}[(\alpha-1) \mathrm{xf}+(\rho-1-\mathrm{x} / \lambda) \mathrm{F}] .
$$

When $\beta \geq 1$, the first term in (4) is less or equal to 0 . Also,

$$
\begin{aligned}
& \frac{\mathrm{d}}{\mathrm{dx}}[(\alpha-1) \mathrm{xf}+(\rho-1-\mathrm{x} / \lambda) \mathrm{F}] \\
& =(\alpha-1) \mathrm{f}+(\alpha-1) \times \mathrm{xf} \frac{\rho-1-\mathrm{x} / \lambda}{\mathrm{x}} \\
& -\mathrm{F} / \lambda+(\rho-1-\mathrm{x} / \lambda) \mathrm{f} \\
& =(\alpha \rho-1) \mathrm{f}-\alpha \mathrm{xf} / \lambda-\mathrm{F} / \lambda
\end{aligned}
$$

which is negative when $\alpha \rho \leq 1$. This implies that $(\alpha-1) x f+(\rho-1-x / \lambda) F \quad$ is $\quad$ a decreasing function. Because $(\alpha-1) x f+(\rho-1-x / \lambda) F=0$ when $x=0$, the second term in (4) is therefore negative. That is, $g^{\prime}(x)$ is negative. By (2) and the fact that $\lim _{x \rightarrow \infty} g(x)=0, g(x)$ has a reversed-J shape for the cases when $\rho \alpha \leq 1$ and $\beta \geq 1$ with maximum occurring at $x=0$.

When $\alpha \leq 1$ and $\rho \leq 1$ regardless of $\beta$, one can see that $g(x)$ has a reversed-J shape by rewriting $g(x)$ as

$$
g(x)=\frac{1}{B(\alpha, \beta)} \frac{f(x)}{1-F(x)} F(x)^{\alpha-1}[1-F(x)]^{\beta} .
$$

Because the cdf $F$ is an increasing function and the hazard function $f /(1-F)$ of the gamma distribution function is a decreasing function when $\rho \leq 1, g(x)$ is therefore a decreasing function with $\lim _{x \rightarrow 0} g(x)=\infty$ when $\alpha \leq 1$ and $\rho \leq 1$. 

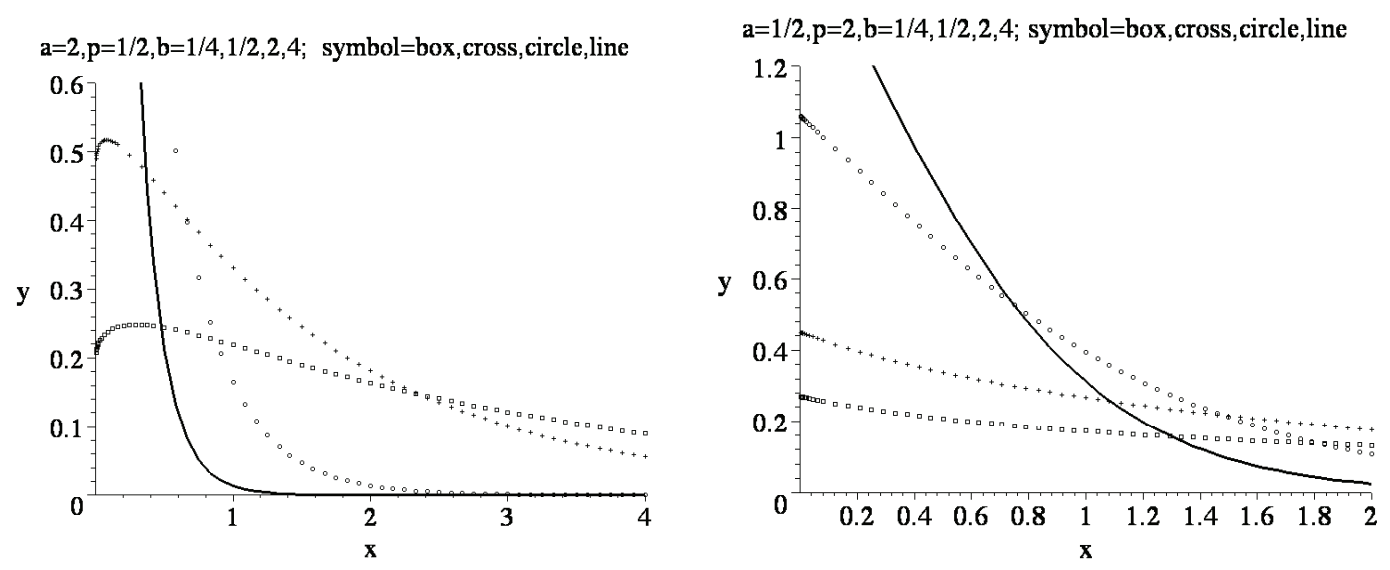

$a=4, p=0.25$ and $b=.25,5,2,4$. symbol=box, cross, circle, line

$p=4, a=0.25$, and $b=.25,5,2,4$. symbol=box, cross, circle, line
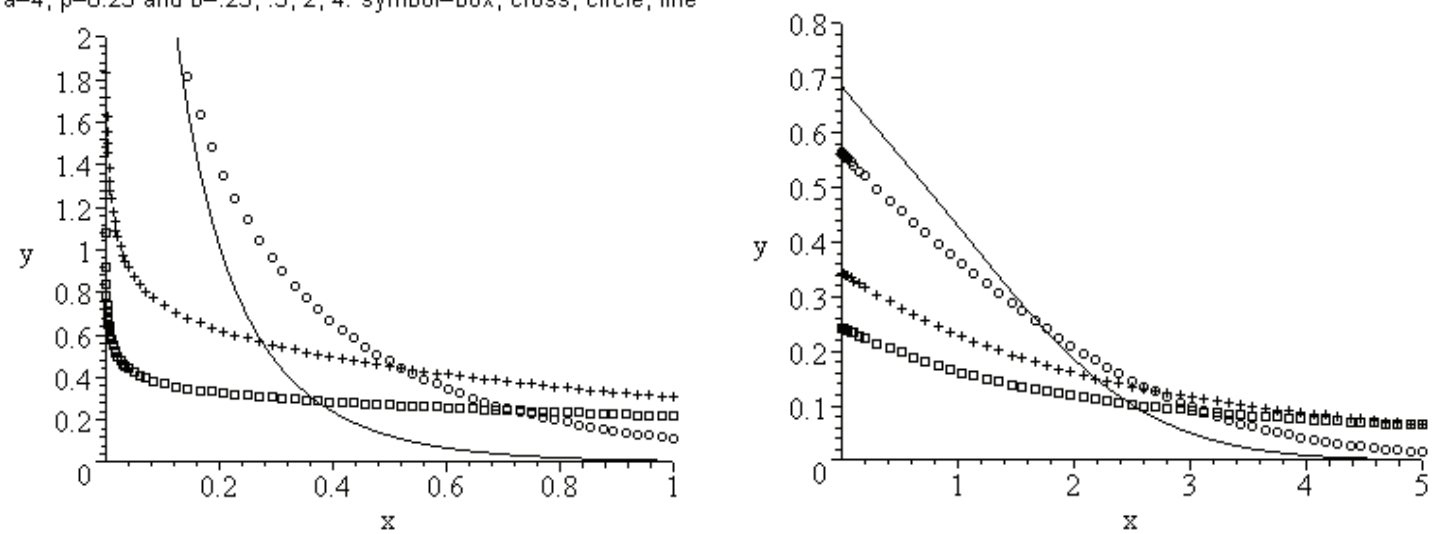

Figure 1. Plot of the density function $g(x)$ when $\rho \alpha=1$, and $\beta=0.25,0.5,2,4$

Next, graphical results are shown to examine the cases when $\beta<1$ and $\alpha \rho \leq 1$ with $\alpha$ or $\rho$ greater than 1 . Figure 1 represents cases when $\alpha \rho=1$. Figure 2 contains cases when $\alpha \rho<1$. Note that $\mathrm{a}=\alpha, \mathrm{b}=\beta$, and $\mathrm{p}$ $=\rho$ in all figures in this article. 


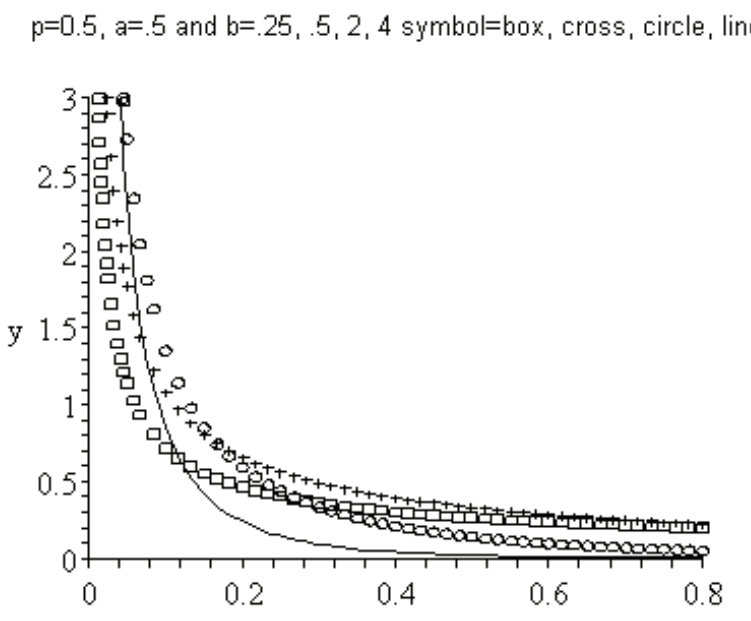

$p=3, a=.25$ and $b=.25, .5,2,4$ symbol=box, cross, circle, line

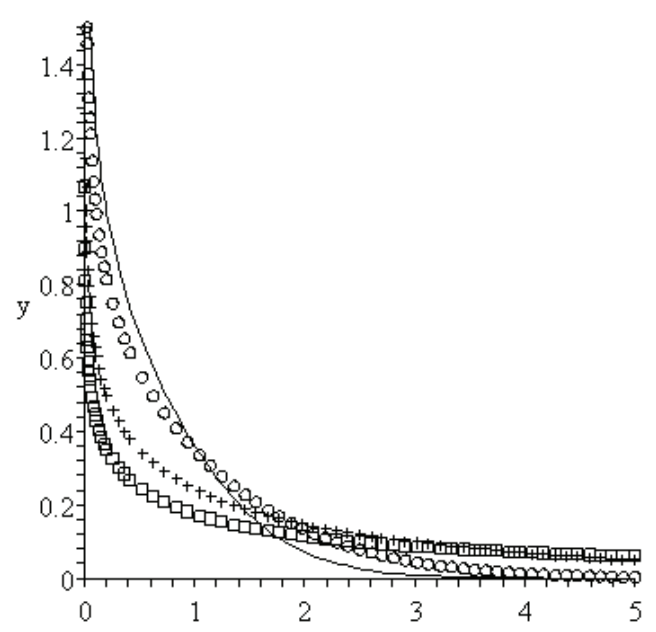

$p=0.75, a=.25$ and $b=25,5,2,4$ symbol=box, cross, circle, line

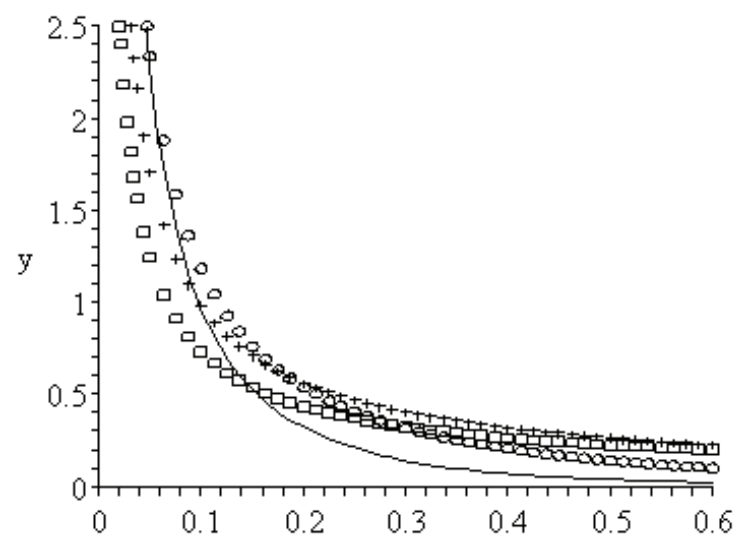

$p=.25, a=3$ and $b=.25,5,2,4$ symbol=box, cross, circle, line

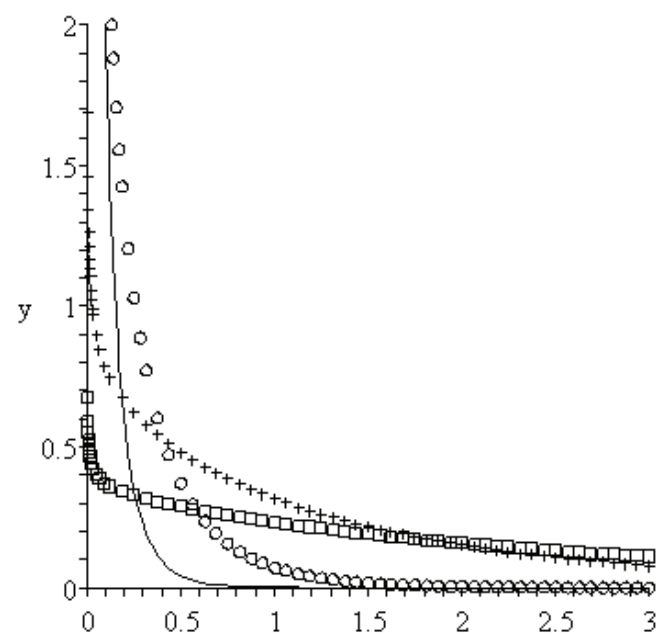

Figure 2. Plot of the density function $g(x)$ when $\rho \alpha<1$, and $\beta=0.25,0.5,2,4$

When $\rho \alpha=1$ and $\beta \geq 1$, the betagamma distribution appears to have a reversed-J shape. Figure 1 also shows that when $\alpha=2$ and $\rho=0.5$, it has a non-zero mode for $\beta$ values of 0.25 and 0.5 .

When $\rho \alpha<1$ and $\beta<1$, it is found that $g(x)$ is not necessarily a reverse J-shape, it can be bimodal (with one mode at 0 ). Figure 3 shows two such cases. The top two are for $\rho=0.25, \alpha=3.9$, and $\beta=0.5$; the bottom graph is for $\rho=2, \alpha=0.49$, and $\beta=0.01$. Note that the horizontal axis of the first plot ranges from 0 to 0.01 and the one of the second plot ranges from 0.01 to 2 . Tables $1-4$ give the 2nd non-zero mode in addition to the mode at $x=0$ for some examples when $\alpha \rho<1$ and $\rho<1$. The empty cells are cases where $g(x)$ is reverse $\mathrm{J}$-shaped and the only mode is at $x=0$. 
$\mathrm{a}=3.9, \mathrm{~b}=0.5$ and $\mathrm{p}=0.25$

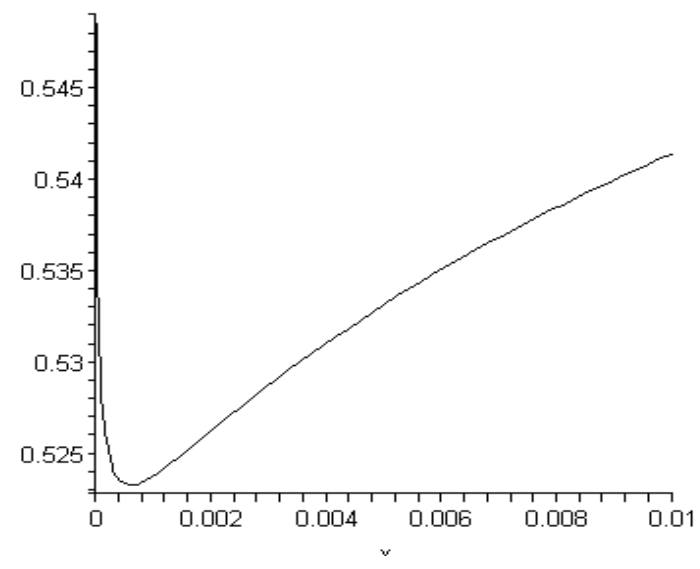

$\mathrm{a}=0.49, \mathrm{~b}=0.01$ and $\mathrm{p}=2$ $\mathrm{a}=3.9, \mathrm{~b}=0.5$ and $\mathrm{p}=0.25$

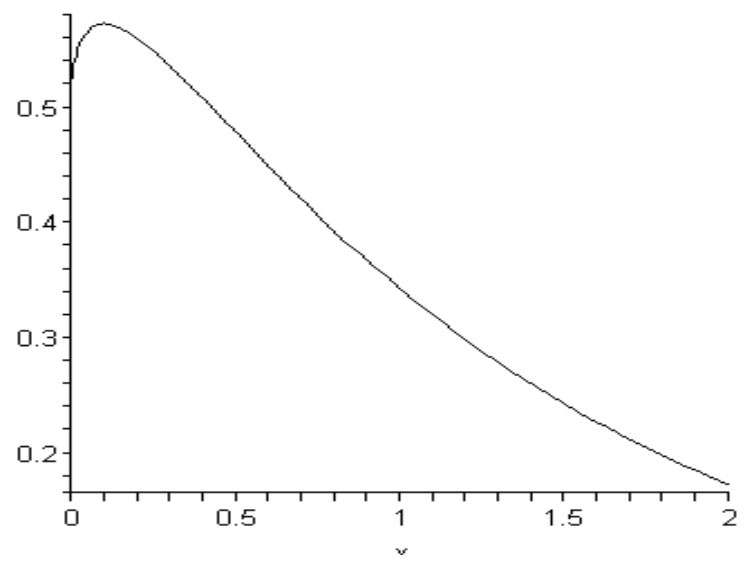

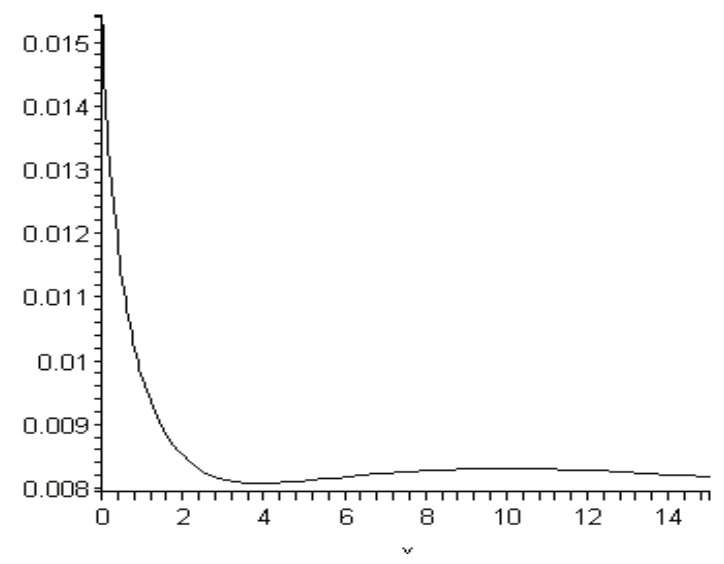

Figure 3. Graphs of $B G(\alpha, \beta, \rho, 1)$ 
Table 1. Nonzero 2nd mode of $B G(\alpha, \beta, \rho, 1)$ with $\rho=1 / 2$.

\begin{tabular}{|r|l|l|l|l|l|l|l|l|l|l|}
\hline & $\beta=0.01$ & 0.1 & 0.2 & 0.3 & 0.4 & 0.5 & 0.6 & 0.7 & 0.8 & 0.9 \\
\hline$\alpha=1.8$ & 1.04 & & & & & & & & & \\
\hline 1.9 & 1.31 & .529 & .234 & & & & & & & \\
\hline 1.95 & 1.42 & .616 & .319 & .167 & .074 & & & & & \\
\hline 1.99 & 1.50 & .678 & .374 & .219 & .013 & .071 & .035 & .012 & & \\
\hline
\end{tabular}

Table 2(a). Nonzero 2nd mode of $B G(\alpha, \beta, \rho, 1)$ with $\rho=1 / 4$.

\begin{tabular}{|c|l|l|l|l|l|l|l|l|l|l|}
\hline & $\beta=0.01$ & 0.1 & 0.2 & 0.3 & 0.4 & 0.5 & 0.6 & 0.7 & 0.8 & 0.9 \\
\hline$\alpha=3.01$ & & & & & & & & & & \\
\hline 3.02 & .365 & & & & & & & & & \\
\hline 3.1 & .571 & & & & & & & & & \\
\hline 3.2 & .714 & & & & & & & & & \\
\hline 3.3 & .826 & .274 & & & & & & & & \\
\hline 3.4 & .923 & .382 & & & & & & & & \\
\hline 3.5 & 1.01 & .460 & .193 & & & & & & & \\
\hline 3.6 & 1.09 & .526 & .266 & .103 & & & & & & \\
\hline 3.7 & 1.16 & .585 & .323 & .173 & .064 & & & & & \\
\hline 3.8 & 1.22 & .638 & .373 & .222 & .124 & .052 & & & & \\
\hline 3.9 & 1.29 & .687 & .417 & .264 & .165 & .097 & .049 & & & \\
\hline
\end{tabular}

Table 2(b). Nonzero 2nd mode of $B G(\alpha, \beta, \rho, 1)$ with $\rho=1 / 4$.

\begin{tabular}{|c|l|l|l|l|l|l|l|l|l|}
\hline & $\beta=0.91$ & 0.92 & 0.93 & 0.94 & 0.95 & 0.96 & 0.97 & 0.98 & 0.99 \\
\hline$\alpha=3.99$ & .004 & .003 & .001 & & & & & & \\
\hline 3.995 & .005 & .004 & .003 & .002 & .001 & & & & \\
\hline
\end{tabular}

Table 3. Nonzero 2nd mode of $B G(\alpha, \beta, \rho, 1)$ with $\rho=1 / 6$

\begin{tabular}{|r|c|c|c|c|c|c|c|c|c|c|}
\hline & $\beta=.01$ & 0.1 & 0.2 & 0.3 & 0.4 & 0.5 & 0.6 & 0.7 & 0.8 & 0.9 \\
\hline$\alpha=5.8$ & 1.21 & .67 & .41 & .27 & .17 & .10 & .05 & & & \\
\hline 5.9 & 1.24 & .69 & .44 & .29 & .19 & .12 & .07 & .036 & .020 & \\
\hline 5.99 & 1.27 & .72 & .48 & .31 & .21 & .14 & .09 & .052 & .026 & .008 \\
\hline
\end{tabular}

Table 4. Nonzero 2 nd mode of $B G(\alpha, \beta, \rho, 1)$ with $\rho=2$

\begin{tabular}{|r|l|l|l|l|l|l|}
\hline & $\beta=0.01$ & 0.015 & 0.1 & 0.2 & 0.3 & 0.4 \\
\hline$\alpha=0.48$ & & & & & & \\
\hline 0.49 & 9.85 & & & & & \\
\hline 0.499 & 9.86 & 7.656 & & & & \\
\hline
\end{tabular}


Note that, for example, when $\alpha=0.48$, $\beta=0.01$, and $\rho=2, g(x)$ has an inversed-J shape and therefore does not have a nonzero mode. The range of $\beta$ where $g(x)$ is bimodal appears to widen as $\alpha$ increases. When bimodality occurs, the nonzero mode increases as the parameter $\alpha$ increases and decreases as the parameter $\beta$ increases. The bimodality property of beta-gamma distribution is not independent of the gamma parameters $(\alpha, \rho)$. The bimodality property also exists for betanormal (Famoye, Lee, \& Eugene, 2004).

Modes when $\alpha \rho>1$

The second derivative of the logarithm of $g(x)$ is given by

$$
\begin{aligned}
& \frac{\rho-1-x / \lambda}{x}\left[\frac{(\alpha-1) f}{F}+\frac{(1-\beta) f}{1-F}+\frac{\rho-1-x / \lambda}{x}\right] \\
& -\left[\frac{(\rho-1-x / \lambda)^{2}}{x^{2}}+\frac{(\alpha-1) f^{2}}{F^{2}}-\frac{(1-\beta) f^{2}}{(1-F)^{2}}+\frac{\rho-1}{x^{2}}\right]
\end{aligned}
$$

The first term equals to 0 at the mode $x_{m}$. Hence, when $x=x_{m}$,

$$
\begin{aligned}
& \frac{d^{2} \ln g}{d x^{2}}= \\
& -\left[\begin{array}{l}
\frac{(\rho-1-x)^{2}}{x^{2}}+\frac{(\alpha-1) f^{2}}{F^{2}} \\
+\frac{(\beta-1) f^{2}}{(1-F)^{2}}+\frac{\rho-1}{x^{2}}
\end{array}\right] .
\end{aligned}
$$

When $\beta \geq 1, \alpha \geq 1$, and $\rho \geq 1$, (5) is less than 0 at $x=x_{m}$. In this case, since there must be a minimum between any two maxima and that $\lim _{x \rightarrow 0} g(x)=0$ and $\lim _{x \rightarrow \infty} g(x)=0$, it is concluded that $g(x)$ is unimodal with a concave shape.

When $\beta \geq 1$ and $\alpha \rho>1$ with $\alpha<1$ or $\rho<1$, though not being shown mathematically, graphs of such cases indicate that beta-gamma density function $g(x)$ is also unimodal with a concave shape. Based on numerous graphs, the density functions $g(x)$ is unimodal when $\alpha \rho>1$ regardless the value of $\beta$. The following illustrates some examples when $\alpha \rho>1$. 

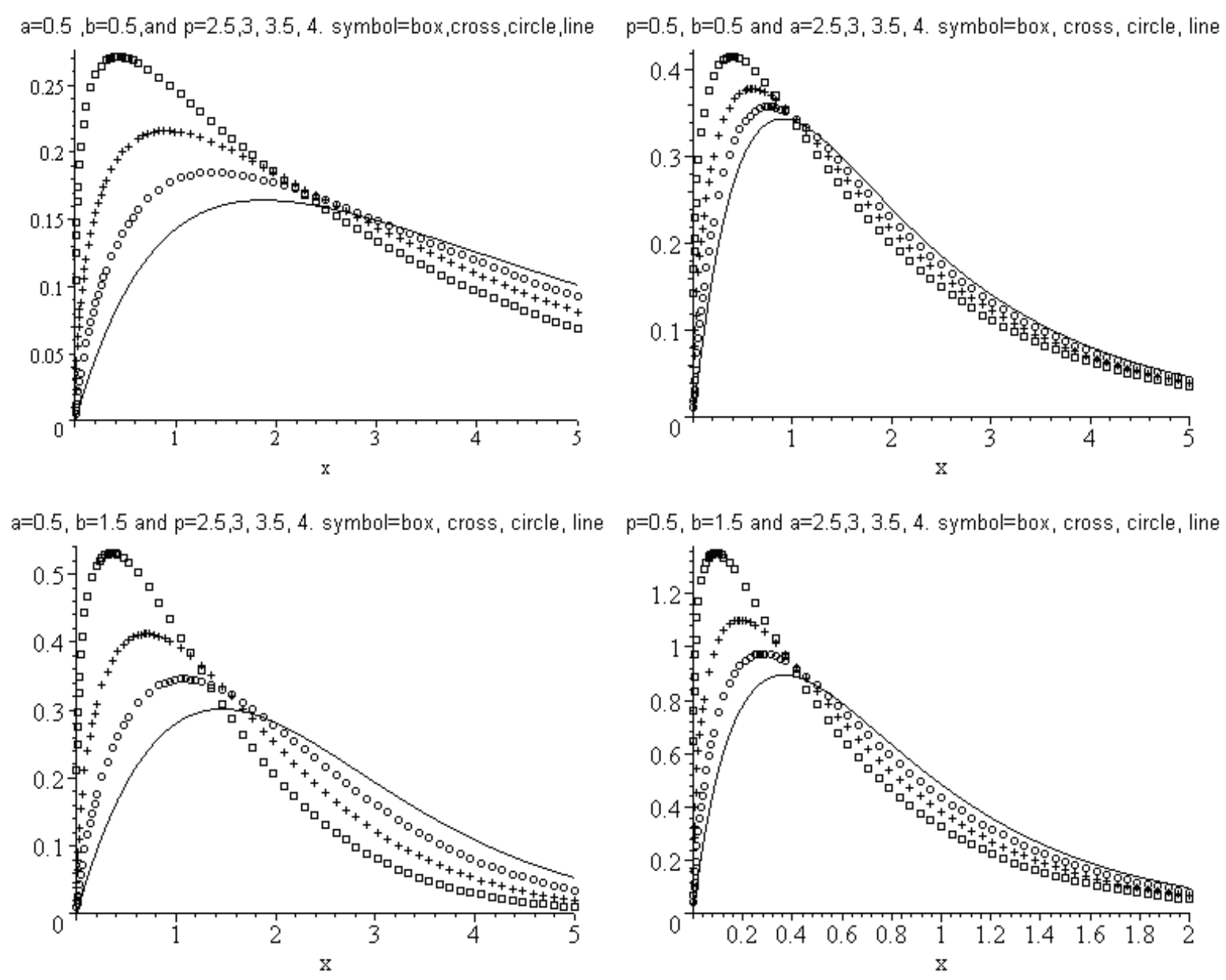

Figure 4. Plot of the density function $g(x)$ when $\rho \alpha>1$, and $\beta=0.5,1.5$

In this case $g(x)$ is unimodal with a concave shape and the mode is nonzero. Tables 5 and 6 tabulate modes for $B G(\alpha, \beta, 2,1)$ and $B G(\alpha, \beta, 1 / 2,1)$ when $\rho \alpha>1$.

The results indicate that when $\rho \alpha>1$ the mode increases as $\alpha$ increases and that the mode decreases as $\beta$ increases for both $B G(\alpha, \beta, 2,1)$ and $B G(\alpha, \beta, 1 / 2,1)$; see also Figure 4. For other cases when $\alpha \rho>1$, this pattern holds for other values of parameters $\rho$ and $\lambda$ though the computation results are not reported here.

Percentiles of $g(x)$

The $50^{\text {th }}, 75^{\text {th }}, 90^{\text {th }}$, and $95^{\text {th }}$ percentiles of $B G(\alpha, \beta, \rho, 1)$ are computed and tabulated in the following Tables 7-9. 
Table 5. Modes for $B G(\alpha, \beta, 2,1)$ when $\rho \alpha>1$

\begin{tabular}{lcccccccc} 
& $\beta=0.2$ & 0.5 & 1 & 1.5 & 2 & 2.5 & 5 & 10 \\
\hline$\alpha=1$ & 2.236 & 1.414 & 1.000 & .8165 & .7071 & .6325 & .4472 & .3162 \\
$\alpha=1.5$ & 3.206 & 2.162 & 1.555 & 1.270 & 1.097 & .9775 & .6815 & .4744 \\
$\alpha=2$ & 3.729 & 2.628 & 1.938 & 1.598 & 1.386 & 1.238 & .8644 & .5994 \\
$\alpha=2.5$ & 4.087 & 2.963 & 2.229 & 1.856 & 1.618 & 1.451 & 1.018 & .7063 \\
$\alpha=5$ & 5.055 & 3.915 & 3.111 & 2.672 & 2.379 & 2.163 & 1.570 & 1.107 \\
$\alpha=10$ & 5.913 & 4.787 & 3.964 & 3.498 & 3.176 & 3.947 & 2.228 & 1.626 \\
\hline
\end{tabular}

Table 6. Modes for $B G(\alpha, \beta, 1 / 2,1)$ when $\rho \alpha>1$.

\begin{tabular}{lcccccccc} 
& $\beta=0.2$ & 0.5 & 1 & 1.5 & 2 & 2.5 & 5 & 10 \\
\hline$\alpha=2.5$ & 0.832 & .3919 & .1692 & .0903 & .0545 & .0359 & .0291 & .0154 \\
$\alpha=5$ & 1.788 & 1.129 & .7150 & .5137 & .3923 & .3114 & .1337 & .0735 \\
$\alpha=10$ & 2.653 & 1.798 & 1.286 & 1.016 & .8411 & .7155 & .3925 & .1812 \\
\hline
\end{tabular}

Table 7. Percentiles of $B G(\alpha, \beta, \rho, 1)$ with $\rho=1 / 2$

\begin{tabular}{|c|c|c|c|c|c|}
\hline$\alpha$ & $\beta$ & 50 th & 75 th & 90th & 95th \\
\hline 0.25 & $\begin{array}{l}0.25 \\
0.5 \\
1 \\
2 \\
4\end{array}$ & $\begin{array}{l}.2275 \\
.0235 \\
.0031 \\
.0005 \\
.0001\end{array}$ & $\begin{array}{l}2.011 \\
.4372 \\
.0831 \\
.0169 \\
.0038\end{array}$ & $\begin{array}{l}5.260 \\
1.684 \\
.4479 \\
.1116 \\
.0277\end{array}$ & $\begin{array}{l}7.855 \\
2.846 \\
.8765 \\
.2445 \\
.0654\end{array}$ \\
\hline 0.5 & $\begin{array}{l}0.25 \\
0.5 \\
1 \\
2 \\
4\end{array}$ & $\begin{array}{l}.9346 \\
.2275 \\
.0508 \\
.0115 \\
.0027\end{array}$ & $\begin{array}{l}3.194 \\
1.054 \\
.3014 \\
.0802 \\
.0207\end{array}$ & $\begin{array}{l}6.550 \\
2.530 \\
.8588 \\
.2632 \\
.0752\end{array}$ & $\begin{array}{l}9.171 \\
3.716 \\
1.358 \\
.4522 \\
.1370\end{array}$ \\
\hline 1 & $\begin{array}{l}0.25 \\
0.5 \\
1 \\
2 \\
4\end{array}$ & $\begin{array}{c}1.735 \\
.6617 \\
.2275 \\
.0706 \\
.0202\end{array}$ & $\begin{array}{l}4.163 \\
1.735 \\
.6617 \\
.2275 \\
.0706\end{array}$ & $\begin{array}{r}7.568 \\
3.317 \\
1.353 \\
.5022 \\
.1678\end{array}$ & $\begin{array}{l}10.21 \\
4.570 \\
1.921 \\
.7405 \\
.2576\end{array}$ \\
\hline 2 & $\begin{array}{l}0.25 \\
0.5 \\
1 \\
2 \\
4\end{array}$ & $\begin{array}{l}2.473 \\
1.205 \\
.5531 \\
.2275 \\
.0816\end{array}$ & $\begin{array}{l}4.979 \\
2.405 \\
1.123 \\
.4817 \\
.1824\end{array}$ & $\begin{array}{l}8.413 \\
4.044 \\
1.899 \\
.8367 \\
.3306\end{array}$ & $\begin{array}{l}11.06 \\
5.316 \\
2.501 \\
1.115 \\
.4504\end{array}$ \\
\hline 4 & $\begin{array}{l}0.25 \\
0.5 \\
1 \\
2 \\
4\end{array}$ & $\begin{array}{l}3.160 \\
1.787 \\
.9914 \\
.5073 \\
.2275\end{array}$ & $\begin{array}{l}5.710 \\
3.057 \\
1.649 \\
.8444 \\
.3872\end{array}$ & $\begin{array}{l}9.166 \\
4.731 \\
2.478 \\
1.261 \\
.5869\end{array}$ & $\begin{array}{l}11.82 \\
6.016 \\
3.102 \\
1.570 \\
.7351\end{array}$ \\
\hline
\end{tabular}


Table 8. Percentiles of $B G(\alpha, \beta, \rho, 1)$ with $\rho=1$

\begin{tabular}{llllll}
\hline$\alpha$ & $\beta$ & 50 th & 75 th & 90 th & 95 th \\
\hline 0.25 & 0.25 & .6939 & 3.106 & 6.752 & 9.535 \\
& 0.5 & .1882 & 1.050 & 2.710 & 4.070 \\
& 1 & .0645 & .3804 & 1.067 & 1.685 \\
& 2 & .0265 & .1577 & .4517 & .7249 \\
& 4 & .0120 & .0718 & .2065 & .3330 \\
0.5 & 0.25 & 1.763 & 4.466 & 8.125 & 10.90 \\
& 0.5 & .6925 & 1.919 & 3.704 & 5.077 \\
& 1 & .2877 & .8267 & 1.661 & 2.328 \\
& 2 & .1285 & .3729 & .7592 & 1.075 \\
& 4 & .0512 & .1758 & .3588 & .5086 \\
& 0.25 & 2.773 & 5.545 & 9.210 & 11.98 \\
& 0.5 & 1.386 & 2.773 & 4.605 & 5.991 \\
& 1 & .6931 & 1.386 & 2.303 & 2.996 \\
& 2 & .3466 & .6931 & 1.151 & 1.498 \\
& 4 & .1733 & .3466 & .5756 & .7489 \\
& 0.25 & 3.644 & 6.436 & 10.10 & 12.88 \\
& 0.5 & 2.115 & 3.565 & 5.366 & 6.720 \\
& 1 & 1.228 & 2.010 & 2.970 & 3.676 \\
& 2 & .6931 & 1.120 & 1.631 & 2.000 \\
& 4 & .3766 & .6055 & .8768 & 1.071 \\
& 4 & 4.428 & 7.229 & 10.90 & 13.68 \\
& 0.25 & 2.836 & 4.312 & 6.167 & 7.556 \\
& 1 & 1.838 & 2.668 & 3.650 & 4.363 \\
& 2 & 1.159 & 1.641 & 2.187 & 2.571 \\
& 4 & .6931 & .9706 & 1.278 & 1.490 \\
\hline
\end{tabular}


Table 9. Percentiles of $B G(\alpha, \beta, \rho, 1)$ with $\rho=2$

\begin{tabular}{|c|c|c|c|c|c|}
\hline$\alpha$ & $\beta$ & 50th & 75th & 90th & 95th \\
\hline$\overline{0.25}$ & $\begin{array}{l}0.25 \\
0.5 \\
1 \\
2 \\
4\end{array}$ & $\begin{array}{l}1.678 \\
.7450 \\
.4035 \\
.2482 \\
.1631\end{array}$ & $\begin{array}{l}4.874 \\
2.220 \\
1.142 \\
.6713 \\
.4277\end{array}$ & $\begin{array}{l}9.049 \\
4.397 \\
2.244 \\
1.273 \\
.7860\end{array}$ & $\begin{array}{l}12.09 \\
6.021 \\
3.094 \\
1.729 \\
1.050\end{array}$ \\
\hline 0.5 & $\begin{array}{l}0.25 \\
0.5\end{array}$ & $\begin{array}{l}3.197 \\
1.678\end{array}$ & $\begin{array}{l}6.479 \\
3.404\end{array}$ & $\begin{array}{l}10.58 \\
5.597\end{array}$ & $\begin{array}{l}13.58 \\
7.194\end{array}$ \\
\hline & $\begin{array}{l}1 \\
2 \\
4\end{array}$ & $\begin{array}{l}.9613 \\
.5961 \\
.3893\end{array}$ & $\begin{array}{c}1.887 \\
1.128 \\
.7157\end{array}$ & $\begin{array}{l}3.063 \\
1.782 \\
1.102\end{array}$ & $\begin{array}{l}3.922 \\
2.254 \\
1.374\end{array}$ \\
\hline 1 & $\begin{array}{l}0.25 \\
0.5 \\
1 \\
2 \\
4\end{array}$ & $\begin{array}{l}4.472 \\
2.693 \\
1.678 \\
1.078 \\
.7095\end{array}$ & $\begin{array}{l}7.710 \\
4.472 \\
2.693 \\
1.678 \\
1.078\end{array}$ & $\begin{array}{l}11.76 \\
6.638 \\
3.890 \\
2.365 \\
1.487\end{array}$ & $\begin{array}{l}14.74 \\
8.212 \\
4.744 \\
2.845 \\
1.767\end{array}$ \\
\hline 2 & $\begin{array}{l}0.25 \\
0.5 \\
1 \\
2 \\
4\end{array}$ & $\begin{array}{l}5.519 \\
3.653 \\
2.473 \\
1.678 \\
1.135\end{array}$ & $\begin{array}{l}8.710 \\
5.425 \\
3.518 \\
2.320 \\
1.536\end{array}$ & $\begin{array}{l}12.72 \\
7.560 \\
4.712 \\
3.023 \\
1.963\end{array}$ & $\begin{array}{l}15.69 \\
9.116 \\
5.557 \\
3.505 \\
2.250\end{array}$ \\
\hline 4 & $\begin{array}{l}0.25 \\
0.5 \\
1 \\
2 \\
4\end{array}$ & $\begin{array}{l}6.434 \\
4.549 \\
3.296 \\
2.376 \\
1.678\end{array}$ & $\begin{array}{l}9.587 \\
6.299 \\
4.344 \\
3.036 \\
2.103\end{array}$ & $\begin{array}{l}13.57 \\
8.408 \\
5.526 \\
3.744 \\
2.543\end{array}$ & $\begin{array}{l}16.53 \\
9.949 \\
6.359 \\
4.225 \\
2.834\end{array}$ \\
\hline
\end{tabular}

The percentiles increase as $\alpha$ increases and decrease as $\beta$ increases with fixed $\rho$, which is consistent with the results of modes. As seen in all figures, the beta-gamma distribution is skewed to the right, one would expect that the mode to be less than the median.

\section{Moments}

The closed form solutions of moments for $B G(\alpha, \beta, \rho, \lambda)$ exist only when $\alpha$ and $\beta$ are integers. The closed form solution for the $n$th moment of $B G(\alpha, \beta, \rho, \lambda)$ is derived in Theorem 1 for the cases when $\alpha$ and $\beta$ are integers in this section. The first four moments are also numerically computed for various parameters.

Theorem 1: When $\alpha, \beta$ are integers, the $n$th moment of the beta-gamma random variable $B G(\alpha, \beta, \rho, \lambda)$ is given by 
$\frac{1}{B(\alpha, \beta)} \sum_{j=0}^{\beta-1}(-1)^{j}\left(\begin{array}{l}\beta-1 \\ j\end{array}\right)\left\{\sum_{k=0}^{\alpha+j-1}(-1)^{k}\left(\begin{array}{l}\alpha+j-1 \\ k\end{array}\right) I_{n, k}\right\}$

where

$$
I_{n, k}=\int_{0}^{\infty} x^{n} f(x)(1-F)^{k} d x
$$

The proof is given in Appendix. The follow Corollary gives $E(X)$ and $E\left(X^{2}\right)$ that are used to obtain variance.

Corollary 1: When $\alpha=2, \beta=1$ and $\rho$ is an integer, $E(X)$ and $E\left(X^{2}\right)$ are given by:

$$
\begin{aligned}
E(X) & =2 \lambda \rho-\sum_{i=0}^{\rho-1} \frac{\lambda(\rho+i) !}{2^{\rho+i}(\rho-1) ! i !} \\
& =2 \lambda \rho-\rho \lambda \sum_{i=0}^{\rho-1} \frac{\left(\begin{array}{l}
\rho+i \\
\rho
\end{array}\right)}{2^{\rho+i}} ;
\end{aligned}
$$

$$
E\left(X^{2}\right)=2 \lambda^{2}(\rho+1) \rho
$$

$$
=\rho(\rho+1) \lambda^{2}\left[2-\sum_{i=0}^{\rho-1} \frac{\left(\begin{array}{l}
\rho+i+1 \\
\rho+1
\end{array}\right)}{2^{\rho+i+1}}\right]
$$

The proof is given in Appendix.

Applying (6), the first four moments of

\begin{tabular}{|c|c|c|c|c|c|}
\hline$\alpha$ & $\beta$ & mean & std & skewness & kurtosis \\
\hline \multirow[t]{4}{*}{1} & 1 & 2.000 & 1.414 & 1.415 & 6.005 \\
\hline & 2 & 1.250 & .8292 & 1.261 & 5.329 \\
\hline & 4 & .8047 & .5048 & 1.120 & 4.768 \\
\hline & 10 & .4660 & .2757 & .9672 & 4.220 \\
\hline \multirow[t]{4}{*}{2} & 1 & 2.750 & 1.479 & 1.207 & 5.347 \\
\hline & 2 & 1.824 & .8975 & 1.010 & 4.588 \\
\hline & 4 & 1.215 & .5585 & .8693 & 4.117 \\
\hline & 10 & .7150 & .3063 & .7319 & 3.736 \\
\hline \multirow[t]{4}{*}{4} & 1 & 3.547 & 1.494 & 1.106 & 5.094 \\
\hline & 2 & 2.503 & .9356 & .8595 & 4.238 \\
\hline & 4 & 1.747 & .5987 & .7038 & 3.779 \\
\hline & 10 & 1.062 & .3345 & .5752 & 3.481 \\
\hline \multirow[t]{4}{*}{10} & 1 & 4.623 & 1.500 & 1.057 & 5.007 \\
\hline & 2 & 3.503 & .9505 & .7650 & 4.063 \\
\hline & 4 & 2.618 & .6278 & .5759 & 3.577 \\
\hline & 10 & 1.705 & .3654 & .4363 & 3.302 \\
\hline
\end{tabular}
$B G(\alpha, \beta, \rho, 1)$ for a certain combinations of the parameters are evaluated and given in Tables 10 and 11.

Table 10. The mean, std, skewness and kurtosis of $B G(\alpha, \beta, \rho, 1)$ with $\rho=2$. 
Table 11. The mean, std, skewness and kurtosis of $B G(\alpha, \beta, \rho, 1)$ with $\rho=1 / 2$

\begin{tabular}{lrllll}
\hline$\alpha$ & $\beta$ & mean & std & skewness & kurtosis \\
\hline 1 & 1 & .5000 & .7071 & 2.829 & 15.00 \\
& 2 & .1814 & .2828 & 3.287 & 18.66 \\
& 4 & .0604 & .1038 & 3.834 & 26.22 \\
& 10 & .0124 & .0237 & 4.688 & 39.71 \\
& 1 & .8180 & .8468 & 2.172 & 10.28 \\
& 2 & .3523 & .3830 & 2.290 & 11.11 \\
& 4 & .1356 & .1586 & 2.544 & 13.14 \\
& 10 & .0319 & .0413 & 3.032 & 17.96 \\
& 1 & 1.235 & .9584 & 1.762 & 8.011 \\
& 2 & .6280 & .4830 & 1.669 & 7.422 \\
& 4 & .2868 & .2289 & 1.718 & 7.614 \\
& 10 & .0820 & .0712 & 1.955 & 9.080 \\
& & & & & \\
& 1 & 1.900 & 1.057 & 1.468 & 6.683 \\
& 2 & 1.154 & .5878 & 1.218 & 5.491 \\
& 4 & .6508 & .3228 & 1.097 & 4.941 \\
& 10 & .2505 & .1290 & 1.119 & 4.968 \\
\hline
\end{tabular}

Based on the numerical results, the mean and standard deviation appear to increase with $\alpha$ for a fixed $\beta$; and skewness and kurtosis appear to decrease as $\alpha$ increases for a fixed $\beta$ in both cases when $\rho=2$ and $\rho=1 / 2$. Based on Figure 4, the density function has a heavier right tail as $\alpha$ increases. The mean and standard deviation decrease as $\beta$ decreases for a fixed $\alpha$. Although the skewness and kurtosis decrease with $\beta$ when $\rho=2$ as shown in Table 10 , the skewness and kurtosis increase with $\beta$ when $\rho=1 / 2$ and $\alpha \rho \leq 1$, as shown in Table 11. However, no clear pattern is noticed when $\alpha \rho>1$.

\section{Reliability and Hazard Functions}

The reliability and hazard functions of the beta-gamma distribution are derived in this section. The reliability function, $R(x)=1-P[X \leq x]$, at time $x$ defined to be the probability that a unit $X$ survives beyond time $x$. For a beta-gamma random variable, it is given by

$$
\begin{aligned}
& 1-\frac{1}{\mathrm{~B}(\alpha, \beta)} \int_{0}^{\mathrm{x}} \mathrm{F}^{\alpha-1}(1-\mathrm{F})^{\beta-1} \mathrm{dF}(\mathrm{t}) \\
& =1-\frac{1}{\mathrm{~B}(\alpha, \beta)} \int_{0}^{\mathrm{F}(\mathrm{x})} \mathrm{t}^{\alpha-1}(1-\mathrm{t})^{\beta-1} \mathrm{dt}
\end{aligned}
$$

where $f$ and $F$ are the density function and cdf of the gamma random variable with parameters $\rho$ and $\lambda$, respectively. The hazard function defined to be a instantaneous measure of failure at time $x$ given survival to time $x$ is equal to 


$$
\begin{aligned}
& H(x)=\frac{g(x)}{R(x)}= \\
& \frac{\frac{1}{B(\alpha, \beta)} F^{\alpha-1}(1-F)^{\beta-1} f(x)}{1-\frac{1}{B(\alpha, \beta)} \int_{0}^{x} F^{\alpha-1}(1-F)^{\beta-1} f(x) d x} .
\end{aligned}
$$

Lemma 2:
(a) $\quad \lim _{x \rightarrow 0} H(x)=\lim _{x \rightarrow 0} g(x)$
(b) $\quad \lim _{x \rightarrow \infty}=\beta / \lambda$

The proof is given in Appendix.

$p=0.5, a=.5$ and $b=.25, .5,2$ symbol=box, cross, line

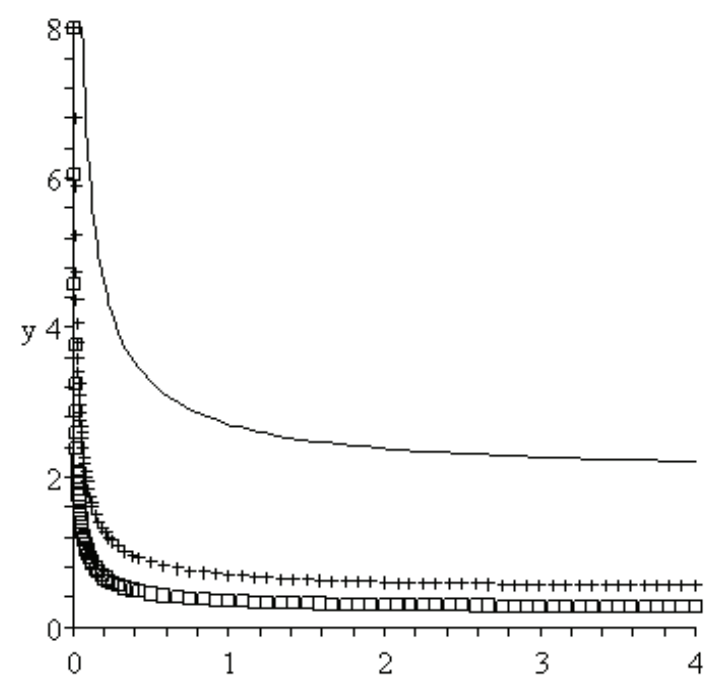

The hazard functions of $B G(\alpha, \beta, \rho, 1)$ are plotted. Cases with $\alpha \rho<1$ are presented in Figure 5 and cases with $\alpha \rho \geq 1$ are given in Figure 6. The graphs in the first column represent the cases $\alpha \rho=1$ with $\beta=1 / 2,1$, and 2; and those in the second column represent the cases when $\alpha \rho>1$ with $\beta=1 / 2,1$, and 2 in Figure 6. $p=.25 a=3$ and $b=.25,5,2$ symbol=box, cross, line

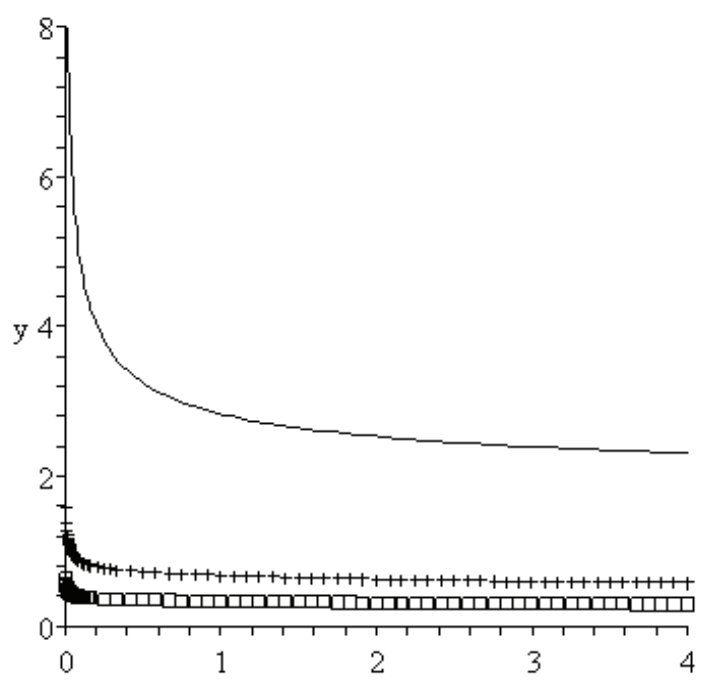

Figure 5. Hazard Function of $B G(\alpha, \beta, \rho, 1)$ when $\alpha \rho<1$ 
$\mathrm{a}=1 / 2, \mathrm{p}=2, \mathrm{~b}=1 / 2$, cross; $\mathrm{a}=1, \mathrm{p}=1, \mathrm{~b}=1 / 2$, circle; $\mathrm{a}=2, \mathrm{p}=1 / 2, \mathrm{~b}=1 / 2$, line

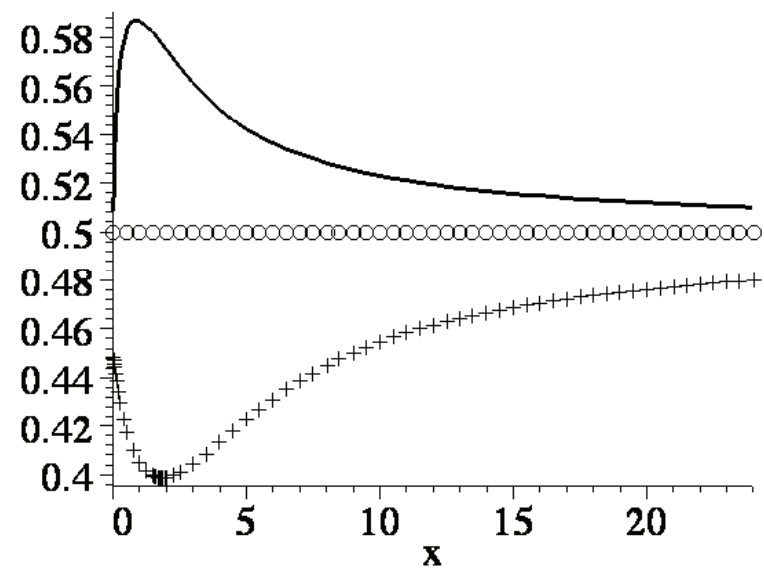

$\mathrm{a}=1 / 2, \mathrm{p}=2, \mathrm{~b}=1$,cross; $\mathrm{a}=1, \mathrm{p}=1, \mathrm{~b}=1$, circle; $\mathrm{a}=2, \mathrm{p}=1 / 2, \mathrm{~b}=1$,line;

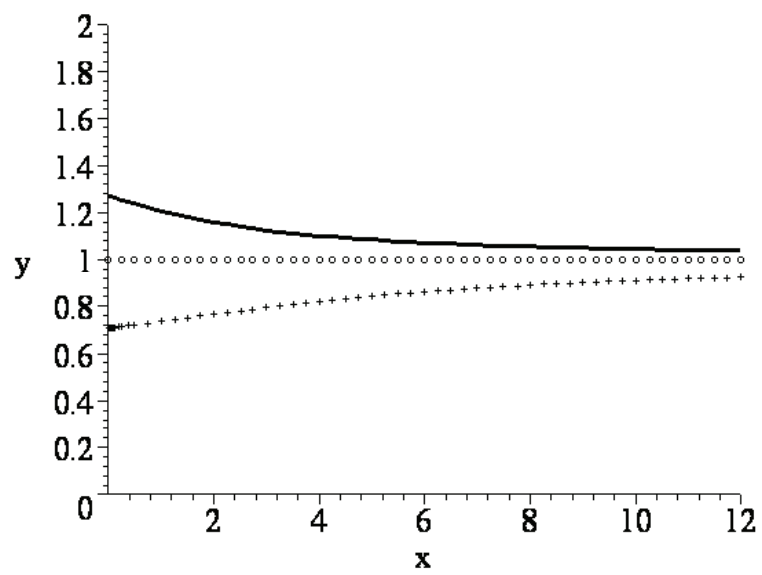

$a=1 / 2, p=2, b=2$, cross; $a=1, p=1, b=2$, circle; $a=2, p=1 / 2, b=2$,line;

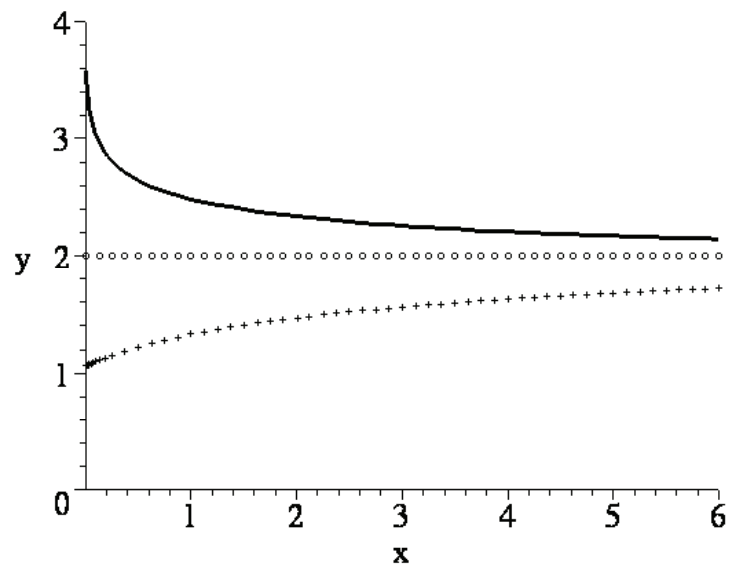

$a=1 / 2, p=4, b=1 / 2$, cross; $a=1, p=2, b=1 / 2$, circle; $a=2, p=1, b=1 / 2$, line

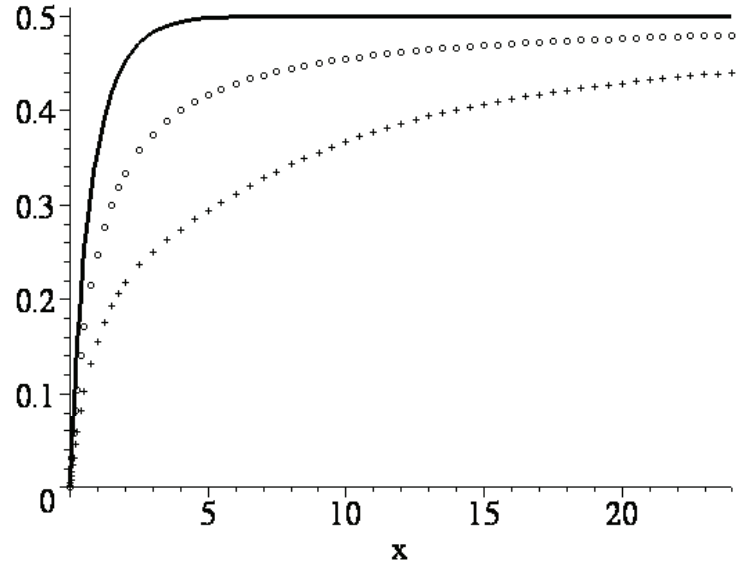

$\mathrm{a}=1 / 2, \mathrm{p}=4, \mathrm{~b}=1$, cross; $\mathrm{a}=1, \mathrm{p}=2, \mathrm{~b}=1$, circle; $\mathrm{a}=2, \mathrm{p}=1, \mathrm{~b}=1$,line;

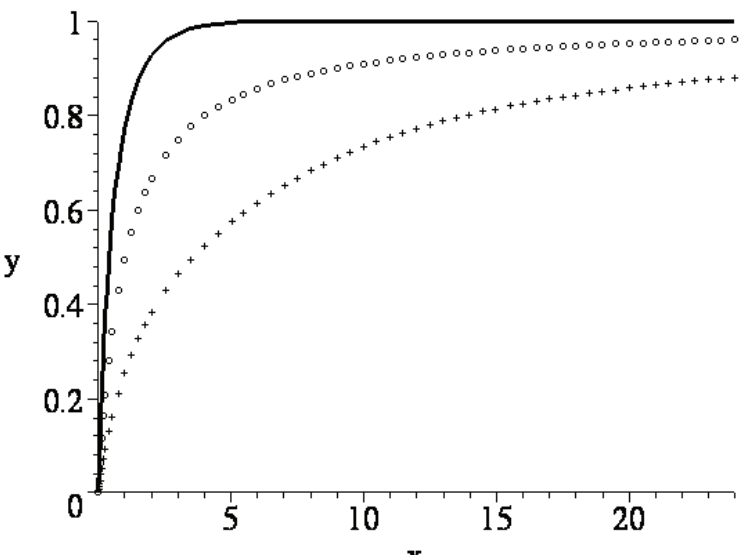

$\mathrm{a}=1 / 2, \mathrm{p}=4, \mathrm{~b}=2$, cross; $\mathrm{a}=1, \mathrm{p}=2, \mathrm{~b}=\mathbf{2}$, circle; $\mathrm{a}=2, \mathrm{p}=1, \mathrm{~b}=2$, line;

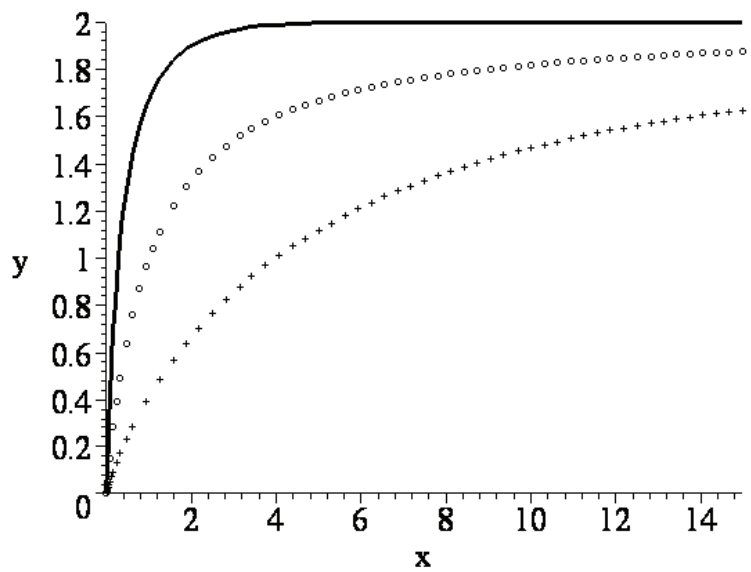

Figure 6. Hazard Function of $B G(\alpha, \beta, \rho, 1)$ when $\alpha \rho \geq 1$ 
As stated in Lemma 2, the curves of the hazard functions start at the values given in Lemma 1 and go to the value of $\beta$ as $x$ goes to $\infty$ regardless the values of other parameters. When $\alpha \rho<1$ and $\beta \geq 1$ (see also Figure 2), $g(x)$ has a reversed $\mathrm{J}$ shape and the trends of hazard functions for $\beta=1$ and $\beta=2$ (both $\beta>1$ ) are similar (Figure 5). When $\alpha \rho=1$ and $\beta=1 / 2$, the hazard function has a nonzero maximum or minimum. The hazard function is constant when $\alpha=\rho=\beta=1$, sine $g(x)$ is the exponential distribution. Within each plot, a larger $\alpha$ value seems to result in a larger value of the hazard function. When $\alpha \rho>1, g(x)$ has a nonzero mode (see also Figure 4 ) and the corresponding hazard function is nondecreasing.

When $\alpha=\beta=1$, it is Gamma function. $\lim _{x \rightarrow \infty}=1 / \lambda$, which is different from that of beta-gamma. Also, the hazard function of the beta-gamma can handle bathtub cases where gamma can not. Therefore, the beta-gamma distribution is more flexible. This is especially important when the beta parameter is not near one.

Parameter Estimation Using Maximum Likelihood Method

Let $x_{1}, x_{2}, \ldots \ldots x_{n}$ be a random sample of size $n$ from a beta-gamma distribution defined in (1.1), the log-likelihood function $l(\alpha, \beta, \rho, \lambda)$ is then given by

$$
\begin{gathered}
n \log \frac{\Gamma(\alpha+\beta)}{\Gamma(\alpha) \Gamma(\beta)}+(\alpha-1) \sum_{i=1}^{n} \log F\left(x_{i}\right) \\
+(\beta-1) \sum_{i=1}^{n} \log \left(1-F\left(x_{i}\right)\right)+\sum_{i=1}^{n} \log f\left(x_{i}\right),
\end{gathered}
$$

where $f(x)$ and $F(x)$ are the pdf and cdf of the gamma distribution with parameters $\rho$ and $\lambda$, respectively. Let $\psi(z)=d \Gamma(z) / d z$ be the digamma function. The equations for solving the maximum likelihood estimates of $\alpha, \beta, \rho$ and $\lambda$ are given in Appendix.

The example in the next section, initial estimates of $\rho$ and $\lambda$ is first computed by assuming the data set follows gamma distribution with $\alpha=1$ and $\beta=1$, the results from MLE of $(\rho, \lambda)$ along with $\alpha=1$ and $\beta=1$ then are used as the initial values for solving the equations (A.3) to (A.6).

\section{Applications of the Beta-Gamma Distribution}

An application of the proposed distribution is presented using the data sets given in Park, Leslie, and Mertz (1964), Park (1954), Moffa and Costantino (1977). Costantino and Desharnais (1981) established a gamma-state probability distribution for adult numbers in continuously growing populations of the flour beetle Tribolium. The hypothesis that the data set is from a beta-gamma distributed population is tested using the observed frequency distributions of adult numbers for Tribolium castaneum and Tribolium Confusum.

The beta-gamma distribution is fitted to the ten data sets discussed above, and the results are compared to those from gamma distribution and beta-normal distribution proposed by Eugene (2001) where the maximum likelihood method was used. Table 12 tabulates the resulting chi-square values form the goodnessof-fit test for the 10 data sets, and for illustration of the computations Tables 13 and 14 contains results for two of the ten data sets (Data set \# 6 and \#10). The expected numbers are calculated using the respective distribution with the parameters set at their maximum likelihood estimates. The chi-square goodness-of-fit test is then employed to make a comparison between the observed and expected number of observations under each distribution. Note that a class interval with an expected number less than 5 is combined with the adjacent class to avoid inflating the chi-square test statistic. 
Table 12. The resulting $\chi^{2}$ values (p-value, d.f.) from the goodness-of-fit tests for the 10 data sets.

\begin{tabular}{|l|l|l|l|}
\hline Data set & Gamma & Beta-Normal & Beta-Gamma \\
\hline$\# 1$ & $24.03(0.0043,9)$ & $5.04(0.6545,7)$ & $7.88(0.3433,7)$ \\
\hline$\# 2$ & $48.16(0,12)$ & $27.02(0.0026,10)$ & $20.50(0.0249,10)$ \\
\hline$\# 3$ & $129.18(0,17)$ & $74.85(0,15)$ & $72.63(0,15)$ \\
\hline$\# 4$ & $78.07(0,11)$ & $25.39(0.0030,9)$ & $28.36(0.0008,9)$ \\
\hline$\# 5$ & $23.62(0.0144,11)$ & $19.99(0.0180,9)$ & $17.89(0.0365,9)$ \\
\hline$\# 6$ & $10.72(0.3793,10)$ & $7.42(0.4913,8)$ & $7.05(0.5312,8)$ \\
\hline$\# 7$ & $21.67(0.0169,10)$ & $10.56(0.2280,8)$ & $12.89(0.1157,8)$ \\
\hline$\# 8$ & $55.71(0,9)$ & $25.05(0.0007,7)$ & $22.28(0.0023,7)$ \\
\hline$\# 9$ & $25.02(0.2463,21)$ & $16.85(0.6001,19)$ & $16.54(0.6210,19)$ \\
\hline$\# 10$ & $17.19(0.3076,15)$ & $17.07(0.1959,13)$ & $15.01(0.3067,13)$ \\
\hline
\end{tabular}

It is of no surprise that the proposed beta-gamma distribution fits better than the gamma distribution for all the data sets. Seven of the ten data sets, the beta-gamma distribution fits better than the beta-normal distribution based on the chi-squares values. Note that, for example, the data set in Table 14 appears to have a long right tail, it is reasonable that betagamma distribution performed the best. 


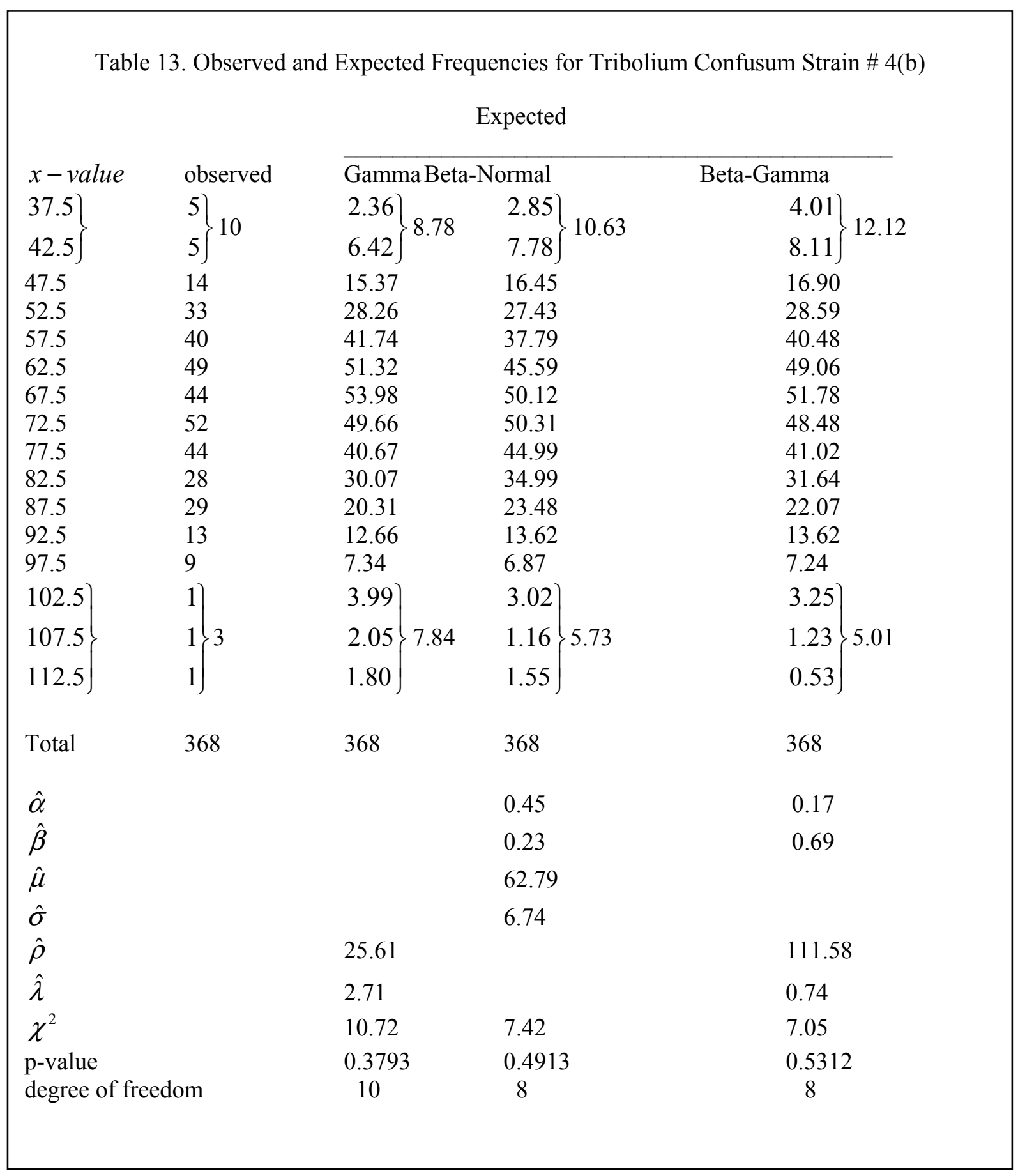


Table 14. Observed and Expected Frequencies for Tribolium Castaneum at $24^{\circ} \mathrm{C}$ (b)

\section{Expected}

\begin{tabular}{|c|c|c|c|c|}
\hline$x$-value & observed & Gamma & Beta-Normal & Beta-Gamma \\
\hline 25 & $0)$ & 0.02 & 0.12 & 0.03 \\
\hline 35$\}$ & $0\} 3$ & 0.443 .43 & 0.754 .25 & 0.463 .69 \\
\hline 45 & 3 & 2.97 & 3.38 & 3.20 \\
\hline 55 & 9 & 11.51 & 11.18 & 12.30 \\
\hline 65 & 39 & 29.71 & 28.07 & 31.23 \\
\hline 75 & 53 & 57.05 & 55.08 & 58.77 \\
\hline 85 & 77 & 87.54 & 87.09 & 88.39 \\
\hline 95 & 105 & 112.64 & 114.43 & 111.90 \\
\hline 105 & 135 & 125.81 & 128.66 & 123.66 \\
\hline 115 & 114 & 125.09 & 127.23 & 122.40 \\
\hline 125 & 113 & 112.87 & 113.37 & 110.58 \\
\hline 135 & 92 & 93.80 & 92.91 & 92.45 \\
\hline 145 & 59 & 72.63 & 71.18 & 72.29 \\
\hline 155 & 54 & 52.89 & 51.60 & 53.29 \\
\hline 165 & 38 & 36.51 & 35.72 & 37.27 \\
\hline 175 & 22 & 24.03 & 23.75 & 24.87 \\
\hline 185 & 17 & 15.17 & 15.22 & 15.91 \\
\hline 195 & 6 & 9.22 & 9.43 & 9.80 \\
\hline 205 & 10 & 5.42 & 5.65 & 5.83 \\
\hline 215 & 3 & 3.09 & 3.28 & 3.36 \\
\hline 225 & 2 & 1.71 & 1.85 & 1.88 \\
\hline 235 & 06 & 0.926 .68 & 1.017 .18 & $1.03 \quad 7.33$ \\
\hline 245 & $1\}$ & $0.49\}$ & $0.53\}$ & 0.55 \\
\hline 255 & 0 & 0.25 & 0.27 & 0.29 \\
\hline 265 & 0 & 0.22 & 0.24 & 0.22 \\
\hline$\hat{\alpha}$ & & & 12.34 & 0.82 \\
\hline$\hat{\beta}$ & & & 0.68 & 0.79 \\
\hline$\hat{\mu}$ & & & 27.33 & \\
\hline$\hat{\sigma}$ & & & 47.01 & \\
\hline$\hat{\rho}$ & & 13.86 & & 17.23 \\
\hline$\hat{\lambda}$ & & 8.50 & & 6.74 \\
\hline$\chi^{2}$ & & 17.19 & 17.07 & 15.01 \\
\hline p-value & & 0.3076 & 0.1959 & 0.3067 \\
\hline degree of fi & om & 15 & 13 & 13 \\
\hline
\end{tabular}




\section{Conclusion}

A beta-gamma distribution is proposed that include the gamma, exponential, and betaexponential distributions as its special cases. When $\alpha \rho>1$, it is unimodal with a concave shape. When $\alpha \rho \leq 1$ and $\beta \geq 1$, it has a reversed-J shape. When $\alpha<1$ and $\rho<1$, it also has a reversed-J shape. When $\alpha \rho=1$ and $\beta<1$, it can be reverse J-shaped or unimodal with a concave shape. When $\alpha \rho<1$ and $\beta<1$, $g(x)$ has a reversed-J shape except when $\alpha \rho$ is close to 1 with $\alpha>1$ or $\rho>1$ for a range of $\beta$ values of less than one, in which it is bimodal with a mode of zero and a nonzero mode.

Note that the beta-normal distribution in Eugene, et al (2002) can be bimodal with two nonzero modes; the beta-gamma can be bimodal with a mode of zero and a nonzero mode. Closed forms of moments are derived when parameters are integers. The mean and standard deviation increase with $\alpha$ and decrease with $\beta$.

The hazard function of the proposed beta-gamma distribution appears to be versatile in the sense it could be constant, nondecreasing, nonincreasing, concave, and convex. This property is potentially useful in real word problems. The estimation of the parameters can be computed via maximum likelihood method. The proposed beta-gamma distribution is a generalization of the widely used gamma distribution and is at least as efficient as the beta-normal if not better.
References

Costantino, R. F. \& Desharnais, R. A. (1981). Gamma distributions of adult numbers for tribolium populations in the region of their steady states. Journal of Animal Ecology, 50, 667-681.

Eugene, N., Lee, C. \& Famoye, F. (2002). Beta-normal distribution and its applications. Communications in StatisticsTheory and Methods, 31(4), 497-512.

Famoye, F., Lee, C. \& Eugene, N. (2004). Beta-normal distribution. Bimodality properties and application. Journal of Modern Applied Statistical Methods, 3(1), 85-103.

Farewell, V. T. (1977). A study of distributional shape in life testing. Technometrics, 19(1), 69-75.

Gupta, A.K.; Nadarajah, S. (2004). On the moments of the beta normal distribution. Communication in Statistics-Theory and Methods, 33.

Lawless, J. F. (1980). Inference in the generalized gamma and log gamma distributions. Technometrics, 22(3), 409-419.

Maynard, J. (2003). Unpublished doctoral dissertation. Central Michigan University.

McDonald, J. B. (1984). Some generalized functions for the size distribution of income. Econometrica, 52, 647-663.

Moffa, A. M. \& Costantino, R. F. (1977). Genetic analysis of a population of tribolium IV. Polymorphism and demographic equilibrium, Genetics, 87, 785-805.

Park, T., Leslie, P. H. \& Mertz, D. B. (1964). Genetics strains and competition in population of tribolium. Physio. Zool., 37, 97162.

Park, T. (1954). Experimental studies of interspecies competition. II. Temperature, humidity, and competition in two species of tribolium, Physio. Zool., 27, 177-238. 


\section{Appendix}

Proof of Lemma 1

Using the Taylor's expansions of $e^{-x / \lambda}$, the gamma density function is

$$
\begin{aligned}
& f(x)=\frac{x^{\rho-1}}{\lambda^{\rho} \Gamma(\rho)} e^{-x / \lambda} \\
& \quad=\frac{x^{\rho-1}}{\lambda^{\rho} \Gamma(\rho)}\left[1-\frac{x}{\lambda}+\frac{x^{2}}{2 ! \lambda^{2}}+\cdots \cdots+\frac{(-1)^{n} x^{n}}{n ! \lambda^{n}}+O\left(x^{n+1}\right)\right]=\frac{x^{\rho-1}}{\lambda^{\rho} \Gamma(\rho)}+O\left(x^{\rho}\right),
\end{aligned}
$$

and $F(x)=\int_{0}^{x} f(x) d x=x^{\rho} / \rho \lambda^{\rho} \Gamma(\rho)+O\left(x^{\rho+1}\right)$. For simplicity of presentation, let $f=f(x)$, $g=g(x), F=F(x)$ and $F^{c}=[F(x)]^{c}$. Using (A.1), the density function $g(x)$ in (1) becomes

$$
\begin{aligned}
& \frac{x^{\rho-1} e^{-x / \lambda}}{\lambda^{\rho} \Gamma(\rho) B(\alpha, \beta)}\left[\frac{x^{\rho}}{\rho \lambda^{\rho} \Gamma(\rho)}+O\left(x^{\rho+1}\right)\right]^{\alpha-1}(1-F)^{\beta-1} \\
= & \frac{x^{\rho-1+\rho(\alpha-1)} e^{-x / \lambda}}{\Gamma^{\alpha}(\rho) B(\alpha, \beta) \rho^{\alpha-1} \lambda^{\rho \alpha}}[1+O(x)]^{\alpha-1}(1-F)^{\beta-1} \\
= & \frac{x^{\rho-1} e^{-x / \lambda}}{\Gamma^{\alpha}(\rho) B(\alpha, \beta) \rho^{\alpha-1} \lambda^{\rho \alpha}}[1+O(x)]^{\alpha-1}(1-F)^{\beta-1} .
\end{aligned}
$$

Lemma 1 can now be readily seen because $F$ is a cdf and $\lim _{x \rightarrow 0} F(x)=0$. 
Proof of Theorem 1

When $\alpha$ and $\beta$ are integers, the $n$th moment of the beta-gamma random variable with density function in

(1) is

$$
\begin{aligned}
E\left(X^{n}\right) & =\int_{0}^{\infty} x^{n} \frac{1}{B(\alpha, \beta)} F(x)^{\alpha-1}[1-F(x)]^{\beta-1} f(x) d x \\
& =\frac{1}{B(\alpha, \beta)} \sum_{j=0}^{\beta-1}(-1)^{j}\left(\begin{array}{l}
\beta-1 \\
j
\end{array}\right) \int_{0}^{\infty} x^{n} F^{\alpha-1} F^{j} f(x) d x \\
& =\frac{1}{B(\alpha, \beta)} \sum_{j=0}^{\beta-1}(-1)^{j}\left(\begin{array}{l}
\beta-1 \\
j
\end{array}\right) \int_{0}^{\infty} x^{n} F^{\alpha+j-1} f(x) d x . \\
& =\frac{1}{B(\alpha, \beta)} \sum_{j=0}^{\beta-1}(-1)^{j}\left(\begin{array}{l}
\beta-1 \\
j
\end{array}\right) \int_{0}^{\infty} x^{n}[1-(1-F)]^{\alpha+j-1} f(x) d x \\
& =\frac{1}{B(\alpha, \beta)} \sum_{j=0}^{\beta-1}(-1)^{j}\left(\begin{array}{l}
\beta-1 \\
j
\end{array}\right)\left\{\sum_{k=0}^{\alpha+j-1}(-1)^{k}\left(\begin{array}{l}
\alpha+j-1 \\
k
\end{array}\right) I_{n, k}\right\},
\end{aligned}
$$

where

$$
I_{n, k}=\int_{0}^{\infty} x^{n} f(x)(1-F)^{k} d x
$$

Proof: of Corollary 1

When $\alpha=2, \beta=1$,

$$
E\left(X^{n}\right)=\frac{1}{B(2,1)} \sum_{k=0}^{1}(-1)^{k}\left(\begin{array}{l}
1 \\
k
\end{array}\right) I_{n, k}=\frac{1}{B(2,1)}\left[I_{n, 0}-I_{n, 1}\right]=2 I_{n, 0}-2 I_{n, 1} .
$$


The first term $I_{n, 0}$ is given by

$$
\int_{0}^{\infty} \frac{x^{n+\rho-1}}{\lambda^{\rho} \Gamma(\rho)} e^{-x / \lambda} d x=\int_{0}^{\infty} \frac{\lambda^{n+\rho}\left(\frac{x}{\lambda}\right)^{n+\rho-1}}{\lambda^{\rho} \Gamma(\rho)} e^{-x / \lambda} d\left(\frac{x}{\lambda}\right)=\int_{0}^{\infty} \frac{\lambda^{n+\rho} t^{n+\rho-1}}{\lambda^{\rho} \Gamma(\rho)} e^{-t} d(t)=\frac{\lambda^{n} \Gamma(n+\rho)}{\Gamma(\rho)}
$$

and $I_{n, 1}=\int_{0}^{\infty} x^{n} f(x)(1-F) d x$ is

$$
\begin{aligned}
& \int_{0}^{\infty} \frac{x^{\rho-1}}{\lambda^{\rho} \Gamma(\rho)} x^{n} e^{-x / \lambda}\left[e^{-x / \lambda}+(x / \lambda) e^{-x / \lambda}+\ldots \ldots+\frac{(x / \lambda)^{\rho-1} e^{-x / \lambda}}{\Gamma(\rho)}\right] d x \\
& =\int_{0}^{\infty} \frac{(2 x / \lambda)^{n+\rho-1}(\lambda / 2)^{n+p-1} e^{-2 x / \lambda}\left[1+x / \lambda+\ldots . .+\frac{(x / \lambda)^{\rho-1}}{\Gamma(\rho)}\right] d(2 x / \lambda)(\lambda / 2)}{\lambda^{\rho} \Gamma(\rho)} \\
& =\frac{\lambda^{n}}{2^{n+p} \Gamma(\rho)} \int_{0}^{\infty} t^{n+p-1} e^{-t}\left[1+t / 2+\ldots \ldots+\frac{(t / 2)^{\rho-1}}{\Gamma(\rho)}\right] d(t) \\
& =\frac{\lambda^{n}}{2^{n+p} \Gamma(\rho)}\left[\int_{0}^{\infty} t^{n+p-1} e^{-t} d t+\int_{0}^{\infty} t^{n+p-1} e^{t}(t / 2) d t+\ldots \ldots+\int_{0}^{\infty} t^{n+p-1} e^{-t}(t / 2)^{\rho-1} / \Gamma(\rho) d t\right] \\
& =\frac{\lambda^{n} \Gamma(n+\rho)}{2^{n+\rho} \Gamma(\rho)}+\frac{\lambda^{n} \Gamma(n+\rho+1)}{2^{n+\rho+1} \Gamma(\rho)}+\ldots+\frac{\lambda^{n} \Gamma(n+2 \rho-1)}{2^{n+2 \rho-1} \Gamma^{2}(\rho)} \\
& =\sum_{i=0}^{\rho-1} \frac{\lambda^{n} \Gamma(n+\rho+i)}{2^{n+\rho+i} \Gamma(\rho) \Gamma(i+1)} .
\end{aligned}
$$

When $\rho$ is an integer, $I_{n, 0}=\lambda^{n}(n+\rho-1) \cdots(\rho+1) \rho$ and $I_{n, 1}=\sum_{i=0}^{\rho-1} \frac{\lambda^{n}(n+\rho+i-1) !}{2^{n+\rho+i}(\rho-1) ! i !}$.

Substituting $I_{n, 0}$ and $I_{n, 1}$ into (A.2), the results of (7) and (8) are obtained. 
Proof of Lemma 2:

(a) As $x$ goes $0, \lim _{x \rightarrow 0} H(x)$ is

$$
\lim _{x \rightarrow 0} \frac{\frac{1}{B(\alpha, \beta)} F^{\alpha-1}(1-F)^{\beta-1} f(x)}{1-\frac{1}{B(\alpha, \beta)} \int_{0}^{x} F^{\alpha-1}(1-F)^{\beta-1} f(x) d x}=\lim _{x \rightarrow 0} g(x)
$$

which is given in Lemma 1.

Proof: (b) As $x$ goes to $\infty$, by L'Hospital Rule, $\lim _{x \rightarrow \infty} H(x)$ is

$$
\begin{aligned}
& \lim _{x \rightarrow \infty} \frac{(\alpha-1) F^{\alpha-2} f(x)(1-F)^{\beta-1} f(x)+F^{\alpha-1}(\beta-1)(1-F)^{\beta-2}\left(-f^{2}\right)+F^{\alpha-1}(1-F)^{\beta-1} f^{\prime}}{-F^{\alpha-1}(1-F)^{\beta-1} f} \\
& =\lim _{x \rightarrow \infty}\left[\frac{(1-\alpha) f(x)}{F(x)}+\frac{(\beta-1) f(x)}{1-F(x)}-\frac{f^{\prime}(x)}{f(x)}\right] \\
& =\frac{0}{1}+(\beta-1) \lim _{x \rightarrow \infty} \frac{f^{\prime}(x)}{-f(x)}-\lim _{x \rightarrow \infty} \frac{f^{\prime}(x)}{f(x)} \\
& =-\beta \lim _{x \rightarrow \infty} \frac{f^{\prime}(x)}{f(x)}=-\beta \lim _{x \rightarrow \infty} \frac{\frac{-x^{\rho-1} e^{-\frac{x}{\lambda}}}{\lambda}+(\rho-1) x^{\rho-2} e^{-\frac{x}{\lambda}}}{x^{\rho-1} e^{-\frac{x}{\lambda}}} \\
& =-\beta \lim _{x \rightarrow \infty}\left(-\frac{1}{\lambda}+\frac{\rho-1}{x}\right)=\frac{\beta}{\lambda} .
\end{aligned}
$$

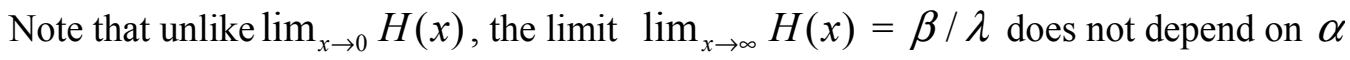
and $\rho$. In other word, the instantaneous failure rate will not depend on $\alpha$ and $\rho$ in the long run. 
The Equations for Solving the Maximum Likelihood Estimates:

$0=\frac{\partial l}{\partial \alpha}=n \psi(\alpha+\beta)-n \psi(\alpha)+\sum_{i=1}^{n} \log F\left(x_{i}\right)$.

$0=\frac{\partial l}{\partial \beta}=n \psi(\alpha+\beta)-n \psi(\beta)+\sum_{i=1}^{n} \log \left(1-F\left(x_{i}\right)\right)$.

$0=\frac{\partial l}{\partial \rho}=(\alpha-1) \sum_{i=1}^{n} \frac{1}{F\left(x_{i}\right)} \frac{\partial F\left(x_{i}\right)}{\partial \rho}-(\beta-1) \sum_{i=1}^{n} \frac{1}{1-F\left(x_{i}\right)} \frac{\partial F\left(x_{i}\right)}{\partial \rho}+\sum_{i=1}^{n} \frac{1}{f\left(x_{i}\right)} \frac{\partial f\left(x_{i}\right)}{\partial \rho}$,

$\frac{\partial f}{\partial \rho}=\frac{e^{-x / \lambda}\left[x^{\rho-1}(\log x) \lambda^{\rho} \Gamma(\rho)-x^{\rho-1}\left(\lambda^{\rho} \Gamma^{\prime}(\rho)+\lambda^{\rho}(\log \lambda) \Gamma(\rho)\right)\right]}{\lambda^{2 \rho} \Gamma^{2}(\rho)}$

$=f(x) \frac{(\log x) \lambda^{\rho} \Gamma(\rho)-\lambda^{\rho} \Gamma^{\prime}(\rho)-\lambda^{\rho}(\log \lambda) \Gamma(\rho)}{\lambda^{\rho} \Gamma(\rho)}=f(x)[\log x-\psi(p)-\log \lambda]$,

$\frac{\partial F}{\partial \rho}=\int_{0}^{x} f(x)[\log x-\psi(p)-\log \lambda] d x$.

$0=\frac{\partial l}{\partial \lambda}=(\alpha-1) \sum_{i=1}^{n} \frac{1}{F\left(x_{i}\right)} \frac{\partial F\left(x_{i}\right)}{\partial \lambda}-(\beta-1) \sum_{i=1}^{n} \frac{1}{1-F\left(x_{i}\right)} \frac{\partial F\left(x_{i}\right)}{\partial \lambda}+\sum_{i=1}^{n} \frac{1}{f\left(x_{i}\right)} \frac{\partial f\left(x_{i}\right)}{\partial \lambda}$,

$\frac{\partial f}{\partial \lambda}=\frac{x^{\rho-1}}{\Gamma(\rho)} \frac{\frac{x}{\lambda^{2}} e^{-x / \lambda} \lambda^{\rho}-e^{-x / \lambda} \rho \lambda^{\rho-1}}{\lambda^{2 \rho}}=f(x)\left(x / \lambda^{2}-\rho / \lambda\right) ; \frac{\partial F}{\partial \lambda}=\int_{0}^{x} f(x)\left(x / \lambda^{2}-\rho / \lambda\right) d x$

The SAS IML optimization methods can be employed to solve the loglikelihood equations (A.3) - (A.6) iteratively. The lengthy and tedious second derivatives required in the algorithm are not presented here, but are available upon request. 


\section{On the Product of Maxwell and Rice Random Variables}

\author{
M Shakil \\ Miami Dade College
}

\author{
B. M. Golam Kibria \\ Florida International University
}

The distributions of the product of independent random variables arise in many applied problems. These have been extensively studied by many researchers. In this paper, the exact distributions of the product $|X Y|$ have been derived when $X$ and $Y$ are Maxwell and Rice random variables respectively, and are distributed independently of each other. The associated cdfs, pdfs, and kth moments have been given.

Key words: Maxwelll distribution, products, Rice distribution.

\section{Introduction}

The distributions of the product $|X Y|$, when $X$ and $Y$ are independent random variables, arise in many applied problems of biology, economics, engineering, genetics, hydrology, medicine, number theory, order statistics, physics, psychology, etc, (see, for example, Cigizoglu \& Bayazit (2000), Galambos \& Simonelli (2005), Grubel (1968), Ladekarl, et al. (1997), and Rokeach \& Kliejunas (1972), among others, and references therein). The distributions of the product $|X Y|$, when $X$ and $Y$ are independent random variables and come from the same family, have been extensively studied by many researchers, (see, for example, Bhargava \& Khatri (1981), Malik \& Trudel (1986), Rathie \& Rohrer (1987), Springer \& Thompson (1970), Stuart (1962), and Wallgren (1980), among others,). In recent years, there has been a great interest in the study of the above

M. Shakil is an Assistant Professor in the Department of Mathematics at Miami Dade College. E-mail: mshakil@mdc.edu. B. M. Golam Kibria is an Associate Professor in the Department of Statistics at Florida International University. E-mail: kibriag@fiu.edu. The second author is grateful to the $\mathrm{Fu}$ Jen Catholic University, Professor W. L. Pearn, National Chiao-Tung University, and National Science Council of Taipei for providing financial support and research facilites. kind when $X$ and $Y$ belong to different families, (see, for example, Nadarajah (2005), and Nadarajah \& Kotz (2005), among others). In this paper, the distributions of the product $|X Y|$, when $X$ and $Y$ are independent random variables having Maxwell and Rice distributions respectively, have been investigated.

The derivation of the cdf, pdf, and kth moment of $Z=|X Y|$ involve some special functions, which are defined as follows, (see, for example, Abramowitz \& Stegun, 1970, Gradshteyn \& Ryzhik, 2000, and Prudnikov, et al., 1986, among others, for details). The series

$$
\begin{aligned}
& \mathrm{p}_{\mathrm{q}}\left(\alpha_{1}, \alpha_{2}, \cdots, \alpha_{\mathrm{p}} ; \beta_{1}, \beta_{2}, \cdots, \beta_{\mathrm{q}} ; \mathrm{z}\right) \\
& =\sum_{\mathrm{k}=0}^{\infty}\left\{\frac{\left(\alpha_{1}\right)_{\mathrm{k}}\left(\alpha_{2}\right)_{\mathrm{k}} \cdots\left(\alpha_{\mathrm{p}}\right)_{\mathrm{k}}}{\left(\beta_{1}\right)_{\mathrm{k}}\left(\beta_{2}\right)_{\mathrm{k}} \cdots\left(\beta_{\mathrm{q}}\right)_{\mathrm{k}}} \frac{\mathrm{z}^{\mathrm{k}}}{\mathrm{k} !}\right\},
\end{aligned}
$$

is called a generalized hypergeometric series of order $(p, q)$, where $(\alpha)_{k}$ and $(\beta)_{k}$ represent Pochhammer symbols. For $p=1$ and $q=2$, we have generalized hypergeometric function ${ }_{1} F_{2}$ of order $(1,2)$, given by

$$
{ }_{1} F_{2}\left(\alpha_{1} ; \beta_{1}, \beta_{2} ; z\right)=\sum_{k=0}^{\infty}\left\{\frac{\left(\alpha_{1}\right)_{k}}{\left(\beta_{1}\right)_{k}\left(\beta_{2}\right)_{k}} \frac{z^{k}}{k !}\right\} .
$$

The integral 


$$
\Gamma(\alpha)=\int_{0}^{\infty} t^{\alpha-1} e^{-t} d t, \alpha>0
$$

is defined as a (complete) gamma function, whereas the integrals

$$
\gamma(\alpha, x)=\int_{0}^{x} t^{\alpha-1} e^{-t} d t, \alpha>0,
$$

and

$$
\Gamma(\alpha, x)=\int_{x}^{\infty} t^{\alpha-1} e^{-t} d t, \alpha>0,
$$

are respectively known as incomplete gamma and complementary incomplete gamma functions. For negative values, gamma function can be defined as

$\Gamma\left(-n+\frac{1}{2}\right)=\frac{(-1)^{n} 2^{n} \sqrt{\pi}}{1.3 .5 \ldots(2 n-1)}$, where $n \geq 0$

is an integer (e.g., Andrews, et al., 1999, and Bohr \& Mollerup, 1922).The error function is defined by

$$
\operatorname{erf}(x)=\frac{2}{\sqrt{\pi}} \int_{0}^{x} e^{-u^{2}} d u
$$

whereas the complementary error, $\operatorname{erfc}(x)$, is defined as

$$
\operatorname{erfc}(x)=\frac{2}{\sqrt{\pi}} \int_{x}^{\infty} e^{-u^{2}} d u=1-\operatorname{erf}(x)
$$

The modified Bessel function of first kind, $I_{v}(x)$, for a real number $v$, is defined by

$$
I_{v}(x)=\left(\frac{1}{2} x\right)^{v} \sum_{k=0}^{\infty} \frac{\left(\frac{1}{4} x^{2}\right)^{k}}{(k !) \Gamma(v+k+1)},
$$

where $\Gamma($.$) denotes gamma function. Also,$

$$
I_{v}(x)=\frac{1}{\Gamma(v+1)}\left(\frac{x}{2}\right)^{v} e^{-x}{ }_{1} F_{1}\left(\frac{1}{2}+v, 1+2 v ; 2 x\right),
$$

where ${ }_{1} F_{1}$ denotes the confluent hypergeometric function. When $v=0$, modified Bessel function of first kind, $I_{0}(x)$, of order 0 is obtained as follows:

$$
I_{0}(x)=\sum_{k=0}^{\infty} \frac{\left(\frac{1}{4} x^{2}\right)^{k}}{(k !)^{2}} \ldots
$$

For

$$
\operatorname{Re}\left(v+\frac{1}{2}\right)>0,|\arg (z)|<\frac{\pi}{2}
$$

or

$$
\operatorname{Re}(z)=0
$$

and

$$
v=0,
$$

the modified Bessel function of second kind of order $v$ is given by

$$
K_{v}(x)=\frac{\left(\frac{z}{2}\right)^{v} \Gamma\left(\frac{1}{2}\right)}{\Gamma\left(v+\frac{1}{2}\right)} \int_{1}^{\infty} e^{-z t}\left(t^{2}-1\right)^{v-\frac{1}{2}} d t
$$

For

$$
|\arg (z)|<\frac{\pi}{2}, \operatorname{Re}\left(z^{2}\right)
$$

one would have

$$
K_{v}(x)=\frac{1}{2}\left(\frac{z}{2}\right)^{v} \int_{0}^{\infty} \frac{e^{-t-\frac{z^{2}}{4 t}}}{(t)^{v+1}} d t .
$$

For non-integer $v$, 


$$
K_{v}(x)=\frac{\pi\left\{I_{-v}(x)-I_{v}(x)\right\}}{2 \sin (v \pi)}
$$

The following Lemmas will also be needed in our calculations.

LEMMA 1 (Gradshteyn \& Ryzhik (2000), Equation (3.381.4), Page 317). For $\operatorname{Re}(\mu)>0$, and $\operatorname{Re}(v)>0$,

$$
\int_{0}^{\infty} t^{v-1} e^{-\mu t} d t=\frac{1}{\mu^{v}} \Gamma(v)
$$

LEMMA 2 (Prudnikov et al. (1986), Volume 2, Equation (2.8.5.15), Page 106). For $a>0$,

$$
\begin{aligned}
& \int_{0}^{\infty} \mathrm{t}^{\alpha-1} \mathrm{e}^{-\mathrm{p} / \mathrm{t}^{2}} \operatorname{erf}(\mathrm{ct}) \mathrm{dt} \\
= & \frac{\mathrm{c} \mathrm{p}^{(\alpha+1) / 2}}{\sqrt{\pi}} \Gamma\left(-\frac{\alpha+1}{2}\right){ }_{1} \mathrm{~F}_{2}\left(\frac{1}{2} ; \frac{3}{2}, \frac{3+\alpha}{2} ; \mathrm{c}^{2} \mathrm{p}\right) \\
- & \frac{1}{\sqrt{\pi} \alpha \mathrm{c}^{\alpha}} \Gamma\left(\frac{\alpha+1}{2}\right){ }_{1} \mathrm{~F}_{2}\left(-\frac{\alpha}{2} ; 1-\frac{\alpha}{2}, \frac{1-\alpha}{2} ; \mathrm{c}^{2} \mathrm{p}\right)
\end{aligned}
$$

LEMMA 3 (Prudnikov et al., 1986, Volume 2, Equations 2.10.3.14, Page 151). For $\operatorname{Re}(\alpha)<0, \operatorname{Re}(p)>0, \operatorname{Re}(v)>0$, and $\operatorname{Re}(c)>0$,

$$
\begin{aligned}
& \int_{0}^{\infty} x^{\alpha-1} e^{-p / x} \gamma(v, c x) d x \\
& =\frac{c^{v}(p)^{\alpha+v}}{v} \Gamma(-\alpha-v){ }_{1} F_{2}(v ; v+1,1+\alpha+v ; c p) \\
& -\frac{\Gamma(\alpha+v)}{\alpha c^{\alpha}}{ }_{1} F_{2}(-\alpha ; 1-\alpha, 1-\alpha-v ; c p)
\end{aligned}
$$

where ${ }_{1} F_{2}$ denotes generalized hypergeometric function of order $(1,2)$, (see definition above).

LEMMA 4 (Gradshteyn \& Ryzhik (2000), Equation (3.471.9), Page 340). For $\operatorname{Re}(\beta)>0, \quad \operatorname{Re}(\gamma)>0$,

$$
\int_{0}^{\infty} x^{v-1} e^{-\frac{\beta}{x}-\gamma x} d x=2\left(\frac{\beta}{\gamma}\right)^{\frac{v}{2}} K_{v}(2 \sqrt{\beta \gamma})
$$

where $K_{v}($.$) denotes modified Bessel function$ of the second kind, (see definition above).

Distribution of the Product $|\mathrm{XY}|$

Let $X$ and $Y$ be Maxwell and Rice random variables respectively, distributed independently of each other and defined as follows.

Maxwell Distribution:

A continuous random variable $X$ is said to have a Maxwell distribution if its pdf $f_{X}(x)$ and cdf $F_{X}(x)$ are, respectively, given by

$$
f_{X}(y)=\sqrt{\frac{2}{\pi}} a^{3 / 2} x^{2} e^{-a x^{2} / 2}, x>0, a>0 \ldots
$$

and

$$
\begin{aligned}
F_{X}(x) & =\frac{2 \gamma\left(\frac{3}{2}, \frac{1}{2} a x^{2}\right)}{\sqrt{\pi}} \\
& =\operatorname{erf}\left(\sqrt{\frac{a}{2}} x\right)-\sqrt{\frac{2 a}{\pi}} x e^{-a x^{2} / 2}
\end{aligned}
$$

where $\gamma(a, x)$ and $\operatorname{erf}(x)$ denote incomplete gamma and error functions respectively, (see definition above).

Rice Distribution: A continuous random variable $Y$ is said to have a Rice distribution if its pdf $f_{Y}(y)$ is given by

$$
f_{Y}(y)=\frac{y}{\sigma^{2}} e^{-\left(y^{2}+v^{2}\right) / 2 \sigma^{2}} I_{0}\left(\frac{y v}{\sigma^{2}}\right), \quad y>0, \sigma>0, v \geq 0 \ldots
$$

where $I_{0}(y)$ denotes the modified Bessel function of the first kind, (see definition above). 
For $|v|=0$, the expression (4) reduces to a Rayleigh distribution. In what follows, we consider the derivation of the distribution of the product $|X Y|$, when $X$ and $Y$ are Maxwell and Rice random variables respectively, distributed independently of each other and defined as above. An explicit expression for the cdf of $|X Y|$ in terms of hypergeometric function has been derived in Theorem 1. In Theorem 2, another explicit expression for the cdf of $|X Y|$ in terms of hypergeometric function and modified Bessel function of the second kind $K_{v}(x)$ has been derived.

\section{Theorem 1}

Suppose $X$ is a Maxwell random variable with pdf $f_{X}(x)$ as given in (2) and cdf $F_{X}(x)=P(X \leq x)$ given by (3) in terms of the incomplete gamma function. Also, suppose $Y$ is a Rice random variable with pdf $f_{Y}(y)$ given by (4) in terms of the modified Bessel function of the first kind $I_{0}(y)$. Then the cdf of $Z=|X Y|$ can be expressed as

$$
\begin{aligned}
& \mathrm{F}(\mathrm{z})= \\
& {\left[\frac{\mathrm{e}^{-\mathrm{v}^{2} / 2 \sigma^{2}}}{\sqrt{\pi} \sigma^{2}}\right] \sum_{\mathrm{k}=0}^{\infty} \frac{\mathrm{v}^{2 \mathrm{k}}}{2^{2 \mathrm{k}} \sigma^{4 \mathrm{k}}(\mathrm{k} !)^{2}}} \\
& {\left[\frac{\Gamma\left(\mathrm{k}-\frac{1}{2}\right) 2^{\mathrm{k}-1} \mathrm{a}^{\frac{3}{2}} \sigma^{2 \mathrm{k}-1} \mathrm{z}^{3}}{3}{ }_{1} \mathrm{~F}_{2}\left(\frac{3}{2} ; \frac{5}{2}, \frac{3}{2}-\mathrm{k} ; \frac{\mathrm{az}}{4 \sigma^{2}}\right)\right.} \\
& \left.+\frac{\Gamma\left(\frac{1}{2}-\mathrm{k}\right) \mathrm{a}^{\mathrm{k}+1} \mathrm{z}^{2(\mathrm{k}+1)}}{2^{\mathrm{k}+1}(\mathrm{k}+1)}{ }_{1} \mathrm{~F}_{2}\left(\mathrm{k}+1 ; \mathrm{k}+2, \mathrm{k}+\frac{1}{2} ; \frac{\mathrm{az}}{4 \sigma^{2}}\right)\right]
\end{aligned}
$$

where ${ }_{1} F_{2}($.$) denotes hypergeometric function$ of order $(1,2)$, (see definition above).

Proof

Using the expressions (3) for cdf of Maxwell random variable $X$ and the expression
(4) for pdf of Rice random variable $Y$, the cdf $F(z)=\operatorname{Pr}(|X Y| \leq z)$ can be expressed as

$$
\begin{aligned}
& F(z) \\
& =\operatorname{Pr}\left(|X| \leq \frac{z}{|Y|}\right) \\
& =\int_{0}^{\infty} F_{X}\left(\frac{z}{y}\right) f_{Y}(y) d y \\
& =\left[\frac{2 \mathrm{e}^{-v^{2} / 2 \sigma^{2}}}{\sqrt{\pi} \sigma^{2}}\right] \int_{0}^{\infty} \mathrm{ye}^{-y^{2} / 2 \sigma^{2}} \gamma\left(\frac{3}{2}, \frac{a z^{2}}{2 y^{2}}\right) I_{0}\left(\frac{v y}{\sigma^{2}}\right) d y
\end{aligned}
$$

where $y>0, z>0, a>0, \sigma>0, v \geq 0$. The proof of Theorem 1 easily follows by using definition (1) of modified Bessel function of first kind, $I_{0}(x)$, of order 0 , and Lemma 3 in the integral (5) above.

Theorem 2

Suppose $X$ is a Maxwell random variable with pdf $f_{X}(x)$ as given in (2) and cdf $F_{X}(x)=P(X \leq x)$ given by (3) in terms of the error function. Also, suppose $Y$ is a Rice random variable with pdf $f_{Y}(y)$ given by (4) in terms of the modified Bessel function of the first kind $I_{0}(y)$. Then the cdf of $Z=|X Y|$ can be expressed as

$$
\begin{aligned}
& F(z)=\left[\frac{\sqrt{a} e^{-v^{2} / 2 \sigma^{2}}}{\sqrt{\pi} \sigma^{2}}\right] \sum_{k=0}^{\infty} \frac{v^{2 k}}{2^{2 k} \sigma^{4 k}(k !)^{2}} \\
& {\left[\left(k+\frac{1}{2}\right) 2^{k} \sigma^{2 k+1} z_{1} F_{2}\left(\frac{1}{2} ; \frac{3}{2}, \frac{1}{2}-k ; \frac{a z^{2}}{4 \sigma^{2}}\right)\right.} \\
& +\frac{\Gamma\left(-k-\frac{1}{2}\right) a^{k+\frac{1}{2}} z^{2(k+1)}}{2^{k+2}(k+1)}{ }_{1} F_{2}\left(k+1 ; k+2, k+\frac{3}{2} ; \frac{a z^{2}}{4 \sigma^{2}}\right) \\
& -\sqrt{2}(\sqrt{a} \sigma)^{k+\frac{1}{2}} z^{k+\frac{3}{2}} K_{k+\frac{1}{2}}\left(\frac{\sqrt{a} z}{\sigma}\right)
\end{aligned}
$$


where ${ }_{1} F_{2}($.$) denotes hypergeometric function$ of order $(1,2)$, and $K_{k}($.$) denotes the$ modified Bessel functions of the second kind of order $k$, (see definition above).

Proof

Using the expressions (3) for cdf of Maxwell random variable $X$ and the expression (4) for pdf of Rice random variable $Y$, the cdf $F(z)=\operatorname{Pr}(|X Y| \leq z)$ can be expressed as

$\mathrm{F}(\mathrm{z})$

$=\operatorname{Pr}\left(|X| \leq \frac{z}{|Y|}\right)$
$=\int_{0}^{\infty} F_{X}\left(\frac{z}{y}\right) f_{Y}(y) d y$
$=\left[\frac{e^{-v^{2} / 2 \sigma^{2}}}{\sigma^{2}} \int_{0}^{\infty} \mathrm{ye}^{-y^{2} / 2 \sigma^{2}}\left\{\begin{array}{l}\operatorname{erf}\left(\sqrt{\frac{a}{2}} \frac{z}{y}\right) \\ -\sqrt{\frac{2 a}{\pi}} \frac{z}{y} e^{-\frac{a z^{2}}{2 y^{2}}}\end{array}\right\} I_{0}\left(\frac{v y}{\sigma^{2}}\right) d y \ldots\right.$

where $y>0, z>0, a>0, \sigma>0, v \geq 0$. The proof of Theorem 2 easily follows by using the definition (1) of modified Bessel function of the first kind, $I_{0}(x)$, of order 0 , substituting $y=\frac{1}{t}$ in the first term and $y^{2}=u$ in the second term of the integral (6) above, and then using Lemmas 2 and 4 respectively.

PDF of the Product $Z=|X Y|$, and kth Moment of RV $Z=|X Y|$

In what follows, without loss of generality, for simplicity of computations, this section discusses the derivation of the pdf of the product $Z=|X Y|$, when $X$ and $Y$ are Rice and Maxwell random variables distributed according to (4) and (2), respectively, and independently of each other. An explicit expression for the pdf of the product $Z=|X Y|$ in terms of the modified Bessel function of the second kind $K_{v}(x)$ has been derived in Theorem 3. The expression for the kth moment of $\mathrm{RV} Z=|X Y|$ in terms of gamma functions has been derived in Theorem 4 .

Theorem 3

Suppose $X$ and $Y$ are Rice and Maxwell random variables having pdf given by (4) and (2), respectively. Then the pdf of $Z=|X Y|$ can be expressed as

$$
\begin{aligned}
& f_{Z}(z)=\left(\sqrt{\frac{2}{\pi}} e^{-v^{2} / 2 \sigma^{2}}\right) \\
& \sum_{n=0}^{\infty} \frac{v^{2 n} a^{n+2}}{\sigma^{2 n+1}(n !)^{2}} K_{n+\frac{1}{2}}\left(\frac{\sqrt{a} z}{\sigma}\right)^{\cdots}
\end{aligned}
$$

where $K_{n+\frac{1}{2}}($.$) denotes the modified Bessel$ functions of the second kind of order $n+\frac{1}{2}$, (see definition above).

Proof

The pdf of $Z=|X Y|$ can be expressed as

$$
\begin{aligned}
& f_{Z}(z) \\
& =\int_{0}^{\infty} \frac{1}{y} f_{X}\left(\frac{z}{y}\right) f_{Y}(y) d y \\
& =\left(\sqrt{\frac{2}{\pi}} \frac{a^{\frac{3}{2}}}{\sigma^{2}} e^{-v^{2} / 2 \sigma^{2}} z\right) \\
& \int_{0}^{\infty} e^{-\frac{z^{2}}{2 \sigma^{2} y^{2}}-\frac{a y^{2}}{2}} I_{0}\left(\frac{v z}{\sigma^{2} y}\right) d y, \ldots
\end{aligned}
$$

where $y>0, z>0, a>0, \sigma>0, v \geq 0$. The proof of Theorem 3 easily follows by using the definition (1) of modified Bessel function of the 
first kind, $I_{0}(x)$, of order 0 , substituting $y^{2}=\frac{1}{t}$, and then using Lemma 4 in the integral (8) above.

\section{Theorem 4}

If $Z$ is a random variable with pdf given by (7), then its $k$ th moment can be expressed as

$$
\begin{gathered}
E\left(Z^{k}\right)=\left(\frac{2^{k-\frac{1}{2}} e^{-v^{2} / 2 \sigma^{2}}}{\sqrt{\pi}}\right) \\
\sum_{n=0}^{\infty} \frac{v^{2 n} a^{n-\frac{k}{2}+\frac{3}{2}}}{\sigma^{2 n-k}(n !)^{2}} \Gamma\left(\frac{2 k-2 n+1}{4}\right) \Gamma\left(\frac{2 k+2 n+3}{4}\right)
\end{gathered}
$$

Proof

$$
\begin{aligned}
& E\left(Z^{k}\right)=\left(\sqrt{\frac{2}{\pi}} e^{-v^{2} / 2 \sigma^{2}}\right) \\
& \sum_{n=0}^{\infty} \frac{v^{2 n} a^{n+2}}{\sigma^{2 n+1}(n !)^{2}} \int_{0}^{\infty} z^{k} K{ }_{n+\frac{1}{2}}\left(\frac{\sqrt{a} z}{\sigma}\right) d z \ldots
\end{aligned}
$$

By using the equation (6.621.3 / page 712) from Gradshteyn and Ryzhik (2000), in the integral (9) above, the result of Theorem 4 easily follows.

\section{Conclusion}

This article has derived the exact distributions of the product of two independent random variables $X$ and $Y$, where $X$ and $Y$ have Maxwell and Rice distributions respectively. The pdf and kth moment of the product of two variables are also given. The distribution is obtained as a function of hypergeometric of order $(1,2)$, where as the pdf has been obtained as a function of Bessel of the second kind. We hope the findings of the article will be useful for the practitioners which are indicated in the introduction of the article.

\section{References}

Abramowitz, M., \& Stegun, I. A. (1970). Handbook of mathematical functions, with formulas, graphs, and mathematical tables. Dover, N.Y.

Andrews, G. E., Askey, R., \& Roy, R. (1999). Special functions. Cambridge: Cambridge University Press.

Bhargava, R. P., \& Khatri, C. G. (1981). The distribution of product of independent beta random variables with application to multivariate analysis. Annals of the Institute of Statistical Mathematics, 33, 287 - 296.

Bohr, H., \& Mollerup, I. (1922). Loerbog I matematisk Analyse, 3. Kopenhagen.

Cigizoglu, H. K., \& Bayazit, M. (2000). A generalized seasonal model for flow duration curve. Hydrological Processes, 14, 1053 - 1067.

Galambos, J., \& Simonelli, I. (2005). Products of random variables:Applications to problems of physics and to arithmetical functions. Boca Raton / Atlanta: CRC Press.

Gradshteyn, I. S., \& Ryzhik, I. M. (2000). Table of integrals, series, and products (6th ed.). San Diego: Academic Press.

Grubel, H. G. (1968). Internationally diversified portfolios: Welfare gains capital flows. American Economic Review, 58, 1299 1314.

Ladekarl, M., Jensen, V., \& Nielsen, B. (1997). Total number of cancer cell nuclei and mitoses in breast tumors estimated by the optical disector. Analytical and Quantitative Cytology and Histology, 19, $329-337$.

Malik, H. J., \& Trudel, R. (1986). Probability density function of the product and quotient of two correlated exponential random variables. Canadian Mathematical Bulletin, 29, 413-418.

Nadarajah, S. (2005). On the product and ratio of Laplace and Bessel random variables. Journal of Applied Mathematics, 4, $393-402$.

Nadarajah, S., \& Kotz, S. (2005). On the product and ratio of Pearson Type VII and Laplace random variables. Austrian Journal of Statistics, 34(1), 11-23. 
Prudnikov, A. P., Brychkov, Y. A., \& Marichev, O. I. (1986). Integrals and series (Volumes 1, 2, \& 3). Amsterdam: Gordon and Breach Science Publishers.

Rathie, P. N., \& Rohrer, H. G. (1987). The exact distribution of products of independent random variables. Metron, 45, 235245.

Rokeach, M., \& Kliejunas, P. (1972). Behavior as a function of attitude-toward-object and attitude-toward-situation. Journal of Personality and Social Psychology, 22, 194201.
Springer, M. D. \& Thompson, W. E. (1970). The distribution of products of beta, gamma and Gaussian random variables. SIAM Journal on Applied Mathematics, 18, 721 - 737.

Stuart, A. (1962). Gamma-distributed products of independent random variables. Biometrika, 49, 564 - 565.

Wallgren, C. M. (1980). The distribution of the product of two correlated $\mathrm{t}$ variates. Journal of the American Statistical Association, $75,996-1000$. 


\title{
Optimal Lp-Metric for Minimizing Powered Deviations in Regression
}

\author{
Stan Lipovetsky \\ GfK Custom Research North America \\ Minneapolis, Minnesota
}

Minimizations by least squares or by least absolute deviations are well known criteria in regression modeling. In this work the criterion of generalized mean by powered deviations is suggested. If the parameter of the generalized mean equals one or two, the fitting corresponds to the least absolute or the least squared deviations, respectively. Varying the power parameter yields an optimum value for the objective with a minimum possible residual error. Estimation of a most favorable value of the generalized mean parameter shows that it almost does not depend on data. The optimal power always occurs to be close to 1.7 , so these powered deviations should be used for a better regression fit.

Key words: Regression, absolute and squared deviations, $L p$-metric, gamma-function.

\section{Introduction}

The criterion of generalized mean by powered deviations is considered for regression modeling. Usually regressions are constructed by minimization of squared deviations of the observations to a theoretical surface, although some other measures, particularly, absolute deviations are also applied in regression, multidimensional scaling, clustering, and other distance-based techniques (Armstrong \& Frome, 1976; Hastie \& Tibshirani, 1990; McCullagh \& Nelder, 1997; Venables \& Ripley, 1997). Robust regression modeling and kernel smoothing use different measures of distance for smaller and bigger deviations (Huber, 1972, 1981; Hill \& Holland, 1977; Hampel et al., 1986; Ripley, 1996). Particularly, the $L p$-metric, or the generalized mean, is widely used as so called $M$ estimator (Maximum likelihood) for robust evaluations (Ramsay, 1977; Sposito, 1982).

In other fields it is also called $L p$-metric for operators spaces, vector and matrix norms, Hölder's mean, power mean, exponential mean,

Dr. Stan Lipovetsky, GfK Custom Research GfK Custom Research North America, 8401 Golden Valley Road, Minneapolis, Minnesota 55427-0900. Email address: stan.lipovetsky@gfk.com
Kolmogorov's mean, or Minkowski distance (Hardy, Littelwood, \& Polya, 1934; Daykin \& Eliezer, 1969; Borwein \& Borwein, 1987; Korn \& Korn, 1988; Alvarez, 1992; Rooij \& Heiser, 2005). Power means are related to Box-Cox transformation often used in applied statistics aims (Weisberg, 1985; McCullagh \& Nelder, 1997; Tishler \& Lipovetsky, 1997, 2000; Lipovetsky \& Conklin, 2000).

If the parameter of the generalized mean equals one or two, $p=1$ or $p=2$, the fitting corresponds to the least absolute $L_{1}$ or the least squared $L_{2}$ deviations, respectively. Theoretical properties of the $L p$-metrics in the range from 1 to 2 were studied in works on approximation theory, Banach's conjecture, and random processes (Breiman, 1968; Fletcher et al., 1971; Kanter, 1973). It is also known due to Jensen's inequality that a generalized mean of a lower power is smaller than a generalized mean of a larger power (Beckenbach, 1946; Korn \& Korn, 1988) that is true for the constant set of the averaging values. However, the estimates of the model parameters and the corresponding residual errors depend on a power parameter, so the better generalized power mean can be reached for a smaller power value. In the literature, known numerical simulations indicated that the minimal residuals correspond to the $p$-powered deviations close to $L_{1.5}$ or $L_{1.8}$ metrics (Gentleman, 1965; Forsythe, 1972; Ramsay, 1977). 
In the current work, trying an objective of least powered deviations in a wide range of the power parameter, it was possible to find an optimum value for the objective by minimizing the residual error. Numerical estimation of an optimum value of the generalized mean parameter indicates a remarkable outcome - this optimum value is almost a constant that does not depend on the data. Analytical derivation shows that the optimal metric parameter is defined via the gamma function of this parameter, and the optimal value occurs to be close to $p \approx 1.7$. Thus, the optimum metric for fitting any data can be suggested - it is neither the mostly used squared deviations $L_{2}$, nor the absolute deviations $L_{1}$, but the intermediate powered deviations of $L_{1.7}$.

Powered Deviations in Regression Modeling

Consider a multiple linear regression model of the dependent variable $y$ by $n$ independent variables $x_{1}, x_{2}, \ldots, x_{n}$ :

$$
y_{i}=a_{0}+a_{1} x_{i 1}+a_{2} x_{i 2}+\ldots+a_{n} x_{i n}+\varepsilon_{i},
$$

where $i$ denotes observations $(i=1,2, \ldots, N)$, and $\varepsilon_{i}$ are deviations of the empirical values $y_{i}$ from the theoretical model. Least squares minimization corresponds to the objective:

$$
S^{2}=\frac{1}{N} \sum_{i=1}^{N} \varepsilon_{i}^{2}=\frac{1}{N} \sum_{i=1}^{N}\left(y_{i}-a_{0}-a_{1} x_{i 1}-\ldots-a_{n} x_{i n}\right)^{2}
$$

This distance is equivalent to the squared Euclidean norm of the errors, or the $L_{2}$ metric. Absolute deviations minimization corresponds to the objective of the mean module:

$$
S^{1}=\frac{1}{N} \sum_{i=1}^{N}\left|\varepsilon_{i}\right|=\frac{1}{N} \sum_{i=1}^{N}\left|y_{i}-a_{0}-a_{1} x_{i 1}-\ldots-a_{n} x_{i n}\right| .
$$

It is the Hamming distance (also known as Manhattan, or taxi-driver distance), or $L_{1}$ metric.
Generalized powered mean of the deviations can be expressed as follows:

$$
\left.S^{2 q}=\frac{1}{N} \sum_{i=1}^{N}\left(\varepsilon_{i}^{2}\right)^{q}=\frac{1}{N} \sum_{i=1}^{N}\left(y_{i}-a_{0}-a_{1} x_{i 1}-\ldots-a_{n} x_{i n}\right)^{2}\right)^{q}
$$

In this definition, if power parameter $q$ equals one, than the generalized mean (4) is reducing to the squared mean (2). If $q$ equals one half, the generalized mean (4) is presented as a square root of squared deviation that coincides with absolute value of the deviations in the objective (3). The definition (4) emphasizes that only positive items are summed, and the parameter $p$ of $L p$ metric equals doubled $q$-parameter. Then (4) can be simplified by using $2 q$ parameter, and represented as the power-mean deviation itself:

$$
\begin{aligned}
& S=\left(\frac{1}{N} \sum_{i=1}^{N} \varepsilon_{i}^{2 q}\right)^{\frac{1}{2 q}} \\
& =\left(\frac{1}{N} \sum_{i=1}^{N}\left(y_{i}-\sum_{j=0}^{n} a_{j} x_{i j}\right)^{2 q}\right)^{\frac{1}{2 q}} .
\end{aligned}
$$

where the intercept's variable $x_{0}$ identically equals one.

For a given value of power parameter $q$, minimization of the objective (5) by the parameters of regressions yields a system of the first order partial derivatives:

$$
\begin{aligned}
\mathrm{U}_{\mathrm{k}} & =\frac{\partial \mathrm{S}}{\partial \mathrm{a}_{\mathrm{k}}} \\
& =\left(\frac{1}{\mathrm{~N}} \sum_{\mathrm{i}=1}^{\mathrm{N}} \varepsilon_{\mathrm{i}}^{2 \mathrm{q}}\right)^{\frac{1}{2 \mathrm{q}}-1}\left[\frac{-1}{\mathrm{~N}} \sum_{\mathrm{i}=1}^{\mathrm{N}} \mathrm{x}_{\mathrm{ik}} \varepsilon_{\mathrm{i}}^{2 \mathrm{q}-1}\right]=0
\end{aligned}
$$

with errors defined as in (5):

$$
\varepsilon_{i}=y_{i}-\sum_{j=0}^{n} x_{i j} a_{j}
$$


Non-linear system of equations (6) can be solved numerically by the Newton-Raphson procedure in the Iteratively Re-Weighted Least Squares (IRLS) approach (Bender, 2000; Lipovetsky \& Conklin, 2005). For this algorithm the elements of Hessian, or the matrix of second derivatives, are constructed using the derivatives of (6):

$$
\begin{aligned}
\mathrm{H}_{\mathrm{mk}} & =\frac{\partial^{2} \mathrm{~S}}{\partial \mathrm{a}_{\mathrm{m}} \partial \mathrm{a}_{\mathrm{k}}} \\
& =\frac{\partial \mathrm{U}_{\mathrm{k}}}{\partial \mathrm{a}_{\mathrm{m}}} \\
& =\frac{2 \mathrm{q}-1}{\mathrm{~N}}\left(\frac{1}{\mathrm{~N}} \sum_{\mathrm{i}=1}^{\mathrm{N}} \varepsilon_{\mathrm{i}}^{2 \mathrm{q}}\right)^{\frac{1}{2 \mathrm{q}}-1} \mathrm{G}_{\mathrm{mk}}
\end{aligned}
$$

where the elements $G_{m k}$ are defined by the expression:

$$
\begin{aligned}
& \mathrm{G}_{\mathrm{mk}}=\sum_{\mathrm{i}=1}^{\mathrm{N}} \mathrm{x}_{\mathrm{im}} \mathrm{x}_{\mathrm{ik}} \varepsilon_{\mathrm{i}}^{2 \mathrm{q}-2} \\
& -\left(\sum_{\mathrm{i}=1}^{\mathrm{N}} \varepsilon_{\mathrm{i}}^{2 \mathrm{q}}\right)^{-1}\left(\sum_{\mathrm{i}=1}^{\mathrm{N}} \mathrm{x}_{\mathrm{im}} \varepsilon_{\mathrm{i}}^{2 \mathrm{q}-1}\right)\left(\sum_{\mathrm{i}=1}^{\mathrm{N}} \mathrm{x}_{\mathrm{ik}} \varepsilon_{\mathrm{i}}^{2 \mathrm{q}-1}\right)^{.}
\end{aligned}
$$

Newton-Raphson procedure for finding vector of coefficients $a(5)$ can be presented as:

$$
a^{(t+1)}=a^{(t)}-H^{-1} U
$$

where $t$ denotes iteration steps, $H^{-1}$ is the inverted Hessian, and $U$ is the gradient-vector with the elements (6). The round parentheses in (6) and in (8) contain the same constant that is canceled in the expression (10), and also the constant $N$ is canceled, so (10) can be reduced to:

$$
a^{(t+1)}=a^{(t)}+(2 q-1)^{-1} G^{-1} X^{\prime} \mathcal{E}^{2 q-1},
$$

where $G^{-1}$ is the inverted matrix of elements (9), $X^{\prime}$ denotes the transposed matrix of all the regressors in (5), and $X^{\prime} \varepsilon^{2 q-1}$ is matrix notation for the sum in the squared parentheses (6).

It is convenient to introduce a diagonal matrix of powered errors by all observations:

$$
\begin{aligned}
\mathrm{W} & =\operatorname{diag}\left(\varepsilon^{2 \mathrm{q}-2}\right) \\
& =\operatorname{diag}\left(\varepsilon_{1}^{2 \mathrm{q}-2}, \varepsilon_{2}^{2 \mathrm{q}-2}, \ldots, \varepsilon_{\mathrm{N}}^{2 \mathrm{q}-2}\right)
\end{aligned}
$$

where $\varepsilon$ is the $N$-th order vector-column of the deviations (7). Then (9) in the matrix form is:

$$
G=X^{\prime} W X-\frac{1}{\varepsilon^{\prime} W \varepsilon}\left(X^{\prime} W \varepsilon\right)\left(X^{\prime} W \varepsilon\right)^{\prime} .
$$

The subtracted outer product in (13) is arranged of the vector $X^{\prime} W \varepsilon$ of the weighted product of regressors and residuals. Such a product is always close to zero due to the relations of orthogonality between regressors $x$ and residual errors $\mathcal{E}$. This property is exact for linear and approximate for a nonlinear regression (Lipovetsky \& Conklin, 2006).

It is always advisable to keep in only the stable part of the Hessian (Becker \& Le Cun, 1988), so it makes sense to reduce (13) to the main first item of the weighted second moment matrix $X^{\prime} W X$. Then the solution (11) can be simplified to:

$$
a^{(t+1)}=a^{(t)}+(2 q-1)^{-1}\left(X^{\prime} W X\right)^{-1} X^{\prime} W \varepsilon,
$$

where due to (12) the equality $X^{\prime} \varepsilon^{2 q-1}=X^{\prime} W \mathcal{E}$ is used. It is interesting to note that the exact expression (14) yields if instead of the mean deviation objective $S(5)$ the powered-deviation $S^{2 q}$ objective (4) is minimized. With (7) in the matrix form, the expression (14) becomes:

$$
\begin{aligned}
a^{(t+1)}= & \left(X^{\prime} W X\right)^{-1}\left(X^{\prime} W X\right) a^{(t)} \\
& +(2 q-1)^{-1}\left(X^{\prime} W X\right)^{-1} X^{\prime} W\left(y-X a^{(t)}\right), \\
= & \left(X^{\prime} W X\right)^{-1} X^{\prime} W z^{(t)}
\end{aligned}
$$

where the working variable is denoted as: 


$$
\begin{aligned}
z^{(t)} & =X a^{(t)} \\
& +(2 q-1)^{-1}\left(y-X a^{(t)}\right) \\
& =(2 q-1)^{-1}\left(y+(2 q-2) X a^{(t)}\right)
\end{aligned}
$$

The working variable (16) is a combination of the empirical dependent variable (vector $y$ ) and the predicted values of the dependent variable (vector $X a^{(t)}$ ) at any $t$-th iteration step. The right-hand side (15) shows that the solution is presented as a weighted linear regression of the dependent variable $z^{(t)}$ by all the predictors, so (15)-(16) define the IRLS algorithm.

It is interesting to note that if $q=1$ then $z^{(t)}$ (16) is reducing to the constant vector $y$, and $W(12)$ is reducing to the scalar matrix of identical ones, so the problem (5) and solution (15) coincide with a regular linear regression. For $q=0.5$ the Hessian (8) degenerates to zero, so the approach (10) does not work, and the methods of linear programming are mostly applied. The process of minimization (5)-(16) can include the power parameter $q$ as well. However, the residuals are usually only weakly dependable on this parameter. So, it is better to find parameters of regression for each fixed $q$, trying $q$ in a wide range of its values.

To explain the results on stability of the power parameter that yields the minimum residual errors in regression modeling, assume the normal distribution for the residual errors using the probability density function:

$$
f(\varepsilon)=\frac{1}{\sqrt{2 \pi} \sigma} \exp \left(\frac{-\varepsilon^{2}}{2 \sigma^{2}}\right),
$$

where $\varepsilon$ are the residuals (7) and $\sigma$ is the standard error. For a new random variable of the powered error $\delta=\varepsilon^{2 q}$, its probability density function can be defined by the technique of variables transformation (Hogg \& Craig, 1969), that yields:

$$
f(\delta)=\frac{1}{\sqrt{2 \pi} \sigma \cdot 2 q} \delta^{\frac{1}{2 q}-1} \exp \left(\frac{-\delta^{1 / q}}{2 \sigma^{2}}\right) .
$$

Such a distribution corresponds to a badness of fit function for $M$-estimates in robust regression (Huber, 1972, 1981; Ramsay, 1977). Approximation of the generalized powered mean (4) by the integral of the random variable $\delta=\varepsilon^{2 q}$ (18), can be expressed as follows:

$$
\begin{aligned}
S^{2 q} & =\frac{1}{N} \sum_{i=1}^{N}\left(\varepsilon_{i}^{2}\right)^{\mathrm{q}}=\frac{1}{N} \sum_{i=1}^{N} \delta_{i} \approx \int_{-\infty}^{\infty} \delta f(\delta) \mathrm{d} \delta \\
& =\frac{1}{\sqrt{2 \pi} \sigma \mathrm{q}} \int_{0}^{\infty} \delta^{\mathrm{v}-1} \exp \left(-\mu \delta^{\mathrm{b}}\right) \mathrm{d} \delta
\end{aligned}
$$

with the parameters denoted as:

$$
v=\frac{1}{2 q}+1, \quad \mu=\frac{1}{2 \sigma^{2}}, \quad b=\frac{1}{q} .
$$

The integral in (19) can be expressed via gamma function (Gradshteyn \& Ryzhik, 1965; Gordon, 1994):

$$
\int_{0}^{\infty} \delta^{v-1} \exp \left(-\mu \delta^{b}\right) d \delta=\frac{1}{|b|} \mu^{-v / b} \Gamma\left(\frac{v}{b}\right),
$$

so (19) can be simplified to:

$$
\begin{aligned}
S^{2 q} & =\frac{1}{\sqrt{2 \pi} \sigma q} \cdot q\left(2 \sigma^{2}\right)^{q+1 / 2} \Gamma\left(q+\frac{1}{2}\right) \\
& =\frac{2^{q} \sigma^{2 q}}{\sqrt{\pi}} \Gamma\left(q+\frac{1}{2}\right)
\end{aligned}
$$

For the case $q=1$, when the generalized power mean (4) is reducing to the least squares, the expression (22) is simplifying to:

$$
\begin{aligned}
S^{2} & =\frac{2 \sigma^{2}}{\sqrt{\pi}} \Gamma\left(1+\frac{1}{2}\right) \\
& =\frac{2 \sigma^{2}}{\sqrt{\pi}} \cdot \frac{1}{2} \Gamma\left(\frac{1}{2}\right), \\
& =\frac{2 \sigma^{2}}{\sqrt{\pi}} \cdot \frac{\sqrt{\pi}}{2} \\
& =\sigma^{2}
\end{aligned}
$$


where the properties $\Gamma(1+x)=x \Gamma(x)$ and $\Gamma(1 / 2)=\sqrt{\pi}$ of gamma function are applied (Abramowitz \& Stegun, 1974). The result (23) proves that the residual mean error estimates the theoretical standard error of the distribution (17). For the case $q=1 / 2$, when the generalized power mean (4) reduces to the least absolute deviations, the expression (22) is:

$$
S=\frac{\sqrt{2} \sigma}{\sqrt{\pi}} \Gamma(1)=\sqrt{\frac{2}{\pi}} \sigma \cong 0.8 \sigma .
$$

It is the mean absolute deviation that equals about $80 \%$ of the standard deviation (see Abraham \& Ledolter, 1983, p. 133). For a positive $x$, gamma function reaches its minimum $\Gamma(x)=0.886$ at the point $x=1.462$ (Abramowitz \& Stegun, 1974). The $q$ value (22) is by 0.5 less at this point, or $q=0.962$, so $p=2 q=1.924$ suggests a better powered approximation than the least squares with $p=2$. Taking the $2 q$-th root of the expression (22) shows that the generalized residual mean $S$ is proportional to the value of the standard error $\sigma$ itself. The residual mean $S$ in the units of $\sigma$, can be presented up to a constant as the $2 q$-th root of the gamma function:

$$
\frac{S}{\sigma}=\left(\Gamma\left(q+\frac{1}{2}\right)\right)^{\frac{1}{2 q}} .
$$

This function reaches its minimum at the value $q \approx 0.83$. A difference between theoretical estimate and empirical numerical trying for the best power parameter can be explained by a not exactly normal distribution of the empirical residual errors assumed in the theoretical derivation. Thus, the metric of the smallest residual deviation (4) or (22) equals $p=2 q \approx 1.7$. Although the evaluation via gamma function is a rough approximation, but it supports the empirical results that not the least-squares but a slightly-less-than-least- squares powered deviations produce minimum residual error estimations.

Numerical Example

For an illustration of the regular numerical output the data on cars technological solutions is used. This data is given in (Chambers \& Hastie, 1992), and is available in the statistical package (S-PLUS'2000, 1999, cu.summary file). The data contains the following variables of dimensions and mechanical specifications of 111 various cars, supplied by manufacturers or measured by Consumers Union reports: Weight (y) - pounds (considered in hundreds); Length $\left(x_{1}\right)$ - inches; WheelBase $\left(x_{2}\right)$ - length of wheelbase, inches; Width $\left(x_{3}\right)$ - inches; Height $\left(x_{4}\right)$ - height of car, inches; FrontHd $\left(x_{5}\right)-$ distance between the car's head-liner and the head of a $5 \mathrm{ft}$. 9in. front seat passenger, inches; RearHd $\left(x_{6}\right)$ - a similar distance for the rear seat passenger, inches; FrtLegRoom $\left(x_{7}\right)$ - maximum front leg room, inches; RearSeating $\left(x_{8}\right)$ - rear fore-and-aft seating room, inches; FrtShld $\left(x_{9}\right)-$ front shoulder room, inches; RearShld $\left(x_{10}\right)-$ rear shoulder room, inches; Turning $\left(x_{11}\right)_{-}^{-}$ radius of the turning circle, feet; Disp $\left(x_{12}\right)$ - the engine displacement, cubic inches; HP $\left(x_{13}\right)-$ the net horsepower; Tank $\left(x_{14}\right)$ - fuel refill capacity, gallons; HPrevs $\left(x_{15}\right)$ - the red line, or the maximum safe engine speed, rpm. The weight can be considered as an aggregate that has a strong impact on a car's cumulative characteristics, such as mileage per gallon (correlation with weight equals -0.87 ), and price (correlation with weight equals 0.70 ).

Regressions were constructed by powered deviations (5) with various values of the parameter $q$. Several best by the residual characteristics models are presented in Table 1. Each column of Table 1 corresponds to a particular value of $q$-parameter and contains the coefficients of regression (beginning from the 
Table 1. Regressions by several minimized powered deviations.

\begin{tabular}{|c|c|c|c|c|c|c|c|c|c|c|}
\hline$q$ & 0.83 & 0.84 & 0.85 & 0.86 & 0.87 & 0.88 & 0.89 & 0.9 & 1.0 & 1.1 \\
\hline$a_{0}$ & -50.076 & -48.400 & -47.617 & -49.880 & -50.114 & -50.275 & -50.458 & -50.641 & -52.388 & -54.017 \\
\hline$a_{1}$ & 0.147 & 0.160 & 0.150 & 0.135 & 0.135 & 0.135 & 0.135 & 0.135 & 0.135 & 0.135 \\
\hline$a_{2}$ & 0.081 & 0.071 & 0.053 & 0.086 & 0.087 & 0.087 & 0.087 & 0.088 & 0.089 & 0.089 \\
\hline$a_{3}$ & 0.279 & 0.288 & 0.353 & 0.321 & 0.319 & 0.318 & 0.317 & 0.317 & 0.314 & 0.315 \\
\hline$a_{4}$ & 0.259 & 0.324 & 0.355 & 0.361 & 0.362 & 0.363 & 0.364 & 0.364 & 0.370 & 0.375 \\
\hline$a_{5}$ & -0.431 & -0.098 & -0.283 & -0.331 & -0.330 & -0.327 & -0.323 & -0.319 & -0.286 & -0.256 \\
\hline$a_{6}$ & 0.708 & 0.238 & 0.091 & 0.098 & 0.092 & 0.088 & 0.083 & 0.079 & 0.042 & 0.011 \\
\hline$a_{7}$ & 0.348 & 0.305 & 0.137 & 0.169 & 0.170 & 0.170 & 0.171 & 0.172 & 0.181 & 0.190 \\
\hline$a_{8}$ & -0.142 & -0.135 & -0.123 & -0.129 & -0.129 & -0.129 & -0.129 & -0.129 & -0.129 & -0.129 \\
\hline$a_{9}$ & 0.018 & -0.107 & -0.105 & -0.075 & -0.073 & -0.071 & -0.069 & -0.067 & -0.054 & -0.045 \\
\hline$a_{10}$ & -0.001 & 0.008 & 0.019 & 0.017 & 0.017 & 0.017 & 0.017 & 0.017 & 0.018 & 0.019 \\
\hline$a_{11}$ & -0.029 & 0.002 & 0.040 & 0.066 & 0.068 & 0.068 & 0.069 & 0.069 & 0.073 & 0.077 \\
\hline$a_{12}$ & -0.013 & -0.019 & -0.009 & -0.008 & -0.008 & -0.008 & -0.008 & -0.008 & -0.008 & -0.008 \\
\hline$a_{13}$ & 0.060 & 0.060 & 0.050 & 0.049 & 0.048 & 0.048 & 0.048 & 0.048 & 0.049 & 0.049 \\
\hline$a_{14}$ & 0.123 & 0.226 & 0.159 & 0.138 & 0.138 & 0.138 & 0.138 & 0.137 & 0.132 & 0.125 \\
\hline$a_{15}$ & -0.001 & -0.001 & -0.001 & -0.001 & -0.001 & -0.001 & -0.001 & -0.001 & -0.001 & -0.001 \\
\hline$S^{2 q}$ & 45 & 1.430 & 1.280 & 1.266 & 1.279 & 1.292 & 1.305 & 1.319 & 1.468 & 1.646 \\
\hline$S$ & 1.446 & 1.237 & 1.157 & 1.147 & 1.152 & 1.157 & 1.161 & 1.166 & 1.212 & 1.254 \\
\hline Sabs & 1.234 & 1.015 & 0.966 & 0.963 & 0.963 & 0.963 & 0.964 & 0.964 & 0.970 & 0.976 \\
\hline Ssqr & 1.543 & 1.327 & 1.229 & 1.213 & 1.213 & 1.213 & 1.213 & 1.212 & 1.212 & 1.212 \\
\hline Mean & -0.733 & -0.371 & -0.122 & 0.000 & 0.011 & 0.012 & 0.011 & 0.010 & 0.000 & -0.010 \\
\hline$S^{2 q}$ cent & 1.465 & 1.327 & 1.269 & 1.266 & 1.279 & 1.292 & 1.305 & 1.319 & 1.468 & 1.646 \\
\hline$S$ cent & 1.259 & 1.183 & 1.150 & 1.147 & 1.152 & 1.157 & 1.161 & 1.166 & 1.212 & 1.254 \\
\hline Sabs cent & 1.043 & 0.965 & 0.966 & 0.963 & 0.963 & 0.964 & 0.964 & 0.965 & 0.970 & 0.975 \\
\hline Ssqr cent & 1.357 & 1.274 & 1.223 & 1.213 & 1.213 & 1.213 & 1.213 & 1.212 & 1.212 & 1.212 \\
\hline
\end{tabular}

intercept $a_{0}$ ) that are slowly varying across the power parameter $q$ values. Below the coefficients, several estimates for the residual errors are presented: the powered residual $S^{2 q}$ (4), the residual deviation $S$ (5), the absolute residual Sabs (3), and the residual standard error Ssqr (corresponds to square root of (2) for mean square root deviation). Note that the last two estimates are obtained by the corresponding set of the regression coefficients. The three of the residual error measures $-S^{2 q}, S$, and $S a b s$ have minimum at the value around $q=0.86$. The residual mean square root error $S s q r$, of course, reaches its minimum at the point $q=1$ that corresponds the least square solution (2). Behavior of these four error measures is shown in Figure 1 in a wide range of $q$. After initial decreasing and oscillating for $q$ below 0.86 , the $S^{2 q}, S$, and $S a b s$ curves reach their minima, and then with $q$ increase they grow as well. The residual mean square root error $S s q r$ is very flat beginning from the same threshold $q=0.86$.

The bottom section of Table 1 presents the estimate of mean value of the deviations (7), and all four residual error estimates centered by this mean value (the error estimates are denoted as $S^{2 q}$ cent, $S$ cent, Sabs cent, and Ssqr cent). It is 
interesting to see that the mean of the deviations is at first negative, than for bigger $q$ values the mean grows and reaches zero at about $q=0.86$, then it stays positive till the next reach of zero at the value $q=1$. So, these two values of $q$ produce minimum centered residual error estimates. The mean deviation and the four centered measures of the residual errors are shown in Figure 2 in a range of $q$ values. The behavior of the residual mean stabilizes with $q$ above 0.86 . All centered error measures change similarly but more flatly than those of non-centered measures from the previous graph, also with a threshold at the point of about $q=0.86$. The obtained results on the minimum of $S^{2 q}, S$, and Sabs errors in the vicinity of the parameter value about $0.83-0.87$ are amazingly constant. In numerous regressions by different data sets the same power region of $q$ is obtained for the minimum residual errors by the powered deviations.

Fig.1: Residual error estimates

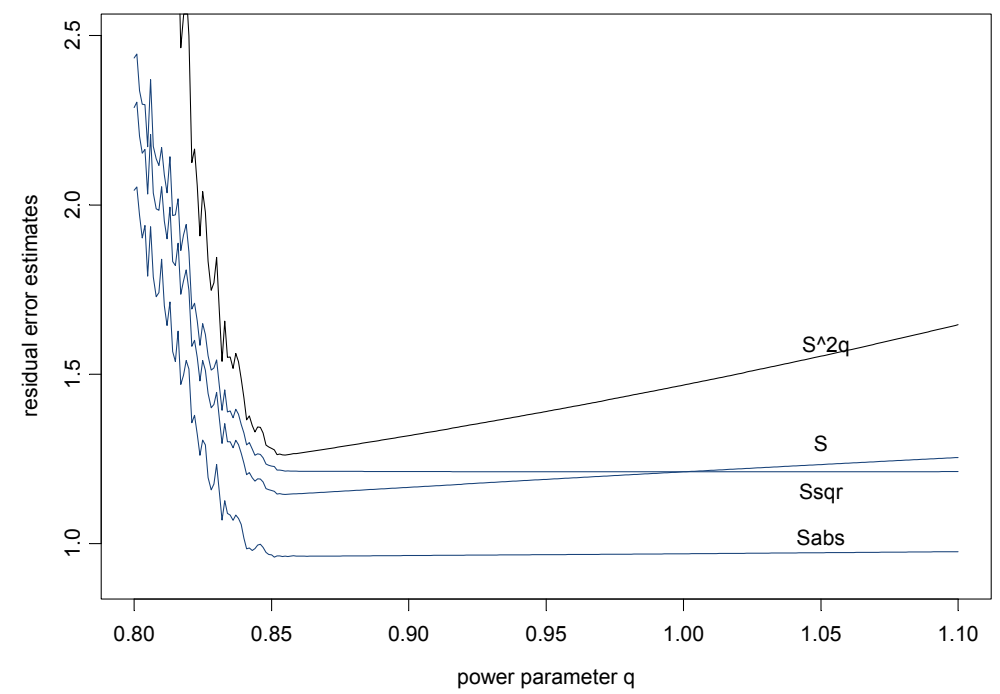

Fig.2: Residual mean and centered error estimates

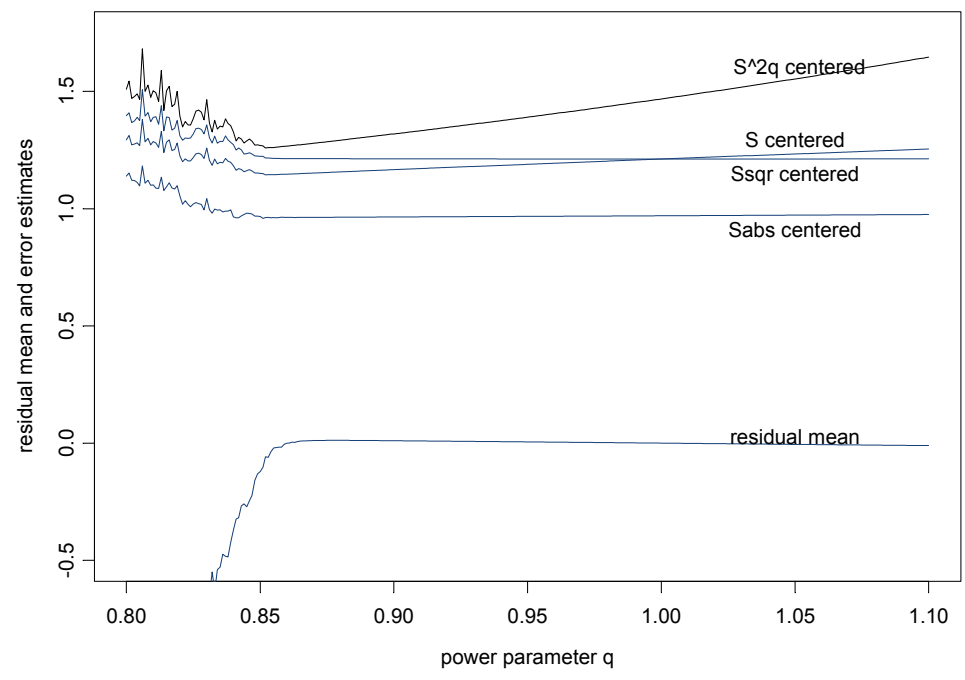




\section{Conclusion}

The generalized powered deviations were considered to estimate minimum possible residual error and the corresponding value of the power parameter. Numerical estimations performed in the work support the analytical result that the best optimization objective corresponds to the metric in the vicinity of $L_{1.7}$. Although change of coefficients and residuals in regressions by different power parameter is moderate, a metric close to the optimum $L_{1.7}$ can be applied for tuning the model. The objective of powered deviations can serve both to the theoretical investigation and practical application in numerous problems of regression modeling.

\section{References}

Abraham, B. \& Ledolter, J. (1983). Statistical methods for forecasting. New York, N.Y.: Wiley.

Abramowitz, M. \& Stegun, I. A. (Eds.), (1974). Handbook of mathematical functions. National Bureau of Standards. New York, N.Y.: Dover.

Alvarez, S. A. (1992). $L^{p}$ Arithmetic, The American Mathematical Monthly, 99, 556662.

Armstrong, R. D. \& Frome, E. L. (1976). A comparison of two algorithms for absolute deviation curve fitting. Journal of the American Statistical Association, 71, 328-330.

Becker, S. \& Le Cun, Y. (1988). Improving the convergence of back-propagation learning with second order methods. In: Touretzky, D. S., Hinton, G. E., \& Sejnowski, T. J. (eds.), Proceedings of the 1988 Connectionist Models Summer School, 29-37, Morgan Kaufmann, San Mateo, CA.

Beckenbach, E. F. (1946). An inequality of Jensen. The American Mathematical Monthly, 53, 501-505.

Bender, E. A. (2000). Mathematical methods in artificial intelligence, IEEE Computer Society Press, Los Alamitos, CA.

Borwein, J. M. \& Borwein, P. B. (1987). The way of all means. The American Mathematical Monthly, 94, 519-522.
Breiman, L. (1968). Probability. Reading, M.A.: Addison-Wesley.

Chambers, J. M. \& Hastie, T. J. (1992). Statistical models in S. Wadsworth \& Brooks, Pacific Grove, CA.

Daykin, D. E. \& Eliezer, C. J. (1969). Elementary proofs of basic inequalities. The American Mathematical Monthly, 76, 543-546.

Fletcher, R., Grant, J. A., \& Hebden, M. D. (1971). The calculation of linear best $L p$ approximations. Computer Journal, 14, 276279.

Forsythe, A. B. (1972). Robust estimation of straight line regression coefficients by minimizing pth power deviations. Technometrics, 14, 159-166.

Gentleman, W. M. (1965). Robust estimation of multivariate location by minimizing $p$-th power deviations, Ph.D. thesis, Dept. of Mathematics, Princeton University.

Gordon, L. (1994). A stochastic approach to the gamma function. The American Mathematical Monthly, 101, 858-865.

Gradshteyn, I. S. \& Ryzhik, I. M. (1965). Table of integrals, series, and products. London: Academic Press.

Hampel, F., Ronchetti, E. M., Rousseeuw, P. J., \& Stahel, W. A. (1986). Robust statistics: The approach based on influence functions. New York, N.Y.: Wiley.

Hardy, G. H., Littelwood, J. E., \& Polya, G. (1934). Inequalities. Cambridge: Cambridge University Press.

Hastie, T. J. \& Tibshirani, R. J. (1990). Generalized additive models. London: Chapman and Hall.

Hill, R. W. \& Holland, P. W. (1977). Two robust alternatives to least-squares regression. Journal of the American Statistical Association, 72, 828-833.

Hogg, R. V. \& Craig, A. T. (1969). Introduction to mathematical statistics. New York, N.Y.: Macmillan.

Huber, P. J. (1972). Robust statistics: A review. Annals of Mathematical Statistics, 43, 1041-1067.

Huber, P. J. (1981). Robust statistics. New York, N.Y.: Wiley. 
Kanter, M. (1973). Stable laws and the imbedding of $L^{p}$ spaces. The American Mathematical Monthly, 80, 403-407.

Korn, G. A. \& Korn, T. M. (1988). Mathematical handbook for scientists and engineers. New York, N.Y.: McGraw-Hill.

Lipovetsky, S. \& Conklin, M. (2000). Box-Cox generalization of logistic and algebraic binary response models. International Journal of Operations and Quantitative Management, 6, 276-285.

Lipovetsky, S. \& Conklin, M. (2005). Latent class regression model in IRLS approach. Mathematical and Computer Modelling, 42, 301-312.

Lipovetsky, S. \& Conklin, M. (2005). Ridge regression in two parameter solution. Applied Stochastic Models in Business and Industry, 21, 525-540.

McCullagh, P. \& Nelder, J. A. (1997). Generalized linear models. London: Chapman and Hall.

Ramsay, J. O. (1977). Comparative study of several robust estimates of slope, intercept, and scale in linear regression. Journal of the American Statistical Association, 72, 608615.
Ripley, B. D. (1996). Pattern recognition and neural networks. Cambridge: Cambridge University Press.

Rooij de, M. \& Heiser, W. J. (2005). Graphical representations and odds ratios in a distance-association model for the analysis of cross-classified data. Psychometrika, 70, 99-122.

Sposito, V. A. (1982). On unbiased Lp regression estimators. Journal of the American Statistical Association, 77, 652-653.

S-PLUS'2000 (1999). MathSoft Inc., Seattle, WA.

Tishler, A. \& Lipovetsky, S. (1997). The flexible CES-GBC family of cost functions: Derivation and application. The Review of Economics and Statistics, LXXIX, 638-646.

Tishler, A. \& Lipovetsky, S. (2000). A globally concave, monotone and flexible cost function: Derivation and application. Applied Stochastic Models in Business and Industry, 16, 279-296.

Weisberg, S. (1985). Applied Linear Regression. New York, N.Y.: Wiley.

Venables, W. N. \& Ripley, B. D. (1997). Modern applied statistics with S-PLUS. New York, N.Y.: Springer. 


\title{
LQ-Moments for Statistical Analysis of Extreme Events
}

\author{
Ani Shabri \\ Universiti Teknologi Malaysia \\ Abdul Aziz Jemain \\ Universiti Kebangsaan Malaysia
}

Statistical analysis of extremes is conducted for predicting large return periods events. LQ-moments that are based on linear combinations are reviewed for characterizing the upper quantiles of distributions and larger events in data. The LQ-moments method is presented based on a new quick estimator using five points quantiles and the weighted kernel estimator to estimate the parameters of the generalized extreme value (GEV) distribution. Monte Carlo methods illustrate the performance of LQ-moments in fitting the GEV distribution to both GEV and non-GEV samples. The proposed estimators of the GEV distribution were compared with conventional L-moments and LQ-moments based on linear interpolation quantiles for various sample sizes and return periods. The results indicate that the new method has generally good performance and makes it an attractive option for estimating quantiles in the GEV distribution.

Key words: LQ-moments, L-moments, quick estimator, generalized extreme value, weighted kernel.

\section{Introduction}

Statistical analysis of extremes is often interested for predicting large return period events. Thus, the more relevant analysis is the upper quantiles of the distributions and the extreme sample events (Wang, 1997). The method of classical moments (MOM) is mostly used because of its relative ease of application but it is generally not as efficient as the maximum likelihood (ML) method estimates and it is too sensitive to the upper quantiles of distributions (Vogel \& Fennessey, 1993).

The ML method is the most important method because it leads to efficient parameter estimators with Gaussian asymptotic

Ani Shabri is a Lecturer at the Department of Mathematics. His research interests are the analysis of flood frequency and time series. Email address: ani_sabri@hotmail.com. Abdul Abdul Aziz Jemain is Professor in the School of Mathematical Science. His researcher interests include multivariate method, social science, flood frequency analysis and multi-criteria decision making. Email address: azizj@pkrisc.cc.ukm.my. distributions. However, this method sometimes under-estimates and so causes large bias and variance of extreme upper quantile and does not always work well in small samples (Park, 2005).

The L-moments (LMOM), certain linear functions of the expectations of order statistics, were introduced and comprehensively reviewed by Hosking (1990). Hosking (1990) presented the LMOM estimators for some common distributions and demonstrates that in some cases, the LMOM method may give even better fit than ML method. Hosking and Wallis (1997) illustrated that LMOM are efficient in estimating parameters of a wide range of distributions. In general, the bias of small sample estimates of higher-order LMOM is fairly small as compared to traditional moment estimates. This method has become a standard procedure in hydrology for estimating the parameters of certain statistical distributions. The LMOM have found wide applications in such fields of applied research as civil engineering, meteorology, hydrology, quality control and engineering (Sankarasubramanian \& Srinivasan, 1999; Karvanen, 2005).

Mudolkar and Hutson (1998) extended LMOM to new moment like entitiles called LQmoments (LQMOM). The LQMOM are constructed by using functional defining the quick estimators, such as the median, trimean or Gastwirth, in places of expectations in LMOM. 
The LQMOM that are based on the quick estimators, namely the trimean and the linear interpolation quantile estimator are used to fit a GEV to observed flood frequencies. They found the LQMOM are often easier to compute than LMOM, and in general behave similarly to the LMOM.

In this article, LQMOM that are based on the trimean and the linear interpolation quantile (LIQ) estimator are reviewed for characterizing the upper part of distributions and larger events in data. The objective of this article is to revisit the LQMOM, presents the LQMOM method based on the new quick estimator using five-points quantiles and the weighted kernel estimator (WK5) to estimate the parameters of the generalized extreme value (GEV) distribution. Estimation of the GEV distribution by using LQMOM is formulated. The performance of the LQMOM based on the new estimator is compared to LMOM and LIQ methods, by using both GEV and non-GEV simulated sample data.

\section{Definition of LQ-Moment}

Let $X_{1}, X_{2}, \ldots, X_{n}$ be a random sample from a continuous distribution function $F($.) with quantile function $Q(u)=F^{-1}(u)$, and let $X_{1: n} \leq X_{2: n} \leq \ldots \leq X_{n: n}$ denote the corresponding order statistics. Hosking (1990) defined the $r$ th L-moment $\lambda_{r}$ as

$$
\lambda_{r}=\frac{1}{r} \sum_{k=0}^{r-1}(-1)^{k}\left(\begin{array}{c}
r-1 \\
k
\end{array}\right) E\left(X_{r-k: r}\right), \quad r=1,2, \ldots
$$

Mudholkar and Hutson (1998) suggested a robust modification in which the mean of the distribution of $X_{r-k: r}$ in (1) is replaced by its median or some others population location measure. In particular, they defined the $r$ th LQmoments $\xi_{r}$ as

$$
\xi_{r}=\frac{1}{r} \sum_{k=0}^{r-1}(-1)^{k}\left(\begin{array}{c}
r-1 \\
k
\end{array}\right) \tau_{p, \alpha}\left(X_{r-k: r}\right), \quad r=1,2, \ldots
$$

where $\tau_{p, \alpha}\left(X_{r-k: r}\right)$ is a quick measure of the location of the sampling distribution of the order $X_{r-k: r}$. They introduced $\tau_{p, \alpha}$ based on a three-points quantiles of the sample calculated from the order statistics and defined as

$$
\begin{aligned}
& \tau_{\mathrm{p}, \alpha}\left(\mathrm{X}_{\mathrm{r}-\mathrm{k}: \mathrm{r}}\right) \\
& =\mathrm{pQ}_{\mathrm{X}_{\mathrm{r}-\mathrm{k} \mathrm{r}}}(\alpha) \\
& +(1-2 \mathrm{p}) \mathrm{Q}_{\mathrm{X}_{\mathrm{r}-\mathrm{k} \mathrm{r}}}(1 / 2) \\
& +\mathrm{pQ}_{\mathrm{X}_{\mathrm{r}-\mathrm{k} \mathrm{r}}}(1-\alpha)
\end{aligned}
$$

where $0 \leq \alpha \leq 1 / 2,0 \leq p \leq 1 / 2 . \tau_{p, \alpha}$ is called the median for $p=0, \alpha=1$, the trimean for $p=1 / 4, \alpha=1 / 4$ and Gastwirth for $p=0.3, \alpha=1 / 3$.

The quick measures of location $\tau_{p, \alpha}$ for five-points quantiles is defined as

$$
\begin{aligned}
& \tau_{\mathrm{p}, \alpha}\left(\mathrm{X}_{\mathrm{r}-\mathrm{k}: \mathrm{r}}\right) \\
& =\mathrm{pQ}_{\mathrm{X}_{\mathrm{r}-\mathrm{k} . \mathrm{r}}}(\alpha) \\
& +\mathrm{p} \mathrm{Q}_{\mathrm{X}_{\mathrm{r}-\mathrm{k} . \mathrm{r}}}(5 \alpha) \\
& +(1-4 \mathrm{p}) \mathrm{Q}_{\mathrm{X}_{\mathrm{r}-\mathrm{k} \cdot \mathrm{r}}}(1 / 2) \\
& +\mathrm{pQ}_{\mathrm{X}_{\mathrm{r}-\mathrm{k} \cdot \mathrm{r}}}(1-5 \alpha) \\
& +\mathrm{pQ}_{\mathrm{X}_{\mathrm{r}-\mathrm{k} \mathrm{r}}}(1-\alpha)
\end{aligned}
$$

where $0 \leq \alpha \leq 0.1$ and $0 \leq p \leq 1 / 4$.

The first four LQ-moments of the random variable $X$ are defined as

$$
\begin{gathered}
\xi_{1}=\tau_{p, \alpha}(X) \\
\xi_{2}=\frac{1}{2}\left[\tau_{p, \alpha}\left(X_{2: 2}\right)-\tau_{p, \alpha}\left(X_{1: 2}\right)\right] \\
\xi_{3}=\frac{1}{3}\left[\tau_{p, \alpha}\left(X_{3: 3}\right)-2 \tau_{p, \alpha}\left(X_{2: 3}\right)+\tau_{p, \alpha}\left(X_{1: 3}\right)\right] \\
\xi_{4}=\frac{1}{4}\left[\tau_{p, \alpha}\left(X_{4: 4}\right)-3 \tau_{p, \alpha}\left(X_{3: 4}\right)\right. \\
\left.+3 \tau_{p, \alpha}\left(X_{2: 4}\right)-\tau_{p, \alpha}\left(X_{1: 4}\right)\right]
\end{gathered}
$$


The skewness and kurtosis based upon the ratios of LQ-moments to be called LQ skewness and LQ kurtosis are given respectively by

$$
\eta_{3}=\xi_{3} / \xi_{2}
$$

and

$$
\eta_{4}=\xi_{4} / \xi_{2}
$$

Estimation of LQ-moments

For samples of size $n$, the $r$ th sample LQ-moment $\xi_{r}$ is given by

$$
\hat{\xi}_{r}=\frac{1}{r} \sum_{k=0}^{r-1}(-1)^{k}\left(\begin{array}{c}
r-1 \\
k
\end{array}\right) \hat{\tau}_{p, \alpha}\left(X_{r-k: r}\right), \quad r=1,2, \ldots
$$

where the quick estimator $\hat{\tau}_{p, \alpha}\left(X_{r-k: r}\right)$ of the location of the order statistic $X_{r-k: r}$ for fivepoints quantiles is given by

$$
\begin{aligned}
& \hat{\tau}_{\mathrm{p}, \alpha}\left(\mathrm{X}_{\mathrm{r}-\mathrm{k}: \mathrm{r}}\right) \\
& =\mathrm{pQ}\left[\mathrm{B}_{\mathrm{r}-\mathrm{k}: \mathrm{r}}^{-1}(\alpha)\right]+\mathrm{pQ}\left[\mathrm{B}_{\mathrm{r}-\mathrm{k}: \mathrm{r}}^{-1}(5 \alpha)\right] \\
& +(1-4 \mathrm{p}) \mathrm{Q}\left[\mathrm{B}_{\mathrm{r}-\mathrm{k}: \mathrm{r}}^{-1}(1 / 2)\right] \\
& +\mathrm{pQ}\left[\mathrm{B}_{\mathrm{r}-\mathrm{k}: \mathrm{r}}^{-1}(1-5 \alpha)\right] \\
& +\mathrm{pQ}\left[\mathrm{B}_{\mathrm{r}-\mathrm{k}: \mathrm{r}}^{-1}(1-\alpha)\right]
\end{aligned}
$$

where $B_{r-k: r}^{-1}(\alpha)$ is the quantile of a beta random variable with parameter $r-k$ and $k+1$, and $Q($.) denotes the quantile estimator. The sample LQ skewness and LQ kurtosis are given respectively by

$$
\hat{\eta}_{3}=\hat{\xi}_{3} / \hat{\xi}_{2}
$$

and

$$
\hat{\eta}_{4}=\hat{\xi}_{4} / \hat{\xi}_{2}
$$

The Quantile Estimator

David and Nagaraja (2003), Sheather and Marron (1990), Huang and Brill (1999) and Huang (2001) discussed several quantile estimators for estimating the values of the population quantile. In this study, only the linear interpolation quantile estimator and the weighted kernel quantile estimator are presented.
The Linear Interpolation Quantile Estimator

Mudholkar and Hutson (1998) proposed the simplest quantile function estimator based on the linear interpolation (LIQ). This quantiles is used commonly in statistical packages such as MINITAB, SAS, IMSL and S-PLUS. The LIQ estimator is given by

$$
\begin{aligned}
& Q(u)=(1-\varepsilon) X_{\left[n^{\prime} u\right]: n}+\varepsilon X_{\left[n^{\prime} u\right]+1: n} \\
& 0<u<1
\end{aligned}
$$

where

$$
\varepsilon=n^{\prime} u-\left[n^{\prime} u\right] \text { and } n^{\prime}=n+1 .
$$

The Weighted Kernel Quantile Estimator

A popular class of $\mathrm{L}$ quantile estimators is called kernel quantile estimators has been widely applied (Sheather \& Marron, 1990; Huang \& Brill, 1999; Huang, 2001). The L quantile estimators is given by

$$
Q(u)=\sum_{i=1}^{n}\left[\int_{(i-1) / n}^{i / n} K_{h}(t-u) d t\right] X_{i: n}
$$

where $K$ is a density function symmetric about 0 and

$$
K_{h}(\bullet)=(1 / h) K(\bullet / h)
$$

The approximation of the $\mathrm{L}$ quantile estimator is called as the weighted kernel quantile estimator (WKQ) is given by

$$
Q(u)=\sum_{i=1}^{n}\left[n^{-1} K_{h}\left(\sum_{j=1}^{i} w_{j, n}-u\right)\right] X_{i: n}, 0<u<1
$$

where

$$
w_{i, n}= \begin{cases}\frac{1}{2}\left(1-\frac{n-2}{\sqrt{n(n-1)}}\right), & i=1, n, \\ \frac{1}{\sqrt{n(n-1)}}, & i=2,3, \ldots, n-1 .\end{cases}
$$




$$
h=[u(1-u) / n]^{1 / 2}
$$

and $K(t)=(2 \pi)^{-1 / 2} \exp \left(-1 / 2 t^{2}\right)$ is the Gaussian Kernel.

Generalized Extreme Value

The generalized extreme value (GEV) distribution has been used widely and importantly in the modeling of extreme events in several areas including hydrology, meteorology, finance and insurance, and reliability engineering (Park, 2005). It was recommended for at-site flood frequency analysis in the United Kingdom, for rainfall frequency and for sea waves in the United States. Many studies in regional frequency have used the GEV distribution (Hosking et al., 1985b; Chowdhury et al., 1991). In practice, it has been used to model a wide variety of natural extremes, including floods, rainfall, wind speeds, and wave height. Mathematically, the GEV distribution is very attractive because its inverse has a closed form, and parameters are easily estimated by LMOM (Martin \& Stedinger, 2000). The GEV distribution has cumulative distribution function (CDF)

$$
\begin{aligned}
F(x) & =\exp \left\{-\left[1-k\left(\frac{x-\mu}{\sigma}\right)\right]^{1 / k}\right\} k \neq 0 \\
& =\exp \left\{-\exp \left[-\frac{(x-\mu)}{\sigma}\right]\right\} \quad k=0
\end{aligned}
$$

where $\mu+\sigma / k \leq x<\infty$ for $k<0$ and $-\infty<x \leq \mu+\sigma / k$ for $k>0$. Here, $\mu, \sigma$, and $k$ are location, scale, and shape parameters, respectively. Quantiles function of GEV distribution are given in terms of the parameters and the cumulative probability $F$ by

$$
Q(F)=\mu+\sigma Q_{0}(F)
$$

where

$$
\begin{aligned}
Q_{0}(F) & =\left[1-(-\log F)^{k}\right] / k \quad k \neq 0 \\
& =-\ln (-\ln (F)) \quad k=0
\end{aligned}
$$

L-Moments of GEV Distribution

The LMOM estimators for GEV distribution (Martins \& Stedinger, 2000) are

$$
\begin{aligned}
& \hat{k}=7.8590 c+2.9544 c^{2}, \\
& c=2 /\left(3+\hat{\tau}_{3}\right)-\log (2) / \log (3), \\
& \hat{\alpha}=\frac{\hat{\lambda}_{2} \hat{k}}{\left(1-2^{-\hat{k}}\right) \Gamma(1+\hat{k})}, \\
& \hat{\mu}=\hat{\lambda}_{1}-\frac{\hat{\alpha}}{\hat{k}}\{1-\Gamma(1+\hat{k})\}
\end{aligned}
$$

The $\hat{k}$ function is a very good approximation for $\hat{k}$ in the range $(-0.5,0.5)$. The LMOM estimators $\hat{\lambda}_{1}, \hat{\lambda}_{2}, \hat{\lambda}_{3}$ and $\hat{\tau}_{3}=\hat{\lambda}_{3} / \hat{\lambda}_{2}$ were obtained by using an unbiased estimator of the first three probability weighted moment (PWM) defined as

$$
\beta_{r}=\mu+\frac{\alpha}{k}\left[1-(r+1)^{-k} \Gamma(1+k)\right] /(r+1) .
$$

The unbiased estimator of $\beta_{r}$ is

$$
\begin{aligned}
& b_{r}=\sum_{i=1}^{n} \frac{(i-1)(i-2)(i-3) \ldots(i-r)}{n(n-1)(n-2) \ldots(n-r)} X_{i: n} \\
& r=0,1,2, \ldots
\end{aligned}
$$

where the $X_{i: n}$ are the ordered observations from a sample of size and

$$
\lambda_{1}=\beta_{0}, \lambda_{2}=2 \beta_{1}-\beta_{0} \text {, and } \lambda_{3}=6 \beta_{2}-6 \beta_{1}+\beta_{0} \text {. }
$$

The LQ moments of GEV Distribution

The LQ-moment estimators for the GEV distribution behave similarly to the L-moments. 
From equations (5)-(9) and equation (22), the first three LQ-moments of the GEV distribution for the quick estimator based on five-points quantiles can be written as

$$
\begin{gathered}
\xi_{1}=\mu+\sigma t_{p, \alpha}\left(X_{1: 1}\right) \\
\xi_{2}=\frac{1}{2} \sigma\left[t_{p, \alpha}\left(X_{2: 2}\right)-t_{p, \alpha}\left(X_{1: 2}\right)\right] \\
\xi_{3}=\frac{1}{3} \sigma\left[t_{p, \alpha}\left(X_{3: 3}\right)-2 t_{p, \alpha}\left(X_{2: 3}\right)+t_{p, \alpha}\left(X_{1: 3}\right)\right] \\
\eta_{3}=\frac{\frac{1}{3}\left[t_{p, \alpha}\left(X_{3: 3}\right)-2 t_{p, \alpha}\left(X_{2: 3}\right)+t_{p, \alpha}\left(X_{1: 3}\right)\right]}{\frac{1}{2}\left[t_{p, \alpha}\left(X_{2: 2}\right)-t_{p, \alpha}\left(X_{1: 2}\right)\right]}
\end{gathered}
$$

where

$$
\begin{aligned}
& \mathrm{t}_{\mathrm{p}, \alpha}\left(\mathrm{X}_{\mathrm{r}-\mathrm{k}: \mathrm{r}}\right) \\
& =\mathrm{pQ}_{0}\left[\mathrm{~B}_{\mathrm{r}-\mathrm{k}: \mathrm{r}}^{-1}(\alpha)\right]+\mathrm{pQ}_{0}\left[\mathrm{~B}_{\mathrm{r}-\mathrm{k}: \mathrm{r}}^{-1}(5 \alpha)\right] \\
& +(1-4 \mathrm{p}) \mathrm{Q}_{0}\left[\mathrm{~B}_{\mathrm{r}-\mathrm{k}: \mathrm{r}}^{-1}(1 / 2)\right] \\
& +\mathrm{pQ}_{0}\left[\mathrm{~B}_{\mathrm{r}-\mathrm{k}: \mathrm{r}}^{-1}(1-5 \alpha)\right]+\mathrm{pQ}_{0}\left[\mathrm{~B}_{\mathrm{r}-\mathrm{k}: \mathrm{r}}^{-1}(1-\alpha)\right]
\end{aligned}
$$

and

$$
Q_{0}(F)=\left[1-(-\log F)^{k}\right] / k
$$

The LQMOM estimators $\hat{\mu}$, $\hat{\sigma}$ and $\hat{k}$ of the parameters are the solution of (28)-(30), when $\xi_{r}$ are replaced by their estimators $\hat{\xi}_{r}$. The relationship between $\eta_{3}$ and $k$ from Eq. (31) (for example $p=0.2$ and $\alpha=0.05$ ) is shown in Figure 1. The following approximation relationships between the value of $k$ and $\eta_{3}$ obtained through regression analysis

$$
\begin{aligned}
& \hat{\mathrm{k}}=0.2801-1.7130 \hat{\eta}_{3}+0.8377 \hat{\eta}_{3}^{2} \\
& -1.0491 \hat{\eta}_{3}^{3}+0.6495 \hat{\eta}_{3}^{4}-0.2934 \hat{\eta}_{3}^{5} \\
& -0.1268 \hat{\eta}_{3}^{6}+0.2765 \hat{\eta}_{3}^{7}-0.0963 \hat{\eta}_{3}^{8}
\end{aligned}
$$

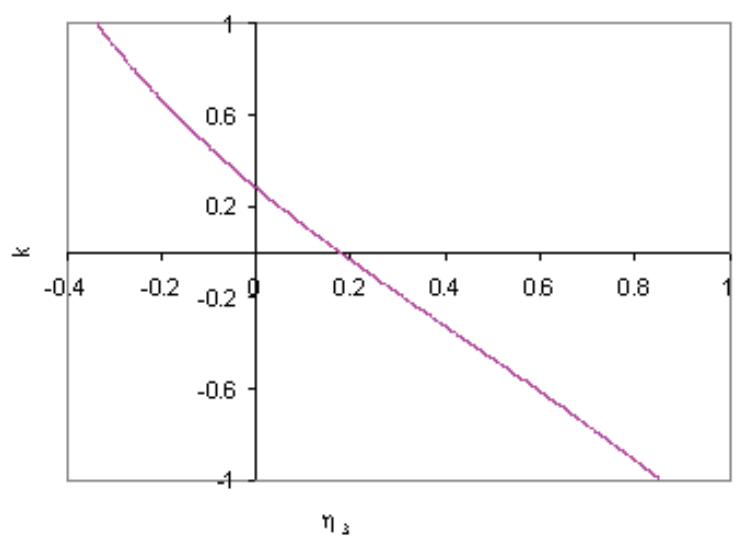

Figure 1: Relationship between $\eta_{3}$ and $k$ for the GEV distribution. 
The $\hat{k}$ function is a very good approximation for $\hat{k}$ in the range $[-1.0,1.0]$ and $\hat{\eta}_{3}$ in the range $[-0.336,0.854]$. Once the value of $\hat{k}$ is obtained, $\hat{\sigma}$ and $\hat{\mu}$ can be estimated successively from Equation (29) and (28) as

$$
\begin{gathered}
\hat{\sigma}=\frac{2 \hat{\xi}_{2}}{\left[\hat{t}_{p, \alpha}\left(X_{2: 2}\right)-\hat{t}_{p, \alpha}\left(X_{1: 2}\right)\right]} \\
\hat{\mu}=\hat{\xi}_{1}-\hat{\sigma} \hat{t}_{p . \alpha}\left(X_{1: 1}\right)
\end{gathered}
$$

\section{Monte Carlo Simulations}

Monte Carlo simulations have been carried out to investigate the effect of LQmoments based on WK5 with $p=0.2$ and $\alpha=0.05$ on the high quantiles estimation.

Simulation Study For Parent Distribution Function Known

It is still useful to look at how estimation is affected by various methods when the distribution function is known, although the true underlying distribution function is never known in practice. In this study, the GEV
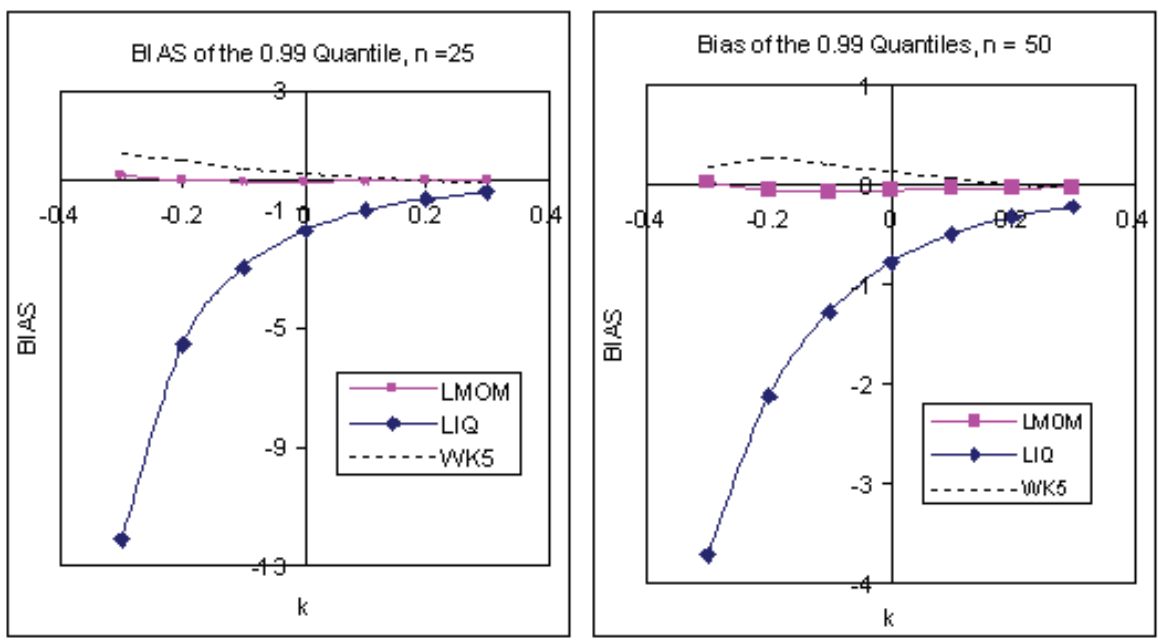

Figure 2. Bias of $\mathrm{Q}(\mathrm{F}=0.99)$ Estimator Using L Moments and LQ Moments Based on WK5 and LIQ, Fitting the GEV Distribution to Generated GEV Samples For $\mathrm{n}=25$ and $\mathrm{n}=50$ distribution is used to generate GEV samples. Monte Carlo simulations were performed for sample sizes $15,25,50$ and 100 , and parameters of GEV are $\mu=0$ and $\sigma=1$ with different values of $k$ between -0.4 and 0.4 . The samples are fitted by the GEV distribution function using the method of LMOM, LIQ, and WK5.

For each sample size, 10,000 replicates were generated, and quantile estimators of $Q(F), F=0.90,0.98,0.99$, and 0.999, are examined in terms of the BIAS and root mean square error (RMSE). Results for BIAS for different quantiles show a very similar pattern. Only the result for $Q(F), F=0.99$ is presented here and is shown in Figure 2. For the extreme quantiles, the LMOM estimator consistently shows the lowest BIAS followed by WK5 and LIQ estimator for samples sizes of 25 and 50.

RMSE has been obtained for quantiles $Q(F), F=0.9,0.98,0.99$, and 0.999, estimated by using LMOM, LIQ, and WK5. Results are presented in Table 1 in terms of estimation efficiency in relation to using WK5 defined as

$$
\phi=\frac{\text { RMSE using WK5 }}{\text { RMSE using LMOM or LIQ }}
$$


Table 1: Efficiency of Q(F), F = 0.9, 0.98, 0.99, and 0.999 Estimated By Using LMOM, LIQ, and WK5, Fitting the GEV Distribution Based on Generated GEV Samples

\begin{tabular}{|c|c|c|c|c|c|c|c|c|c|c|c|}
\hline \multirow[b]{3}{*}{$\mathrm{n}$} & \multirow[b]{3}{*}{$\mathrm{F}$} & \multicolumn{10}{|c|}{$k$} \\
\hline & & \multicolumn{2}{|c|}{-0.3} & \multicolumn{2}{|c|}{-0.1} & \multicolumn{2}{|c|}{0} & \multicolumn{2}{|c|}{0.1} & \multicolumn{2}{|c|}{0.3} \\
\hline & & LMOM & LIQ & LMOM & LIQ & LMOM & LIQ & LMOM & LIQ & LMOM & LIQ \\
\hline \multirow[t]{4}{*}{15} & 0.9 & 0.99 & 0.00 & 1.10 & 0.64 & 1.14 & 0.72 & 1.19 & 0.80 & 1.34 & 0.92 \\
\hline & 0.98 & 1.15 & 0.00 & 0.87 & 0.18 & 0.83 & 0.26 & 0.81 & 0.29 & 0.79 & 0.30 \\
\hline & 0.99 & 1.19 & 0.00 & 0.80 & 0.09 & 0.77 & 0.17 & 0.76 & 0.21 & 0.84 & 0.27 \\
\hline & 0.999 & 1.23 & 0.00 & 0.70 & 0.00 & 0.75 & 0.04 & 0.87 & 0.09 & 1.24 & 0.20 \\
\hline \multirow[t]{4}{*}{25} & 0.9 & 1.22 & 0.71 & 1.10 & 0.73 & 1.13 & 0.77 & 1.17 & 0.82 & 1.28 & 0.89 \\
\hline & 0.98 & 1.21 & 0.18 & 0.94 & 0.34 & 0.91 & 0.35 & 0.88 & 0.35 & 0.84 & 0.35 \\
\hline & 0.99 & 1.22 & 0.07 & 0.90 & 0.24 & 0.87 & 0.27 & 0.85 & 0.28 & 0.87 & 0.31 \\
\hline & 0.999 & 1.31 & 0.00 & 0.87 & 0.07 & 0.88 & 0.11 & 0.93 & 0.13 & 1.19 & 0.25 \\
\hline \multirow[t]{4}{*}{50} & 0.9 & 1.15 & 0.80 & 1.08 & 0.77 & 1.09 & 0.78 & 1.12 & 0.79 & 1.20 & 0.80 \\
\hline & 0.98 & 1.19 & 0.50 & 1.01 & 0.47 & 0.97 & 0.45 & 0.94 & 0.43 & 0.88 & 0.39 \\
\hline & 0.99 & 1.23 & 0.41 & 0.99 & 0.40 & 0.95 & 0.39 & 0.92 & 0.37 & 0.88 & 0.35 \\
\hline & 0.999 & 1.53 & 0.14 & 1.01 & 0.22 & 0.97 & 0.24 & 0.95 & 0.25 & 1.02 & 0.31 \\
\hline \multirow[t]{4}{*}{100} & 0.9 & 0.96 & 0.85 & 1.04 & 0.79 & 1.04 & 0.77 & 1.06 & 0.76 & 1.09 & 0.73 \\
\hline & 0.98 & 0.88 & 0.69 & 0.75 & 0.57 & 0.66 & 0.52 & 0.71 & 0.49 & 0.90 & 0.42 \\
\hline & 0.99 & 0.91 & 0.67 & 0.72 & 0.53 & 0.62 & 0.48 & 0.67 & 0.44 & 0.89 & 0.38 \\
\hline & 0.999 & 1.33 & 0.62 & 0.79 & 0.41 & 0.64 & 0.37 & 0.67 & 0.35 & 0.91 & 0.34 \\
\hline
\end{tabular}

Values $\phi<1$ indicated that the WK5 method is superior to the other methods. Table 1 shows the $\phi$ of the estimators for LMOM, and LIQ estimators compared to WK5 method for $k=$ $-0.3,-0.1,0,0.1,0.3$. For the estimation of $Q(F), F>0.9$, WK5 in many cases leads to higher efficiency especially for $k>-0.3$. The LIQ estimators lead to lower efficiency than LMOM for all $n$ and $k$.

\section{Parent Distribution Function Unknown}

In practice, the true distribution function is never known. Thus, it will be even more useful to look how estimation is affected by various methods when the assumed distribution function differs from the parent distribution function. In this study Kappa distribution was used to generate the random samples data.

Hosking and Wallis (1993) used the kappa distribution to generate artificial data for assessing the goodness of fit of different distributions in their study on regional frequency analysis. The cumulative distribution function of the Kappa distribution four-parameter is

$$
F(x)=\left\{\begin{array}{lll}
\left\{1-h[1-k(x-\varsigma) / \sigma]^{1 / k}\right\}^{1 / h} & \text { if } & k \neq 0, h \neq 0, \\
\exp \left\{-[1-k(x-\varsigma) / \sigma]^{1 / k}\right\} & \text { if } & k \neq 0, h=0, \\
\{1-h \exp [-(x-\varsigma) / \sigma]\}^{1 / h} & \text { if } & k=0, h \neq 0, \\
\exp \{-\exp [-(x-\varsigma) / \sigma]\} & \text { if } & k=0, h=0 .
\end{array}\right.
$$

where $\varsigma$ is a location parameter, $\sigma$ is a scale parameter, and $h$ and $k$ are shape parameters (Park and Park, 2002). The quantile function of the kappa distribution is

$$
Q(F)=\varsigma+\sigma\left\{1-\left[\left(1-F^{h}\right) / h\right]^{k}\right\} / k .
$$

This distribution is a special cases of the generalized logistic (GL) $(h=-1$ and $k \neq 0)$, generalized extreme-value

(GEV) 
Table 2: Efficiency of Q(F), F = 0.9, 0.98, 0.99, and 0.999 Estimated By Using LMOM and WK5, Fitting the GEV Distribution Based on Generated Kappa Samples

\begin{tabular}{|c|c|c|c|c|c|c|c|c|c|}
\hline \multirow[b]{2}{*}{$\underline{n}$} & \multirow[b]{2}{*}{$\mathrm{F}$} & \multicolumn{2}{|c|}{ GL } & \multicolumn{2}{|c|}{ EXP } & \multicolumn{2}{|c|}{ GP } & \multicolumn{2}{|c|}{ Uniform } \\
\hline & & LMOM & WK5 & LMOM & WK5 & LMOM & WK5 & LMOM & WK5 \\
\hline \multirow[t]{4}{*}{15} & 0.9 & 1.358 & 1.555 & 0.596 & 0.637 & 0.316 & 0.369 & 0.233 & 0.081 \\
\hline & 0.98 & 4.322 & 4.444 & 1.335 & 1.212 & 0.589 & 0.446 & 0.169 & 0.123 \\
\hline & 0.99 & 6.990 & 6.981 & 2.069 & 1.582 & 0.908 & 0.560 & 0.249 & 0.205 \\
\hline & 0.999 & 33.875 & 32.832 & 9.033 & 4.714 & 3.125 & 1.713 & 0.544 & 0.499 \\
\hline \multirow[t]{4}{*}{25} & 0.9 & 1.051 & 1.178 & 0.465 & 0.505 & 0.250 & 0.301 & 0.208 & 0.093 \\
\hline & 0.98 & 3.422 & 3.669 & 1.051 & 0.993 & 0.460 & 0.348 & 0.146 & 0.091 \\
\hline & 0.99 & 5.502 & 5.936 & 1.657 & 1.347 & 0.730 & 0.455 & 0.214 & 0.157 \\
\hline & 0.999 & 24.892 & 30.109 & 7.056 & 4.459 & 2.436 & 1.489 & 0.431 & 0.373 \\
\hline \multirow[t]{4}{*}{50} & 0.9 & 0.776 & 0.829 & 0.353 & 0.382 & 0.186 & 0.229 & 0.192 & 0.111 \\
\hline & 0.98 & 2.572 & 2.850 & 0.753 & 0.763 & 0.346 & 0.252 & 0.127 & 0.063 \\
\hline & 0.99 & 4.088 & 4.681 & 1.219 & 1.087 & 0.583 & 0.353 & 0.186 & 0.115 \\
\hline & 0.999 & 16.568 & 23.406 & 5.321 & 4.072 & 1.965 & 1.253 & 0.351 & 0.264 \\
\hline \multirow[t]{4}{*}{100} & 0.9 & 0.566 & 0.586 & 0.271 & 0.284 & 0.145 & 0.177 & 0.184 & 0.121 \\
\hline & 0.98 & 1.884 & 2.108 & 0.547 & 0.569 & 0.273 & 0.184 & 0.119 & 0.056 \\
\hline & 0.99 & 2.989 & 3.461 & 0.939 & 0.874 & 0.496 & 0.303 & 0.174 & 0.103 \\
\hline & 0.999 & 11.563 & 15.713 & 4.377 & 3.702 & 1.723 & 1.178 & 0.318 & 0.229 \\
\hline
\end{tabular}

$(h=0$ and $k \neq 0)$, generalized Pareto (GP) LMOM, and WK5 method. The WK5 almost

$(h=1$ and $k \neq 0)$, Gumbel (EV1) always perform better than LMOM except when

( $h=0$ and $k=0)$, uniform (U) $(h=1$ and $k=1)$ and exponential (EXP) $(h=0$ and $k=1)$ distributions (Sing et al, 2002).

In order to evaluate the performance of the four-parameter estimation methods for GEV distribution, different parameters of kappa distribution were considered for simulation with values of the shape parameter $(h, k)$ were set $(-1,-0.3)$ for $\mathrm{GL},(1,0.3)$ for $\mathrm{GP},(1,1)$ for $\mathrm{U}$ and $(0,1)$ for EXP distribution. The location, $\varsigma$ and scale, $\sigma$ parameters were set 0 and 1 , respectively. For this purpose, 10000 random samples of $n=15,25,50$, and 100 are used. The performance of the LQ-moments using WK5 are only considered to compare with LMOM because the LIQ estimator always has lower efficiency in comparison to the other estimators.

Table 2 shows the RMSE of the $F=0.9$, $0.98,0.99$, and 0.999 quantile estimators for the data are generated by the GL distribution for $n>15$.

Figure 3 shows the BIAS of $Q(F), F=$ 0.99 estimators for $n=25$ and 100 . The results are quite similar. In term of BIAS the WK5 method is clearly superior to the LMOM method except when the data are from the GL distribution for $n=25$.

Data Analysis

To illustrate the use of the GEV distribution for fitting data sets by various methods (LMOM, LQ moments using LIQ, and WK5), two sets of annual maximum flood series for the Feather River at Oroville and the Blackstone River at Woonsocket, were taken from Mudholkar and Hutson (1998). The parameter estimates for each data set, using various methods, are given in Table 3 . Observed and computed frequency curves for the two data sets are plotted in Figure 4. The observed data values are plotted against the corresponding 
EV1 reduced variates using the Cunnane plotting position.
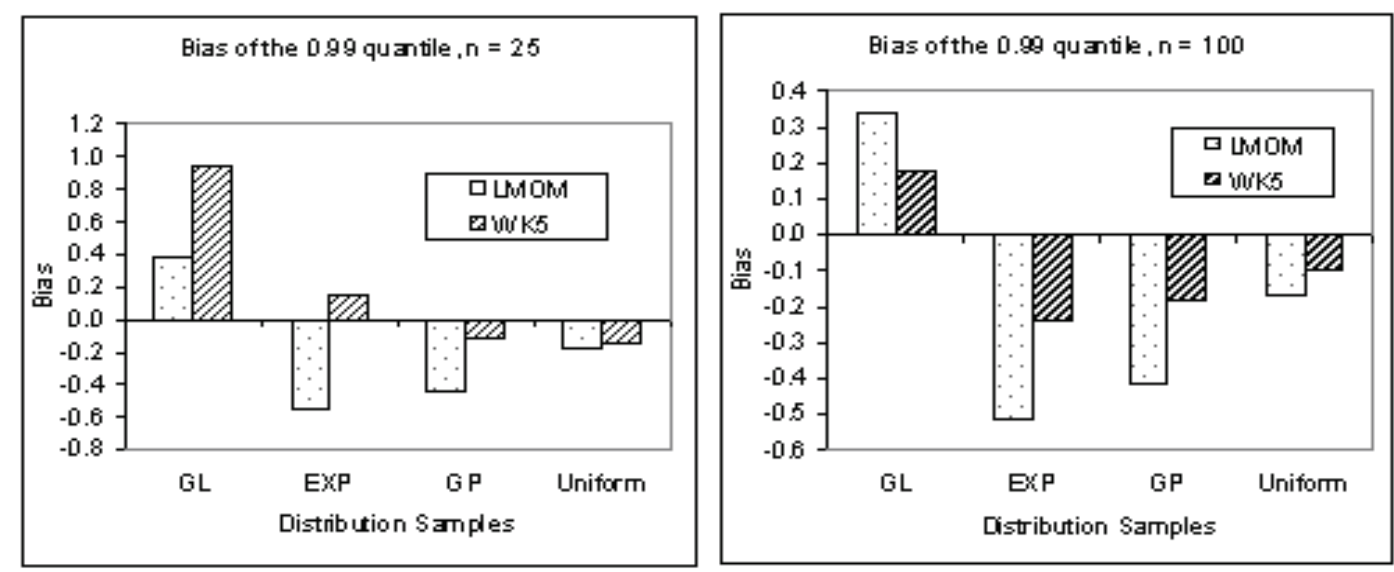

Figure 3: Bias of $\mathrm{Q}(\mathrm{F}=0.99)$ Estimator Using L-Moments and LQ-Moment Based On WK5, Fitting the GEV Distribution to Generated Kappa Samples For $n=25$ and $n=100$

Table 3: Estimated Values for the GEV Distribution

(a) Blackstone River Data

\begin{tabular}{llll}
\hline Parameter & L Moments & \multicolumn{2}{l}{ LQ Moment Method } \\
\cline { 2 - 4 } & Method & LIQ & WK5 \\
\hline$\mu$ & 4257.0 & 4495.0 & 4064.1 \\
$\sigma$ & 1443.2 & 1213.4 & 1955.1 \\
$k$ & -0.479 & -0.468 & -0.359 \\
10 year flood $\left(\mathrm{ft}^{3} / \mathrm{s}\right)$ & 10096.0 & 9335.6 & 10833.7 \\
50 year flood & 20764.5 & 18006.5 & 20717.1 \\
100 year flood & 28153.6 & 24232.2 & 27011.1 \\
1000 year flood & 83546.4 & 67657.9 & 63607.2 \\
\hline
\end{tabular}

(b) Feather River Data

\begin{tabular}{llll}
\hline Parameter & $\begin{array}{l}\text { L Moments } \\
\text { Method }\end{array}$ & LQ Moment Method \\
\cline { 3 - 4 } & 44893.6 & 43537.8 & WK5 \\
\hline$\mu$ & 37335.8 & 40146.3 & 46385.7 \\
$\sigma$ & -0.094 & -0.119 & 34804.1 \\
$k$ & 138501.2 & 147176.7 & -0.093 \\
10 year flood $\left(\mathrm{ft}^{3} / \mathrm{s}\right)$ & 221017.6 & 243047.3 & 146897.9 \\
50 year flood & 259959.9 & 289615.9 & 235293.5 \\
100 year flood & 408508.6 & 474246.2 & 276951.9 \\
1000 year flood & & & 435565.3 \\
\hline
\end{tabular}


similarly to the L-moments. Results from fitting the GEV distribution function to generated GEV samples show that LQ-moments using WK5
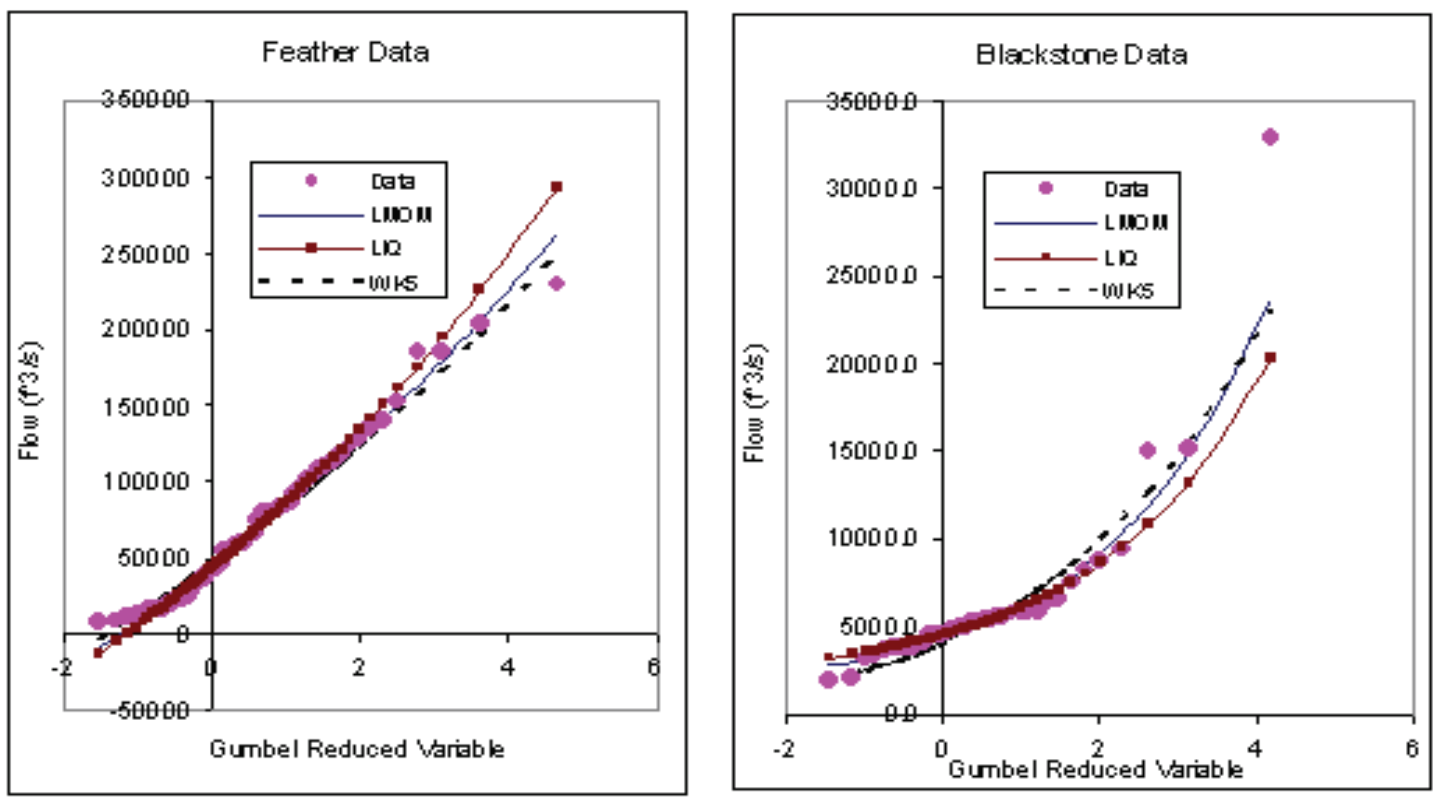

Figure 4: Fitting the GEV Distribution To Annual Maximum Flows At Blackstone River And Feather River.

For Feather River data, the frequency curves obtained by the WK5 lie much closer to the data than LMOM and LIQ methods. For the Blackstone River data, the frequency curves of the WK5 and LMOM methods are steeper than those of LIQ method, however the fitting of these methods are in serious error, especially for the larger flows.

\section{Conclusion}

The LQ-moments are constructed by using a function that defines the quick estimators, such as the median, trimean or Gastwirth, in places of expectations in L-moments have are reexamined. The quick estimators based on five points quantiles using weighted kernel estimators are introduced for characterizing the upper quantiles of distributions and larger events in a sample. The parameters of the GEV distribution are estimated by matching LQmoments to their sample estimates behave almost always perform better than L-moments but has more BIAS than L-moments method. Results from fitting the GEV distribution function to samples generated from the Kappa distribution show that the WK5 lead to reduced BIAS and in many cases, higher efficiency compared to the other methods. The LIQ estimator leads to poorer estimation of high quantiles in terms of BIAS and RMSE.

This study has demonstrated that the conventional L-moment is not optimal for the estimation of GEV distribution. The new method of estimation, denoted the LQ-moments based on WK5 method, in many cases represents higher efficiency in high quantile estimation compared the L-moments method. The simplicity and generally good performance of this method make it an attractive option for estimating quantiles in the GEV distribution. Although the linear interpolation quantile estimator commonly used in most statistical software packages and in the LQ-moments 
method, but it does not perform as good as the WK5 in estimating the parameters of the GEV distribution.

\section{References}

Chowdhury, J. U., Stedinger, J. R., Lu, L. H. (1991). Goodness of fit tests for regional generalized extreme value flood distribution. Water Resources Research. 27(7), 1765-1776.

David, H. A. \& Nagaraja, H. N. (2003). Order Statistic. New York: Wiley.

Hosking, J. R. M. (1990). L-moments: Analysis and Estimation of Distribution Using Liner Combinations of Order Statistics. Journal of the Royal Statistical Society, Series B. 52, 105-124.

Hosking, J. R. M. \& Wallis, J. R. (1993). Some statistics useful in regional frequency analysis. Water Resources Research. 29(2), 271-281.

Hosking, J. R. M. \& Wallis, J. R. (1997). Regional Frequency Analysis: An approach based on L-Moments Cambridge: Cambridge University Press.

Hosking, J. R. M., Wallis, J. R., \& Wood, E. F. (1985a). Estimation of the generalized extreme-value distribution by the method of probability weighted moments. Technometrics, 27(3), 251-261.

Huang, M. L., Brill, P. (1999). A Level Crossing Quantile Estimation Method. Statistics \& Probability Letters, 45, 111-119.

Huang, M. L. (2001). On a Distribution-

Free Quantile Estimator. Computational Statistics \& Data Analysis, 37, 477-486.

Karvane, J. (2006). Estimation of quantile mixtures via L-moments and trimmed L-moments. Computational Statistics \& Data Analysis, 51, 947-959.
Martins, E. S., Stedinger, J. R. (2000). Generalized Maximum-Likelihood Generalized Extreme-Value Quantile Estimators for Hydrologic Data. Water Resources Research, 36(3), 737-744.

Mudholkar, G. S. \& Hutson, A. D. (1998). LQ moments: Analogs of L-Moments. Journal of Statistical Planning and Inference, 71, 191-208.

Park, J. S. (2006). A Simulation-Based Hyperparameter Selection for Quantile Estimation of the Generalized Extreme Value Distribution. Mathematics and Computers in Simulation, 70(4), 366-376.

Park, J. S. \& Park, B. J. (2002). Maximum Likelihood Estimation of the FourParameter Kappa Distribution Using The Penalty Method. Computers and Geosciences, 28, 65-68.

Sankarasubramanian, A. \& Srinivasan K. (1999). Investigation and Comparison of Sampling Properties of L-Moments and Conventional Moments. Journal of Hydrology, 218, 13-24.

Sheather, S. J. \& Marron, J. S. (1990). Kernel Quantile Estimators. Journal of the American Statistical Association, 85, 410-416.

Vogel, R. M. \& Fennessey, N. M. (1993). L-Moment diagrams should replace product moment diagrams. Water Resources Research, 29(6), 1745-1752.

Wang, Q. J. (1997). LH moments for statistical analysis of extreme events. Water Resources Research, 33(12), 2841-2848. 


\section{A Spline-Based Lack-Of-Fit Test for Independent Variable Effect in Poisson Regression}

\author{
Chin-Shang Li \\ St. Jude Children's Research Hospital
}

\author{
Wanzhu Tu \\ Indiana University School of Medicine
}

In regression analysis of count data, independent variables are often modeled by their linear effects under the assumption of log-linearity. In reality, the validity of such an assumption is rarely tested, and its use is at times unjustifiable. A lack-of-fit test is proposed for the adequacy of a postulated functional form of an independent variable within the framework of semiparametric Poisson regression models based on penalized splines. It offers added flexibility in accommodating the potentially non-loglinear effect of the independent variable. A likelihood ratio test is constructed for the adequacy of the postulated parametric form, for example log-linearity, of the independent variable effect. Simulations indicate that the proposed model performs well, and misspecified parametric model has much reduced power. An example is given.

Key words: B-splines, likelihood ratio test, loglinear model, penalized likelihood, Poisson regression model.

Introduction

The Poisson regression model is among the most frequently used statistical tools in event count analysis. It has been successfully used in numerous applications (e.g. McCullagh \& Nelder, 1989; Cameron \& Trivedi, 1998). In its parametric form, the model is constructed as follows: Let $Y_{i}$ be the number of occurrences of an event of interest for the $i$ th subject and $x_{i}$ be

Chin-Shang $\mathrm{Li}$ is an Associate Member in the Department of Biostatistics at St. Jude Children's Research Hospital, Memphis, Tennessee. Email him at chinshang.li@stjude.org. Wanzhu Tu is an Associate Professor in the Division of Biostatistics at Indiana University School of Medicine, and a research scientist at Indiana University Center for Aging Research and Regenstrief Institute, Indianapolis, Indiana. Email him at wtu1@iupui.edu. This research was supported in part by grants R01 HD042404, HL69399 (W. Tu), and CA21765 (C.S. Li) from the National Institutes of Health and by the American Lebanese Syrian Associated Charities (C.S. Li). an independent variable, $i=1 \ldots, n$. Assuming that $Y_{i}$ follows a Poisson distribution, i.e., $Y_{i} \sim \operatorname{Poisson}\left(\mu\left(x_{i}\right)\right)$ with mean $\mu\left(x_{i}\right)$, write the Poisson density as follows:

$$
f\left(y_{i} \mid x_{i}\right)=\frac{\exp \left(-\mu\left(x_{i}\right)\right) \mu\left(x_{i}\right)^{y_{i}}}{y_{i} !}
$$

where $\mu\left(x_{i}\right)=E\left[Y_{i} \mid x_{i}\right]$ is the mean function, $i=1 \ldots, n$.

Under this formulation, the model depicts the dependency of the event counts on $x_{i}$ via a logarithmic link function,

$$
\log \left(\mu\left(x_{i}\right)\right)=h\left(x_{i}, \boldsymbol{\beta}\right)
$$

where $h(; ; \boldsymbol{\beta})$ is a known functional form apart from the parameter vector $\boldsymbol{\beta}$. In other words, the model assumes a log parametric form for the independent variable effect. Although the theory does not restrict $h(\cdot ; \boldsymbol{\beta})$ to a linear form, in practice, however, most analysts choose to use $h(x ; \boldsymbol{\beta})=\beta_{0}+\beta_{1} x$, which is often referred to as the loglinear model. Despite its popularity, the 
validity of this postulated loglinear form is rarely verified, possibly due to the lack of readily accessible testing procedures. In addition, the consequences of a misspecified functional form of the independent variable are not well studied.

An alternative approach is to replace the linear predictor $h(\cdot ; \boldsymbol{\beta})$ with a regression spline. This approach is semiparametric in nature because it has not only a parametric component for the data distribution (Poisson in the case of counts), but also a nonparametric component involving the predictor (e.g. Ruppert et al., 2003). Such an approach is known to enhance the modeling flexibility in regression analysis. Regression spline techniques have been used frequently to estimate independent variable effects in generalized linear models (e.g., Eilers \& Marx, 1996). But testing procedures based on regression splines have attracted considerably less attention.

The purpose of this article is to construct a general test for the inference concerning the adequacy of a given functional form of the independent variable effect in count data analysis. The proposed test contributes to the existing literature of count data analysis by providing a practical way for the determination of functional forms of independent variables.

Example

Patients with chronic diseases, such as congestive heart failure (CHF), must take medications regularly to prevent disease exacerbation that requires costly health care services such as emergency department (ED) visits and hospitalization admissions. In a study on medication adherence in older adults with $\mathrm{CHF}$, participants were monitored for their medication use during a 1-year study period. Eligible participants were English-speaking, 50 years of age or older, had a diagnosis of CHF, and were currently prescribed for at least one cardiovascular medication, including angiotensin-converting enzyme inhibitors, angiotensin II-receptor antagonists, $\beta$-adrenergic receptor antagonists, digoxin, loop and nonloop diuretics, and an aldosterone antagonist. Upon enrollment, participants were provided electronic medication container lids for their cardiovascular medications. The electronic container lids automatically recorded the dates and times of the lid openings (Tu et al., 2005). Assuming that the patient took the prescribed amount of medication each time the lid was opened, patient's medication adherence to the prescribed drug was calculated as the percentage of dose taken during the observation period according to the prescribed regimen.

For example, if 30 openings were recorded during a 1-month period for a b.i.d. (twice a day) drug, the medication adherence was $r=30 / 60=50 \%$, meaning the patient took only half of the medicine that he was supposed to take. Although $100 \%$ is the target level for medication adherence, values that are significantly less or more than $100 \%$ would represent suboptimal medication-taking behavior on the part of the patient. Therefore, in pharmacy practice, researchers often calculate the patient's deviation in medication consumption from the target level $(|1-r|)$ and report an adjusted adherence $\mathrm{x}=1-|1-r|$ as a percentage between 0 and $100 \%$. For a more detailed discussion (see Hope et al., (2004).

When a patient was on multiple cardiovascular drugs, his overall adjusted medication adherence was summarized as the average level of the adjusted medication adherence values for all of the study drugs. An important issue of this study is to understand the relationship between adjusted medication adherence and disease exacerbation. Herein, the number of ED visits during the 1-year follow-up period is used as the primary outcome of interest.

For the purpose of illustration, consider a subset of the study data: 93 subjects who belong to the New York Heart Association (NYHA) Class III. The NYHA classification is one of the most commonly used clinical classification systems for patients with heart failure. Typical NYHA Class III patients experience a marked limitation of physical activities, such as walking one to two blocks on the level or climbing more than one flight of stairs under normal conditions. Patients are comfortable at rest, but more than usual physical activity causes fatigue, palpitation, dyspnea, anginal pain, or a combination thereof. 


\section{Methodology}

Semiparametric Poisson Model

Using the previously introduced notation, we write the response variables as $Y_{i} \sim \operatorname{Poisson}\left(\mu\left(x_{i}\right)\right)$ for $i=1 \ldots, n$. To model the effect of the independent variable $x_{i}$ on the response variable, link the mean function $\mu\left(x_{i}\right)$ to $x_{i}$ via $g\left(\mu\left(x_{i}\right)\right)=h\left(x_{i}\right)$, where $g(\cdot)$ is a monotone differentiable link function, and $h(\cdot)$ is the predictor function. To differentiate the proposed model from the traditional loglinear predictor, write $g\left(\mu\left(x_{i}\right)\right)=s\left(x_{i}\right)$, where $s\left(x_{i}\right)$ is a smooth function to be estimated from the data. Under the commonly used log-link function, there is a semiparametric Poisson regression model:

$$
g(\mu(x))=\log (\mu(x))=s(x) .
$$

Equivalently, write $\mu(x)=\exp (s(x))$. Because the B-spline basis is numerically more stable for the representation of a spline function than the truncated-power basis is, to approximate the unknown function $s(\cdot)$, parameterize it with the cubic B-splines as

$$
s(x)=\sum_{k=1}^{K} \theta_{k} B_{k}(x)
$$

where $B_{k}$ are the cubic B-spline basis functions for $s ; \theta_{k}$ are spline coefficients; and $K=q+4$, where $q$ is the number of knots; see de Boor (1978) for details of computation of B-splines of any degree from B-splines of a lower degree. These knots were chosen to be equally spaced with respect to the quantiles of the distinct values of $x_{i}$ and set $q=\min [($ number of distinct values of $x / 6), 30]$, where $[a]$ is the greatest integer less than or equal to $a$; for reference on the selection of $q$, (see Ruppert, 2002). Let $\mathbf{B}_{x_{i}}=\left(B_{1}\left(x_{i}\right), \ldots, B_{K}\left(x_{i}\right)\right)^{T}$ and $\boldsymbol{\theta}=\left(\theta_{1}, \ldots, \theta_{K}\right)^{T}$ and expressing $s$ in vector notation as $s\left(x_{i}\right)=\mathbf{B}_{x_{i}}^{T} \boldsymbol{\theta}$, rewrite the Poisson density in $(1.1)$ as

$$
f\left(y_{i} \mid x_{i}\right)=\frac{\exp \left[-\exp \left(\mathbf{B}_{x_{i}}^{T} \boldsymbol{\theta}\right)\right] \exp \left(\mathbf{B}_{x_{i}}^{T} \boldsymbol{\theta}\right)^{y_{i}}}{y_{i} !}
$$

Parameter Estimation

To estimate $\boldsymbol{\theta}$ and prevent overfitting, employ a penalized likelihood approach with a discrete approximation to the integrated squared second derivative of $s, \int s^{\prime \prime}(x)^{2} d x$, which is used as a measure of its roughness. Therefore, the penalized log-likelihood $\ell(\boldsymbol{\theta})-(1 / 2) \int s^{\prime \prime}(x)^{2} d x$ is approximated by

$$
\ell_{p e n}(\boldsymbol{\theta})=\ell(\boldsymbol{\theta})-\frac{1}{2} \lambda \boldsymbol{\theta}^{T} \mathbf{K} \boldsymbol{\theta},
$$

where the log-likelihood $\ell(\boldsymbol{\theta})$ is

$$
\ell(\boldsymbol{\theta})=\sum_{i=1}^{n}\left\{y_{i} \mathbf{B}_{x_{i}}^{T} \boldsymbol{\theta}-\exp \left(\mathbf{B}_{x_{i}}^{T} \boldsymbol{\theta}\right)-\log y_{i} !\right\} .
$$

$\lambda$ is a smoothing parameter to be chosen. It is used to govern the tradeoff between goodness-of-fit and smoothness; $\boldsymbol{\theta}^{T} \mathbf{K} \boldsymbol{\theta}=\sum_{k=1}^{K}\left(\Delta^{2} \theta_{k}\right)^{2}$ for $\Delta^{2} \theta_{k}=\theta_{k}-2 \theta_{k-1}+\theta_{k-2}$ and $\quad \mathbf{K}=\mathbf{D}_{2}^{T} \mathbf{D}_{2}$ for $\mathbf{D}_{2}$ being the matrix representation of the difference operator $\Delta^{2}$. For details of similar operation, see Eilers \& Marx (1996). To estimate $\boldsymbol{\theta}$, set the first-order partial derivatives of the penalized log-likelihood in (3.4) with respect to $\boldsymbol{\theta}$ equal to $\mathbf{0}$, $q(\boldsymbol{\theta})=\partial \ell_{p e n}(\boldsymbol{\theta}) / \partial \boldsymbol{\theta}=0$. Then, solve iteratively the following weighted least-squares equations in matrix notation:

$$
(\mathbf{B W B}+\lambda \mathbf{K}) \boldsymbol{\theta}=\mathbf{B}^{T} \mathbf{W y}^{*},
$$


where

$$
\begin{gathered}
\mathbf{B}=\left(\mathbf{B}_{x_{i}}^{T}, \ldots, \mathbf{B}_{x_{i}}^{T}\right)^{T}, \\
\mathbf{W}=\operatorname{diag}\left(\exp \left(\mathbf{B}_{\mathrm{x}_{1}}^{\mathrm{T}} \boldsymbol{\theta}\right), \ldots, \exp \left(\mathbf{B}_{\mathrm{x}_{\mathrm{n}}}^{\mathrm{T}} \boldsymbol{\theta}\right)\right),
\end{gathered}
$$

and

$$
\mathbf{y}^{*}=\left(y_{1}^{*}, \ldots, y_{n}^{*}\right)^{T}
$$

is the working response vector for

$$
\mathrm{y}_{\mathrm{i}}^{*}=\frac{\mathrm{y}_{\mathrm{i}}-\exp \left(\mathbf{B}_{\mathrm{x}_{\mathrm{i}}^{\mathrm{T}}}^{\mathrm{T}} \boldsymbol{\theta}\right)}{\exp \left(\mathbf{B}_{\mathrm{x}_{\mathrm{i}}^{\mathrm{T}}}^{\mathrm{\theta}}\right)}+\mathbf{B}_{\mathrm{x}_{\mathrm{i}}}^{\mathrm{T}} \boldsymbol{\theta} .
$$

The concept of the effective number of degrees of freedom (Edf) is used to choose the value of the smoothing parameter $\lambda, \operatorname{Edf}=\operatorname{tr}\left(\mathbf{S}_{\lambda}\right)-1$, where

$$
\mathbf{S}_{\lambda}=\mathbf{B}(\mathbf{B W B}+\lambda \mathbf{K})^{-1} \mathbf{B}^{T} \mathbf{W}
$$

is called the smoother matrix (see Hastie \& Tibshirani, 1990).

The solution to (3.5), denoted by $\hat{\boldsymbol{\theta}}_{\text {pen }}$, is called a maximum penalized likelihood estimate of $\boldsymbol{\theta}$. The cubic B-spline fitted mean function is $\hat{\mu}_{\mathrm{B}}=\exp \left(\hat{s}_{B}(x)\right)=\exp \left(\mathbf{B}_{\mathrm{x}}^{\mathrm{T}} \hat{\boldsymbol{\theta}}_{p e n}\right)$.

Inference

Let $\mathbf{Q}(\boldsymbol{\theta})=\partial^{2} \ell_{p e n}(\boldsymbol{\theta}) / \partial \boldsymbol{\theta} \partial \boldsymbol{\theta}^{T}$ be the second-order partial derivatives of the penalized $\log$-likelihood in (3.4) with respect to $\boldsymbol{\theta}$. Then, approximate the covariance matrix of $\hat{\boldsymbol{\theta}}_{\text {pen }}$ as $\operatorname{cov}\left(\hat{\boldsymbol{\theta}}_{\text {pen }}\right) \approx\{-\mathbf{Q}(\boldsymbol{\theta})\}^{-1} \operatorname{var}\{\mathbf{q}(\boldsymbol{\theta})\}\{-\mathbf{Q}(\boldsymbol{\theta})\}^{-1}$ and, hence, estimate it as follows:

$$
\operatorname{cov}\left(\hat{\boldsymbol{\theta}}_{p e n}\right) \approx\{-\mathbf{Q}(\hat{\boldsymbol{\theta}})\}^{-1}
$$

From (3.6), obtain a confidence interval for $s(x)$ by computing the estimated variance of $\hat{s}_{B}(x)$ as follows:

$$
\operatorname{vâr}\left(\hat{s}_{B}(x)\right)=\mathbf{B}_{x}^{T} \operatorname{cov}\left\{\hat{\boldsymbol{\theta}}_{p e n}\right\} \mathbf{B}_{x}
$$

where

$$
\hat{s}_{B}(x)=\mathbf{B}_{x}^{T} \hat{\boldsymbol{\theta}}_{p e n} .
$$

Accordingly, a confidence interval for $\exp (s(x))$ can be obtained by using the delta method and the estimated variance of $\hat{s}_{B}(x)$.

The proposed estimation method is used with deviance, or log-likelihood ratio statistic, to assess the adequacy of a postulated parametric form of the model in (1.2), i.e., testing the following parametric null hypothesis:

$$
\mathrm{H}_{0}: \log (\mu(\cdot))=h(\cdot ; \boldsymbol{\beta})
$$

Then, test for the lack of fit of the postulated parametric model in (3.7) by using the log-likelihood ratio test (LRT) statistic

$$
-2 \log \frac{\sup \prod_{i=1}^{n} \exp \left\{-\exp \left[h\left(x_{i} ; \boldsymbol{\beta}\right)\right]\right\} \exp \left[y_{i} h\left(x_{i} ; \boldsymbol{\beta}\right)\right]}{\prod_{i=1}^{n} \exp \left\{-\exp \left[\mathbf{B}_{x_{i}}^{T} \hat{\boldsymbol{\beta}}_{p e n}\right]\right\} \exp \left[y_{i} \mathbf{B}_{x_{i}}^{T} \hat{\boldsymbol{\beta}}_{p e n}\right]}
$$

and comparing its value to its asymptotic limiting chi-square distribution with $D$ degrees of freedom, where $D=$ Edf - the number of the postulated parametric model parameters +1 (see Hastie \& Tibshirani, 1990). This is called a smoothing log-likelihood ratio test (SLRT). By comparing the likelihood of the postulated parametric model $h(\cdot ; \boldsymbol{\beta})$ with that of a B-spline model, the SLRT allows for inference on the adequacy of the postulated model. For example, the SLRT can be used to assess the appropriateness of the loglinear effect of $x$ by testing

$$
\mathrm{H}_{0}: \log (\mu(x))=\beta_{0}+\beta_{1} x
$$

versus

$$
\mathrm{H}_{a}: \log (\mu(x))=s(x)
$$


where $s(x)$ is a general nonlinear smooth function.

\section{Simulation Study}

A Monte Carlo simulation study is conducted to assess the performance of the SLRT. The values of the independent variable are equally spaced design points $x_{i}=(2 i-1) / 2 n, i=1, \ldots, n=100 ; 1000$ data sets were generated for each configuration of the experiments. The goal is to test the adequacy of the following loglinear model:

$$
\mathrm{H}_{0}: \log (\mu(x ; \boldsymbol{\beta}))=\beta_{0}+\beta_{1} x .
$$

To assess the empirical power of the SLRT, data were generated from the model in (1.1) with the following logarithmic mean functions:

$$
\log (\mu(x ; \boldsymbol{\beta}, \gamma))=\beta_{0}+\beta_{1} x+\gamma \sin (3 \pi x)
$$

where

$$
\boldsymbol{\beta}=\left(\beta_{0}, \beta_{1}\right)=(1.159,-0.675),
$$

and
Note that $\gamma=0$ corresponds to the null hypothesis. In this simulation study, the $\boldsymbol{\beta}$ values were chosen to reflect the estimated coefficient values of the example data, and the value of the smoothing parameter $\lambda$ was chosen by fixing $\operatorname{Edf}=\operatorname{tr}\left(\mathbf{S}_{\lambda}\right)-1=4$ while estimating the cubic B-spline coefficients. The null model is rejected if the observed value of the SLRT statistic in (3.8) with $h(x ; \boldsymbol{\beta})=,\beta_{0}+\beta_{1} x$, exceeds the 0.95 -quantile of the chi-square distribution with $D=3$ degrees of freedom. This is called an asymptotic smoothing log-likelihood ratio test (ASLRT).

An obvious variant of the ASLRT is to approximate the 0.95-quantile of the distribution of the SLRT statistic via 200 Monte Carlo simulations for each sample and reject the null model if the observed value of the SLRT statistic exceeds the approximated 0.95-quantile. This test is called the Monte Carlo smoothing log-likelihood ratio test (MSLRT).

For the purpose of comparative evaluation, it is important to establish a benchmark for the power of the inference when the true model is known. To do so, consider the following parametric likelihood ratio test for $\gamma=0$ under the assumption that the true model is known:

$$
\gamma=0,0.05,0.1, \ldots, 0.5
$$

$$
-2 \log \frac{\sup \prod_{i=1}^{n} \exp \left\{-\exp \left[\beta_{0}+\beta_{1} x_{i}\right]\right\} \exp \left[y_{i}\left(\beta_{0}+\beta_{1} x_{i}\right)\right]}{\prod_{i=1}^{n} \exp \left\{-\exp \left[a_{0}+a_{1} x_{i}+a_{2} x_{i}^{2}\right]\right\} \exp \left[y_{i}\left(a_{0}+a_{1} x_{i}+a_{2} x_{i}^{2}\right)\right]} .
$$


Because this test directly compares the likelihood of the postulated model (4.1) with the true parametric model (4.2) which generates the data, the parametric test in (4.3) is referred to as an exactly specified parametric log-likelihood ratio test (ESPLRT). The null model is rejected if the observed value of the LRT statistic in (4.3) was greater than the 0.95 -quantile of the chisquare distribution with one degree of freedom. When the null model is appropriate, the exactly specified test ESPLRT in (4.3) follows an asymptotic chi-square distribution with degree of freedom and should have the best power among all competitors. This asymptotically optimal test, however, provides only a benchmark for the power comparison and is of limited practical values, because its use requires specific knowledge of the form of the true model.

In practical data analysis, when there are clear indications that an independent variable effect is not linear, data analysts often attempt to alleviate the lack-of-fit by including a quadratic term. To assess the potential power loss associated with such practice when a wrong model is used, consider a misspecified quadratic parametric model, $\log (\mu(x))=a_{0}+a_{1} x+a_{2} x^{2}$, and use it to construct a parametric likelihood ratio test:

Because the misspecified parametric model $\log (\mu(x))=a_{0}+a_{1} x+a_{2} x^{2}$ is used to fit data generated under the true model $\log (\mu(x ; \boldsymbol{\beta}, \gamma))=\beta_{0}+\beta_{1} x+\gamma \sin (3 \pi x)$, in (4.2), this parametric test is referred to as a misspecified parametric likelihood ratio test (MSPLRT). By comparing the empirical power of the MSPLRT with that of the benchmark test, ESPLRT, and other competitors, it is possible to assess the power loss when a misspecified quadratic model is used in data analysis.

The empirical powers of the four candidate tests, ESPLRT, MSPLRT, ASLRT, and MSLRT, are depicted in Figure 1. While the empirical significance level of the ASLRT tends to be slightly higher than the nominal level 0.05 , its power is the closest to that of the exactly specified parametric likelihood ratio test (ESPLRT), the benchmark test, among all three other candidates. A close second is the Monte Carlo version of the smoothing likelihood ratio test, MSLRT. On the other hand, the misspecified parametric likelihood ratio test MSPLRT suffers a severe loss of power, highlighting the consequences of making inference under misspecified parametric regression models.

Example Data Analysis

The practical use of the proposed semiparametric Poisson regression model and lack-of-fit tests is illustrated by examining the effect of the adjusted medication adherence on ED utilization in a study of 93 NYHA Class III patients. Consider the number of all-cause ED visits as the response variable. All-cause ED visits include ED admissions for any reason. The use of all-cause ED visits in this analysis is justified, because acute exacerbation in patients with heart failure does not always occur in the form of CHF, coronary artery disease, or cardiovascular diseases. Sometimes, it results in complications in other organs, which would be recorded as noncardiovascular-related conditions in the medical records. The adjusted medication adherence to all prescribed cardiovascular medications is the independent variable of interest. Therefore, do not restrict the effect of the overall adjusted medication adherence $(x)$ to be loglinear. Instead, use the proposed inference procedure to test for the loglinear effect of the independent variable $\mathrm{x}$ on the number of $\mathrm{ED}$ visits. That is, test the following hypotheses:

$$
H_{0}: \log (\mu(x))=\beta_{0}+\beta_{1} x \text {, }
$$

versus

$$
H_{a}: \log (\mu(x))=s(x)
$$

In this example, $\mathrm{Edf}=\operatorname{tr}\left(\mathbf{S}_{\lambda}\right)-1=4$ is fixed to choose the value of $\lambda$ for the estimation of cubic B-spline coefficients. By using the smoothing log-likelihood ratio test (SLRT) statistic with an asymptotic chi-square distribution with 3 degrees of freedom, it is found that that the independent variable has a significantly non-loglinear effect on the number of ED visits (ASLRT p-value $=0.029$ ).

The distribution of the SLRT statistic is approximated through 200 Monte Carlo simulations. The p-value from the Monte Carlo based test MSLRT is 0.015 , which is the 


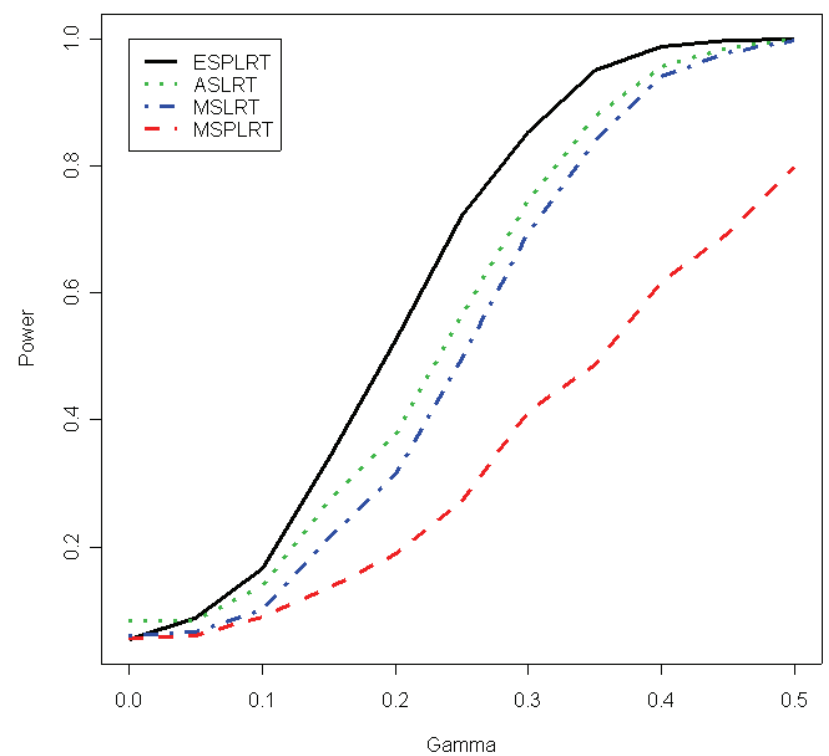

Figure 1: Empirical power curve comparison of the ESPLRT, ASLRT, MSLRT, and MSPLRT for the null model $\mathrm{H}_{0}: \log (\mu(x ; \boldsymbol{\beta}))=\beta_{0}+\beta_{1} x \quad$ and alternative model $\log (\mu(x ; \boldsymbol{\beta}, \gamma))=\beta_{0}+\beta_{1} x+\gamma \sin (3 \pi x)$, where $\boldsymbol{\beta}=\left(\beta_{0}, \beta_{1}\right)^{T}=(1.159,-0.675)^{T}$. Abbreviations: ASLRT, asymptotic smoothing log-likelihood ratio test; ESPLRT, the exactly specified parametric log-likelihood ratio test; MSLRT, Monte Carlo smoothing log-likelihood ratio test; MSPLRT, misspecified parametric log-likelihood ratio test.

proportion of simulated values of the test statistic exceeding the observed value of the SLRT statistic 9.0033. Therefore, the SLRT based on the Monte Carlo simulations again confirmed the lack of $\log$ linearity in the medication adherence effect. Figure 2 shows the cubic B-spline fitted mean function $\mu(x)$ and $95 \%$ point-wise confidence interval for the mean function and the parametrically fitted mean function $\hat{\mu}(x)=\exp (1.159-0.675 x)$; Figure 3 further confirms that the cubic B-spline fitted function and $95 \%$ point-wise confidence interval for the functional form of the effect of $x$ and the parametrically fitted function $1.159-0.675 x$.

From the perspective of pharmacotherapy, the lack of log linearity in the effect of medication adherence is perhaps not entirely surprising: underconsumption of cardiovascular drugs often leads to decompensation in patients with $\mathrm{CHF}$, and overdosing can cause dangerous hypotension. Both are likely to result in increased ED use. Because the adherence data are in the adjusted form $\quad(x=100(1-|1-r|) \%) \quad$ where overconsumption of the medication was converted to a percentage less than $100 \%$, a deviation from the target level $(100 \%)$ could be the results of overconsumption as well as underconsumption. Figures 2 and 3 showed an increase in ED admission when adjusted medication adherence around 0.8 , possibly caused by the folding of the raw adherence measure. Therefore, the loglinear relationship forced by the parametric Poisson regression model would not be adequate and the proposed semiparametric model would provide a relief in such a data situation. 


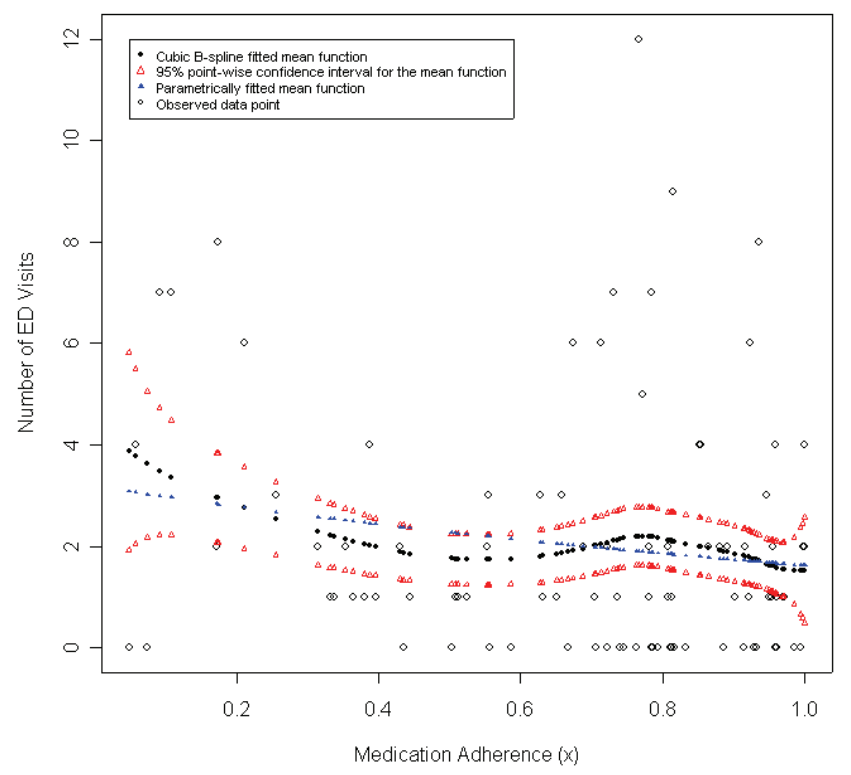

Figure 2: Cubic B-spline fitted mean function $\hat{\mu}_{B}(x)$ and $95 \%$ point-wise confidence interval for the mean function $\mu(x)$ of medication adherence $(x)$ and parametrically fitted mean function $\hat{\mu}(x)=\exp (1.159-0.675 x)$ for $\mu(x)$.

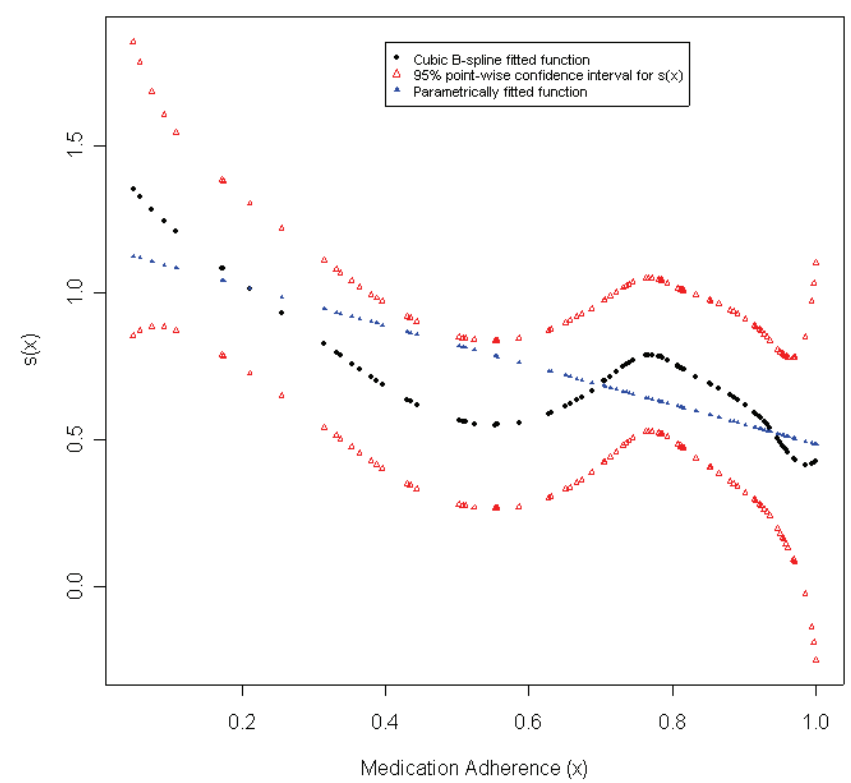

Figure 3: Cubic B-spline fitted function $\hat{s}_{B}(x)$ and $95 \%$ point-wise confidence interval for the functional form $s(x)$ of the effect of medication adherence $(x)$ and parametrically fitted function $1.159-0.675 x$ for the effect of $x$. 


\section{Conclusion}

Cubic B-spline basis functions can be used in Poisson regression analysis in a flexible manner, without imposing any particular functional form on the effects of independent variables. An easily implementable estimation procedure was examined for the coefficients of cubic B-spline basis functions by using a penalized likelihood approach. Fitting B-splines is usually not more difficult than that of a polynomial regression.

Because the selection of the number and locations of the knots is an important issue, it is the topic of much research in nonparametric regression methods (Ruppert, 2002; Lindstrom, 1999). Knots were chosen to be equally spaced with respect to the quantiles of the distinct values of the independent variable and set the number of chosen knots to be $\min \left[\left(\frac{\text { number of distinct values of covariate }}{6}\right), 30\right]$

(Ruppert, 2002).

This study has shown that by smoothing the effect of an independent variable, the proposed method allows for a test of the lack of fit of a postulated parametric model by the use of likelihood ratio method. As shown in the simulation study, the proposed test has the ability to detect more general alternative models and is superior to parametric likelihood ratio tests unless the true model is known. This is of great practical importance, because in most real data applications, the true parametric forms of independent variable effects are usually unknown. Therefore, investigators must consider the consequences of statistical inferences under misspecified parametric regression models. Specifically, this simulation study showed that the common practice of adding a quadratic term to the linear predictor could severely undercut the power of inference.

The scope was restricted to count data following Poisson distribution. But, as many have observed, count data often exhibit greater variability than that is provided by the Poisson distribution. In the presence of extra-Poisson variation, one could use regression models based on negative binomial distribution (Tu \& Piegorsch, 2003). This research can be extended by linking the negative binomial mean $\mu(x)$ to smooth function $s(x)$. The testing procedure associated with the extended model is currently under investigation.

\section{References}

Cameron, A. C. \& Trivedi, P. K. (1998). Regression analysis of count data. New York: Cambridge University Press.

de Boor, C. (1978). A practical guide to splines. New York: Springer-Verlag.

Eilers, P. H. C. \& Marx, B. D. (1996). Flexible smoothing with B-splines and penalties. Statistical Science, 11, 89-121.

Hastie, T. J. \& Tibshirani, R. J. (1990). Generalized additive models. New York: Chapman \& Hall.

Hope, C., Wu, J., Tu, W., Young, J., \& Murray, M. D. (2004). Association of medication knowledge, skill and emergency department visits in older adults with heart failures. American Journal of Health-System Pharmacy, 61, 2043-2049.

Lindstrom, M. (1999). Penalized estimation of free-knot splines. Journal of Computational and Graphical Statistics, 8, 333-352.

McCullagh, P. \& Nelder, J. A. (1989). Generalized linear model (2nd ed.). New York: Chapman \& Hall.

Ruppert, D. (2002). Selecting the number of knots for penalized splines. Journal of Computational and Graphical Statistics, 11, 735-757.

Ruppert, D., Wand, M. P. \& Carroll, R. J. (2003). Semiparametric regression. New York: Cambridge University Press.

Tu, W., Morris, A.B., Li, J., Wu, J., Young, J., Brater, D. C. \& Murray, M. D. (2005). Association between adherence measurements of metoprolol and healthcare utilization in older patients with heart failure. Clinical Pharmacology and Therapeutics, 77, 189-201.

Tu, W. \& Piegorsch, W. W. (2003). Empirical Bayes analysis of a hierarchical Poisson regression model. Journal of Statistical Planning and Inference, 111(1-2), 235-248. 


\title{
Using the Fractional Imputation Methodology to Evaluate Variance due to Hot Deck Imputation in Survey Data
}

\author{
Adriana Pérez \\ The University of Texas Health Science Center at Houston
}

This article examines empirically the effect on the variance estimate due to the use of hot deck imputation with a nearest neighbor donor in comparison with the pairwise fractional hot deck imputation methodology in the 1999 Survey of Doctorate Recipients.

Key words: Ignorability, missing at random, item nonresponse, serpentine sorting, nearest neighbor, successive difference replication

\section{Introduction}

Imputation is commonly used to deal with nonresponse and incomplete data in surveys. Usually, the use of imputed values as observed values produces appropriate estimates of smooth statistics (totals, means, proportions, etc) as well as non-smooth statistics (quantiles, etc), if the imputation does not cause severe systematic bias. However, the dangers are well known of not correcting the variance estimates to reflect the uncertainty due to missing data. This may lead to larger underestimation as the proportion of imputed values increases when treating the imputed values as observed. Over the years, a number of methods have been suggested in the statistical literature to overcome these issues(Kalton \& Kasprzyk, 1986; Brick \& Kalton, 1996; Groves et al., 2004).

Among other reasons, imputation techniques typically are not used with survey data because their users are unfamiliar with techniques of analyzing missing data. Due to operational convenience, most of the commonly

Adriana Pérez is an Associate Professor of Biostatistics in the School of Public Health. Email: adriana.perez@uth.tmc.edu. used statistical packages do not incorporate adjustments for missing data into their analysis. For simplicity, often an entire observation with one missing variable response is eliminated.

Following Shao and Steel's (1999) description, two general perspectives exist to obtain variance estimators for large complex sample surveys after imputation: design-based and model-assisted perspective (including multiple imputation). Paraphrasing their definitions: the variance estimate in a designbased perspective accounts for repeated sampling from a fixed finite population and uniform nonresponse within an imputation cell.

Using the model-assisted perspective, the variance estimate is with respect to the sample design and response as well as to the model used for the imputation method (Särndal, Swensson, \& Wretman, 1992; Shao et al., 1999). Variance estimators under a multiple imputation perspective (Rubin, 1987), are reasonable using Bayesian inference but are not applicable for design-based or deterministic imputation methods (Shao, 2002). The model-assisted perspective variance estimation methods will not be discussed any further.

Several variance estimation methods exist under the design-based perspective after imputation. Two examples are linearization methods (i.e., Taylor series expansions (Chen \& Shao, 1997; Chen \& Shao, 2000; Kim, 2001)) 
and replication methods (i.e., Jackknife (Rao \& Shao, 1992), bootstrap (Shao \& Sitter, 1996) and balanced half samples (Lee, Rancourt, \& Särndal, 1995; Rao \& Shao, 1996; Shao, Chen, \& Chen, 1998; Shao \& Chen, 1999; Kim, 2001; Kim \& Fuller, 2004). Lee, Rancourt and Särndal (2002) discussed the differences between these approaches. All these methods provide adequate estimates. The choice depends on the users, the need for the estimation of variance components, the computational burden, the adaptability of the sampling fraction and the response mechanism (Lee et al., 2002).

This article is focused on the effect on the variance estimates in the 1999 Survey of Doctorate Recipients (SDR) (National Science Foundation, Directorate for Social, \& Division of Science Resources Statistics, 2002). In the next section, the 1999 SDR survey methods will be discussed. Next, a description of the aspects of nearest neighbor hot deck imputation method, fractional imputation, successive difference replication method and the effect of multiple weighting stages will be provided. All these methods are used here to evaluate the variance estimates of this survey. This study extends the proposal of pairwise fraction imputation by Kim and Fuller (1999) on the use of variance estimation with pairwise fractional hot deck imputation and the successive difference replication method.

The 1999 Survey of Doctoral Recipients (SDR)

The 1999 SDR is a National Science Foundation (NSF) survey conducted by the U.S. Census Bureau (National Science Foundation et al., 2002). The population of interest for this survey includes individuals who earned a doctoral degree from a United States (U.S.) institution in Science and Engineering (S\&E) fields, are less than 76 years old and planned to stay in the U.S. after their degree(US Bureau of the Census, Demographic Statistical Methods Division, \& Health Surveys and Supplements
Branch, 2003a). The SDR provides information about demographic and employment characteristics of the nation's science and engineering doctorate holders. The sampling frame consists of the doctorates records file which contains all research doctorate recipients from U.S. universities since 1920 (National Science Foundation et al., 2002).

The 1999 SDR survey sample size was 40,000. The sample was systematically selected from three groups using the probability proportional to size selection methodology. The three groups were the new cohort (doctoral recipients between July 1996 and June 1998), the nearly new cohort (doctoral recipients between July 1992 and June 1996) and the old cohort (doctoral recipients prior to July 1992) (National Science Foundation et al., 2002).

The sampling strata consisted of 240 strata for the old and nearly new cohorts and were defined by demographic group, degree field and sex. The same 240 strata (six of which were empty) defined the sampling strata for the new cohort (US Bureau of the Census et al., 2003a).

Item non-response was observed in this survey in all variables except seven. All seven were critical variables and had to be filled in order for the response to be considered complete. Hence, two imputation methods were used: logical imputation and hot deck imputation. Logical imputation was used when the answer to a question could be determined by the answer to another question either within the same survey year or from a prior survey round (US Bureau of the Census, Demographic Statistical Methods Division, \& Health Surveys and Supplements Branch, 2001a). Logical imputation will not be addressed further in this article.

Hot deck imputation was implemented using a nearest neighbor donor. The auxiliary variables selected to identify the pool of donors were determined by prediction models for each 
variable in the survey with item nonresponse. A serpentine sorting on the auxiliary variables was implemented to determine the nearest neighbor donor response (US Bureau of the Census et al., 2001a). This survey allowed for the use of information from any one donor a maximum of four times. The missing mechanism and the tentative reasons for missing values in this survey is likely missing at random (Perez, 2003).

Base weights of the 1999 SDR data were computed by the U.S. Census Bureau (US Bureau of the Census, Demographic Statistical Methods Division, \& Health Surveys and Supplements Branch, 2001b). To obtain the final weights, the base weights underwent several adjustments to correct for duplicates, frame ineligibles, never earned doctorate case and control totals. Included in these weighting adjustments were a non-interview adjustment and a ratio adjustment via a raking methodology (US Bureau of the Census et al., 2001b). Variance estimates were calculated using successive difference replication methods with 160 replicates (US Bureau of the Census, Demographic Statistical Methods Division, \& Health Surveys and Supplements Branch, 2003b; Sukasih \& Jang, 2003). Point and variance estimates are currently reported using imputation values as observed values (National Science Foundation et al., 2002).

\section{Methodology}

Nearest Neighbor Hot Deck Imputation

Hot deck imputation refers to the process where missing responses or items are replaced by values selected from respondents within the same survey. The respondent selected as a donor is chosen by using observable values from auxiliary variables. The 1999 SDR survey used the hot deck imputation method based on imputation cells (US Bureau of the Census et al., 2001a). This means that in using auxiliary variables known for respondents and nonrespondents, the sample was divided into cells. Sorting was performed within each imputation cell and a neighboring case was selected as a donor for each missing value. Then, the missing value was replaced by the selected value within that cell (Chen et al., 2000).
Fractional imputation

Fractional imputation identifies the method where each missing response or item is replaced by several imputed values drawn from the responding values in an imputation cell (Fay, 1996; Kim et al., 1999). Fractional imputation provides an adjustment method for variance estimation in design-based estimators in the presence of missing values (da Silva \& Opsomer, 2002).

Fractional imputation estimators were designed to reduce the imputation variance (Kim et al., 2004) by using more than one donor for a recipient and increasing the weight of the donor for each missing item by a value equal to a fraction of the original weight of the missing observation. Respondents who are not donors retain their original weights. Pairwise fractional hot deck imputation is a special case of fractional imputation where two distinct donors are selected for each missing item. The assumption for this method is that there are at least two donors in each imputation cell (Kim et al., 1999).

The Successive Difference Replication Method

The current approach in calculating the 1999 SDR variance estimates is the successive difference replication method (SDRM). Wolter (1984) developed the basic theory of the successive difference method and later Fay and Train (Fay \& Train, 1995) extended this theory with replicates generating the SDRM. The variance estimator is calculated based on the squared differences between neighboring sample cases. The SDRM produces variance estimates with a greater number of degrees of freedom than other replication methods. To create the replicates, the SDRM variance estimator uses an orthogonal Hadamard matrix. Because the 1999 SDR used 160 replicates, a 160x160 Hadamard matrix was formed.

\section{Notation}

Paraphrasing, Kim and Fuller's (2004) notation: let $P$ be a finite population containing indices $1, \ldots, N . P$ is stratified into $H$ strata with $N_{h}$ units in the $h$-th stratum. $n_{h} \geq 2$ units are selected following some probability sampling plan called the sampling mechanism. 
Let $S$ denote the sample. According to the sampling plan, survey weights $w_{i}, i \in S$ are constructed. This expectation is in respect to $S$. Let $\mathrm{Y}$ be a variable of interest and $Y=\left(y_{1}, y_{2}, \ldots ., y_{N}\right)$ denotes the population vector. The response mechanism ( $I$ ) identifies the probability mechanism of the responses obtained in the sample. $I_{i}=1$ if $y_{i}$ is a respondent and $I_{i}=0$ otherwise. Let the population characteristic of interest be $\theta_{N}=\theta\left(y_{1}, \ldots, y_{N}\right)$ and let $\hat{\theta}$ be a linear estimator of $\theta_{N}$ based on the full sample, $\hat{\theta}=\sum_{i \in S} w_{i} y_{i}$.

The SDRM variance estimator for $\hat{\theta}$ can be defined without loss of generality as (ignoring the finite population correction factor) in equation (1):

$$
V_{S D R M}(\hat{\theta})=\frac{4}{k} \sum_{r=1}^{k}\left(\hat{\theta}^{(r)}-\hat{\theta}\right)^{2}
$$

where $r$ is the replicate sample $(r=1, \ldots, k) . k$ is the total number of replicate samples, $\hat{\theta}^{(r)}$ is the $r$-th replicate of $\hat{\theta}$ and can be written as: $\hat{\theta}^{(r)}=\sum_{i \in S} w_{i}^{(r)} y_{i}, \quad$ where $w_{i}^{(r)}$ denotes the replicate weight for the $\mathrm{i}$-th unit of the $r$-th replicate.

In the imputation procedure, let $a_{i j}$ be the number of times that $y_{i}$ is used as a donor for the missing $y_{j} . S_{R}$ is the set of indices of the sample respondents and $S_{M}$ is the set of indices of the sample nonrespondents. Let us define $a=\left\{a_{i j} ; i \in S_{R}, j \in S_{M}\right\}$, then the distribution of $a$ is called the imputation mechanism. In addition, when $y_{i}$ is used as a donor for element $\mathrm{j}$, let $w_{i j}^{\bullet}$ be the fraction of the original weight for element $\mathrm{j} . w_{i j}^{\bullet}$ is called the imputation fraction (Fuller \& Kim, 2001; Kim et al., 2004). $w_{i i}^{\bullet}=1$ for $i \in S_{R}$ and $w_{i i}^{\bullet}=0$ for $i \in S_{M}$. The $a_{i j}$ are nonnegative and the sum of the imputation fractions of the donors for a missing item is mandatory to be one: $\sum_{i \in S_{R}} a_{i j} w_{i j}^{\bullet}=1, \forall j \in S$. In the case of a pairwise fractional hot deck imputation, the imputation fractions, $w_{i j}^{\cdot}$, are equal to 0.5 . A linear estimator using fractional hot deck imputation can be written as in equation (2):

$$
\hat{\theta}_{I}=\sum_{i \in S_{R}}\left(w_{i}+\sum_{j \in S_{M}} a_{i j} w_{i j}^{\bullet} w_{j}\right) y_{i}
$$

The term in parenthesis equation (2) is called the imputation adjustment weight. Kim and Fuller(1999) demonstrated that the linear estimator $\hat{\theta}_{I}$ is unbiased and consistent under an ignorable response mechanism. These authors also estimated the variance of this fractional hot deck imputation in terms of the imputation cells.

Variance Estimation After Pairwise Fractional Hot Deck Imputation

Extending the idea of variance estimation after imputation (Kim, 2002; Kim et al., 1999), if the imputed values from the pairwise fractional hot deck imputation are treated as true values and apply the successive difference replication method then the variance estimator can be expressed as in equation (3):

$$
V_{S D R M, I}(\hat{\theta})=\frac{4}{k} \sum_{r=1}^{k}\left(\hat{\theta}_{I}^{(r)}-\hat{\theta}_{I}\right)^{2}
$$

where $\hat{\theta}_{I}^{(r)}$ is the $r$-th replicate of $\hat{\theta}_{I}$ and can be written as $\hat{\theta}_{I}^{(r)}=\sum_{i \in S_{R}}\left(w_{i}^{(r)}+\sum_{j \in S_{M}} a_{i j} w_{i j}^{\bullet} w_{j}^{(r)}\right) y_{i}$, where $w_{i}^{(r)}$ denotes the replicate weight for the ith unit of the $r$-th replicate and $w_{j}^{(r)}$ denotes the replicate weight for the $\mathrm{j}$-th unit of the $r$-th replicate. Because $a_{i j}$ and $w_{i j}^{\cdot}$ are the imputation mechanism and imputation fraction, respectively, they will take on the same value across all replicates. This is to ensure the correct calculation of the imputation adjustment weight. 
Effect of Multiple Weighting Stages On Variance Estimation After Imputation

Frequently, multiple stages of weighting adjustments are implemented in survey (Valliant, 2004). The main aim of the weighting plan is to produce final weights that reduce the nonresponse bias in the survey estimates, balance for noncoverage, and adjust sample estimates to control totals. Each stage introduces a different source of variability in an estimator that may perhaps be important to reflect when estimating variances. The advantage of variance estimation through replication is that it can explicitly account for all the stages in estimation by repeating each adjustment separately for each replicate. This concept will be evaluated in this study.

\section{Methods Implemented On 1999 SDR Data}

As mentioned previously, this research focuses on variance estimation after imputation of the 1999 SDR. The pairwise fractional hot deck imputation procedure was evaluated and compared to the variance estimates with the ones obtained when treating the imputed values as observed. Five variables were selected: Race, Hispanic, Gender, Citizenship, and Median Basic Annual Salary of the doctoral scientist and engineers. The Woodruff (1952) method was used for calculating the median and its corresponding standard error was estimating using the program described by Gossett et al (2002). Employment status is a variable without missing data that was used in forming estimates for this study. Separate replicates were computed for each variable of interest as the response mechanism differs for each one. Employment, in combination with the aforementioned variables, was used to calculate 19 survey estimates.

After identifying two donors per missing value for each of the variables selected, the imputation adjustment weight was calculated. However, this imputation adjustment weight can be calculated at three stages of the weighting adjustment process: using the base weights, using the weights after the noninterview adjustment or using the final weights (US Bureau of the Census et al., 2001b). It was decided that all three stages should be explored and the corresponding replicates needed for the
SDRM under all three weighting stages were calculated for evaluation purposes. The three weighting stages being evaluated are discussed in Methods B, C and D below. Method A is the nearest neighbor hot deck imputation used in the 1999 SDR, and did not include an imputation weighting adjustment.

- Method A: The original sampling weights based on the one donor hot deck imputation methodology were used and the imputed values were treated as observed values. The imputation weight adjustment was not used in this method.

- Method B: The base weights were used to obtain the imputation adjustment weights. The imputation adjusted weights were then adjusted to include the non-interview and raking adjustments.

- Method C: The base weights were used to obtain the non-interview adjusted weights. The non-interview adjusted weights were then used to determine the imputation adjustment weights. Finally, the raking adjustments were the final weighting step in the weighting process for this method.

- Method D: After applying the noninterview and raking adjustments to the base weights to create the final weights, the final weights were then used to obtain the imputation adjustment weight.

This empirical evaluation will allow for a determination of the stage of the weighting process at which the imputation weighting adjustment should be performed. In addition, it will allow for an evaluation of the impact of using a single hot deck imputation versus a pairwise fractional hot deck imputation.

After the replicates were computed, the point estimates and their corresponding standard errors were obtained. Statistics combining employment status with variables with missing values used the imputation adjustment weight 
for the variable with missing values. As an example, when the employed male estimate was formed, the imputation adjustment weight reflected the adjustments due to the gender variable being imputed.

The standard errors ( $S E$ ) which do not take the imputation adjustment into account (Method A) were compared with the standard errors which take into account the imputation adjustment (Methods B, C and D). To assess this comparison, the relative difference (RD) was used. For example, when comparing method B versus method $A$ the $R D$ is in equation (4):

$$
R D=100 \% * \frac{S E_{B}\left(\hat{\theta}_{I}\right)-S E_{A}\left(\hat{\theta}_{I}\right)}{S E_{A}\left(\hat{\theta}_{I}\right)}
$$

The RD measures the magnitude of over or under estimation of the alternative method $\mathrm{B}$ compared with the current baseline method A. It is important to highlight that all $S E$ are estimates of standard errors instead of true standard errors and furthermore all are subject to sampling errors.

\section{Results}

The imputation rates in the 1999 SDR are relatively low and are provided in table 1 . Table 1 presents the point estimates for the 19 estimates selected on the doctoral scientists and engineers for methods A through D. As expected due to the low imputation rates, the point estimates did not vary significantly with either method across all the statistics selected.

Table 2 presents the variance estimates with methods $\mathrm{A}$ through $\mathrm{D}$; and includes the relative variances comparing each method $\mathrm{B}, \mathrm{C}$ and $\mathrm{D}$ to method $\mathrm{A}$. The results in table 2 suggest that (i) the variance estimator is lower when the pairwise fractional imputation methods is used and (ii) there is no preference on the weighting stage of the adjustments, except for the median of the basic annual salary where a $17 \%$ reduction on its variance is obtained using method D.

\section{Conclusion}

The purpose of this article was to perform the pairwise fractional hot deck imputation to evaluate the effect on the variance estimates due to the imputation procedure. The use of this method shows a lower variance in comparison to the single hot deck imputation method which treated the imputed values as observed values. This is achieved in most of the variables of interest. Exceptions are Naturalized U.S. citizen and employed Naturalized U.S. citizen. For these exceptions, the relative difference is slight at most $(1.1 \%)$ when compared with the hot deck imputation method.

Nevertheless, the effort involved may argue that the need of having an imputation adjustment weight for each variable may not have been necessary in this particular survey with its low imputation rates. Interestingly, this empirical evaluation confirms the disadvantage pointed out by Kim (2002) that its computation can be cumbersome for a large dataset such as the 1999 SDR.

There are limitations to the empirical evaluation. i) The dataset does not have a serious missing data problem which does not allow us to determine clearly which method should be preferred under what conditions. ii) Separate replicates were computed for each variable of interest, assuming an independent univariate missingness pattern. Neither the nearest neighbor hot deck nor the pairwise fractional hot deck imputation methods allows incorporation of multivariate missingness variables to estimate their replicates. iii) The true variance of the SDR data is unknown; for that reason this empiric investigation does not quantify the true relative efficiency.

Further investigation is needed on how to obtain an imputation adjustment weight for the entire survey, as well as how to use/obtain imputation adjustment weights for statistics where more than one variable with missing data are required. Monte Carlo simulations identifying the true variance for a pseudo SDR population as well as incorporating several patterns and missing data mechanisms beyond missing completely at random need to be explored. 
Table 1. Doctoral scientist and engineers in 1999: Point estimates using four methods. Method A: hot deck imputation using one donor and treating the imputed values as observed values. Method B: pairwise fractional hot deck imputation using the base weight to obtain the imputation adjustment weight. Method C: pairwise fractional hot deck imputation using the noninterview weight to obtain the imputation adjustment weight. Method D: pairwise fractional hot deck imputation using the final weight to obtain the imputation adjustment weight.

\begin{tabular}{|c|c|c|c|c|c|c|c|}
\hline & \multirow{2}{*}{ Statistic/Variable } & \multirow{2}{*}{$\begin{array}{l}\text { Sample } \\
\text { Size }\end{array}$} & \multirow{2}{*}{$\operatorname{IR}(\%) *$} & \multicolumn{4}{|c|}{ Point Estimates } \\
\hline & & & & $\mathbf{A}$ & B & C & D \\
\hline & Total & & & & & & \\
\hline 1 & All & 31,318 & & 626,698 & 626,699 & 626,699 & 626,698 \\
\hline \multirow[t]{2}{*}{2} & Hispanic & 1,623 & 1.89 & 15,007 & 14,787 & 14,787 & 15,045 \\
\hline & Race & & 0.89 & & & & \\
\hline 3 & White! & 22,949 & & 508,447 & 508,859 & 508,863 & 508,417 \\
\hline 4 & African American & 1,567 & & 14,179 & 14,081 & 14,082 & 14,182 \\
\hline 5 & Asian or Pacific Islander & 4,847 & & 87,034 & 86,823 & 86,818 & 87,075 \\
\hline \multirow[t]{2}{*}{6} & American Indian/Alaskan Native & 332 & & 2,032 & 2,009 & 2,011 & 2,017 \\
\hline & Gender & & 0.01 & & & & \\
\hline 7 & Male & 22,432 & & 476,495 & 476,511 & 476,511 & 476,503 \\
\hline 8 & Female & 8,886 & & 150,204 & 150,188 & 150,188 & 150,196 \\
\hline 9 & Employed Male & 19,835 & & 419,869 & 419,884 & 419,884 & 419,876 \\
\hline \multirow[t]{2}{*}{10} & Employed Female & 7,910 & & 133,494 & 133,480 & 133,480 & 133,486 \\
\hline & Citizenship & & 0.93 & & & & \\
\hline 11 & Native Born U.S. Citizen & 24,837 & & 491,928 & 491,940 & 491,927 & 491,930 \\
\hline 12 & Naturalized U.S. Citizen & 3,676 & & 70,921 & 70,843 & 70,851 & 70,943 \\
\hline 13 & Non-U.S. Citizen. Permanent Resident & 2,124 & & 48,938 & 48,984 & 48,981 & 48,919 \\
\hline 14 & Non-U.S. Citizen. Temporary Resident & 681 & & 14,911 & 14,921 & 14,930 & 14,907 \\
\hline 15 & Employed Native Born U.S. Citizen & 21,794 & & 429,085 & 429,459 & 429,454 & 429,507 \\
\hline 16 & Employed Naturalized U.S. Citizen & 3,243 & & 62,507 & 62,460 & 62,461 & 62,540 \\
\hline 17 & $\begin{array}{l}\text { Employed Non-U.S. Citizen. Permanent } \\
\text { Resident }\end{array}$ & 2,045 & & 47,264 & 47,321 & 47,318 & 47,258 \\
\hline 18 & $\begin{array}{l}\text { Employed Non-U.S. Citizen. Temporary } \\
\text { Resident }\end{array}$ & 663 & & 14,507 & 14,527 & 14,536 & 14,514 \\
\hline 19 & $\begin{array}{l}\text { Median Basic Annual Salary of Full Time } \\
\text { Employed }\end{array}$ & 25,686 & 4.27 & 70,000 & 68,000 & 68,000 & 68,000 \\
\hline
\end{tabular}

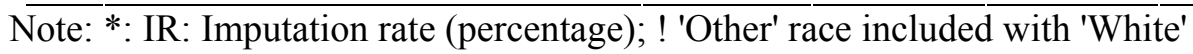


Table 2. Doctoral scientist and engineers in 1999: Standard error estimates and relative differences using four methods. Method A: hot deck imputation using one donor and treating the imputed values as observed values. Method B: pairwise fractional hot deck imputation using the base weight to obtain the imputation adjustment weight. Method C: pairwise fractional hot deck imputation using the noninterview weight to obtain the imputation adjustment weight. Method D: pairwise fractional hot deck imputation using the final weight to obtain the imputation adjustment weight.

\begin{tabular}{|c|c|c|c|c|c|c|c|c|}
\hline & \multirow[b]{2}{*}{ Statistic/Variable } & \multicolumn{4}{|c|}{ Standard Error } & \multicolumn{3}{|c|}{ Relative Difference } \\
\hline & & $\mathbf{A}$ & B & $\mathbf{C}$ & D & $\frac{B-A}{A}$ & $\frac{C-A}{A}$ & $\frac{D-A}{A}$ \\
\hline & Total & & & & & & & \\
\hline 1 & All & 732.2 & 732.1 & 732.1 & 732.2 & 0.00 & 0.00 & 0.00 \\
\hline \multirow[t]{2}{*}{2} & Hispanic & 427.0 & 416.4 & 416.3 & 421.3 & -0.02 & -0.03 & -0.01 \\
\hline & Race & & & & & & & \\
\hline 3 & White! & $1,001.0$ & 992.9 & 993.8 & 994.1 & -0.01 & -0.01 & -0.01 \\
\hline 4 & African American & 360.7 & 350.5 & 350.4 & 352.7 & -0.03 & -0.03 & -0.02 \\
\hline 5 & Asian or Pacific Islander & 819.8 & 814.7 & 813.8 & 819.0 & -0.01 & -0.01 & 0.00 \\
\hline \multirow[t]{2}{*}{6} & American Indian/Alaskan Native & 161.1 & 160.1 & 160.0 & 159.5 & -0.01 & -0.01 & -0.01 \\
\hline & Gender & & & & & & & \\
\hline 7 & Male & 694.5 & 693.9 & 693.9 & 694.2 & 0.00 & 0.00 & 0.00 \\
\hline 8 & Female & 374.8 & 374.1 & 374.1 & 374.4 & 0.00 & 0.00 & 0.00 \\
\hline 9 & Employed Male & $1,164.1$ & $1,162.0$ & $1,162.0$ & $1,163.0$ & 0.00 & 0.00 & 0.00 \\
\hline \multirow[t]{2}{*}{10} & Employed Female & 689.0 & 689.1 & 689.1 & 689.0 & 0.00 & 0.00 & 0.00 \\
\hline & Citizenship & & & & & & & \\
\hline 11 & Native Born U.S. Citizen & 686.9 & 682.8 & 683.0 & 686.5 & -0.01 & -0.01 & 0.00 \\
\hline 12 & Naturalized U.S. Citizen & 856.3 & 865.6 & 864.7 & 857.3 & 0.01 & 0.01 & 0.00 \\
\hline 13 & Non-U.S. Citizen. Permanent Resident & 787.0 & 784.9 & 783.6 & 783.9 & 0.00 & 0.00 & 0.00 \\
\hline 14 & Non-U.S. Citizen. Temporary Resident & 471.3 & 471.0 & 471.0 & 468.3 & 0.00 & 0.00 & -0.01 \\
\hline 15 & Employed Native Born U.S. Citizen & $1,253.6$ & $1,239.7$ & $1,239.1$ & $1,247.6$ & -0.01 & -0.01 & 0.00 \\
\hline 16 & Employed Naturalized U.S. Citizen & 873.4 & 875.9 & 875.2 & 872.2 & 0.00 & 0.00 & 0.00 \\
\hline 17 & $\begin{array}{l}\text { Employed Non-U.S. Citizen. Permanent } \\
\text { Resident }\end{array}$ & 797.8 & 791.6 & 790.3 & 791.1 & -0.01 & -0.01 & -0.01 \\
\hline 18 & $\begin{array}{l}\text { Employed Non-U.S. Citizen. Temporary } \\
\text { Resident }\end{array}$ & 486.5 & 486.3 & 486.5 & 483.8 & 0.00 & 0.00 & -0.01 \\
\hline 19 & $\begin{array}{l}\text { Median Basic Annual Salary of Full } \\
\text { Time Employed }\end{array}$ & 1,519 & 1,326 & 1,324 & 1,266 & -0.13 & -0.13 & -0.17 \\
\hline
\end{tabular}

Note: ! 'Other' race included with 'White' 


\section{References}

Brick, J. M. \& Kalton, G. (1996). Handling missing data in survey research. Statistical Methods in Medical Research, 5, 215238.

Chen, J. \& Shao, J. (1997). Biases and variances of survey estimators based on nearest neighbor imputation. American Statistical Association, 365-370.

Chen, J. \& Shao, J. (2000). Nearest neighbor imputation for survey data. Journal of Official Statistics, 16, 113-131.

da Silva, D. N. \& Opsomer, J. D. (2002). Estimation after fractional imputation under a nonparametric response mechanism. http://www.stat.iastate.edu/preprint/abstracts/20 02-01.pdf.

Fay, R. E. (1996). Alternative paradigms for the analysis of imputed survey data. Journal of the American Statistical Association, 91, 490498.

Fay, R. E. \& Train, G. F. (1995). Aspects of survey and model-based postcensal estimation of income and poverty characteristics for states and counties. American Statistical Association, 154-159.

Fuller, W. A. \& Kim, J. K. (2001). Hot deck imputation for the response model.

Gossett, J. M., Simpson, P., Parker, J. G., \& Simon, W. L. (2002). How complex can complex survey analysis be with SAS ${ }^{\circledR}$ ? In SUGI 27 Proceedings, SAS users group international 27th annual conference Apr 14-17, Orlando, FL. http://www2.sas.com/proceedings /sugi27/p266-27.pdf

Groves, R. M., Fowle F. J .Jr, Couper, M. P., Lepkowski, J. M., Singer, E., \& Tourangeau, R. (2004). Survey Methodology. (1st ed.) New Jersey: John Wiley \& Sons, Inc.

Kalton, G. \& Kasprzyk, D. (1986). The treatment of missing survey data. Survey Methodology, 12, 1-16.

Kim, J. K. (2001). Variance estimation after imputation. Survey Methodology, 27, 7583.

Kim, J. K. (2002). Variance imputation for nearest neighbor imputation with application to census long form data. American Statistical Association, 1857-1862.
Kim, J. K. \& Fuller, W. A. (2004). Fractional hot deck imputation. Biometrika, 91, 559-578.

Kim, J. K. \& Fuller, W. A. (1999). Jackknife variance estimation after hot deck imputation. American Statistical Association, 825-830.

Lee, H., Rancourt, E., \& Särndal, C. E. (1995). Variance estimation in the presence of imputed data for the generalized estimation system. American Statistical Association, 384389.

Lee, H., Rancourt, E., \& Särndal, C. E. (2002). Variance estimation from survey data under single imputation. In Groves, R. M., Dillman, D. A, Eltinge, J. L, \& Little, R. J. A. (Eds.), Survey Nonresponse. New York: John Wiley \& Sons, 315-328.

National Science Foundation, Directorate for Social, B. a. E. S., \& Division of Science Resources Statistics (2002). Characteristics of doctoral scientists and engineers in the United States: 1999. Detailed statistical tables. (NSF 02-328 ed.) Arlington, VA.

Perez A. Missing Data Analysis on the Science Resources Statistics Surveys. Focus on 1999 NSRCG, NSCG and SDR. 7-3-2003. National Science Foundation. Division of Science Resources Statistics. Arlington, VA, Powerpoint presentation.

Rao, J. N. K. \& Shao, J. (1992). Jackknife variance estimation with survey data under hot deck imputation. Biometrika, 79, 811822.

Rao, J. N. K. \& Shao, J. (1996). On balanced half-sample variance estimation in stratified random sampling. Journal of the American Statistical Association, 91, 343-348.

Rubin, D. B. (1987). Multiple imputation for nonresponse in surveys. New York: John Wiley \& Sons, Inc.

Särndal, C. E., Swensson, B., \& Wretman, J. (1992). Model assisted survey sampling. New York: Springer Verlag.

Shao, J. (2002). Replication methods for variance estimation in complex surveys with imputed data. In Groves, R. M., Dillman, D. A., Eltinge, J. L., \& Little, R. J. A. (Eds.), Survey Nonresponse. New York: John Wiley \& Sons. 
Shao, J. \& Chen, Y. (1999). Approximate balanced half sample and related replication methods for imputed survey data. Sankhya: The Indian Journal of Statistics.Series $B, 61,187-201$.

Shao, J., Chen, Y., \& Chen, Y. (1998). Balanced repeated replication for stratified multistage survey data under imputation. Journal of the American Statistical Association, 93, 819-831.

Shao, J. \& Sitter, R. R. (1996). Bootstrap for imputed survey data. Journal of the American Statistical Association, 91, 12781288.

Shao, J. \& Steel, P. (1999). Variance estimation for survey data with composite imputation and nonnegligible sampling fractions. Journal of the American Statistical Association, 94, 254-265.

Sukasih, A. S. \& Jang, D. (2003). Monte Carlo study on the successive difference replication method for non-linear statistics. American Statistical Association, 4141-4147.

US Bureau of the Census, Demographic Statistical Methods Division, \& Health Surveys and Supplements Branch (2003a). Sample design for the 1999 survey of doctorate recipients Washington, DC: US department of commerce.

US Bureau of the Census, Demographic Statistical Methods Division, \& Health Surveys and Supplements Branch (2001a). Imputation specification for the 1999 survey of doctorate recipients Washington, DC: US department of commerce.
US Bureau of the Census, Demographic Statistical Methods Division, \& Health Surveys and Supplements Branch (2001b). The 1999 Survey of Doctorate Recipients Weighting Plan (Rep. No. SDR99-WT-1). Washington, DC: US department of commerce.

US Bureau of the Census, Demographic Statistical Methods Division, \& Health Surveys and Supplements Branch (2003b). Generalized variance parameters for the 2001 survey of doctorate recipients. Direct calculation of variance estimates, generalized variance functions, generalized variance parameters, standard errors and their use (SDR01-VAR-3) Washington, DC: United States department of commerce; Economic and Statistics Administration; US Census Bureau.

Valliant, R. (2004). The effect of multiple weighting steps on variance estimation. Journal of Official Statistics, 20, 1-18.

Wolter, K. M. (1984). An investigation of some estimators of variance for systematic sampling. Journal of the American Statistical Association, 79, 781-790.

Woodruff, R. S. (1952). Confidence intervals for medians and other position measures. Journal of the American Statistical Association, 47, 635-646. 


\title{
Practical Unit-Root Analysis Using Information Criteria: Simulation Evidence
}

\author{
Kosei Fukuda \\ Nihon University
}

The information-criterion-based model selection method for detecting a unit root is proposed. The simulation results suggest that the performances of the proposed method are usually comparable to and sometimes better than those of the conventional unit-root tests. The advantages of the proposed method in practical applications are also discussed.

Key words: Information criteria, model selection, Monte Carlo simulation, pre-testing problem, unit root

\section{Introduction}

Since the seminal work of Dickey and Fuller (1979), numerous alternative methods have been developed to improve the size and power properties of unit-root tests. However, little attention has been paid to two practical problems encountered in unit-root tests. Consider the following augmented DF (ADF) regression for an observed time series $y_{t}(t=1, \ldots, T)$ :

$$
\Delta y_{t}=\mu+\beta t+\rho y_{t-1}+\sum_{i=1}^{k} \phi_{i} \Delta y_{t-i}+e_{t}
$$

where $e_{t} \sim \operatorname{NID}\left(0, \sigma^{2}\right)$. Three model classes and the corresponding $t$-statistics (denoted by $\hat{\tau}$ ) for a unit root are obtained by considering parameter restrictions on (1).

Kosei Fukuda is Associate Professor, College of Economics, at Nihon University, Tokyo, Japan. He had served as an economist in the Economic Planning Agency of the Japanese Government (1986-2000). Email him at fukuda@eco.nihonu.ac.jp or at kosefuku@crocus.ocn.ne.jp.
Model 1 (Statistics, Null, and Alternative)

$$
\hat{\tau} \quad \mu=\beta=\rho=0 \quad \mu=\beta=0 \text { and } \rho<0
$$

Model 2 (Statistics, Null, and Alternative)

$$
\hat{\tau}_{\mu} \quad \mu=\beta=\rho=0 \quad \beta=0 \text { and } \rho<0
$$

Model 3 (Statistics, Null, and Alternative)

$$
\hat{\tau}_{\tau} \quad \beta=\rho=0 \quad \rho<0
$$

Although there is no discussion on a statistical method for selecting a suitable model class from among these three alternatives, different statistics can lead to different conclusions. For example, in the seminal work of Nelson and Plosser (1982), the result obtained applying Model 3 suggested that the annual time series of U.S. unemployment rate is generated from the trend-stationary process. Forecasting the unemployment rate with the trend-stationary model in the very long horizon would provide a value less than zero or more than 1. If Model 1 is applied in place of Model 3, the null hypothesis of a unit root cannot be rejected. Which conclusion should be embraced? As discussed by Phillips (2005), there is little guidance from economic theory about the source and nature of the trending behavior. Thus, model selection criteria are expected to be applied in selecting a suitable model from among three alternatives.

Furthermore, the pretesting problem also arises. In the conventional ADF regression, the lag length $k$ is selected by applying the $\mathrm{Ng}$ and 
Perron (1995) (NP) general-to-specific procedure. In this procedure, given the maximum lag length $k_{\max }$, by working backward from $k=k_{\max }$, the first value of $k$ is selected such that the $t$-statistic on $\hat{\phi}_{k}$ is significant. Thus, in total, hypothesis testing has to be implemented at two stages. Every test has a nonzero frequency of rejecting the null hypothesis, thus causing Type I errors to accumulate. As discussed by Krolzig and Hendry (2001), it is important to distinguish between the individual test sizes and the overall test size.

These two practical problems have remained even in the recent literature. For example, although Elliott et al. (1996) have proposed more powerful unit root tests, there is no criterion in selecting deterministic components and there is the pretesting problem in selecting the lag length. The ADF test still has the most popularity even now in empirical analyses, while it is the oldest unit-root test and has low power. Thus, the ADF test remains a benchmark method in the present study.

The purpose of this article is to propose an information criterion (IC)-based model selection method for detecting a unit root in order to provide a solution to the above two problems. In this method, the following three steps are taken. First, several alternative models are considered by changing the model class (Models 1, 2, and 3 with and without a unit root) and the lag length, and each model is estimated with the corresponding IC. Second, the best model is selected from among the alternative models by using the minimum IC procedure. Finally, on the basis of the selected model, it is determined whether the observed data contain a unit root. In this article, the Akaike information criterion (AIC) proposed by Akaike (1974) and the Bayesian information criterion (BIC) proposed by Schwartz (1978) are applied. The AIC and BIC for Model (1) are obtained as follows:

$$
\begin{gathered}
\mathrm{AIC}=(T-k-1) \ln \hat{\sigma}^{2}+2 p, \\
\mathrm{BIC}=(T-k-1) \ln \hat{\sigma}^{2}+p \ln (T-k-1),
\end{gathered}
$$

where $p$ denotes the number of parameters. In the full model (Model 3 without a unit root), $p=3+k$, and the other values of $p$ are obtained in correspondence with the number of parameter restrictions.

Simulation Studies: The Case Where the Model Specification is Known

Unlike the ADF tests described earlier, the DF likelihood ratio (DFLR, Dickey \& Fuller, 1981) tests are now considered. This is because the AIC and BIC are both penalized likelihoods. However, DF argued that the limiting distribution of the LR test statistics is too complex to provide an analytical solution. DF presented empirical distributions using Monte Carlo simulations. In their simulation study, the following three cases are considered:

Null Alternative

Case 1: $y_{t}=y_{t-1}+e_{t} \quad y_{t}=\mu+\rho y_{t-1}+e_{t}$

Case 2: $y_{t}=y_{t-1}+e_{t} \quad y_{t}=\mu+\beta t+\rho y_{t-1}+e_{t}$

Case 3: $y_{t}=\alpha+y_{t-1}+e_{t} \quad y_{t}=\mu+\beta t+\rho y_{t-1}+e_{t}$.

In the subsequent study presented in this section, it is assumed that the model specification is known in each case. In the Monte Carlo simulation, the assumed data generating process (DGP) is

$$
y_{t}=\theta y_{t-1}+e_{t} \text { and } e_{t} \sim \mathrm{NID}(0,1) .
$$

Each experiment is performed as follows. First, artificial time series are generated from the assumed DGP. Second, in each case, the DFLR test and the IC-based model selection are performed. Finally, the presence or absence of a unit root is determined in each method. In the DFLR test, three significance levels- $-10 \%, 5 \%$, and $1 \%$ - are applied. In each experiment, three values of $\theta(0.9,0.95,1)$ and two values of $T$ $(100,250)$ are considered. The number of replications in each experiment is 5,000. 
Table 1 shows the frequency count of selecting stationary models. In Case 1, the frequency count of incorrectly selecting stationary models, which corresponds to test size in the terminology of hypothesis testing, is high in the AIC-based method. In the case of $T=100$, this count is 0.41 and it is 0.43 in the case of $T=250$. On the other hand, the performances of the BIC-based method are comparable to those of the DFLR tests. Interestingly, in the case of $T=100$, the performances of the BIC-based method are identical to those of the DFLR tests at the 5\% significance level. This is because the penalty on the likelihood of the stationary model in the BIC-based method is accidentally identical to that in the DFLR test. In Case 2, similar results are obtained. However, in Case 3, the frequency count of incorrectly selecting stationary models is slightly high in the BIC-based method in the case of $T=100$. Thus, it can be concluded that the performances of the BIC-based method are roughly comparable to those of the DFLR tests.

The Case Where Only the Lag Length is Unknown

The IC-based method is compared with the ADF tests using the NP lag length selection. The DGP considered here is partially similar to that considered by NP. Artificial time series are generated using the following process:

$$
y_{t}=\rho y_{t-1}+\sum_{i=1}^{3} \phi_{i} \Delta y_{t-i}+e_{t},
$$

where $e_{t} \sim \operatorname{NID}(0,1)$. Three values of $\rho-$ $\rho=0.85,0.95,1$-are considered, and four vectors of $\left(\phi_{1}, \phi_{2}, \phi_{3}\right)-\left(\phi_{1}, \phi_{2}, \phi_{3}\right)=(0.6,0,0)$, $(-0.6,0,0),(0.4,0.2,0),(0.2,0.2,0.2)$-are considered. The maximum lag length $k_{\max }$ is assumed to be $k_{\max }=8$. Given the observed

Table 1. Frequency count of selecting stationary models

\begin{tabular}{ccccccc}
\hline \multirow{2}{*}{$\theta$} & \multirow{5}{c}{$T$} & \multicolumn{5}{c}{ Methods } \\
\cline { 3 - 7 } & & $10 \%$ & $5 \%$ & $1 \%$ & AIC & BIC \\
\hline 0.9 & 100 & 0.39 & 0.24 & 0.06 & 0.86 & 0.24 \\
0.9 & 250 & 0.99 & 0.93 & 0.64 & 1.00 & 0.80 \\
0.95 & 100 & 0.15 & 0.08 & 0.01 & 0.56 & 0.08 \\
0.95 & 250 & 0.54 & 0.36 & 0.11 & 0.95 & 0.20 \\
1 & 100 & 0.10 & 0.05 & 0.01 & 0.41 & 0.05 \\
1 & 250 & 0.10 & 0.05 & 0.01 & 0.43 & 0.02 \\
\multicolumn{7}{c}{ Null: $y_{t}=y_{t-1}+e_{t}$, Alternatix: $y_{t}=\mu+\beta t+\rho y_{t-1}+e_{t} \cdot$} \\
0.9 & 100 & 0.18 & 0.09 & 0.02 & 0.78 & 0.10 \\
0.9 & 250 & 0.82 & 0.64 & 0.28 & 1.00 & 0.41 \\
0.95 & 100 & 0.09 & 0.04 & 0.01 & 0.57 & 0.04 \\
0.95 & 250 & 0.25 & 0.13 & 0.03 & 0.88 & 0.05 \\
1 & 100 & 0.10 & 0.05 & 0.01 & 0.58 & 0.05 \\
1 & 250 & 0.10 & 0.05 & 0.01 & 0.58 & 0.02 \\
\multicolumn{7}{c}{ Null: $y_{t}=\alpha+y_{t-1}+e_{t}$, Alternatix : $y_{t}=\mu+\beta t+\rho y_{t-1}+e_{t}}$. \\
0.9 & 100 & 0.28 & 0.15 & 0.04 & 0.96 & 0.39 \\
0.9 & 250 & 0.90 & 0.77 & 0.41 & 1.00 & 0.88 \\
0.95 & 100 & 0.13 & 0.07 & 0.01 & 0.83 & 0.21 \\
0.95 & 250 & 0.36 & 0.22 & 0.06 & 0.98 & 0.33 \\
1 & 100 & 0.11 & 0.06 & 0.01 & 0.75 & 0.18 \\
1 & 250 & 0.09 & 0.05 & 0.01 & 0.74 & 0.08 \\
\hline
\end{tabular}

Note: DGP: $y_{t}=\theta y_{t-1}+e_{t}$. 
time series, the lag length selection is performed as follows. In the ADF tests, by working backward from $k=k_{\max }$, the first value of $k$ is selected such that the $t$-statistic on $\hat{\phi}_{k}$ is significant. Three significance levels- $10 \%, 5 \%$, and $1 \%$ - are applied in the case of the ADF unit-root tests and the $t$-tests for the lag length selection.

In the IC-based method, alternative models are considered by changing the lag length as $k=0,1, \ldots, k_{\max }$, and the best model is selected from $\left(1+k_{\max }\right)$ alternative models. Two values of $T(100,250)$ are considered, and each experiment is replicated 5,000 times.
Table 2 shows the frequency count of selecting stationary models. First, the size distortion does not occur, while it is well known that the incorrect lag length can cause the size distortion. As shown by NP, the general-tospecific procedure in selecting the lag length can prevent the size distortion. Second, the pretesting problem is not shown, similar to the results obtained by NP. Finally, the performances of the BIC-based method are roughly comparable to those of the ADF 5\% tests in the case of $T=100$ and to those of the ADF $1 \%$ tests in the case of $T=250$.

Table 2. Frequency count of selecting stationary models

\begin{tabular}{|c|c|c|c|c|c|c|c|c|}
\hline \multicolumn{4}{|c|}{ Parameters } & \multicolumn{5}{|c|}{ Methods } \\
\hline$\rho$ & $\phi_{1}$ & $\phi_{2}$ & $\phi_{3}$ & $10 \%$ & $5 \%$ & $1 \%$ & AIC & $\mathrm{BIC}$ \\
\hline \multicolumn{9}{|c|}{$T=100$} \\
\hline 0.85 & 0.6 & 0 & 0 & 0.99 & 0.98 & 0.97 & 1.00 & 0.97 \\
\hline 0.85 & -0.6 & 0 & 0 & 0.78 & 0.63 & 0.26 & 0.89 & 0.49 \\
\hline 0.85 & 0.4 & 0.2 & 0 & 0.98 & 0.97 & 0.90 & 0.99 & 0.95 \\
\hline 0.85 & 0.2 & 0.2 & 0.2 & 0.98 & 0.94 & 0.64 & 0.99 & 0.78 \\
\hline 0.95 & 0.6 & 0 & 0 & 0.86 & 0.76 & 0.43 & 0.94 & 0.66 \\
\hline 0.95 & -0.6 & 0 & 0 & 0.35 & 0.19 & 0.05 & 0.44 & 0.11 \\
\hline 0.95 & 0.4 & 0.2 & 0 & 0.84 & 0.68 & 0.27 & 0.92 & 0.51 \\
\hline 0.95 & 0.2 & 0.2 & 0.2 & 0.80 & 0.63 & 0.22 & 0.87 & 0.37 \\
\hline 1 & 0.6 & 0 & 0 & 0.12 & 0.05 & 0.01 & 0.20 & 0.03 \\
\hline 1 & -0.6 & 0 & 0 & 0.11 & 0.05 & 0.01 & 0.19 & 0.04 \\
\hline 1 & 0.4 & 0.2 & 0 & 0.11 & 0.06 & 0.01 & 0.19 & 0.04 \\
\hline 1 & 0.2 & 0.2 & 0.2 & 0.11 & 0.05 & 0.01 & 0.20 & 0.06 \\
\hline \multicolumn{9}{|c|}{$\mathrm{T}=250$} \\
\hline 0.85 & 0.6 & 0 & 0 & 1.00 & 1.00 & 1.00 & 1.00 & 1.00 \\
\hline 0.85 & -0.6 & 0 & 0 & 1.00 & 0.99 & 0.95 & 1.00 & 0.95 \\
\hline 0.85 & 0.4 & 0.2 & 0 & 1.00 & 1.00 & 1.00 & 1.00 & 1.00 \\
\hline 0.85 & 0.2 & 0.2 & 0.2 & 1.00 & 1.00 & 1.00 & 1.00 & 0.99 \\
\hline 0.95 & 0.6 & 0 & 0 & 1.00 & 1.00 & 0.98 & 1.00 & 0.96 \\
\hline 0.95 & -0.6 & 0 & 0 & 0.76 & 0.55 & 0.20 & 0.87 & 0.28 \\
\hline 0.95 & 0.4 & 0.2 & 0 & 1.00 & 1.00 & 0.96 & 1.00 & 0.96 \\
\hline 0.95 & 0.2 & 0.2 & 0.2 & 1.00 & 0.99 & 0.92 & 1.00 & 0.93 \\
\hline 1 & 0.6 & 0 & 0 & 0.11 & 0.05 & 0.01 & 0.20 & 0.02 \\
\hline 1 & -0.6 & 0 & 0 & 0.10 & 0.05 & 0.01 & 0.19 & 0.02 \\
\hline 1 & 0.4 & 0.2 & 0 & 0.11 & 0.05 & 0.01 & 0.19 & 0.02 \\
\hline 1 & 0.2 & 0.2 & 0.2 & 0.11 & 0.05 & 0.01 & 0.19 & 0.02 \\
\hline
\end{tabular}

Note: DGP: $y_{t}=\rho y_{t}+\sum_{i=1}^{3} \phi_{i} \Delta y_{t-i}+e_{i}$ 
The Case Where the Model Class as Well as the Lag Length is Unknown

In this phase, the model class (Models 1, 2 , and 3 in Section 1) as well as the lag length is unknown. Thus, the ADF tests should determine which model class is applied. No study has been devoted to this problem. Furthermore, there is little guidance from economic theory about the source and nature of the trending behavior. In the present study, therefore, the selection of the model class is performed based on statistical tests. Motivated by NP, general-to-specific modeling is performed in this subsection as follows. First, using Model 3, the ADF regression is performed with the NP lag length selection. If the $t$-statistic on $\hat{\beta}$ of the selected model is significant, Model 3 is obtained and the unit-root test is implemented. Otherwise, the ADF regression is performed using Model 2. If the $t$-statistic on $\hat{\mu}$ of the selected model is significant, Model 2 is obtained and the unit-root test is implemented. Otherwise, the ADF unitroot test is performed using Model 1. Three significance levels-10\%, 5\%, and $1 \%$-are applied in the case of the ADF unit-root tests, the $t$-tests for the lag length selection, and the $t$-tests for $\hat{\beta}$ and $\hat{\mu}$. If each test is independent at three stages and is evaluated at the $10 \%$ significance level, the overall rejection probability under the null is $1-(1-0.1)^{3}=0.271$, which is substantial.

In this simulation, artificial time series are generated using the following process:

$$
y_{t}=\mu+\beta t+\rho y_{t-1}+\sum_{i=1}^{3} \phi_{i} \Delta y_{t-i}+e_{t},
$$

where $e_{t} \sim \mathrm{NID}(0,1)$. In order to obtain the stationary Models $1-3$, the parameter vectors considered are $(\mu=0, \beta=0),(\mu=1, \beta=0)$, and $(\mu=0, \beta=0.1)$, respectively. With regard to the nonstationary models, the parameter vectors considered are $(\mu=0, \beta=0)$ and $(\mu=0.1, \beta=0)$. The other parameter setting is implemented as follows. Two values of $\rho-\rho=0.95,1$-are considered, and four vectors of $\left(\phi_{1}, \phi_{2}, \phi_{3}\right)$ - $\left(\phi_{1}, \phi_{2}, \phi_{3}\right)=(0.6,0,0),(-0.6,0,0),(0.4,0.2,0)$, $(0.2,0.2,0.2)$-are considered. The maximum lag length $k_{\max }$ is assumed to be $k_{\max }=8$. Two values of $T(100,250)$ are considered, and each experiment is replicated 5,000 times.

Table 3 shows the frequency count of selecting stationary models. Unlike in the preceding subsection, in this case, the pretesting problem is clearly shown. Consider the results of applying the $10 \%$ significance level for eight unit-root processes with $T=100$. The frequency count of incorrectly selecting stationary models is from $26 \%$ to $31 \%$. In the preceding subsection, it was shown that the lag length selection has little effect on the results of the unit-root tests under the assumption of the known model class. The size distortion is caused by the assumed method for selecting the model class. The selection of the model class with statistically significant deterministic components such as $\mu$ and $\beta$ has a bias toward selecting a stationary model. The same results are obtained in the case of $T=250$; however, in this case, the extent of size distortion is smaller. The frequency count of incorrectly selecting stationary models by the BIC-based method is from $12 \%$ to $19 \%$ in the case of $T=100$ and from $5 \%$ to $9 \%$ in the case of $T=250$. The performances of the BIC-based method are roughly comparable to those of the ADF 5\% tests in the case of $T=100$ and to those of the ADF 2.5\% tests (not shown here) in the case of $T=250$.

In particular, in the case of the trending process, it can be concluded that the BIC-based method outperforms the hypothesis-testing method. For example, compare the two cases of $\left(\rho, \mu, \beta, \phi_{1}, \phi_{2}, \phi_{3}\right)=(1,0.1,0,0.6,0,0) \quad$ and $(0.95,0,0.1,0.6,0,0)$ with $T=100$. In the case of the $10 \%$ significance level, the frequency counts of incorrectly and correctly selecting stationary models are 0.27 and 0.45 , respectively. On the other hand, in the case of the BIC-based method, the frequency counts of incorrectly and correctly selecting stationary models are 0.15 and 0.49 , respectively. In the terminology of hypothesis testing, the BICbased method shows lower size and higher 
Table 3. Frequency count of selecting stationary models

\begin{tabular}{|c|c|c|c|c|c|c|c|c|c|c|}
\hline \multicolumn{6}{|c|}{ Parameters } & \multicolumn{5}{|c|}{ Methods } \\
\hline$\rho$ & $\mu$ & $\beta$ & $\phi_{1}$ & $\phi_{2}$ & $\phi_{3}$ & $10 \%$ & $5 \%$ & $1 \%$ & AIC & $\mathrm{BIC}$ \\
\hline \multicolumn{11}{|c|}{$T=100$} \\
\hline 0.95 & 0 & 0 & 0.6 & 0 & 0 & 0.84 & 0.78 & 0.46 & 0.98 & 0.72 \\
\hline 0.95 & 0 & 0 & -0.6 & 0 & 0 & 0.45 & 0.29 & 0.07 & 0.76 & 0.18 \\
\hline 0.95 & 0 & 0 & 0.4 & 0.2 & 0 & 0.83 & 0.71 & 0.29 & 0.96 & 0.55 \\
\hline 0.95 & 0 & 0 & 0.2 & 0.2 & 0.2 & 0.80 & 0.66 & 0.25 & 0.94 & 0.40 \\
\hline 0.95 & 1 & 0 & 0.6 & 0 & 0 & 0.64 & 0.48 & 0.18 & 0.91 & 0.34 \\
\hline 0.95 & 1 & 0 & -0.6 & 0 & 0 & 0.29 & 0.17 & 0.04 & 0.65 & 0.08 \\
\hline 0.95 & 1 & 0 & 0.4 & 0.2 & 0 & 0.58 & 0.40 & 0.11 & 0.88 & 0.22 \\
\hline 0.95 & 1 & 0 & 0.2 & 0.2 & 0.2 & 0.55 & 0.36 & 0.10 & 0.84 & 0.15 \\
\hline 0.95 & 0 & 0.1 & 0.6 & 0 & 0 & 0.45 & 0.32 & 0.10 & 0.96 & 0.49 \\
\hline 0.95 & 0 & 0.1 & -0.6 & 0 & 0 & 0.36 & 0.24 & 0.09 & 1.00 & 1.00 \\
\hline 0.95 & 0 & 0.1 & 0.4 & 0.2 & 0 & 0.41 & 0.26 & 0.07 & 0.93 & 0.35 \\
\hline 0.95 & 0 & 0.1 & 0.2 & 0.2 & 0.2 & 0.39 & 0.24 & 0.06 & 0.91 & 0.27 \\
\hline 1 & 0 & 0 & 0.6 & 0 & 0 & 0.31 & 0.19 & 0.04 & 0.75 & 0.13 \\
\hline 1 & 0 & 0 & -0.6 & 0 & 0 & 0.30 & 0.17 & 0.04 & 0.72 & 0.12 \\
\hline 1 & 0 & 0 & 0.4 & 0.2 & 0 & 0.30 & 0.17 & 0.03 & 0.74 & 0.13 \\
\hline 1 & 0 & 0 & 0.2 & 0.2 & 0.2 & 0.28 & 0.16 & 0.04 & 0.76 & 0.16 \\
\hline 1 & 0.1 & 0 & 0.6 & 0 & 0 & 0.27 & 0.16 & 0.04 & 0.77 & 0.15 \\
\hline 1 & 0.1 & 0 & -0.6 & 0 & 0 & 0.26 & 0.15 & 0.03 & 0.74 & 0.14 \\
\hline 1 & 0.1 & 0 & 0.4 & 0.2 & 0 & 0.27 & 0.15 & 0.04 & 0.76 & 0.15 \\
\hline 1 & 0.1 & 0 & 0.2 & 0.2 & 0.2 & 0.26 & 0.14 & 0.04 & 0.78 & 0.19 \\
\hline \multicolumn{11}{|c|}{$\mathrm{T}=250$} \\
\hline 0.95 & 0 & 0 & 0.6 & 0 & 0 & 1.00 & 1.00 & 0.99 & 1.00 & 1.00 \\
\hline 0.95 & 0 & 0 & -0.6 & 0 & 0 & 0.73 & 0.57 & 0.21 & 0.94 & 0.31 \\
\hline 0.95 & 0 & 0 & 0.4 & 0.2 & 0 & 1.00 & 1.00 & 0.96 & 1.00 & 0.99 \\
\hline 0.95 & 0 & 0 & 0.2 & 0.2 & 0.2 & 0.99 & 0.99 & 0.92 & 1.00 & 0.95 \\
\hline 0.95 & 1 & 0 & 0.6 & 0 & 0 & 0.97 & 0.96 & 0.85 & 1.00 & 0.89 \\
\hline 0.95 & 1 & 0 & -0.6 & 0 & 0 & 0.41 & 0.25 & 0.07 & 0.81 & 0.07 \\
\hline 0.95 & 1 & 0 & 0.4 & 0.2 & 0 & 0.97 & 0.94 & 0.73 & 1.00 & 0.77 \\
\hline 0.95 & 1 & 0 & 0.2 & 0.2 & 0.2 & 0.97 & 0.92 & 0.67 & 1.00 & 0.70 \\
\hline 0.95 & 0 & 0.1 & 0.6 & 0 & 0 & 0.92 & 0.88 & 0.66 & 1.00 & 0.94 \\
\hline 0.95 & 0 & 0.1 & -0.6 & 0 & 0 & 1.00 & 1.00 & 0.99 & 1.00 & 1.00 \\
\hline 0.95 & 0 & 0.1 & 0.4 & 0.2 & 0 & 0.90 & 0.83 & 0.55 & 1.00 & 0.84 \\
\hline 0.95 & 0 & 0.1 & 0.2 & 0.2 & 0.2 & 0.89 & 0.80 & 0.50 & 1.00 & 0.79 \\
\hline 1 & 0 & 0 & 0.6 & 0 & 0 & 0.24 & 0.14 & 0.03 & 0.71 & 0.06 \\
\hline 1 & 0 & 0 & -0.6 & 0 & 0 & 0.24 & 0.14 & 0.03 & 0.70 & 0.05 \\
\hline 1 & 0 & 0 & 0.4 & 0.2 & 0 & 0.25 & 0.13 & 0.03 & 0.71 & 0.05 \\
\hline 1 & 0 & 0 & 0.2 & 0.2 & 0.2 & 0.25 & 0.15 & 0.03 & 0.73 & 0.06 \\
\hline 1 & 0.1 & 0 & 0.6 & 0 & 0 & 0.19 & 0.10 & 0.02 & 0.76 & 0.08 \\
\hline 1 & 0.1 & 0 & -0.6 & 0 & 0 & 0.18 & 0.10 & 0.02 & 0.74 & 0.07 \\
\hline 1 & 0.1 & 0 & 0.4 & 0.2 & 0 & 0.20 & 0.11 & 0.02 & 0.77 & 0.08 \\
\hline 1 & 0.1 & 0 & 0.2 & 0.2 & 0.2 & 0.19 & 0.11 & 0.02 & 0.76 & 0.09 \\
\hline
\end{tabular}

Note: DGP: $y_{t}=\mu+\beta t+\rho y_{t}+\sum_{i=1}^{3} \phi_{i} \Delta y_{t-i}+e_{t}$. 
power than the hypothesis-testing method. Similar results are obtained in other trending process of $(\rho=1, \mu=0.1) \quad$ and $(\rho=0.95, \beta=0.1)$.

\section{Conclusion}

This article focused on the two problems encountered in the conventional unit-root tests: the absence of a criterion for selecting a suitable model class and the presence of the pretesting problem. In order to provide a solution to these problems, the IC-based model selection method was proposed. In this method, alternative models with and without a unit root are considered by changing the model class and the lag length. All the possible models are estimated and the corresponding IC values are stored. Finally, the best model is selected from among the alternatives. Thus, on the basis of the selected model, it is determined whether the observed time series contain a unit root. The simulation results suggested that the performances of the BIC-based method are usually comparable to and sometimes better than those of the DFLR and ADF unit-root tests.

In comparison with the conventional hypothesis testing methods, this BIC-based model selection method has two advantages. First, by the introduction of the minimum BIC procedure, the subjective judgment required in the hypothesis testing procedure for determining the levels of significance is completely eliminated, thus enabling a semiautomatic execution. The well-known criticism of the ICbased method is that it cannot control the test size. However, as shown in Table 3, the conventional hypothesis testing method causes the pretesting problem and cannot control the overall test size.

Second, the selection of the model class can be performed automatically and consistently using the IC-based method. Furthermore, flexible time-series modeling, such as the introduction of measurement error (Fukuda, 2005a) and/or regime switching (Fukuda, 2005b), is applicable in the proposed method, and the efficacy of a model change can be consistently evaluated via the minimum BIC procedure. In the case of hypothesis testing, different models require different statistics; this makes time-series analyses very complex.

\section{References}

Akaike, H. (1974). A new look at the statistical model identification. IEEE Transactions on Automatic Control AC-19, 716723.

Dickey, D. A. \& Fuller, W. A. (1979). Distribution of the estimators for autoregressive time series with a unit root. Journal of the American Statistical Association, 74, 427-431.

Dickey, D. A. \& Fuller, W. A. (1981). Likelihood ratio statistics for autoregressive time series with a unit root. Econometrica, 49, 1057-1072.

Elliot, G., Rothnberg, T. J., \& Stock, J. H. (1996). Efficient tests for an autoregressive unit root. Econometrica, 64, 813-836.

Fukuda, K. (2005a). Unit-root detection allowing for measurement error. Statistics and Probability Letters, 74, 373-377.

Fukuda, K. (2005b). Detection of regime switches between stationary and nonstationary processes and economic forecasting. Journal of Forecasting, 24, 255267.

Krolzig, H.-M. \& Hendry, D.F. (2001). Computer automation of general-to-specific model selection procedures. Journal of Economic Dynamics and Control, 25, 831-866.

Nelson, C. R. \& Plosser, C. I. (1982). Trends and random walks in macroeconomic time series: some evidence and implications. Journal of Monetary Economics, 10, 139-162.

Ng, S. \& Perron, P. (1995). Unit root tests in ARMA models with data dependent methods for the selection of the truncation lag. Journal of the American Statistical Association, 90, 268-281.

Phillips, P. C. B. (2005). Challenges of trending time series econometrics. Mathematics and Computers in Simulation, 68, 401-416.

Schwarz, G. (1978). Estimating the dimension of a model. Annals of Statistics 6, 461-464. 


\title{
A Fano-Huffman Based Statistical Coding Method
}

\author{
Aladdin Shamilov Senay Asma \\ Anadolu University, Turkey
}

Statistical coding techniques have been used for lossless statistical data compression, applying methods such as Ordinary, Shannon, Fano, Enhanced Fano, Huffman and Shannon-Fano-Elias coding methods. A new and improved coding method is presented, the Fano-Huffman Based Statistical Coding Method. It holds the advantages of both the Fano and Huffman coding methods. It is more easily applicable than the Huffman coding methods and it is more optimal than Fano coding method. The optimality with respect to the other methods is realized on the basis of English, German, Turkish, French, Russian and Spanish.

Key words: Fano-Huffman based statistical coding method, probability distribution of language, entropy, information, optimal code.

\section{Introduction}

Problem Statement

Huffman's algorithm is a well-known encoding method that generates an optimal prefix encoding scheme, in the sense that the average code word length is minimum. As opposed to this, Fano's method has not been used so much because it generates prefix encoding schemes that can be sub-optimal (Rueda \& Oommen, 2004).

In this article, an improved coding method is presented, which has been named the Fano-Huffman Based Statistical Coding method and applications of this method. This method holds the both advantages of Fano and Huffman coding method. So, it is more easily applicable than the Huffman coding method and is more optimal than Fano coding method. The optimality of the mentioned coding method with

Aladdin Shamilov is a Professor in the Department of Statistics. Email: asamilov@anadolu.edu.tr. Senay Asma is a Research Assistant in the Department of Statistics. Email: senayyolacan@anadolu.edu.tr. respect to the other coding methods is realized on the basis of English, German, Turkish, French, Russian and Spanish.

The classical coding methods and the concept of optimality are described in the section titled Classical Coding Methods and Optimality.

An improved coding method, FanoHuffman Based Coding Method by which encoding schemes, which are arbitrarily close to the optimum, can be easily constructed, is introduced in the section called Fano-Huffman Based Statistical Coding Method.

In the following section, the tables of constructed binary codes are given and comparisons of considered methods in sense of optimality are made.

In the conclusion, the interpretation of optimality of these results is made subject to classical coding methods and suggestions are given.

\section{Overview}

Assume that a source alphabet, $S=\left\{s_{1}, s_{2}, \ldots s_{n}\right\}$, whose probabilities of occurrence are $\mathrm{P}=\left\{\mathrm{p}_{1}, \mathrm{p}_{2}, \ldots, \mathrm{p}_{\mathrm{n}}\right\}$, and a code alphabet, $A=\left\{a_{1}, a_{2}, \ldots a_{r}\right\}$ is given. The propose of this study is the generation of an encoding scheme, $\left\{s_{i} \rightarrow w_{i}\right\}$, in such a way 
that $\bar{l}=\sum_{i=1}^{n} p_{i} l_{i}$ is minimized, where $l_{i}$ is the length of $w_{i}$.

Information theory has important applications in probability theory, statistics and communication systems. Lossless encoding methods used to solve this problem include Huffman's algorithm (Huffman, 1952), Shannon's method (Shannon \& Weaver, 1949), arithmetic coding (Sayood, 2000), Fano's method (Hankerson, Harris, \& Johnson, 1998), enhanced Fano-based coding algorithm (Rueda $\&$ Oomen, 2004) etc. Adaptive versions of these methods have been proposed, and can be found in (Faller, 1973; Gallager, 1978; Hankerson et al., 1998; Knuth, 1985; Rueda, 2002; Sayood, 2000). The survey is necessarily brief as this is a well-reputed field.

Also, assume that the source is memoryless or zeroth-order, which means that the occurrence of the next symbol is independent of any other symbol that has occurred previously. Higher-order models include Markov models (Hankerson et al., 1998), dictionary techniques (Ziv \& Lempel, 1977; Ziv \& Lempel, 1978), prediction with partial matching (Witten, Moffat, \& Bell, 1999), grammar based compression (Kieffer \& Yang, 2000), etc., and the techniques introduced here are also readily applicable for such structure models.

\section{Classical Coding Methods and Optimality}

In this section, the fundamental steps of classical coding methods are described and the concept of optimality of codes is expounded.

\section{Classical coding methods}

Suppose that source alphabet (alphabet of language) $\mathrm{S}=\left\{\mathrm{S}_{1}, \mathrm{~S}_{2}, \ldots, \mathrm{S}_{\mathrm{n}}\right\}$ and its probability distribution $\mathrm{P}=\left\{\mathrm{p}_{1}, \mathrm{p}_{2}, \ldots, \mathrm{p}_{\mathrm{n}}\right\}$ are given.

\section{Ordinary Coding Method}

This method requires the following steps:

(a) Determine number $\ell$ satisfying the inequality $\ell \geq \log _{2} \mathrm{~N}$, where $\ell$ is the length of codeword and $\mathrm{N}$ is the the number of symbols in source alphabet;

(b) frequency;

Enumerate letter ignoring the

(c)

Convert numbers determined by

(b) from base 10 to base 2 such that $\ell$ is the length of converted number (Roman, 1997).

\section{Shannon Coding Method \\ Construction of Shannon Codes is provided by steps:} Put $\quad P=\left\{p_{1}, p_{2}, \ldots, p_{n}\right\} \quad$ in ascending order $p_{1} \geq p_{2} \geq \ldots \geq p_{n}$; Calculate $\ell_{\mathrm{i}}=\left\lceil\log _{2} \mathrm{p}_{\mathrm{i}}^{-1}\right\rceil$ the length of codeword, $i=1,2, \ldots, n$; $\mathrm{F}_{1}=0 \quad$ and $\quad \mathrm{F}_{\mathrm{k}}=\sum_{\mathrm{i}=1}^{\mathrm{k}-1} \mathrm{p}_{\mathrm{i}}, \quad 2 \leq \mathrm{k} \leq \mathrm{n}$. Then calculate $F_{i}, i=1,2, \ldots, n$; Convert dyadic fraction $F_{i}$ to binary form by using Koblitz's trick, then select first $\ell_{\mathrm{i}}$ bits as a code corresponding to $\mathrm{s}_{\mathrm{i}}$ (Hankerson et. al., 2003).

Fano Coding Method This method involves the steps:

(a) Perform the probabilities of symbols in source alphabet in ascending order $\mathrm{p}_{1} \geq \mathrm{p}_{2} \geq \ldots \geq \mathrm{p}_{\mathrm{n}}$

(b)

Divide the set of symbols into two subsets such that the sum of the probabilities of occurrences of symbols in each subset are equal or almost equal. Then, assign a 0 to first subset and a 1 to second;

(c) Repeat step (a) until all subsets have a single element (Венцель, 1969). 
Enhanced Fano Coding Method steps:

This method proposed the following

(a) Consider the source alphabet $\mathrm{S}=\left\{\mathrm{s}_{1}, \mathrm{~s}_{2}, \ldots, \mathrm{s}_{\mathrm{n}}\right\} \quad$ whose probability distribution of occurrences is $\mathrm{P}=\left\{\mathrm{p}_{1}, \mathrm{p}_{2}, \ldots, \mathrm{p}_{\mathrm{n}}\right\}$, where $\mathrm{p}_{1} \geq \mathrm{p}_{2} \geq \ldots \geq \mathrm{p}_{\mathrm{n}} ;$

(b) Obtain $\phi: \mathrm{s}_{1} \rightarrow \mathrm{w}_{1}, \ldots, \mathrm{s}_{\mathrm{n}} \rightarrow \mathrm{w}_{\mathrm{n}}$ the encoding scheme by Fano's method;

(c) Rearrange $\mathrm{w}_{1}, \mathrm{w}_{2}, \ldots, \mathrm{w}_{\mathrm{n}}$ into $\mathrm{w}_{1}^{\prime}, \mathrm{w}_{2}^{\prime}, \ldots, \mathrm{w}_{\mathrm{n}}^{\prime}$ such that $\ell_{\mathrm{i}}^{\prime} \leq \ell_{\mathrm{j}}^{\prime}$ for all $\mathrm{i}<\mathrm{j}$, and simultaneously maintain $\mathrm{s}_{1}, \mathrm{~s}_{2}, \ldots, \mathrm{s}_{\mathrm{n}}$ in the same order, to yield the encoding scheme: $\phi^{\prime}: \mathrm{s}_{1} \rightarrow \mathrm{w}_{1}^{\prime}, \ldots, \mathrm{s}_{\mathrm{n}} \rightarrow \mathrm{w}_{\mathrm{n}}^{\prime} \quad$ (Rueda \& Oommen, 2004).

\section{Huffman Coding Method}

This method is bottom-up while the others are top-down. It can be explained more clearly as follows:

(a) Sort symbols of source alphabet in decreasing order of their probabilities;

(b) Merge the two least-probable letter into a single output whose probability is the sum of the corresponding probabilities;

(c) Go to step (a) if the number of remaining outputs is more than 2 ;

(d) Assign a 0 and a 1 arbitrarily as code words for the two remaining outputs;

(e) Append the current codeword with a 0 and a 1 to obtain the codeword the preceding outputs and repeat step (e) if an output is the result of the merger of two outputs in a preceding step. Stop if no output is preceded by another output in a preceding step (Aazhang, 2004).

Shannon-Fano-Elias Coding Method

This method can be explained by steps: (a) Perform the source alphabet $\mathrm{S}=\left\{\mathrm{S}_{1}, \mathrm{~s}_{2}, \ldots, \mathrm{S}_{\mathrm{n}}\right\} \quad$ whose probability distribution of occurrences is $\mathrm{P}=\left\{\mathrm{p}_{1}, \mathrm{p}_{2}, \ldots, \mathrm{p}_{\mathrm{n}}\right\}$ and the order of probabilities isn't important;

(b) Obtain the cumulative distribution by the function $\mathrm{F}(\mathrm{s})=\sum_{\mathrm{a} \leq \mathrm{s}} \mathrm{p}(\mathrm{a})$;

(c) Consider modified cumulative distribution function $\overline{\mathrm{F}}(\mathrm{s})=\sum_{\mathrm{a}<\mathrm{s}} \mathrm{p}(\mathrm{a})+\frac{1}{2} \mathrm{p}(\mathrm{s})$, where $\overline{\mathrm{F}}(\mathrm{s})$ denotes the sum of probabilities of all symbols less than s plus half the probability of the symbols;

(d) Obtain the length of codeword by the formula $\ell_{\mathrm{i}}(\mathrm{s})=\left\lceil\log \frac{1}{\mathrm{p}(\mathrm{s})}\right\rceil+1$, where $\lceil$. denotes rounding up;

(e) Convert dyadic fraction $\overline{\mathrm{F}}(\mathrm{s})$ to binary form by using Koblitz's trick such that the codeword has $\ell_{\mathrm{i}}(\mathrm{s})$ bits (Cover \& Thomas, 1991).

The concept of optimality of codes There exists a uniquely decodable code whose codeword lengths are given by the sequence $\left\{1_{i}\right\}_{i=1}^{n}$ if Kraft inequality $\sum_{i=1}^{n} 2^{-l_{i}} \leq 1$ holds. Due to Kraft inequality (Cover, 1991), the conditions for optimal codes are as follows:

(a) The average codeword length $\bar{\ell}=\sum_{i=1}^{n} p_{i} \ell_{i}$ of an optimal code for a source $\mathrm{S}$ is greater than or equal to its entropy $H(S)=-\sum_{i=1}^{n} p_{i} \log _{2} p_{i}$; 
(b) The average codeword length $\bar{\ell}$ of an optimal code for a source $\mathrm{S}$ is strictly less than $\mathrm{H}(\mathrm{S})+1$.

For source alphabet $S=\left\{\mathrm{s}_{1}, \mathrm{~s}_{2}, \ldots, \mathrm{s}_{\mathrm{n}}\right\}$ whose probability distribution of occurrences is $\mathrm{P}=\left\{\mathrm{p}_{1}, \mathrm{p}_{2}, \ldots, \mathrm{p}_{\mathrm{n}}\right\}$, the average codeword length is given by $\bar{\ell}$, and entropy of the source alphabet is given by $H(S)$.

Under these conditions, it is required to transmit as well as possible information by using codes consists of fewer bits. So, this problem can be considered as optimization problem which is consist of minimizing $\bar{\ell}=\sum_{\mathrm{i}=1}^{\mathrm{n}} \mathrm{p}_{\mathrm{i}} \ell_{\mathrm{i}}$

subject to constraint $\sum_{i=1}^{n} D^{-l_{i}} \leq 1$, where $\mathrm{D}$ is dimension of codebook, i.e. if the codebook is $\{0,1\}$ then $\mathrm{D}=2$ etc.

This problem is solved by using Langrange Multipliers, and the following result is obtained:

$$
\begin{gathered}
l_{i}^{*}=-\log _{D} p_{i} \\
\bar{l}=\sum_{i=1}^{n} p_{i} l_{i}^{*}=-\sum_{i=1}^{n} p_{i} \log _{D} p_{i}=H_{D}(S) \\
\bar{l}=H_{D}(S) .
\end{gathered}
$$

But it isn't possible to find an interger number for codeword length that satisfies (2.1). For this reason, it is necessary to obtain the entropy lower bound (Cover \& Thomas, 1991; Roman, 1997) satisfying the following inequality:

$$
\bar{l}=\sum_{i=1}^{n} p_{i} l_{i}^{*} \geq H_{D}(S)
$$

Moreover, if $\mathrm{S}$ is a stationary stochastic process,

$$
\bar{l} \rightarrow H(S),
$$

where $\mathrm{H}(\mathrm{S})$ is the entropy rate of the process.

Under the mentioned knowledge, the information per symbol (letter) is given by $I_{\mathrm{inf} / \text { letter }}=\frac{H(S)}{\bar{\ell}}$ and the optimality criteria for codes is considered as $I_{\mathrm{inf} / \text { letter }} \rightarrow 1$ (Венцель, 1969). Moreover, the optimality means that if the text is coded by an optimal coding method, the number of $1 \mathrm{~s}$ and the number of $0 \mathrm{~s}$ are nearly equal in sence of maximum entropy. Hence, the optimal codes means that they transmit nearly maximum information since $1 \mathrm{~s}$ and 0 s aren't always equal probable.

Fano-Huffman Based Statistical Coding Method In this section, a new and improved coding method is proposed, which can be considered as a hybrid method that holds the both advantages of Fano and Huffman coding methods.

It is well known that Fano coding method is a suboptimal procedure for constructing a source code (Rueda \& Oommen, 2004). In this method, the source symbols and their probabilities are sorted in a non-increasing order of the probabilities and then the set of symbols is divided into two subsets such that the sum of the probabilities of occurrences of symbols in each subset are equal or almost equal. The main advantage of this method is the division of the set of symbols. Because, it requires pure computations. Hence, the first goal of the improved coding method is to hold this advantage.

Huffman coding method is a optimal procedure (Cover \& Thomas, 1991). In this method, the source symbols and their probabilities are also sorted in decreasing order and then the two least-probable symbols are merged into a single output whose probability is the sum of the corresponding probabilities. Thus, by this recursive procedure, the optimal Huffman codes are constructed. The advantage of this coding method is that the procedure is from bottom to top. In this way, the short code 
words are attain to the symbols that occur frequently and long code words are attain to the symbols that occur rarely. This advantage of Huffman coding method constitutes the second goal of the improved coding method.

Considering the advantages of these two coding procedure a hybrid coding method is presented. So, the coding method is more easily applicable than the Huffman coding methods and is more optimal than Fano coding method. The codes performed by that coding method are prefix codes and satisfy the sibling property.

The Fano-Huffman based statistical coding method is now proposed in the following form:

(a) Perform the probabilities of symbols in source alphabet in ascending order $\mathrm{p}_{1} \geq \mathrm{p}_{2} \geq \ldots \geq \mathrm{p}_{\mathrm{n}}$

(b) Choose k such that $\left|\sum_{i=1}^{k} p_{i}-\sum_{i=k+1}^{m} p_{i}\right|$ is minimized. This number $\mathrm{k}$ divides the source symbols into two sets of almost equal probability.

(c) Merge the two least-probable letter in each set into a single output whose probability is the sum of the corresponding probabilities;

(d) Go to step (c) if the number of remaining outputs is more than 2 ;

(e) Assign a 0 and a 1 arbitrarily as codewords for the two remaining outputs; (f) Append the current codeword with a 0 and a 1 to obtain the codeword the preceding outputs and repeat step (e) If no output is preceded by another output in a preceding step merge the two least-probable subset into a single output whose probability is the sum of the corresponding probabilities;

(g) Stop if no output is preceded by another output in a preceding step.

Note that, according to step (b) due to size of source alphabet, the set of symbols can be divided into more subsets $\left(2^{\mathrm{n}}, \mathrm{n}=1,2, \ldots\right)$ of equal or almost equal probabilities.

The advantages of the proposed method arise from the comparisons of this method with the other aforesaid coding methods. The applications of this method and comparisons are given in the following section.

Tables, Computational Details and Comparisons

In this section, in order to indicate the advantages of our proposed method, FanoHuffman Based statistical coding method, we compare it with the traditional coding methods. Various binary codes for English, German, Turkish, French, Russian and Spanish symbols are constructed in sense of optimality.

French, German, Spanish and English symbols (letters) are the Latin characters consisting of 26 letters which are given in Table $1 \mathrm{a}$.

The probabilities of French, German and Spanish symbols (letters) were established in 1939 by Fletcher Pratt (Stephens, 2002; Pratt, 1939), the probabilities of English symbols (letters) were established by Nam Phamdo (2001) and they are given in Table $1 \mathrm{~b}$.

Table 1a. French, German, Spanish and English Symbols

$\begin{array}{lllllllllllll}\text { A } & \text { B } & \text { C } & \text { D } & \text { E } & \text { F } & \text { G } & \text { H } & \text { I } & \text { J } & \text { K } & \text { L } & \text { M } \\ \text { a } & \text { B } & \text { c } & \text { d } & \text { e } & \text { F } & \text { g } & \text { h } & \text { i } & \text { j } & \text { k } & \text { l } & \text { m } \\ & & & & & & & & & & & & \\ \text { N } & \text { O } & \text { P } & \text { Q } & \text { R } & \text { S } & \text { T } & \text { U } & \text { V } & \text { W } & \text { X } & \text { Y } & \text { Z } \\ \text { n } & \text { O } & \text { p } & \text { q } & \text { r } & \text { s } & \text { t } & \text { u } & \text { v } & \text { w } & \text { X } & \text { y } & \text { Z }\end{array}$


Table 1b. Probabilities of French, German, Spanish and English Symbols

\begin{tabular}{lllll}
\hline Symbols & English & French & German & Spanish \\
\hline A & 0.065174 & 0.08147 & 0.06506 & 0.12529 \\
B & 0.012425 & 0.00876 & 0.02566 & 0.01420 \\
C & 0.021734 & 0.03063 & 0.02837 & 0.04679 \\
D & 0.034984 & 0.04125 & 0.05414 & 0.05856 \\
E & 0.104144 & 0.17564 & 0.16693 & 0.13676 \\
F & 0.019788 & 0.00959 & 0.02044 & 0.00694 \\
G & 0.015861 & 0.01051 & 0.03647 & 0.01006 \\
H & 0.049289 & 0.00721 & 0.04064 & 0.00704 \\
I & 0.055809 & 0.07559 & 0.07812 & 0.06249 \\
J & 0.000903 & 0.00598 & 0.00191 & 0.00443 \\
K & 0.005053 & 0.00041 & 0.01879 & 0.00004 \\
L & 0.033149 & 0.05783 & 0.02825 & 0.04971 \\
M & 0.020212 & 0.02990 & 0.03005 & 0.03150 \\
N & 0.056451 & 0.07322 & 0.09905 & 0.06712 \\
O & 0.059630 & 0.05289 & 0.02285 & 0.08684 \\
P & 0.013765 & 0.02980 & 0.00944 & 0.02505 \\
Q & 0.000861 & 0.01361 & 0.00055 & 0.00875 \\
R & 0.049756 & 0.06291 & 0.06539 & 0.06873 \\
S & 0.051576 & 0.08013 & 0.06765 & 0.07980 \\
T & 0.072936 & 0.07353 & 0.06742 & 0.04629 \\
U & 0.022513 & 0.05991 & 0.03703 & 0.03934 \\
V & 0.008290 & 0.01557 & 0.01069 & 0.00895 \\
W & 0.017127 & 0.00020 & 0.01396 & 0.00023 \\
X & 0.001369 & 0.00350 & 0.00022 & 0.00221 \\
Y & 0.014598 & 0.00116 & 0.00032 & 0.00895 \\
Z & 0.000784 & 0.00072 & 0.01002 & 0.00523 \\
\# & 0.191818 & - & - & - \\
\hline & & & &
\end{tabular}

Turkish Source Alphabet consists of 29 symbols (letters). The capital and small letters of the Turkish Alphabet are given in Table 2a.

Probabilities of occurrence of Turkish symbols (letters) are given in Table 2b (Shamilov \& Yolacan, 2005; Dalkilic \& Dalkilic, 2002). Considered probabilities have been constituted from a corpus consist of words from many variety of fields, i. e. scientific articles, newspapers, poetics etc., 12.5 million characters in total.

Russian uses Cyrillic alphabet consisting of 32 symbols (letters) which are given in Table 3a. Probabilities of Russian symbols are given in Table 3b., where \# denotes the space symbol (Венцель, 1969; Yaglom \& Yaglom, 1966). 
Table 2a. Turkish Source Alphabet

$\begin{array}{lllllllllllllll}\text { A } & \text { B } & \text { C } & \text { Ç } & \text { D } & \text { E } & \text { F } & \text { G } & \breve{G} & \text { H } & \text { I } & \text { I } & \text { J } & \text { K } & \\ \text { a } & \text { b } & \text { C } & \text { ç } & \text { d } & \text { e } & \text { f } & \text { g } & \breve{g} & \text { h } & 1 & \text { i } & \text { j } & \text { k } & \\ & & & & & & & & & & & & & & \\ \text { L } & M & N & O & O ̈ & \text { P } & \text { R } & \text { S } & \text { Ş } & \text { T } & \text { U } & \text { Ü } & \text { V } & \text { Y } & \text { Z } \\ 1 & \text { m } & \text { N } & \text { o } & \ddot{O} & \text { p } & \text { r } & \text { s } & \text { Ş } & \text { t } & 1 & \text { ü } & \text { v } & \text { y } & \text { Z }\end{array}$

Table 2b. Probabilities of Turkish Symbols

\begin{tabular}{llllll}
\hline Letter & Frequency & Letter & Frequency & Letter & Frequency \\
\hline $\mathrm{A}$ & 0.1026 & $\mathrm{I}$ & 0.0444 & $\mathrm{R}$ & 0.0604 \\
$\mathrm{~B}$ & 0.0237 & I & 0.0723 & $\mathrm{~S}$ & 0.0264 \\
$\mathrm{C}$ & 0.0084 & $\mathrm{~J}$ & 0.0003 & $\mathrm{~S}$ & 0.0157 \\
$\mathrm{C}$ & 0.0102 & $\mathrm{~K}$ & 0.0407 & $\mathrm{~T}$ & 0.0287 \\
$\mathrm{D}$ & 0.0400 & $\mathrm{~L}$ & 0.0530 & $\mathrm{U}$ & 0.0284 \\
$\mathrm{E}$ & 0.0782 & $\mathrm{M}$ & 0.0320 & Ü & 0.0171 \\
$\mathrm{~F}$ & 0.0038 & $\mathrm{~N}$ & 0.0633 & $\mathrm{~V}$ & 0.0087 \\
$\mathrm{G}$ & 0.0114 & $\mathrm{O}$ & 0.0214 & $\mathrm{Y}$ & 0.0295 \\
$\breve{G}$ & 0.0092 & Ö & 0.0074 & $\mathrm{Z}$ & 0.0130 \\
$\mathrm{H}$ & 0.0096 & $\mathrm{P}$ & 0.0073 & $\#$ & 0.1329 \\
\hline
\end{tabular}

Table 3a. Russian Symbols (Cyrillic alphabet)
$\begin{array}{lllllllllllllll}\text { А } & Б & \text { В } & \text { Г } & \text { Д } & \text { Е } & Ж & 3 & И & \breve{~} & \text { К } & Л & \text { M } & \text { Н } & \text { О }\end{array}$

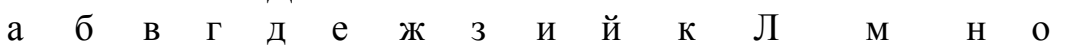

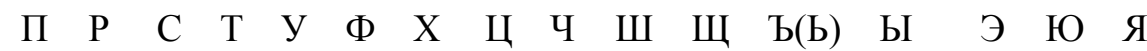

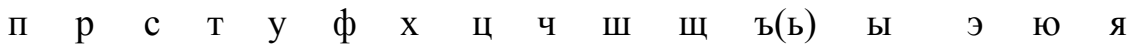

Table 3b. Probabilities of Russian Symbols

\begin{tabular}{|c|c|c|c|}
\hline Symbols & Probabilities & Symbols & Probabilities \\
\hline A & 0.064 & $\mathrm{P}$ & 0.041 \\
\hline Б & 0.015 & $\mathrm{C}$ & 0.047 \\
\hline B & 0.039 & $\mathrm{~T}$ & 0.056 \\
\hline$\Gamma$ & 0.014 & $\mathrm{y}$ & 0.021 \\
\hline Д & 0.026 & $\Phi$ & 0.002 \\
\hline E & 0.074 & X & 0.009 \\
\hline Ж & 0.008 & Ц & 0.004 \\
\hline 3 & 0.015 & Ч & 0.013 \\
\hline И & 0.064 & Ш & 0.006 \\
\hline Й & 0.010 & Щ & 0.003 \\
\hline K & 0.029 & $\mathrm{~b}(\mathrm{~b})$ & 0.015 \\
\hline Л & 0.036 & Ы & 0.016 \\
\hline M & 0.026 & $Э$ & 0.003 \\
\hline $\mathrm{H}$ & 0.056 & Ю & 0.007 \\
\hline $\mathrm{O}$ & 0.095 & Я & 0.019 \\
\hline$\Pi$ & 0.024 & \# & 0.145 \\
\hline
\end{tabular}


In order to construct binary codes for English, German, Turkish, French, Russian and Spanish, the classical coding methods are applied to considered source alphabets. Consequently, the constructed binary codes are given respectively in Tables 4-9. Moreover, Fano-Huffman Based statistical coding method is also applied to considered languages. Binary Codes constructed by Fano-Huffman based statistical coding are given in Table 10.

Table 4 Binary Codes for Probability Distrubution of English Symbols

\begin{tabular}{|c|c|c|c|c|c|c|c|c|}
\hline $\begin{array}{l}\text { English } \\
\text { Alphabet }\end{array}$ & No & $\begin{array}{l}\text { Ordinary } \\
\text { Codes }\end{array}$ & $\begin{array}{l}\text { S-F-E } \\
\text { Codes }\end{array}$ & $\begin{array}{l}\text { Ordered } \\
\text { Alphabet }\end{array}$ & $\begin{array}{l}\text { Shannon } \\
\text { Codes }\end{array}$ & Fano Codes & $\begin{array}{l}\text { Enhanced } \\
\text { Fano } \\
\text { Codes }\end{array}$ & $\begin{array}{l}\text { Huffman } \\
\text { Codes }\end{array}$ \\
\hline A & 0 & 00000 & 00001 & \# & 000 & 000 & 000 & 001 \\
\hline B & 1 & 00001 & 00010010 & E & 0011 & 001 & 001 & 100 \\
\hline $\mathrm{C}$ & 2 & 00010 & 0001011 & $\mathrm{~T}$ & 0100 & 010 & 010 & 0101 \\
\hline $\mathrm{D}$ & 3 & 00011 & 000111 & A & 0101 & 0110 & 0110 & 0011 \\
\hline $\mathrm{E}$ & 4 & 00100 & 00101 & $\mathrm{O}$ & 01101 & 0111 & 0111 & 0111 \\
\hline $\mathrm{F}$ & 5 & 00101 & 0011111 & $\mathrm{~N}$ & 01111 & 1000 & 1000 & 0000 \\
\hline G & 6 & 00110 & 0100010 & I & 10001 & 1001 & 1001 & 1000 \\
\hline $\mathrm{H}$ & 7 & 00111 & 010011 & $\mathrm{~S}$ & 10011 & 1010 & 1010 & 0010 \\
\hline I & 8 & 01000 & 010110 & $\mathrm{R}$ & 10100 & 10110 & 10110 & 1010 \\
\hline $\mathrm{J}$ & 9 & 01001 & 011000010011 & $\mathrm{H}$ & 10110 & 10111 & 10111 & 0110 \\
\hline $\mathrm{K}$ & 10 & 01010 & 011000011 & $\mathrm{D}$ & 11000 & 11000 & 11000 & 01101 \\
\hline $\mathrm{L}$ & 11 & 01011 & 011001 & $\mathrm{~L}$ & 11001 & 11001 & 11001 & 01011 \\
\hline M & 12 & 01100 & 0110110 & $\mathrm{U}$ & 110100 & 11010 & 11010 & 01110 \\
\hline $\mathrm{N}$ & 13 & 01101 & 011101 & $\mathrm{C}$ & 110110 & 110111 & 11100 & 11110 \\
\hline $\mathrm{O}$ & 14 & 01110 & 100001 & M & 110111 & 110110 & 110110 & 011101 \\
\hline $\mathrm{P}$ & 15 & 01111 & 10001111 & $\mathrm{~F}$ & 111000 & 11100 & 110111 & 111101 \\
\hline Q & 16 & 10000 & 100100011001 & W & 111001 & 111010 & 111010 & 011011 \\
\hline $\mathrm{R}$ & 17 & 10001 & 100110 & G & 111011 & 111011 & 111011 & 001111 \\
\hline $\mathrm{S}$ & 18 & 10010 & 101001 & Y & 1111000 & 111100 & 111100 & 101111 \\
\hline $\mathrm{T}$ & 19 & 10011 & 10110 & $\mathrm{P}$ & 1111010 & 111101 & 111101 & 011111 \\
\hline $\mathrm{U}$ & 20 & 10100 & 1100000 & B & 1111011 & 111110 & 111110 & 111111 \\
\hline $\mathrm{V}$ & 21 & 10101 & 11000101 & $\mathrm{~V}$ & 1111101 & 1111110 & 1111110 & 0111011 \\
\hline W & 22 & 10110 & 1100100 & $\mathrm{~K}$ & 11111100 & 11111110 & 11111110 & 01111011 \\
\hline $\mathrm{X}$ & 23 & 10111 & 110001010110 & $\mathrm{X}$ & 1111111000 & 111111110 & 111111110 & 011111011 \\
\hline $\mathrm{Y}$ & 24 & 11000 & 11001100 & $\mathrm{~J}$ & 11111110100 & 1111111110 & 1111111110 & 1011111011 \\
\hline Z & 25 & 11001 & 110011101100 & Q & 11111110110 & 11111111110 & 11111111110 & 0111111011 \\
\hline \# & 26 & 11010 & 1110 & Z & 11111110111 & 11111111111 & 11111111111 & 1111111011 \\
\hline
\end{tabular}


Table 5. Binary Codes for Probability Distrubution of German Symbols

\begin{tabular}{lllllllll}
\hline $\begin{array}{l}\text { German } \\
\text { Alphabet }\end{array}$ & No & $\begin{array}{l}\text { Ordinary } \\
\text { Codes }\end{array}$ & $\begin{array}{l}\text { S-F-E } \\
\text { Codes }\end{array}$ & $\begin{array}{l}\text { Ordered } \\
\text { Alphabet }\end{array}$ & $\begin{array}{l}\text { Shannon } \\
\text { Codes }\end{array}$ & Fano Codes & $\begin{array}{l}\text { Enhanced } \\
\text { Fano } \\
\text { Codes }\end{array}$ & $\begin{array}{l}\text { Huffman } \\
\text { Codes }\end{array}$ \\
\hline A & 0 & 00000 & 00001 & E & 000 & 000 & 000 & 000 \\
B & 1 & 00001 & 0001001 & N & 0010 & 001 & 001 & 001 \\
C & 2 & 00010 & 0001101 & I & 0100 & 010 & 010 & 0100 \\
D & 3 & 00011 & 001001 & S & 0101 & 0110 & 0110 & 0010 \\
E & 4 & 00100 & 0100 & T & 0110 & 0111 & 0111 & 1010 \\
F & 5 & 00101 & 0101100 & R & 0111 & 1000 & 1000 & 0110 \\
G & 6 & 00110 & 011000 & A & 1000 & 1001 & 1001 & 0011 \\
H & 7 & 00111 & 011010 & D & 10011 & 1010 & 1010 & 0111 \\
I & 8 & 01000 & 01111 & H & 10101 & 10110 & 10110 & 0101 \\
J & 9 & 01001 & 10000100010 & U & 10110 & 10111 & 10111 & 01100 \\
K & 10 & 01010 & 1000011 & G & 10111 & 11000 & 11000 & 01110 \\
L & 11 & 01011 & 1000110 & M & 110001 & 11001 & 11001 & 11110 \\
M & 12 & 01100 & 1001010 & $\mathrm{C}$ & 110011 & 11010 & 11010 & 01011 \\
N & 13 & 01101 & 10100 & L & 110101 & 11011 & 11011 & 11011 \\
O & 14 & 01110 & 1011010 & B & 110111 & 11100 & 11100 & 01111 \\
P & 15 & 01111 & 10111000 & O & 111000 & 111010 & 111010 & 01101 \\
Q & 16 & 10000 & 101110011111 & F & 111010 & 111011 & 111011 & 011100 \\
R & 17 & 10001 & 11000 & K & 111011 & 111100 & 111100 & 111100 \\
S & 18 & 10010 & 11010 & W & 1111001 & 111101 & 111101 & 011111 \\
T & 19 & 10011 & 11100 & V & 1111011 & 1111100 & 1111100 & 011101 \\
U & 20 & 10100 & 111100 & Z & 1111101 & 1111101 & 1111101 & 111101 \\
V & 21 & 10101 & 11111000 & P & 1111110 & 1111110 & 1111110 & 0111111 \\
W & 22 & 10110 & 11111011 & J & 1111111100 & 11111110 & 11111110 & 01111111 \\
X & 23 & 10111 & 11111101001011 & Q & 11111111100 & 111111110 & 111111110 & 011111111 \\
Y & 24 & 11000 & 1111110100111 & Y & 111111111011 & 1111111110 & 1111111110 & 0111111111 \\
Z & 25 & 11001 & 11111110 & X & 1111111111001 & 1111111111 & 1111111111 & 1111111111 \\
\hline
\end{tabular}

Table 6. Binary Codes for Probability Distrubution of Turkish Symbols

\begin{tabular}{|c|c|c|c|c|c|c|c|c|}
\hline $\begin{array}{l}\text { Turkish } \\
\text { Alphabet }\end{array}$ & No & $\begin{array}{l}\text { Ordinary } \\
\text { Codes }\end{array}$ & $\begin{array}{l}\text { S-F-E } \\
\text { Codes }\end{array}$ & $\begin{array}{l}\text { Ordered } \\
\text { Alphabet }\end{array}$ & $\begin{array}{l}\text { Shannon } \\
\text { Codes }\end{array}$ & Fano Codes & $\begin{array}{l}\text { Enhanced } \\
\text { Fano } \\
\text { Codes }\end{array}$ & $\begin{array}{l}\text { Huffman } \\
\text { Codes }\end{array}$ \\
\hline A & 0 & 00000 & 00001 & \# & 000 & 000 & 000 & 001 \\
\hline B & 1 & 00001 & 0001110 & A & 0010 & 001 & 001 & 000 \\
\hline $\mathrm{C}$ & 2 & 00010 & 00100001 & E & 0011 & 0100 & 0100 & 0011 \\
\hline Ç & 3 & 00011 & 00100011 & İ & 0101 & 0101 & 0101 & 0111 \\
\hline $\mathrm{D}$ & 4 & 00100 & 001010 & $\mathrm{~N}$ & 0110 & 0110 & 0110 & 0101 \\
\hline $\mathrm{E}$ & 5 & 00101 & 00111 & $\mathrm{R}$ & 01110 & 0111 & 0111 & 0010 \\
\hline $\mathrm{F}$ & 6 & 00110 & 0100001111 & $\mathrm{~L}$ & 10000 & 1000 & 1000 & 0110 \\
\hline G & 7 & 00111 & 01000101 & I & 10010 & 1001 & 1001 & 0100 \\
\hline$\breve{G}$ & 8 & 01000 & 01001000 & $\mathrm{~K}$ & 10011 & 10100 & 10100 & 01011 \\
\hline $\mathrm{H}$ & 9 & 01001 & 01001010 & $\mathrm{D}$ & 10100 & 10101 & 10101 & 01111 \\
\hline I & 10 & 01010 & 010100 & M & 10110 & 10110 & 10110 & 01101 \\
\hline İ & 11 & 01011 & 01100 & $\mathrm{Y}$ & 101110 & 10111 & 10111 & 01010 \\
\hline $\mathrm{J}$ & 12 & 01100 & 0110100111111 & $\mathrm{~T}$ & 101111 & 11000 & 11000 & 11010 \\
\hline $\mathrm{K}$ & 13 & 01101 & 011011 & U & 110001 & 11001 & 11001 & 01110 \\
\hline $\mathrm{L}$ & 14 & 01110 & 011110 & $\mathrm{~S}$ & 110011 & 11010 & 11010 & 01100 \\
\hline M & 15 & 01111 & 100001 & B & 110101 & 110110 & 110110 & 11100 \\
\hline $\mathrm{N}$ & 16 & 10000 & 10010 & $\mathrm{O}$ & 110110 & 110111 & 110111 & 011011 \\
\hline $\mathrm{O}$ & 17 & 10001 & 1001110 & Ü & 111000 & 111000 & 111000 & 011101 \\
\hline Ö & 18 & 10010 & 101000001 & Ş & 111001 & 111001 & 111001 & 011110 \\
\hline $\mathrm{P}$ & 19 & 10011 & 101000101 & Z & 1110100 & 111010 & 111010 & 111110 \\
\hline $\mathrm{R}$ & 20 & 10100 & 101010 & G & 1110110 & 111011 & 111011 & 0111011 \\
\hline $\mathrm{S}$ & 21 & 10101 & 1011011 & $\mathrm{C}$ & 1110111 & 1111000 & 1111000 & 0011111 \\
\hline Ș & 22 & 10110 & 1011101 & $\mathrm{H}$ & 1111000 & 1111001 & 1111001 & 1011111 \\
\hline $\mathrm{T}$ & 23 & 10111 & 1100000 & $\breve{G}$ & 1111010 & 1111010 & 1111010 & 0111111 \\
\hline $\mathrm{U}$ & 24 & 11000 & 1100100 & V & 1111011 & 1111011 & 1111011 & 1111111 \\
\hline Ü & 25 & 11001 & 1100111 & $\mathrm{C}$ & 1111100 & 1111100 & 1111100 & 0111101 \\
\hline $\mathrm{V}$ & 26 & 11010 & 11010001 & Ö & 11111011 & 1111101 & 1111101 & 1111101 \\
\hline $\mathrm{Y}$ & 27 & 11011 & 1101011 & $\mathrm{P}$ & 11111101 & 1111110 & 1111110 & 01111011 \\
\hline $\mathrm{Z}$ & 28 & 11100 & 11011100 & $\mathrm{~F}$ & 111111101 & 11111110 & 11111110 & 011111011 \\
\hline \# & 29 & 11101 & 1110 & $\mathrm{~J}$ & 111111111110 & 11111111 & 11111111 & 111111011 \\
\hline
\end{tabular}


Table 7. Binary Codes for Probability Distrubution of French Symbols

\begin{tabular}{|c|c|c|c|c|c|c|c|c|}
\hline $\begin{array}{l}\text { French } \\
\text { Alphabet }\end{array}$ & No & $\begin{array}{l}\text { Ordinary } \\
\text { Codes }\end{array}$ & $\begin{array}{l}\text { S-F-E } \\
\text { Codes }\end{array}$ & $\begin{array}{l}\text { Ordered } \\
\text { Alphabet }\end{array}$ & $\begin{array}{l}\text { Shannon } \\
\text { Codes }\end{array}$ & Fano Codes & $\begin{array}{l}\text { Enhanced } \\
\text { Fano } \\
\text { Codes }\end{array}$ & $\begin{array}{l}\text { Huffman } \\
\text { Codes }\end{array}$ \\
\hline A & 0 & 00000 & 00001 & $\mathrm{E}$ & 000 & 000 & 000 & 00 \\
\hline B & 1 & 00001 & 00010101 & A & 0010 & 001 & 001 & 0101 \\
\hline $\mathrm{C}$ & 2 & 00010 & 0001101 & $\mathrm{~S}$ & 0100 & 010 & 010 & 0001 \\
\hline $\mathrm{D}$ & 3 & 00011 & 001001 & I & 0101 & 0110 & 0110 & 1001 \\
\hline E & 4 & 00100 & 0011 & $\mathrm{~T}$ & 0110 & 0111 & 0111 & 0011 \\
\hline $\mathrm{F}$ & 5 & 00101 & 01010111 & $\mathrm{~N}$ & 0111 & 1000 & 1000 & 1011 \\
\hline G & 6 & 00110 & 01011010 & $\mathrm{R}$ & 1000 & 1001 & 1001 & 0111 \\
\hline $\mathrm{H}$ & 7 & 00111 & 010111001 & $\mathrm{U}$ & 10011 & 1010 & 1010 & 0110 \\
\hline I & 8 & 01000 & 01100 & $\mathrm{~L}$ & 10101 & 1011 & 1011 & 0010 \\
\hline $\mathrm{J}$ & 9 & 01001 & 011100011 & $\mathrm{O}$ & 10111 & 1100 & 1100 & 1010 \\
\hline $\mathrm{K}$ & 10 & 01010 & 0111001001100 & $\mathrm{D}$ & 11001 & 11010 & 11010 & 01101 \\
\hline $\mathrm{L}$ & 11 & 01011 & 011110 & $\mathrm{C}$ & 110101 & 11011 & 11011 & 01111 \\
\hline M & 12 & 01100 & 1000010 & M & 110111 & 11100 & 11100 & 01110 \\
\hline $\mathrm{N}$ & 13 & 01101 & 10010 & $\mathrm{P}$ & 111001 & 111010 & 111010 & 11110 \\
\hline $\mathrm{O}$ & 14 & 01110 & 101000 & $\mathrm{~V}$ & 1110110 & 111011 & 111011 & 011111 \\
\hline $\mathrm{P}$ & 15 & 01111 & 1010110 & Q & 1111000 & 111100 & 111100 & 0111101 \\
\hline $\mathrm{Q}$ & 16 & 10000 & 10110010 & $\mathrm{G}$ & 1111010 & 1111010 & 1111010 & 0011101 \\
\hline $\mathrm{R}$ & 17 & 10001 & 10111 & $\mathrm{~F}$ & 1111011 & 1111011 & 1111011 & 1011101 \\
\hline $\mathrm{S}$ & 18 & 10010 & 11001 & B & 1111100 & 1111100 & 1111100 & 0111111 \\
\hline $\mathrm{T}$ & 19 & 10011 & 11100 & $\mathrm{H}$ & 11111011 & 1111101 & 1111101 & 1111111 \\
\hline $\mathrm{U}$ & 20 & 10100 & 111100 & $\mathrm{~J}$ & 11111101 & 1111110 & 1111110 & 01111101 \\
\hline $\mathrm{V}$ & 21 & 10101 & 11111101 & $\mathrm{X}$ & 111111101 & 11111110 & 11111110 & 011111101 \\
\hline W & 22 & 10110 & 11111111000101 & $\mathrm{Y}$ & 1111111111 & 111111110 & 111111110 & 0111111101 \\
\hline $\mathrm{X}$ & 23 & 10111 & 1111111110 & Z & 00000000001 & 1111111110 & 1111111110 & 01111111101 \\
\hline $\mathrm{Y}$ & 24 & 11000 & 00000000001 & $\mathrm{~K}$ & 000000000101 & 11111111110 & 11111111110 & 011111111101 \\
\hline Z & 25 & 11001 & 000000000110 & $\mathrm{~W}$ & 0000000001110 & 11111111111 & 11111111111 & 111111111101 \\
\hline
\end{tabular}

Table 8. Binary Codes for Probability Distrubution of Russian Symbols

\begin{tabular}{|c|c|c|c|c|c|c|c|c|}
\hline $\begin{array}{l}\text { Russian } \\
\text { Alphabet }\end{array}$ & No & $\begin{array}{l}\text { Ordinary } \\
\text { Codes }\end{array}$ & $\begin{array}{l}\text { S-F-E } \\
\text { Codes }\end{array}$ & $\begin{array}{l}\text { Ordered } \\
\text { Alphabet }\end{array}$ & $\begin{array}{l}\text { Shannon } \\
\text { Codes }\end{array}$ & Fano Codes & $\begin{array}{l}\text { Enhanced } \\
\text { Fano } \\
\text { Codes }\end{array}$ & $\begin{array}{l}\text { Huffman } \\
\text { Codes }\end{array}$ \\
\hline $\mathrm{A}$ & 0 & 00000 & 00010 & $\#$ & 000 & 000 & 000 & 000 \\
\hline Б & 1 & 00001 & 00010010 & $\mathrm{O}$ & 0010 & 001 & 001 & 001 \\
\hline B & 2 & 00010 & 000110 & $\mathrm{E}$ & 0011 & 0100 & 0100 & 011 \\
\hline$\Gamma$ & 3 & 00011 & 00100000 & A & 0101 & 0101 & 0101 & 0010 \\
\hline Д & 4 & 00100 & 0010001 & И & 0110 & 0110 & 0110 & 1010 \\
\hline $\mathrm{E}$ & 5 & 00101 & 00110 & $\mathrm{~T}$ & 01110 & 0111 & 0111 & 0111 \\
\hline Ж & 6 & 00110 & 00111100 & $\mathrm{H}$ & 01111 & 1000 & 1000 & 0011 \\
\hline 3 & 7 & 00111 & 00111111 & $\mathrm{C}$ & 10001 & 1001 & 1001 & 0101 \\
\hline И & 8 & 01000 & 01001 & $\mathrm{P}$ & 10011 & 10100 & 10100 & 00100 \\
\hline Й & 9 & 01001 & 01010010 & B & 10100 & 10101 & 10101 & 01100 \\
\hline K & 10 & 01010 & 0101011 & Л & 10101 & 10110 & 10110 & 01110 \\
\hline Л & 11 & 01011 & 011000 & K & 101101 & 10111 & 10111 & 01011 \\
\hline M & 12 & 01100 & 0110100 & M & 101111 & 11000 & 11000 & 01101 \\
\hline $\mathrm{H}$ & 13 & 01101 & 011100 & Д & 110001 & 110010 & 11010 & 11101 \\
\hline $\mathrm{O}$ & 14 & 01110 & 10000 & $\bar{\Pi}$ & 110011 & 110011 & 110010 & 010100 \\
\hline$\Pi$ & 15 & 01111 & 1001010 & $\mathrm{y}$ & 110100 & 11010 & 110011 & 110100 \\
\hline $\mathrm{P}$ & 16 & 10000 & 100111 & Я & 110101 & 110110 & 110110 & 011100 \\
\hline $\mathrm{C}$ & 17 & 10001 & 101010 & Ы & 110111 & 110111 & 110111 & 011110 \\
\hline $\mathrm{T}$ & 18 & 10010 & 101101 & 3 & 1110000 & 111000 & 111000 & 001111 \\
\hline $\mathrm{y}$ & 19 & 10011 & 1011111 & $\mathrm{~b}(\mathrm{~b})$ & 1110010 & 111001 & 111001 & 101111 \\
\hline$\Phi$ & 20 & 10100 & 1100001011 & Б & 1110100 & 111010 & 111010 & 011111 \\
\hline $\mathrm{X}$ & 21 & 10101 & 11000100 & $\Gamma$ & 1110110 & 111011 & 111011 & 011011 \\
\hline Ц & 22 & 10110 & 110001011 & Ч & 1110111 & 111100 & 111100 & 111011 \\
\hline Ч & 23 & 10111 & 11001000 & Й & 1111001 & 1111010 & 1111010 & 0111100 \\
\hline Ш & 24 & 11000 & 110010100 & $\mathrm{X}$ & 1111010 & 1111011 & 1111011 & 0111110 \\
\hline щ & 25 & 11001 & 1100101110 & Ж & 1111100 & 1111100 & 1111100 & 1111110 \\
\hline $\mathrm{b}(\mathrm{b})$ & 26 & 11010 & 11001101 & Ю & 11111010 & 1111101 & 1111101 & 0111111 \\
\hline Ы & 27 & 11011 & 1101000 & Ш & 11111011 & 11111100 & 11111100 & 01111100 \\
\hline$\ni$ & 28 & 11100 & 1101010001 & Ц & 11111101 & 11111101 & 11111101 & 11111100 \\
\hline Ю & 29 & 11101 & 110101011 & Щ & 111111100 & 11111110 & 11111110 & 01111111 \\
\hline Я & 30 & 11110 & 1101100 & $\ni$ & 111111110 & 111111110 & 111111110 & 011111111 \\
\hline \# & 31 & 11111 & 1110 & $\Phi$ & 111111111 & 111111111 & 111111111 & 111111111 \\
\hline
\end{tabular}


Table 9. Binary Codes for Probability Distrubution of Spanish Symbols

\begin{tabular}{|c|c|c|c|c|c|c|c|c|}
\hline $\begin{array}{l}\text { Spanish } \\
\text { Symbols }\end{array}$ & No & $\begin{array}{l}\text { Ordinary } \\
\text { Codes }\end{array}$ & $\begin{array}{l}\text { S-F-E } \\
\text { Codes }\end{array}$ & $\begin{array}{l}\text { Ordered } \\
\text { Alphabet }\end{array}$ & $\begin{array}{l}\text { Shannon } \\
\text { Codes }\end{array}$ & Fano Codes & $\begin{array}{l}\text { Enhanced } \\
\text { Fano } \\
\text { Codes }\end{array}$ & $\begin{array}{l}\text { Huffman } \\
\text { Codes }\end{array}$ \\
\hline $\mathrm{A}$ & 0 & 00000 & 0001 & $\mathrm{E}$ & 000 & 000 & 000 & 000 \\
\hline B & 1 & 00001 & 00100001 & A & 001 & 001 & 001 & 001 \\
\hline $\mathrm{C}$ & 2 & 00010 & 001010 & $\mathrm{O}$ & 0100 & 010 & 010 & 0010 \\
\hline $\mathrm{D}$ & 3 & 00011 & 001101 & $\mathrm{~S}$ & 0101 & 0110 & 0110 & 1010 \\
\hline E & 4 & 00100 & 0101 & $\mathrm{R}$ & 0110 & 0111 & 0111 & 0110 \\
\hline $\mathrm{F}$ & 5 & 00101 & 011000101 & $\mathrm{~N}$ & 0111 & 1000 & 1000 & 0100 \\
\hline G & 6 & 00110 & 01100100 & I & 10010 & 1001 & 1001 & 1100 \\
\hline $\mathrm{H}$ & 7 & 00111 & 011001101 & $\mathrm{D}$ & 10100 & 1010 & 1010 & 0101 \\
\hline I & 8 & 01000 & 011011 & $\mathrm{~L}$ & 10101 & 1011 & 1011 & 0011 \\
\hline $\mathrm{J}$ & 9 & 01001 & 011110000 & $\mathrm{C}$ & 10111 & 11000 & 11000 & 1011 \\
\hline $\mathrm{K}$ & 10 & 01010 & 0111100011111011 & $\mathrm{~T}$ & 11001 & 11001 & 11001 & 0111 \\
\hline $\mathrm{L}$ & 11 & 01011 & 011111 & $\mathrm{U}$ & 11010 & 11010 & 11010 & 01110 \\
\hline M & 12 & 01100 & 100010 & M & 11011 & 11011 & 11011 & 01101 \\
\hline $\mathrm{N}$ & 13 & 01101 & 10010 & $\mathrm{P}$ & 111001 & 11100 & 11100 & 01111 \\
\hline $\mathrm{O}$ & 14 & 01110 & 10101 & B & 1110110 & 111010 & 111010 & 011110 \\
\hline $\mathrm{P}$ & 15 & 01111 & 1011100 & $\mathrm{G}$ & 1111000 & 111011 & 111011 & 011111 \\
\hline Q & 16 & 10000 & 10111100 & $\mathrm{Y}$ & 1111001 & 111100 & 111100 & 111111 \\
\hline $\mathrm{R}$ & 17 & 10001 & 11000 & $\mathrm{~V}$ & 1111010 & 1111010 & 1111010 & 0111110 \\
\hline S & 18 & 10010 & 11011 & $\mathrm{Q}$ & 1111011 & 1111011 & 1111011 & 1111110 \\
\hline $\mathrm{T}$ & 19 & 10011 & 111010 & $\mathrm{H}$ & 11111001 & 1111100 & 1111100 & 0011101 \\
\hline $\mathrm{U}$ & 20 & 10100 & 111101 & $\mathrm{~F}$ & 11111011 & 1111101 & 1111101 & 1011101 \\
\hline $\mathrm{V}$ & 21 & 10101 & 11111010 & $\mathrm{Z}$ & 11111101 & 1111110 & 1111110 & 0111101 \\
\hline W & 22 & 10110 & 11111100000110 & $\mathrm{~J}$ & 11111110 & 11111110 & 11111110 & 01111101 \\
\hline $\mathrm{X}$ & 23 & 10111 & 1111110001 & $\mathrm{X}$ & 111111111 & 111111110 & 111111110 & 011111101 \\
\hline $\mathrm{Y}$ & 24 & 11000 & 11111101 & W & 0000000001000 & 1111111110 & 1111111110 & 0111111101 \\
\hline Z & 25 & 11001 & 111111111 & $\mathrm{~K}$ & 000000000101001 & 1111111111 & 1111111111 & 1111111101 \\
\hline
\end{tabular}

Table 10. Binary Codes Constructed by Fano-Huffman Based Statistical Coding Method

\begin{tabular}{|c|c|c|c|c|c|c|c|c|}
\hline $\begin{array}{l}\text { Turkish } \\
\text { Alphabet }\end{array}$ & $\begin{array}{l}\text { Fano- } \\
\text { Huffman } \\
\text { based } \\
\text { Codes for } \\
\text { Turkish } \\
\text { symbols }\end{array}$ & $\begin{array}{l}\text { Russian } \\
\text { Alphabet }\end{array}$ & $\begin{array}{l}\text { Fano- } \\
\text { Huffman } \\
\text { based Codes } \\
\text { for Russian } \\
\text { symbols }\end{array}$ & $\begin{array}{l}\text { English, } \\
\text { French, } \\
\text { German, } \\
\text { Spanish } \\
\text { Alphabet }\end{array}$ & $\begin{array}{l}\text { Fano- } \\
\text { Huffman } \\
\text { based } \\
\text { Codes for } \\
\text { English } \\
\text { symbols }\end{array}$ & $\begin{array}{l}\text { Fano- } \\
\text { Huffman } \\
\text { based } \\
\text { Codes for } \\
\text { French } \\
\text { symbols }\end{array}$ & $\begin{array}{l}\text { Fano-Huffman } \\
\text { based } \\
\text { Codes for } \\
\text { German } \\
\text { symbols }\end{array}$ & $\begin{array}{l}\text { Fano-Huffman } \\
\text { based } \\
\text { Codes for } \\
\text { Spanish } \\
\text { symbols }\end{array}$ \\
\hline $\mathrm{A}$ & 110 & $\mathrm{~A}$ & 1101 & $\mathrm{~A}$ & 0101 & 0001 & 0010 & 011 \\
\hline B & 10111 & Б & 011100 & $\mathrm{~B}$ & 111100 & 0001100 & 11110 & 111000 \\
\hline $\mathrm{C}$ & 0100101 & B & 11000 & $\mathrm{C}$ & 110000 & 11100 & 01010 & 1110 \\
\hline Ç & 0011001 & $\Gamma$ & 011010 & $\mathrm{D}$ & 11000 & 10000 & 0110 & 0010 \\
\hline $\mathrm{D}$ & 01001 & Д & 10110 & E & 0001 & 11 & 001 & 101 \\
\hline $\mathrm{E}$ & 0000 & $\mathrm{E}$ & 0101 & $\mathrm{~F}$ & 101000 & 1100000 & 001000 & 1011010 \\
\hline $\mathrm{F}$ & 010000001 & Ж & 1010100 & G & 001100 & 0100000 & 00100 & 0110000 \\
\hline G & 1000001 & 3 & 001100 & $\mathrm{H}$ & 00000 & 1001100 & 10000 & 0011010 \\
\hline$\breve{G}$ & 0111001 & И & 0011 & I & 1010 & 0101 & 111 & 1100 \\
\hline $\mathrm{H}$ & 1011001 & Й & 1100000 & $\mathrm{~J}$ & 1010000100 & 11000000 & 01101110 & 00111010 \\
\hline I & 1111 & K & 01010 & $\mathrm{~K}$ & 00000100 & 010101000000 & 101000 & 1110111010 \\
\hline$\dot{I}$ & 1000 & Л & 00100 & $\mathrm{~L}$ & 10100 & 0110 & 11010 & 0110 \\
\hline $\mathrm{J}$ & 110000001 & M & 00110 & M & 001000 & 01010 & 10100 & 01010 \\
\hline K & 10001 & $\mathrm{H}$ & 0010 & $\mathrm{~N}$ & 0010 & 1000 & 011 & 0100 \\
\hline $\mathrm{L}$ & 1011 & $\mathrm{O}$ & 111 & $\mathrm{O}$ & 1101 & 1110 & 000000 & 111 \\
\hline M & 10101 & $\Pi$ & 000000 & $\mathrm{P}$ & 011100 & 11010 & 0101110 & 010000 \\
\hline $\mathrm{N}$ & 0010 & $\mathrm{P}$ & 10000 & Q & 0110000100 & 0000000 & 011101110 & 1011000 \\
\hline $\mathrm{O}$ & 100001 & $\mathrm{C}$ & 1110 & $\mathrm{R}$ & 1110 & 0100 & 1100 & 1001 \\
\hline Ö & 1100101 & $\mathrm{~T}$ & 1011 & S & 0110 & 1001 & 0101 & 0001 \\
\hline $\mathrm{P}$ & 00000001 & $\mathrm{y}$ & 001000 & $\mathrm{~T}$ & 1001 & 1101 & 1101 & 00000 \\
\hline $\mathrm{R}$ & 1010 & $\Phi$ & 110100000 & $\mathrm{U}$ & 010000 & 0010 & 11000 & 01000 \\
\hline S & 00111 & $X$ & 0010100 & $\mathrm{~V}$ & 1000100 & 101100 & 0100000 & 0011000 \\
\hline Ş & 011101 & Ц & 01111100 & W & 100100 & 110101000000 & 001110 & 0110111010 \\
\hline $\mathrm{T}$ & 00011 & $\mathrm{\varphi}$ & 111010 & $\mathrm{X}$ & 0010000100 & 001000000 & 1111101110 & 010111010 \\
\hline $\mathrm{U}$ & 10011 & Ш & 00100000 & $\mathrm{Y}$ & 101100 & 1101000000 & 0111101110 & 1110000 \\
\hline$\ddot{U}$ & 000101 & щ & 11111100 & $\mathrm{Z}$ & 1110000100 & 00101000000 & 1100000 & 1111010 \\
\hline V & 1111001 & $\mathrm{~b}(\mathrm{~b})$ & 101100 & \# & 11 & & & \\
\hline Y & 01101 & Ы & 110100 & & & & & \\
\hline Z & 111101 & $Э$ & 010100000 & & & & & \\
\hline \multirow[t]{3}{*}{ \# } & 100 & Ю & 0111100 & & & & & \\
\hline & & Я & 101000 & & & & & \\
\hline & & \# & 001 & & & & & \\
\hline
\end{tabular}


In order to determine the information per letter for considered alphabets due to the mentioned coding methods, the following stages are presented:

1) The entropy of each mentioned languages $H(S)$ is calculated.

2) The codeword length of each codes shown in Tables 4-10 is obtained by counting the bits of the code words and thus average codeword length $\bar{\ell}$ is computed for each coding methods.

3) The information per letter $\mathrm{I}_{\mathrm{inf} / \text { letter }}=\frac{\mathrm{H}(\mathrm{S})}{\bar{\ell}}$ is get for interpretation of optimality of codes.
The results of these stages are given in Table 11. As previously presented, the optimality criteria for codes is $\mathrm{I}_{\mathrm{i} / \mathrm{s}} \rightarrow 1$. Obviously, it is seen from Table 11 that, binary codes constructed for each symbols of different alphabet by Fano-Huffman based statistical coding method is more optimal than Fano coding method and is as optimal as constructed by Huffman coding method but it is more easily applicable than Huffman coding method. Also, the improved coding method is more optimal than the others. Moreover, if a file is coded by Fano-Huffman based codes then the dimension of the file will be less than the files coded by the other considered coding methods. Hence, this means faster communication.

Table 11 Information per letter sent by constructed binary codes

\begin{tabular}{llllllll}
\hline $\begin{array}{l}\text { Source } \\
\text { Alphabet }\end{array}$ & $\begin{array}{l}\text { Ordinary } \\
\text { Codes } \\
\text { (bits) }\end{array}$ & $\begin{array}{l}\text { Shannon } \\
\text { Codes } \\
\text { (bits) }\end{array}$ & $\begin{array}{l}\text { Fano } \\
\text { Codes } \\
\text { (bits) }\end{array}$ & $\begin{array}{l}\text { Improved } \\
\text { Fano } \\
\text { Codes } \\
\text { (bits) }\end{array}$ & $\begin{array}{l}\text { Shannon } \\
\text { Fano } \\
\text { Elias } \\
\text { Codes } \\
\text { (bits) }\end{array}$ & $\begin{array}{l}\text { Huffman } \\
\text { Codes } \\
\text { (bits) }\end{array}$ & $\begin{array}{l}\text { Fano-Huffman } \\
\text { based Codes } \\
\text { (bits) }\end{array}$ \\
\hline English & 0.8145 & 0.8801 & 0.9834 & 0.9839 & 1.0792 & 0.9905 & 0.9888 \\
Turkish & 0.8732 & 0.9075 & 0.9937 & 0.9937 & 1.0955 & 0.9939 & 0.9939 \\
French & 0.7971 & 0.8885 & 0.9854 & 0.9854 & 1.0911 & 0.9899 & 0.9899 \\
German & 0.8190 & 0.9100 & 0.9901 & 0.9901 & 1.1083 & 0.9915 & 0.9901 \\
Spanish & 0.8032 & 0.9150 & 0.9909 & 0.9909 & 1.1161 & 0.9924 & 0.9916 \\
Russian & 0.8839 & 0.9085 & 0.9925 & 0.9936 & 1.2142 & 0.9936 & 0.9936 \\
\hline
\end{tabular}




\section{Conclusion}

It is seen that, binary codes constructed by FanoHuffman based statistical coding method carry information per letter as much as codes constructed by Huffman coding method. However, by this coding method the less subset you divide the more optimal codes you obtain. Thus, this result make Fano-Huffman based statistical coding method preferred coding methods as Huffman coding method for each of the considered languages. Fano-Huffman based statistical coding method takes less time than Huffman coding method to construct binary codes. However, it require more pure computation than Huffman coding method by means of dividing the source alphabet to subsets and this means faster coding.

As it is commonly known, operating system of computers based on American Standard Code for Information Interchange (ASCII) which is ordinary binary codes. Therefore, another main result from this study is the advantage of Fano-Huffman based codes rather than ASCII. Obviously, it can be concluded from this study that ordinary codes are not optimal because they have the highest average codeword length and the least information per letter. Hence, since ASCII codes are ordinary codes, the text coded by them will be larger in size contrary to Fano-Huffman based codes. So, ASCII codes are not preferred codes.

Consequently, Fano-Huffman based codes can be used in computer systems for data compression rather than ASCII for faster communication. Because, if a file is coded by Fano-Huffman based codes then the dimension of the file will be less than file coded by ASCII but it will transmit the same information by using codes consist of less bits.

\section{References}

Aazhang, B. (2004). http://cnx.rice.edu/content/m10176/latest/, Creative Commons.

Cover, T. M. \& Thomas, J. A. (1991). Elements of information theory. USA: John Wiley \& Sons, Inc.
Faller, N. (1973). An adaptive system for data compression. In 7th Asilomar conference on circuits, systems, and computers , 593-597.

Gallager, R. (1978). Variations on a theme by Huffman. IEEE Transactions on Information Theory, 24(6), 668-674.

Hankerson, D., Harris, G. A. \& Johnson, P. D. (2003). Introduction to information theory and data compression (2nd ed.). Boca Raton, FL: Chapman \& Hall/CRC Press.

Hankerson, D., Harris, G. \& Johnson, P. (1998). Introduction to information theory and data compression. CRC Press.

Huffman, D. (1952). A method for the construction of minimum redundancy codes. Proceedings of IRE, 40(9), 1098-1101.

Kieffer, J. C., \& Yang, E. (2000). Grammar-bassed codes: a new class of universal lossless source codes. IEEE Transactions on Information Theory, 46(3), 737-754.

Knuth, D. (1985). Dynamic Huffman coding. Journal of Algorithms, 6, 163-180.

Roman, S. (1997). Introduction to Coding and Information Theory. New York: Springer-Verlag.

Rueda, L. (2002). Advances in data compression and pattern recognition. $\mathrm{PhD}$ thesis, School of Computer Science, Carleton University, Ottawa, Canada.

Rueda, L. G. \& Oommen B. J. (2004). A Nearly-Optimal Fano-Based Coding Algorithm. Information Processing and Management, 40, 257-268.

Sayood, K. (2000). Introduction to data compression ( $2^{\text {nd }}$ ed.). Morgan Kaufmann.

Венцель, Е. С. (1969). Теория Вероятностей, Москва.

Pratt, F. (1939). Secret and urgent: The story of codes and ciphers. Blue Ribbon Books.

Phamdo, N. (2001). http://diwww.epfl.ch/mantra/CoursINF

OII/Web/compression/english.html, State University of New York.

Stephens, D. (2002). http://www.santacruzpl.org/readyref/files/g1/ltfrqsp.shtml, Santa Cruz Public Libraries, California. 
Shamilov A. and Yolacan S. (2005). Various binary codes for probability distribution of Turkish letters. International Conference Ordered Statistical Data: Approximations, Bounds and Characterizations, pp.70 Izmir, Turkey.

Shannon, C. E., \& Weaver, W. (1949). The mathematical theory of communications. University of Illinois Press.

Witten, I., Moffat, A. \& Bell, T. (1999). Managing gigabytes: Compressing and indexing documents and images (2nd ed.). Morgan Kaufmann.
Yolacan, S. (2005). Statistical properties of different languages based on entropy and information theory. Anadolu University Graduate School of Sciences, Master of Science Thesis (at turkish), Eskisehir.

Ziv, J. \& Lempel, A. (1977). A universal algorithm for sequential data compression. IEEE Transactions on Information Theory, 23(3), 337-343.

Ziv, J. \& Lempel, A. (1978). Compression of individual sequences via variable-rate coding. IEEE Transactions on Information Theory, 25(5), $530-536$. 


\title{
A Comparison of One-High-Threshold and Two-High-Threshold Multinomial Models of Source Monitoring
}

\author{
Mahesh Menon Todd S. Woodward \\ Riverview Hospital, Coquitlam, British Columbia, Canada
}

A data simulation study comparing the one-high-threshold (1HT) and two-high-threshold (2HT) multinomial models suggested that $2 \mathrm{HT}$ models are more likely to misestimate the underlying parameter values, due to inflation of some parameters ( $b$ and $d$ ), and deflation of others (D).

Key words: Multinomial modeling, source monitoring, data simulation

\section{Introduction}

Source monitoring and reality monitoring studies have proven to be extremely useful in understanding a variety of memory processes in the normal and clinical populations (Brebion, Gorman, Amador, Malaspina, \& Sharif, 2002; Hoffman, 1997; Johnson, Hashtroudi, \& Lindsay, 1993; Keefe, Arnold, Bayen, McEvoy, \& Wilson, 2002; Lindsay, Johnson, \& Kwon, 1991). Consider a simple source monitoring experiment with two sources: A and B. Single words are presented in a random fashion from the two sources, and the final recognition test consists of a mix of old $\mathrm{A}$ and $\mathrm{B}$ items along with new distracters $\mathrm{N}$. The analysis of data from such a study typically examines item detection (the number of previously presented items that are correctly identified as being old), source recognition (the number of times the source of the item was correctly attributed), and

Mahesh Menon is also affiliated with the Schizophrenia PET Research Group, Centre for Addiction \& Mental Health, Toronto, Canada. Email him at mahesh@cantab.net. Todd S. Woodward is also affiliated with the Department of Psychology, Simon Fraser University, Burnaby, British Columbia, Canada. Email him at toddswoodward@gmail.com. the false positive error rates (the number of new items that are incorrectly identified as being old).

However, traditional methods of analysis are unable to separate guessing biases and meta-cognitive response strategies from true item and source recognition. For example, when subjects notice that they are recognizing too few items from the (less memorable) external source, they tend to compensate by increasing the number of external-source guesses (Batchelder \& Riefer, 1990). Therefore, in order to accurately measure externalizations, increases in strategic external guesses must be excluded. Similarly, to accurately estimate source recognition, it must be separable from both biases and strategic guessing. Multinomial modeling allows these distinct cognitive mechanisms can be disentangled. Multinomial modeling is a statistically sophisticated, yet simple method of separating item recognition, source recognition and response biases in discrimination tasks such as source monitoring, allowing estimation of guessing strategies and biases separately from source-discrimination processes.

Multinomial models attempt to explain discrete responses in a particular psychological paradigm by postulating latent cognitive processes that combine in different ways to determine the response category. The basic idea is that any given response category may occur as a consequence of one or more processing sequences, where each processing sequence is characterized by a series of successful or unsuccessful processing events. The processing 
sequences are represented in a tree structure (see Figure 1). The root (or initial node) represents the beginning of the processing sequence, the intermediate nodes represent stages involving a choice between two or more processing events, and the terminal nodes correspond to the observable response categories. The application of multinomial models to source monitoring has been reviewed in detail elsewhere (Batchelder \& Riefer, 1999; Bayen, Murname, \& Erdfelder, 1996).

One theoretical divide with implications for future research and interpretability of prior research findings lies in the selection of the basic model used for the analyses. Bayen et al. (1996) described three classes of models: the one-low- threshold (1LT), one-high-threshold (1HT) and two-high-threshold (2HT) models. The 1LT model has been used in some studies (Macmillan \& Creelman, 1991), but the lack of a recognition bias parameter greatly weakens the LT model as a general purpose model of source monitoring (Bayen et al., 1996). The major theoretical debate therefore remains between the use of the 1HT and 2HT classes of models.

In 1HT models there is a single high threshold that divides the decision space into two discrete areas that correspond to detect as old and undetected. In 1HT models, it is assumed that only old items can cross the high threshold. If the threshold is crossed on presentation of a test item, the item is detected as old. If the threshold is not crossed, the item is said to be in an undetected state. All the new items and the old items that do not cross the threshold are categorized as old or new only on the basis of guessing. The probability with which an undetected item is guessed as being old is labeled $b$ (see Figure 1).

In a 2HT model, there are two high thresholds that divide decision space into three discrete areas that correspond to detect as old, detect as new and undetected. It is assumed that only old items can cross the detect as old threshold, and only new items can cross the detect as new threshold. If either threshold is crossed on presentation of a test item, the item is detected as either old or new, depending on which threshold was crossed. If neither threshold is crossed, the item goes undetected, and is guessed to be new or old. As can be seen from
Figure 1, the 2HT source monitoring model can be constructed from the $1 \mathrm{HT}$ model by adding a parameter (labeled $D_{\mathrm{N}}$ ) indicating the probability that a new item will be detected as new. Conversely, the 1HT model described earlier can be derived from the $2 \mathrm{HT}$ model by imposing the restriction $D_{\mathrm{N}}=0$. A $1 \mathrm{HT}$ model may thus always be regarded as a special case of a $2 \mathrm{HT}$ model, where the probability of crossing the second threshold is zero for all classes of items.

The nature of this debate centers around three issues. The primary theoretical issue is one of how an item is recognized as being old or new. In 2HT model space, the detection of an item as new requires it to cross a discrete threshold, which would determine it to be previously unseen. The 1HT model instead argues that the failure to cross the detection threshold for the item being old would imply that it remains unrecognized, and in the absence of a false positive recognition, the person would conclude that the item is new.

The second issue is an empirical one, stated as follows: for a given set of data, does including a $D_{\mathrm{N}}$ parameter affect the values of the other parameters (even though it is thought to represent a distinct, independent cognitive process), and if so, what is the nature of these changes?

A third issue lies in the interpretability of the parameters in the two classes of models. Specifically, in the 1HT and 2HT models (but not the 1LT model), the $b$ parameter represents the probability of guessing that a word is a target item, when it has not actually crossed one of the thresholds. This parameter is reflected in two separate scenarios: (1) for previously seen words, it is an indicator of the tendency to guess that the word is old even when it has not been detected as being old, and (2) for new words, it is an indicator of the tendency to make false positive errors. It is assumed that these two scenarios are underpinned by the same cognitive process, and hence are assigned a common parameter. But how representative are the $b$ parameters generated by the $1 \mathrm{HT}$ and $2 \mathrm{HT}$ model of this cognitive process?

The empirical question of how estimating a $D_{\mathrm{N}}$ parameter affects the values of the other parameters by way of a data simulation of a three-source source monitoring 


\begin{tabular}{|c|c|}
\hline One-high-threshold model (1HT) & Two-high-threshold model (2HT) \\
\hline 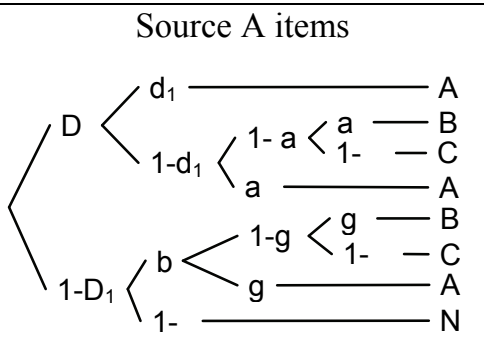 & 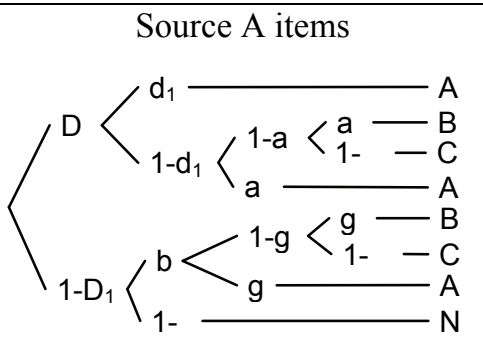 \\
\hline 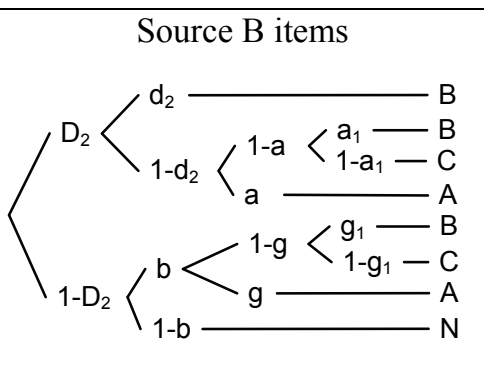 & 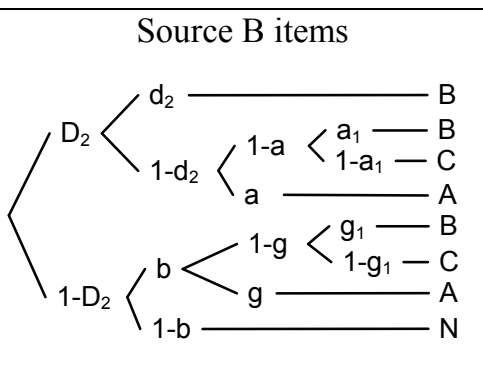 \\
\hline 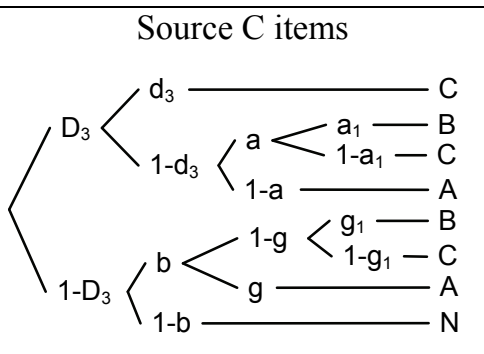 & 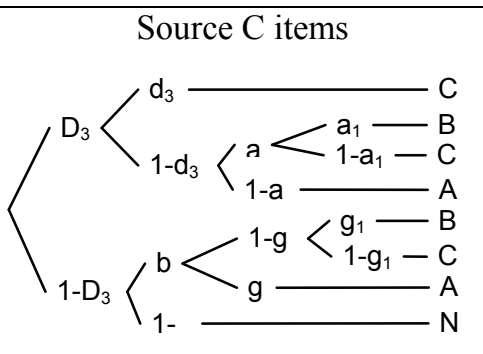 \\
\hline $\begin{array}{c}\text { New items } \\
\left\langle\begin{array}{l}\mathrm{b}<\mathrm{i-g}<\mathrm{g}_{1}-\mathrm{B} \\
\mathrm{g}-\mathrm{C}-\mathrm{g}_{1}-\mathrm{C} \\
\mathrm{A}-\mathrm{B}\end{array}\right.\end{array}$ & 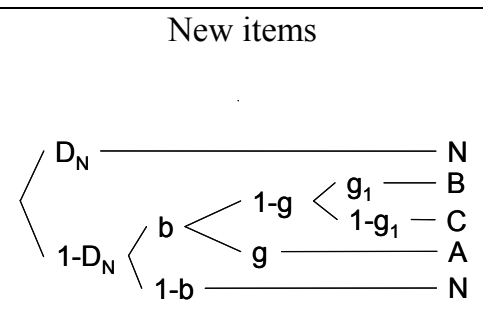 \\
\hline
\end{tabular}

Figure 1 One- and two-high-threshold models of source monitoring.

Notes. $\mathrm{A}=$ Source $\mathrm{A}$ item; $\mathrm{B}=$ Source $\mathrm{B}$ item; $\mathrm{C}=$ Source $\mathrm{C}$ item; $\mathrm{N}=$ distracter item; Item recognition parameters: $D_{1}=$ probability of detecting an item from source A; $D_{2}=$ probability of detecting an item from source B; $D_{3}=$ probability of detecting an item from source C; $D_{N}=$ probability of detecting that a distracter item is new; Source recognition parameters: $d_{l}=$ probability of correctly discriminating the source of an item from source $\mathrm{A} ; d_{2}=$ probability of correctly discriminating the source of an item from source $\mathrm{B} ; d_{3}=$ probability of correctly discriminating the source of an item from source C; Guessing biases: $a=$ probability of guessing that a detected item is from source $\mathrm{A} ; a_{l}=$ probability of guessing that a detected item is from source $\mathrm{B} ; b=$ probability of guessing that an undetected item is old; $g=$ probability of guessing that an undetected item is from source A; $g_{l}=$ probability of guessing that an undetected item is from source B. 
study was addressed. The three source model was used as it allows for greater flexibility with model specification, which is not limited by the available degrees of freedom (Keefe et al., 2002; Riefer, Hu, \& Batchelder, 1994; Woodward et al., 2006). The data simulation results are also pertinent to the theoretical and interpretational issues mentioned above, and these issues are addressed in the Discussion section.

The simulation of the to-be-analyzed data involved generating frequency tables from tree models for which all parameter values were set, with the exception of $D_{\mathrm{N}}$, which was varied between 0 and .90 . Varying the $D_{\mathrm{N}}$ parameter in this fashion allowed us to simulate a situation where, as is assumed by the 1HT model, there is no cognitive process that is captured by $D_{\mathrm{N}}$, as well as three other situations whereby detection of new items is the true state of affairs, to varying degrees.

Then, these frequency tables were analyzed using $1 \mathrm{HT}$ and various $2 \mathrm{HT}$ models. Performance was assessed by examining the inflation and deflation of estimated parameter values that occur when the $1 \mathrm{HT}$ and $2 \mathrm{HT}$ underlying assumptions did or did not match the true state of affairs (the true state of affairs being the state where the estimated $D_{\mathrm{N}}$ parameter value matched the generating $D_{\mathrm{N}}$ parameter value). In this fashion, the underlying assumptions (i.e., 1HT or 2HT generating parameter values) and the methods of analysis (i.e., 1HT or 2HT estimated parameter values) were completely crossed. Both the $1 \mathrm{HT}$ and 2HT models were expected to perform well when the method of analysis matched the underlying assumptions determined by the generating parameters, but inflations and deflations in parameter estimates were expected when a mismatch occurred between the method of analysis and the underlying assumptions. The conclusions are based on the net inflation or deflation of parameter values for the $1 \mathrm{HT}$ and 2HT models under these mismatch conditions.

\section{Methodology}

A series of simulated frequency tables of responses from a three-source source monitoring task were created, similar to one used by Keefe and colleagues (Keefe et al., 2002) and one used by Woodward, Menon, and Whitman (in press). The frequency tables were created under the constraint of 100 old items from each of the three sources (labeled A, B and C), and 300 new items. A set of underlying parameter values (i.e., the generating parameters) were specified, and these were used to create sets of response frequencies, under the restrictions of 300 old and 300 new items, as mentioned above. The final probabilities for each source/response combination were computed by multiplying the generating parameters down the processing tree, and summing together the events that lead to each specific source/response combination. For example, for the $1 \mathrm{HT}$ and $2 \mathrm{HT}$ models shown in Figure 1, the probability of participants responding "A" given stimulus A can be arrived at my multiplying the following parameters:

$$
\begin{aligned}
& \mathrm{P}(\text { (“A”| A) } \\
& =D_{1} * d_{1}+D_{1} *\left(1-d_{1}\right) * a+\left(1-D_{1}\right) * b * g
\end{aligned}
$$

The simulated response frequencies were created by multiplying the final probabilities associated with each source/response combination (as specified in Figure 1, and exemplified in Equation 1) by the number of responses for that source. For example, multiplying the summed probability shown in Equation (1) by 100 generates the number of times participants responded " $A$ " for stimuli from source A.

The generating parameter values were fixed across simulated sets of frequencies, with the exception of the $D_{\mathrm{N}}$ parameter (the item recognition parameter for new items), which was varied $(0, .30, .60$ and .90). This method of creating sets of frequencies is similar to the method used by Reifer and Batchelder (1991) in their Monte Carlo simulation. The sets of frequencies created when $D_{\mathrm{N}}=0$ represent patterns of frequencies that would be generated under the assumptions underlying 1HT model, whereas the sets of frequencies created when $D_{\mathrm{N}}$ $>0$ represent patterns of frequencies that would be generated under the assumptions underlying various $2 \mathrm{HT}$ models. The frequency tables generated for the analyses are presented in Appendix A. Note that the only variation in the sets of frequencies generated using the various 
values of $D_{\mathrm{N}}$ occurred in the number of false positive responses generated by the new words.

Then, the data were analyzed using an Excel spreadsheet specialized for multinomial modeling (Dodson, Prinzmetal, \& Shimamura, 1998). In the analysis, the value of the $D_{\mathrm{N}}$ parameter was first fixed at the different values $(0, .30, .60$ and .90$)$ to test the impact of varying degrees of mismatch between the underlying model assumptions (as determined by the generating parameter values) and the methods of analysis. Following this, the $2 \mathrm{HT}$ analysis was carried out allowing all parameters (including $\left.D_{\mathrm{N}}\right)$ to vary freely. In the Excel spreadsheet, the optimum parameter values are found by allowing them to vary in an iterative fashion using the solver function. Starting values for probability estimates were .50 , and the $\log$ likelihood ratio statistic $G^{2}$ was used to assess overall fit. $G^{2}$ asymptotically has a chi-square distribution, and the optimized solution is the one that minimizes the $G^{2}$ value (Riefer \& Batchelder, 1988).

\section{Results}

The estimated parameter values are presented as a function of varying generating and estimating $D_{\mathrm{N}}$ parameter values in Table 1 , and in Figures 2-6. From these results it is apparent that, within each set of response frequencies, varying the value of the $D_{\mathrm{N}}$ parameter (which in theory should only affect the recognition of new items) affected the value of the other parameters in a variety of important ways. As was expected, all models were very accurate when the method of analysis matched the underlying assumption (i.e., when the estimating $D_{\mathrm{N}}$ was equal to the generating $D_{\mathrm{N}}$ ). However, in the case of mismatches, occasionally severe inflation and deflation of the underlying parameters occurred. Specifically, underestimation of $D_{\mathrm{N}}$ parameter resulted in overestimation of the other $D$ parameters and underestimation of $b$ and $d$ parameters, whereas overestimation of $D_{\mathrm{N}}$ resulted in underestimation of $D$ and overestimation of $b$ and $d$ parameters.

With respect to the comparative performance of $1 \mathrm{HT}$ and $2 \mathrm{HT}$ models, the most important pattern that can be derived from Table
1 and Figures 2-6 is that the consequences of overestimating $D_{\mathrm{N}}$ are far more severe than the consequences of underestimating $D_{\mathrm{N}}$. As Figures 2-6 show, the changes in the generating parameter values had the least effect when estimated using the 1HT model (i.e., $D_{\mathrm{N}}=0$ ), while the variability of the estimated parameters increased greatly when a $2 \mathrm{HT}$ model is used, particularly with high $D_{\mathrm{N}}$ parameter values.

For example, consider the results based on a generating $D_{\mathrm{N}}$ parameter of .60 (see Table 1). An instructive comparison can be made between when $D_{\mathrm{N}}$ was underestimated by .30 and when $D_{\mathrm{N}}$ was overestimated by .30 for this condition. When the $D_{\mathrm{N}}$ parameter was underestimated by .30 (i.e., estimated $\mathrm{D}_{\mathrm{N}}=.30$ ), $D_{1}$ and $D_{2}$ increased to .70 (from .67), $D_{3}$ increased to .78 (from .75), and the $d_{1}$ and $d_{2}$ parameters decreased to .57 (from .60), a net change of .03 on all these parameters. This also resulted in a reduction in the value of the $b$ parameter to .11 (from .20). However, in the comparison condition when $D_{\mathrm{N}}$ was overestimated by .30 (i.e., estimated $D_{\mathrm{N}}=.90$ ), $D_{1}$ decreased to .40 (from .67), the $d_{1-2}$ parameters increased to 1.0 (from .60, indicating perfect source recognition), a net change of .27 and .40 , respectively. In addition, the $b$ parameter increased to .59 (from .20).

Following the analysis where the estimating $D_{\mathrm{N}}$ parameter was fixed at various values, we also examined the results using an unconstrained 2HT model (i.e., all parameters, including the $D_{\mathrm{N}}$ parameter, were free to vary). The results are shown in Table 1 (bottom row). The unconstrained 2HT model did not retrieve the generating parameters in any of the lower $D_{\mathrm{N}}$ conditions (i.e., generating $D_{\mathrm{N}}=0, .30$ or .60), despite a perfect fit (i.e., very low $G^{2}$ values). Instead the model tended to estimate $D_{\mathrm{N}}$ values that were higher than the generating $D_{\mathrm{N}}$ values, resulting in a corresponding elevation of the $b$ and $d$ parameter values, and a reduction in the $D$ parameter values.

Both 1HT and 2HT models estimated the guessing parameters $a, a_{1}, g$ and $g_{1}$ accurately, and their values did not change with changes in the value of the estimating $D_{\mathrm{N}}$ parameter; therefore, they are not presented here. 
Table 1. Estimated Parameter Values Presented as a Function of Varying Generating and Estimating $D_{\mathrm{N}}$ Parameter Values

\begin{tabular}{|c|c|c|c|c|c|c|c|c|c|c|c|c|c|}
\hline & \multicolumn{13}{|c|}{ Generating $\mathrm{D}_{\mathrm{N}}$ Parameter } \\
\hline & \multicolumn{6}{|c|}{$D_{\mathrm{N}}=0(1 \mathrm{HT}) \mathrm{FP}=.20$} & \multicolumn{7}{|c|}{$D_{\mathrm{N}}=.30 \mathrm{FP}=.14$} \\
\hline Est $D_{\mathrm{N}}$ & $D_{1-2}$ & $D_{3}$ & $d_{1-2}$ & $d_{3}$ & $b$ & Fit & Est $D_{\mathrm{N}}$ & $D_{1-2}$ & $D_{3}$ & $d_{1-2}$ & $d_{3}$ & $b$ & Fit \\
\hline$D_{\mathrm{N}}=0$ & .67 & .75 & .60 & .59 & .20 & $0(.99)$ & $D_{\mathrm{N}}=0$ & .69 & .77 & .58 & .58 & .14 & $0(.99)$ \\
\hline$D_{\mathrm{N}}=.30$ & .63 & .72 & .64 & .61 & .29 & $0(.99)$ & $D_{\mathrm{N}} .30$ & .67 & .75 & .60 & .59 & .20 & $0(.99)$ \\
\hline$D_{\mathrm{N}}=.60$ & .47 & .60 & .85 & .74 & .50 & $0(.98)$ & $D_{\mathrm{N}}=.60$ & .59 & .69 & .68 & .64 & .36 & $0(.99)$ \\
\hline$D_{\mathrm{N}}=.90$ & .37 & .44 & 1.0 & 1.0 & .68 & $62.8(.0)$ & $D_{\mathrm{N}}=.90$ & .38 & .45 & 1.0 & 1.0 & .65 & $26.3(.0)$ \\
\hline$D_{\mathrm{N}}=.53$ & .54 & .66 & .74 & .68 & .43 & $0(.99)$ & $D_{\mathrm{N}}=.61$ & .58 & .69 & .69 & .65 & .37 & $0(.99)$ \\
\hline & \multicolumn{13}{|c|}{ Generating $D_{\mathrm{N}}$ Parameter } \\
\hline & \multicolumn{6}{|c|}{$D_{\mathrm{N}}=.60 \mathrm{FP}=.08$} & \multicolumn{7}{|c|}{$D_{\mathrm{N}}=.90 \mathrm{FP}=.02$} \\
\hline Est $D_{\mathrm{N}}$ & $D_{1-2}$ & $D_{3}$ & $d_{1-2}$ & $d_{3}$ & $b$ & Fit & Est $D_{\mathrm{N}}$ & $D_{1-2}$ & $D_{3}$ & $d_{1-2}$ & $d_{3}$ & $b$ & Fit \\
\hline$D_{\mathrm{N}}=0$ & .71 & .78 & .57 & .56 & .08 & $0(.99)$ & $D_{\mathrm{N}}=0$ & .73 & .80 & .55 & .55 & .02 & $0(.99)$ \\
\hline$D_{\mathrm{N}}=.30$ & .70 & .78 & .57 & .57 & .11 & $0(.99)$ & $D_{\mathrm{N}}=.30$ & .73 & .80 & .55 & .56 & .03 & $0(.99)$ \\
\hline$D_{\mathrm{N}}=.60$ & .67 & .75 & .60 & .59 & .20 & $0(.99)$ & $D_{\mathrm{N}}=.60$ & .72 & .79 & .56 & .56 & .05 & $0(.99)$ \\
\hline$D_{\mathrm{N}}=.90$ & .40 & .52 & 1.0 & .74 & .59 & $2.6(.10)$ & $D_{\mathrm{N}}=.90$ & .67 & .75 & .60 & .58 & .20 & $0(.99)$ \\
\hline$D_{\mathrm{N}}=.73$ & .63 & .72 & .65 & .62 & .30 & $0(.99)$ & $D_{\mathrm{N}}=.91$ & .67 & .75 & .61 & .59 & .21 & $0(.99)$ \\
\hline
\end{tabular}

Note. The generating probabilities used were: $D_{1} / \mathrm{D}_{2}=.67, D_{3}=.75, d_{1} / d_{2}=.60, d_{3}=.60, a=.60, b=.20, g$ $=.60, a_{1}=.50, g_{1}=.50$. FP refers to false positives, and Fit refers to Chi-square values of the final model $(p$ values are bracketed, such that $\mathrm{p}<.05$ indicates poor fit). When estimating, all parameters were free to vary with the exception of $D_{\mathrm{N}}$, which was fixed (Est $D_{\mathrm{N}}$ ). The bottom row displays the results when the $D_{\mathrm{N}}$ parameter was also allowed to vary. The models generated guessing parameters $\left(a, a_{1}, g, g_{1}\right)$ that were identical to the generating probabilities and did not vary across the different simulations, and are therefore not listed in the above table. 


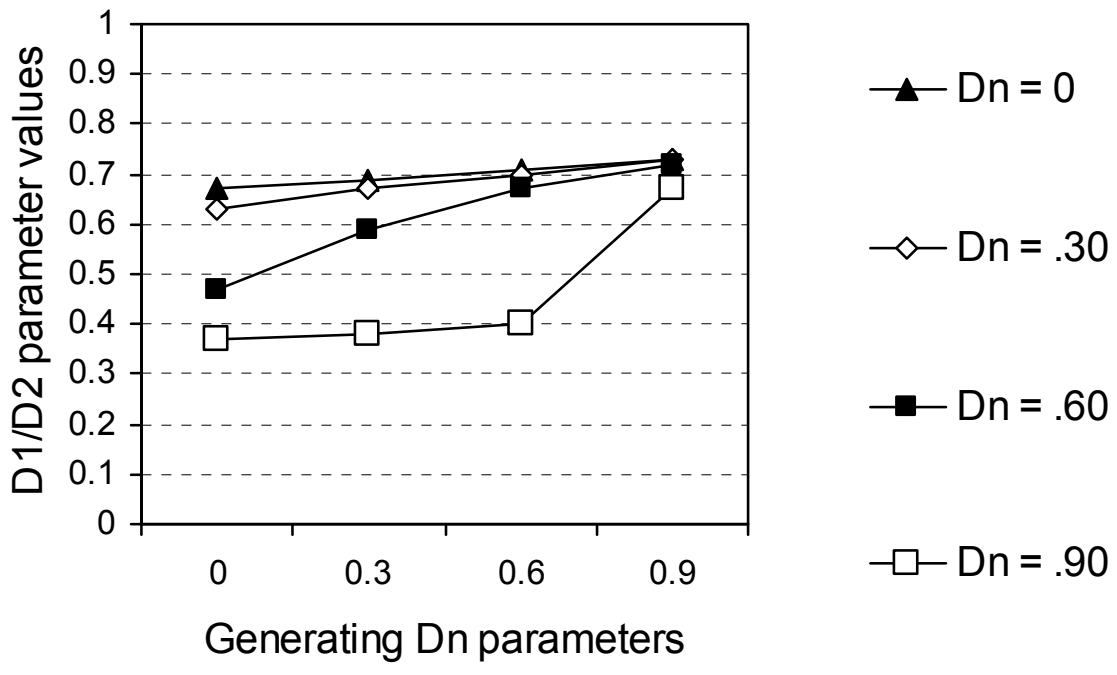

Figure 2 Estimated $D_{1} / D_{2}$ parameter values plotted as a function of generating and estimated $D_{\mathrm{N}}$ parameters (True $D_{1} / D_{2}=.67$ ).

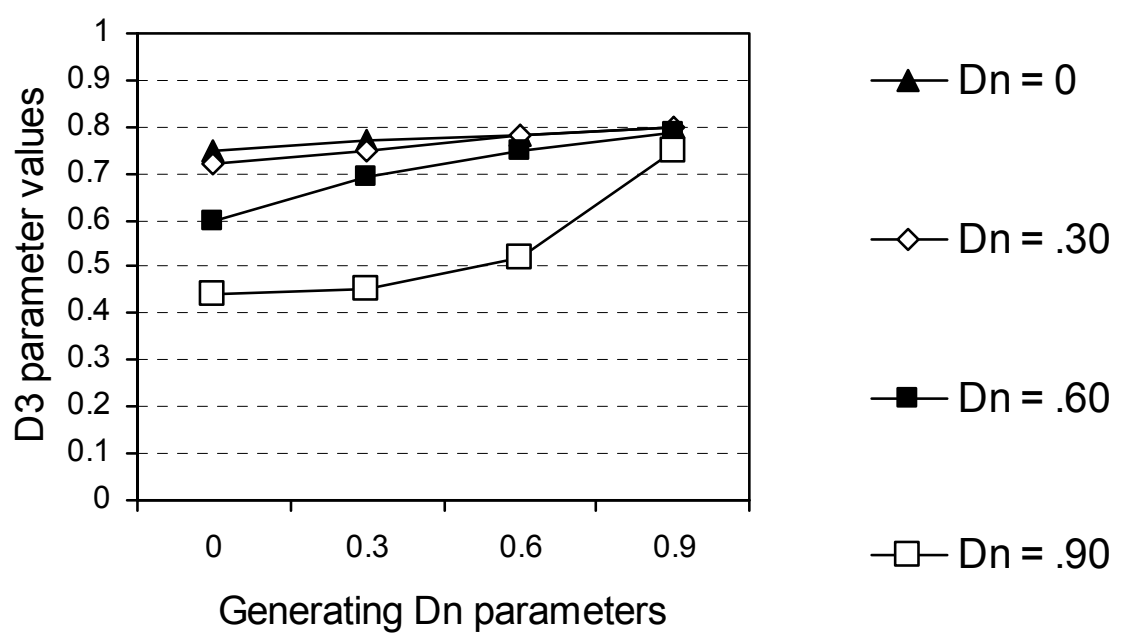

Figure 3. Estimated $D_{3}$ parameter values plotted as a function of generating and estimated $D_{\mathrm{N}}$ parameters (True $D_{3}=.75$ ). 

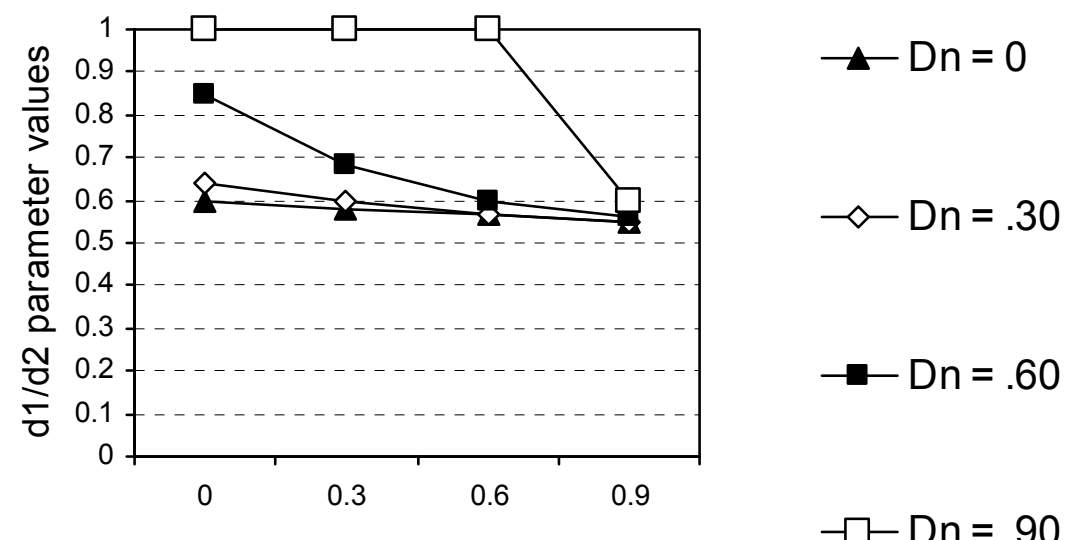

Generating Dn parameters

$-\square-\mathrm{Dn}=.90$

Figure 4 Estimated $d_{1} / d_{2}$ parameter values plotted as a function of generating and estimated $D_{\mathrm{N}}$ parameters (True $d_{1} / d_{2}=.60$ ).
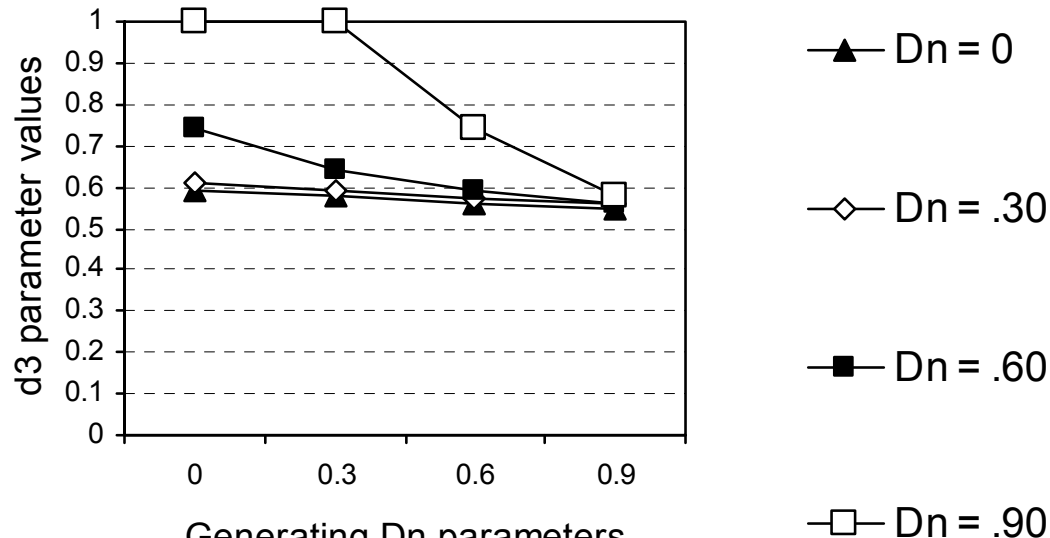

Figure 5. Estimated $d_{3}$ parameter values plotted as a function of generating and estimated $D_{\mathrm{N}}$ parameters (True $d_{3}=.60$ ) 


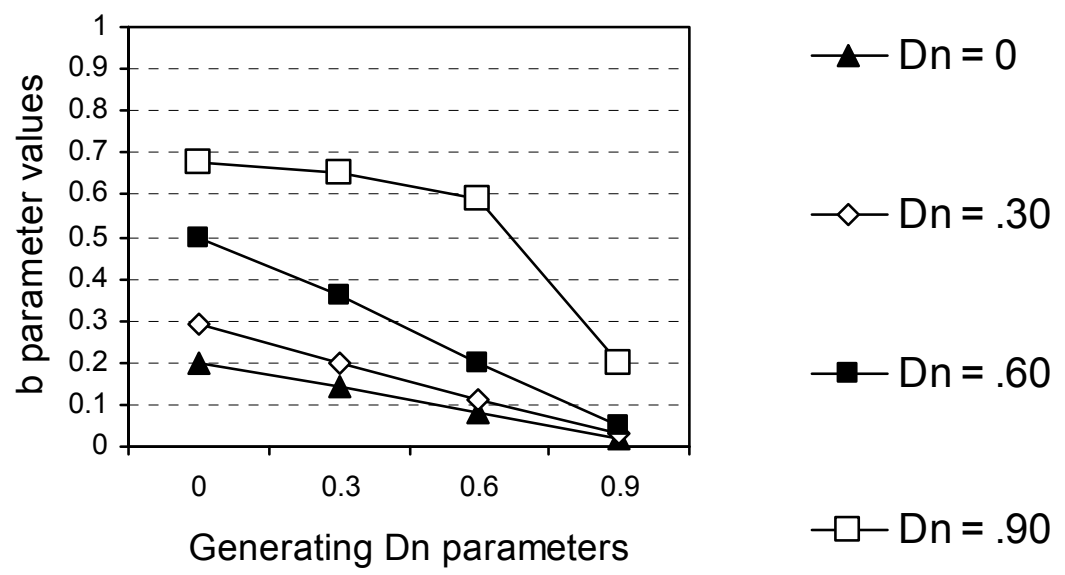

Figure 6. Estimated $b$ parameter values plotted as a function of generating and estimated $D_{\mathrm{N}}$ parameters (True $b=.20$ ).

\section{Conclusion}

This simulation was designed to compare the $1 \mathrm{HT}$ and $2 \mathrm{HT}$ approaches to multinomial modeling on their ability to accurately estimate underlying parameter values under a number of experimental conditions. The results suggest that the 1 HT model is a more conservative choice for the analysis of data from source monitoring tasks, because the $2 \mathrm{HT}$ models are more likely to misestimate the underlying parameters. Specifically, the simulation showed that use of the 2HT models lead to an artificial inflation of the $b$ parameter (probability of guessing that an item not detected as old was actually seen before), which in turn caused inflation of $d$ (source recognition), and deflation of $D$ (item recognition) parameter values. The $1 \mathrm{HT}$ model showed less variability and gave parameter estimates that were closer to the underlying parameter values, even when the underlying assumptions were those held by the 2HT perspective. As the simulation shows, the unconstrained 2HT solution (where the $D_{\mathrm{N}}$ parameter value was not fixed - Table 1 bottom row) typically produced a $D_{\mathrm{N}}$ parameter value that was in excess of the true generating $D_{\mathrm{N}}$ value, which in turn affected many of the other parameters as outlined above. With respect to a behavioral interpretation, the 2HT models produce parameter values that suggest artificially reduced item recognition for old items, increased guessing of undetected old items, and increased source recognition compared to the 1HT model. For instance, with the high estimated $D_{\mathrm{N}}$ parameter values, the $2 \mathrm{HT}$ models suggest a state with perfect source recognition even though item recognition is occurring much less frequently, which is counterintuitive, given that item recognition is generally regarded as a less demanding process than source recognition.

With respect to the theoretical issue mentioned in the Introduction, whether or not the recognition of new items occurs via the new items crossing a definite threshold (as suggested by the 2HT model) or simply by not being recognized as being old (as suggested by the 1HT model) remains open to debate. What the simulation results show is that, given that these theoretical issues cannot currently be resolved, the 1 HT model is a more conservative choice for 
the analysis of data from source monitoring tasks, because the 2HT models are more sensitive to possible mis-estimation of the underlying parameters.

With respect to interpretability of the $b$ parameter (the probability of guessing that an undetected item is actually a target item), as can be seen in Table 1, the results show that only the 1HT model (i.e., $D_{\mathrm{N}}=0$ ) produced a $b$ parameter value equal to the false positive error rate. In the 2HT model, increasing values of $D_{\mathrm{N}}$ resulted in $b$ parameter values that were far higher than the proportion of false positives. As mentioned earlier, the parameter is an indicator of two separate scenarios. For previously seen words, it is an indicator of the tendency to guess that the word is old even when it has not been detected as being old, and for new words, it is the tendency to make false positive errors (i.e., detect them as being old). However, the crucial difference between these two processes is that the tendency to make false positive errors can be estimated (by observing raw frequencies of the number of false positive recognition errors), while the same cognitive process for old words (making fortuitous false positive recognitions, which in turn elevates the hit rate), remains a hidden process.

If both these patterns of false recognition are driven by the same cognitive processes, then the $b$ parameter should reflect both in equal measure. The use of the 1HT model, which generates the value of the $b$ parameter corresponding to the proportion of false positive error rates, has an intuitive appeal from this perspective. The $2 \mathrm{HT}$ model is likely to produce a $b$ parameter that is inflated, possibly leading to the false conclusion that much of the recognition reflected by the raw frequencies occurs through fortuitous guessing. Moreover, an increase in $b$ causes corresponding decreases and increases in the $D$ and $d$ parameters, respectively, leading to the false conclusion that recognition has decreased and source discrimination has increased, respectively.

In an important study that suggested the superiority of the 2HT model over the 1HT model in source monitoring, Bayen et al. (1996) studied the impact of increased distracter similarity on the ability to carry out item detection and source recognition. On the assumption that increased distracter similarity (but not increased source similarity) would decrease item detection, they analyzed their results using $1 \mathrm{HT}$ and $2 \mathrm{HT}$ models. They found that $D$ parameter values decreased with increasing distracter similarity only when the 2HT model was used, and argued on these grounds that the $2 \mathrm{HT}$ is more sensitive to changes in item detection, making it a superior model of source monitoring. However, as Bayen et al. (1996) pointed out, increasing distracter similarity only serves to increase the number of false alarms and not the hit rate (p 205, Appendix C pg 215), casting doubt on their assumption that increasing distracter similarity should decrease item detection. Moreover, the simulation results demonstrate that use of the 2HT model is likely to lead to artificial decreases in the recognition parameter $D$, suggesting that Bayen et al.'s (1996) recommendations may have been based on an artifact of the 2HT model, as opposed to the purported superiority of the 2HT model in detecting an experimental-manipulation-induced true decrease in recognition.

There are two reasons to suggest that the recognition parameters may have been affected by the model selected rather than the experimental manipulation. First, the Bayen et al. (1996) models used one $D$ parameter for both old and new items (i.e. $D_{\mathrm{N}}=D_{1}=D_{2}$ ), and as a result, the increase in false positives, which should cause a decrease in $D_{\mathrm{N}}$ but not in $D_{1}$ or $D_{2}$, affected all three of these parameters. Secondly, due to the reciprocal relationship between $b$ and $D$ in the 2HT model (as can be seen from Figures 2, 3 and 6), an increase in the $b$ parameter (which is often well in excess of the true proportion of false alarms in the experimental data) caused a reduction in the $D$ parameter. That is to say, use of the $2 \mathrm{HT}$ model can lead to apparent decreases in item detection as an artifact of increases in false positives, even in the absence of an experimental manipulation affecting item detection.

In the aforementioned Bayen et al. (1996) study, the 1HT model, on the other hand, showed a slight decrease in the $D$ parameter due to an increase in false alarms, and a significant decrease in the $D$ parameter only when the hit 
rate decreased. This pattern of results would be expected from a model that was appropriately reflecting factors affecting false alarms independently from those affecting item detection.

Bayen et al. (1996) computed decreases in the signal detection measure $d^{\prime}$ for item detection. They showed decreases in $d^{\prime}$ with increasing source similarity, and, based on this measure, concluded that their experimental manipulations had affected the true recognition rate. Batchelder, Riefer and $\mathrm{Hu}$ (1994) and Thomas and Olzak (1992) explicitly pointed out that $d^{\prime}$ can be used as an means to compare item recognition only when false alarm rates are comparable across conditions. For a paradigm such as that used by Bayen et al. (1996), where the manipulation specifically affected the false alarm rates, $d^{\prime}$ is expected to underestimate item detection as the false alarm rate increases. It therefore seems likely that the fundamental assumption of their experimental manipulation (i.e., that increasing distracter similarity causes decreases in item detection) is debatable, and that the decreases in recognition parameters that they observed when employing the 2HT model may have instead reflected an artifact of the 2HT model.

The data simulation indicates that the 1HT model generally provides more accurate estimates of the underlying parameter values than the 2HT model, and is more robust to variation in the generating $D_{\mathrm{N}}$ parameters. In addition, with respect to interpretation, the 1HT model produces a $b$ parameter value that accurately reflects the true proportion of false positives and the 2HT model inflates the $b$ parameter value, which in turn spuriously reduces the item detection parameters and inflates the source recognition parameters. In the light of these considerations, use of the 1HT model over the 2HT model is recommended in the analysis of data from source monitoring studies.

\section{References}

Batchelder, W. H., \& Riefer, D. M. (1990). Multinomial processing models of source monitoring. Psychological Review, 97, 548-564.

Batchelder, W. H., \& Riefer, D. M. (1999). Theoretical and empirical review of multinomial process tree modeling. Psychonomic Bulletin \& Review, 6, 57-86.

Batchelder, W. H., Riefer, D. M., \& Hu, X. G. (1994). Measuring memory factors in source monitoring - reply to kinchla. Psychological Review, 101, 172-176.

Bayen, U. J., Murname, K., \& Erdfelder, E. (1996). Source monitoring, item detection and multinomial models of source monitoring. Journal of Experimental Psychology: Learning, Memory and Cognition, 22, 197- 215.

Brebion, G., Gorman, J. M., Amador, X., Malaspina, D., \& Sharif, Z. (2002). Source monitoring impairments in schizophrenia: Characterisation and associations with positive and negative symptomatology. Psychiatry Research, 112(1), 27-39.

Dodson, C. S., Prinzmetal, W., \& Shimamura, A. P. (1998). Using excel to estimate parameters from observed data: An example from source memory data. Behavior Research Methods, Instruments, \& Computers, 30, 517-526.

Hoffman, H. G. (1997). Role of memory strength in reality monitoring decisions: Evidence from source attribution biases. Journal of experimental Psychology: Learning, Memory and Cognition, 23(2), 371-383.

Johnson, M. K., Hashtroudi, S., \& Lindsay, D. S. (1993). Source monitoring. Psychological Bulletin, 114(1), 3-28.

Keefe, R. S. E., Arnold, M. C., Bayen, U. J., McEvoy, J. P., \& Wilson, W. H. (2002). Source-monitoring deficits for self-generated stimuli in schizophrenia: Multinomial modeling of data from three sources. Schizophrenia Research, 57(1), 51-67. 
Lindsay, D. S., Johnson, M. K., \& Kwon, P. (1991). Developmental changes in memory source monitoring. Journal of Experimental Child Psychology, 52, 297-318.

Macmillan, N. A., \& Creelman, C. D. (1991). Detection theory: A user's guide. Cambridge, England: Cambridge University Press.

Riefer, D. M., \& Batchelder, W. (1988). Multinomial modeling and the measurement of cognitive processes. Psychological Review, 95(3), 318-339.

Riefer, D. M., \& Batchelder, W. (1991). Statistical inference for multinomial processing tree models. In J.-P. Doignon \& J.-C. Falmange (Eds.), Mathematical psychology: Current developments. New York: Springer-Verlag.
Riefer, D. M., Hu, X. G., \& Batchelder, W. H. (1994). Response strategies in source monitoring. Journal of Experimental Psychology-Learning Memory and Cognition, 20(3), 680-693.

Thomas, J. P., \& Olzak, L.A. (1992). Simultaneous detection and identification. In F. G. Ashby (Ed.), Multidimensional models of perception and cognition (pp. 253-277). Hillsdale, NJ: Erlbaum.

Woodward, T. S., Menon, M., \& Whitman, J. C. (in press). Multinomial modelling of source monitoring biases associated with hallucinations and delusions in schizophrenia.

Woodward TS, Menon M, Hu X, Keefe RSE. (2006). Optimization of a multinomial model for investigations of hallucinations and delusions with source monitoring. Schizophrenia Research, 85, 106- 112.

Appendix A

Table of frequencies generated with varying $2 \mathrm{HT}$ assumptions

\begin{tabular}{|c|c|c|c|c|}
\hline \multirow[b]{2}{*}{ Source } & \multicolumn{4}{|c|}{ Response } \\
\hline & Experimenter & Computer & Self & New \\
\hline & & $D_{\mathrm{N}}=0$ & & \\
\hline Experimenter & $50(.50)$ & $10(.10)$ & $13(.13)$ & $26(.26)$ \\
\hline Computer & $10(.10)$ & $50(.50)$ & $13(.13)$ & $26(.26)$ \\
\hline Self & $11(.11)$ & $11(.11)$ & $58(.58)$ & $20(.20)$ \\
\hline New & $18(.06)$ & $18(.06)$ & $24(.08)$ & $240(.80)$ \\
\hline & & $D_{\mathrm{N}}=.30$ & & \\
\hline Experimenter & $50(.50)$ & $10(.10)$ & $13(.13)$ & $26(.26)$ \\
\hline Computer & $10(.10)$ & $50(.50)$ & $13(.13)$ & $26(.26)$ \\
\hline Self & $11(.11)$ & $11(.11)$ & $58(.58)$ & $20(.20)$ \\
\hline New & $13(.04)$ & $13(.04)$ & $17(.06)$ & $258(.86)$ \\
\hline & & $D_{\mathrm{N}}=.60$ & & \\
\hline Experimenter & $50(.50)$ & $10(.10)$ & $13(.13)$ & $26(.26)$ \\
\hline Computer & $10(.10)$ & $50(.50)$ & $13(.13)$ & $26(.26)$ \\
\hline Self & $11(.11)$ & $11(.11)$ & $58(.58)$ & $20(.20)$ \\
\hline New & $7(.02)$ & $7(.02)$ & $10(.03)$ & $276(.92)$ \\
\hline & & $D_{\mathrm{N}}=.90$ & & \\
\hline Experimenter & $50(.50)$ & $10(.10)$ & $13(.13)$ & $26(.26)$ \\
\hline Computer & $10(.10)$ & $50(.50)$ & $13(.13)$ & $26(.26)$ \\
\hline Self & $11(.11)$ & $11(.11)$ & $58(.58)$ & $20(.20)$ \\
\hline New & $2(.007)$ & $2(.007)$ & $2(.007)$ & $294(.98)$ \\
\hline
\end{tabular}

Note. Row percentages are presented in brackets. Correct responses are in bold. 


\title{
Examining Cronbach Alpha, Theta, Omega Reliability Coefficients According to the Sample Size
}

\author{
Ilker Ercan \\ Berna Yazici \\ Uludag University, Turkey \\ Anadolu University, Turkey \\ Deniz Sigirli \\ Uludag University, Turkey \\ Bulent Ediz \\ Ismet Kan \\ Uludag University, Turkey \\ Uludag University, Turkey
}

Differentiations according to the sample size of different reliability coefficients are examined. It is concluded that the estimates obtained by Cronbach alpha and teta coefficients are not related with the sample size, even the estimates obtained from the small samples can represent the population parameter. However, the Omega coefficient requires large sample sizes.

Key words: Cronbach alpha, theta, omega, reliability, scale, sample size.

\section{Introduction}

A scale is needed to measure and that scale must be reliable and valid. The scale's reliability does not matter in the case of measuring the concrete characteristics. But, it is an important problem in the case of measuring the abstract characteristics. So, it is necessary to analyze the reliability of the scales using some statistical

Ilker Ercan is in the Department of Biostatistics. Research interests include reliability analysis, statistical shape analysis, and cluster analysis. Email at ercan@uludag.edu.tr. Berna Yazici is in the Department of Statistics. Research interests include experimential design, regression analysis, and quality control. E-mail at bbaloglu@anadolu.edu.tr Deniz Sigirli in the Department of Biostatistics. Research interests include neural networks. E-mail at sigirli@uludag.edu.tr. Bulent Ediz is in the Department of Biostatistics. Research interests include logistic regression analysis, discriminant analysis, and power analysis. E-mail at ediz@uludag.edu.tr. Ismet Kan is in the Department of Biostatistics. Research interests include applied statistics. E-mail at kan@uludag.edu.tr methods. In making a reliability analysis, the reliability coefficients that are suitable in obtaining the reliability of the scale and the structure of the empirical study must be examined. Sample size is also important to determine the reliability level of the scale. Thus, one of the dimensions that must be examined is the changes in Cronbach alpha, theta, and omega coefficients according to the sample size.

\section{Reliability}

The scale, used to get some information on a defined subject, must have some properties. Reliability, a property that a scale must have, is an indicator of consistency of measurement values obtained from the measurements repeated under the same circumstances (Gay, 1985; Carmines \& Zeller, 1982; Arkin \& Colton, 1970; O’Connor, 1993; Carey, 1988).

The reliability of the scale can be examined by different ways. The reliability of the scale can be examined by applying the scale once, applying the scale twice or applying the equivalent scales once. In case of applying the scale once, the reliability of internal consistency is examined. The reliability coefficient ranges between 0 and 1 . 
Methods of Internal Consistency

If the reliability can be estimated by applying the scale once, the error in reliability estimation will be less than the other reliability estimation methods. In this kind of reliability estimation, wrong management, scoring, temporary changes in personal performance affect the internal consistency, the leading affect will be the content sampling (O'Connor, 1993).

Another method, split-half, denotes the homogeneity indices of the items in the scales. It pertains to the relationship level between the responses of the items and the total scale score (Oncu, 1994). An increase in homogeneity in the set of items increases this reliability estimate (O'Connor, 1993). The idea that the internal consistency methods depend upon is that every measurement tool is constructed to realize an objective and those have known equal weights (Karasar, 2000). The internal consistency methods are preferred because they are economical and easy to apply (Oncu, 1994).

\section{Cronbach Alpha}

The Alpha coefficient method (Cronbach, 1951), is a suitable method that can be used for likert scale items (e.g., 1-3, 1-4, 1-5). Thus, it is not limited to the true-false or correctincorrect format (Oncu, 1994).

Cronbach alpha coefficient is weighted standard variations mean, obtained by dividing the total of the $\mathrm{k}$ items in the scale, to the general variance (Thorndike et al., 1991).

$$
\alpha=\frac{n}{(n-1)}\left[1-\frac{\sum_{i=1}^{n} \sigma_{Y_{i}}^{2}}{\sigma_{x}^{2}}\right]
$$

$\mathrm{n}:$ Number of the items

$$
\begin{aligned}
& \boldsymbol{\sigma}_{Y_{i}}: \mathrm{i}^{\text {th }} \text { item's standard deviation } \\
& \boldsymbol{\sigma}_{X}: \text { General standard deviation }
\end{aligned}
$$

If the items are standardized, coefficient is calculated by using the items' correlation mean or variance-covariances' mean (Carmines \& Zeller, 1982; Ozdamar, 1999a; SPSS, 1991; SPSS, 1999).
Calculation of alpha coefficient due to the correlation mean,

$$
\alpha=\frac{n \bar{\rho}}{1+(n-1) \bar{\rho}}
$$

Calculation of alpha coefficient due to the variance-covariance mean,

$$
\alpha=\frac{n \overline{\sigma_{X X}} / \overline{\sigma_{X}}}{1+(n-1) \overline{\sigma_{X X}} / \overline{\sigma_{X}}}
$$

When the formula for calculating Cronbach alpha using the correlation means between items is examined, it can be seen that it is proportionally related with the number of the items and the mean of the correlation between items (Carmines \& Zeller, 1982). If the correlation between the items is negative, alpha coefficient will also be negative. Because this situation will spoil the scale's additive property, it also causes a spoil in the reliability model and the scale is no more additive (Ozdamar, 1999a). The coefficient is equal to the mean of all probable coefficients using split-half method (Carmines \& Zeller, 1982; Gursakal, 2001).

Theta Coefficient

The Theta coefficient depends on the principal components analysis. In principal components analysis, the components are in descending order due to the variances of $(2 \mathrm{ch})$ of the constructions (Carmines \& Zeller, 1982). The first component is the linear component with the maximum variance. The second component is the linear component with the second maximum variance. Components can be explained by the component variances defined by the percentage values to explain the variance of the original data set in order (Ozdamar, 1999b). Theta coefficient depends on that property. The Theta coefficient, takes into account the eigenvalue that maximum explains the event, is calculated as follows: 


$$
\theta=(N / N-1)\left(1-1 / \lambda_{i}\right)
$$

$\mathrm{N}:$ Number of items

$\lambda_{i}$ : The largest eigenvalue (the first eigenvalue)

\section{Omega Coefficient}

Another coefficient for linear dependencies is the Omega coefficient proposed by Heise and Bohrnstedt (1970). It depends on the factor analysis model. Omega coefficient is modeled on factor analysis. In this type of modeling, in calculating the coefficient, before factoring " 1 " values on diagonal in the correlation matrix are replaced with the communality values. The Omega coefficient can be calculated with two ways, using variancecovariance matrix and correlation matrix (Carmines \& Zeller, 1982).

When studied with variance-covariance matrix,

$$
\Omega=1-\left(\sum \sigma_{\mathrm{i}}^{2}-\sum \sigma_{\mathrm{i}}^{2} \mathrm{~h}_{\mathrm{i}}^{2}\right) /\left(\sum \sum \sigma_{\mathrm{X}_{\mathrm{i}} \mathrm{X}_{\mathrm{j}}}\right)
$$

$\mathrm{h}_{\mathrm{i}}^{2}$ : Communality of the $\mathrm{i}^{\text {th }}$ item

When studied with correlation matrix,

$\Omega=1-\left(a-\sum h_{i}^{2}\right) /(a+2 b)$

a: Number of items

b: Sum of the correlations among items

There are some differences between the Theta and Omega coefficients. They depend on different factor-analytic models. The Theta coefficient depends on principal components model, whereas the Omega coefficient depends on factor analysis model. Therefore, in calculating the eigenvalues for Theta coefficients, the diagonal 1.0 values are used, but in calculating the Omega coefficients, communality values that are not related with 1.0 values are used (Carmines \& Zeller, 1982).

There is a relationship between Alpha, Theta, and Omega coefficients. If the items take parallel values, three coefficients are equal each other and will be 1.0. Otherwise, the relationship of magnitude for the coefficients will be $\alpha<\theta<$ $\Omega$. Among these internal consistency coefficients, $\alpha$ gives the lower bound of the reliability coefficient and $\Omega$ gives the upper bound of the reliability coefficient (Carmines \& Zeller, 1982).

\section{Methodology}

To compare the Alpha, Theta and Omega coefficients, a data set has been used from an instrument developed by Ercan et al. (2004) to measure patient satisfaction in the secondary health-care units. To obtain the effects of different number of items and different sample sizes, 3 different scales are constructed with 39 , 34 , and 30 items by subtracting some items from the scale with 43 items. Because all the subjects did not answer all the items, the subject numbers in the scales are also different. There are 170 subjects answered all of the 43 items, 240 subjects answered all of the 39 items, 230 subjects answered all of the 34 items, and 320 subjects answered all of the 30 items.

After giving a number to each of the subjects, samples are constructed by producing random numbers using MINITAB 13.2 beginning from 10 and increasing 10 units each of those random numbers. The same procedure was repeated 10 times and for each of the samples Cronbach alpha, Theta and Omega reliability coefficients are calculated.

SPSS 13.0 was used for these analyses. Statistical comparisons are performed in order to determine if alpha, theta and omega coefficients change or not according to the sample size and in order to determine the sample size that the reliability coefficients begin to get stable. Before the between group comparisons, the homogeneity of variances is tested using the Levene statistic. If the variances are found to be homogeneous, then analysis of variance 
Table-4.1: The homogeneity test results for the scale with 30 items

\begin{tabular}{ccccc}
\hline & Levene Statistic & $\begin{array}{c}\text { Degree of } \\
\text { Freedom 1 }\end{array}$ & $\begin{array}{c}\text { Degree of } \\
\text { Freedom 2 }\end{array}$ & $\begin{array}{c}\text { Significance } \\
\text { level (p) }\end{array}$ \\
\hline$\alpha$ & 5.631 & 31 & 288 & $<0.001$ \\
\hline$\theta$ & 5.578 & 31 & 288 & $<0.001$ \\
\hline$\Omega$ & 1.531 & 31 & 288 & 0.040 \\
\hline
\end{tabular}

Table-4.2: Significance level in comparison of $\alpha, \theta$ and $\Omega$ reliability coefficients according to different sample sizes using Kruskal-Wallis test for the scale with 30 items

\begin{tabular}{cccc}
\hline & $\alpha$ & $\theta$ & $\Omega$ \\
\hline$\chi^{2}$ & 23.706 & 46.720 & 259.636 \\
\hline Degree of freedom & 31 & 31 & 31 \\
\hline Significance level $(\mathrm{p})$ & 0.822 & 0.035 & $<0.001$ \\
\hline
\end{tabular}

$$
\begin{aligned}
\text { Bonferroni correction: } & \alpha^{*}=1-(1-\alpha)^{1 / \mathrm{k}} \\
& \alpha^{*}=1-(1-0.05)^{1 / 32}=0.0016
\end{aligned}
$$

and Tukey HSD post-hoc comparison test are applied. If the variances are heterogeneous, Kruskal-Wallis and Mann-Withney U tests are applied to make reliability comparisons according to sample size. The level of significance in multiple comparisons is determined after Bonferrroni correction $\left(\alpha^{*}=1-(1-\alpha)^{1 / \mathrm{k}} \mathrm{k}\right.$ : number of groups).

\section{Results}

The results of comparisons $\alpha, \theta$ and $\Omega$ coefficients according to different sample sizes are given in Table 4.1, 4.2, 4.3, 4.4 for the scale with 30 items. 
Table-4.3: Significance level ( $\mathrm{p}$ values $\times 10^{-3}$ ) in comparison of $\theta$ reliability coefficients according to different sample sizes using Mann-Whitney $U$ test for the scale with 30 items $\left(\alpha^{*}=0.0016\right)$.

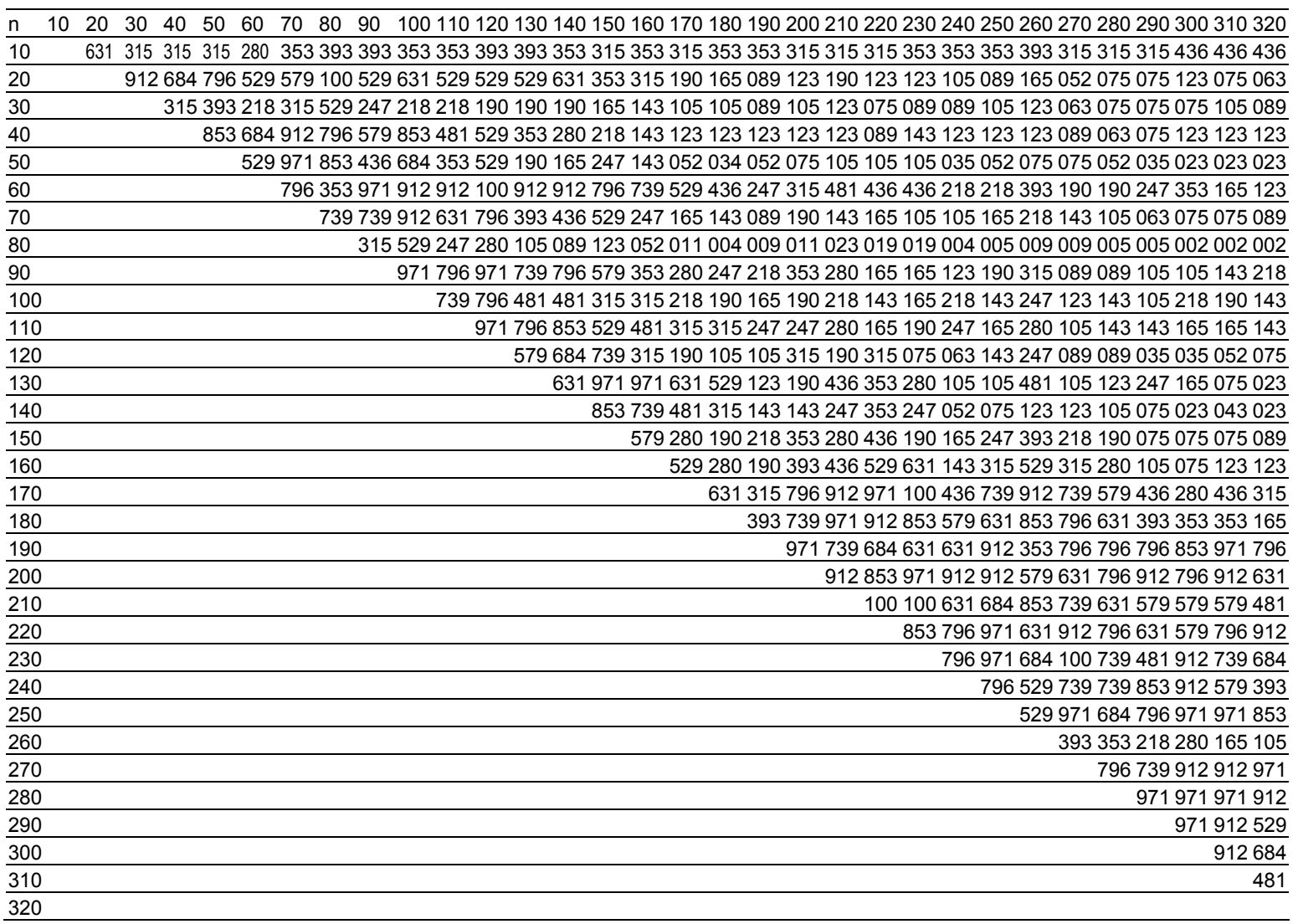


Table-4.4: Significance level ( $\mathrm{p}$ values $\times 10^{-3}$ ) in comparison of $\Omega$ reliability coefficients according to different sample sizes using Mann-Whitney $U$ test for the scale with 30 items $\left(\alpha^{*}=0.0016\right)$.

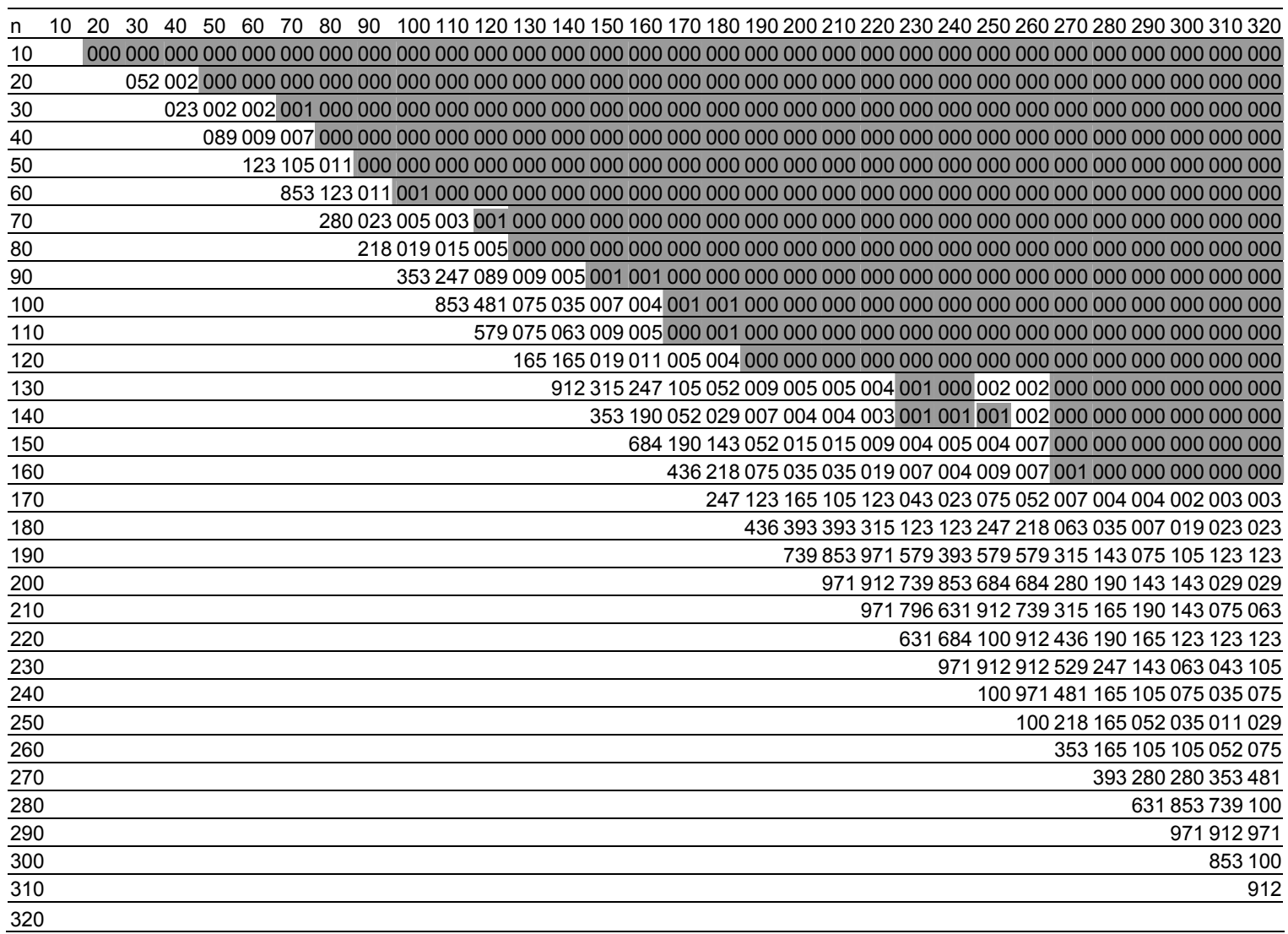


The results of comparisons $\alpha, \theta$ and $\Omega$ coefficients according to different sample sizes are given in Table 4.5, 4.6, 4.7 for the scale with 34 items.

Table-4.5: The homogeneity test results for the scale with 34 items

\begin{tabular}{ccccc}
\hline & $\begin{array}{c}\text { Levene } \\
\text { Statistic }\end{array}$ & $\begin{array}{c}\text { Degree of } \\
\text { freedom 1 }\end{array}$ & $\begin{array}{c}\text { Degree of } \\
\text { freedom 2 }\end{array}$ & $\begin{array}{c}\text { Significance level } \\
(\mathrm{p})\end{array}$ \\
\hline$\alpha$ & 11.003 & 22 & 207 & $<0.001$ \\
\hline$\theta$ & 10.477 & 22 & 207 & $<0.001$ \\
\hline$\Omega$ & 3.238 & 22 & 207 & $<0.001$ \\
\hline
\end{tabular}

Table-4.6: Significance level in comparison of $\alpha, \theta$ and $\Omega$ reliability coefficients according to different sample sizes using Kruskal-Wallis test for the scale with 34 items

\begin{tabular}{cccc}
\hline & $\alpha$ & $\theta$ & $\Omega$ \\
\hline$\chi^{2}$ & 6.329 & 8.960 & 176.741 \\
\hline Degree of freedom & 22 & 22 & 22 \\
\hline Significance level (p) & 1.000 & 0.994 & $<0.001$ \\
\hline
\end{tabular}

$$
\text { Bonferroni correction: } \begin{aligned}
& \alpha^{*}=1-(1-\alpha)^{1 / \mathrm{k}} \\
\alpha^{*} & =1-(1-0.05)^{1 / 23}=0.0022
\end{aligned}
$$


Table-4.7: Significance level ( $\mathrm{p}$ values $\times 10^{-3}$ ) in comparison of $\Omega$ reliability coefficients according to different sample sizes using Mann-Whitney U test for the scale with 34 items $\left(\alpha^{*}=0.0022\right)$

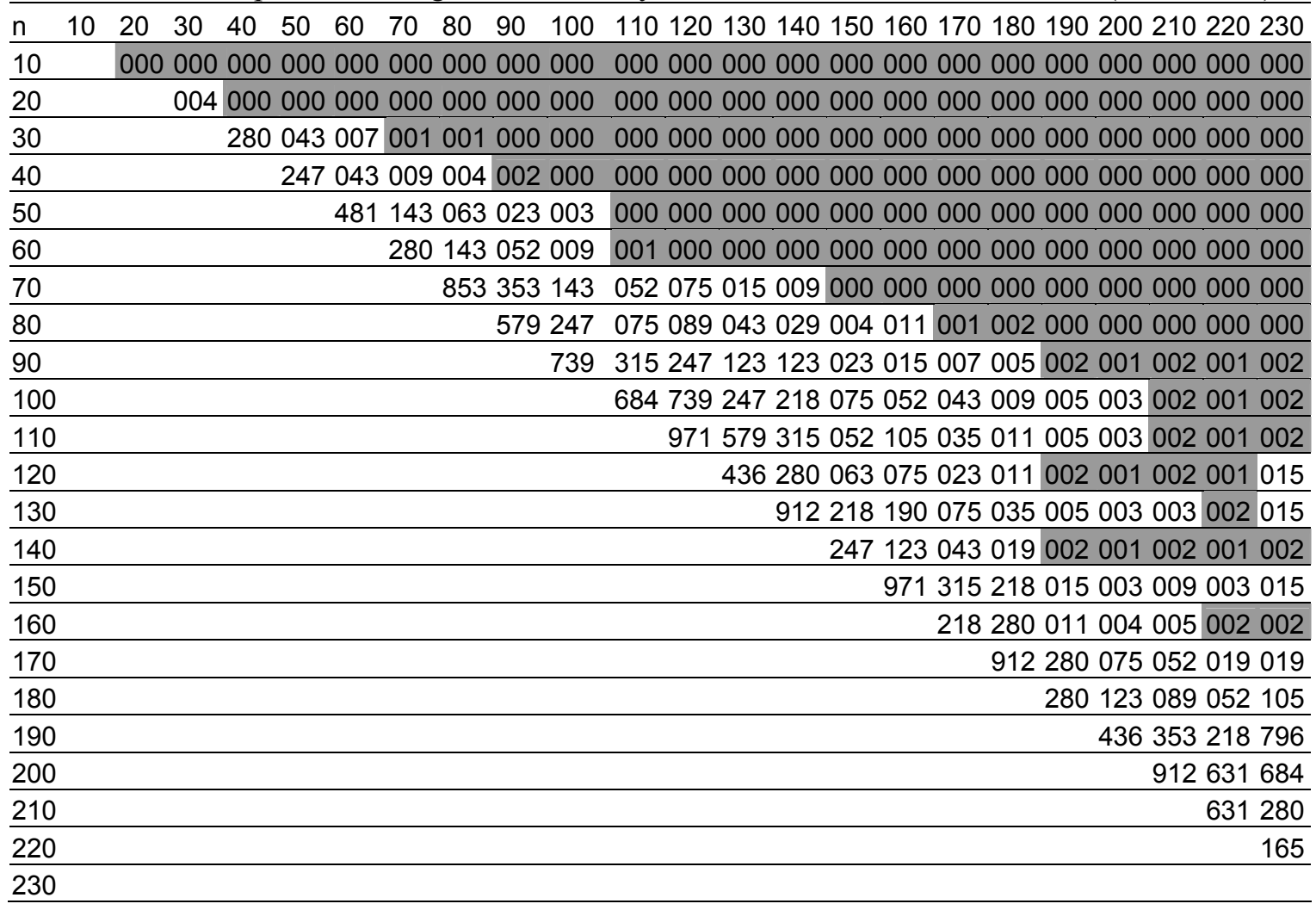


The results of comparisons $\alpha, \theta$ and $\Omega$ coefficients according to different sample sizes are given in Table 4.8, 4.9, 4.10, 4.11 for the scale with 39 items.

Table-4.8: The homogeneity test results for the scale with 39 items

\begin{tabular}{ccccc}
\hline & $\begin{array}{c}\text { Levene } \\
\text { Statistic }\end{array}$ & $\begin{array}{c}\text { Degree of } \\
\text { freedom 1 }\end{array}$ & $\begin{array}{c}\text { Degree of } \\
\text { freedom } 2\end{array}$ & $\begin{array}{c}\text { Significance level } \\
(\mathrm{p})\end{array}$ \\
\hline$\alpha$ & 10.692 & 23 & 216 & $<0.001$ \\
\hline $\boldsymbol{\Omega}$ & 12.048 & 23 & 216 & $<0.001$ \\
\hline & 1.418 & 23 & 216 & 0.104 \\
\hline
\end{tabular}

Table-4.9: Significance level in comparison of $\alpha$ and $\theta$ reliability coefficients according to different sample sizes using Kruskal-Wallis test for the scale with 39 items

\begin{tabular}{ccc}
\hline & $\alpha$ & $\theta$ \\
\hline$\chi^{2}$ & 7.206 & 8.702 \\
\hline Degree of freedom & 23 & 23 \\
\hline Significance level (p) & 0.999 & 0.997 \\
\hline
\end{tabular}

Table-4.10: Significance level in comparison of $\Omega$ reliability coefficients according to different sample sizes by analysis of variance for the scale with 39 items

\begin{tabular}{cccccc}
\hline & $\begin{array}{c}\text { Sum of } \\
\text { Squares }\end{array}$ & $\begin{array}{c}\text { Degrees of } \\
\text { freedom }\end{array}$ & $\begin{array}{c}\text { Sum of } \\
\text { Squares }\end{array}$ & F & $\begin{array}{c}\text { Significance } \\
\text { level (p) }\end{array}$ \\
\cline { 1 - 4 } $\begin{array}{c}\text { Between } \\
\text { groups }\end{array}$ & 0.00536 & 23 & 0.0002329 & & \\
\cline { 1 - 3 } $\begin{array}{c}\text { Within } \\
\text { groups }\end{array}$ & 0.000352 & 216 & 0.00000163 & 142.881 & $<0.001$ \\
\hline Total & 0.00571 & 239 & & & \\
\hline
\end{tabular}

$$
\text { Bonferroni correction: } \begin{aligned}
& \alpha^{*}=1-(1-\alpha)^{1 / \mathrm{k}} \\
\alpha^{*} & =1-(1-0.05)^{1 / 24}=0.0021
\end{aligned}
$$


Table-4.11: Significance level ( $\mathrm{p}$ values $\times 10^{-3}$ ) in comparison of $\Omega$ reliability coefficients according to different sample sizes using Tukey's HSD post-hoc comparison test

for the scale with 39 items $\left(\alpha^{*}=0.0021\right)$.

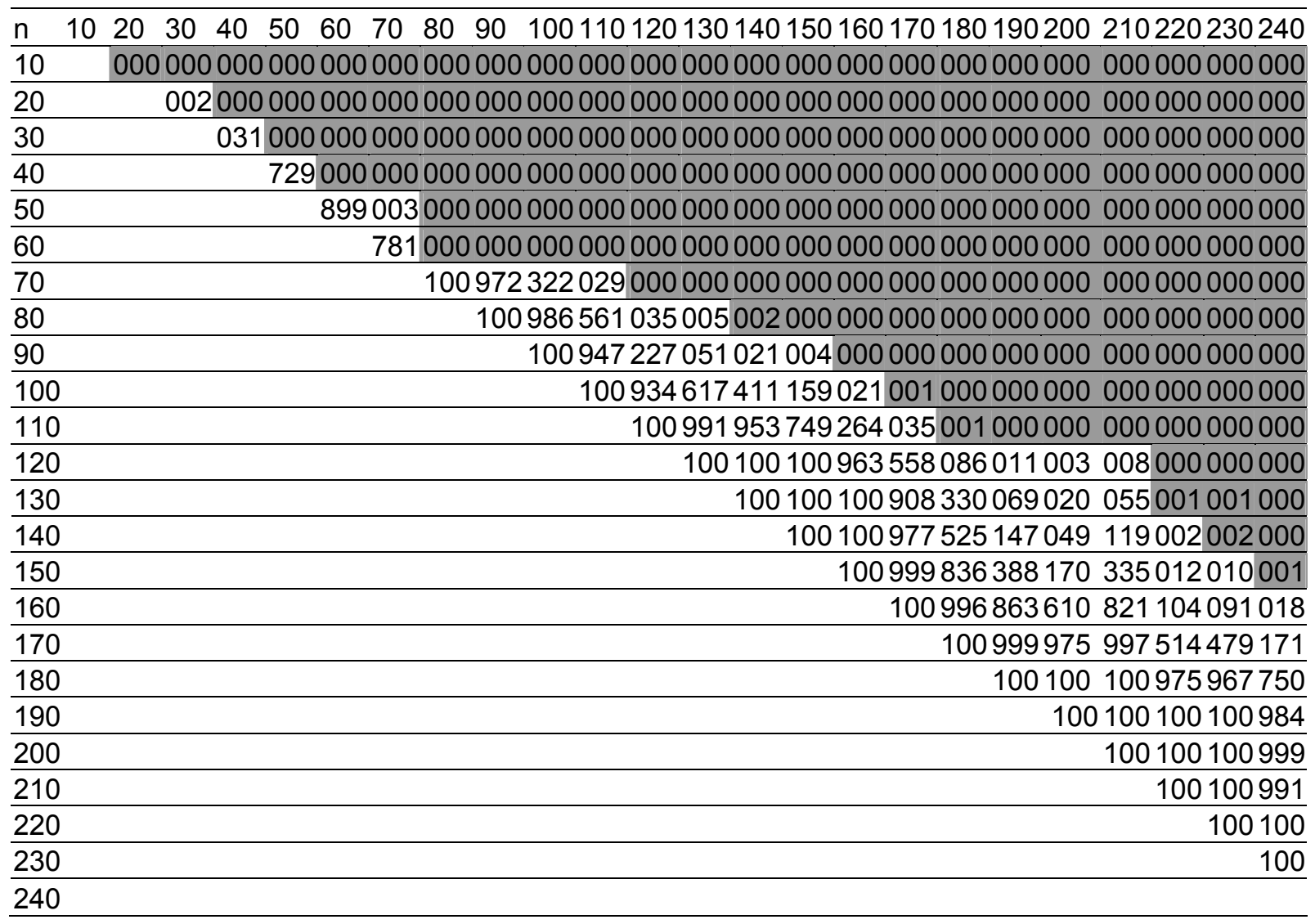


The results of comparisons $\alpha, \theta$ and $\Omega$ coefficients according to different sample sizes are given in Table 4.12, 4.13, 4.14 for the scale with 43 items.

Table-4.12: The homogeneity test results for the scale with 43 items

\begin{tabular}{ccccc}
\hline & $\begin{array}{c}\text { Levene } \\
\text { Statistic }\end{array}$ & $\begin{array}{c}\text { Degree of } \\
\text { freedom } 1\end{array}$ & $\begin{array}{c}\text { Degree of } \\
\text { freedom } 2\end{array}$ & $\begin{array}{c}\text { Significance level } \\
(\mathrm{p})\end{array}$ \\
\hline$\alpha$ & 6.313 & 16 & 153 & $<0.001$ \\
\hline$\theta$ & 7.654 & 16 & 153 & $<0.001$ \\
\hline$\Omega$ & 2.463 & 16 & 153 & 0.002 \\
\hline
\end{tabular}

Table-4.13: Significance level in comparison of $\alpha, \theta$ and $\Omega$ reliability coefficients according to different sample sizes using Kruskal-Wallis test for the scale with 43 items

\begin{tabular}{cccc}
\hline & $\alpha$ & $\theta$ & $\Omega$ \\
\hline$\chi^{2}$ & 11.248 & 7.026 & 141.750 \\
\hline Degree of freedom & 16 & 16 & 16 \\
\hline Significance level $(\mathrm{p})$ & 0.794 & 0.973 & $<0.001$ \\
\hline
\end{tabular}

Bonferroni correction: $\alpha^{*}=1-(1-\alpha)^{1 / \mathrm{k}}$

$$
\alpha^{*}=1-(1-0.05)^{1 / 17}=0.003
$$


Table-4.14: Significance level ( $\mathrm{p}$ values $\times 10^{-3}$ ) in comparison of $\Omega$ reliability coefficients according to different sample sizes using Mann-Whitney $U$ test for the scale with 39 items $\left(\alpha^{*}=0.003\right)$

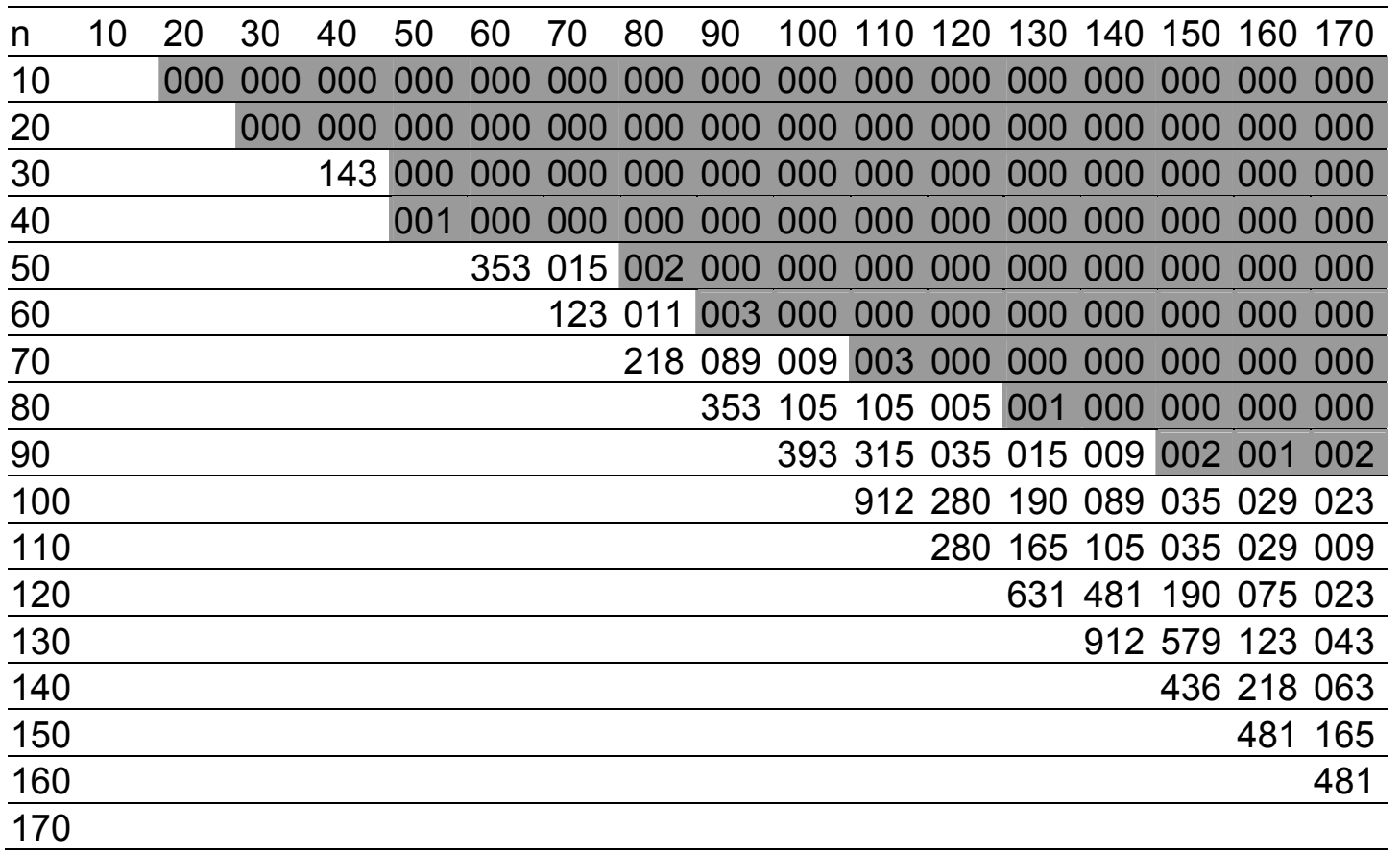

\section{Conclusion}

The answer to the question of sample size in this context is important. The accuracy of reliability coefficients changes according to the sample size. There is high positive correlation between number of items and reliability coefficient as mentioned in Carmines and Zeller (1982). Also, the difference in number of items must be taken into account.

Significant differences are not observed due to the sample size in the commonly used Cronbach Alpha, and with the Theta coefficient which is based on principal components. However, with the Omega coefficient, based on factor analysis, large differences were observed due to the sample size. With an increase in item numbers, however, the Omega coefficient is stabilized even for smaller sample sizes.

Ozdamar (1999a) mentioned that the sample size should be more than 50 in reliability analysis applications. According to the results of this study, that sample size is not important for the Cronbach alpha or theta coefficients, and is stable even for a small number of items (although of course an increase in the number of items will increase the magnitude.) However, in order to estimate the population parameter with Omega coefficient, the item number is important. With an increase in item number, either the consistency of estimation or the reliability level increases.

\section{References}

Arkin, H., \& Colton, P. R. (1970). Statistical methods. New York: Barnes \& Noble Books.

Carey, L. M. (1988). Measuring and evaluating school learning. London: Allyn and Bacon. 
Carmines, E. G., \& Zeller, R. A. (1982). Reliability and validity assessment. Beverly Hills: Sage Publications.

Ercan I, Ediz B \& Kan I. (2004). Saglik Kurumlarinda Teknik Olmayan Boyut icin Hizmet Memnuniyetini Olcebilmek Amaciyla Gelistirilen Olcek (A Scale Developed in order to Evaluate the Non-Technical Side of Service Satisfaction), Uludag University J Medical Faculty, Vol: 30, No. 3, 151-157.

Gay, L. R. (1985). Educational evaluation and measurement. London: A Bell \& Howell.

Gursakal, N. (2001). Sosyal bilimlerde arastirma yontemleri (Research methods in social sciences). Bursa: Uludag Uni. Guclendirme Vakfi Yayini, 178.

Karasar, N. (2000). Bilimsel arastirma yontemleri (Research methods in science). Ankara: Nobel Yayin Dagitim.

O'Connor, R. (1993). Issues in the measurement of health-related quality of life, Working paper 30. Melbourne: NHMRC National Centre for Health Program Evaluation ISBN: 1-875677-26-7.
Oncu, H. (1994). Egitimde olcme ve degerlendirme (Measurement and evaluation in education). Ankara: Matser Basim.

Ozdamar, K. (1999a). Paket programlarla istatistiksel veri analizi-1 (Statistical data analysis by custom softwares1). Eskisehir: Kaan Kitabevi.

Ozdamar, K. (1999b). Paket programlarla istatistiksel veri analizi-2 (Statistical data analysis by custom softwares2). Eskisehir: Kaan Kitabevi.

SPSS Base 10.0 (1999). Application guide. Chicago: SPSS.

SPSS Statistical Algorithms (1991). (2 $2^{\text {nd }}$ ed.). Chicago: SPSS.

Thorndike, R. M., Cunningham, G. K., Thorndike, R. L., \& Hagen, E. P., (1991). Measurement and evaluation in psychology and education $\left(5^{\text {th }}\right.$ ed.). New York: Macmillian Publishing Co. 


\section{Modeling Longitudinal Ordinal Response Variables for Educational Data}

\author{
Ann A. O'Connell \\ The Ohio State University
}

\author{
Heather Levitt Doucette \\ University of Connecticut
}

This article presents applications for the analysis of multilevel ordinal response data through the proportional odds model. Data are drawn from the public-use Early Childhood Longitudinal Study. Results showed that gender, number of family risk characteristics, and age at kindergarten entry were associated with initial reading proficiency ( 0 to 5 scale). The number of family risks and age were associated with time-slopes. Three issues are highlighted: building multilevel ordinal models, interpretation of multilevel effects; and determination of predicted probabilities based on results of the multilevel proportional odds models.

Key words: Proportional odds models, multilevel models, ordinal data.

\section{Introduction}

Prior to the fitting of statistical models to investigate relational characteristics of data, researchers must first consider the much more fundamental process of measurement. Stevens

Ann A.O'Connell, Ed.D., is an Associate Professor in the School of Educational Policy and Leadership at The Ohio State University. Her research interests include data analysis for ordinal-level response variables and multilevel modeling of health and educational outcomes. Heather Levitt Doucette is at the Connecticut State Department of Education and is a graduate student in the Department of Educational Psychology at the University of Connecticut. Her research interests are in assessment and early childhood education. This research was supported by a grant to the first author from the American Educational Research Association which receives funds for its "AERA Grants Program" from the National Science Foundation and the US Department of Education's National Center for Education Statistics and the Office of Educational Research and Improvement (now the Institute for Education Sciences), under NSF Grant \#REC-9980573. Opinions reflect those of the author and do not necessarily reflect those of the granting agencies.
(1946) referred to the measurement process as the development of a model that "represent[s] aspects of the empirical world" (p. 677) that are consistent with the nature of the objects under study. In education as well as the social and behavioral sciences, many outcomes are measured on an ordinal rather than an interval or ratio scale, reflecting of course the underlying nature of the phenomenon under study. As an example of an ordinal scale, consider the Concerns-Based Adoption Model (CBAM), developed to characterize the progression of teacher and administrator concerns regarding implementation of innovations within their classrooms or schools (Hall, George \& Rutherford, 1986; van den Berg, Sleegers \& Pelkmans, 2002, etc.).

Responses on the CBAM correspond to eight ordinal categories, representing progressive stages ranging from self-concern, task-concern to other-concern. This stage-based model is currently being adapted to characterize agency capacity for implementation of evidencebased HIV prevention interventions (O'Connell, Cornman \& Heybruck, 2003). Examples of ordinal scales can be found in many different contexts. Proficiency on statewide educational assessments has been characterized as ordinal, with students identified as below basic, basic, proficient, goal, and advanced in mathematics and reading (Beaudin, 2003). The goals set by No Child Left Behind (http://www.nclb.org/) 
require $100 \%$ of students within schools to attain proficiency in order to demonstrate effectiveness, making an understanding of ordinal measures and their statistical treatment important for schools, teachers, administrators, districts and state personnel.

In fact, most variables that are used to detect educational or behavior change are ordinal in nature. For example, change in proficiency during the kindergarten year in early reading or mathematics can be characterized as ordinal (i.e., achieved or did not achieve a particular level within a hierarchy of proficiency goals, pre- and post-school year); so can frequency of condom use before and after an intervention (never, sometimes, almost always, always). Many health intervention studies have relied on the transtheoretical model to characterize individual change before and after participation (Bowen \& Trotter, 1995; Hedeker \& Mermelstein, 1998; Lauby et al., 1998; Prochaska \& DiClemente, 1983, 1986; Prochaska, DiClemente, \& Norcross, 1992; Prochaska, Redding, Harlow, Rossi, \& Velicer, 1994; Stark et. al, 1996). Other examples include change in severity of illness or physical condition with scale categories such as mild, moderate, and severe (Knapp, 1999), and the common approach of using endorsement of responses to a particular statement (strongly disagree, disagree, neutral, agree, strongly agree) to assess attitudes before and after an event or period of time.

As these examples suggest, the use of ordinal-level variables in education and the social sciences are abundant. This should not be surprising, as Cliff (2003, 1996, and 1993) has consistently pointed out in much of his work on ordinal measurement that the questions we ask of our data are primarily ordinal in nature as well (Did students perform better after a schoolbased intervention?). However, there is inconsistency in the fidelity between ordinal measurement of a behavioral or cognitive outcome and how these quantities are analyzed in statistical models (Cliff, 2003, 1996, 1993; O'Connell, 2000; Clogg \& Shihadeh, 1994; Long, 1997; Agresti, 1996). The accurate interpretation of relationships among variables is dependent on the application of appropriate statistical techniques, yet the treatment of ordinal responses present challenges for many applied researchers in the educational and behavioral sciences. Similar to the field of biomedical and epidemiological research, the underutilization of ordinal regression models in the educational and behavioral sciences may be partially explained by researcher unfamiliarity with software programs capable of fitting these models, confusion about model assumptions and how to investigate these assumptions, and problems in interpretation of model results (Bender \& Benner, 2000). These challenges are multiplied when the study purports to consider change in an ordinal outcome over time. In this paper, the hierarchical generalized linear model (HGLM; Goldstein, 2003; McCullagh \& Nelder, 1989; Raudenbush \& Bryk, 2002) for ordinal responses is demonstrated and explained, using a small number of potential explanatory variables for illustration purposes.

Data applications that characterize an approach to analyzing change over time in ordinal response variables are presented. The data used is drawn from the Early Childhood Longitudinal Study (ECLS), a national database developed and managed through the National Center for Education Statistics (NCES). The ECLS-K (Kindergarten cohort) follows nearly 20,000 students from kindergarten through the first grade, with additional follow-ups in $3^{\text {rd }}$ and $5^{\text {th }}$ grade. The outcome of interest in the models constructed is student proficiency for early reading and literacy assessed across kindergarten and $1^{\text {st }}$ grade, which was measured using six ordinal categories (Table 1). Particular attention is paid to interpretation of the model estimates and assumptions, and the effects of independent variables on proficiency over time. HLM version 6.03 is used for these analyses (Raudenbush, Bryk, Cheong \& Congdon, 2004). The goal is to make a contribution to the applied literature on use and interpretation of hierarchical ordinal models, as well as to highlight the methodological challenges of modeling longitudinal ordinal outcomes. 
Table 1. Percent of Sample Reaching Reading Proficiency Levels Across Four Waves of ECLS-K.

\begin{tabular}{|c|c|c|c|c|}
\hline Proficiency Level & $\begin{array}{l}\text { Baseline } \\
0 \text { months } \\
\underline{\mathrm{n}}=3242\end{array}$ & $\begin{array}{l}8 \text { months } \\
\underline{\mathrm{n}}=3346\end{array}$ & $\begin{array}{l}12 \text { months } \\
\underline{\mathrm{n}}=3380\end{array}$ & $\begin{array}{c}20 \text { months } \\
\underline{\mathrm{n}}=3425\end{array}$ \\
\hline 0. Did not pass level 1 & 28.0 & 4.5 & 2.0 & 0.2 \\
\hline $\begin{array}{l}\text { 1. Identifying upper/lower case } \\
\text { letters }\end{array}$ & 34.6 & 14.8 & 8.3 & 1.1 \\
\hline $\begin{array}{l}\text { 2. Associating letters with } \\
\text { sounds at the beginning of } \\
\text { words }\end{array}$ & 17.2 & 23.3 & 17.6 & 3.0 \\
\hline $\begin{array}{l}\text { 3. Associating letters with } \\
\text { sounds at the end of words }\end{array}$ & 17.0 & 40.9 & 44.0 & 11.8 \\
\hline 4. Recognizing words by sight & 2.1 & 11.3 & 17.5 & 37.9 \\
\hline $\begin{array}{l}\text { 5. Recognizing words in } \\
\text { context }\end{array}$ & 1.2 & 5.2 & 10.6 & 46.0 \\
\hline
\end{tabular}

\section{Methodology}

Context: Proficiency in Early Literacy

In the ECLS-K, proficiency in early literacy is represented as a series of steppingstones, which reflect the skills that form the foundation for further learning in reading (West, Denton, \& Germino-Hausken, 2000). The categorization of early literacy proficiencies represented in the ECLS-K assessment instrument is consistent with the skills that have been identified as the building blocks of reading mastery: phonemic awareness (the understanding that letters represent spoken sounds), phonics (understanding the sounds of letters in combination), fluency, vocabulary and text-comprehension (CIERA, 2001). Six categories of hierarchical skill levels are used to establish the proficiency scale (Table 1). Mastery is defined as passing 3 out of 4 items in a cluster representing each successive proficiency level.

Research has indicated that children who experience difficulty learning to read in the early primary grades tend to begin school with limited proficiency for early-literacy skills (Burns, Snow \& Griffen, 1998). These early skills in reading carry-over to performance at later grades in reading as well as in other subjects, and children who experience difficulties early in school tend to experience continuation of these difficulties as they progress through school (Bayder, Brooks-Gunn, \& Furstenberg, 1993; Butler, Marsh, Sheppard \& Sheppard, 1985; Juel, 1988; McCoach, O'Connell, Reis, \& Levitt, 2006). Even prior to formal schooling, much is happening in the way of literacy skill development via the interaction between life experience and language development. The notion of emergent literacy suggests that children do indeed enter kindergarten with diverse literacy skills that may have an important predictive relationship with later reading abilities (Lonigan, Burgess, \& Anthony, 2000).

Initial data summaries from the Early Childhood Longitudinal Study-Kindergarten (ECLS-K) cohort indicate that some children do enter kindergarten with greater preparedness and readiness to learn relative to other children, perhaps putting them a step ahead of their peers for the important early grades at school (West, Denton, Germino-Hausken, 2000). ECLS-K studies have shown that children entering kindergarten from families with particular characteristics (living in a single parent household, living in a family that receives welfare payments or food stamps, having a 
mother with less than a high school education, or having parents whose primary language is not English) tended to be at risk for low reading skills (Zill \& West, 2001). Pre-kindergarten experiences related to family life, pre-school or daycare and personal characteristics (e.g., gender, persistence) may relate to children's initial proficiency in reading as well as their potential growth in skills and abilities across the kindergarten year and beyond. For example, girls typically enter kindergarten with slightly greater early literacy ability than boys. Childfocused predictors of success and failure in early reading are helpful for understanding how individual children may be at risk for reading difficulties. From a policy and practice perspective it is clearly desirable that teachers, school administrators, parents, and other stakeholders be aware of these individual factors related to entry-level proficiency as well as to growth in proficiency in order to develop curriculum and instructional practices that can promote achievement for all students relative to their kindergarten entry skills.

School and instructional characteristics have also been shown to be associated with student ability in early literacy, but it is not entirely clear how the differing educational experiences of children across schools (teacher and school effects) might affect growth in proficiency. The National Research Council (1998) reviewed predictors of success and failure in early reading at the neighborhood, school, and community level. In the continuing work using the ECLS-K, the effects of specific school-level variables on proficiency have been modeled separately across the four years of available data. These models included frequency of use of ability-grouping in kindergarten, principals' ratings on the success of various teacher instructional practice, attendance at public versus private schools, school socioeconomic status, and neighborhood climate including the presence of racial tensions, litter, drug/alcohol use in the neighborhood, and extent of crime (Levitt \& O'Connell, 2002; McCoach, O'Connell, Levitt \& Reis, 2006; O'Connell \& Levitt, 2002).

Although instructional, organizational and neighborhood effects on children's entrylevel reading ability and growth in reading are critical to understanding how to create and implement effective school-supported teaching strategies, these effects have not been modeled here. Instead, as the purpose of this article is on the methodology for developing and interpreting multilevel models for ordinal responses, the focus herein is on the development and interpretation of two-level models investigating the effect of child-level characteristics on reading growth across four time points (fall and spring of kindergarten, and fall and spring of first grade); extensions to the three-level case are relatively straightforward.

\section{Hierarchical Ordinal Regression Models}

Explanatory models for ordinal outcome data collected during a single time frame have been previously reviewed by O'Connell (2000; 2006) and others (e.g., Agresti, 1989, 1990, 1996; Bender \& Benner, 2000; Clogg \& Shihadeh, 1994; Long, 1997; McCullagh, 1980). This work can be adapted to fit the needs of a hierarchical context. Wong and Mason (1985) and Hedeker and Mermelstein (1998) provided examples of extensions of models for dichotomous and ordinal outcomes for hierarchical data. In addition, the latest version of the HLM program (HLMv6.03; Raudenbush, Bryk, Cheong, and Congdon, 2004) includes options for modeling the cumulative odds for ordinal hierarchical data. An article by Plewis (2002) in the Multilevel Modeling Newsletter describes the fitting of multilevel ordinal data using MLwiN.

The most common ordinal outcome model is the regression-type proportional or cumulative odds (PO) model (Agresti, 1996; Armstrong \& Sloan, 1989; Long, 1997; McCullagh, 1980). In this approach, the (log of the) odds of a response at or below each of the ordinal categories form the quantities of interest. For example, with a six-category ordinal outcome $(K=6)$, the $K-1$ formulas shown in Table 2 would be used to compute the cumulative probabilities and consequently the cumulative odds (note: consistent with the ECLS-K categories, the possible outcomes are 0 through 5). The cumulative probabilities are the probabilities that the response for the $\mathrm{i}^{\text {th }}$ student nested within the $\mathrm{j}^{\text {th }}$ school (or, for longitudinal data, the $\mathrm{i}^{\text {th }}$ student at the $\mathrm{t}^{\text {th }}$ time point) is at or 
Table 2. Cumulative Odds Model for $\mathrm{K}=6(\mathrm{~K}=0,1, \ldots 5)$, Where $\mathrm{R}_{\mathrm{ti}}$ Represents the Proficiency Outcome (Response) for the $\mathrm{I}^{\text {th }}$ Student at the $\mathrm{T}^{\text {th }}$ Wave.

\begin{tabular}{|l|l|l|l|}
\hline Category & Cumulative Probability & Cumulative Odds $\left[Y_{k t i}^{\prime}\right]$ & Probability Comparison \\
\hline $\begin{array}{l}\mathrm{k}=0 \\
\text { (Proficiency 0) }\end{array}$ & $P\left(R_{t i} \leq 0\right)$ & $\frac{P\left(R_{t i}=0\right)}{P\left(R_{t i}>0\right)}$ & $\begin{array}{l}\text { Proficiency 0 versus all } \\
\text { levels above }\end{array}$ \\
\hline $\begin{array}{l}\mathrm{k}=1 \\
\text { (Proficiency 1) }\end{array}$ & $P\left(R_{t i} \leq 1\right)$ & $\frac{P\left(R_{t i} \leq 1\right)}{P\left(R_{t i}>1\right)}$ & $\begin{array}{l}\text { Proficiency 0 and 1 } \\
\text { combined versus all levels } \\
\text { above }\end{array}$ \\
\hline $\begin{array}{l}\mathrm{k}=2 \\
\text { (Proficiency 2) }\end{array}$ & $P\left(R_{t i} \leq 2\right)$ & $\frac{P\left(R_{t i} \leq 2\right)}{P\left(R_{t i}>2\right)}$ & $\begin{array}{l}\text { Proficiency 0,1,2 } \\
\text { combined versus 3, 4, 5 } \\
\text { combined }\end{array}$ \\
\hline $\begin{array}{l}\mathrm{k}=3 \\
\text { (Proficiency 3) }\end{array}$ & $P\left(R_{t i} \leq 3\right)$ & $\frac{P\left(R_{t i} \leq 3\right)}{P\left(R_{t i}>3\right)}$ & $\begin{array}{l}\text { Proficiency 0,1,2,3 } \\
\text { combined versus 4,5 } \\
\text { combined }\end{array}$ \\
\hline $\begin{array}{l}\mathrm{k}=4 \\
\text { (Proficiency 4) }\end{array}$ & $P\left(R_{t i} \leq 4\right)$ & $\frac{P\left(R_{t i} \leq 4\right)}{P\left(R_{t i}>4\right)}$ & $\begin{array}{l}\text { Proficiency 0,1,2,3,4 } \\
\text { versus proficiency 5 }\end{array}$ \\
\hline
\end{tabular}

below a given proficiency level. The odds is a ratio of the probability of an event occurring to the probability of an event not occurring. Accordingly, the cumulative odds $\left[Y_{k t i}^{\prime}\right]$ represent the odds that any given response would be in at most category $k$ (rather than beyond category $k$ ), for the $i^{\text {th }}$ child at the $t^{\text {th }}$ wave of data collection. From Table 2, it may be seen that the cumulative odds, in order, correspond to the probability of being in proficiency level 0 relative to all categories above it; the probability of being in proficiency level 0 or 1 relative to all above it; and so on until arriving at the probability of being in categories $0,1, \ldots 4$ relative to being in category 5 . The $\mathrm{K}^{\text {th }}$ or final cumulative probability would always be 1.0 (being at or below the last possible level), and its probability and associated odds are therefore not included in the table. It is common to refer to the value marking each of these binary comparisons as cutpoints or cumulative splits. For example, the cutpoint for the first comparison is 0 (proficiency level 0 versus above 0 ); the cutpoint for the second comparison is 1 (proficiency 0 and 1 versus above 1), etc.

To better understand how the PO model works, imagine if the separate comparisons indicated in the last column of Table 2 were investigated using corresponding binary (hierarchical) logistic regressions at each of the associated cumulative splits. The simultaneous fitting of each of these separate K-1 (in this example, $\mathrm{K}-1=5$ ) logistic models represents the overall PO approach. For this approach to be valid, a critical assumption must be made of the data. This assumption of proportionality states that the effects of the explanatory variables cannot be statistically different across these cutpoint comparisons. This is also called the cumulative odds assumption or the equal slopes assumption and can be restrictive but is the most common choice for ordinal regression models (Hedeker \& Gibbons, 2006; O'Connell, 2006). For non-hierarchical data, the assumption of equal slopes can easily be tested within SAS or SPSS, for example. However, in a multi-level context direct tests of this assumption are not currently available. Interaction terms can be 
used to test for non-proportionality of some or all of the predictors, or an ad hoc approach can be applied that investigates the consistency of slope estimates across the cumulative splits described in Table 2. Space does not allow for a demonstration of this assessment here; interested readers can find further discussion and examples in O'Connell, Goldstein, Rogers \& Peng (in press), as well as in Hedeker, et al., 2006).

General Model: Students Nested Within Schools.

A brief description of the ordinal HGLM is presented for analyses focused at one point in time; in the next section it is expanded this to cover repeated ordinal measures. For the $i^{\text {th }}$ student in the $j^{\text {th }}$ school, the hierarchical proportional odds model is fit according to the following equations (Raudenbush \& Bryk, 2002):

Student level:

$$
\begin{aligned}
\ln \left(\mathrm{Y}_{\mathrm{kij}}^{\prime}\right) & =\ln \left(\frac{\mathrm{P}\left(\mathrm{R}_{\mathrm{ij}} \leq \mathrm{k}\right)}{\mathrm{P}\left(\mathrm{R}_{\mathrm{ij}}>\mathrm{k}\right)}\right) \\
& =\beta_{0 \mathrm{j}}+\sum_{\mathrm{q}=1}^{\mathrm{Q}} \beta_{\mathrm{qj}} \mathrm{X}_{\mathrm{qij}}+\sum_{\mathrm{k}=2}^{\mathrm{K}-1} \mathrm{D}_{\mathrm{kij}} \delta_{\mathrm{k}}
\end{aligned}
$$

School or Context level:

$$
\beta_{\mathrm{qj}}=\gamma_{\mathrm{q} 0}+\sum_{\mathrm{s}=1}^{\mathrm{S}_{\mathrm{q}}} \gamma_{\mathrm{qs}} \mathrm{W}_{\mathrm{sj}}+\mathrm{u}_{\mathrm{qj}}
$$

where $\left[Y_{k i j}^{\prime}\right]$ represents the cumulative odds for each category $k$, with $k=1 \ldots \mathrm{K}-1$ levels of the ordinal response and $q=1 \ldots Q$ independent variables at the student level. For these models, the term on the left side of equation (1) is the log of the cumulative odds for each category $k$, and is referred to as the logit for the cumulative distribution. The terms on the right can be interpreted similar to any logistic regression model, with the $\beta_{q j}$ representing the expected change in the logit for each one unit change in the $q^{\text {th }}$ explanatory variable, $X_{q}$. Its exponentiation will provide the estimate of the cumulative odds for that variable. However, an important difference between an ordinal model and a binary logistic regression model is that with K-1 ways to characterize the cumulative odds, the slope parameters for each of the independent variables are restricted to be constant across all the separate possible cumulative splits derived according to the second column of Table 2. That is, the model assumes that the effect of any independent variable can be represented by a common cumulative odds ratio, $\exp (\beta)$; this is the assumption of proportional odds. If this assumption does not hold, then the PO model is not a plausible one for the data and less restrictive models should be investigated.

The collection of estimates at the far right of equation (1) are referred to as thresholds or delta coefficients, and they operate as deviations from the baseline intercept for each of the K-1 separate binary comparisons beyond the first, with $\beta_{0 j}$ as the baseline intercept (i.e., for the first cumulative comparison). $D_{k i j}$ is the indicator variable for each category beyond the first. In other words, each cumulative comparison has its own intercept, while the effects of the explanatory variables are assumed to be constant across each comparison.

Changes Over Time in an Ordinal Response.

When data are gathered over time, methodologies for the treatment of ordinal outcomes need to be combined with methods that address the multilevel nature of longitudinal data. As with other studies of growth, change was modeled in the logit as a linear effect. With only four time points, this approach is reasonable (Murray, 1998). At level one, the repeated measures are modeled over time, and at level two student characteristics are used to look at changes in intercepts or growth trajectories across children. For demonstration purposes, the focus is on the two-level model in this article rather than include a third level for modeling school effects. To investigate child-level variability in baseline (entry) proficiency and in the trajectory of change, we considered the following child-level variables: age at kindergarten entry, gender (boys $=1$ ), attending half-day rather than full-day kindergarten (halfday $=1$ ), previously attending any center-based 
care $($ yes $=1)$, frequency with which parents read books to their child, socio-economic status, count of family risks, and a model-based approach was used to adjust for oversampling of Asian and Pacific Islanders (API) by including API (yes $=1)$ in all preliminary analyses. The general level one and level two models are provided below.

Time level:

$$
\begin{aligned}
\ln \left(Y_{k t i}^{\prime}\right) & =\ln \left(\frac{P\left(R_{t i} \leq k\right)}{P\left(R_{t i}>k\right)}\right) \\
& =\pi_{0 i}+\pi_{1 i} T_{t i}+\sum_{k=2}^{K-1} D_{k t i} \delta_{k}
\end{aligned}
$$

Student level:

$$
\pi_{q j}=\beta_{q 0}+\sum_{s=1}^{S_{q}} \beta_{q s} X_{s i}+u_{q i}
$$

The last term in equation 3 is used to estimate the increasing intercepts for each of the underlying cumulative models, and is described in depth in the next section. Not unsurprisingly, these multilevel ordinal models were difficult to converge. Therefore, each independent variable was considered separately, consistent with Raudenbush and Bryk's (2002) suggestions regarding strategies for building complex multilevel models. Based on these preliminary analyses, two were selected that were found to be associated with proficiency in the simpler (univariate) models, and known to be associated with early literacy: gender and family-risk characteristics. The sum of the number of family-risk characteristics was used as a contextual variable in models predicting baseline proficiency (intercept) as well as change in proficiency (slope) over time. Once the contextual model was derived, age at kindergarten entry was included to control for age-effects. These few variables were selected to illustrate how contextual models may be developed and interpreted for longitudinal ordinal outcomes, and below an application of the PO model is presented in the prediction of change in reading proficiency across four years of data using the ECLS-K.
The following section describes the process by which the repeated measures and hierarchical ordinal models were developed.

\section{Procedures}

A sample of $n=3440$ children were selected from the ECLS-K. Since the primary purpose of this presentation is to illustrate the application of a multilevel approach to ordinal data, the sample was limited to children who did not change schools from kindergarten to firstgrade, had four waves of data (a 30\% subsample of the original data were included in a fall firstgrade wave of data collection), were first-time kindergarteners only (no repeaters were included), and had no missing observations on the child-level (level-2) characteristics investigated for this study (gender, family-risk, and age at kindergarten entry). These criteria were applied to minimize complexity of the statistical design regarding number of data points available per child, convergence issues, and concerns regarding the impact of crossclassification of children changing schools during the study period. The resulting data set represents a sample of first-time kindergarteners assessed twice in kindergarten and twice in first grade.

HGLM, the non-linear counterpart to hierarchical linear modeling (HLM), was used to model the ordinal outcomes (Raudenbush and Bryk, 2002). The most general case of an HGLM for ordinal data assumes proportional odds across successive cumulative categories. Proportionality implies that the effect of an independent variable remains constant across the cumulative categories of the outcome variable.

In the PO model, the likelihood (or odds) of an observation falling into category $k$ or below is assessed over time. Similar to the familiar logistic regression model, the PO analysis predicts a transformation of the odds, i.e., the logit, which is the $\log$ of the odds. A logit of zero corresponds to an odds of 1.0, which implies that there is no difference between the probability of being in a certain category (or below) and being above that category $(.5 / .5=1.0, \ln (1.0)=0)$. A positive logit implies that the likelihood of being in lower categories is greater (e.g., .7/.3 = 2.33, $\log (2.33)=.847)$; and a negative logit implies 
that the likelihood of being in higher categories is greater (e.g., $.3 / .7=.429, \log (.429)=-.847$ ).

Using the HLM program, the desired data structure is similar to that in other multilevel analyses of longitudinal data. The level-one data file represents the repeated measures outcomes, and contains the proficiency score as an ordinal-level response variable for each child at each of the four time points. With 3440 children, there would be at most $4 \times 3440$ or 13,760 observations at level one. Some children were missing proficiency scores at some point during the four waves of data collection; thus there were 13,393 observations overall at level one for the analytic sample. The level-two data contains the child-level characteristics, including gender, the number of family risk characteristics, and age at kindergarten entry. Although three level ordinal models are now available in HLMv6.03 (Raudenbush et al., 2004), the models presented in this article illustrate the assessment of child-level effects (level two) on changes in proficiency over time (level one), and work is continuing on how these models might be extended to incorporate school effects as a third level.

Although many different models were investigated, only three are reported here. The final models include a random coefficients model (Table 4), with time in months as the sole predictor of proficiency (more precisely, as the predictor of the logits for the cumulative odds for proficiency). Next, a contextual model was developed using gender and the number of risk factors as the explanatory child-level variables at level 2 (Table 4). This contextual model was designed to illustrate how the effects of gender and the number of family risk factors may moderate the change in cumulative odds over time. These effects were included as predictors of the intercepts or baseline values and as predictors of the slope for time. This model was then adjusted to include age at kindergarten entry (grand mean centered) as a control variable for predicting both the intercept and the slope from level one, as well as deleted nonstatistically significant predictors. Results of this final model are provided in Table 5.

The random coefficients analysis looks at the thresholds between (cumulative) adjacent proficiency levels and estimates the odds of a person being in proficiency level $k$ or below over time. If changes in proficiency can be reliably detected over time, the effect of time on the logit should be negative, so that the likelihood of being in higher categories increases over time. With a six-category outcome $(\mathrm{k}=0,1,2,3,4,5)$ and time measured in months from baseline $(\mathrm{t}=0,8,12,20)$, five models are fit simultaneously, as shown below.

Level one:

$$
\begin{aligned}
& \ln \left(Y_{0 t i}^{\prime}\right)=\pi_{0 i}+\pi_{1 i}(\text { time })_{t i} \\
& \ln \left(Y_{1 t i}^{\prime}\right)=\pi_{0 i}+\pi_{1 i}(\text { time })_{t i}+\delta_{2} \\
& \ln \left(Y_{2 t i}^{\prime}\right)=\pi_{0 i}+\pi_{1 i}(\text { time })_{t i}+\delta_{3} \\
& \ln \left(Y_{3 t i}^{\prime}\right)=\pi_{0 i}+\pi_{1 i}(\text { time })_{t i}+\delta_{4} \\
& \ln \left(Y_{4 t i}^{\prime}\right)=\pi_{0 i}+\pi_{1 i}(\text { time })_{t i}+\delta_{5}
\end{aligned}
$$

Level two:

$$
\begin{aligned}
& \pi_{0 i}=\beta_{00}+u_{0 i} \\
& \pi_{1 i}=\beta_{10}+u_{1 i}
\end{aligned}
$$

In the collection of equations for level one, the terms on the left, $\ln \left(Y_{3 t i}^{\prime}\right)$ for example, represents the $\log$ of the odds for being in category 3 or below (rather than beyond category 3), consistent with the approach described in Table 2.

The critical assumption of proportional odds implies that the effect of time is constant across the cumulative splits identified through the level one model. The level one effects, $\pi_{0 i}$ and $\pi_{1 i}$, represent, respectively, the baseline estimates (at the first wave of data collection (entry into kindergarten)) for the log of the odds of being in category $k$ or below, and the effect of time (slope) on these logits. These intercepts and slopes are free to vary from person to person. This variability is captured by the level two random effects, $u_{0 i}$ and $u_{1 i}$, with variance components, respectively, of $\tau_{00}$ and $\tau_{11}\left(\operatorname{var}\left(u_{0 i}\right)\right.$ $=\tau_{00}$ and $\left.\operatorname{var}\left(u_{1 i}\right)=\tau_{11}\right)$. The thresholds, $\delta_{2}$ to $\delta_{5}$, represent the differences in the logit for each successive cumulative category relative to the 
first logit; for example, in this sample the estimate at baseline for the $\log$ (odds) of being in category 3 or below would be $\beta_{00}+\delta_{3}$.

The first contextual model analysis considers the effects of gender $(1=$ male $)$ and the number of risk characteristics (0 through 4$)$ on the baseline logits and the slopes. The level one model remains the same as (5), but now the level-two models used to describe the effects of gender and number of family risks on the intercept and slope are:

$$
\begin{aligned}
& \pi_{0 i}=\beta_{00}+\beta_{01}(\text { gender })_{i}+\beta_{02}(\text { risknum })_{i}+u_{0 i} \\
& \pi_{1 i}=\beta_{10}+\beta_{11}(\text { gender })_{i}+\beta_{12}(\text { risknum })_{i}+u_{1 i}
\end{aligned}
$$

Finally, in the second contextual model analysis age at kindergarten entry was included (grand mean centered) in the level two models for both the intercepts and the slopes. The gender effect was deleted from the model for time-slopes due to lack of statistically significant results for gender in a preliminary run.

\section{Results}

Table 1 contains the proportion of children classified into each literacy proficiency level from kindergarten through first grade. Table 3 shows the proportion of children making specific transitions in literacy proficiency across the four waves. Most children made a positive change across the kindergarten year; most did not change during the summer between kindergarten and first grade, but then children tended to increase again by one or two proficiency levels across the first grade year.

Results of the random coefficients model are provided in Table 4 . These results show that overall across children, the expected $\log$ odds of being in proficiency level 0 at baseline is negative $\left(\beta_{00}=-1.73, \mathrm{p}<.01\right)$, which implies that at baseline it is more likely for a child to be at least in level 1 or higher. There is a statistically significant linear trend in the cumulative logits for time $\left(\beta_{10}=-.41, \mathrm{p}<.01\right)$, indicating that as a child progresses in school, the likelihood of being at or below category 0 decreases (stated differently, the negative slope for time implies that the probability of being beyond category 0 is increasing with time). This is consistent with what we see in Tables 1 and 3 . At baseline, children are more likely to be beyond category 0 , and this likelihood increases over time. The model estimates are predicted logits. To transform to odds and then to probabilities, odds $=\exp (\beta)$, and probability $=$ odds/ $(1+$ odds $)$ are used. For this example, the odds at baseline of a child being in proficiency level 0 or below is $\exp (-1.73)=.1773$; this corresponds to a probability of $.1773 /(1+.1773)$ $=.15$. For this random coefficients model containing no child-level predictor variables, $15 \%$ of children would be predicted to be at or below category 0 at baseline. For the predicted logit of being at or below category 0 at time 2 ( 8 months), the model estimates the logit as: -1.73 $+(-.41)(8)=-5.01$. Thus, at the end of kindergarten, the model predicts that the odds of being in category 0 or below is decreased (exp($5.01)=.0067)$, and the associated probability of being at or below proficiency category 0 at the end of kindergarten is .007 , or $.7 \%$.

To examine the model predictions at other splits in the cumulative hierarchy, for example, model predictions for being at or below category three at baseline, $\delta_{4}$ is used in addition to the baseline intercept and the slope (see equation 5). For this data, the new intercept, or threshold, becomes $\beta_{00}+\delta_{4}=-1.73+7.86=$ 6.13. Accordingly, the probability of a child being at or below proficiency level 3 at baseline is $.998 \mathrm{pr} 99.8 \%$. At time 2 (8 months), the predicted logit is $\beta_{00}+\beta_{10} *(8$ months $)+\delta_{4}=-$ $1.73+(-.41) *(8)+7.86=2.85$, where $\exp (2.85)$ $=17.29$, and the predicted probability of being at or below proficiency category three at the end of kindergarten is .945 , or $94.5 \%$. These predictions, based on a model with no explanatory variables, are reasonably consistent with the data in Table 1.

Finally, reviewing the variance components for the model, it may be seen that considerable variation remains in the intercepts, $\tau_{00}=8.35, \mathrm{p}<.01$, as well as in the slopes, $\tau_{11}=$ $.003, \mathrm{p}<.01$.

The first contextual model (Table 4) describes the effect of gender and the number of family risk factors on the baseline logits and the 
Table 3. Change in Proficiency Across the Kindergarten (K1 \& K2) and First Grade (FG1 \& FG2) Years.

\begin{tabular}{crrr}
\hline Raw Change in Proficiency & K2-K1 & FG1-K2 & FG2-FG1 \\
& & & \\
-3 & 0.0 & 0.1 & 0.0 \\
-2 & 0.3 & 0.8 & 0.1 \\
-1 & 1.7 & 7.7 & 0.7 \\
0 & 21.3 & 46.5 & 18.5 \\
1 & 33.6 & 32.6 & 40.9 \\
2 & 27.5 & 8.3 & 29.9 \\
3 & 9.0 & 1.0 & 7.1 \\
4 & 0.5 & 0.0 & 0.8 \\
5 & 0.01 & 0.0 & 0.0 \\
\hline
\end{tabular}

Table 4. Multilevel Ordinal Models for Prediction of Proficiency Using Four Waves Of ECLS-K; Ivs are Gender and Number of Family Risks.

\begin{tabular}{|c|c|c|c|c|}
\hline Effect & $\begin{array}{l}\text { Coeff. } \\
\text { (s.e.) }\end{array}$ & $\begin{array}{c}\mathrm{t} \\
(\mathrm{df})\end{array}$ & $\begin{array}{l}\text { Coeff. } \\
\text { (s.e.) }\end{array}$ & $\begin{array}{c}\mathrm{t} \\
(\mathrm{df})\end{array}$ \\
\hline \multicolumn{5}{|l|}{ Intercept $\left(\pi_{0 \mathrm{i}}\right)$} \\
\hline$\beta_{00}$ & $\begin{array}{l}-1.73 \\
(.068)\end{array}$ & $\begin{array}{l}-25.41 * * \\
(3439)\end{array}$ & $\begin{array}{l}-2.56 \\
(.097)\end{array}$ & $\begin{array}{l}-26.48 * * \\
(3437)\end{array}$ \\
\hline$\beta_{01}(\operatorname{gender}(\mathrm{M}=1))$ & & & $\begin{array}{l}0.62 \\
(.114)\end{array}$ & $\begin{array}{l}5.48 * * \\
(3437)\end{array}$ \\
\hline$\beta_{02}$ (number of risks) & & & $\begin{array}{l}1.07 \\
(.078)\end{array}$ & $\begin{array}{l}13.75 * * \\
(3437)\end{array}$ \\
\hline \multicolumn{5}{|l|}{ Time Slope $\left(\pi_{1 \mathrm{i}}\right)$} \\
\hline$\beta_{10}$ & $\begin{array}{l}-.41 \\
(.004)\end{array}$ & $\begin{array}{l}-98.46 * * \\
(3439)\end{array}$ & $\begin{array}{l}-.41 \\
(.005)\end{array}$ & $\begin{array}{l}-77.45 * * \\
(3437)\end{array}$ \\
\hline$\beta_{11}($ gender $(\mathrm{M}=1))$ & & & $\begin{array}{l}-.001 \\
(.005)\end{array}$ & $\begin{array}{l}-0.21 \\
(3437)\end{array}$ \\
\hline$\beta_{12}$ (number of risks) & & & $\begin{array}{l}-.01 \\
(.003)\end{array}$ & $\begin{array}{l}-2.18 * \\
(3437)\end{array}$ \\
\hline \multicolumn{5}{|l|}{ For Thresholds: } \\
\hline$\delta_{2}$ & $\begin{array}{l}2.75 \\
(.053)\end{array}$ & $\begin{array}{l}51.71 * * \\
(13387)\end{array}$ & $\begin{array}{l}2.78 \\
(.054)\end{array}$ & $\begin{array}{l}51.58 * * \\
(13383)\end{array}$ \\
\hline$\delta_{3}$ & $\begin{array}{l}4.69 \\
(.060)\end{array}$ & $\begin{array}{l}77.28 * * \\
(13387)\end{array}$ & $\begin{array}{l}4.71 \\
(.061)\end{array}$ & $\begin{array}{l}77.03 * * \\
(13383)\end{array}$ \\
\hline$\delta_{4}$ & $\begin{array}{l}7.86 \\
(.077)\end{array}$ & $\begin{array}{l}101.46 * * \\
(13387)\end{array}$ & $\begin{array}{l}7.88 \\
(.079)\end{array}$ & $\begin{array}{l}101.17 * * \\
(13383)\end{array}$ \\
\hline$\delta_{5}$ & $\begin{array}{l}10.32 \\
(.091)\end{array}$ & $\begin{array}{l}112.88 * * \\
(13387)\end{array}$ & $\begin{array}{l}10.35 \\
(.092)\end{array}$ & $\begin{array}{l}112.61 * * \\
(13383)\end{array}$ \\
\hline
\end{tabular}

Note: $* \mathrm{p}<.05 ; * * \mathrm{p}<.01$

Random Coefficients Model Contextual Model

\begin{tabular}{lrrrrrr}
\hline \multicolumn{1}{c}{ Random Effects } & Variance & $d f$ & Chi-square & Variance & $d f$ & Chi-square \\
\hline Variance in Base- K1 $\left(\tau_{\mathrm{oo}}\right)$ & 8.346 & 3391 & $10350.03 * *$ & 7.75 & 3389 & $10025.82 * *$ \\
Variance in Time slope $\left(\tau_{11}\right)$ & .003 & 3392 & $3615.27 * *$ & .003 & 3392 & $3626.38^{* *}$ \\
\hline
\end{tabular}

Note: $* \mathrm{p}<.05 ; * * \mathrm{p}<.01$ 
slopes for time. Gender has a statistically significant effect on the baseline logits $\left(\beta_{01}=\right.$ $.62, \mathrm{p}<.01)$. Being a boy tends to increase the logit, making the likelihood of being in higher proficiency categories lower for boys relative to girls. The number of risk factors also has a statistically significant effect on the baseline $\operatorname{logit}\left(\beta_{02}=1.07, \mathrm{p}<.01\right)$. Because the logit is positive, it may be seen that as the number of family-risk characteristics increases, the likelihood that a child would be in lower proficiency categories (i.e., at or below any category $k$ ) increases, relative to a child with fewer risks.

Attention is now turned to interpretation of the effects of gender and the number of risk characteristics on the slope for time. $\beta_{10}=-.41$ may be interpreted as the estimated slope for girls with out any family risks. Controlling for the number of risk factors, gender has no effect on the slopes $\left(\beta_{11}=-.001, \mathrm{p}>.05\right)$; thus gender does not affect the rate of change in proficiency. The number of risk factors does impact rate of change $\left(\beta_{12}=-.01, \mathrm{p}<.05\right)$. On the surface this would suggest that the likelihood is greater that a child with more risk characteristics improves over time even beyond that of a child with fewer risks. However, on closer inspection of the model predictions - particularly in terms of predicted probabilities across the four time points of being at or below any category $k-$ it is seen that children with increased family risks tend not to improve as readily over time as their non-risk peers.

This complexity of ordinal model interpretation can be overcome by estimating outcomes for discrete cases of children. For example, substituting into the prediction model, a female child (gender $=0$ ) from a family with 0 risk characteristics would be expected to have a predicted logit for the first cumulative comparison (proficiency level 0 or below) at baseline (time $=0$ ) of -2.56 , which corresponds to a cumulative odds of $\exp (-2.56)=.08$ and cumulative probability of being at or below proficiency category 0 of .072 , or $7.2 \%$. For a girl at baseline from a family with 1 risk characteristic, the predicted logit is -1.49 , corresponding to a cumulative odds of .23 , and a probability of .187 or $18.7 \%$. This is a large proportion of girls estimated to be at or below proficiency level 0 (rather than beyond category 0 ), given the addition of just one risk factor. In fact, the odds ratio for the variable number of risks is $\exp (1.07)=2.92$. The model suggests that, at baseline, the odds of being at or below any category increases by a factor of 2.92 for every one unit increase in a child's number of family risks. Baseline is the simplest case for making predictions; moving to time 2 at 8 months, the model estimates now need to include gender and family risk effects on the effect of time, but the process of estimating outcomes is similar to the process demonstrated above. Based on the parameter estimates from the model, probability predictions for being at or below proficiency category 0 at time 2 ( 8 months) are $.29 \%, 1.56 \%$, and $13.24 \%$ for girls with 0,1 , and 4 family risk factors, respectively.

The variance estimates for this contextual model indicates that variability in the baseline logits and in the time slopes continues to be statistically different from zero, which suggests that additional variables may be useful in understanding proficiency growth (initial status and rate of change). Table 5 provides the model estimates for an adjusted contextual model. In this modified model, age at kindergarten entry (grand-mean centered) is included in the models, and gender is removed from the level 2 models for the slope due to its lack of contribution to that model. The predictions for baseline or initial proficiency remain fairly similar to the contextual model estimates in Table 4. All three predictors contribute to the prediction of the baseline logits, with age at kindergarten entry having a negative effect $\left(\beta_{03}=-.13, \mathrm{p}<.01\right)$. This implies that for older children at kindergarten entry, the probability of being in higher categories of proficiency increases. After adjusting for age at kindergarten entry, the number of family risks is still a statistically significant predictor of the trajectory (slope) in the proficiency logits from baseline through the end of first grade $\left(\beta_{11}=\right.$ $.01, \mathrm{p}<.05)$, with little change in magnitude from the previous model. In addition, age at kindergarten entry is positively related to the time slopes $\left(\beta_{12}=.002, \mathrm{p}<.01\right)$; based on model predictions, older children tend to improve over time more readily than their younger peers. 
Table 5. Multilevel Ordinal Model for Prediction of Proficiency Controlling for Kindergarten Entry Age; Ivs are Gender, Number of Family Risks, and Age at Kindergarten Entry.

Contextual Model 2

\begin{tabular}{|c|c|c|c|}
\hline Effect & Coeff (s.e.) & $t(d f)$ & $\mathrm{p}$ \\
\hline \multicolumn{4}{|l|}{ Intercept $\left(\pi_{0 \mathrm{i}}\right)$} \\
\hline$\beta_{00}$ & $-2.58(.089)$ & $-28.91 * *(3436)$ & .000 \\
\hline$\beta_{01}($ gender $(M=1))$ & $.67(.089)$ & $7.514 * *(3436)$ & .000 \\
\hline$\beta_{02}$ (number of risks) & $1.05(.077)$ & $13.65 * *(3436)$ & .000 \\
\hline$\beta_{03}$ (age at $\mathrm{K}$ entry) & $-.13(.014)$ & $-9.09 * *(3436)$ & .000 \\
\hline \multicolumn{4}{|l|}{ Time Slope $\left(\pi_{1 \mathrm{i}}\right)$} \\
\hline$\beta_{10}$ & $-.41(.005)$ & $-90.98 * *(3437)$ & .000 \\
\hline$\beta_{11}$ (number of risks) & $-.01(.004)$ & $-2.08 *(3437)$ & .037 \\
\hline$\beta_{12} \quad$ (age at K entry) & $.002(.001)$ & $3.72 * *(3437)$ & .000 \\
\hline \multicolumn{4}{|l|}{ For Thresholds: } \\
\hline$\delta_{2}$ & $2.76(.054)$ & $51.54 * *(13382)$ & .000 \\
\hline$\delta_{3}$ & $4.71(.061)$ & $76.99 * *(13382)$ & .000 \\
\hline$\delta_{4}$ & $7.88(.078)$ & $101.10 * *(13382)$ & .000 \\
\hline$\delta_{5}$ & $10.35(.092)$ & $112.53 * *(13382)$ & .000 \\
\hline
\end{tabular}

Note: $* \mathrm{p}<.05 ; * * \mathrm{p}<.01$

Random Components

\begin{tabular}{lccc}
\hline \multicolumn{1}{c}{ Random Effects } & Variance & $d f$ & Chi-square $(p)$ \\
\hline Variance in Base- K1 $\left(\tau_{\mathrm{oo}}\right)$ & 7.47 & 3388 & $9818.72(\mathrm{p}=.000)^{* *}$ \\
Variance in Time slope $\left(\tau_{11}\right)$ & .003 & 3392 & $3611.19(\mathrm{p}=.005)^{* *}$ \\
\hline
\end{tabular}

Note: ${ }^{*} \mathrm{p}<.05 ;{ }^{* *} \mathrm{p}<.01$

Despite the addition of entry age to both the intercept and slope models, however, significant variability remains in the initial status and the growth trajectories across children $\left(\tau_{00}=7.47, \mathrm{p}\right.$ $<.01 ; \tau_{11}=.003, \mathrm{p}<01$ ).

Table 6 provides predictions based on the random coefficients model and the final contextual model for the probability of a child being at or below proficiency level 3 across all four waves, and contains the actual proportion of children for comparison. Probabilities decline over time, as expected, because it is hoped that children are moving beyond category three by the end of first grade. Among the notable comparisons possible based on this simple table is the predicted probability at the end of first grade for a hypothetical male child of average age with no family risk characteristics (prob =
.097) relative to the predicted probability for a male average-age child with four family risk characteristics (prob $=.763$ ). Recall that these probabilities are cumulative, and represent the probabilities of being at or below proficiency category 3. These differences are quite large. Further, at the end of first grade, the likelihood that boys do not achieve proficiency in the highest categories in comparison to girls' likelihood is large as well. These predicted probabilities help to make clear the utility of hierarchical ordinal models for understanding effects of child-demographic variables on growth in proficiency for early literacy skills in a way that the basic interpretation of parameter estimates from the models in Tables 5 and 6 cannot easily do. 
Table 6. Probability Predictions (at or Below Category 3) for Each Time Point Based on Models in Tables 5 And 6 (Age is Grand Mean Centered).

\begin{tabular}{|l|c|c|c|c|}
\hline & $\begin{array}{c}\text { K-entry } \\
(0 \text { months })\end{array}$ & $\begin{array}{c}\text { K-completion } \\
(8 \text { months })\end{array}$ & $\begin{array}{c}\text { FG-entry } \\
(12 \text { months })\end{array}$ & $\begin{array}{c}\text { FG-completion } \\
\text { (20 months })\end{array}$ \\
\hline $\begin{array}{l}\text { At or below Category 3: } \\
\text { Actual Data }\end{array}$ & .967 & .835 & .719 & .161 \\
\hline Random Coefficients Model & .998 & .945 & .770 & .112 \\
\hline $\begin{array}{l}\text { Contextual Model 2 } \\
\text { Female } \\
\text { Average age } \\
\text { Family Risks =0 }\end{array}$ & .995 & .883 & .594 & .052 \\
\hline $\begin{array}{l}\text { Female } \\
\text { Average age } \\
\text { Family Risks }=4\end{array}$ & .999 & .997 & .984 & .622 \\
\hline $\begin{array}{l}\text { Male } \\
\text { Average age } \\
\text { Family Risks }=0\end{array}$ & .997 & .936 & .741 & .097 \\
\hline $\begin{array}{l}\text { Male } \\
\text { Average age } \\
\text { Family Risks =4 }\end{array}$ & .999 & .997 & .992 & .763 \\
\hline
\end{tabular}

\section{Conclusion}

These examples illustrate the application and interpretation of ordinal regression models to longitudinal data. Given that ordinal responses are best analyzed using ordinal methods, it is important that educational statisticians add these techniques to their toolkit. The ECLS provides a rich data set for investigating many challenging statistical issues. However, some issues need more clarity before these models can be effectively applied.

In this article, the focus has been on the cumulative odds or proportional odds model; however, this assumption may not always hold. Other options are routinely available for researchers dealing with single-level ordinal response data such as the continuation ratio model or non-proportional odds models (Agresti, 1989, 1990; Armstrong \& Sloan, 1989; Cox, 1972; Greenland, 1994; Goodman, 1983;
McCullagh, 1980; O'Connell, 2000, 2006). In addition, multilevel software programs are somewhat limited in terms of ordinal model methodology, and the default model may often be based on the (untested) assumption of proportional odds. Ultimately, the choice for what approach to take should be guided by theory or an a-priori expectation of which approach would be most appropriate for a given situation (Agresti, 1990; Armstrong \& Sloan, 1989). It is hoped that this article has helped to familiarize applied researchers with some of these issues as well as with the interpretation of multilevel ordinal models. Yet, further work is necessary to clarify model fitting for multilevel ordinal data when the assumption of proportional odds is violated, and for when three-level models might offer the best structure for the research data being analyzed. 


\section{References}

Agresti, A. (1989). Tutorial on modeling ordered categorical response data. Psychological Bulletin, 105, 290-301.

Agresti, A. (1990). Categorical data analysis. New York: Wiley.

Agresti, A. (1996). An introduction to categorical data analysis. New York: John Wiley \& Sons.

Armstrong, B. G. \& Sloan, M. (1989). Ordinal regression models for epidemiologic data. American Journal of Epidemiology, 129(1), 191-204.

Beaudin, B. (2003). "NCLB Act of 2001: Connecticut State Department of Education." Invited presentation to the Neag School of Education, University of Connecticut.

Bender, R. \& Benner, A. (2000). Calculating ordinal regression models in SAS and S-PLUS. Biometrical Journal, 42(6), p. $677-$ 699.

Bayder, N., Brooks-Gunn, J., \& Furstenberg, F. F. (1993). Early warning signs of functional illiteracy: Predictors in childhood and adolescence. Child Development, 64, 815829.

Burns, M. S, Griffen, P., \& Snow, C.E.(Eds.) (1998). Preventing reading difficulties in young children. Washington, D.C.: National Academy of Sciences - National Research Council.

Butler, S., Marsh, H. W., Sheppard, M. J, \& Sheppard, J. L. (1985). Seven-year longitudinal study of the early prediction of reading achievement. Journal of Educational Psychology, 77(3), 349-361.

Center for the Improvement of Early Reading Achievement (CIERA). (2001). Put reading first: The research building blocks for teaching children to read, kindergarten through grade 3. Washington, DC: Government Printing Office.

Cliff, N. (1993). What is and isn't measurement. In G. Keren \& C. Lewis (Eds.), $A$ handbook for data analysis in the social and behavioral sciences: Methodological issues. Hillsdale, NJ: LEA, p. 59-93.
Cliff, N. (1996). Ordinal methods for behavioral data analysis. Mahwah, NJ: Lawrence Erlbaum Associates.Cliff, N. (1996). Answering ordinal questions with ordinal data using ordinal statistics. Multivariate Behavioral Research, 3(3), 331-350.

Cliff, N. \& Keats, J. A. (2003). Ordinal mesurement in the behavioral sciences. Mahwah, NJ: Lawrence Erlbaum Associates.

Clogg, C. C., Shihadeh, E. S. (1994). Statistical models for ordinal variables. Thousand Oaks, CA: Sage.

Cox, D. R. (1972). Regression Models and life tables (with discussion). Journal of the Royal Statistical Society B, 74, 187-220.

Goldstein, H. (2003). Multilevel statistical models ( $3^{\text {rd }}$ ed.). London: Edward Arnold.

Goodman, L. A. (1983). The analysis of dependence cross-classifications having ordered categories, using log-linear models for frequencies and log-linear models for odds. Biometrics, 39, 149-160.

Greenland, S. (1994). Alternative models for ordinal logistic regression. Statistics in Medicine, 13, 1665-1677.

Hall, G., George, A. \& Rutherford, W. (1986). Measuring stages of concern about the innovation: A manual for use of the SoC Questionnaire. Austin, TX: Southwest Educational Development Laboratory.

Hedeker, D., \& Gibbons, R. D. (2006). Longitudinal data analysis. New York: John Wiley and Sons.

Hedeker, D. Mermelstein, R. J.(1998). A multilevel thresholds of change model for analysis of stages of change data. Multivariate Behavioral Research, 33(4), 427-455.

Huynh, C-L (2002). "Regression models of ordinal response data: Analytical methods and goodness-of-fit tests." Paper presented at the annual meeting of the AERA, New Orleans, LA, April 1-5, 2002.

Juel, C. (1988). Learning to read and write: A longitudinal study of 54 children from first through fourth grades. Journal of Educational Psychology, 80(4) 437-447. 
Lauby, J. L., Semaan, S., Cohen, A., Levition, L., Gielen, A., Pulley, L., Walls, C., and O'Campo, P. (1998) Self-efficacy, decisional balance and stages of change for condom use among women at risk for HIV infection. Health Education Research: Theory and Practice, 13(3), 343-356.

Levitt, H. \& O'Connell, A. A. (2002). "Differential Outcomes on Measures of Reading Ability for Full-Day Versus Half-day Kindergarteners." Paper presented at the $33^{\text {rd }}$ Annual Northeastern Educational Research Association Annual Meeting, October 23-25, Kerhonkson, NY.

Long, J. S. (1997). Regression models for categorical and limited dependent variables. Thousand Oaks, CA: Sage.

Lonigan, C. J. Burgess, S. R., Anthony, J. L.(2000). Development of emergent literacy and early reading skills in preschool children: Evidence from a latent-variable longitudinal study. Developmental Psychology, 36(5), 596613.

McCoach, D. B., O'Connell., A. A., Levitt, H. A., \& Reis, S. M. (2006). Ability grouping across kindergarten using an early childhood longitudinal study. Journal of Educational Research, 99(6), 339-346.

McCoach, D. B., O'Connell., A. A., Reis, S. M., \& Levitt, H. A. (2006). Growing readers: A hierarchical linear model of children's reading growth during the first two years of school. Journal of Educational Psychology, 98(1), 14-28.

McCullagh, P. (1980). Regression models with ordinal data (with discussion). Journal of the Royal Statistical Society, B, 42, 109-142.

McCullagh, P. \& Nelder, J. (1989). Generalized linear models (2nd ed.). London: Chapman \& Hall.

Murray, D. M. (1998). Design and analysis of group-randomized trials. New York: Oxford University Press.

National Research Council (1998). Preventing reading difficulties in young children. Washington, DC: National Academy Press.
O'Connell, A. A., (2000). Methods for modeling ordinal outcome variables. Measurement and Evaluation in Counseling and Development, 33(3), 170-193.

O'Connell, A.A. (2006). Logistic regression models for ordinal response variables. Thousand Oaks: Sage.

O'Connell, A. A., Cornman, D., \& Heybruck, K. (2003). "Measuring CBO capacity for implementation of evidence-based HIV prevention interventions." Paper submitted to APHA.

O'Connell, A. A. \& Levitt, H. (2002). "Modeling Ordinal Response Variables for Multilevel Analyses." Paper presented at the $33^{\text {rd }}$ Annual Northeastern Educational Research Association Annual Meeting, October 23-25, Kerhonkson, NY.

O’Connell, A. A., Goldstein, J., Rogers, H. J., \& Peng, C. Y. J. (in press). Multilevel logistic models for dichotomous and ordinal data. In A. A. O'Connell and D. B. McCoach (Eds.), Multilevel modeling of educational data. Greenwich, CT: Information Age Publishing.

Plewis, I. (2002). Modeling ordinal data using MlwiN. Multilevel Modelling Newsletter, 14(1). Available online at http://multilevel.ioe.ac.uk/publref/newsletters.ht $\mathrm{ml}$.

Prochaska, J. O., \& DiClemente, C. C. (1983). Stages and processes of self-change of smoking: toward an integrative model. Journal of Consulting and Clinical Psychology, 51(3), 390-395.

Prochaska, J. O. \& DiClemente, C. C. (1986). Towards a comprehensive model of change. In W.R. Miller \& N. Heather (Eds.), Treating addictive behaviors: Processes of change (pp. 3-27). New York: Plenum Press.

Prochaska, DiClemente, \& Norcross (1992). In search of how people change: Applications to addictive behavior. American Psychologist, 47(9), 1102-1114.

Prochaska, J. O., Redding, C. A., Harlow, L. L., Rossi, J. S., Velicer, W. F. (1994). The transtheoretical model of change and HIV prevention: A review. Health Education Quarterly, 21(4), 471-486. 
Raudenbush, S., Bryk, A., Cheong, Y. F., \& Congdon, R. (2004). HLM 6: Hierarchical linear and nonlinear modeling. Lincolnwood, IL: Scientific Software International.

Raudenbush, S. W. \& Bryk, A. S. (2002). Hierarchical linear models: applications and data analysis methods (2nd ed.). Newbury Park, CA: Sage.

Stevens, S. S. (1946) On the theory of scales of measurement. Science, 103(2684), 677680 .

Stark, M. J., Tesselaar, H. M., O'Connell, A. A., Cohen, A., Person, B., Walls, C. \& Galvotti. (1996). Psychosocial factors associated with the stages of change for condom use among women at risk for HIV/STDs: Implications for intervention development. Journal of Consulting and Clinical Psychology, 66(6), 967-978. van den Berg, R., Sleegers, P.\& Pelkmans, T. (2002). Research into interventions from a cultural-individual innovation perspective. Studies in Educational Evaluation, 28, 147-175.

West, J., Denton, K., Germino-Hausken, E. (2000). America's kindergartners: findings from the early childhood longitudinal study, kindergarten class of 1998-99: Fall 1998. Washington, DC: United States Department of Education, National Center for Education Statistics. (NCES 2000-070)

Wong, G. Y., Mason, W. M. (1985). The hierarchical logistic regression model for multilevel analysis. Journal of the American Statistical Association, 80(391), 513-524.

Zill, N. \& West, J. (2001). Entering kindergarten: A portrait of American children when they begin school. Washington, DC: United States Department of Education, National Center for Education Statistics. (NCES 2001-035). 


\title{
Estimation of Risk for Developing Cardiac Problem in Patients of Type 2 Diabetes as Obtained by the Technique of Density Estimation
}

\author{
Ajit Mukherjee \\ Ajit Mathur \\ Rakesh Mittal \\ Division of Reproductive Health and Nutrition, Indian Council of Medical Research \\ New Delhi, India
}

High levels of cholesterol and triglyceride are known to be strongly associated with development of cardiac problem in patients of type 2 diabetes. In a hospital-based study, patients showing ECG positive were compared with those who were not. The observations on cholesterol and triglyceride were considered for estimation of risk for developing the cardiac problem. The technique of density estimation employing Epanechnikov kernel was used for estimating bivariate probability density functions with respect to observations on cholesterol and triglyceride of the two groups. Using the odds form of Bayes' rule, the estimates of posterior odds were computed.

Key words: Density estimation, kernel, logistic regression, probability density function.

\section{Introduction}

The technique of Density Estimation is a nonparametric approach and involves no assumptions as it deals directly with the experimental data. The method of density estimation describes the probability distribution of people with respect to the parameter under investigation. This technique has found favour with many applied statisticians in the past. Scott, Gotto, Cole, and Gorry (1978) used density

Ajit Mukherjee, Ph.D. in Mathematical Statistics, is Deputy Director General in Division of Reproductive Health and Nutrition, Indian Council of Medical Research, Ansari Nagar, Post Box 4911, New Delhi-110029, India. He has over 30 publications to his credit in both national and international journals and is the holder of a prestigious National Award Email: mukherjeeajit@hotmail.com. Ajit Mathur, M.Sc., is also Deputy Director General in Division of Reproductive Health and Nutrition. $\mathrm{He}$ has 25 publications to his credit. Email:ajiticmr@hotmail.com. Rakesh Mittal, M. D., is Deputy Director General (Senior Grade), also in Division of Reproductive Health and Nutrition. He has about 30 publications and books to his credit.E-mail:mittrak@hotmail.com estimation for assessing plasma lipids as collateral risk factors in coronary artery disease. Bithell (1990) gave an application of this technique in Geographical Epidemiology. Mukherjee, Kumar, Mittal, and Saxena (2002) used density estimation for estimating risk of developing goiter in an endemic area. Silverman (1986) provided an excellent account of various approaches to Density Estimation in his book.

High levels of cholesterol and triglyceride are known to be strongly associated with development of cardiac problem in patients of type 2 diabetes. However, the extent of risk posed by elevated levels of these two risk factors in patients of type 2 diabetes has not been studied extensively. The present article describes an alternative methodology whereby risk of developing cardiac problem in patients of type 2 diabetes can be estimated using cholesterol and triglyceride as risk factors.

\section{Methodology}

In a hospital-based study conducted by Indian Council of Medical Research in 1989-92, 4637 patients of Non Insulin Dependent Diabetes Mellitus (NIDDM) also known as Type2 Diabetes were enrolled. Various bio-chemical investigations and electrocardiogram (ECG) were carried out at regular intervals. The 311 
patients showing ECG positive and thereby indicating coronary artery disease $(\mathrm{CAD}+)$, formed the first group. The remaining patients numbering 4326 formed the second group. The Epanechnikov kernel, which is known to have $100 \%$ efficiency in terms of mean integrated square error (Silverman, 1986), was employed in the technique of density estimation for estimating probability density functions of the patients falling in the two groups with respect to their cholesterol and triglyceride levels. Using the odds form of Bayes' rule, the estimate of odds ratio (OR) was obtained. A simulation study was undertaken and 100 estimates of OR were generated using the approach of density estimation giving a mean estimated odds ratio and an estimate of standard deviation.

Let the number of patients in the first group be denoted by $N_{C A D+}$ and that in the second group by $N_{N I D}$. Let $\mathrm{x}$ and y in general denote the observations on cholesterol and triglyceride of the patients with $x_{i}$ and $y_{i}$ being the observations on the ith patient. Then the bivariate kernel density estimator for the first group is given by

$$
\begin{aligned}
& \mathrm{f}_{\mathrm{CAD}+} \equiv \mathrm{f}_{\mathrm{CAD}+}(\mathrm{x}, \mathrm{y}) \\
& =\frac{1}{\mathrm{~N}_{\mathrm{CAD}+} \mathrm{h}_{\mathrm{x}} \mathrm{h}_{\mathrm{y}}} \sum_{\mathrm{i}=1}^{\mathrm{N}_{\mathrm{CAD}+}} \mathrm{K}\left(\frac{\mathrm{x}-\mathrm{x}_{\mathrm{i}}}{\mathrm{h}_{\mathrm{x}}}\right) \mathrm{K}\left(\frac{\mathrm{y}-\mathrm{y}_{\mathrm{i}}}{\mathrm{h}_{\mathrm{y}}}\right)
\end{aligned}
$$

the quantities $h_{x}$ and $h_{y}$ are called bandwidths of the function $f_{C A D}+$ and are appropriately chosen.

The function $\mathrm{K}(\mathrm{z})$ which is known as Epanechnikov kernel, is defined as follows:

$$
K(z)=\left\{\begin{array}{l}
\frac{3}{4}\left(1-\frac{1}{5} z^{2}\right) / \sqrt{5}, \text { if }|z|<\sqrt{5} \\
0, \text { otherwise }
\end{array}\right.
$$

Similarly, the Epanechnikov kernel density estimator for the other group namely, $f_{N I D} \equiv f_{N I D}(x, y)$ can also be worked out.

The Likelihood Ratio (LR) will then be given by

$$
L R=\frac{f_{C A D+}}{f_{N I D}} .
$$

Further, the odds form of Bayes' rule states that

$$
O^{\prime}(D)=O(D) * L R
$$

where $O^{\prime}(D)$ is the posterior odds and $O(D)$ is prior odds and is given as $O(D)=\frac{N_{C A D+}}{N_{N I D}}$.

For the present set of data, $N_{C A D+}=311$ and $N_{N I D}=4326$. For estimating $f_{C A D+}$, the optimum values of $h_{x}$ and $h_{y}$ were obtained by objectively starting the process of smoothing with $h_{x}=h_{y}=5$ giving an increment of 5 until $h_{x}=h_{y}=35$. Thereafter, the process of smoothing was continued by giving a unit increment. The estimates of density stabilized with values of $h_{x}$ and $h_{y}$ at 40, 41,42, 43 to five decimal places. Hence, $h_{x}=h_{y}=40$ was accepted as an optimum value of $h_{x}$ and $h_{y}$.

Similarly, the values of $h_{x}$ and $h_{y}$ for estimating $f_{N I D}$ were also obtained to be 40 each. The estimate of probability density function for the first group i.e., $f_{C A D+}$ is as depicted in Figure 1.

Computation of OR

Consider the following transformation

$$
\begin{gathered}
P=\frac{1}{1+e^{-Y}}, \text { where } \\
Y=\beta_{0}+\beta_{1} X_{1}+\beta_{2} X_{2}+---+\beta_{k} X_{k}+\varepsilon .
\end{gathered}
$$

It can be shown that 
Fig.1. Bivariate Probability Density Function of patients with CAD+ at different values of Cholesterol and Triglyceride using Epanechnikov Kernel

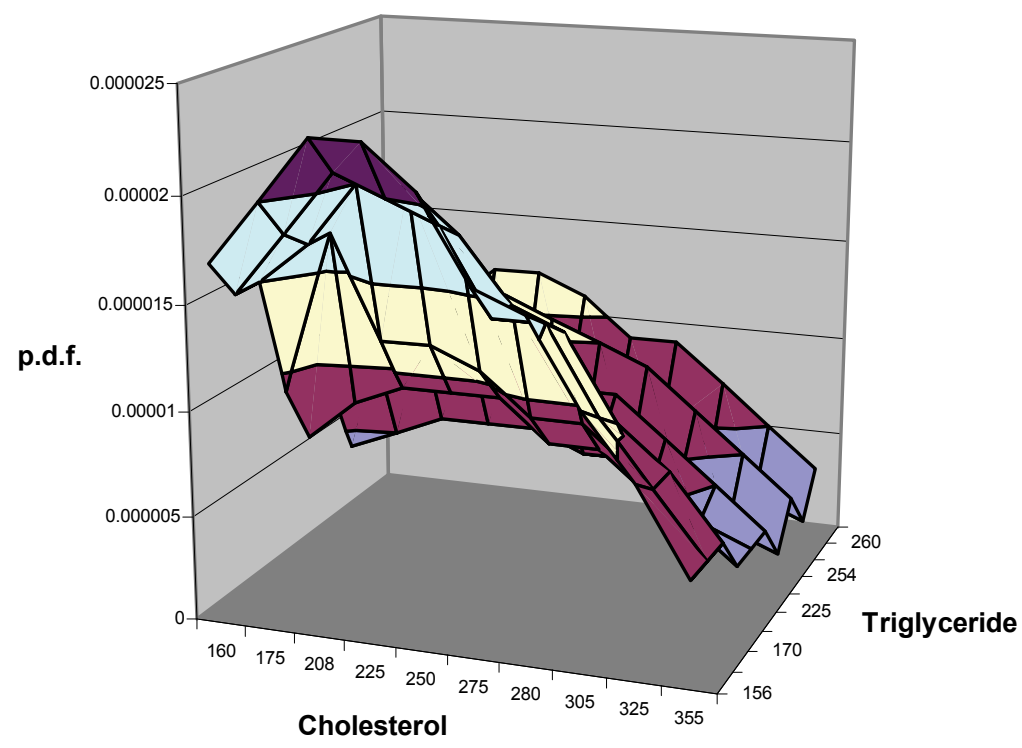

Q0.00002-0.000025 口0.000015-0.00002 口0.00001-0.000015 口0.000005-0.00001 口0-0.000005

$$
\log \left(\frac{P}{1-P}\right)=Y
$$

or

$\log \left(\frac{P}{1-P}\right)=\beta_{0}+\beta_{1} X_{1}+\beta_{2} X_{2}+---+\beta_{k} X_{k}+\mathcal{E}$

Thus $\beta_{i}$ gives average quantum of change in $\log$ (odds) per unit change in $X_{i}, i=1,2, \ldots . ., k$ and $e^{\beta_{i}}$ gives the odds ratio with respect to $X_{i}$ keeping other predictors at constant levels.

\section{Results}

Keeping cholesterol fixed at 250 and varying the value of triglyceride from 209 to 254 , the posterior odds at three pairs of values of cholesterol and triglyceride viz., (250,209), $(250,254)$ and $(250,260)$ were worked out to be respectively $0.0629,0.08047$ and 0.08549 . Thus, keeping cholesterol fixed at 250 and increasing triglyceride by a margin of 45 and 51 units from 209 , led to respectively 1.28 and 1.34 times increase in odds for developing a cardiac problem. Further, considering first two of the above three pairs of values of cholesterol and triglyceride, the following would be obtained:

$$
\beta_{Y}=\frac{\log (0.08047)-\log (0.0629)}{45}
$$

or

$$
\beta_{Y}=0.005474
$$


Therefore $\mathrm{OR}$ is given by $e^{\beta_{Y}}=1.005489$. Drawing a simple random sample of 100 consecutive pairs of values of cholesterol and triglyceride and using the above methodology, 100 estimates of OR were obtained with a mean value of OR as 1.0025 and S.D. of 0.0027 giving $95 \%$ C.I. as $\{1.0020,1.0031\}$. The OR as estimated by Logistic Regression model was 1.0029 with a $95 \%$ C.I. of $\{0.9984,1.0074\}$.

\section{Conclusion}

It is seen that with the technique of density estimation employing Epanechnikov kernel, it is possible to obtain an estimate of the probability density function of the patients of type 2 diabetes falling in the two groups with respect to their cholesterol and triglyceride levels. It has also been demonstrated in the present article, how the posterior odds vary with increasing levels of triglyceride keeping cholesterol at a constant high level, which ultimately led to an estimate of odds ratio (Table 1).

From table 1, it is clear that the estimate of odds ratio as obtained by the method of density estimation is in close proximity to the estimate as obtained by the method of logistic regression. Thus, the risk of developing a cadiac problem can also be alternatively estimated by using the technique of density estimation.
References

Bithell, J. F (1990). An application of density estimation to geographical epidemiology. Statistics in Medicine, 9, 691701.

Hennekens, C. H. \& Burning, J. E. (1987). In (S. L. Mayrent, Ed.) Epidemiology in medicine. Boston, MA: Little, Brown and Co.

Mukherjee, A., Kumar, S., Mittal, R. \& Saxena, N. C. (2002). An application of the technique of density estimation in geographical epidemiology of goitre in the Muzaffarpur district of Bihar state of India. Statistics in Medicine, 21, 2403-2407.

Scott, D. W., Gotto, A. M., Cole, J.S., \& Gorry, G. A. (1978). Plasma lipids as collateral risk factors in coronary artery disease: A study of 371 males with chest pain. Journal of Chronic Diseases, 31, 337-345.

Silverman, B. W (1986). Density estimation for statistics and data analysis. London: Chapman and Hall.

Table 1. Estimates of Odds Ratio as obtained by the application of Epanechnikov kernel in Density Estimation and Logistic Regression

\begin{tabular}{|c|c|c|}
\hline Method & Odds Ratio & 95\% C.I. \\
\hline Logistic Model & 1.0029 & $0.9984-1.0074$ \\
\hline Density Estimation: & & $1.002-1.0031$ \\
\hline Epanechnikov Kernel & 1.0025 & \\
\hline
\end{tabular}




\title{
Multinomial Logistic Regression Model for the Inferential Risk Age Groups for Infection Caused by Vibrio cholerae in Kolkata, India
}

\author{
Krishnan Rajendran Thandavarayan Ramamurthy Dipika Sur \\ National Institute of Cholera \& Enteric Diseases \\ West Bengal, India
}

\begin{abstract}
Multinomial Logistic Regression (MLR) modeling is an effective approach for categorical outcomes, as compared with discriminant function analysis and log-linear models for profiling individual category of dependent variable. To explore the yearly change of inferential age groups of acute diarrhoeal patients infected with Vibrio cholerae during 1996-2000 by MLR, systematic sampling data were generated from an active surveillance study. Among 1330 V.cholerae infected cases, the predominant age category was up to 5 years accounting for $478(30.5 \%)$ cases. The independent variables V.cholerae $\mathrm{O} 1(\mathrm{p}<0.001)$ and non-O1 and non-O139 $(\mathrm{p}<0.001)$ were significantly associated with children under 5 years age group. V.cholerae $\mathrm{O} 139$ inferential age group was $>40$ years. The infection mediated by V.cholerae $\mathrm{O} 1 \mathrm{had}$ significantly decreasing trend $\operatorname{Exp}(\mathrm{B})$ year wise from 1996 to $2000(\mathrm{p}<0.001, \mathrm{p}<0.001, \mathrm{p}<0.001, \mathrm{p}<$ 0.001 and $\mathrm{p}<0.001$, respectively). MLR model showed that up to 5 year's age children are more vulnerable to infection caused by V.cholerae $\mathrm{O} 1$.
\end{abstract}

Key words: MLR, Vibrio cholerae, $\operatorname{exp(B),~explanatory,~dependent,~categorical~}$

\section{Introduction}

Study design and data sources

Cholera is an epidemic disease in developing countries which has been the focus of intensive research for many years. This water borne disease is typified by severe watery diarrhea, vomiting and dehydration of the different serogroups of $V$. cholerae, serogroups $\mathrm{O} 1, \mathrm{O} 139$ and non-O1, non-O139 colonize in the small intestine and produce enterotoxin

The authors thank all the scientists and Research Fellows of National Institute of Cholera and Enteric Diseases, Kolkata for helping to generate the laboratory data, and Infectious Diseases Hospitals, Kolkata, for taking part in the active surveillance program. Comments or question about this article should be directed to the first author at: rajenk20@yahoo.com or rajenk20@hotmail.com, or Division of Epidemiology, National Institute of Cholera \& Enteric Diseases, P-33, Scheme-XM, CIT.Road, Beliaghata, Kolkata 700 010, West Bengal, India. responsible for watery diarrhea. Until 19th century, cholera was confined in the Indian subcontinent (Epstein, 1993; Islam et al., 1994) and from this region, it has spread to many parts of the world causing seven pandemics. (Codeco, 2001; Faruque et al., 1998; Banerjee \& Hazra, 1974). In 1992, a newly described non-O1 serogroup of $V$. cholerae designated O139 Bengal, caused unusual cholera outbreaks in India (Ramamurthy et al., 1993). Total eradication of this organism is very unlikely because of its propensity and acquaintance in the coastal ecosystem (Sack et al., 2004). In cholera endemic regions, severe cholera affects one in every 10-50 individuals, the highest attack rates of disease being in children of two to four years age (Cash et al., 1974). About 5.5 millions cases of cholera occur annually in Asia and Africa, $8 \%$ severe enough to be hospitalized, and $20 \%$ of the severe cases resulting in deaths, totaling approximately 120000/year (Mahalanabis et al., 1992; Noah \& Mahony, 1998).

Classification and prediction are the more common practices in applied medical research. Mathematical model is widely used for prediction of disease outcomes. Discriminate analysis is mainly used for classification and 
logistic regression and the dependent variable is binary or strict with two category. In a few studies, the relative predictivity of these methods were employed as an outcome variable that had more than two groups with unequal sizes. These models have been investigated when reducing bias by promoting the efficiency of the parameter estimation when the dependent variable has more than two groups. In this study Multivariate Logistic regression model was employed to identify inferential age group at greatest risk for diarrhea.

\section{Materials And Methods}

During 1996 to 2000, systematic sampling was done from every 5th hospitalized diarrhea patients attending the Infectious Diseases Hospital, Kolkata, India in two randomly selected days of the week. Samples were collected in the form of stool or rectal swab and sent to the laboratory for the isolation of common enteric pathogens within 3 hrs. The enteric pathogens were isolated and identified by standard laboratory methods (World Health Organization, 1987; Garg et al., 2000).

\section{Data Management}

The pre-designed proforma describing case demographics, symptoms etc. were checked manually and sent to data management center. The data were entered into pre-designed format of the proforma in EPI-info (6.0 version) with inbuilt entry validation checking facilitated program, by two trained data entry professionals in two separate computers. Data were randomly checked and matched to derive consistency and validity. The edited data was exported to SPSS version 4.0, and the final analysis was done using the SPSS.10. In this study, the inferential age groups was explored for three different serogroups of V.cholerae, O1, O139 and non-O1, non-O139 among culture positive cases by MLR and also to know the year wise changing pattern by parametric estimation through Odds Ratio(OR) $((\operatorname{Exp}(\mathrm{B}))$. The proposed objective of the study was to determine the likelihood of age to have infection by V.cholerae O1, $\mathrm{O} 139$ and non-O1, nonO139, serogroups.

The age groups were classified into 6 categories viz. up to 5 years, above 5-10 years, above 10-20 years, above 20-30 years, above 30 40 years and more than 40 years and were coded as 1-6, respectively. The relationship between the risk dependent variable and each of the three categorical explanatory variables in the serogroup are shown in Table 1 . Infection by any serogroup of V.cholerae was classified in numbers as 1 for organism present and 2 for its absence.

To describe categorical dependent variables and one or more categorical or dichotomous or continuous explanatory variables, Logistic regression was found suitable if dependent is strict with two categories. The conceptualized objective in this study was to employ MLR which may be more efficient and reliable to obtain the probability estimation of concerned patient population. In addition, MLR explores estimation of the net effects of a set of explanatory variables on the dependent variable (Cabrera, 1994; Demaris, 1992; Menard, 2000).

\section{Data Analysis}

The MLR model involves categorical dependent variable (more than two) Y. e.g. six categories of age group and 3 explanatory (V.cholerae serogroups) variables $\mathrm{x}_{1} \mathrm{x}_{2}$ and $\mathrm{x}_{3}$ $\left(\mathrm{x}_{1}=\mathrm{O} 1, \mathrm{x}_{2}=\mathrm{O} 139\right.$ and $\mathrm{x}_{3}=$ non-O1, non-O139).

Let $\mathrm{P}_{1}=$ the probability of up to 5 years age group at risk $(\mathrm{Y}=1), \mathrm{P}_{2}=$ the probability of above 5-10 years age group at risk $(\mathrm{Y}=2) \mathrm{P}_{3}=$ the probability of above 10-20 years age group at risk $(\mathrm{Y}=3), \mathrm{P}_{4}=$ the probability of above 20 30 years age group at risk $(\mathrm{Y}=4), \mathrm{P}_{5}=$ the probability of above 30-40 years age group at risk $(\mathrm{Y}=5)$ and $\mathrm{P}_{6}=$ the probability of more than 40 years age group at risk $(Y=6)$. The modality of MLR relates to the log of odds (or logit) of $\mathrm{Y}$ to the explanatory variable $\mathrm{x}_{1}$ in linear form as

$$
\begin{aligned}
& \mathrm{Pi}=\mathrm{A}+\mathrm{Px}_{\mathrm{i}} \\
& \operatorname{Probit}\left(\mathrm{P}_{\mathrm{i}}\right)=\text { intercept }+ \text { R. co-eff }\left(\mathrm{x}_{\mathrm{i}}\right)
\end{aligned}
$$

The model explores

$$
\operatorname{Prob}(\mathrm{y}=\mathrm{j})=\frac{\mathrm{e}^{\Sigma \beta_{\mathrm{jk}}{ }_{\mathrm{k}}}}{-1+\sum \mathrm{e}^{\Sigma \beta_{\mathrm{jk}}{ }_{\mathrm{k}}}}
$$


Table 1: Distribution of V.cholerae O1, O139 and non-O1, non-O139 among different age groups of patients during 1996-2000.

\begin{tabular}{|c|c|c|c|c|c|c|c|c|c|c|c|}
\hline \multirow{2}{*}{$\begin{array}{l}\text { Age groups } \\
\text { (in years) }\end{array}$} & \multirow[t]{2}{*}{ V.cholerae serogroup } & \multicolumn{2}{|c|}{1996} & \multicolumn{2}{|c|}{1997} & \multicolumn{2}{|c|}{1998} & \multicolumn{2}{|c|}{1999} & \multicolumn{2}{|c|}{2000} \\
\hline & & No & $\%$ & No & $\%$ & No & $\%$ & No & $\%$ & No & $\%$ \\
\hline \multirow{3}{*}{ Upto 5} & $(n=301) O 1$ & 70 & 23.3 & 43 & 14.3 & 93 & 30.9 & 54 & 17.9 & 41 & 13.6 \\
\hline & $(\mathrm{n}=55) 0139$ & 14 & 25.4 & 16 & 29.1 & 7 & 12.7 & 15 & 27.3 & 3 & 5.5 \\
\hline & $\begin{array}{l}(\mathrm{n}=109) \text { Non-O1, } \\
\text { Non-O139 }\end{array}$ & 8 & 7.3 & 13 & 11.9 & 29 & 26.6 & 38 & 34.9 & 21 & 19.3 \\
\hline \multirow{3}{*}{$>5-10$} & $(\mathrm{n}=90) \mathrm{O} 1$ & 18 & 20.0 & 12 & 13.3 & 34 & 37.8 & 15 & 16.7 & 11 & 12.2 \\
\hline & $(n=27) 0139$ & 6 & 22.2 & 12 & 44.4 & 2 & 7.5 & 3 & 11.1 & 4 & 14.8 \\
\hline & $\begin{array}{l}(\mathrm{n}=14) \text { Non-O1, } \\
\text { Non-O139 }\end{array}$ & 2 & 14.3 & 3 & 21.4 & 5 & 5.7 & 2 & 14.3 & 2 & 14.3 \\
\hline \multirow{3}{*}{$>10-20$} & $(n=87) O 1$ & 19 & 21.8 & 6 & 6.9 & 29 & 33.3 & 21 & 24.2 & 12 & 13.8 \\
\hline & $(\mathrm{n}=52) 0139$ & 8 & 15.4 & 24 & 46.1 & 6 & 11.5 & 11 & 21.2 & 3 & 5.8 \\
\hline & $\begin{array}{l}(\mathrm{n}=39) \text { Non-O1, } \\
\text { Non-O139 }\end{array}$ & 6 & 15.4 & 15 & 38.4 & 12 & 30.8 & 3 & 7.7 & 3 & 7.7 \\
\hline \multirow{3}{*}{$>20-30$} & $(\mathrm{n}=100) \mathrm{O}$ & 20 & 20.0 & 12 & 12.0 & 44 & 44.0 & 13 & 13.0 & 11 & 11.0 \\
\hline & $(n=87) 0139$ & 20 & 23.0 & 29 & 33.3 & 18 & 20.7 & 18 & 20.7 & 2 & 2.3 \\
\hline & $\begin{array}{l}(\mathrm{n}=59) \text { Non-O1, } \\
\text { Non-O139 }\end{array}$ & 15 & 25.4 & 18 & 30.5 & 14 & 23.7 & 7 & 11.9 & 5 & 8.5 \\
\hline \multirow{3}{*}{$>30-40$} & $(\mathrm{n}=40) \mathrm{O} 1$ & 8 & 20.0 & 9 & 22.5 & 12 & 30.0 & 8 & 20.0 & 3 & 7.5 \\
\hline & $(\mathrm{n}=51) 0139$ & 11 & 21.6 & 19 & 37.2 & 8 & 15.7 & 7 & 13.7 & 6 & 11.8 \\
\hline & $\begin{array}{l}(\mathrm{n}=29) \text { Non-O1, } \\
\text { Non-O139 }\end{array}$ & 7 & 24.1 & 6 & 20.7 & 7 & 24.1 & 5 & 17.2 & 4 & 13.8 \\
\hline \multirow{3}{*}{$>40$} & $(\mathrm{n}=63) \mathrm{O} 1$ & 12 & 19.1 & 6 & 9.5 & 24 & 38.1 & 14 & 22.2 & 7 & 11.1 \\
\hline & $(n=84) 0139$ & 20 & 23.8 & 25 & 29.8 & 18 & 21.4 & 16 & 19.0 & 5 & 6.0 \\
\hline & $\begin{array}{l}(\mathrm{n}=43) \text { Non-O1, } \\
\text { Non-O139 }\end{array}$ & 7 & 16.3 & 9 & 20.9 & 13 & 30.2 & 11 & 25.6 & 3 & 7.0 \\
\hline
\end{tabular}




$$
\mathrm{P}_{\mathrm{ij}}=\log \frac{\mathrm{P}\left(\Delta_{\mathrm{i}}\right)}{\mathrm{P}\left(\Delta_{6}\right)}=\text { intercept }+\operatorname{parameter}\left(V_{k}\right)
$$

$\mathrm{i}, \mathrm{j}, \mathrm{k}>0 \quad \mathrm{i}=$ age 1 to age $5, \mathrm{j}=1996$ to $2000, k=$ O1, O139 and non O1, non O139, $V=V$. cholerae and $\Delta_{6}=$ age 6 .

The intercept (initial level) terms are simple logit for positive V.cholerae O1. The first intercept is the $\log$ of ratio of the probability of a positive in up to 5 years to the probability of a positive in $>40$ years. Hence, co-efficient for positive cases reveal the relationship between the logits and V.cholerae O1. Because the coefficient is positive and significantly different from 0 that V.cholerae $\mathrm{O} 1$ positives are more likely associated with upto5 years age group as compared to $>40$ years age group.

Result

During 1996-2000, a total of 1330 V.cholerae stool culture positive cases formed the test set in this analysis, of which 681(51.20 $\%), 356(26.8 \%)$ and $293(22 \%)$ were positive for V.cholerae O1, O139 and non-O1, non-O139 respectively. The age was coded as 6 categories in which $465(35.0 \%), 131(9.8 \%), 178(13.4 \%)$, $246(18.5 \%) 120(9.0 \%)$ and $190(14.3 \%)$ were in the age groups upto 5 years, $>5-10$ years, $>10-20$ years, $>20-30$ years, $>30-40$ years, $>40$ years respectively. The analysis was made to explore inferential age group. The predominant infected age category was upto 5 years age group. Overall, explanatory variables V.cholerae O1 $(\mathrm{p}<0.001, \mathrm{OR}=3.48,95 \%$ confidence interval (CI): 2.44, 4.90) was highly significant with children under five years of age. V.cholerae O139 was detected for more than 40 years age group ( $\mathrm{p}<0.001, \mathrm{OR}=2.99,95 \% \mathrm{CI}: 1.29,4.98)$ and under five years old children were associated with V.cholerae non-O1, non-O139 $(\mathrm{p}<0.001, \mathrm{OR}=2.50,95 \% \mathrm{CI}: 1.38,4.55)$. As per the above equation 3 the result shows

Predicted logit $(\mathrm{Y} 1 \leq 5$ years $)=.302+1.247$

(V. cholerae $\mathrm{O} 1$ )
Predicted logit $(\mathrm{Y} 1 \leq 5$ years $)=1.335+(-) 1.550$

(V. cholerae $\mathrm{O} 139)$

Predicted logit $(\mathrm{Y} 1 \leq 5$ years $)=.898+(-) .055$

( $V$. cholerae non-O1, non-O139)

Similarly, a prediction can be made for other age groups.

Overall, the chi-square test of proportional odds assumption was significant (degrees of freedom(df) $=5: \quad p<0.001$ ), indicating that the model is fit. Table 2 depicts only the predominantly affected age group with respective $V$. cholerae serogroup. Data on nonsignificant age group were not shown to avoid multiple tables. The explanatory variables are compared individually with dependent variable age. According to MLR models, the log of the Odds of an up to 5 years age group shows risk of infection positively related to the serogroup $V$. cholerae $\mathrm{O} 1$, in all years (Table 2). It was also shown a decreasing slope (rate of change) during the consecutive years. The respective years of OR for $V$. cholerae O139 has increased in 1998 and declined, though there was no significant association in all years with its inferential age group $>40$ years. In the case of $V$. cholerae nonO1, non-O139, year wise significance was not detected but the more vulnerable age group was $<5$ years.

\section{Conclusion}

Generally, Logistic Regression analysis (LR) is a common statistical technique which could be used to predict the likelihood of a categorical or binary or dichotomous outcome variables. In epidemiological studies, the dependent variable is presence or absence of a disease. The LR model has been applied in social science (Janik \& Kravitz, 1994). Most of the microbiological laboratory generated data are not being utilized with proper statistical techniques owing to lack of appropriate guidelines for application. This study exploited the usefulness of MLR as a tool in statistical modeling and detecting the inferential risk age groups for $V$. cholerae mediated infection for 1330 culture positive cases from 1996-2000. 
Table 2. Multinomial Logistic Regression Models exploring significant risk age group of cholera infection during 1996-2000.

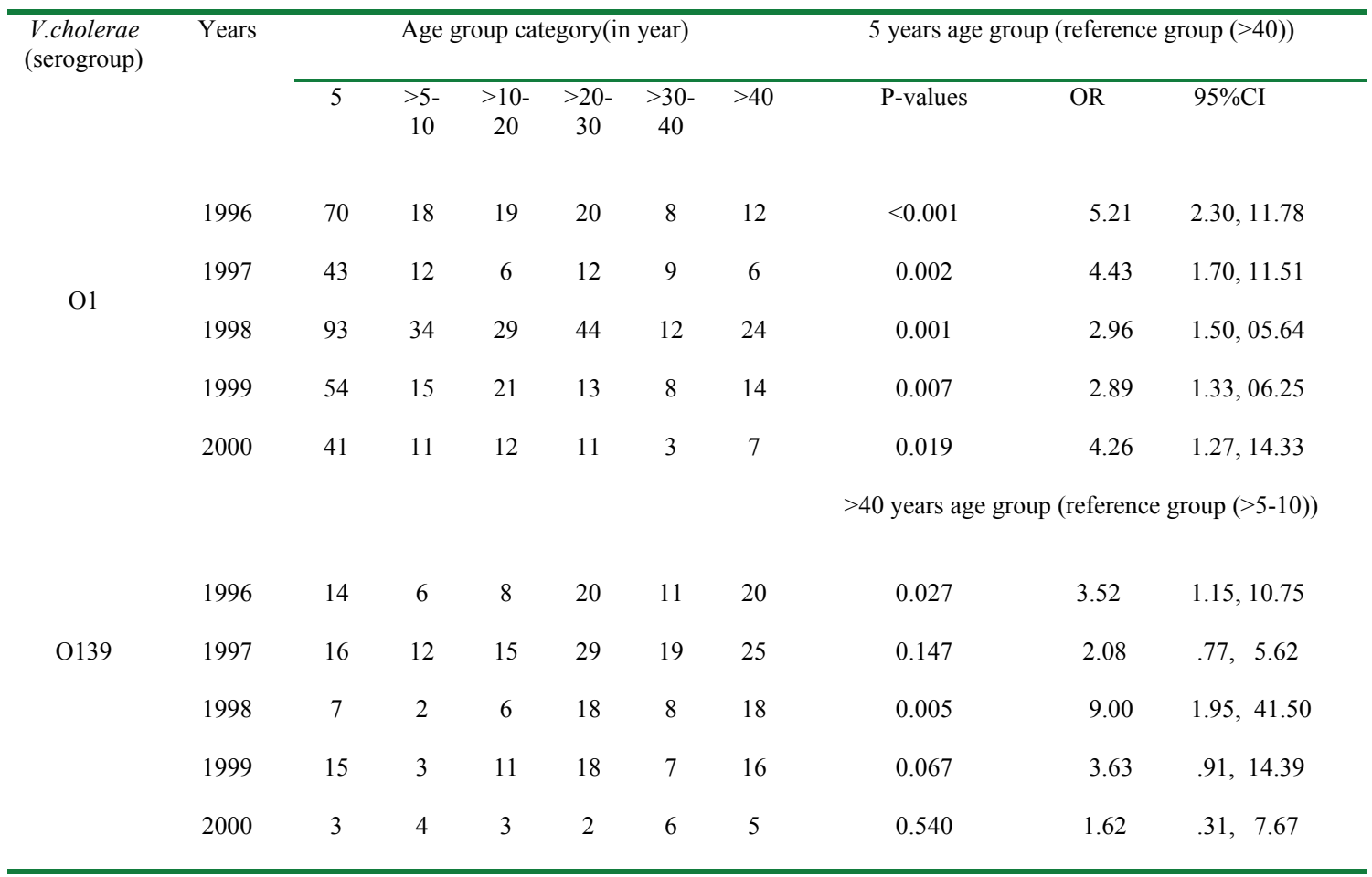

The MLR requires the dependent variables to be non-metric, dichotomous, nominal and ordinal, satisfy the level of measurement and independent variable to be metric or dichotomous. The minimum number of cases per independent variable is 10 using a guideline provided by Homen and Lameshow (2000), in which the MLR predicts and provides a set of co-efficient for each of the two comparisons. The co-efficient for the reference group are all zeros, similar to the co-efficient of the reference group for a dummy-coded variable. Dependent variable will be defined as groups, where the equations can be used to compute the probability and predict the groups associated with the highest probability. The predicted group membership can then be compared to the actual group membership to obtain a measure of classification accuracy.

The emphasis is given on MLR utility because (a) application for categorical outcomes in multivariate techniques are very few, including Logistic Regression, discriminant function analysis and log-linear models, (b) the MLR does not make any assumptions of normality, linearity, and homogeneity of variance for the independent variables (Hosmer \& Lemeshow , 2000; Peng \& Nichols, 2003; Clayton \& Hills, 1993). (c) MLR does not impose these requirements, it is preferable to use discriminant analysis when the data does not satisfy these assumptions. (d) a more useful measure to assess the utility of MLR is classification accuracy and (e) because the laboratory data generally exist either in 
dichotomous or an ordinal form of variable that can be explored in the form of Odds Ratio. The LR and MLR are best methods for the above format of data structure.

This study explained how effectively the MLR models are useful in the epidemiology of cholera and overall model evaluations. The likelihood Ratio was examined to improve the MLR model over null models. An intercept is the only model that serves as a good baseline with no predictors. According to MLR model, the test yielded significance and was more effective than the null model.

In the tests of individual predictors, the Wald chi-square statistic was tested using individual B coefficients to inclined relationship with dependent variables. The goodness of fit statistics assess fitness of logistic model against actual classification i.e. six levels of age group category in the MLR model. The two measures were almost similar in overall estimation, which is similar to Ordinary Least Square (OLS) regression. No equivalents of this concept for MLR explains variance, and for this reason the Pseudo R-Square reported to be complementary to others, which has more useful evaluative indices such as tests of individual regression coefficients (Peng et al., 2001).

The advantage of inferential test of the goodness of fit was suggested by Begg and Gray (1984) for multinomial logistic models. In the validation of predicted probabilities, the MLR model predicts the logit of levels of degrees of inferential risk age group from independent variables. The logit is probability/1-probability, which can be transformed later to the probability scale according to the equation 2 (Rabins \& Dickinson, 1985; Peterson \& Harrell, 1990; Greenland, 1987; Savitz, 1992). The predicted probability of inferential risk age group is evaluated and compared with actual risk age to determine various levels of age groups.

Reference category was fixed based on the occurrence of positive cases in the age groups in which the cases were low. The main aim of the selection of reference category was to interrogate the age group favored by the pathogen. In V.cholerae O1, greater than 40 years age was selected as reference category owing to less incidence rate. The interesting trend of V.cholerae $\mathrm{O} 139$ was higher incidence rate in older age group and lower in $>5-10$ years age group that served as reference category to explore existing relation. The parameter estimation of all age groups with different serogroups of V.cholerae was newly conceptualized by comparing reference category of age group with positive cases of respective serogroups. The above equation gives the ratio of comparing categories with reference category in the intercept.

The MLR supported the statistical significance of the three independent variables in different age groups for five consecutive years (1996-2000). Importantly, V.cholerae O1 infection mostly occurs upto5 years age group, which is highly significant. Infection caused by V.cholerae $\mathrm{O} 139$ showed the significant risk age group was $>40$ years, which is a more interesting trend. V.cholerae non-O1, non-O139 was not significantly associated with any age group, but the highest risk age group was less than 5 years age. The effectiveness of MLR model was supported by multiple indices, including models for overall test of all explanatory variables and significance test of each explanatory variables. In the categorical outcomes, logistic regression is more flexible and less restrictive than discriminant function analysis and log-linear models (Wacholder, 1986; Peng et al., 2002).

Few studies describes the application of Multinomial logistic regression methods. In this finding, we found that MLR is an effective model for profiling greatest risk age groups due to infection caused by different serogroups of V.cholerae. Microbiologists and epidemiologists can employ this model for laboratory data.

\section{Reference}

Banerjee, B. \& Hazra, B. (1974). Geoecology of cholera in West Bengal: A study in medical geography. Jayati Hazra Publishers, Calcutta.

Begg, C. B. \& Gray, R. (1984). Calculation of polychotomous logistic regression parameters using individualized regression. Biometrika, 71, 11-18.

Cabrera, A. F. (1994). Logistic regression analysis in higher education: An 
applied perspective. Higher Education: Handbook of Theory and Research, 10, 225-256.

Cash, R. A., Music, S. I., Libonati, J. P., Snyder, M. J., Wenzel, R. P. \& Hornick, R. B. (1974). Response of man to infection with Vibrio cholerae. I. Clinical, serologic and bacteriologic responses to a known inoculum. The Journal of infectious diseases, 129, 45-52.

Clayton, D. \& Hills, M. (1993). Statistical models in epidemiology. Oxford, England: Oxford University Press.

Codeco, C. T. (2001). Endemic and epidemic dynamics of cholera: the role of the aquatic reservoir. BMC infectious diseases, $1,1$.

Demaris, A. (1992). Logit modeling: Practical application. Newbury Park, CA: Sage.

Epstein, P. R. (1993). Algal blooms in the spread and persistence of cholera. Biosystems, 31, 209-221.

Faruque, S. M., Albert, M. J. \& Mekalanos, J. J. (1998). Epidemiology, genetics and ecology of toxigenic Vibrio cholerae. Microbiology and Molecular Biology Reviews, 62, 1301-1314.

Garg, P., Chakraborty, S., Basu, I., Datta, S., Rajendran, K., Bhattacharya, T., Yamasaki, S., Bhattacharya, S. K., Takeda, Y., Nair, G. B., \& Ramamurthy, T. (2000). Expanding Multiple antibiotic resistance among clinical strains of Vibrio cholerae isolated from 1992-7 in Calcutta, India. Epidemiology and infection, 124, 393-399.

Greenland, S. (1987). Interpretation and choice of effect measures in epidemiologic analysis. American Journal of Epidemiology, $125,761-768$.

Hosmer, D. W. \& Lemeshow, S. (2000). Applied logistic regression $\left(2^{\text {nd }}\right.$ ed.). New York: John Wiley \& Sons Inc.

Islam, M. S., Miah, M. A., Hasan, M. K., Sack, R. B. \& Albert, M. J. (1994). Detection of non-culturable Vibrio cholerae $\mathrm{O} 1$ associated with a cyanobacterium from an aquatic environment in Bangladesh. Transactions of the Royal Society of Tropical Medicine and Hygiene, 88, 298-299.

Janik, J. \& Kravitz, H. M. (1994). Linking work and domestic problems with police suicide. Suicide \& life-threatening behavior, 24, 267-74.
Mahalanabis, D., Molla, A. M., \& Sack, D. A. (1992). Cholera management. In; D Barua, WB Greenough III, eds. Cholera. New York: Plenum Medical Book Company, 129-154.

Menard, S. (2000). Coefficient of determination for multiple logistic regression analysis. The American Statistician, 54, 17-24.

Noah, N. \& Mahony, M. O. (1998). Communicable disease epidemiology and control. John Wiley \& Sons Ltd.

Peng, C. Y., Manz, B. D. \& Keck, J. (2001). Modeling categorical variables by logistic regression. American Journal of Health Behavior, 25, 278-284.

Peng, C. Y., So, T. S., Stage, H. F. K. \& St. John, E. P. (2002). The use and interpretation of logistic regression in higher education journals: 1988-1999. Research in Higher Education, 43, 259-293.

Peng, C. J. \& Nichols, R. N. (2003). Using multinomial logistic models to predict adolescent behavioral risk. Journal of Modern Applied Statistical Methods, 2, 538-1554.

Peterson, B. \& Harrell, F. (1990). Partial proportional odds models for ordinal response variables. Applied Statistics, 39, 205-217.

Rabins, P. K. \& Dickinson, K. P. (1985). Child support and welfare dependence: A multinomial logit analysis. Demography, 22(3), 367-380.

Ramamurthy, T., Garg, S., Sharma, R., Bhattacharya, S. K., Nair, G. B., Shimada, T., Takeda, T., Karasawa, T., Kurazano, H., Pal, A., \& Takeda, Y. (1993). Emergence of novel strain of Vibrio cholerae with epidemic potential in southern and eastern India. Lancet, 703-704.

Sack, D. A., Sack, R. B., Nair, G. B. \& Siddique, A. K. (2004). Cholera. Lancet, 363, 223-233.

Savitz, D. A. (1992). Measurements, estimates, and inferences in reporting epidemiologic study results [editorial]. American Journal of Epidemiology ,135, 223-224.

Wacholder, S. (1986). Binamial regression in GLIM: estimation risk ratios and risk differences. American Journal of Epidemiology, 123, 174-84.

World Health Organization. (1987). Manual for laboratory investigation of acute enteric infection, CDD/33.3. WHO, Geneva, Switzerland. 


\title{
Early Scholars \\ A Comparison of Two Rank Tests for Repeated Measures Designs
}

\author{
Tian Tian Rand Wilcox \\ University of Southern California
}

This article compares the small-sample properties of the Agresti-Pendergast and the ATS rank-based method, as described in Brunner, Domh, and Langer (2002), for comparing $J$ dependent groups. The results indicate that the Type I error of the Agresti-Pendergast method is more conservative when $J=2$, but under most conditions, the ATS method performs best in terms of both Type I errors and power.

Key words: rank tests, repeated measures

\section{Introduction}

The classic rank-based method for comparing $J$ dependent groups is Friedman's test. Consider a random sample of $n$ vectors from some $J$-variate distribution. As is well-known, Friedman's test assigns ranks to the values within each vector and is based on a compound symmetry assumption under the hypothesis of no treatment effect (e.g., Brunner, Domhof, \& Langer, p. 68). That is, the distribution is assumed to be invariant under all permutations, which implies that the variances and covariances are equal. Two attempts at improving test between Friedman's and are based in part by assigning ranks to the pooled data instead (Iman, 1974; Quade, 1979). Subsequently, Agresti and Pendergast (1986) proposed a rank-based test that was found to provide better control over the probability of a Type I error and better power. (For relevant theoretical results, see Kepner \& Robinson, 1988.) Two alternative methods are

Tian Tian is a graduate student in psychology. She specializes in quantitative methods. Rand Wilcox is Professor of Psychology. He is the author of seven books on statistics. His research interests are robust methods. Email him at rwilcox@almaak-01.usc.edu. described by Brunner, Domhof and Langer (2002, section 7.2.2). The first, based on a Waldtype statistic, is known to be rather unsatisfactory when the sample size is relatively small. The second is an ANOVA-type statistic (ATS) that was found to be preferable to the Wald-type statistic, but no results were provided about how it compares to the Agresti-Pendergast technique. The goal in this article is to compare their small-sample properties via simulations. The results indicate that the ATS method performs better than the Agresti-Pendergast technique for most of conditions.

Description of the Methods

$$
\text { Let } \quad \mathbf{X}_{1}, \ldots, \mathbf{X}_{n}, \quad \text { where }
$$
$\mathbf{X}_{k}=\left(X_{k 1}, \ldots, X_{k J}\right)^{\prime}, k=1, \ldots, n$, be a random sample from a $J$-variate distribution with distribution $\mathbf{F}=\left(F_{1}, \ldots, F_{J}\right)^{\prime} . \quad$ In the event sampling is from a discrete distribuition, the $j^{\text {th }}$ marginal distribution is taken to be $F_{j}(x)=\frac{1}{2}\left[F_{j}^{+}(x)+F_{j}^{-}(x)\right]$, where $F_{j}^{+}$ and denote $F_{j}^{-}$are the right continuous and the left continuous version of the distribution function, respectively. That is, $F_{j}^{-}(x)=P\left(X_{j}<x\right)$ and $F_{j}^{+}(x)=P\left(X_{j} \leq x\right)$. The total number of observations is $N=n \times J$ and the null hypothesis is $H_{0}: F_{1}=\ldots=F_{J}$. 
Agresti-Pendergast Test

Let $R_{i j}$ be the midrank of $X_{i j}$ among all $N$ observations. The midrank is determined by means of the so-called counting functions

$$
\begin{gathered}
c^{-}(x)=\left\{\begin{array}{ll}
0, & x \leq 0 \\
1, & x>0
\end{array},\right. \\
c^{+}(x)=\left\{\begin{array}{ll}
0, & x<0 \\
1, & x \geq 0
\end{array},\right.
\end{gathered}
$$

and

$$
c(x)=\frac{1}{2}\left[c^{+}(x)+c^{-}(x)\right] .
$$

The midrank of $X_{i j}$ among the $N$ random variables in the $i^{\text {th }}$ row and the $j^{\text {th }}$ colomn can be written as $R_{i j}=\frac{1}{2}+\sum_{k=1}^{n} \sum_{l=1}^{J} c\left(X_{i j}-X_{k l}\right)$. Let $\overline{\mathbf{R}}_{j}=\frac{1}{n} \sum_{i=1}^{n} R_{i j} \quad$ for $\quad 1 \leq j \leq J$. The estimated covariance matrix $\mathbf{S}$ of the ranks, which has entries $s_{i j}$, is

$$
s_{i j}=\frac{1}{n-J+1} \sum_{i=1}^{n}\left(\mathbf{R}_{i j}-\overline{\mathbf{R}}_{j}\right)\left(\mathbf{R}_{i j}-\overline{\mathbf{R}}_{k}\right) .
$$

Under general conditions, the asymptotic distribution of $\mathbf{R}^{\prime}=\left(\overline{\mathbf{R}}_{1}, \ldots, \overline{\mathbf{R}}_{J}\right)$ is multivariate normal. Let $v=E(\mathbf{R})$ and

$$
\mathbf{C}_{1}=\left(\begin{array}{cccccc}
1 & -1 & 0 & \ldots & 0 & 0 \\
0 & 1 & -1 & \ldots & 0 & 0 \\
. & . & . & . & . & . \\
0 & 0 & 0 & \ldots & 1 & -1
\end{array}\right)
$$

The null hypothesis $H_{0}: F_{1}=\ldots=F_{J}$ implies that $\mathbf{C}_{1} v=0$ and the test statistic is

$$
F=\frac{n}{J-1}\left(\mathbf{C}_{1} \mathbf{R}\right)^{\prime}\left(\mathbf{C}_{1} \mathbf{S C}_{1}{ }^{\prime}\right)^{-1} \mathbf{C}_{1} \mathbf{R}
$$

which has, approximately, an $\mathrm{F}$ distribution with degrees of freedom $J-1$ and $(J-1)(n-1)$ when null hypothesis is true.

ATS

Following the notation in Brunner et al. (2002), let $\mathbf{I}_{J}=\operatorname{diag}\{1, \ldots, 1\} \quad$ be the $J$ dimensional identity matrix, let $\mathbf{J}_{J}$ denote the $J$ by- $J$ matrix of $1 \mathrm{~s}$, and let

$$
\mathbf{C}_{2}=\mathbf{I}_{J}-\frac{1}{J} \mathbf{J}_{J}=\left(\begin{array}{ccc}
1 & \ldots & 0 \\
\ldots & \ldots & \ldots \\
0 & \ldots & 1
\end{array}\right)_{J \rtimes J}-\frac{1}{J}\left(\begin{array}{ccc}
1 & \ldots & 1 \\
\ldots & \ldots & \ldots \\
1 & \ldots & 1
\end{array}\right)_{J \rtimes J} .
$$

The null hypothesis $H_{0}: F_{1}=\ldots=F_{J}$ is equivalent to

$$
H_{0}: \mathbf{C}_{2} \mathbf{F}=\left(\begin{array}{c}
F_{1}-\bar{F} \\
\ldots \\
F_{J}-\bar{F}
\end{array}\right)=\left(\begin{array}{c}
0 \\
\ldots \\
0
\end{array}\right)=0
$$

Let

$$
\mathbf{R}_{i}=\left(R_{i 1}, \ldots, R_{i J}\right), \overline{\mathbf{R}}=\frac{1}{n} \sum_{i=1}^{n} \mathbf{R}_{i},
$$

and let

$$
\hat{\mathbf{V}}_{n}=\frac{1}{N^{2}(n-1)} \sum_{i=1}^{n}\left(\mathbf{R}_{i}-\overline{\mathbf{R}}\right)\left(\mathbf{R}_{i}-\overline{\mathbf{R}}\right)^{\prime}
$$

denote an estimate of the covariance matrix $V_{n}$. For the ATS method, the test statistic is

$$
F_{n}\left(\mathbf{C}_{2}\right)=\frac{n}{N^{2} \operatorname{trace}\left(\mathbf{C}_{2} \hat{\mathbf{V}}_{n}\right)} \sum_{j=1}^{J}\left(\overline{\mathbf{R}}_{. j}-\frac{N+1}{2}\right)^{2} .
$$

Under $H_{0}: \mathbf{C}_{2} \mathbf{F}=0$, the distribution of $F_{n}$ can be approximated by an $\mathrm{F}$ distribution with 
degrees of freedom $\hat{f}=\frac{\left[\operatorname{trace}\left(\mathbf{C}_{2} \hat{\mathbf{V}}_{n}\right)\right]^{2}}{\operatorname{trace}\left(\mathbf{C}_{2} \hat{\mathbf{V}}_{n} \mathbf{C}_{2} \hat{\mathbf{V}}_{n}\right)}$ and $\infty$.

Simulation Results

This section reports simulation results on the small-sample properties of the AgrestiPendergast and ATS methods. The simulations were run with MATLAB 7.1. A correlation matrix with a common correlation $\rho$ was used and observations were generated from $J$-variate normal distribution $(J=2,3,4)$. Because any order preserving transformation of the data does not alter the results, the simulation results apply to a wide range of non-normal distributions. The sample sizes were taken to be $n=10,20$, and 30 and correlations used were $\rho=0,0.2,0.5,0.8$ resulting in 36 conditions. A total of 1,000 replications were used to estimate the Type I error probabilities, denoted by $\hat{\alpha}$, and estimated power, which is denoted by $\hat{\gamma}$. When studying power, the mean of the marginal distribution of the first group was increased from zero to one. The results are given in Table 1.

Table 1. Estimated Type I error probabilities and powers for the ATS and Agresti-Pendergast methods, based on 1,000 replications

\begin{tabular}{|c|c|c|c|c|c|c|c|c|c|c|}
\hline \multirow{2}{*}{$n$} & \multirow{2}{*}{$J$} & & \multicolumn{2}{|c|}{$\rho=0$} & \multicolumn{2}{|c|}{$\rho=0.2$} & \multicolumn{2}{|c|}{$\rho=0.5$} & \multicolumn{2}{|c|}{$\rho=0.8$} \\
\hline & & & $\hat{\alpha}$ & $\hat{\gamma}$ & $\hat{\alpha}$ & $\hat{\gamma}$ & $\hat{\alpha}$ & $\hat{\gamma}$ & $\hat{\alpha}$ & $\hat{\gamma}$ \\
\hline \multirow{6}{*}{10} & \multirow[b]{2}{*}{2} & ATS & 0.095 & 0.595 & 0.085 & 0.688 & 0.081 & 0.843 & 0.082 & 0.989 \\
\hline & & Agresti-Pendergast & 0.064 & 0.495 & 0.050 & 0.593 & 0.050 & 0.775 & 0.053 & 0.975 \\
\hline & \multirow{2}{*}{3} & ATS & 0.062 & 0.585 & 0.069 & 0.669 & 0.065 & 0.853 & 0.062 & 0.998 \\
\hline & & Agresti-Pendergast & 0.092 & 0.581 & 0.080 & 0.641 & 0.084 & 0.813 & 0.074 & 0.994 \\
\hline & \multirow{2}{*}{4} & ATS & 0.072 & 0.579 & 0.065 & 0.646 & 0.066 & 0.849 & 0.047 & 0.996 \\
\hline & & Agresti-Pendergast & 0.111 & 0.611 & 0.116 & 0.671 & 0.107 & 0.814 & 0.107 & 0.988 \\
\hline \multirow{6}{*}{20} & \multirow{2}{*}{2} & ATS & 0.065 & 0.862 & 0.072 & 0.913 & 0.059 & 0.994 & 0.067 & 1 \\
\hline & & Agresti-Pendergast & 0.053 & 0.833 & 0.053 & 0.895 & 0.046 & 0.990 & 0.050 & 1 \\
\hline & \multirow[b]{2}{*}{3} & ATS & 0.058 & 0.885 & 0.052 & 0.949 & 0.063 & 0.997 & 0.066 & 1 \\
\hline & & Agresti-Pendergast & 0.065 & 0.883 & 0.056 & 0.936 & 0.070 & 0.993 & 0.067 & 1 \\
\hline & \multirow{2}{*}{4} & ATS & 0.052 & 0.900 & 0.056 & 0.936 & 0.054 & 0.993 & 0.058 & 1 \\
\hline & & Agresti-Pendergast & 0.070 & 0.897 & 0.088 & 0.921 & 0.072 & 0.992 & 0.072 & 1 \\
\hline \multirow{6}{*}{30} & \multirow{2}{*}{2} & ATS & 0.060 & 0.963 & 0.071 & 0.975 & 0.065 & 1 & 0.050 & 1 \\
\hline & & Agresti-Pendergast & 0.050 & 0.954 & 0.060 & 0.971 & 0.051 & 1 & 0.042 & 1 \\
\hline & \multirow[b]{2}{*}{3} & ATS & 0.058 & 0.984 & 0.063 & 0.995 & 0.049 & 0.999 & 0.060 & 1 \\
\hline & & Agresti-Pendergast & 0.062 & 0.973 & 0.062 & 0.988 & 0.051 & 0.999 & 0.064 & 1 \\
\hline & \multirow{2}{*}{4} & ATS & 0.045 & 0.991 & 0.058 & 0.994 & 0.057 & 1 & 0.043 & 1 \\
\hline & & Agresti-Pendergast & 0.057 & 0.986 & 0.076 & 0.990 & 0.068 & 1 & 0.059 & 1 \\
\hline
\end{tabular}



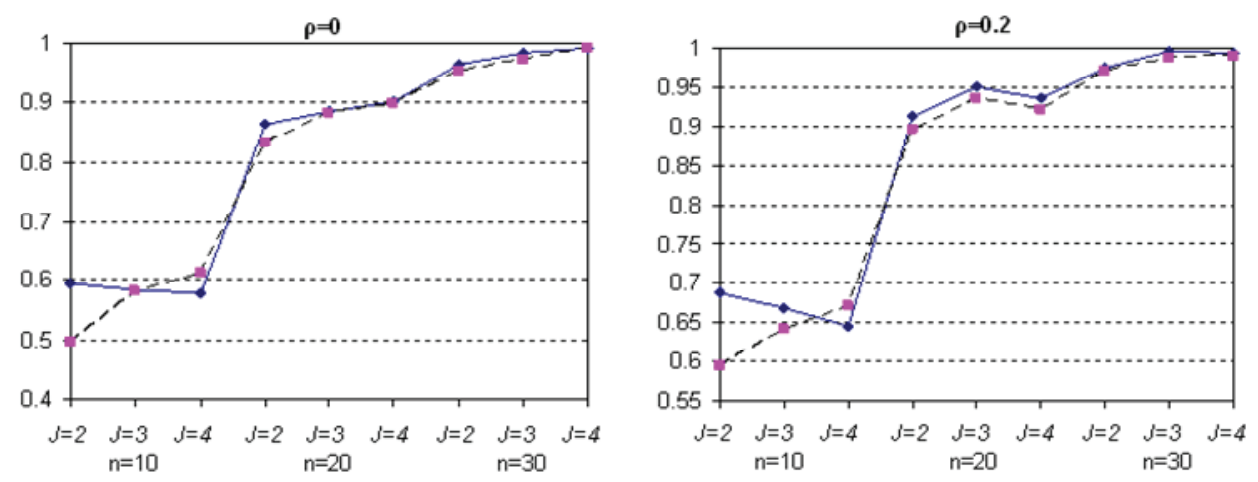

$\longrightarrow-$ ATS $-\rightarrow--$ Agresti-Pendergast
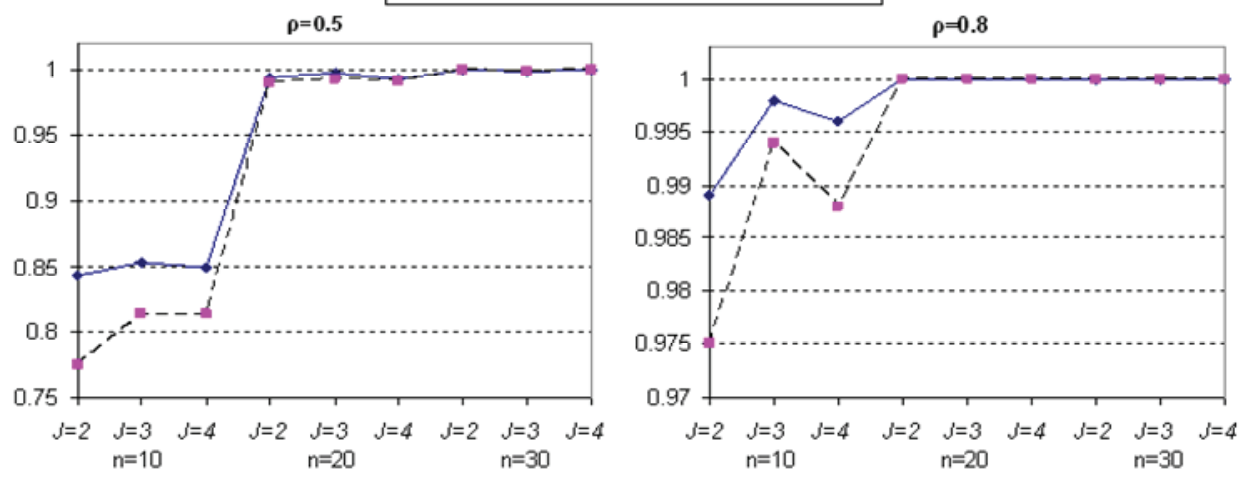

Figure 1 Plots of Power vs. Sample size for ATS test and Agresti-Pendergast test (multinormal)

As can be seen, for $n \geq 20$, the $\hat{\alpha}$ values are reasonably close to the nominal value of 0.05 for both the Agresti-Pendergast and ATS methods. With a fixed $n$ and $\rho, \hat{\alpha}$ decreases with $J$ increasing when using ATS, while $\hat{\alpha}$ increases for the Agresti-Pendergast test. For instance, when $\rho=0.2$ and $n=30$, the $\hat{\alpha}$ values are $0.071(J=2), 0.062(J=3)$, and 0.058 $(J=4)$ for ATS, and for the Agresti-Pendergast test $\hat{\alpha}$ values are $0.060(J=2), 0.062(J=3)$, and $0.076(J=4)$, For $n=10$ and $J=2$, the ATS method can be unsatisfactory in terms of Type I errors, the estimate exceeding .075. Otherwise, ATS is generally preferable to the Agresti-Pendergast test. Also, for $n=10$ and $J>2$, now the Agresti-Pendergast method performs poorly in terms of Type I errors; the ATS method is preferable.
Table 2 gives the basic descriptive statistics of estimated the Type I errors and power for the two methods. As can be seen from the table, the Type I errors for ATS have smaller variances.

Table 2 Descriptive statistics of estimated Type I errors and powers for Agresti-Pendergast test and ATS

\begin{tabular}{|c|c|c|c|c|}
\cline { 2 - 5 } \multicolumn{1}{c|}{} & \multicolumn{2}{c}{ Type I error $\hat{\alpha}$} & \multicolumn{2}{c|}{ Power $\hat{\gamma}$} \\
\cline { 2 - 5 } \multicolumn{1}{c|}{} & mean & STD & Mean & STD \\
\hline ATS & 0.0625 & 0.0112 & 0.9061 & 0.1370 \\
\hline $\begin{array}{c}\text { Agresti- } \\
\text { Pendergast }\end{array}$ & 0.0681 & 0.0192 & 0.8931 & 0.1485 \\
\hline
\end{tabular}



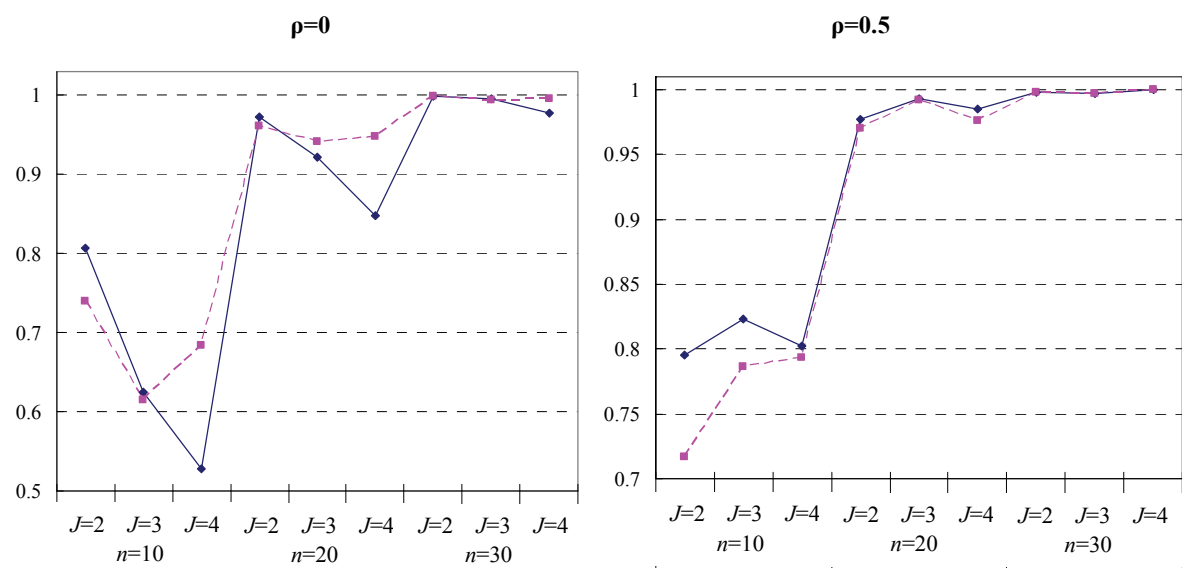

$\longrightarrow$ ATS ---- Agresti-Pendergast

Figure 2 Plots of Power vs. Sample size for Brunner-Puri test and Agresti-Pendergast test (Bin(10, 0.4))

Figure 1 contains the estimated powers for all of the conditions. To make it clear, the four $\rho$ s are listed separately. As indicated, ATS is generally preferable.

The discrete case, where tied values occur, was also considered. For the goal of creating a reasonable number of tied values, the distribution used here is Binomial $(10,0.4)$. Figure 2 gives the plots of power vs. sample size in this case. As can be seen, ATS has higher power than Agresti-Pendergast for $\rho=0.5$. For the independent case, the choice of method is less clear, with the Agresti-Pendergast offering a bit of an advantage in some instances.

\section{Conclusion}

In summary, the simulations show that in many situations, there is little separating ATS and Agresti-Pendergast. However, there are situations where ATS is preferable to AgrestiPendergast in terms of both Type I errors and power. The main exception is the case $J=2$ and $n=10$, where the Agresti-Pendergast performs reasonably well in terms of Type I errors, while ATS does not.

\section{References}

Agresti, A. \& Pendergast, J. (1986). Comparing mean ranks for repeated measures data. Communications in Statistics: Theory and Method, 15, 1417-1433.

Brunner, E., Domhof, S. \& Langer, F., (2002). Nonparametric analysis of longitudinal data in factorial experiments. New York: John Wiley \& Sons.

Iman, R. L. (1974). Use of a t-statistic as an approximation to the exact distribution of the Wilcoxon signed rank statistic. Communications in Statistics, 3, 795 -806.

Kepner, J. L. \& Robinson, D. H. (1988). Nonparametric methods for detecting treatment effects in repeated-measures designs. Journal of the American Statistical Association, 83, 456461.

Quade, D. (1979). Using weighted rankings in the analysis of complete blocks with additive block effects. Journal of the American Statistical Association, 74, 680-683. 


\title{
Algorithms and Code \\ JMASM26: Hettmansperger and Mckean Linear Model Aligned Rank Test for the Single Covariate and One-Way ANCOVA Case (SAS)
}

\author{
Paul A. Nakonezny \\ The University of Texas Southwestern Medical Center \\ Robert D. Shull \\ Formerly of Geisinger Health Plan
}

\begin{abstract}
A SAS program (SAS 9.1.3 release, SAS Institute, Cary, N.C.) is presented to implement the Hettmansperger and McKean (1983) linear model aligned rank test (nonparametric ANCOVA) for the single covariate and one-way ANCOVA case. As part of this program, SAS code is also provided to derive the residuals from the regression of $\mathrm{Y}$ on X (which is step 1 in the Hettmansperger and McKean procedure) using either ordinary least squares regression (proc reg in SAS) or robust regression with MM estimation (proc robustreg in SAS).
\end{abstract}

Key words: Aligned ranks, ANCOVA, SAS, nonparametric

\section{Introduction}

Parametric analysis of covariance (ANCOVA) was introduced by Sir Ronald A. Fisher in 1932. The design goal of ANCOVA is to use the relationship between a dependent variable and covariate to adjust the dependent variable scores in order to reduce unexplained error variance (error variance in the dependent variable is reduced by an amount that can be accounted for by a covariate) and, hence, to provide a more precise estimate of treatment effects and a more powerful test of the hypothesis (Fisher, 1932; Harwell \& Serlin, 1988; Maxwell \& Delaney, 1990).

In order to provide a more sensitive test of the hypothesis, parametric ANCOVA must satisfy a set of underlying statistical assumptions (Elashoff, 1969; Huitema, 1980), which include (a) a linear relationship between the covariate (X) and the dependent variable (Y), (b) covariate independent of treatment, (c) equality of group

Paul A. Nakonezny is Assistant Professor of Biostatistics in the Department of Clinical Sciences, Division of Biostatistics. Email: paul.nakonezny@utsouthwestern.edu. Robert D. Shull is a Quantitative Psychologist who earned a Ph.D. from the Department of Psychology at the University of Oklahoma. conditional variances (homogeneity of variance), (d) equality of group regression slopes (homogeneity of regression slopes), (e) normality of the distribution of Y scores for each $X$ value within each group (conditional normality), and (f) independence of errors. However, in practical research settings, such as field experiments, it is not always possible to satisfy all of these statistical assumptions. If these underlying statistical assumptions are not tenable, then robustness and power of the parametric ANCOVA model could be threatened, and a nonparametric ANCOVA procedure should be considered.

Nonparametric ANCOVA models, which are less restrictive in their statistical assumptions, represent an alternative to the usual parametric ANCOVA. A variety of nonparametric ANCOVA models have been proposed, including those procedures developed by (1) Quade (1967); (2) McSweeney and Porter (1971); (3) Burnett and Barr (1977); (4) Rogosa (1980); (5) Conover and Iman (1982); (6) Hettmansperger and McKean (1983); and (7) Puri-Sen-Harwell-Serlin (1989). The nonparametric ANCOVA models are similar in that each model involves a ranking procedure to transform the original scores. Each model, however, is not similar with respect to robustness and power (Olejnik \& Algina, 1984, 1985; Harwell \& Serlin, 1988; Rheinheimer \& Penfield, 2001). 
Previous Monte Carlo studies that have examined the robustness and power of the nonparametric ANCOVA models have, in general, found that the Hettmansperger and McKean (1983) method (which is an aligned rank test) is robust and powerful when the underlying statistical assumptions of the parametric ANCOVA are not tenable (such as conditional non-normality, unequal regression slopes, and variance heterogeneity-even in the presence of unequal group sample sizes) (Harwell \& Serlin, 1988; Rheinheimer \& Penfield, 2001).

A SAS program (SAS 9.1.3 release, SAS Institute, Cary, N.C.) is presented for implementing the Hettmansperger and McKean (1983) linear model aligned rank test for the single covariate and one-way ANCOVA case. The SAS program is presented in Appendix A.

Hettmansperger and McKean

Nonparametric ANCOVA Method

A brief description of the Hettmansperger and McKean (1983) nonparametric linear model ANCOVA method is presented. For a single covariate and grouping variable, the hypothesis tested based on ranks in the linear model (for the omnibus between-subjects main effect of group) is:

$$
\mathrm{H}_{0}: \rho_{y} D_{1}, \ldots, D_{K-1} \cdot X=0,
$$

where group $\mathrm{K}$ and $\rho_{y} D_{1}, \ldots, D_{K-1} \cdot X$ is the rank correlation between the dependent variable $\mathrm{Y}$ and the K-1 group membership variables $D_{K}$ with the effects of the covariate $\mathrm{X}$ removed. Under this null hypothesis, all groups are assumed to possess identical expected covariateadjusted mean ranks.

Hettmansperger and McKean (1983) proposed an aligned rank test (of the hypothesis specified in Equation 1) that involves the following steps: (a) calculate the least squares residuals from the regression of $\mathrm{Y}$ on $\mathrm{X}$, where $\mathrm{Y}$ is the single dependent variable and $\mathrm{X}$ is the covariate; (b) rank the raw score residuals from low to high (1 to $\mathrm{N})$; (c) weight the ranked residuals $\left(R_{i}^{\prime}\right)$ using $R_{i}^{\prime}=$ $\sqrt{12}\left[R_{i} /(N+1)-0.5\right]$, where $\mathrm{R}_{\mathrm{i}}$ is the ranked raw score residuals, $\mathrm{N}$ is the total number of observations, 12 and 0.5 are constants; and (d) perform a parametric ANOVA (with least squares estimation) on the weighted ranked residuals $\left(R_{i}^{\prime}\right)$, while maintaining the original group membership.

The sum of squares between groups,

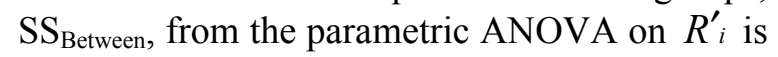
the aligned rank Test Statistic, which is asymptotically distributed as a central $\chi^{2}$ with $\mathrm{K}$ 1 degrees of freedom. Hettmansperger and McKean (1983) originally proposed the use of least squares residuals from the regression of $Y$ on $\mathrm{X}$ (step 1 in their procedure). If outliers are present in the data, however, then the residuals from the regression of $\mathrm{Y}$ on $\mathrm{X}$ (step 1 in the procedure) should be derived using robust regression. The current article, therefore, provides SAS code to derive the residuals using either ordinary least squares regression (proc reg in SAS) or robust regression with MM estimation (proc robustreg in SAS). The reader is referred to Rousseeuw and Leroy (1987) and Yohai (1987) for a discussion of robust estimates for regression.

Although the SAS program in the current article only addressed the single covariate and one-way ANCOVA case (for the one-tailed test of the omnibus between-subjects main effect of group), the Hettmansperger and McKean (1983) aligned rank test can be extended to the multiple covariate case and twoway/higher-order factorial ANCOVA case. A separate aligned rank test, however, is required for each main effect and interaction effect tested. The reader is referred to Adichie (1978), Hettmansperger and McKean (1983), and Hettmansperger (1984) for an expanded discussion of the aligned rank test.

\section{References}

Adichie, J. N. (1978). Rank tests of subhypotheses in the general linear regression. Annals of Statistics, 6, 1021-1026.

Burnett, T. D., \& Barr, D. R. (1977). A nonparametric analogy of analysis of covariance. Educational and Psychological Measurement, 37, 341-348. 
Conover, W. J., \& Iman, R. L. (1982). Analysis of covariance using the rank transformation. Biometrics, 38, 715-724.

Elashoff, J. D. (1969). Analysis of covariance: A delicate instrument. American Educational Research Journal, 6, 383-401.

Fisher, R. A. (1932). Statistical methods for research workers. Edinburgh: Oliver \& Boyd.

Harwell, M. R., \& Serlin, R. C. (1988). An empirical study of a proposed test of nonparametric analysis of covariance. Psychological Bulletin, 104, 267-280.

Harwell, M. R., \& Serlin, R. C. (1989). A nonparametric test statistic for the general linear model. Journal of Educational Statistics, $14,351-371$.

Hettmansperger, T. P., \& McKean J. W. (1983). A geometric interpretation of inferences based on ranks in the linear model. Journal of the American Statistical Association, 78, 885893.

Hettmansperger, T. P. (1984). Statistical inference based on ranks. New York: Wiley.

Huitema, B. E. (1980). The analysis of covariance and alternatives. New York: Wiley.

Maxwell, S. E., \& Delaney, H. D. (1990). Designing experiments and analyzing data. Belmont, CA: Wadsworth Publishing Company.

McSweeney, M., \& Porter, A. C. (1971, April). Small sample properties of nonparametric index of response and rank analysis of covariance. Paper presented at the annual meeting of the American Educational Research Association, New York.
Olejnik, S. F., \& Algina, J. (1984). Parametric ANCOVA and the rank transform ANCOVA when the data are conditionally nonnormal and heteroscedastic. Journal of Educational Statistics, 9, 129-149.

Olejnik, S. F., \& Algina, J. (1985). A review of nonparametric alternatives to analysis of covariance. Evaluation Review, 9, 51-83.

Puri, M. L., \& Sen, P. K. (1969). Analysis of covariance based on general rank scores. Annals of Mathematical Statistics, 40, 610-618.

Quade, D. (1967). Rank analysis of covariance. Journal of the American Statistical Association, 62, 1187-1200.

Rousseeuw, P.J. \& Leroy, A.M. (1987). Robust regression and outlier detection. New York: John Wiley \& Sons, Inc.

Rheinheimer, D. C., \& Penfield, D. A. (2001). The effects of Type I error rates and power on the ANCOVA $F$ test and selected alternatives under nonnormality and variance heterogeneity. The Journal of Experimental Education, 69, 373-391.

Rogosa, D. (1980). Comparing nonparallel regression lines. Psychological Bulletin, 88, 307-321.

SAS. Cary, NC: Statistical Analysis System Institute.

Yohai, V. J. (1987). High Breakdown Point and High Efficiency Robust Estimates for Regression. Annals of Statistics, 15, 642-656. 


\section{Appendix A}

The following SAS program (SAS 9.1.3 release) can be used to perform the Hettmansperger and McKean (1983) aligned rank test for the single covariate and one-way ANCOVA case (one-tailed test of the omnibus between-subjects main effect of group).

/* Step 1: Read internal data into SAS data set hett */

/* Note. You may also read data from an external file into a SAS data set using Proc Import */

data hett;

input subject group X Y;

cards;

$<$ your data $>$

;

/* Step 2: Use Proc Reg to compute and output the least squares residuals from the regression of $\mathrm{Y}$ on $\mathrm{X}$, where $\mathrm{Y}$ is the dependent variable and $\mathrm{X}$ is the single covariate */

proc reg;

model $\mathrm{Y}=\mathrm{X}$;

output out=res $\mathrm{r}=$ residual;

run;

proc print data $=$ res;

/* Alternative Step 2: If outliers are present in the data, then the residuals should be derived using robust regression. Use Proc Robustreg to compute and output the robust residuals, with MM estimation, from the regression of $\mathrm{Y}$ on $\mathrm{X} * /$

proc robustreg method $=\mathrm{mm}$;

model $\mathrm{Y}=\mathrm{X}$;

output out $=$ res $\mathrm{r}=$ residual;

run;

proc print data $=$ res;

/* Step 3: Use Proc Rank to rank the raw score residuals from low to high (1 to $\mathrm{N})$ */

proc rank data $=$ res out $=$ rank; var residual;

/* Step 4: Weight the ranked residuals $\left(R^{\prime}{ }_{i}\right)$ using $R_{i}^{\prime}=\sqrt{12}\left[R_{i} /(N+1)-0.5\right]$, where $\mathrm{R}_{\mathrm{i}}$ is the ranked raw score residuals and $\mathrm{N}$ is the total number of observations $* /$

data weight; set rank nobs $=\mathrm{n}$; rename residual $=$ RankedResiduals; weight $=12 * * .5 *($ residual $/(\mathrm{n}+1)-.5)$;

run;

proc print data=weight; 
/* Step 5: Perform a parametric ANOVA on the weighted ranked residuals $\left(R_{i}^{\prime}\right)$, while maintaining the original group membership */

proc glm; class group;

model weight= group;

ods output modelanova $=$ ma $($ where $=($ hypothesistype $=3)$ );

run;

/* Step 6: One-tailed test of the omnibus between-subjects main effect of group

Dependent $=$ dependent variable $=$ weighted ranked residuals $\left(R_{i}^{\prime}\right)$

Source $=$ omnibus main effect of group

$\mathrm{DF}=\mathrm{K}-1$ degrees of freedom

HettMckeanChisqValue $=$ aligned rank test statistic $\left(\right.$ which is the $\left.\mathrm{SS}_{\text {Between }}\right)$

ProbChisq $=$ p-value of the obtained test statistic

CriticalValueChisq $=$ chi-square critical value with $\mathrm{K}-1$ degrees of freedom, $\alpha=.05 * /$

data ma;

set ma;

rename ss=HettMckeanChisqValue;

ProbChisq=sdf('chisquare',ss,df);

CriticalValueChisq=quantile('chisquare',.95,df);

run;

title 'The Hettmansperger and McKean Nonparametric One-Way ANCOVA (aligned rank test) Procedure';

proc print data=ma noobs; var Dependent Source DF HettMckeanChisqValue ProbChisq CriticalValueChisq;

run; 


\title{
JMASM27: An Algorithm for Implementing Gibbs Sampling for 2PNO IRT Models (Fortran)
}

\author{
Yanyan Sheng Todd C. Headrick \\ Southern Illinois University-Carbondale
}

A Fortran 77 subroutine is provided for implementing the Gibbs sampling procedure to a normal ogive IRT model for binary item response data with the choice of uniform and normal prior distributions for item parameters. The subroutine requires the user to have access to the IMSL library. The source code is available at http://www.siu.edu/ epse1/sheng/Fortran/, along with a stand alone executable file.

Key words: IRT, two-parameter normal ogive model, MCMC, Gibbs sampling, Fortran.

\section{Introduction}

Item response theory (IRT) describes a probabilistic relationship between correct responses on a set of test items and a latent variable, where the influence of items and persons on the responses is modeled by distinct sets of parameters. Common IRT models include the two-parameter normal ogive (2PNO; Lawley, 1943, 1944; Lord, 1952, 1953a, 1953b) model such that the probability of person $i$ obtaining a correct response for item $j$, where $i=1, \ldots, n$ and $j=1, \ldots, k$, is defined as

$$
\begin{aligned}
P\left(y_{i j}=1\right) & =\Phi\left(\alpha_{j} \theta_{i}-\gamma_{j}\right) \\
& =\int_{-\infty}^{\alpha_{j} \theta_{i}-\gamma_{j}} \frac{1}{\sqrt{2 \pi}} e^{\frac{-t^{2}}{2}} d t,
\end{aligned}
$$

Yanyan Sheng is an Assistant Professor of Measurement and Statistics. Her areas of research interest are psychometrics, IRT and Bayesian hierarchical models, and adaptive testing. Email her at ysheng@siu.edu. Todd C. Headrick is Associate Professor of Statistics. Address: Section on Statistics and Measurement, Department of EPSE, 222-J Wham Building, Mail Code 4618, Southern Illinois UniversityCarbondale, IL, 62901. His areas of research interest are statistical computing, nonparametric statistics, and optimization. Email him at headrick@siu.edu. where $\gamma_{j}$ and $\alpha_{j}$ denote item parameters and $\theta_{i}$ denotes the continuous person trait. In the model, items are assumed to vary in terms of location, $\gamma_{j}$, as well as slope, $\alpha_{j}$. Simultaneous estimation of both item and person parameters results in statistical complexities in the estimation task of IRT models, which have made estimation procedures a primary focus of psychometric research over decades (e.g., Birnbaum, 1969; Bock \& Aitkin, 1981; Molenaar, 1995). Recent attention has been focused on Markov chain Monte Carlo (MCMC; e.g., Chib \& Greenberg, 1995) techniques, which have demonstrated to be useful for complex estimation problems in many areas of applied statistics. Albert (1992) was the first to apply an MCMC algorithm, known as Gibbs sampling (Casella \& George, 1992; Gelfand \& Smith, 1990; Geman \& Geman, 1984), to the 2PNO model, where he adopted non-informative priors for item parameters.

As Albert's (1992) focus was on investigating the applicability of Gibbs sampling to IRT, he did not specifically consider the situations where informative priors are adopted for item parameters. However, in some applications, they are more preferred than vague priors. For example, when comparing several candidate models, Bayes factors are commonly adopted in the Bayesian framework, but they are not defined with non-informative priors (Gelman et al., 2003). In this case, the program given by Albert (1992) does not provide a solution. Moreover, given that MCMC is computationally 
demanding in drawing a sufficiently long chain to ensure convergence, a major problem in applied IRT is the accessibility of efficient MCMC programs. Researchers have either used WinBUGS (e.g., Bazán, Branco \& Bolfarinez, 2006; DeMars, 2005) to implement MCMC for IRT models, or coded the sampler in S-Plus (e.g., Patz \& Junker, 1999) or MATLAB (e.g., Albert, 1992). They noted that each execution consumed many hours, and hence was computationally expensive. This fact makes it impractical for users to utilize these programs for various applications of IRT. They further limit researchers in conducting Monte Carlo studies, or developing more complicated IRT models. It is then anticipated that Fortran will provide a better solution, as it is the fastest programming language for numerical computing (Brainerd, 2003).

In view of the above, the purpose of this article is to provide a Fortran subroutine that obtains the posterior estimates (and their associated standard errors) of item and person parameters in the 2PNO IRT model. The subroutine will have the option of specifying non-informative and informative priors for item parameters.

\section{Methodology}

The Gibbs Sampling Procedure

To implement Gibbs sampling to the 2PNO model defined in (1), a latent continuous random variable $Z$ is introduced so that $Z_{i j} \sim$ $N\left(\alpha_{j} \theta_{i}-\gamma_{j}, 1\right)$ (Albert, 1992; Tanner \& Wong, 1987). With prior distributions assumed for $\theta_{i}$ and $\xi_{j}$, where $\xi_{j}=\left(\alpha_{j}, \gamma_{j}\right)^{\prime}$, the joint posterior distribution of $(\boldsymbol{\theta}, \boldsymbol{\xi})$ is hence

$$
p(\boldsymbol{\theta}, \boldsymbol{\xi} \mid \mathbf{y}) \propto f(\mathbf{y} \mid \mathbf{Z}) p(\mathbf{Z} \mid \boldsymbol{\theta}, \boldsymbol{\xi}) p(\boldsymbol{\theta}) p(\boldsymbol{\xi}),
$$

where $f(\mathbf{y} \mid \mathbf{Z})$ is the likelihood function.

With a normal prior for $\theta_{i}$ and noninformative priors for $\alpha_{j}$ and $\gamma_{j}$ so that $\theta_{i} \sim N\left(\mu, \sigma^{2}\right), \alpha_{j}>0$ and $p\left(\gamma_{j}\right) \propto 1$, the full conditional distributions of $Z_{i j}, \theta_{i}$, and $\xi_{j}$ can be derived in closed forms as follows:

$$
\begin{gathered}
Z_{i j} \mid \bullet \sim\left\{\begin{array}{c}
N_{(0, \infty)}\left(\alpha_{j} \theta_{i}-\gamma_{j}, 1\right), \text { if } y_{i j}=1 \\
N_{(-\infty, 0)}\left(\alpha_{j} \theta_{i}-\gamma_{j}, 1\right), \text { if } y_{i j}=0
\end{array}\right. \\
\theta_{i} \mid \bullet \sim N\left(\frac{\sum_{j}\left(Z_{i j}+\gamma_{j}\right) \alpha_{j}+\mu}{\frac{1}{\sigma^{2}}+\sum_{j} \alpha_{j}^{2}}, \frac{1}{\frac{1}{\sigma^{2}}+\sum_{j} \alpha_{j}^{2}}\right) \\
\xi_{j} \mid \bullet \sim N\left(\left(\mathbf{x}^{\prime} \mathbf{x}\right)^{-1} \mathbf{x}^{\prime} \mathbf{Z}_{j},\left(\mathbf{x}^{\prime} \mathbf{x}\right)^{-1}\right) I\left(\alpha_{j}>0\right),
\end{gathered}
$$

where $\mathbf{x}=[\theta,-1]$. Alternatively, informative conjugate priors can be assumed for $\alpha_{j}$ and $\gamma_{j}$ so that $\alpha_{j} \sim N_{(0, \infty)}\left(\mu_{\alpha}, \sigma_{\alpha}^{2}\right), \gamma_{j} \sim N\left(\mu_{\gamma}, \sigma_{\gamma}^{2}\right)$. In this case, the full conditional distribution of $\xi_{j}$ is derived as

$$
\begin{array}{r}
\xi_{j} \mid \bullet \sim N\left(\left(\mathbf{x}^{\prime} \mathbf{x}+\boldsymbol{\Sigma}_{\xi}^{-1}\right)^{-1}\left(\mathbf{x}^{\prime} \mathbf{Z}_{j}+\boldsymbol{\Sigma}_{\xi}^{-1} \boldsymbol{\mu}_{\xi}\right)\right. \\
\left.\left(\mathbf{x}^{\prime} \mathbf{x}+\boldsymbol{\Sigma}_{\xi}^{-1}\right)^{-1}\right) I\left(\alpha_{j}>0\right)
\end{array}
$$

where $\boldsymbol{\mu}_{\xi}=\left(\mu_{\alpha}, \mu_{\gamma}\right)^{\prime}$ and $\boldsymbol{\Sigma}_{\xi}=\left(\begin{array}{cc}\sigma_{\alpha}^{2} & 0 \\ 0 & \sigma_{\gamma}^{2}\end{array}\right)$. Hence, with starting values $\theta^{(0)}$ and $\xi^{(0)}$, observations $\left(Z^{(l)}, \theta^{(l)}, \xi^{(l)}\right)$ can be simulated from the Gibbs sampler by iteratively drawing from their respective full conditional distributions specified in (3), (4) and (5) (or equations 3,4 , and 6$)$. To go from $\left(Z^{(l-1)}, \theta^{(l-1)}\right.$, $\left.\xi^{(l-1)}\right)$ to $\left(Z^{(l)}, \theta^{(l)}, \xi^{(l)}\right)$, it takes three transition steps:
1. Draw $Z^{(l)} \sim p\left(Z \mid y, \theta^{(l-1)}, \xi^{(l-1)}\right)$;
2. Draw $\theta^{(l)} \sim p\left(\theta \mid Z^{(l)}, \xi^{(l-1)}\right)$;
3. Draw $\xi^{(l)} \sim p\left(\xi \mid Z^{(l)}, \theta^{(l)}\right)$.

This iterative procedure produces a sequence of $\left(\theta^{(l)}, \xi^{(l)}\right), l=0, \ldots, L$. To reduce the effect of the starting values, early iterations in the Markov chain are set as burn-ins to be discarded. Samples from the remaining iterations are then used to summarize the posterior density of item 
parameters $\xi$ and ability parameters $\theta$. As with standard Monte Carlo, with large enough samples, the posterior means of $\xi$ and $\theta$ are considered as estimates of the true parameters. However, their standard deviations tend to underestimate the posterior standard deviations, as subsequent samples in Gibbs sampler are autocorrelated (e.g., Albert, 1992; Patz \& Junker, 1999). One approach to calculating them is through batching (Ripley, 1987). That is, with a long chain of samples being separated into contiguous batches of equal length, the posterior mean and standard deviation for each parameter are then estimated to be the sample mean and standard deviation of these batch means respectively. Thus, the standard error of the estimate is a ratio of the standard deviation and the square root of the number of batches.

\section{The Fortran Subroutine}

The subroutine initially sets the starting values for the parameters, so that $\theta_{i}^{(0)}=0$, $\alpha_{i}^{(0)}=2 \quad$ and $\quad \gamma_{i}^{(0)}=-\Phi^{-1}\left(\sum_{i} y_{i j} / n\right) \sqrt{5}$

(Albert, 1992). It then iteratively draws random samples for $Z$ and $\theta$ from their respective full conditional distributions specified in (3) and (4) with $\mu=0$ and $\sigma^{2}=1$. Samples for $\xi_{j}$ are simulated either from (5), where uniform priors are assumed for $\xi_{j}$, or from (6), where normal priors are adopted with $\mu_{\alpha}=\mu_{\gamma}=0$ and $\sigma_{\alpha}^{2}=\sigma_{\gamma}^{2}=1$. The algorithm continues until all the $L$ samples are simulated. It then discards the early burn-in samples, and computes the posterior estimates and standard errors for the model parameters, $\theta, \alpha$ and $\gamma$, using batching.

For example, for a 2000-by-10 (i.e., $n=$ $2,000$ and $k=10)$ dichotomous (0-1) data matrix simulated using the item parameters shown in the first two columns of Table 1, the Gibbs sampler was implemented so that 10,000 samples were simulated with the first 5,000 taken to be burn-in. The remaining 5,000 samples were separated into 5 batches, each with 1,000 samples. Two sets of the posterior means for $\alpha$ and $\gamma$, as well as their standard errors, were obtained assuming the uniform or normal prior distributions described previously, and are displayed in the rest of the table. It is noted that the item parameters were estimated with enough accuracy and the two sets of posterior estimates differ only slightly from each other, signifying that the results are not sensitive to the choice of priors for $\xi$. For this example, each implementation took less than 13 minutes. Although 10,000 iterations are long enough for the Markov chain to reach the stationary distribution, one may easily increase the length of the chain to be as long as 50,000, which takes about 60-90 minutes for each execution.

\section{Conclusion}

This Fortran subroutine leaves it to the user to choose between uniform and normal priors for the item parameters, $\alpha$ and $\gamma$. In addition, the user can change the source code so that the prior distribution for $\theta_{i}$ assumes different location, $\mu$ and scale, $\sigma^{2}$. Similarly, $\mu_{\alpha}, \sigma_{\alpha}^{2}$, and $\mu_{\gamma}, \sigma_{\gamma}^{2}$ can be modified to reflect different prior beliefs on the distributions for the item parameters. It is noted that convergence can be assessed by comparing the marginal posterior mean and standard deviation of each parameter computed for every 1,000 samples after the burn-ins. Similar values provide a rough indication of similar marginal posterior densities, which further indicates possible convergence of the Gibbs sampler (Gelfand, Hills, Racine-Poon \& Smith, 1990; Hoijtink \& Molenaar, 1997). 
Table 1. Posterior estimates and their standard errors for $\alpha$ and $\gamma$ with uniform priors and normal priors.

\begin{tabular}{|c|c|c|c|c|c|}
\hline \multirow{3}{*}{$\begin{array}{c}\text { Parameters } \\
\alpha\end{array}$} & \multirow[b]{3}{*}{$\gamma$} & \multicolumn{3}{|c|}{ Posterior estimates } & \\
\hline & & \multicolumn{2}{|c|}{ uniform priors } & \multicolumn{2}{|c|}{ normal priors } \\
\hline & & $\begin{array}{c}\hat{\alpha} \\
(\mathrm{SE})\end{array}$ & $\begin{array}{c}\hat{\gamma} \\
(\mathrm{SE})\end{array}$ & $\begin{array}{c}\hat{\alpha} \\
(\mathrm{SE})\end{array}$ & $\begin{array}{c}\hat{\gamma} \\
(\mathrm{SE})\end{array}$ \\
\hline \multirow[t]{2}{*}{0.0966} & -0.7997 & 0.1147 & -0.8258 & 0.1120 & -0.8223 \\
\hline & & (.0009) & $(.0010)$ & $(.0012)$ & $(.0006)$ \\
\hline \multirow[t]{2}{*}{0.0971} & -0.5321 & 0.1291 & -0.5441 & 0.1285 & -0.5449 \\
\hline & & (.0003) & $(.0006)$ & $(.0022)$ & $(.0004)$ \\
\hline \multirow[t]{2}{*}{0.4589} & 0.8583 & 0.4412 & 0.9289 & 0.4416 & 0.9278 \\
\hline & & $(.0031)$ & $(.0021)$ & $(.0031)$ & $(.0017)$ \\
\hline \multirow[t]{2}{*}{0.9532} & 0.7237 & 1.1335 & 0.8906 & 1.1124 & 0.8801 \\
\hline & & $(.0088)$ & $(.0032)$ & (.0074) & $(.0041)$ \\
\hline \multirow[t]{2}{*}{0.0771} & -0.8184 & 0.0517 & -0.8063 & 0.0510 & -0.8056 \\
\hline & & $(.0005)$ & $(.0005)$ & $(.0007)$ & $(.0007)$ \\
\hline \multirow[t]{2}{*}{0.4891} & -0.5834 & 0.4761 & -0.6186 & 0.4726 & -0.6188 \\
\hline & & $(.0023)$ & $(.0006)$ & $(.0023)$ & $(.0004)$ \\
\hline \multirow[t]{2}{*}{0.8599} & 0.3629 & 0.7960 & 0.3484 & 0.8015 & 0.3501 \\
\hline & & $(.0028)$ & $(.0013)$ & $(.0036)$ & $(.0022)$ \\
\hline \multirow[t]{2}{*}{0.9427} & -0.9010 & 0.9230 & -0.8661 & 0.9189 & -0.8618 \\
\hline & & $(.0060)$ & $(.0017)$ & $(.0083)$ & $(.0049)$ \\
\hline \multirow[t]{2}{*}{0.2727} & -0.9339 & 0.3981 & -0.9497 & 0.3948 & -0.9465 \\
\hline & & $(.0027)$ & $(.0018)$ & $(.0028)$ & $(.0023)$ \\
\hline \multirow[t]{2}{*}{0.6532} & -0.3978 & 0.6562 & -0.3785 & 0.6544 & -0.3781 \\
\hline & & $(.0016)$ & $(.0008)$ & $(.0015)$ & $(.0009)$ \\
\hline
\end{tabular}

\section{References}

Albert, J. H. (1992). Bayesian estimation of normal ogive item response curves using Gibbs sampling. Journal of Educational Statistics, 17, 251-269.

Bazán, J. L., Branco, M. D, \& Bolfarinez, H. (2006). A Skew Item Response Model. Bayesian Analysis, 1, 861-892.

Birnbaum, A. (1969).Statistical theory for logistic mental test models with prior distribution of ability. Journal of Mathematical Psychology, 6, 258-276.

Bock, R. D., \& Aitkin, M. (1981). Marginal maximum likelihood estimation of item parameters: application of an EM algorithm. Psychometrika, 46, 443-459.

Brainerd, W. (2003). The importance of Fortran in the $21^{\text {st }}$ century. Journal of Modern Statistical Methods, 2, 14-15.
Casella, G., \& George, E. I. (1992). Explaining the Gibbs sampler. The American Statistician, 46(3), 167-174.

Chib, S., \& Greenberg, E. (1995). Understanding the Metropolis-Hastings algorithm. The American Statistician, 49(4), 327-335.

DeMars, C. E. (2005). "Guessing" parameter estimates for multidimensional IRT models. Paper presented at the annual meeting of the American Educational Research Association, Montreal.

Gelfand, A. E., \& Smith, A. F. M. (1990). Sampling-based approaches to calculating marginal densities. Journal of the American Statistical Association, 85, 398-409.

Gelfand, A. E., Hills, S. E., Racine-Poon, A., \& Smith, A. F. M. (1990). Illustration of Bayesian inference in normal data models using Gibbs sampling. Journal of the American Statistical Association, 85, 315-331. 
Geman, S. and D. Geman (1984). Stochastic relaxation, Gibbs distributions, and the Bayesian restoration of images. IEEE Trans. Pattern Analysis and Machine Intelligence 6: 721-741.

Gelman, A., Carlin, J. B., Stern, H. S., \& Rubin, D. B. (2003). Bayesian data analysis. Boca Raton: Chapman \& Hall/CRC.

Hoijtink, H., \& Molenaar, I. W. (1997). A multidimensional item response model: Constrained latent class analysis using posterior predictive checks. Psychometrika, 62, 171-189.

Lawley, D. N. (1943). On problems connected with item selection and test construction. Proceedings of the Royal Society of Edinburgh, 61, 273-287.

Lawley, D. N. (1944). The factorial analysis of multiple item tests. Proceedings of the Royal Society of Edinburgh, 62, 74-82.

Lord, F.M. (1952). A theory of test scores. Psychometric Monograph No. 7.
Lord, F.M. (1953a). An application of confidence intervals and of maximum likelihood to the estimation of an examinee's ability. Psychometrika, 18, 57-75.

Lord, F.M. (1953b). The relation of test score to the trait underlying the test. Educational and Psychological Measurement, 13, 517-548.

Molenaar, I. W. (1995). Estimation of item parameters. In G. H. Fischer \& I. W. Molenaar (Eds.), Rasch models: Foundations, recent developments, and applications (pp. 3951). NewYork: Springer-Verlag.

Patz, R. J., \& Junker, B. W. (1999). A straightforward approach to Markov chain Monte Carlo methods for item response models. Journal of Educational and Behavioral Statistics, 24, 146-178.

Ripley, B. D. (1987). Stochastic simulation. New York: Wiley.

Tanner, M. A., and W. H. Wong (1987). The calculation of posterior distribution by data augmentation (with discussion). Journal of the American Statistical Association, 82, 528-550.

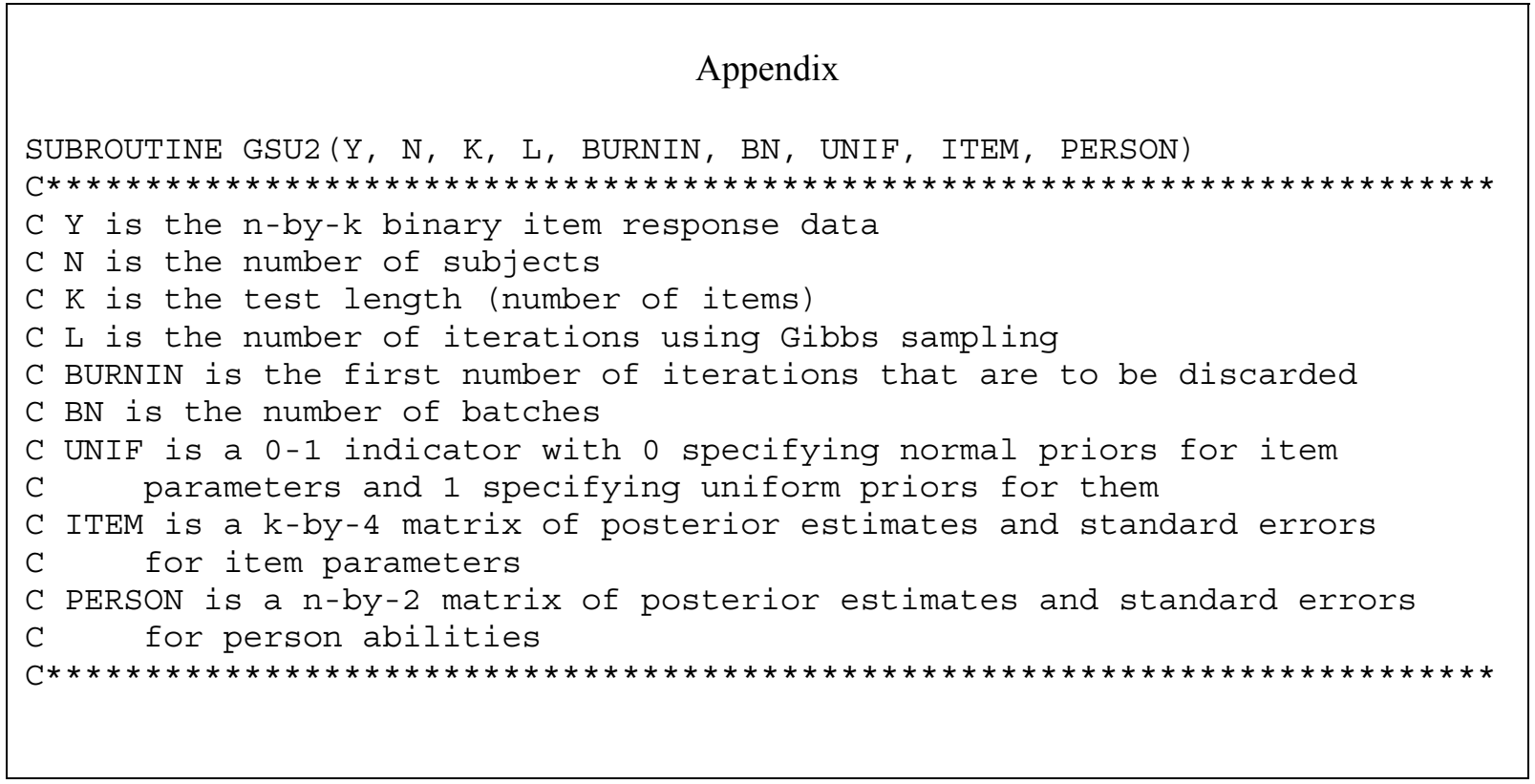




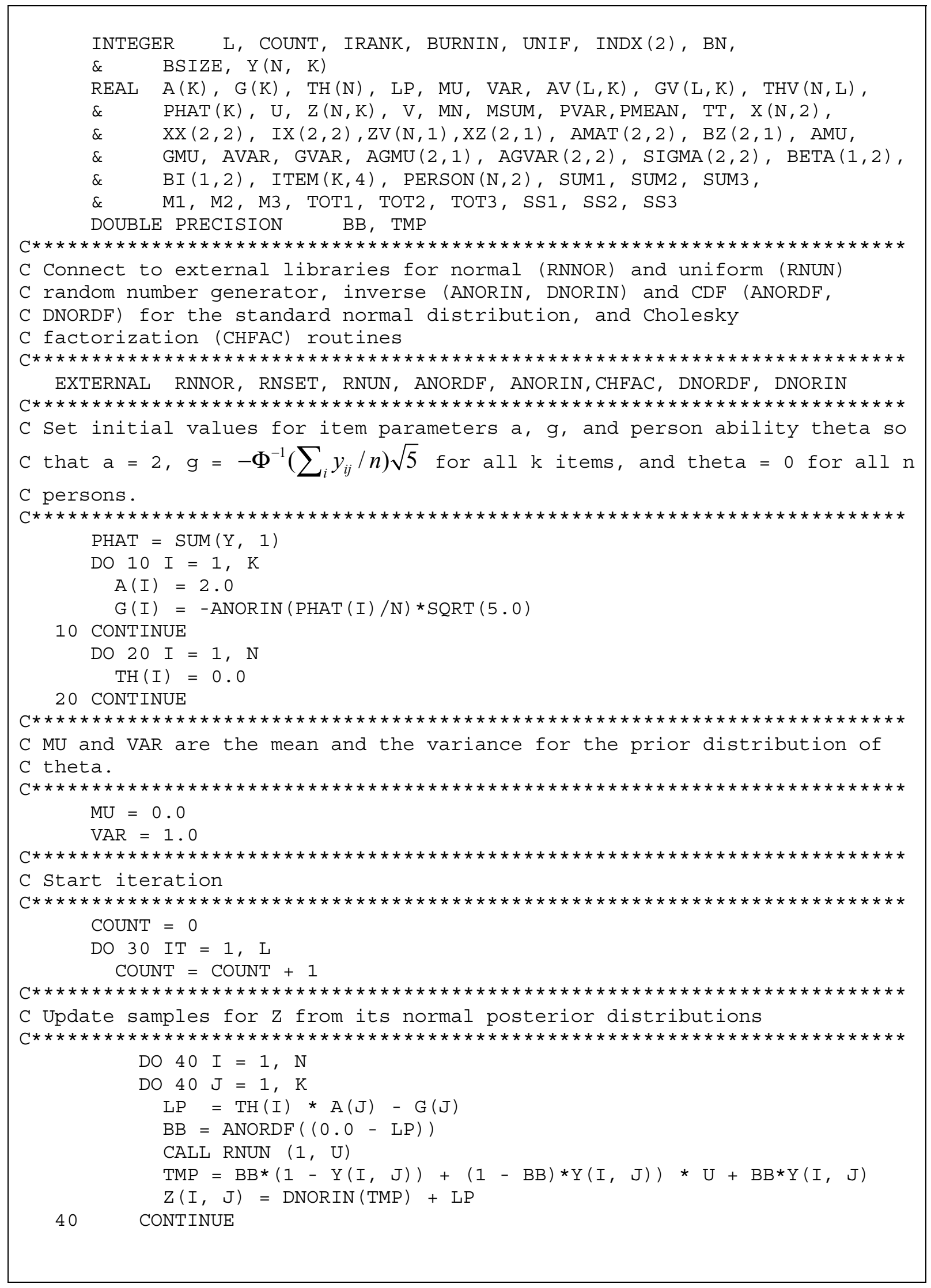




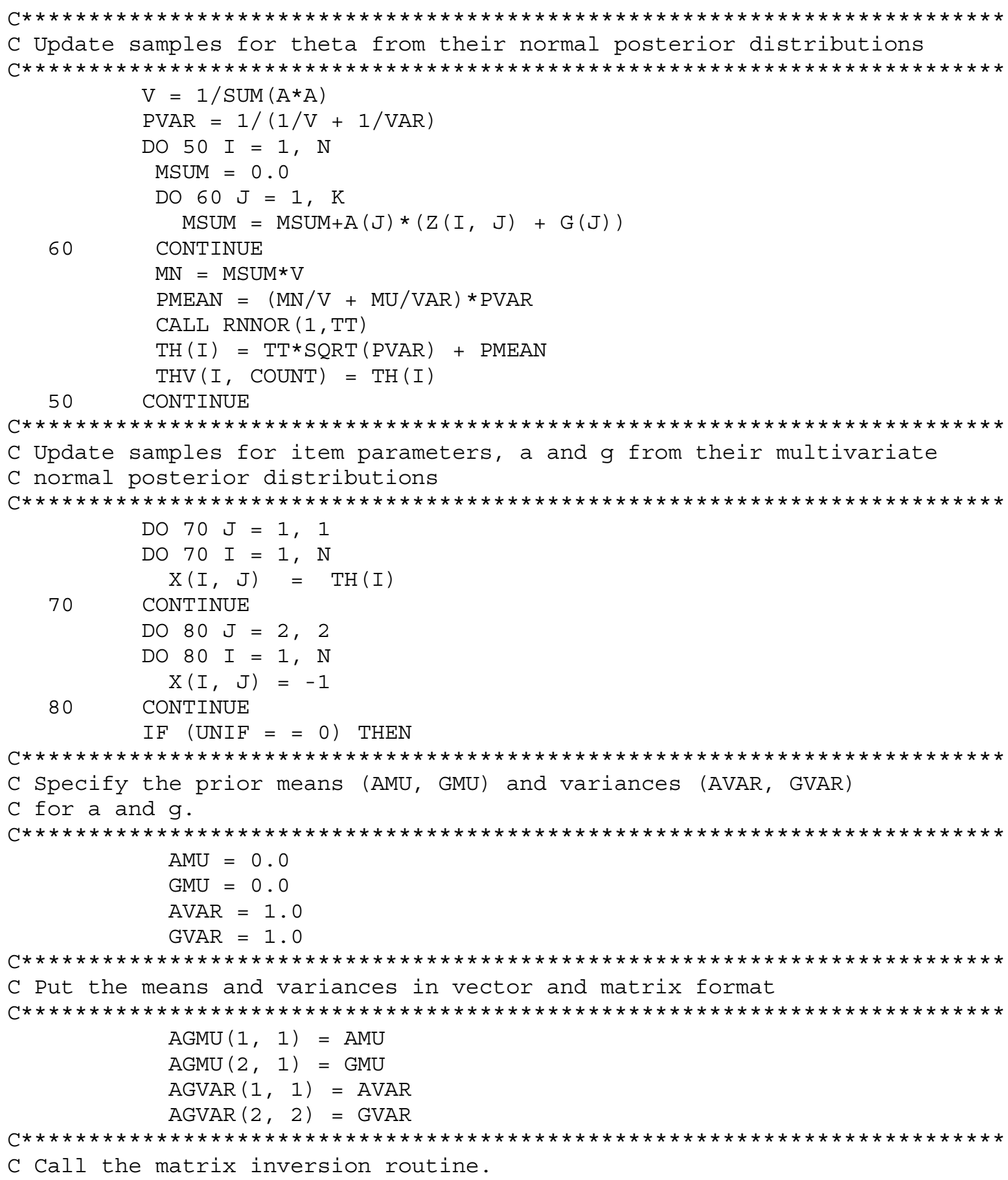


C Invert matrix AGVAR with the inverse stored in SIGMA

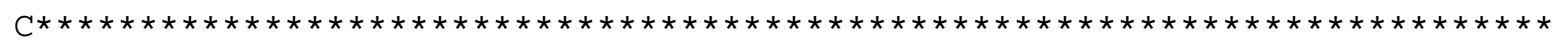

CALL MIGS (AGVAR, 2, SIGMA, INDX)

$\mathrm{XX}=$ MATMUL $($ TRANSPOSE $(\mathrm{X}), \mathrm{X})+$ SIGMA

ELSE IF (UNIF = = 1) THEN

$\mathrm{XX}=\operatorname{MATMUL}($ TRANSPOSE $(\mathrm{X}), \mathrm{X})$ END IF

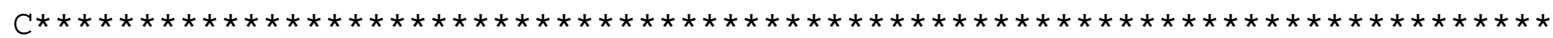

C Call the matrix inversion routine.

C Invert matrix XX with the inverse stored in IX

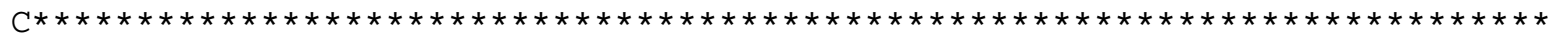
CALL MIGS (XX, 2, IX, INDX)

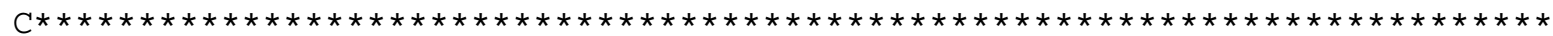

C Call the Cholesky factorization routine. Compute the Cholesky

$C$ factorization of the symmetric definite matrix IX and store the

C result in AMAT

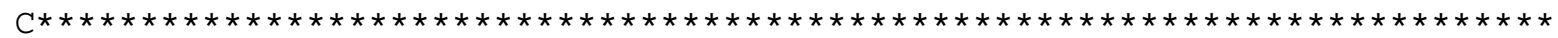

CALL CHFAC (2, IX, 2, 0.00001, IRANK, AMAT, 2)

$\mathrm{DO} 90 \mathrm{~J}=1, \mathrm{~K}$

DO $100 \mathrm{I}=1, \mathrm{~N}$

$\mathrm{ZV}(I, 1)=\mathrm{Z}(\mathrm{I}, \mathrm{J})$

100

CONTINUE

IF $($ UNIF $=0$ ) THEN

$\mathrm{XZ}=\operatorname{MATMUL}(\mathrm{SIGMA}, \mathrm{AGMU})+\operatorname{MATMUL}(\mathrm{TRANSPOSE}(\mathrm{X}), \mathrm{ZV})$

ELSE IF (UNIF = = 1) THEN

$\mathrm{XZ}=\operatorname{MATMUL}($ TRANSPOSE $(\mathrm{X}), \mathrm{ZV})$

END IF

$\mathrm{BZ}=\operatorname{MATMUL}(\mathrm{IX}, \mathrm{XZ})$

$A(J)=0$

DO WHILE (A (J) .LE.0)

CALL RNNOR (2, BI)

$\mathrm{BETA}=\mathrm{MATMUL}(\mathrm{BI}, \mathrm{AMAT})+\mathrm{TRANSPOSE}(\mathrm{BZ})$;

$A(J)=\operatorname{BETA}(1,1)$

$\mathrm{G}(\mathrm{J})=\operatorname{BETA}(1,2)$

END DO

$\mathrm{AV}(\mathrm{COUNT}, \mathrm{J})=\mathrm{A}(\mathrm{J})$

$\mathrm{GV}(\mathrm{COUNT}, \mathrm{J})=\mathrm{G}(\mathrm{J})$

90 CONTINUE

30 CONTINUE

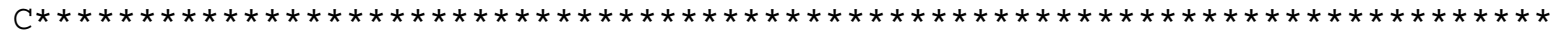

C Calculate the posterior means and SEs for a, 9 and theta and store them

$\mathrm{C}$ in ITEM and PERSON

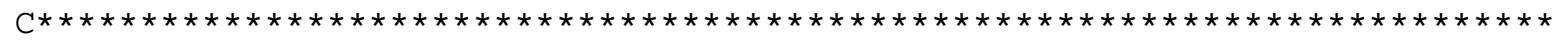

BSIZE $=(\mathrm{L}-\mathrm{BURNIN}) / \mathrm{BN}$

$\mathrm{DO} 110 \mathrm{~J}=1, \mathrm{~K}$

COUNT $=$ BURNIN

TOT1 $=0.0$

TOT2 $=0.0$

$\mathrm{SS} 1=0.0$

$\mathrm{SS} 2=0.0$

DO $120 \mathrm{M}=1, \mathrm{BN}$

SUM1 $=0.0$

SUM2 $=0.0$

DO $130 \mathrm{I}=1, \mathrm{BSIZE}$

COUNT $=$ COUNT +1

SUM1 $=$ SUM1 $+A V(C O U N T, J)$

SUM2 $=$ SUM2 + GV $($ COUNT, J $)$ 


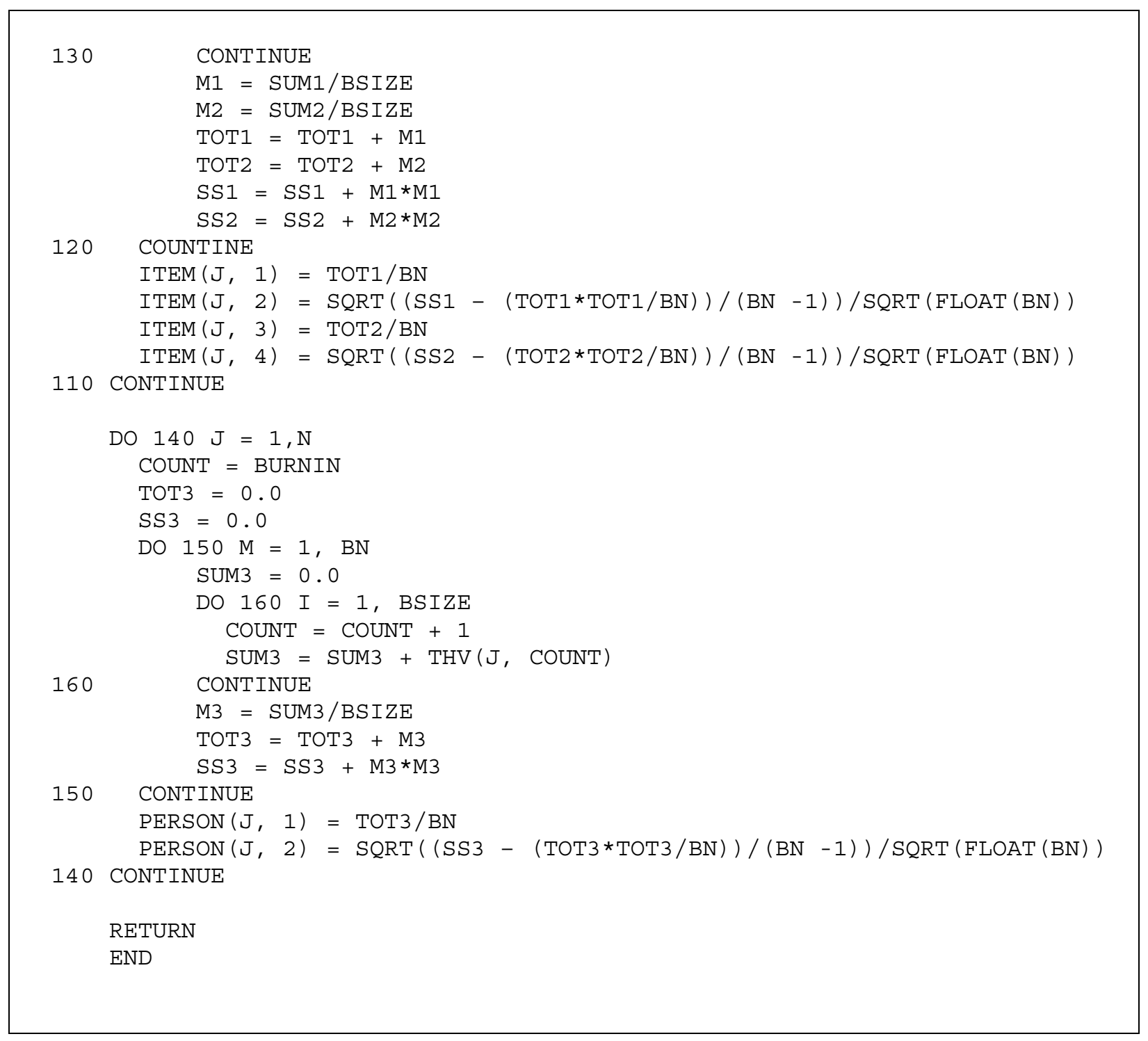




\title{
Translations, Ephemerals, \& Biographies Mathematics in Volume I of Scripta Universitatis
}

\author{
Shlomo S. Sawilowsky \\ Wayne State University
}

Immanuel Velikovsky's journal, Scripta Universitatis, edited by Albert Einstein and first published in 1923, played a significant role in the establishment of the library, and hence, Hebrew University in Jerusalem. The inaugural issue contained an article by the French mathematician Jacques Hadamard. Excerpts from Velikovsky's diary pertaining to the rationale for the creation of the journal, and the interest in Jewish scholars such as Hadamard, are translated here.

Key words: Scripta Universitatis, Velikovsky, Hadamard, Hebrew University

\section{Introduction}

Immanuel Velikovsky (June 10, 1895 November 17, 1979) studied medicine intermittently from 1913 through 1918 at various universities (Montpelier, Edinburgh, \& Kharkov), eventually receiving the M. D. in 1921 from the University of Moscow. He was the author of Worlds in Collision (1950), Ages in Chaos (1952), Earth in Upheaval (1956), Oedipus and Akhnaton (1960), Peoples of the Sea (1977), Ramses II and His Times (1978), Mankind in Amnesia (1982, posthumously by his wife, Elisheva Kramer Velikovsky), and Stargazers and Gravediggers (1983, also posthumously).

While doing post-doctoral work at Charité and the Kaiser Wilhelm Academie in Berlin in 1922 - 1923, he founded the journal Scripta Universitatis. This scholarly work comprised of two parts: (1) Mathematics and Physics, and (2) Judaica and Orientalia. It has been considered the impetus leading to the establishment of Hebrew University in Jerusalem. Jewish authors of note from around the world contributed articles in their native tongue, which were published in one side of the

Shlomo S. Sawilowsky is Professor and Program Coordinator of Educational Evaluation and Research, College of Education. Email him atshlomo@wayne.edu.
Journal, while the other side of the journal contained a Hebrew translation of their work. A scan of the Hebrew cover of the first issue is presented in Figure 1, and the English cover of the same issue in Figure 2.

Velikovsky appointed Albert Einstein (March 14, 1879 - April 18, 1955) to serve as Editor. Einstein also contributed an article, with co-author Jacob Gommer from the University of Berlin, titled "Beweis der Nichtexistenz eines überall regulären zentrisch symmetrischen Feldes nach der Feld-Theorie von Th. Kaluza." Velikovsky relied on Einstein to recruit other notable Jewish scholars to submit to the journal.

Unfortunately, the two of them later parted ways. On July 5, 1946, Velikovsky met with Einstein in Princeton to discuss the extraterrestrial role of Venus in Earth's catastrophic planetary development that would become the premise of his Worlds in Collision (1950). Einstein found the theory to be preposterous, canceled further meetings with Velikovsky, and written communication between them was subsequently strained.

His elder daughter, Shulamit Velikovsky Kogan (b. 1925), gifted a copy of Scripta to a cataloger of her father's archives. She also included typeset excerpts from Velikovsky's letters written in the 1920s to his father (Figure 3 ) who lived in Israel. The excerpts are in diary format and pertain to the development of Scripta, written while Velikovsky was in Berlin and Leipzig. Autobiographical information in 
English pertaining to Scripta may also be found in Velikovsky (1978).

Among these excerpts, material regarding Albert Einstein and the mathematician Jacques Salomon Hadamard (December 8, 1865 - October 17, 1963) is translated below from Hebrew to (flowing American) English. A translation of Hadamard's contributed article in Volume 1 of Scripta will appear in the next issue of the Journal of Modern Applied Statistical methods.

\section{References}

Velikovsky, I. (1978). Genesis of the first Jerusalem 'Scripta'. Jewish Family Quarterly, 26, 15-19.

\section{August 20, 1922}

Yesterday I visited Prof. Einstein, the purpose of which was to invite him to be the Editor of the [Physics and] Mathematics section [of Scripta Universitatis]. We spent a long time in conversation. He accepted upon himself to write to two important Jewish scholars, both close personal friends, who had yet to join us in this endeavor. They are the greatest mathematician in France, Hadamard, and the greatest biologist in America, Jacques Loeb. Because Einstein is now the Editor, I will bring to him, in the coming days, the hand written manuscripts in Mathematics [and Physics] to determine which to accept for publication. Afterward, I will invite editors for other sections. For Biology, I would like Wasserman.

December 17, 1922

In a few weeks, we will send as many as 500 circulars to a large number of scholars. I hope we will be able to thereby increase the number of participants, specifically Jewish scholars with international reputations, such as Volterra and Enriques (Italian mathematicians), Hadamard (French mathematician), Bergson (French philosopher), L. R. Flexner and J. Loeb (American biologists), and A. Michelson (American physicist). If these seven scholars will agree participate, it will become apparent to the Jewish world, and throughout the world for that matter, that the Jewish people have made important contributions to science, despite the fact that we are scattered all over the world.

\section{January 2, 1923}

There was a very important event that happened in our work this week. We were joined by the French scholar Hadamard, a famous mathematician. Einstein and I wrote him a letter of invitation to submit. In his answer he agreed, and attached a hand-written manuscript. Based on this, I will now have a much easier time to get other scholars in France to participate. Everyone knows that Einstein contributes to projects that promote Zionism, but when Hadamard joined us, this is something entirely new. Thus, getting such scholars to participate in the [Hebrew University] Jerusalem project was a worthwhile effort.*

[Footnote material added by Velikovsky much later] *Indeed, from that time, Hadamard became an active participant in Zionist activities, giving assistance to different projects in Israel. Many scholars who later became members of the board of directors of the Jerusalemite [Hebrew] University were initially attracted by their participation with Scripta Universitatis. 


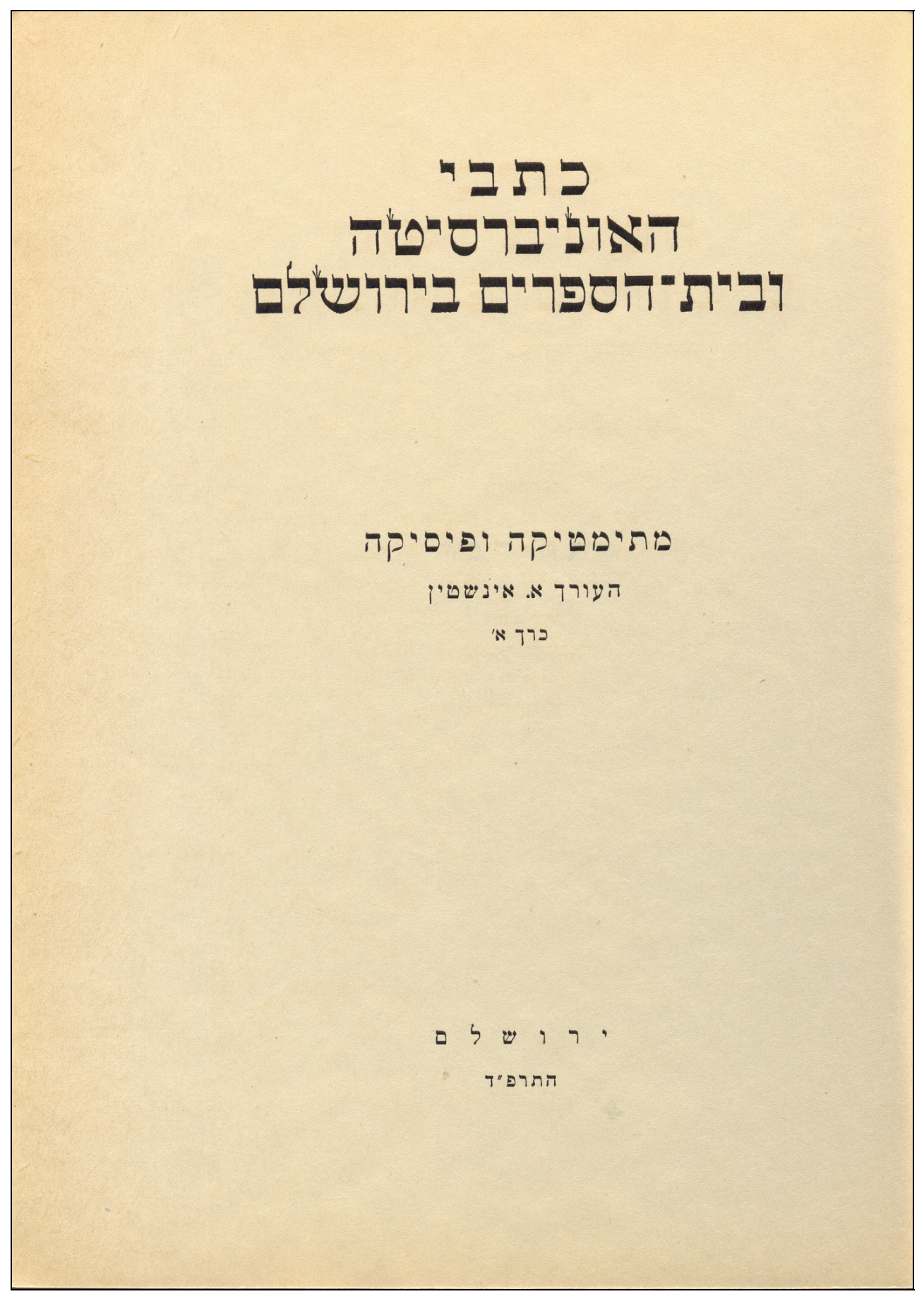

Figure 1. Cover of the Hebrew side of Volume I of Scripta Universitatis, indicating the Editor as Albert Einstein, and the journal was published in Jerusalem in the Jewish year 5684 (1923-1924). From the private collection of Shlomo S. Sawilowsky. 


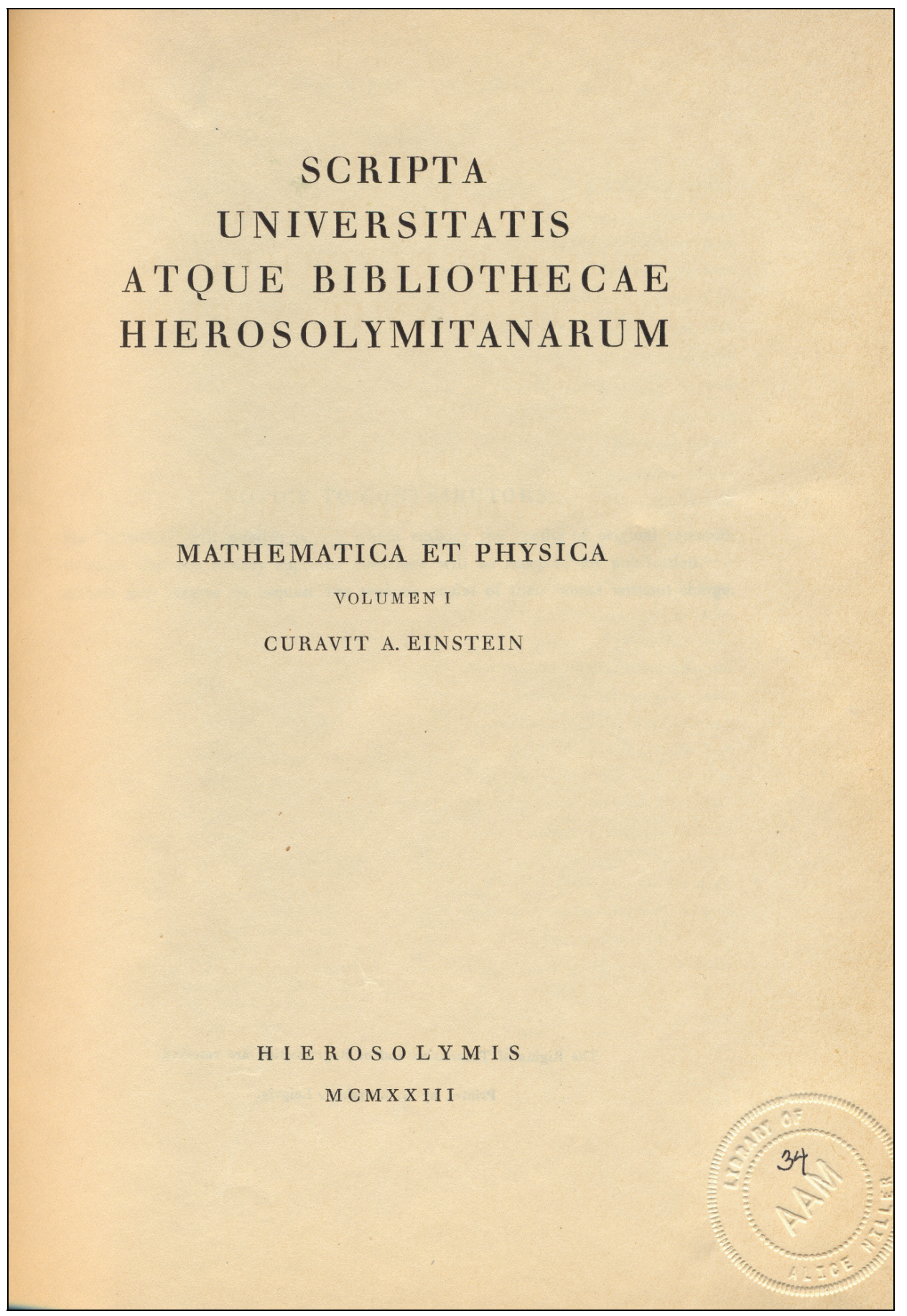

Figure 2. Cover of the English side of Volume I of Scripta Universitatis.

From the private collection of Shlomo S. Sawilowsky. 


\begin{tabular}{|l|l|}
\hline inchdes exeerps & A rave took \\
from I. Velikorsky's & -to a rare person, \\
letters to his father & in thanks and \\
while eding & appreciation for \\
Seripta Universitatis & cataloguing my \\
& father's archive. \\
\hline
\end{tabular}

Figure 3. Two hand written notes with signature from Shulamit Velikovsky Kogan. From the private collection of Shlomo S. Sawilowsky. 


\section{Two Years in the Making...}

\section{Intel ${ }^{\oplus}$ Visual Fortran 8.0}

The next generation of Visual Fortran is here! Intel Visual Fortran 8.0 was developed jointly by Intel and the former DEC/Compaq Fortran engineering team.

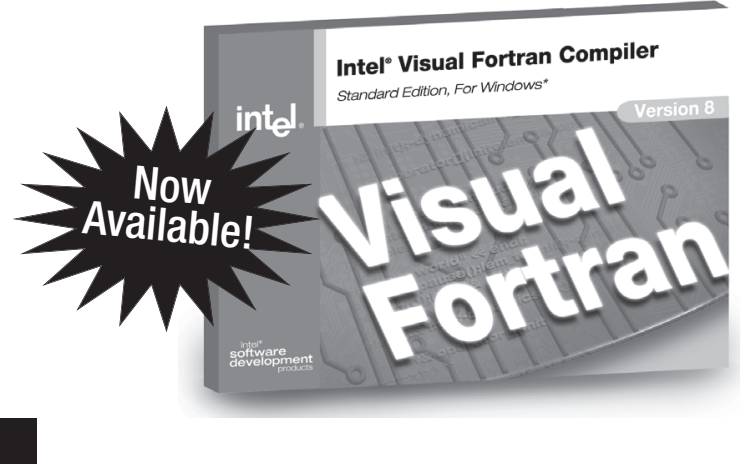

\section{Visual Fortran Timeline}

1997 DEC releases

Digital Visual Fortran 5.0

1998 Compaq acquires DEC and releases DVF 6.0

1999 Compaq ships CVF 6.1

2001 Compaq ships CVF 6.6

2001 Intel acquires CVF engineering team

2003 Intel releases

Intel Visual Fortran 8.0

Intel Visual Fortran $\mathbf{8 . 0}$

- CVF front-end + Intel back-end

- Better performance

- OpenMP Support

- Real*16

\section{Performance}

Outstanding performance on Intel architecture including Intel ${ }^{\circledR}$ Pentium $^{\circledR} 4$, Intel ${ }^{\circledR}$ Xeon $^{\mathrm{TM}}$ and Intel Itanium ${ }^{\circledR} 2$ processors, as well as support for Hyper-Threading Technology.

\section{Compatibility}

- Plugs into Microsoft Visual Studio* .NET

- Microsoft PowerStation4 language and library support

- Strong compatibility with Compaq* Visual Fortran

\section{Support}

1 year of free product upgrades and Intel Premier Support

"The Intel Fortran Compiler 7.0 was first-rate, and Intel Visual Fortran 8.0 is even better. Intel has made a giant leap forward in combining the best features of Compaq Visual Fortran and Intel Fortran. This compiler... continues to be a 'must-have' tool for any Twenty-First Century Fortran migration or software development project."

-Dr. Robert R. Trippi
Professor Computational Finance
University of California, San Diego

FREE trials available at: programmersparadise.com/intel

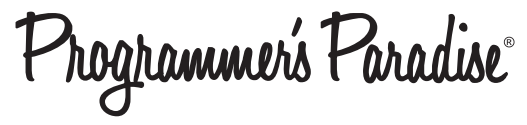

To order or request additional information call: 800-423-9990

Email: intel@programmers.com 
NCSS

329 North 1000 East

Kaysville, Utah 84037

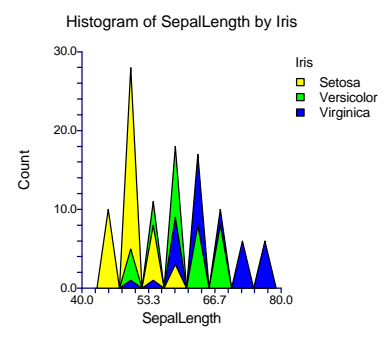

\section{Announcing NCSS 2004 Seventeen New Procedures}

NCSS 2004 is a new edition of our popular statistical NCSS package that adds seventeen new procedures.

\section{New Procedures}

Two Independent Proportions

Two Correlated Proportions

One-Sample Binary Diagnostic Tests

Two-Sample Binary Diagnostic Tests

Paired-Sample Binary Diagnostic Tests

Cluster Sample Binary Diagnostic Tests

Meta-Analysis of Proportions

Meta-Analysis of Correlated Proportions

Meta-Analysis of Means

Meta-Analysis of Hazard Ratios

Curve Fitting

Tolerance Intervals

Comparative Histograms

ROC Curves

Elapsed Time Calculator

T-Test from Means and SD's

Hybrid Appraisal (Feedback) Model

\section{Documentation}

The printed, 330-page manual, called

NCSS User's Guide $V$, is available for

$\$ 29.95$. An electronic (pdf) version of

the manual is included on the distribution

$\mathrm{CD}$ and in the Help system.

\section{Two Proportions}

Several new exact and asymptotic techniques were added for hypothesis testing (null, noninferiority, equivalence) and calculating confidence intervals for the difference, ratio, and odds ratio. Designs may be independent or paired. Methods include: Farrington \& Manning, Gart \& Nam, Conditional \& Unconditional Exact, Wilson's Score, Miettinen \& Nurminen, and Chen.
Meta-Analysis

Procedures for combining studies measuring paired proportions, means, independent proportions, and hazard ratios are available. Plots include the forest plot, radial plot, and L'Abbe plot. Both fixed and random effects models are available for combining the results.

\section{Curve Fitting}

This procedure combines several of our curve fitting programs into one module. It adds many new models such as Michaelis-Menten. It analyzes curves from several groups. It compares fitted models across groups using computerintensive randomization tests. It computes bootstrap confidence intervals.

\section{Tolerance Intervals}

This procedure calculates one and two sided tolerance intervals using both distribution-free (nonparametric) methods and normal distribution (parametric) methods. Tolerance intervals are bounds between which a given percentage of a population falls.

\section{Comparative Histogram}

This procedure displays a comparative histogram created by interspersing or overlaying the individual histograms of two or more groups or variables. This allows the direct comparison of the distributions of several groups.

\section{Random Number Generator} Matsumoto's Mersenne Twister random number generator (cycle length > $10 * * 6000)$ has been implemented.

\section{Binary Diagnostic Tests}

Four new procedures provide the specialized analysis necessary for diagnostic testing with binary outcome data. These provide appropriate specificity and sensitivity output. Four experimental designs can be analyzed including independent or paired groups, comparison with a gold standard, and cluster randomized.

\section{ROC Curves}

This procedure generates both binormal and empirical (nonparametric) ROC curves. It computes comparative measures such as the whole, and partial, area under the ROC curve. It provides statistical tests comparing the AUC's and partial AUC's for paired and independent sample designs.

\section{Hybrid (Feedback) Model}

This new edition of our hybrid appraisal model fitting program includes several new optimization methods for calibrating parameters including a new genetic algorithm. Model specification is easier. Binary variables are automatically generated from class variables.

\section{Statistical Innovations Products}

Through a special arrangement with

Statistical Innovations (S.I.), NCSS

customers will receive $\$ 100$ discounts on:

Latent GOLD ${ }^{\circledR}$ - latent class modeling

SI-CHAID $®$ - segmentation trees

GOLDMineR ${ }^{\circledR}$ - ordinal regression

For demos and other info visit: www.statisticalinnovations.com 
Please rush me the following products:

Qty

NCSS 2004 CD upgrade from NCSS 2001, \$149.95

$\$$

NCSS 2004 User’s Guide V, \$29.95

$\$$

NCSS 2004 CD, upgrade from earlier versions, $\$ 249.95 \ldots \ldots \ldots . . \$$

NCSS 2004 Deluxe (CD and Printed Manuals), \$599.95 ........... \$

PASS 2002 Deluxe, $\$ 499.95$

$\$$

Latent Gold ${ }^{\circledR}$ from S.I., $\$ 995$ - $\$ 100$ NCSS Discount $=\$ 895 \ldots . . . \$$

GoldMineR ${ }^{\circledR}$ from S.I., \$695 - \$100 NCSS Discount $=\$ 595 \ldots . . \$$

CHAID ${ }^{\circledR}$ Plus from S.I., $\$ 695$ - $\$ 100$ NCSS Discount $=\$ 595 \ldots . \$$

Approximate shipping--depends on which manuals are ordered (U.S: \$10 ground, \$18 2-day, or \$33 overnight) (Canada \$24) (All other countries \$10) (Add \$5 U.S. or \$40 International for any S.I. product) ........ \$_

Total...........

CALL: (800) 898-6109 FAX: (801) 546-3907

ONLINE: Www.ncss.com

MAIL: NCSS, 329 North 1000 East, Kaysville, UT 84037

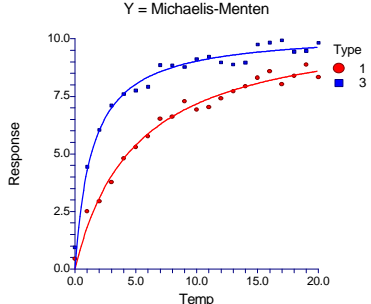

ROC Curve of Fever

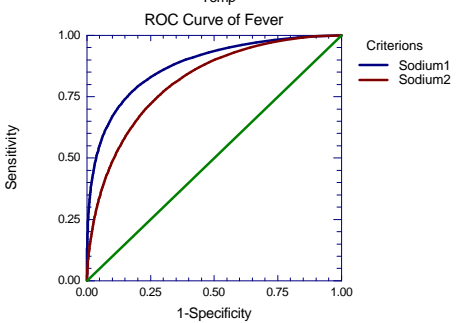

Histogram of SepalLength by lirs

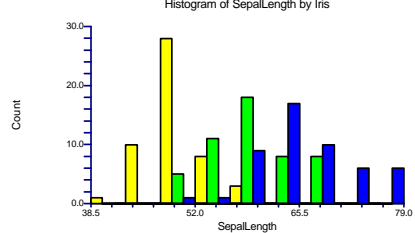

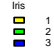

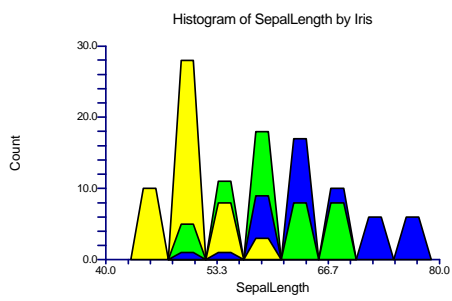

My Payment Option:

Check enclosed

Please charge my: _ VISA _ MasterCard

Amex Purchase order attached

Card Number Exp

Signature

Telephone:

Email:

Ship to:

NAME

ADDRESS

ADDRESS

ADDRESS

CITY STATE

ZIP/POSTAL CODE COUNTRY

\section{Statistical and Graphics Procedures Available in NCSS 2004}

Analysis of Variance / T-Tests Analysis of Covariance

Analysis of Variance

Barlett Variance Test

Crossover Design Analysis

Factorial Design Analysis

Friedman Test

Geiser-Greenhouse Correction

General Linear Models

Mann-Whitney Test

MANOVA

Multiple Comparison Tests

One-Way ANOVA

Paired T-Tests

Power Calculations

Repeated Measures ANOVA

T-Tests - One or Two Groups

T-Tests - From Means \& SD's

Wilcoxon Test

Time Series Analysis

ARIMA / Box - Jenkins

Decomposition

Exponential Smoothing

Harmonic Analysis

Holt - Winters

Seasonal Analysis

Spectral Analysis

Trend Analysis

*New Edition in 2004
Regression / Correlation Survival / Reliability All-Possible Search

Experimental Designs

Balanced Inc. Block

Box-Behnken

Central Composite

D-Optimal Designs

Fractional Factoria

Latin Squares

Placket-Burman

Response Surface

Screening

Taguchi Canonical Correlation Correlation Matrices Cox Regression

Kendall's Tau Correlation Linear Regression Logistic Regression Multiple Regression Nonlinear Regression PC Regression Poisson Regression Response-Surface Ridge Regression Robust Regression Stepwise Regression Spearman Correlation Variable Selection

Accelerated Life Tests

Cox Regression

Cumulative Incidence Exponential Fitting

Extreme-Value Fitting

Hazard Rates

Kaplan-Meier Curves

Life-Table Analysis

Lognormal Fitting

Log-Rank Tests

Probit Analysis

Proportional-Hazards

Reliability Analysis

Survival Distributions

Time Calculator*

Weibull Analysis

Quality Control

Xbar-R Chart

$C, P, N P, U$ Charts

Capability Analysis

Cusum, EWMA Chart

Individuals Chart

Moving Average Chart

Pareto Chart

R \& R Studies

Cluster Analysis

Factor Analysis

Hotelling's T-Squared

Item Analysis

Loglinear Models
Curve Fitting

Bootstrap C.I.'s*

Built-In Models

Group Fitting and Testing

Model Searching

Nonlinear Regression

Randomization Tests*

Ratio of Polynomials

User-Specified Models

Miscellaneous

Area Under Curve

Bootstrapping

Chi-Square Test

Confidence Limits

Cross Tabulation

Data Screening

Fisher's Exact Test

Frequency Distributions

Mantel-Haenszel Test

Correspondence Analysis

Discriminant Analysis

Item Response Analysis

MANOVA

Multi-Way Tables

Multidimensional Scaling

Principal Components
Meta-Analysis* Independent Proportions ${ }^{\star}$ Correlated Proportions* Hazard Ratios* Means*

Binary Diagnostic Tests* One Sample*

Two Samples* Paired Samples Clustered Samples*

Proportions

Tolerance Intervals* Two Independent

Two Correlated ${ }^{*}$

Exact Tests*

Exact Confidence Intervals ${ }^{\star}$

Farrington-Manning ${ }^{\star}$

Fisher Exact Test

Gart-Nam* Method

McNemar Test

Miettinen-Nurminen*

Wilson's Score* Method

Equivalence Tests*

Noninferiority Tests ${ }^{\star}$

Mass Appraisal

Comparables Reports Hybrid (Feedback) Model ${ }^{\star}$ Nonlinear Regression

Sales Ratios 
"Perfection is achieved, not when there is nothing more to add, but when there is nothing left to take away."

- Antoine de Saint Exupery

F is a carefully crafted subset of the most recent version of Fortran, the world's most powerful numeric language.

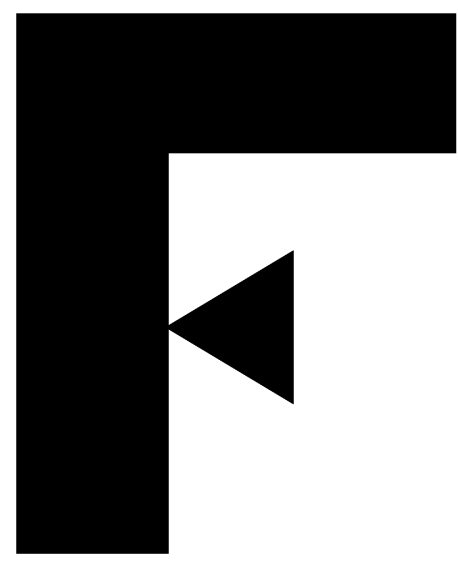

Using F has some very significant advantages:

- Programs written in F will compile with any Fortran compiler

- $\mathrm{F}$ is easier to use than other popular programming languages

- F compilers are free and available for Linux, Windows, and Solaris

- Several books on F are available

- F programs may be linked with C, Fortran 95, or older Fortran 77 programs

F retains the modern features of Fortran-modules and data abstraction, for example-but discards older error-prone facilities of Fortran.

It is a safe and portable programming language.

F encourages Module-Oriented Programming.

It is ideal for teaching a programming language in science, engineering, mathematics, and finance.

It is ideal for new numerically intensive programs.

The Fortran Company

11155 E. Mountain Gate Place, Tucson, AZ 85749 USA $+1-520-256-1455+1-520-760-1397$ (fax) http://www.fortran.com info@fortran.com 


\section{Introducing GGUM2004}

Item Response Theory Models for Unfolding

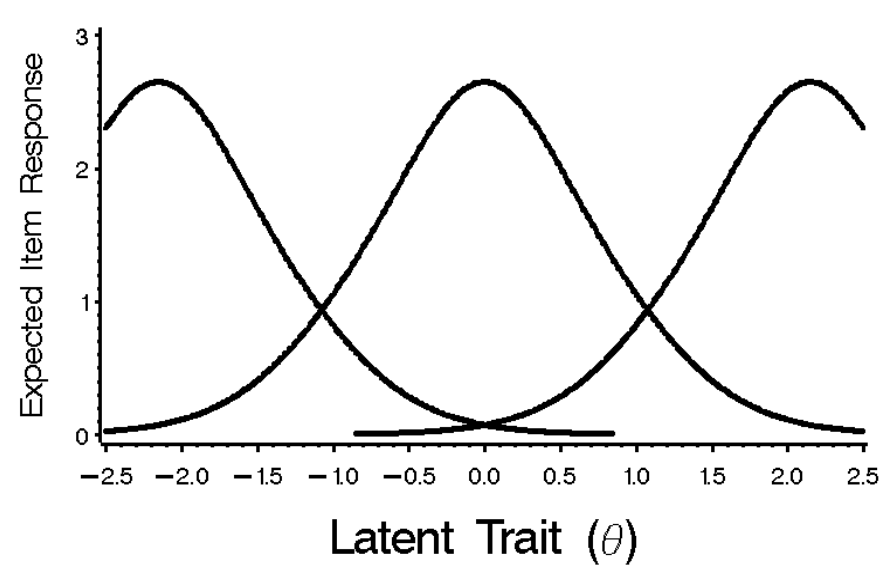

The new GGUM2004 software system estimates parameters in a family of item response theory (IRT) models that unfold polytomous responses to questionnaire items. These models assume that persons and items can be jointly represented as locations on a latent unidimensional continuum. A single-peaked, nonmonotonic response function is the key feature that distinguishes unfolding IRT models from traditional, "cumulative" IRT models. This response function suggests that a higher item score is more likely to the extent that an individual is located close to a given item on the underlying continuum. Such single-peaked functions are appropriate in many situations including attitude measurement with Likert or Thurstone scales, and preference measurement with stimulus rating scales. This family of models can also be used to determine the locations of respondents in particular developmental processes that occur in stages.

The GGUM2004 system estimates item parameters using marginal maximum likelihood, and person parameters are estimated using an expected a posteriori (EAP) technique. The program allows for up to 100 items with 2-10 response categories per item, and up to 2000 respondents. GGUM2004 is compatible with computers running updated versions of Windows 98 SE, Windows 2000, and Windows XP. The software is accompanied by a detailed technical reference manual and a new Windows user's guide. GGUM2004 is free and can be downloaded from:

\section{http://www.education.umd.edu/EDMS/tutorials}

\section{GGUM2004 improves upon its predecessor (GGUM2000) in several important ways:}

- It has a user-friendly graphical interface for running commands and displaying output.

- It offers real-time graphics that characterize the performance of a given model.

- It provides new item fit indices with desirable statistical characteristics.

- It allows for missing item responses assuming the data are missing at random.

- It allows the number of response categories to vary across items.

- It estimates model parameters more quickly.

Start putting the power of unfolding IRT models to work in your attitude and preference measurement endeavors. Download your free copy of GGUM2004 today! 


\section{JOIN DIVISION 5 OF APA!}

The Division of Evaluation, Measurement, and Statistics of the American Psychological Association draws together individuals whose professional activities and/or interests include assessment, evaluation, measurement, and statistics. The disciplinary affiliation of division membership reaches well beyond psychology, includes both members and non-members of APA, and welcomes graduate students.

Benefits of membership include:

- $\quad$ subscription to Psychological Methods or Psychological Assessment (student members, who pay a reduced fee, do not automatically receive a journal, but may do so for an additional $\$ 18$ )

- $\quad$ The Score - the division's quarterly newsletter

- Division's Listservs, which provide an opportunity for substantive discussions as well as the dissemination of important information (e.g., job openings, grant information, workshops)

Cost of membership: $\$ 38$ (APA membership not required); student membership is only $\$ 8$

For further information, please contact the Division's Membership Chair, Yossef Ben-Porath (ybenpora@kent.edu) or check out the Division's website:

http://www.apa.org/divisions/div5/

\section{ARE YOU INTERESTED IN AN ORGANIZATION DEVOTED TO EDUCATIONAL AND BEHAVIORAL STATISTICS?}

Become a member of the Special Interest Group - Educational Statisticians of the American Educational Research Association (SIG-ES of AERA)!

The mission of SIG-ES is to increase the interaction among educational researchers interested in the theory, applications, and teaching of statistics in the social sciences.

Each Spring, as part of the overall AERA annual meeting, there are seven sessions sponsored by SIG-ES devoted to educational statistics and statistics education.

We also publish a twice-yearly electronic newsletter.

Past issues of the SIG-ES newsletter and other information regarding SIG-ES can be found at http://orme.uark.edu/edstatsig.htm

To join SIG-ES you must be a member of AERA. Dues are $\$ 5.00$ per year.

For more information, contact Joan Garfield, President of the SIG-ES, at jbg@umn.edu. 


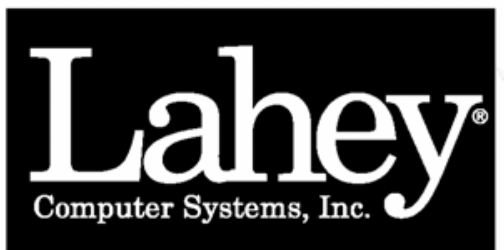

SOFTWARE SOLUTIONS

for Science \& Engineering

\section{Lahey/Fujitsu Fortran}

The standard for Fortran programming from the leader in Fortran language systems

LF95 Fortran for Linux and Windows

Full Fortran 95/90/77 support

Unsurpassed diagnostics

Intel and AMD optimizations
IMSL compatible

Fujitsu SSL2 math library

Wisk graphics package

\section{LF Fortran for the Microsoft ${ }^{\circledR}$.NET Framework - Coming Soon!}

Visual Studio integration

Windows / Web Forms designer

Project and code templates
On-line integrated help

XML Web services

ADO.NET support

Visit www.lahey.com for more information

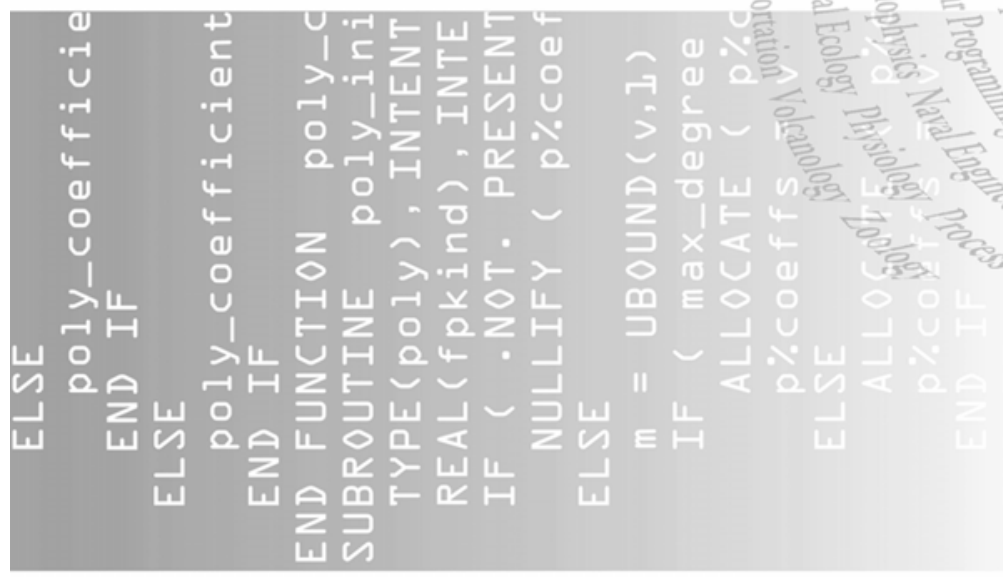

Lahey Computer Systems, Inc.

865 Tahoe Blvd - P.O. Box 6091

Incline Village, NV 89450 USA

1-775-831-2500

www.lahey.com

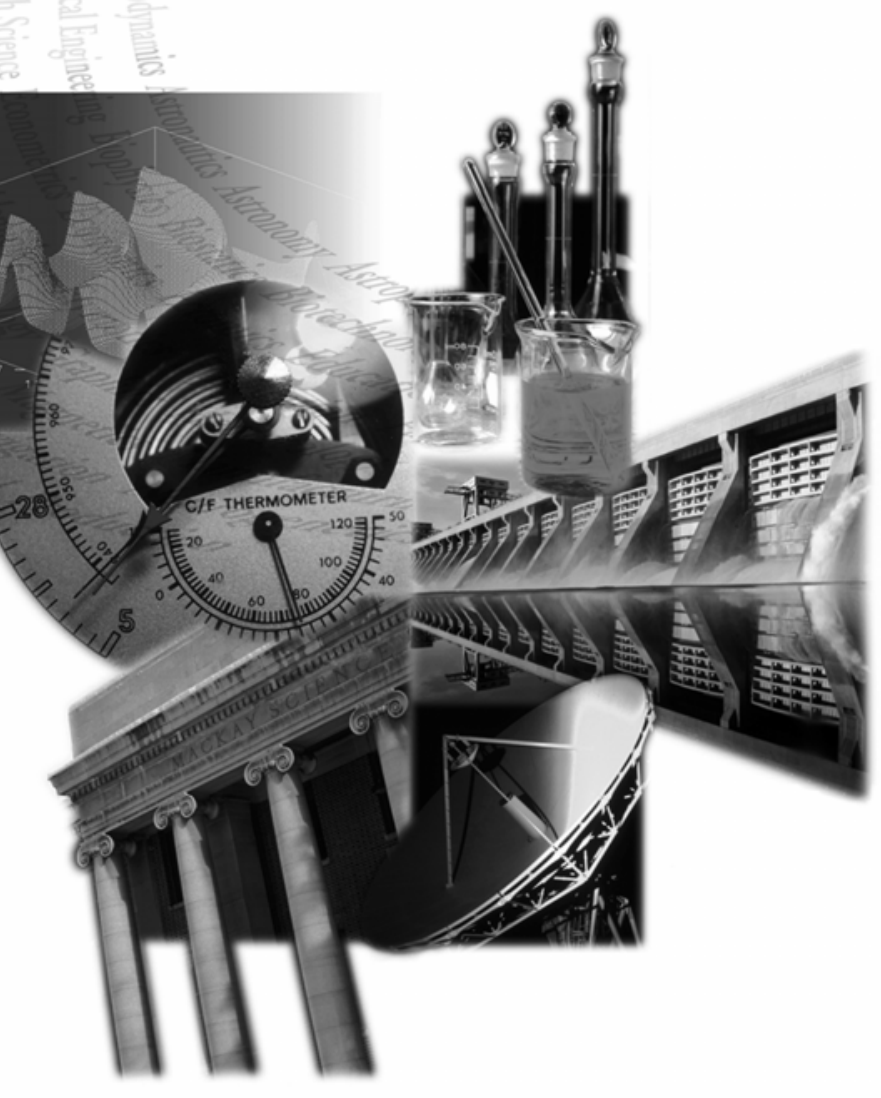




\section{Instructions For Authors}

Follow these guidelines when submitting a manuscript:

1. JMASM uses a modified American Psychological Association style guideline.

2. Submissions are accepted via e-mail only. Send them to the Editorial Assistant at ea@edstat.coe.wayne.edu. Provide name, affiliation, address, e-mail address, and 30 word biographical statements for all authors in the body of the email message.

3. There should be no material identifying authorship except on the title page. A statement should be included in the body of the e-mail that, where applicable, indicating proper human subjects protocols were followed, including informed consent. A statement should be included in the body of the e-mail indicating the manuscript is not under consideration at another journal.

4. Provide the manuscript as an external e-mail attachment in MS Word for the PC format only. (Wordperfect and .rtf formats may be acceptable - please inquire.) Please note that Tex (in its various versions), Exp, and Adobe .pdf formats are designed to produce the final presentation of text. They are not amenable to the editing process, and are NOT acceptable for manuscript submission.

5. The text maximum is 20 pages double spaced, not including tables, figures, graphs, and references. Use 11 point Times Roman font.

6. Create tables without boxes or vertical lines. Place tables, figures, and graphs "in-line", not at the end of the manuscript. Figures may be in .jpg, .tif, .png, and other formats readable by Adobe Illustrator or Photoshop.

7. The manuscript should contain an Abstract with a 50 word maximum, following by a list of key words or phrases. Major headings are Introduction, Methodology, Results, Conclusion, and References. Center headings. Subheadings are left justified; capitalize only the first letter of each word. Sub-subheadings are leftjustified, indent optional.

8. Do not use underlining in the manuscript. Do not use bold, except for (a) matrices, or (b) emphasis within a table, figure, or graph. Do not number sections. Number all formulas, tables, figures, and graphs, but do not use italics, bold, or underline. Do not number references. Do not use footnotes or endnotes.

9. In the References section, do not put quotation marks around titles of articles or books. Capitalize only the first letter of books. Italicize journal or book titles, and volume numbers. Use "\&" instead of "and" in multiple author listings.

10. Suggestions for style: Instead of "I drew a sample of 40" write "A sample of 40 was selected". Use "although" instead of "while", unless the meaning is "at the same time". Use "because" instead of "since", unless the meaning is "after". Instead of "Smith (1990) notes" write "Smith (1990) noted". Do not strike spacebar twice after a period.

\section{Print Subscriptions}

Print subscriptions including postage for professionals are US \$95 per year; for graduate students are US $\$ 47.50$ per year; and for libraries, universities, and corporations are US \$195 per year. Subscribers outside of the US and Canada pay a US $\$ 10$ surcharge for additional postage. Online access is currently free at http://tbf.coe.wayne.edu/jmasm. Mail subscription requests with remittances to JMASM, P. O. Box 48023, Oak Park, MI, 48237. Email journal correspondence, other than manuscript submissions, to jmasm@edstat.coe.wayne.edu.

\section{Notice To Advertisers}

Send requests for advertising information to jmasm@edstat.coe.wayne.edu. 


\section{STATISTICIANS}

\section{HAVE YOU VISITED THE \\ Mathematics Genealogy Project?}

The Mathematics Genealogy Project is an ongoing research project tracing the intellectual history of all the mathematical arts and sciences through an individual's Ph.D. advisor and Ph.D. students. Currently we have over 80,000 records in our database. We welcome and encourage all statisticians to join us in this endeavor.

\section{Please visit our web site}

\section{http://genealogy.math.ndsu.nodak.edu}

The information which we collect is the following:

The full name of the individual, the school where he/she earned a Ph.D., the year of the degree, the title of the dissertation, and, MOST IMPORTANTLY, the full name of the advisor(s). E.g., Fuller, Wayne Arthur; Iowa State University; 1959; A Non-Static Model of the Beef and Pork Economy; Shepherd, Geoffrey Seddon

For additions or corrections for one or two people a link is available on the site. For contributions of large sets of names, e.g., all graduates of a given university, it is better to send the data in a text file or an MS Word file or an MS Excel file, etc. Send such information to:

\section{harry.coonce@ndsu.nodak.edu}

The genealogy project is a not-for-profit endeavor supported by donations from individuals and sales of posters and t-shirts. If you would like to help this cause please send your tax-deductible contribution to: Mathematics Genealogy Project, 300 Minard Hall, P. O. Box 5075, Fargo, North Dakota 58105-5075E 


\section{The easy way to find open access journals}

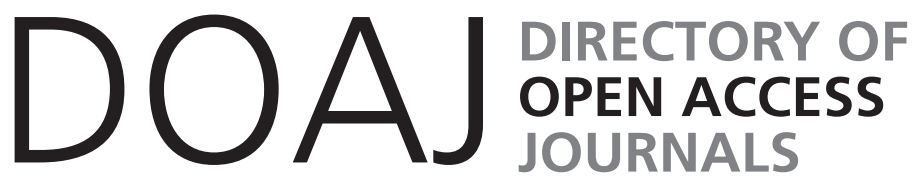

\section{www.doaj.org}

The Directory of Open Access Journals covers free, full text, quality controlled scientific and scholarly journals. It aims to cover all subjects and languages.
Aims
- Increase visibility of open access journals
- Simplify use
- Promote increased usage leading to higher impact

\section{Scope}

The Directory aims to be comprehensive and cover all open access scientific and scholarly journals that use a quality control system to guarantee the content. All subject areas and languages will be covered.

\section{In DOAJ browse by subject}

Agriculture and Food Sciences

Biology and Life Sciences

Chemistry

General Works

History and Archaeology

Law and Political Science

Philosophy and Religion

Social Sciences
Arts and Architecture
Business and Economics
Earth and Environmental Sciences
Health Sciences
Languages and Literatures
Mathematics and statistics
Physics and Astronomy
Technology and Engineering

Contact

Lotte Jørgensen, Project Coordinator

Lund University Libraries, Head Office

E-mail: lotte.jorgensen@lub.lu.se

Tel: +46462223431

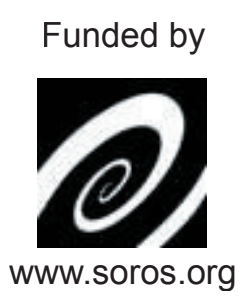

Hosted by

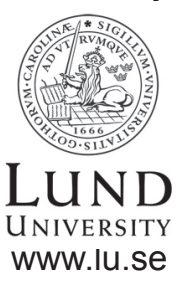

